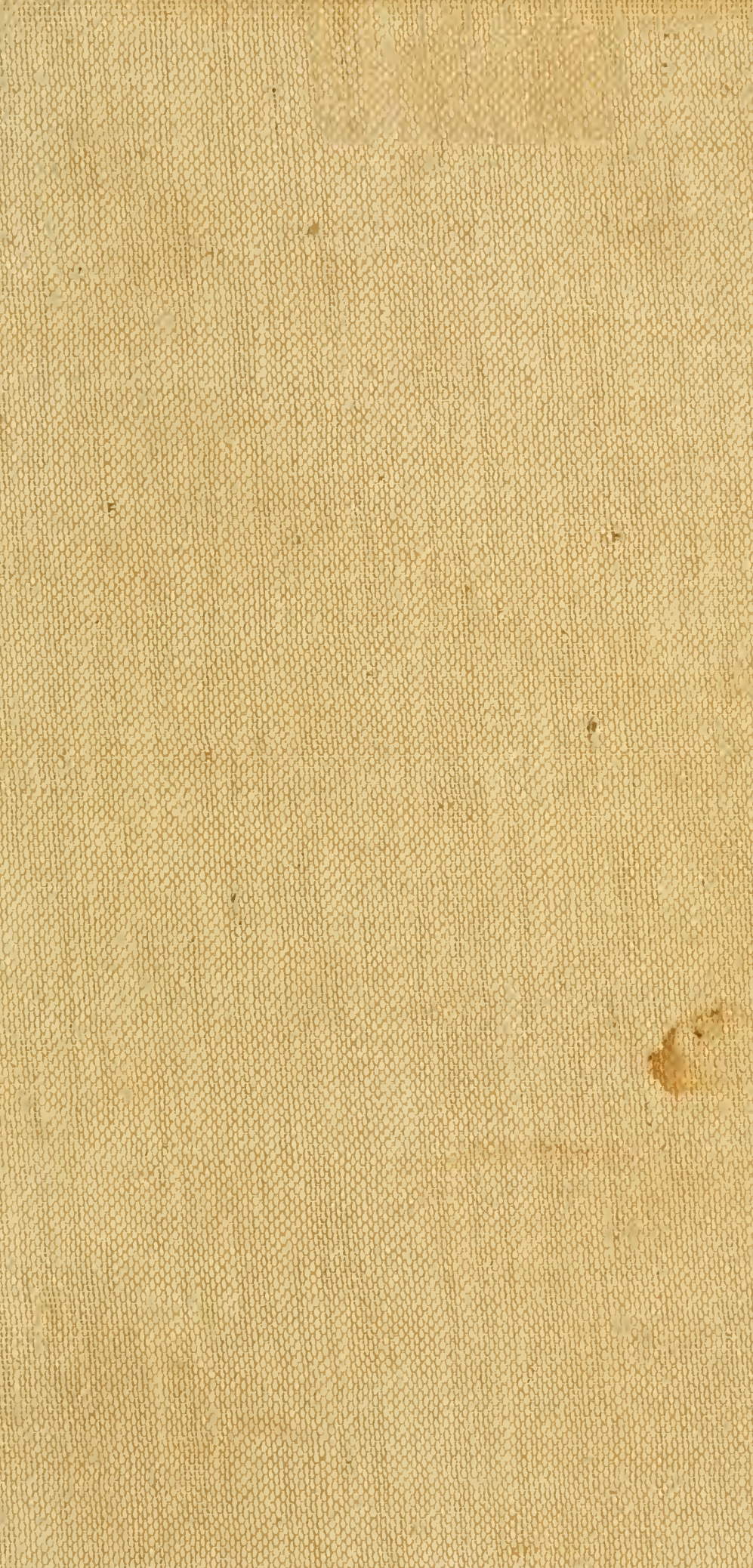




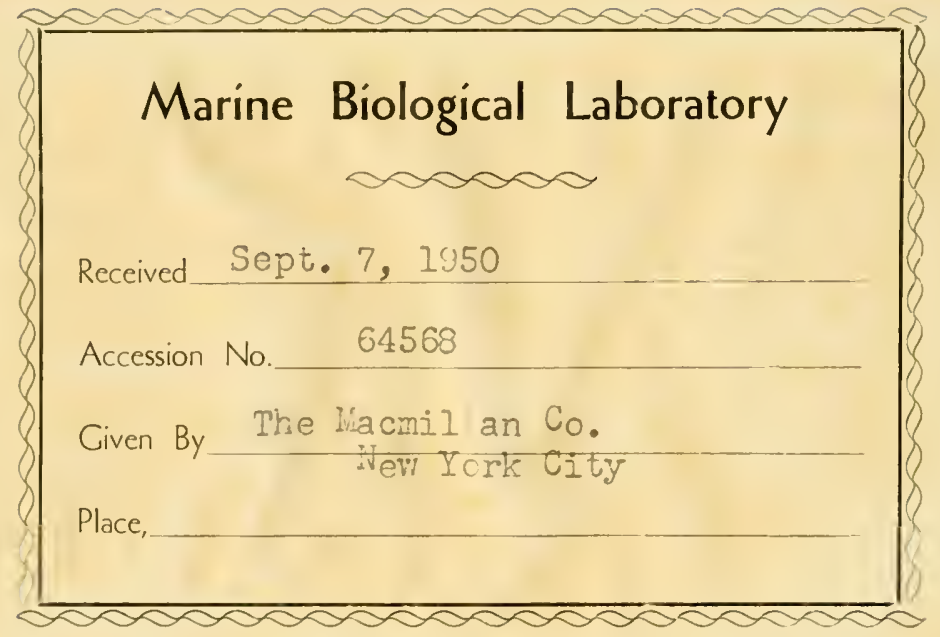




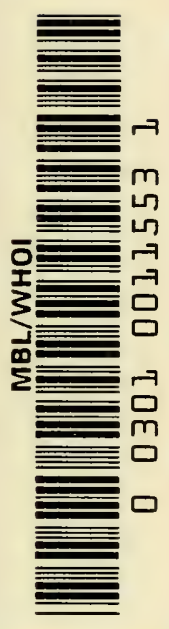




\section{THE ELEMENTS OF GENETICS}

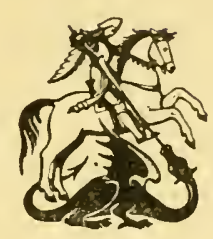


by Dr. C. D. Darlington and Dr. K. Mather ESSAYS ON GENETICS

by Dr. C. D. Darlington

CHROMOSOMES AND PLANT BREEDING

RECENT ADVANCFS IN CYTOLOGY

THE EVOLUTION OF GENETIC SYSTEMS

by Dr. C. D. Darlington and L. F. La Cour THE HANDLING OF CHROMOSOMES

by Dr. C. D. Darlington and E. K. Janaki Ammal CIROMOSOME ATLAS OF CULTIVATED PLANTS

$$
\text { by Dr. K. Mather }
$$

THE MEASUREMENT OF LINKAGE IN HEREDITY STATISTICAL ANALYSIS IN BIOLOGY

BIOMETRICAL GENETICS 


\section{THE ELEMENTS}

\section{$\mathrm{OF}$}

\section{GENETICS}

by

C. D. DARLINGTON

and

K. MATHER

NEW YORK

THE MACMILLAN COMPANY

1950 
FIRST PUBLISIJED IN 1949

SECOND IMPRESSION 1950

This book is copyright under the Berne Convention. No portion of it may be reproduced by any process without written permission.

Inquiries should be addressed to the publisher

PRINTED IN GREAT BRITAIN in 12 point Bembo type BY UNWIN BROTHERS LTD WOKING AND LONDON 
Knowledge of all kinds is good. Conjecture as to things useful is good; but conjecture as to what it would be useless to know, such as whether men went on all four, is very idle.

JOHNSON (Bostvell's Life, I79I)

For to pass the time this book shall be pleasant to read in; but for to give faith and believe that all is true that is contained herein, ye be at liberty. CAxTON (preface to Mallory, I485)

Except ye see signs and wonders, ye will not believe.

JOHN IV. 48 


\section{DEDICATED}

to the compatible and incompatible clements of Linnaeus and Darwin, of Pasteur and Wcismann, of Mendel and Morgan, of Bateson and Pcarson, which, being brought together in fertile combination by the passage of time and the intercourse of nations, have yiclded the abundant harvest of Genctical Science 


\section{PREFACE}

There ARE TWO WAYs of attempting to describe a part of nature in scientific terms. One is to deal with the area which has been exactly mapped by experiment, with the ensuing generalizations and predictions, and to leave the rest cmpty. The other is to go further and use our knowledge of the mapped area to fill in the empty spaces according to the more likely assumptions. The first method is evasive, the second hazardous. We prefer the second and have adopted it. In the present account of Genetics we have tried to use what is known in order to find out what is unknown.

As a consequence there stretches a complete range, in a gentle gradient or cline, from the old theories of Chapter I, which are called Laws of Nature, to the new theories of Chapter I6, which are called Dangerous Speculations. The reader will don his doubting glasses at the point he feels proper. He will do well, however, to recollect that this is the first attempt to represent the whole scope of genetics, the whole of what has always been needed. In the past open hypotheses have been replaced by concealed assumptions and such assumptions are far more dangerous than the statements we have laid before the reader in black and white.

Events have lent a special urgency to the survey of the whole territory of genetics. They have at the same time made it a venture of special opportunity, one which offers unprecedented rewards. Genetics has now reached the point when its central position in the world of science is becoming generally understood. This isthmus is being found to join continents that have hitherto been unknown to one another. Across it now botanists and zoologists may venture to find common ground with bacteriologists and virologists. On its pathways the student of evolution may teach, and learn from, the investigator of cancer and the practical stock-breeder. Within its confines the physical chemist may verify some of his predictions and confound others. Breathing its air, the physiologist and the embryologist may come to agree that plants are organisms not too simple to be used in explaining the more claborate mysteries of animal life. 
It may be, of course, that we have over-simplified our story. It may be that, in secing heredity as the outcome, as well as the material, of adaptive and evolutionary change, and in assuming in it a unity of principle which applies also to development and infection in all plants and animals (even in ourselves), we have travelled too far and too fast. We do not hope to satisfy the critic who prefers the small, the single, and the secluded, department. But we do hope that many, whether wise vetcrans or innocent enthusiasts, who read this book, will share some of the delight we have had in writing it.

C. D. D.

K. M.

John Innes Horticultural Institution, Merton

December 1947

\section{ACKNOWLEDGEMENT}

We are indebted to the various authors and publishers of periodicals and books named in the References to each chapter for the use of their figures in the text.

\section{Note on Frontispiece}

It has been pointed out to us, by Professor R. A. Fisher, that the figures on this page of Mendel's notes suggest factor interaction. Thus the ratio of $343: 92: 166$ in the middle of the page suggests the 9: $3: 4$ ratio characteristic of an $F_{2}$ segregating for two genes related in action by recessive epistasy (see Fig. 38 on page I56). This is all the more likely as the class of $x 66$ is recorded near the top of the page as "weiss" and denoted as W. If this inference of epistasy is correct, the results were perhaps from beans rather than from peas. Mendel mentions work with beans (including the species cross Phaseolus vulgaris $\times$ Ph. multiflorus) in his paper, but gives little detail of the results, which he evidently found difficult to interpret. He makes no mention of any epistatic colour relations in his peas and the notion of epistasy was not introduced until 1907. 


\section{CONTENTS}

Introduction The Aim and Scope of Genetics

PAGE

\section{PARTI INDIVIDUALS}

CHAPTER

I. The Chromosome Mechanism

2. The Mendelian Method

3. Continuous Variation

4. The Biometrical Analysis

5. Bases of Change

6. Consequences of Change

\section{PART II CELLS}

7. Genes, Molecules and Processes

8. The Cytoplasm

9. Development and Differentiation $\quad$ I89

Io. Viruses, Proviruses and the Conflict of Systems

\section{PARTIII POPULATIONS}

II. Adjustment and Balance

I2. Breeding Systems

13. Selection and Variability

14. 'The Breakdown of Continuity

Is. The Growth of Genes

I6. Man and Mankind

Appendix I. Glossary of Genetical Ternıs

Appendix 3. Genetical Books published in the English Language 433 Index 



\section{TEXT FIGURES}

FIGURE

I. Nucleus and cytoplasm in Acetabularia 17

2. Hybrid and merogon sea-urchins 18

3. The chromosome cycle in sexual reproduction 24

4. The chromosomes of Drosoplila and Crocus 26

5. The salivary gland chromosomes of Drosophila 27

6. Meiosis and crossing-over 32

7. Segregation in peas 39

8. Recombination in Chlamydomonas 44

9. Maps of the X chromosome in Drosoplila melanogaster 47

I0. Sex-linked inheritance 48

I I. Autosexing in poultry 50

12. Partial sex-linkage in fishes 5 I

13. The sex chromosomes of Drosophila and man 53

14. The normal frequency distribution of stature in man 57

Is. Relation between genotype and phenotype in polygenic inheritance

16. Polygenic segregation in Epilaclita

I7. Segregation for corolla length in Nicotiana 7 I

18. The components of variation in height of Antirrhinum plants 87

19. The origin of triploids in Ascaris 97

20. The results of chromosome breakage and reunion 101

21. The Bar duplication in Drosophila 108

22. Chimaeras in Solanum I I I

23. A gynandromorph in Drosophila $\quad$ I I 2

24. The modes of origin of gynandromorphs II 3

25. Pleiotropic action of a lethal gene in the rat II

26. The Agouti series of allelomorphs in the mouse $\quad$ I 8

27. Meiosis in polyploids $\quad 122$

28. The pollen grain chromosomes of Crepis capillaris $\quad \mathbf{2} 4$

29. Interchange, and meiosis in the interchange hybrid $\quad$ I28

30. Chromosome pairing in structural hybrids $\quad$ I29

3I. Inversion distinguishing Drosophiln melanogaster and simulans I3 I

32. Pachytene pairing and linkage map of Oenotlera I33

33. Chromosome balance in Raphano-brassica $\quad$ I36

34. Polyploidy in Aesculus $\quad{ }_{13} 8$

35. Segregation and fertility in polyploids $\quad$ I40

36. Genes, chromosomes and nucleo-proteins I48

37. The $\mathrm{A}, \mathrm{B}, \mathrm{O}$ system of blood groups in man I53

38. The interactions of two genes $\quad$ Is6

39. The synthesis of tryptophane in Neurospora I62

40. The four relations of genes in action 165

4I. Plastid inheritance in barley $\quad$ I72

42. The inheritance of Killer in Paramecium $\quad 176$

43. The inheritance of melibiose fermentation in yeast I80

44. Dauermodification in Pliaseolus $\quad$ I 84 
45. The inheritance of direction of coiling in snails

46. Mitosis in the red and white blood precursor cells of nuan

47. The differentiation of nuclear behaviour in Scilla pollcn grains 196

48. Cleavage and chromosome fragmentation in Ascaris 197

49. Co-operation between pollen grains in Uvularia 199

5o. Types of enıbryo-sac in terıns of cell gradients 200

SI. Haploid competition, including the Renner Effect $20 \mathrm{I}$

52. Nucleus and cytoplasin in differentiation 204

53. The three levels of genetic structure $22 \mathrm{I}$

54. Differing balances in the homo- and hetero-gametic sexes of hybrids

5s. Sex balance in Drosoplita

56. Chromosome behaviour and pollen fertility in Festuca-Lolium hybrids

$\begin{array}{ll}\text { 57. Heterosis and inbreeding depression in maize } & 236 \\ 58 . \text { Incompatibility in sweet cherries } & 244\end{array}$

59. The mechanism of incompatibility 245

6o. Heterothally governed by two loci in fungi 246

6I. The efficiency of heterothally as an outbreeding mechanism $\quad 247$

62. Distyly in Primula 250

63. Tristyly in Lythrum $25 \mathrm{I}$

64. The breakdown of incompatibility in Petunia 257

65. Temperature and sex determination $\quad 264$

66. Sexual and non-sexual forms in Artemia 265

67. The lcaves of triploid and hypo-triploid Taraxacum 266

68. Sexuality and apomixis in Taraxacum species $\quad 267$

69. Types of twinning in plants and animals 270

70. The release of potential variability 280

7I. The states of variability 28I

72. Litter size and fertility in pigs $\quad 284$

73. Selection in Drosoplila 286

74. Seasonal variation of gene frequency in Adalia 295

75. The mechanism of correlated response to selection 299

76. Geographical variation in Microtus 304

$\begin{array}{ll}\text { 77. Isolating mechanisms } & 307\end{array}$

78. Inversion phylogeny in Drosophila 313

79. The isolating effect of inversion 3 IS

80. Three types of interchange evolution $\quad 317$

8I. Genetic changes in species formation 3 I9

82. Cliromosome phylogeny in woody plants 325

83. The Rhesus blood groups in man 333

84. Sex-ratio inversions in Drosophila azteca 337

85. The fatuoid series in oats 339

86. Hetcrocaryosis in fungi 343

87. Mating restrictions in plants, animals and man 357

88, $\mathrm{A}$ and $\mathrm{B}$. The distributions of the $\mathrm{O}$ blood group gene and the

TH sound in Europe $362-3$ 


\section{TABLES}

TABLE

I. Mendel's seven characters in peas 37

2. The composition of $F_{2}$ 's in peas 38

3. The nine genotypic classes in an $F_{2}$ segregating for two factors $4 \mathbf{I}$

4. Weights of mother and daughter beans in Johannsen's expcriments

5. Weights of coloured and white beans in an $F_{2}$

6. Effects of the three large chromosomes on hair production in Drosophila

7. The inheritance of height in Antirrhinum majus $\times$ glutinosum $\quad 86$

8. Balanced and unbalanced complements of chromosomes 98

9. The frequencies of chromosome mutants in tomatoes and newts 99

IO. Primary and sccondary chromosome changes $\mathrm{IO}_{3}$

II. Four allelomorphs governing size of eye in flowers of Prinula sinensis

12. Chromosomes in the pollen of triploid Crepis 123

13. The extra chromosomes in triploid Tulipa and Datura I25

I4. The fertility of male and female gametes from triploids $\quad$ I26

I5. Chiasmata in Primula kewensis $\quad{ }_{37}$

I6. Chromosome numbers in the pollen of Prinula kewensis $\quad$ I39

17. The decay of dauermodification in Phaseolus $\quad 185$

I8. Inheritance of variegation in Scolopendrimm $\quad$ I86

19. The origin and transmission of viruses 219

20. Chromosome behaviour and pollen fertility in Lolimn-Festuca hybrids

2I. Seed setting in the primrose 249

22. Pollen competition in Streptocarpus 254

23. The breakdown of incompatibility in Primula sinensis 255

24. Chromosome numbers in Poa pratensis 269

25. Chiasma formation and chromosome pairing in asynaptic maize 289

26. The geographical distribution of interchanges in Datura 3 I6

27. Species in Paeonia 322

28. Frequencies of first cousin marriages in man 347

29. Percentages of concordance in human one-egg and two-egg twins

30. Tumours in one-egg and two-egg twins 350

31. Frequencies of affection in relatives of tuberculous individuals $35 \mathrm{I}$ 



$$
\begin{aligned}
& V_{1}=3 \%
\end{aligned}
$$

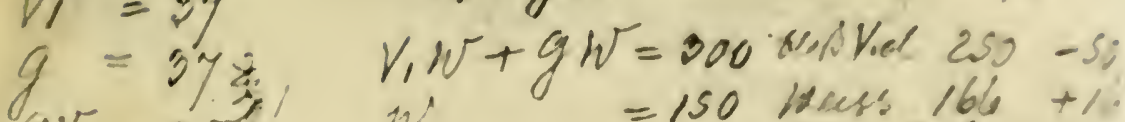

$$
\begin{aligned}
& 1 V_{1}=15 \% \text { H } \quad=150 \text { ines: } 166+1
\end{aligned}
$$

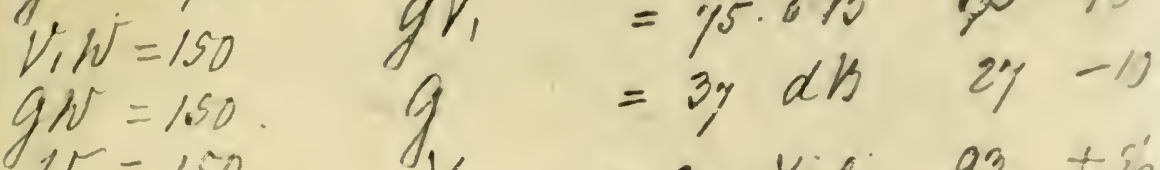

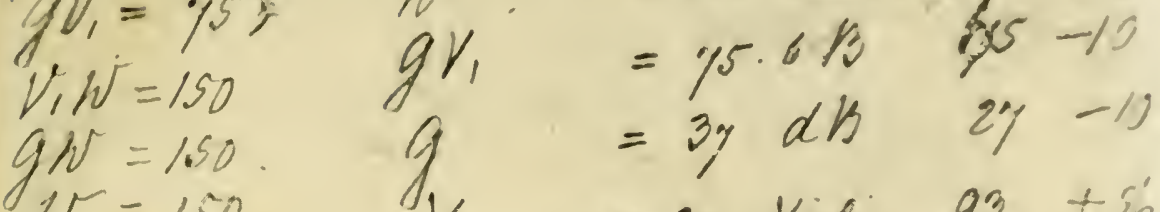

$$
\begin{aligned}
& y=1.50 \\
& =3 y \text { Vil } 93+50
\end{aligned}
$$

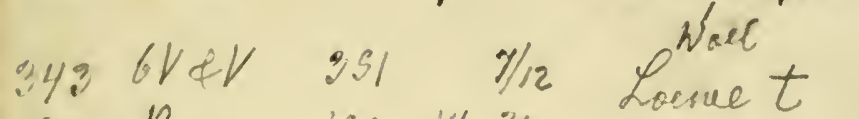

$92 \mathrm{~B}, 100 \mathrm{16} \% / 2$

$166.25150 \frac{1}{4} \frac{3}{12}$ cess

aldine $l$

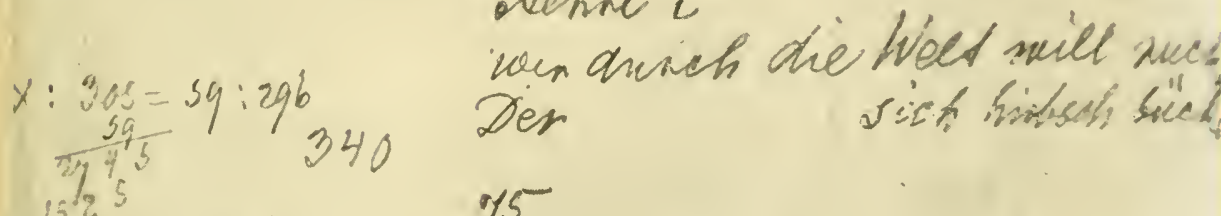

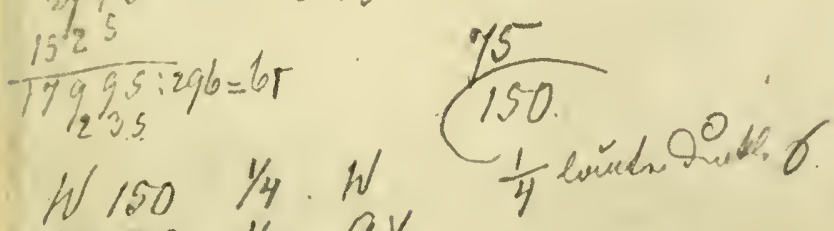

br y $75 \quad 1 / 8 \quad g r_{1}$

$\begin{array}{llll}\text { ab } 3 y & 1 / 16 & g \\ 6 v & 300 & 1 / 2 & g w+V_{1} w\end{array}$

i. $31 / 6.1 \%$

A PAGE FROM THE NOTEBOOK OF GREGOR MENDEL RECORDING THE RESULTS OF HIS CROSSES

(see note on pe)

Reproduced by kind permission of the Curator of the Museum Mendelianum, Brunt 



\section{THE AIM AND SCOPE OF GENETICS}

Genotype and Environment Nucleus and Cyroplasm

Individuals, Cells and Populations

WHEN WE SOW AN ACORN we expect to raise an oak; when we breed dogs we expect to get puppies; and even when we culture a typhoid bacillus we expect to produce more bacteria of a kind that will cause typhoid. In a word, we expect that like will beget like. But the resemblance we look for is not absolute or unconditional. One oak is not exactly like another; nor is one dog like another; nor even, invariably, one typhoid bacillus. The causes of these unlikenesses are sometimes obviously external. Lack of iron, or lack of light, or roo much light, may make the green leaves of a plant turn yellow. Disease, starvation, or training may alter the shape, habits, or abilities of an animal. The causes of other unlikenesses, as of the likenesses, ure internal, although less obviously so. These inborn causes have o be discovered. By comparison and experiment they have to be eparated from the external causes. They have to be defined as naterials or processes whose behaviour and effects we can predict nd control. This is the aim and scope of genetics.

Our first task is thus to separate external from internal causes. eaves may be yellow on account of the conditions under which the lant is grown, that is on account of the environment. But we also now plants that have yellow leaves under any conditions. These uust be yellow on account of their internal or inborn properties. What makes these inborn properties? Popularly we say heredity. 'echnically, we must be stricter and say genotype (the type which reates). The genotype, according to Johannsen, is what gives the bserved development, the appearance, of a plant or animal in a articular environment. This appearance we call the phenotype (the rpe which is seen). Differences between phenotypes (such as our ellow and green leaved plants) may be due to differences between znotypes, to differences between environments, or even to difrences in both acting together. For example, there are strains of 
oats, Avena sativa, which bleach and dic in strong sunlight, but, in a weaker light, seem normal, i.c. have a normal phenotype.

The phenotype is the product of reaction of the genotype and the enviromment. Either can change it, and it is not to be traced to cither scparately. Thus, too, the study of differences in both heredity and environment, by permitting us to separate their effects, must be the foundation of our knowledge of genetics.

The differences between individuals are not the only ones with which heredity is concerned. All organisms undergo development which consists of growth together with the origin of differences between the parts, differentiation as we call it. Heredity depends on a repetition of this development. Similar genotypes give or determine similar sequences of development. Hercdity at once determines that individuals are alike and that their parts are unlike. This is the paradox that baffled our forebears. We can now resolve it by examining the materials and processes concerned.

When we cut the tail off a worm or the top off a dandelion, what is left grows again to replace the lost part: it regencrates. In spite of their differentiation, the parts of the animal or plant have somcthing inborn in them which is still the same. Similarly, fragments, cuttings and grafts, of particular animals and plants, Hydra, the pondweed Elodea, or a variety of apple or pineapple, can be propagated throughout the world with great and predictable uniformity. These vegctative individuals, or clones, demonstratc heredity in its simplest form. They have, as a rule, and so far as we can make out, the same genotype. And since the whole of each clone is derived from a fragment of one individual, we see that the differentiated parts of that individual must have had the same genotype.

With sexual reproduction the matter is different. Here a new individual arises from the fusion of two germ cells, usually from the fertilization of an egg by a sperm. No two individuals formed in this way are absolutcly alikc. In fact, if we find a pair of twin children (or cight armadillos) who are alike, we assume that they have been derived from single fertilized eggs by mere fragmentation and growth. And we call them identical twins (or octuplets).

What is it that remains constant in vegetative individuals, yet is liable to change in sexual reproduction? To know this, we must look at the cclls of which plant and animal bodics are composed. 
and nucleus, show that the nucleus must be deciding what kind of hat is grown. The nucleus seems to bear the genotype (Fig. I).

Sexual reproduction is just such a transplantation as we see in the alga. A sperm nucleus or generative nucleus of a pollen grain enters an egg cell hundreds or thousands of times larger, and fuses with the female nucleus. Thus the nucleus of the fertilized egg or zygote is of mixed origin; but its cytoplasm is almost exclusively from the mother. The new organism develops from this zygote and shows,

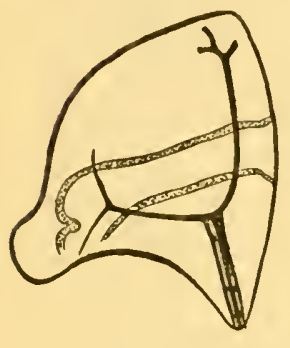

S

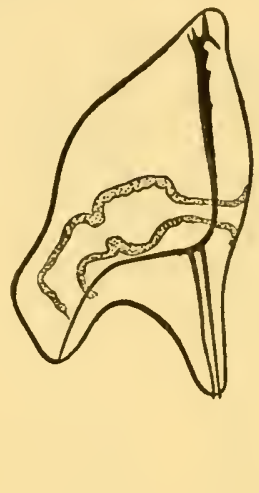

$\mathrm{S} q \times \mathrm{E} \delta$

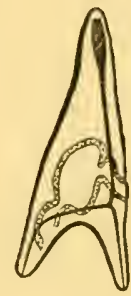

$[S] \times E \sigma^{\prime}$

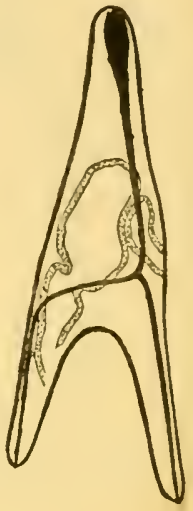

E

Fig. 2.-Larvae of sea urchins: S, Sphaerechinus granulatus; E, Echinus microtuberculatus; $\mathrm{S} q \times \mathrm{E} \delta$, the normal cross; $[\mathrm{S}] \times \mathrm{E} \delta$, the haploid larva produced by the same cross when the nucleus of the egg has been removed. The larva is then scarcely anything other than a dwarf edition of the sperm parent which is the source of its nuclei (after Boveri I889).

where they differ sufficiently, the characters of father and mother in equal measure. Going a step further, Boveri was able to fertilize a piece of egg, lacking a nucleus, of one species of sea urchin with the sperm of another. The resulting larva (termed a merogon) closely resembled a dwarf of its male parent from which it had derived its nuclei and showed no obvious influence of its female parent from which it had derived its cytoplasm (Fig. 2).

We thus see the overriding action of the nucleus. The study of its structure and movements may therefore be expected to tell us why individuals are so constant within themselves in their inborn character and also why they differ from one another. 
Equipped with this knowledge of individuals we can return to consider their parts, to find out what happens within each individual. We can try to discover what the rest of the cell, the cytoplasm, has to do with the nucleus in determining heredity, and how it reacts with the nucleus to produce the differentiation of cells and tissues during development.

And finally we can see the individual as part of something larger. We can recognise its heredity as part of a system of individuals, a population breeding together, descended from a long line of ancestors similarly related, and likely also to give rise to a long line of descendants. From the most minute and transient events we shall come to envisage the vast and enduring laws of change which we associate with the name of evolution.

It is thus in three parts, in relation to individuals, to cells and to populations, that we shall have to unfold the working of genetics.

\section{REFERENCES}

ÅKERMAN, A. I922. Untersuchungen über eine in direkten Sonnenlichte nicht lebensfähige Sippe von Avena sativa. Hereditas, 3: 147-177.

BOVERI, T. I889. Ein geschlechtlich erzeugter Organismus ohne mütterliche Eigenschaften. Sitz. Ber. Ges. Morph. Phys. München, 5. (Translation by T. H. Morgan, 1893. Am. Nat. 27: 222-232.)

HaLdane, J. B. S. 1946. The interaction of nature and nurture. Ann. Eugenics, I3: 197-205.

hämmerLing, J. 1943. Ein- und zweikernige Transplante zwischen Acetabularia mediterranea und A. crenulata. Z.I.A.V., 8I: I I4-180.

Johannsen, w. I9II. The genotype concept of heredity. Am. Nat., 45: I29-159. 



$$
\text { PARTI }
$$

INDIVIDUALS 



\title{
THE CHROMOSOME MECHANISM
}

\author{
Mitosis The Polytene Nucleus Meiosis \\ Reduction and Reconbination
}

\section{Mitosis}

THE GROWTH OF THE ORGANISM depends on the growth and multiplication (or, as we call it paradoxically, division) of its cells and nuclei, which must evidently divide together if each cell is to have its one nucleus. The process by which they divide is known as mitosis, and it is during this process that we are able to study the structure of the nucleus.

The first sign of mitosis is shown by a change in the nucleus. In the undividing cell the nucleus is said to be at rest. It is globular and shows no structure apart from one or two dense storage bodies, the nucleoli. When mitosis begins, dense and stainable threads appear in a more fluid medium. These threads are double. They shorten and thicken by forming a spiral, to give curved or bent double rods, the chromosomes, which are released into the cell when the membrane or boundary of the nucleus disappears.

The release of the chromosomes marks the end of prophase. At the same time, the beginning of metaphase is shown by the appearance of the spindle. The spindle is a liquid-crystal structure whose fibres lie parallel to its axis. The chromosomes, now at their shortest (25 $\mu$ down to I $\mu$ or less), come to lie in the wider middle of the spindle and form a flat plate. We can then see five of their important properties (Fig. 3).

The first is that the number of chromosomes in the mitoses of one individual, and often indeed of one whole species, is constant, whether it be two thousand or only two. The second is that the chromosomes are also constant in shape and relative size. The third property is revealed by the second and is true of all cells in the higher plants and animals except the germ cells: there are two chromosomes of each kind or, if you like, two similar sets. The 


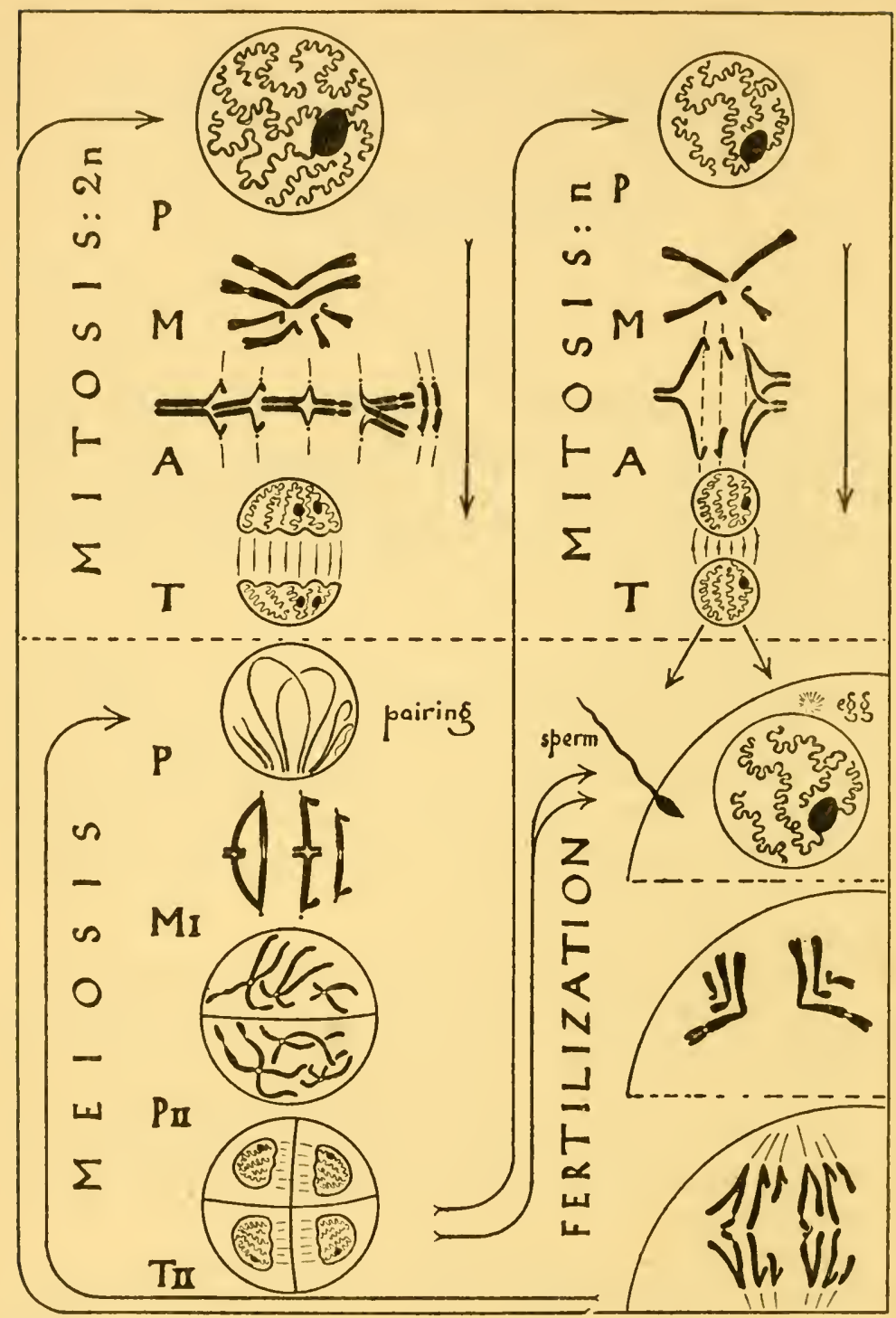

Fig. 3.-The chromosome life cycle in sexually reproducing organisms showing the alternation of haploid and diploid phases of different duration according to the relative position of meiosis and fertilization. Of the three pairs of chromosomes the longest bears the nucleolar organizer. P, M, A, T: pro-, meta-, ana-, and tclo-phase. 
fourth is that each chromosome owes its characteristic shape to the possession of a constant point of attachment to the spindle, marked by a constriction where the chromosome bends. The main body of the chromosome may lie off the spindle and off the plate: only the point of attachment is fixed on the spindle. The fifth is that, except at these attachment constrictions, the chromosomes are double throughout. They consist of pairs of sister chromatids (Fig. 4).

The importance of these structures becomes apparent at the next stage. All the chromosomes simultaneously begin to split at those very points of attachment where they have previously remained single. It can then be seen, after proper treatment, that the point of attachment consists of a minute particle, the centromere. It is the centromere which organizes the developing spindle. It is also the centromere which suddenly divides, or explodes, so that its two halves are driven apart along the fibres of the spindle, that is towards the poles. Each half drags behind it one of the two chromatids of its chromosome. Hence the whole body of the chromosomes lying on the plate is divided into two groups of daughter chromosomes, identical in number, shape and size. The separation of these daughter chromosomes is known as anaphase.

Two daughter nuclei are reformed from the two groups. The chromatids now loosen their packed spirals, and their fine threads are lost once more in the optically homogeneous nucleus. One or more nucleoli reappear in each nucleus. A partition or wall develops across the equator of the spindle and separates the daughter cells. This is telophase. Mitosis is complete.

This course of action shows us that it is through the chromosomes that the nucleus transmits its character to its daughters, that is from cell to cell. It shows us, too, how a complement of chromosomes inside a nucleus is divided into two daughter complements which are distributed to the two daughter nuclei. The significant fact for genetics is that these two complements are always exactly like one another. It was first noted so long ago as 1879 by Flemming, one of the discoverers of mitosis. This observation, however, leaves us with yet another question. How does each chromosome, single as it is when it disappears in telophase, come to be double when it reappears, consisting of two apparently identical halves, in the following prophase? The obvious explanation, the one long ago suggested by 
Boveri, was that the chromosomes remained in being in the resting nucleus. They remained as invisible threads, each of which split lengthwise, or reproduced itself, to give two identical chromatids which reappeared in the following mitosis.

There are many ways of showing that the theory of the reproduction of chromosomes, which at first seemed unnecessary and
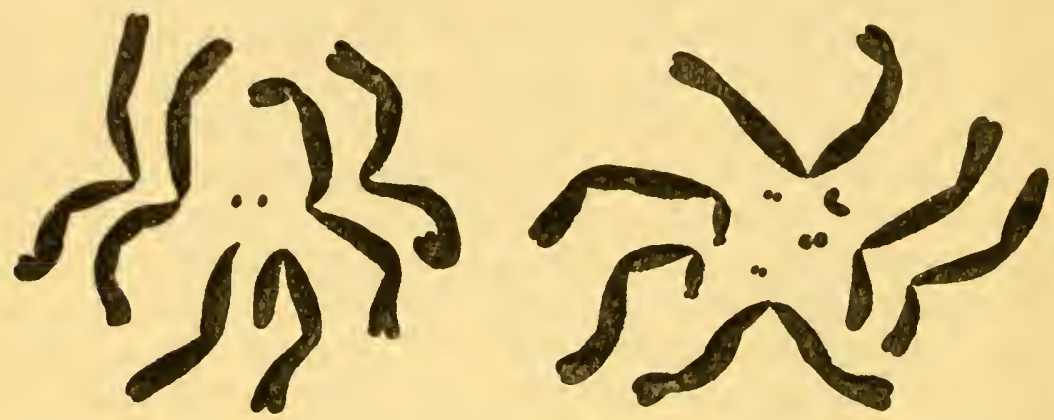

FIG. 4.-Diploid chromosomes at metaphase of mitosis in polar view to show the arrangement in a flat plate. Left, Drosophila melanogaster malc with XY, two large, and one very small autosome pair. Right, Crocus hyemalis with five pairs. All chromosomes have centric constrictions and in addition one Crocus pair has a nucleolar constriction. $\times 3,000$.

fantastic, is both necessary and true. The simplest way is by comparing the uncoiling threads of telophase with those which become visible at prophase. Then we see the uncoiling still going on. There are coils which straighten out and disappear only just before metaphase, and these are obviously relic coils, the outward and visible signs of the inward and invisible coils of the preceding mitosis. As we proceed step by step new evidence of this fundamental principle of continuity will come to light.

\section{The Polytene Nucleus}

There is another kind of indication of what the chromosomes are doing, or can do, in the resting nucleus in certain large gland cells. Such nuclei, for example in the salivary glands of flies, continue growing without ever dividing. Their chromosomes, however, remain stainable and divide again and again inside the nuclei. They reproduce while continuing their ordinary work. Instead of the double chromatids of prophase nucleus, there are then bundles of 
four, eight, sixteen, and higher doublings of threads. Each of these threads, when stained, has a characteristic beaded structure, and the identical beads of the multiplied sister threads form plates. Each polytene, or multiple chromosome, then looks like a banded ribbon

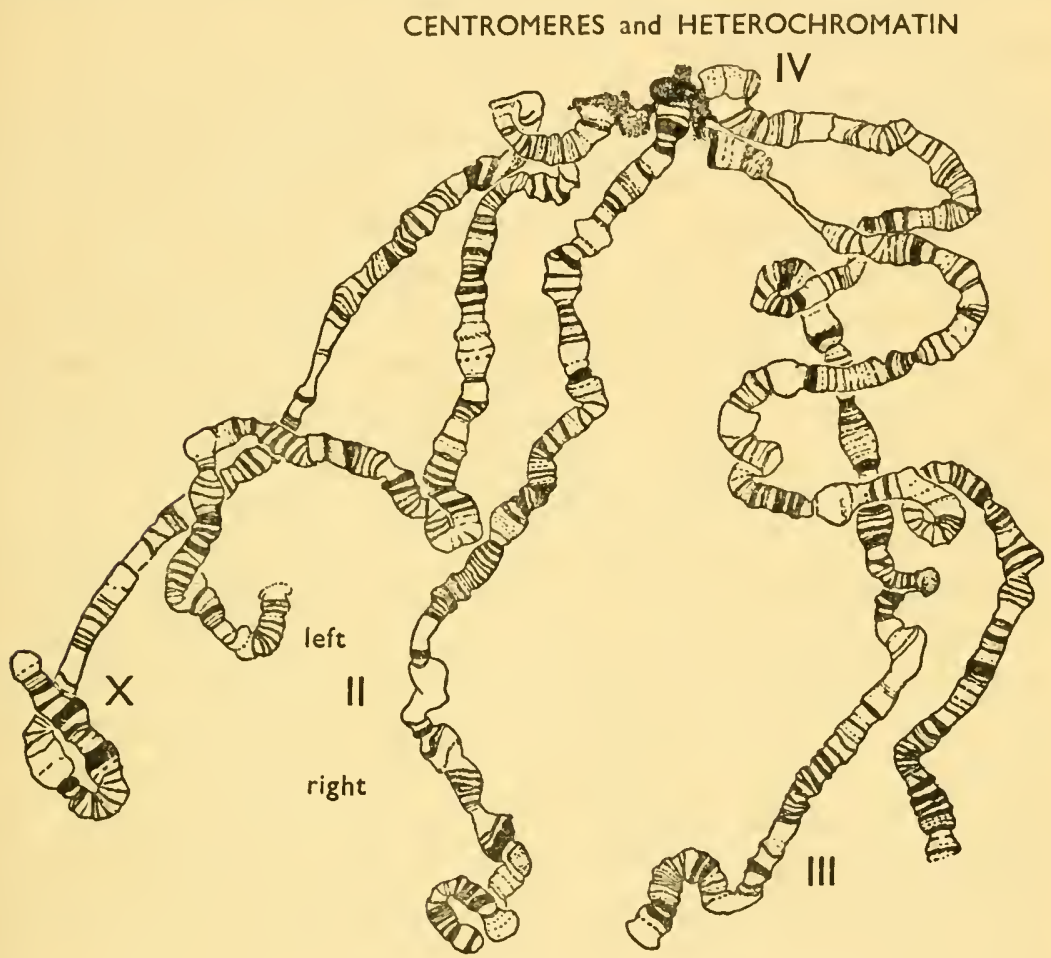

FIG. 5.-The paired and banded polytene chromosomes of a pressed salivary gland nucleus in Drosophila melanogaster. All the centromeres and heterochromatin are agglutinated in a single body at the top. The two arms of each of the long autosomes are marked. $\times$ ea. 600 (after Painter 1934).

as seen from the side. Now these bands, of which there are hundreds in each chromosome, are different from one another, and constant in size, structure and number, just as are the chromosomes in a metaphase plate. They are the chromomeres (Fig. 5).

The linear order of the chromomeres is recognizably constant from nucleus to nucleus and displays the individual permanence of the chromosomes. It shows us how they must maintain their structure as a line of distinct particles within the resting nucleus. It also 
shows us why the chromosomes must reproduce along a longitudinal axis. Only by linear order and longitudinal doubling and splitting can a chromosome, an aggregate of chromomeres, produce daughter chromosomes, daughter aggregates, identical with each other and with itself. The nucleus, therefore, evidently has the means of identical self-propagation.

What do we now know of the nucleus? We saw that it is, in some sense, paramount in the cell; that is, in development and heredity. We now see that it is an aggregate of particles, dissimilar particles, and that this aggregate has the capacity of reproducing itself as an aggregate of the same particles in constant proportions and constant order. It undergoes great transformations in each cycle of nuclear division, but they have a chemical regularity. Underlying this regularity must be a constant molecular structure. It has a complex character but it has the capacity for propagating this character unaltered; in other words it has the capacity of heredity.

The chromosome mechanism shown by mitosis makes it possible to understand the constancy of vegetative growth and propagation. It provides for that uniform genetic character of cells in a tissue and in an organism on which, as we shall see later, their co-operation in development is based. It shows why fragmentation of an organism, from buds, cuttings and vegetative spores, gives daughter individuals which are identical in heredity. It does not, however, explain the differences between individuals, or the variation amongst the progeny of the same individual or pair in sexual reproduction. To do this we must examine the history of the nucleus during the sexual processes.

\section{Meiosis}

We have seen that fertilization involves, or indeed consists in, the fusion of two nuclei. The nucleus of the zygote produced by fertilization thus contains the sum of the chromosomes of egg and sperm. In the nematode Ascaris megalocephala chromosomes from the two germ cells, one from each, combine to give two in the embryo. In the flowering plants, Crepis capillaris or Crocus graveolens, three chromosomes from the pollen nucleus combine with three corresponding ones from the egg nucleus in the embryo sac to give six in the embryo, three obvious pairs. In the formation of these 
germ cells by the parent zygotes there must therefore have been a process of reduction, a halving of the chromosome number to compensate for the addition in fertilization. Such a process, predicted by Weismann in 1887 , is now known or assumed to occur in the cell history wherever there is sexual reproduction. It is called meiosis.

In most algae, fungi and protozoa the diploid or double nucleus produced by fertilization undergoes meiosis at once to give plants or animals with haploid nuclei containing the half number of chromosomes. Elsewhere meiosis is postponed so that a diploid stage is intercalated in the life history. In the mosses and liverworts the small diploid organism or sporophyte is, as it were, parasitic on the larger haploid. In the ferns the diploid has become more important than the haploid. In the flowering plants the haploid phases, the embryosac and pollen grain, are reduced to a parasitic state. Finally, the complete reversal, in which the haploid phase is eliminated as a separate organism and exists only as the germ cells themselves, eggs and sperm, is attained in all the higher animals.

Through all these variations in relation to the life cycle the course and character of meiosis remain the same. It may be concerned with the formation of a fungus spore or a lily pollen grain, the maturation of a spider's egg or a human sperm; but it consists always of two divisions of the nucleus, with only one division of the chromosomes. From its very outset it differs from mitosis. The chromosomes are single when they emerge from the resting stage in the mother cell. Moreover, just as in polytene nuclei, where activity and reproduction are going on at once, the chromosomes appear in the form of strings of chromomeres. As soon as they appear, these strings begin to pair. They come together side by side, chromomere by chromomere. The chromosomes make contact and begin to pair at their ends, or at their centromeres, or at both at the same time. From the contact point the pairing runs along them zip-fashion. Sometimes it runs the whole length. Sometimes it is interrupted so that only the part near the first point of contact is paired: pairing is then localized. After pairing, the chromosomes coil round one another, and so they remain for an indefinite period. This is the thick-thread or pachytene stage.

We then see that the chromosomes of each pair exactly correspond. Evidently one is from the sperm, the other from the egg, which 
fused to give the zygote we are examining. Thus, instead of the diploid, $2 n$, number of chromosomes of mitosis, there is a reduced, haploid, n, number of chromosomes. And instead of the paired sister chromatids of mitosis produced by reproduction, there are paired homologous chromosomes brought together by attraction.

Suddenly attraction gives way to repulsion. All the pairs fall apart. At the same time they are seen to be double. Each chromosome has divided into a pair of clrromatids. The chromosomes seem to remain in contact at certain points and closer examination shows that their chromatids exchange partners at these points. Apparently, under the strain of coiling and at the moment of their origin by division, the chromatids have broken, pairs of partner chromatids breaking at corresponding points. They have uncoiled and rejoined in new combinations. This mechanical transformation is called crossing-over, the observed change of partner is called a chiasma, and the stage is called diplotene.

Some of the coiling of the chromosomes is undone in the formation of chiasmata and some is lost afterwards by still more uncoiling. The chiasmata can then be seen and recorded in number and position. They are as a rule evenly distributed over those lengths of the chromosomes which were paired at pachytene. When pairing has been complete, their distribution is uniform throughout. When it has been localized, the chiasmata are of course similarly localized, near the ends, or near the centromeres, or near both. The kind of distribution of chiasmata is characteristic of each species or even genus of plant or animal, e.g. complete and uniform in Lilium or Zea Mays and pro-terminal in Triton, Tradescantia or Oenothera. In Allium and Fritillaria there are some species with uniform, and others with pro-centric, distribution.

As uncoiling proceeds, the chromosomes thicken and shorten even more than in mitosis. As this happens, adjoining loops between chiasmata come to lic at right angles. The distal chiasmata (nearest the ends) are pushed towards the ends of the chromosomes, which then appear merely to touch. The compact paired, or bivalent, chromosomes, as we may now call them, lie evenly in the nucleus. Small chromosomes, where the centromeres are very close to the nearest chiasma, are drawn into fine threads by the repulsion between their centromeres. This stage is diakinesis. It is followed by the dis- 
appearance of the nucleolus and the nuclear membrane, the appearance of the spindle, and the movement of the bivalents on to the metaphase plate, in other words, by metaphase.

But this, the first metaphase of meiosis, is different from metaphase of an ordinary mitosis in two related and all-important respects. First, individual chromosomes are not now sufficient to themselves. Their centromeres do not orientate themselves separately or individually on the spindle. They orientate themselves only in relation to their partner centromeres. The stretching between partner centromeres, already noticeable at diakinesis, is now exaggerated. Secondly, and consequently, anaphase does not begin by the division of the centromeres. Just as the chromosomes remained undivided at the beginning of prophase, so, in turn, the centromeres remain undivided at the beginning of anaphase. It is not their division which sets the chromosomes moving to the poles. It is merely the lapse of attraction between the paired chromatids where they are hindering the movement apart of the paired chromosomes, i.e. on the far sides of the chiasmata from the centromeres. Such a lapse of attraction occurs at mitosis, but it is hardly noticeable because at mitosis there is nothing to pull the chromatids apart until the centromeres divide. Now the paired centromeres are already repelling one another in readiness for the lapse of attraction. When it occurs, the daughter bivalents, each double except for its centromere, run to opposite poles. The first division is complete.

The situation as the daughter nuclei are formed is a remarkable one. Each nucleus has the haploid number of chromosomes, but the diploid number of chromatids. For the chromosomes are already divided. They are double as they are in the prophase, not in the telophase, of an ordinary mitosis.

Nor are the two chromatids of each chromosome related to one another like those in prophase of mitosis. They are true sister chromatids between the centromere and the nearest chiasma. Beyond that chiasma, until the next one, they are chromatids from partner chromosomes. Looking at it from another angle, we may say that the separation of the first division has been between partners next to the centromere: it has been reductional. Beyond the chiasma it has been between sister chromatids: it has been equational.

An unexampled situation calls for an unexampled remedy. The 

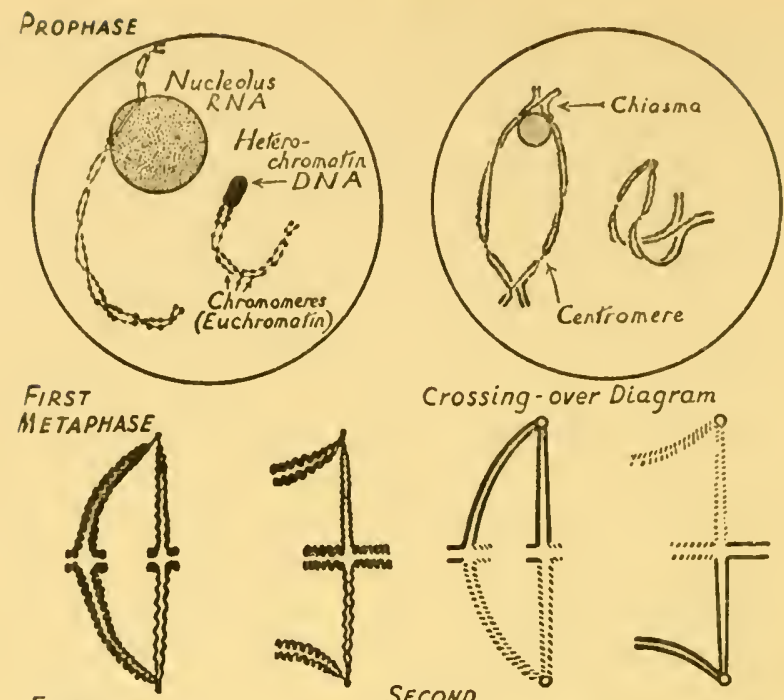

Crossing-over Diagram
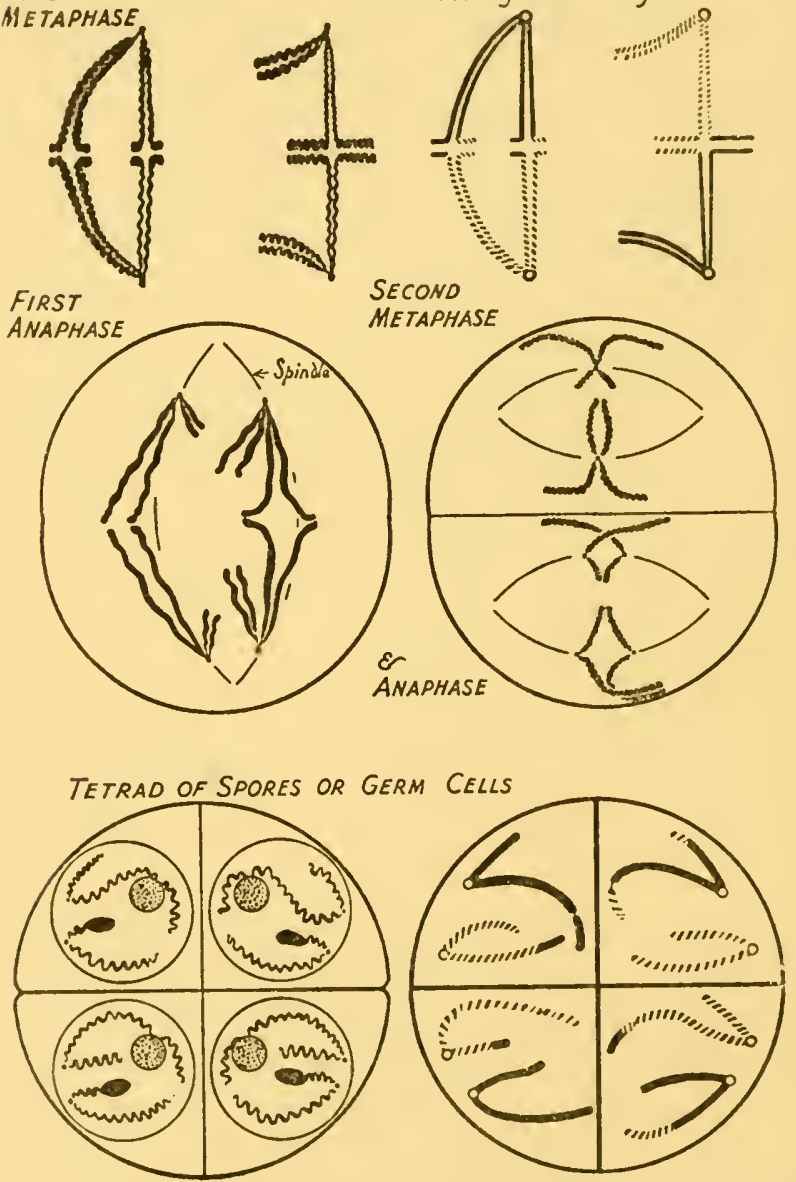

FIG. 6.-The structural and genetical history at meiosis of two pairs of chromosomes, one partner in each hatched, undergoing crossing-over and segregation. Note that all four products differ in the genctical origin of each of their chromosomes even wherc only onc chiasma has been formed in a bivalent (from Darlington 1945). 
daughter nuclei at once divide again. But their chromosomes, already double, do not divide again. They lie on the second metaphase plate and look just like those of an ordinary mitosis, except that their chromatids, which are not true sister chromatids throughout their length, do not lie together throughout their length. Indeed they need not touch at all except at their centromeres. The second meiotic anaphase then enables us to see very clearly the essential character of an anaphase. Separation must depend on the division of the centromeres, because elsewhere the chromatids are already separated. The result is four nuclei, each with the haploid number of chromosomes, now normal single chromosomes, in fact four truly haploid nuclei (Fig. 6).

\section{Reduction and Recombination}

What does meiosis tell us? The chromosomes which pair, indeed the individual chromomeres which pair, are generally of similar size and shape. They must therefore pair because they are like one another. And since the differences in the sizes and shapes of different pairs are constant from generation to generation, the pairing chromosomes must be derived from opposite parents in which they have corresponding structures and functions. They must therefore (ultimately) be of common ancestry, or, as we say, homologous. Furthermore the chromosomes which do not pair, whether from the same parent or from opposite parents, must be different in structure and function from one another. To some extent, the chromomeres which make up each chromosome may be supposed to be carried along by their neighbours in pairing. But, to some extent, they too must be differentiated particles with their own specific attractions for similar homologues. In other words, the chromosomes composing a haploid set must be different from one another and are likely to be made up of specific and different chromomeres in a constant linear arrangement.

What are the results of meiosis? The diploid mother-cell has given rise to four haploid spores or gametes. The number of the chromosomes has been reduced to half. There was formerly a great deal of argument as to whether this reduction took place at the first division or at the second. We now see that it takes place at neither. It takes place by virtue of the fact that no division of the chromo- 
somes intervenes between these two divisions of the nucleus. If either of the divisions fails to separate two daughter nuclei effectively, an unreduced or diploid germ cell is formed. And if both fail, the germ cell or spore is tetraploid.

Now the four germ cells produced by a regular meiosis are alike in being haploid. But in all other respects their nuclei are different. The arrangement of the bivalents at first metaphase has been, as we can show, at random, so that maternal and paternal chromosomes will have been assorted at random. At the same time we have to realize that we can no longer speak of chromosomes as the units they were at mitosis. Their parts have also been reassorted and exchanged or recombined by crossing-over. Those parts which separated reductionally at the first division, separate equationally at the second, and vice versa. Further, since every bivalent has been held together by at least one chiasma, and its chromatids have undergone at least one crossing-over, all its four chromatids distributed to the four nuclei are different combinations. And finally each bivalent in each mother cell differs from all others almost without limit, since each crossing-over can occur at hundreds of different positions. Hence each mother cell will have given rise to four spores or sperms different from one another and different from those produced by any of the other mother cells. It was the consideration that the universal production of four cells at meiosis implied that the four must be universally different that led Janssens in I909 to suppose that crossingover was a universal concomitant of meiosis; that it was associated with the formation of chiasmata; and that it led to the recombination of hereditary differences.

In short, by virtue of crossing-over, no two of the products of one meiosis will be alike. And since crossing-over can probably take place between any two chromomeres, it will be rare indeed for two identical haploid nuclei to be produced from different meiosesunless the pairing chromosomes are themselves identical. Still rarer will it be that two haploid gametes will combine in fertilization to restore the type of either of their parents. Here then we see how variation, the occurrence of differences between individuals, can be maintained from generation to generation in sexual reproduction; and not only maintained, but rearranged and redistributed in such a way as to make every varying individual unique. 


\section{THE CHROMOSOME MECHANISM}

\section{REFERENCES}

BOVERI, T. 1904. Ergebnisse über die Konstitution der Chromatischen Substanz des Zellkeris. Jena.

DARLington, C. D. 1937. Recent Advances in Cytology. 2nd ed. London.

DARLINGTON, C. D. I945. The chemical basis of heredity and development. Discovery, 6: 79-86.

PAINTER, T. S. 1934. A new method for the study of chromosome aberrations and the plotting of chromosome maps in Drosophila molanogaster. Genetics, I9: I75-188.

weismann, A. 1893. The Germplasm. London. 


\title{
THE MENDELIAN METHOD
}

\author{
Mendel's Experiments The Segregation of Factors \\ Linkage The Chromosome Basis \\ Sex Determination and Sex-Linkage
}

WE HAVE NOW SEEN hOW certain visible self-propagating structures are transmitted from cell to cell and from parent to offspring. We have seen the rule and order of these processes. How are they reflected in the gross structures, the phenotypes, of parents and offspring? The answer is given by the mendelian method.

Mendel discovered the two requirements for success in planning a critical experiment on heredity - that is, on sexual heredity. First of all, crosses must be made between visibly and sharply different parents. Secondly, these parents must come from true-breeding lines, otherwise any differences visible in the progeny might be referable, not to the known differences between these parents, but to unknown differences between the ancestors of one of them. Such true-breeding lines he knew from experience could be obtained with certainty only in organisms with regular self-fertilization, i.e. where the male and female gametes which fuse are produced, generation after generation, by the same zygote, by the same or different flowers of one parent plant.

\section{Mendel's Experiments}

The garden pea, Pisum sativum, has varieties which meet these requirements and we cannot do better than describe the experiments Mendel made with them. In 1857 he took 34 varieties, and after two years' trial selected 22 of them for his experiments. These remained constant throughout the work. They gave him seven differences of character (or phenotype, as we may say) distinct enough for his purpose. These differences, or "characters" as they are conveniently called, showed themselves in the course of development in the order given by Table $\mathrm{I}$.

One character, that of purple pigmentation, expresses itself at 
different stages of development. It will be seen that the one structure of the seed contains parts of the parent gencration (in the seed coat) and parts of the offspring (in the cotyledons).

TABLE 1

MENDEL'S SEVEN CHARACTERS IN PEAS

\begin{tabular}{|c|c|c|}
\hline $\begin{array}{l}\text { 1. Cotyledons } \\
\text { 2. Cotyledons } \\
\text { 3. }\left\{\begin{array}{l}\text { Leaf-axils } \\
\text { Petals } \\
\text { Seed-coat }\end{array}\right. \\
\text { 4. Stem } \\
\text { 5. Flowers } \\
\text { 6. Unripe pods } \\
\text { 7. Ripe pods }\end{array}$ & $\begin{array}{l}\text { yellow versus } \text { green } \\
\text { round versus } \text { wrinkled } \\
\text { purple versus } \text { green } \\
\text { purple versus } \text { white } \\
\text { purple versus white } \\
\text { tall versus } \text { dwarf } \\
\text { axial versus terminal } \\
\text { green versus yellow } \\
\text { inflated non-sugary versus } \\
\text { constricted sugary }\end{array}$ & $\begin{array}{l}\text { in the ripe seed } \\
\text { in the ripe seed } \\
\text { in the seedling } \\
\text { in the mature plant } \\
\text { in the mature plant } \\
\text { in the growing plant } \\
\text { in the mature plant } \\
\text { in the mature plant } \\
\text { in the mature plant }\end{array}$ \\
\hline
\end{tabular}

Mendel crossed these differing lines of peas and found that, in each cross, the whole of the seedlings of the first filial generation, or $F_{1}$, resembled one of the parents in respect of each of the character differences. The type which appeared in the crossed plants he termed dominant, and its latent alternative recessive. The dominant is the first of the two alternatives for each character in Table I, but it is important to note that the result is the same whichever way the cross is made: reciprocal crosses are alike.

The $F_{1}$ was uniform, as uniform as the parents had been. A second generation, $F_{2}$, was then raised from self-pollination of the $F_{1}$, and in the $F_{2}$ families the recessive types reappeared together with the dominants. But no transitional or intermediate types appeared amongst them. Furthermore, the dominants and recessives were always in the approximate proportion of three to one. The exact proportion, of course, varied from family to family according to the chances of sampling. The examples of Table 2 , showing the numbers of plants with alternative cotyledon characters, illustrate the principle.

The next question to settle was the breeding properties of the different types, the dominant and the recessive, in the $\mathrm{F}_{2} . \mathrm{F}_{3}$ families were raised from individual $F_{2}$ plants by self-pollination. All the recessives bred true, but only one third of the dominants did so. For example 166 bred true for yellow cotyledons and 353 gave yellow and green types again in the proportion of three to one. 
TABLE 2

COMPOSITION OF F's IN PEAS (MENDEL)

\begin{tabular}{|c|c|cc|}
\hline Families from crossing & No. of $F_{1}$ plants & \multicolumn{2}{|c|}{$F_{2}$} \\
\hline Yollow-green ...... & 258 & 6,022 & 2,001 \\
Round-wrinkled .. & 253 & 5,474 & 1,850 \\
\hline
\end{tabular}

Thus the $\mathrm{F}_{2}$ consisted of three types: purc dominant (like one parent), mixed dominant (like the $F_{1}$ again), pure recessive (like the other parent), and they occurred in the proportions of I : $2:$ I or, expressed as fractions of the whole, $\frac{1}{4}: \frac{1}{2}: \frac{1}{4}$.

\section{The Segregation of Factors}

How was Mendel, and how are we, to understand these simple rules and ratios? In respect of each character the plants of the $\mathrm{F}_{2}$ are all either like one or other of the two parents, or like the $F_{1}$, which is of course itself merely the product of fusion of gametes from those two parents. We need therefore assume only that the $F_{1}$ plants are producing gametes, pollen and eggs, of the types produced by both of the parents. Further, since plants of the two parental types are equally frequent in the $F_{2}$, gametes of the two parental types must be equally frequent in the pollen and eggs of the $F_{1}$.

Take as an example the case of round and wrinkled seeds. Half the pollen (and half the eggs) in $\mathrm{F}_{1}$ are of round type, $R$, and the other half of wrinkled type, $r$. Then $R$ eggs will meet $R$ pollen in $\frac{1}{2} \times \frac{1}{2}$ of the cases to give $R R$ homozygotes (to use Bateson's term), and $r$ eggs will similarly meet $r$ pollen in $\frac{1}{4}$ of the cases to give $r r$ homozygotes. $R$ eggs will mect $r$ pollen in another $\frac{1}{4}$ and $r$ eggs $R$ pollen in the fourth $\frac{1}{4}$; these two types, being both $R r$ heterozygotes, are indistinguishable and together constitute $\frac{1}{2}$ the cases (Fig. 7).

Of course $\frac{1}{2}$ and $\frac{1}{4}$ merely represents the chance of a gamete being of a given kind, or of a zygote arising in a given way. The vagaries of sampling will result in the exact ratios, the theoretical or expected ratios, being rarely realized. 


\section{ROUND}

WRINKLED

$P$

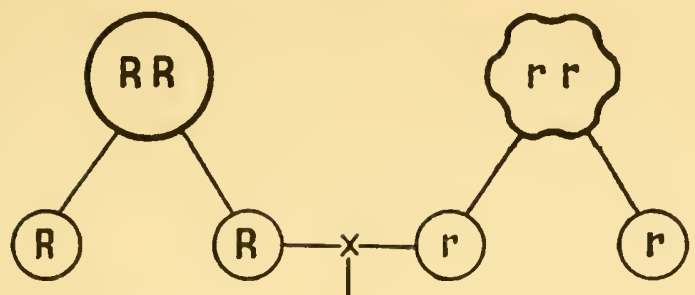

$F_{1}$

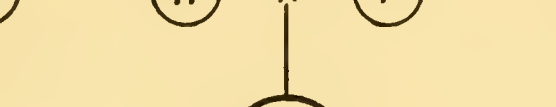

1)

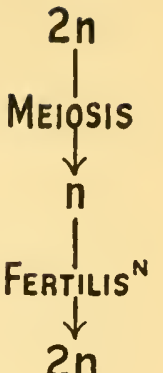

$\mathrm{F}_{2}$

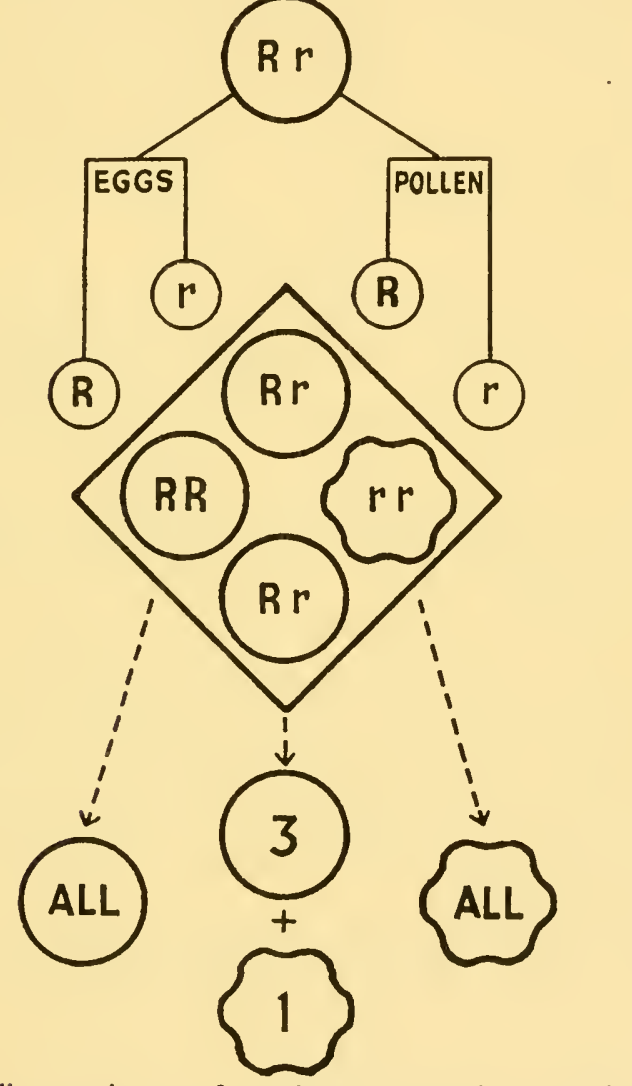

MEIOSIS

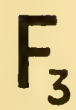

MEIOSIS

GAMETES

AND

FERTILIS $^{N}$

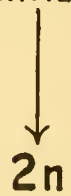

FIG. 7.-Mendel's experiment of crossing round and wrinkled peas. The parent strains (P) were true breeding, giving only one kind of gamete (small circles) each. The hybrid between them $\left(\mathrm{F}_{1}\right)$ was round seeded, but gave two kinds of gamete in equal numbers, one like the gametes from the round parent and the other like the gametes from the wrinkled parent. When pollen of the two kinds fertilizes embryo sacs of the two kinds in random proportions, three round seeded peas are obtained on the average for every wrinkled seeded pea in $F_{2}$. The wrinkled peas and one-third of the round peas of $\mathrm{F}_{2}$ are true breeding like the original parents, but two-thirds of the round peas resemble $F_{1}$ in constitution and again give the $3:$ I segregation in $F_{3}$. The chromosome cycle of reduction and fertilization is shown in relation to Mendel's observations on the right of the diagram, where $2 n$ is the somatic and $n$ the gametic number of chromosomes. 
Now all these statements can be put much more simply if we say that the zygotes are double, and the gametes single, in respect of something which determines each of the characters and differences Mendel was studying. We must then say that the two alternative determinants or factors of each kind in a zygote may be alike or different; but even if different, these alternatives, or allelomorphs as they are called, separate, or segregate, from one another unchanged to give pure gametes; that is each gamete has only one of them, and that one untainted and unblended. This is Mendel's so-called first law of inheritance. In applying this law we may note that it is quite indifferent for its transmission whether a factor is dominant or recessive, whether it comes from the male or the female parent, or whether it goes to male or female gamete.

On this basis we can, as Mendel did, make certain predictions. When the $F_{1}$ is crossed, back-crossed as we say, to one of its parents, offspring will be produced of two kinds in equal numbers. One kind will be like the parent in question and the other like the $F_{1}$. With the dominant-carrying parent the two types will look alike, although they will breed differently $(R R$ and $R r)$. With the recessive-carrying parent they will look different, as well as breed differently ( $r r$ and $R r$ ).

This expectation was realized. In a back-cross of an $F_{1}$ to its roundseed parent, Mendel obtained 192 round seeds, and on testing 177 of these further 87 bred true and 90 proved to be of the $F_{1}$ mixed type. In the back-cross to the wrinkled parent, he obtained 208 seeds; I02 were wrinkled seeds breeding true, i.e. homozygous for the factor $r r$, Io6 were round seeds, all of the mixed type, i.e. heterozygous for the factor $R r$.

So much for single differences. Where the homozygous parents differed in two characters, the factors determining the two differences were independent. They were independent equally in transmission and in action. Thus the $\mathrm{F}_{1}$ showed both dominant characters whether these came from the same or from different parents. And in both cases the $F_{2}$ showed a proportion which was the square of a $3:$ I proportion, in other words $9: 3: 3: \mathrm{I}$.

Thus Mendel crossed round yellow by wrinkled green peas $(R R Y Y \times r r y y)$ and obtained a round yellow $\mathrm{F}_{1}(R r Y y)$. This gave him an $\mathrm{F}_{2}$ of 556 plants in the following numbers: 
Round Yellow Wrinkled Yellow Round Green Wrinkled Green

\section{IS \\ IOI \\ I08 \\ 32}

All but 27 of these plants set seed by selfing and their constitutions proved to be as shown in Table 3 :

TABLE 3

THE NINE GENETICAL CLASSES IN AN F 2 SEGREGATING FOR TWO FACTORS (MENDEL)

\begin{tabular}{|c|c|c|c|c|}
\hline$Y-y^{R-r}$ & $R R$ & $R r$ & $r r$ & Total \\
\hline$Y Y$ & 38 & 60 & 28 & 126 \\
\hline$Y y$ & & & 68 & 271 \\
\hline$y y$ & $\begin{array}{l}35 \\
1\end{array}$ & $\begin{array}{l}67 \\
2\end{array}$ & 30 & 132 \\
\hline Totals & 138 & 265 & 126 & 529 \\
\hline
\end{tabular}

Thus the segregation ratio, as we may call it, is $\mathrm{I}: 2: \mathrm{I}$ for $R-r$ in each of the $Y-y$ classes and vice versa. The factors are recombined at random, so that the whole table represents the square of a $I: 2:$ I ratio.

Applying this square rule, if for one factor there are two kinds of gametes, for two factors there must be four kinds, produced in equal numbers $(R Y, R Y, r Y$, and $r y)$. This conclusion Mendel also verified by back-crosses. He even extended it by testing the independence of three factors, where of course eight kinds of gametes are produced in equal numbers. This principle of independent recombination is known as Mendel's second law.

\section{Linkage}

Since Mendel's work was rediscovered in 1900 , the application of these rules has been studied in nearly all groups of sexually reproducing organisms. They have been universally verified with one modification, itself also universal: different factors are not always independent of one another in their segregation.

In the $\mathrm{F}_{2}$ of a cross between two strains of Sweet Pea, Lathyrus 
odoratus, Bateson and Punnett in 1902 observed segregation for flower colour $(P-p)$ and pollen shape $(L-l)$. Each factor gave a $3: \mathbf{I}$ ratio, three purples to one red, three long-pollen to one roundpollen. But the combinations of these characters were not in the $9: 3: 3:$ I ratio that Mendel had found. They were as follows:
Purple Long
Purple Round
I, 528
I06
Red Long
Red Round
$38 \mathrm{I}$

Evidently the four classes of gamete were not being produced in equal numbers. The double dominant and double recessive classes, $P L$ and $p l$, must have been about seven times as numerous as their alternatives $P l$ and $p L$. Later, other cases were found in which the two classes of gametc with one dominant and one recessive (such as $A b$ and $a B$ ) were in excess.

The reason for this behaviour was made clear by Morgan and his colleagues in their experiments with Drosophila melanogaster in I 910 and later years. In this small fruit fly, which raises its enormous progenies in a ten-day cycle, they studied the inheritance of some hundreds of factors, to which they gave Johannsen's name of genes. These, they found, fell into four groups. A gene from one group would segregate independently of any gene from the other three. But two genes from the same group showed linked inheritance. Certain of the combinations of these genes were more frequent in the gametes than their alternatives. And these favoured combinations (whether dominant with dominant or dominant with recessive) turned out to be the ones which had been present together in the parents.

For example, let us take the case of females produced by crossing the double dominant wild or standard type $B B V g V g$, with the double recessive $b b v g v g$, having a black body and vestigial wings. These females were back-crossed to $b b v g v g$ males and their progeny were as follows:
$B b \mathrm{Vg} v g$
586
$b b V g v g$
106
B b vg vg
$b$ b vg vg
465

Thus the old or parental types of gamete are about five times as common as the new or recombinant types. To be precise, in this experiment, $17 \cdot \mathrm{I}$ per cent of gametes show recombination.

When, however, the doubly heterozygous females came from 
the cross of black ( $b b V g V g$ ) by vestigial ( $B B v g v g$ ), the progeny from back-crossing to the double recessive were as follows:

$\begin{array}{cccc}B b V g v g & b b V g v g & B b v g v g & b b v g v g \\ 338 & 1,552 & 1,315 & 294\end{array}$

The preponderant types of gametes are again the parental ones and now $17 \cdot 9$ per cent show recombination (Morgan I9I4). Thus the percentage of recombination is a property of the two genes recombining; it is a property fixed within narrow linits for each pair of genes (such as $b$ and $v g$ ), and differing between different pairs of genes. All percentages occur up to 50 , this limit being indistinguishable from Mendel's case of independence, which appears when the genes are in different linkage groups.

What then happens when three genes in the same linkage group are recombined in the same experiment? We can obviously work out the recombination percentage or value for each pair of them. Taking a third gene, that for purple eye, $p r, *$ in addition to $b$ and $v g$, Bridges found that it showed $6 \cdot 4$ per cent of recombination with $b$ and 10. 8 per cent with $v g$, while $b$ and $v g$ showed 16.3 per cent in this experiment. Thus two of the values add up to the third, or to a little more. It seems as if the genes were arrayed in a line, thus:

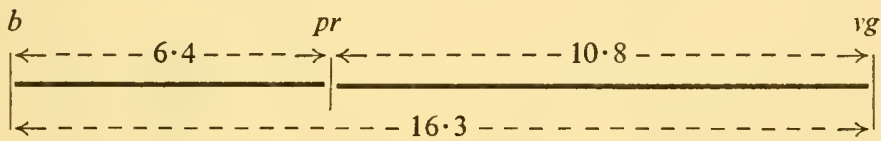

Now with such a linear arrangement, a recombination between $b$ and $p r$ must also give a recombination between $b$ and $v g$. Similarly one between $p r$ and $v g$ must give one between $b$ and $v g$, always provided that the recombinations between $b$ and $p r$ and between $p r$ and $v g$ do not take place simultaneously. Such double recombinations were in fact found in 0.46 per cent of gametes, so giving a deficit 0.92 per cent (each double cancelling two singles) in the observed recombination of $b$ and $v g$ as compared with the sum of the two smaller recombination values.

* In designating the gene as pr, the allelomorph which gives the normal or wild-type red eye is assumed. It is this wild-type allelomorph which we should designate by $\operatorname{Pr}$ on the convention we have been adopting. In the Drosophila system (see Appendix 2) no special symbol is assigned to the wild-type allelomorph, the gene being designated by the variant allelomorph. 

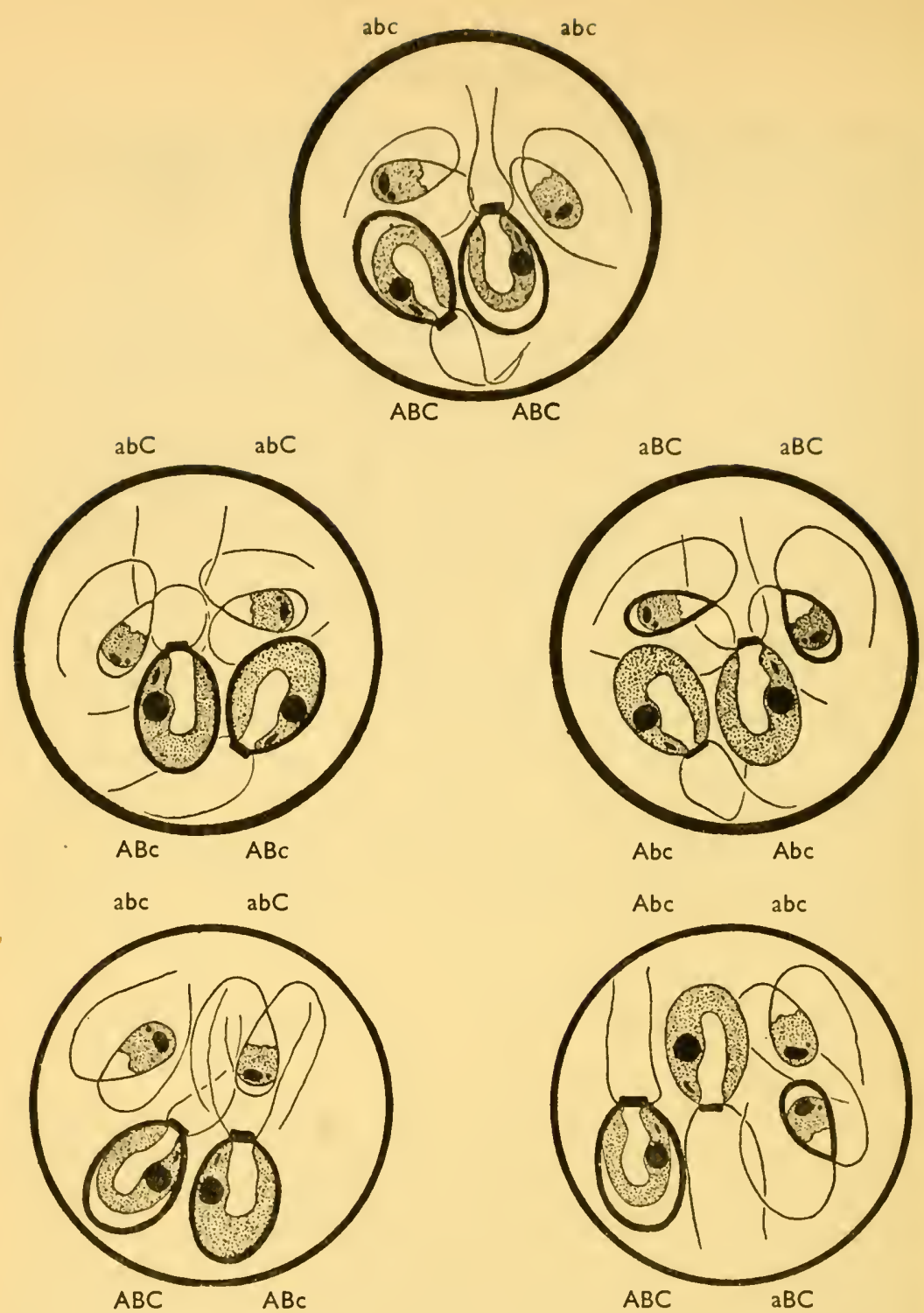

FIG. 8.-Types of haploid spore produced by meicsis in an $F_{1}$ hybrid between two species of the green alga Chlamydomonas. Top: the two spore types of the parents have segregated, i.e. Large $(A)$, Thick-walled $(B)$ and Loose-chloroplast $(C)$ from Small (a), Thin-walled (b) and Adherent-chloroplast (c). Middle Row: two of the results where separation is reductional at the first division for all the three genes and in consequence only two different types are formed. This event ensues whenever there is no chiasma between any of the gencs and the centromeres of the chromosomes that are carrying them. N.B. on the left, recombination is of $C-c$ with $A-a$ and $B-b$; on the right, of $A-a$ with $B-b$ and $C-c$. Bottom Row: two of the results where separation is equational at the first division for at least one of the three genes and in consequence four different types are formed. This event ensues when a chiasma has been formed between the gene in question and the centromere of its chromosome. N.B. in each case the four types include the parental combinations, but they can all be recombinants (with kind permission, after Moewus, 1940, and unpublished). 
Now this proportion, 0.46 per cent, is instructive. If double recombinations occurred in the proportion expected with randomness there would be $(6.4 \times 10.8) / 100$ or 0.69 per cent. The fact that only 0.46 per cent are recovered (i.e. found in the progeny) shows that recombination between $b$ and $p r$ reduces the chance of, or, as Muller put it, interferes with, that between $p r$ and $v g$.

It is an easy step from this kind of experiment to the business of testing and arranging in a line all the genes of one group at intervals proportional to their recombination values. In this way we get a map on which distance between any two points stands for frequency of recombination between the two genes they represent (Fig. 9). From this map the linkage properties of all the mapped genes can be, and nowadays regularly are, predicted in combinations which have not been tried.

\section{The Chromosome Basis}

The system of map prediction is useful but it is not enough. We want to know why it works. It must have a material basis, and this becomes plain when we return to the cell. The chromosomes of the fruit fly fall into four pairs. One of these is a very small pair (Figs. 4 and 5), just as one of the linkage groups contains very few genes. Two of the other chromosomes are roughly equal and somewhat longer than the remaining one. Again just like the linkage maps. Both the chromosomes and the linkage maps are linear. Both the chromosomes and the genes are double in the zygote, single in the gamete. The chromosomes, or rather their constituent chromatids, cross-over and separate in germ cell formation just as the genes segregate and recombine. And the recombination limit of 50 per cent follows simply from the fact that two of the four chromatids cross over at each chiasma.

All in all we have to take it that we are observing the same chromosomes in two different ways, under the microscope and in the breeding experiment, cytologically and genetically. In other organisms than Drosophila the comparison can be carried even further. In maize (Zea mays), for example, not only can each of the ten linkage groups be shown to correspond with one of the ten pairs of chromosomes; these chromosomes can be seen to pair and 
to cross-over with the frequency required by the breeding data. And we shall find later that they can be seen to undergo other changes whose results can be recognized by breeding.

We can now turn back and look at the facts of heredity from the chromosome point of view. Our starting point must be the parental chromosome which can be passed on unchanged to the offspring. Recombination between linked genes is the result of a change in their chromosome, the change always produced by crossing-over. If there is no crossing-over the different chromosomes are recombined as wholes and the individual chromosome passes on from generation to generation as a unit of heredity. All its genes will then go in one block and appear like one gene, a super-gene, having many different effects.

Strange to say this failure of crossing-over actually happens in the male of Drosophila and other flies. At meiosis in the sperm mother cell, the chromosomes pair but no chiasmata are formed and no crossing-over takes place. The chromosomes passed from father to offspring pass as unbroken units. Thus a chromosome can remain unbroken for any number of generations. It is not, however, likely to do so since the chance of being passed to a female, where crossingover will occur, is a half in each generation, for half the flies are females. Of all the chromosomes in flies only $\mathrm{I}$ in $\mathrm{I}, 024$ will have been free from crossing-over for ten generations or more.

To this rule there is one exception. One chromosome is passed down only from father to son. This is known as the Y chromosome and its smaller partner, with which it pairs at meiosis, is known as the $\mathrm{X}$. In the female there are two X's and these correspond to the middle-sized linkage group (Fig. 9). The male, XY, is thus heterozygous for this pair of whole chromosomes, the female homozygous. The male at meiosis produces four sperm from each mother cell; two have an $\mathrm{X}$ and two a $\mathrm{Y}$. At the same time the female produces eggs all with one $\mathrm{X}$.

Thus the cross between male and female is a back-cross for the $\mathrm{X}-\mathrm{Y}$ pair of chromosomes or, if you like, the $\mathrm{X}-\mathrm{Y}$ super-gene, and half the offspring are of each sex. Further, we nust notice, the daughter receives an $\mathrm{X}$ from each parent, the son an $\mathrm{X}$ from his mother and a Y from his father. It would be easy on this evidence to say that the difference between $\mathrm{X}$ and $\mathrm{Y}$ determines sex. It would 


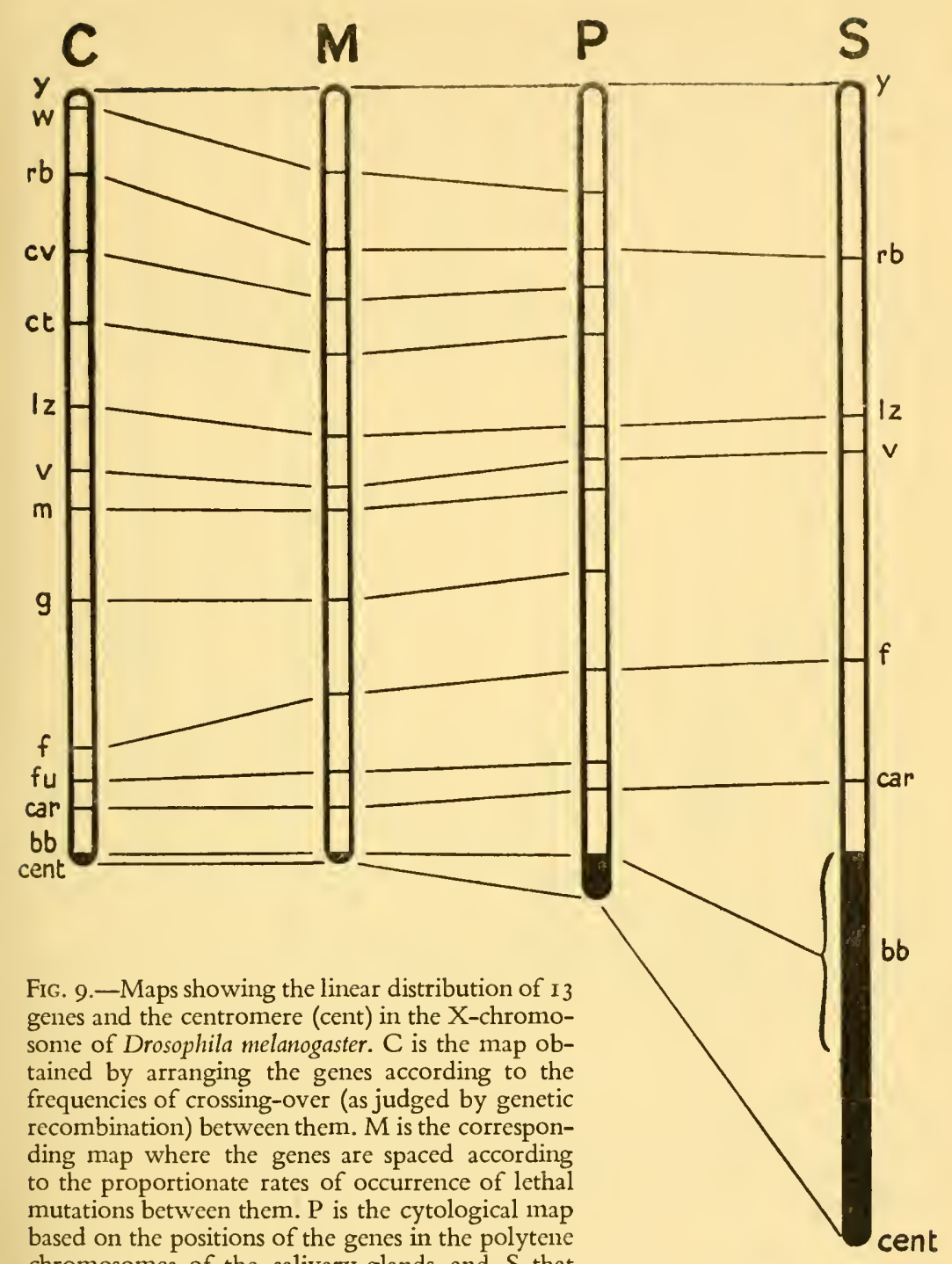
chromosomes of the salivary glands, and $\mathrm{S}$ that based on the positions of the genes in the mitotic chromosomes of the nerve ganglia cells. Only 7 of the genes have been localized on the mitotic map. The large block of heterochromatin near the centromere is shown black. It is hardly detectable in the genetic map, and is much smaller in the polytene than in the mitotic chromosomes. 
be more accurate, however, to say that it determines whether a fertilized egg with a normal outfit of the other chromosomes, the autosomes, shall develop into male or female. How the autosomes affect the issue we shall see in a later chapter.

\section{Sex-Linkage}

Corresponding with this cytologically observed situation is the genetically observed mechanism of sex-linked inheritance. When a wild type, red-eyed, female fly is crossed with a white-eyed male,
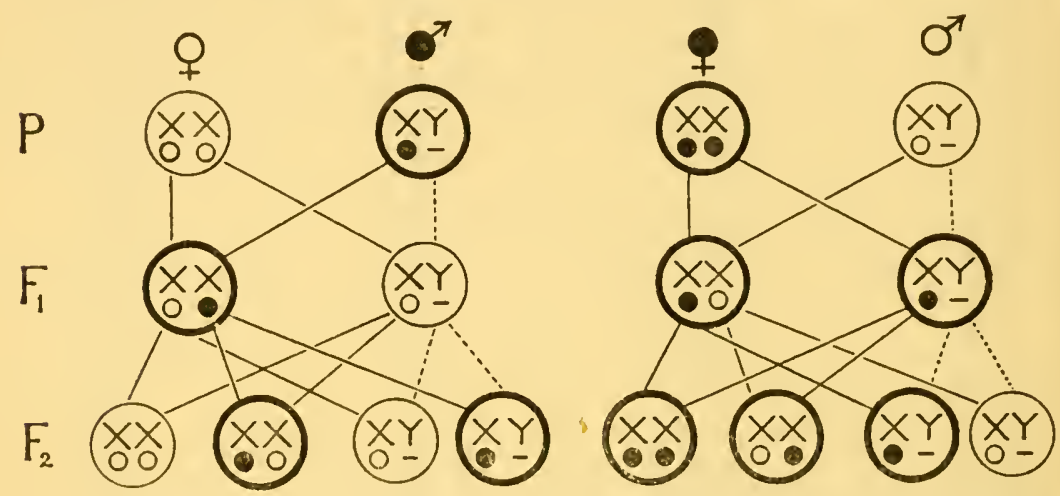

Fig. I0.- Reciprocal crosses showing the transmission and expression of a gene pair in the $\mathrm{X}$ chromosome, having no allelomorph in the $\mathrm{Y}$ and with dominance, e.g. white-eye in Drosophila or haemophilia in man. In the black-yellow difference of the cat, the heterozygote is distinguishable as the tortoiseshell female.

The solid circle below the $\mathrm{X}$ denotes the dominant allelomorph, the empty circle the recessive. The thick outer circle denotes the dominant phenotype. Broken lines show the transmission of the Y.

all the $F_{1}$ are red: white is recessive. In the $F_{2}$ (from crossing brothers and sisters in the $F_{1}$ ) we get three red flies to every white. But all the white-eyed flies are males: the gene is sex-linked.

When the cross is made the other way round, white female being crossed with red male, the sex linkage makes itself apparent in the $F_{1}$. As before, the females are red but the males are now white. In the $\mathrm{F}_{2}$ the males as before are half red and half white, but now so are the females too.

What does this mean? The females in the $F_{1}$ are the same no matter which way the cross is made. The females must therefore inherit the gene equally from both parents. The males in $F_{2}$ are all 
the same although their fathers are of the two kinds. They obtain the gene only from their mothers. Thus we see that the gene follows the path of the $\mathrm{X}$ chromosome. At the same time we see that the $\mathrm{Y}$ has no effect. Where the $\mathrm{X}$ chromosome carries the red-producing form or allelomorph of the gene, the male fly is red-eyed; where the $\mathrm{X}$ carries the white allelomorph the male fly is white-eyed. Figure Io makes this clear.

In mammals, like flies, the male is the heterozygous sex, but in birds it is the female. Sex-linkage is of peculiar and practical interest in both. The gold-silver difference used in sexing crossed chicks depends on a gene in the $\mathrm{X}$ chromosome, and so shows criss-cross inheritance like the red-white eye in the fly. Haemophilia and colourblindness depend on recessive genes in the $\mathrm{X}$ of man and hence appear less often in the XX female. Their inheritance follows the same rules as that of white-eye in flies and their frequencies in the population can be deduced from these rules. Thus Pickford found that populations with 7.8 per cent of colour-blind men have 0.65 per cent of affected women. The tortoiseshell cat is heterozygous for the X-linked gene, one allelomorph of which gives black, the other yellow, when homozygous. In mammals a normal male cannot be heterozygous for an X-linked gene and tortoiseshell males occur only as rare and sterile abnormalities.

In Drosophila the red-white eye difference showed that for the purpose of this sex-linked experiment the Y chromosome might as well be empty. No gene in the $Y$ appeared to correspond with this gene, for no gene interfered with its action. This is a general property of the Y chromosome for, with the sole exception of the gene for "bobbed," the Y simply does not correspond. Nor does it show recognizable differences of its own. That is to say, different males do not carry $\mathrm{Y}$ chromosomes determining differences comparable with that between red and white eyes. What differences they can carry we shall see later.

The fly is typical of a great many insects in the emptiness of the $\mathrm{Y}$ chromosome. In the vertebrates, however, the $\mathrm{Y}$ chromosome shows more correspondence with the $\mathrm{X}$. The corresponding genes are of two kinds. Some of them do not cross-over from one chromosome to the other. For example the $\mathrm{Y}$ chromosome in poultry seems to carry the normal allelomorph of the barring gene 
in the $\mathrm{X}$, but the two chromosomes never exchange allelomorphs in heterozygous females. The barring gene can be used, therefore, to distinguish between the $\mathrm{X}$ and the $\mathrm{Y}$, and so makes possible the "autosexing" breeds as shown in Fig. II.

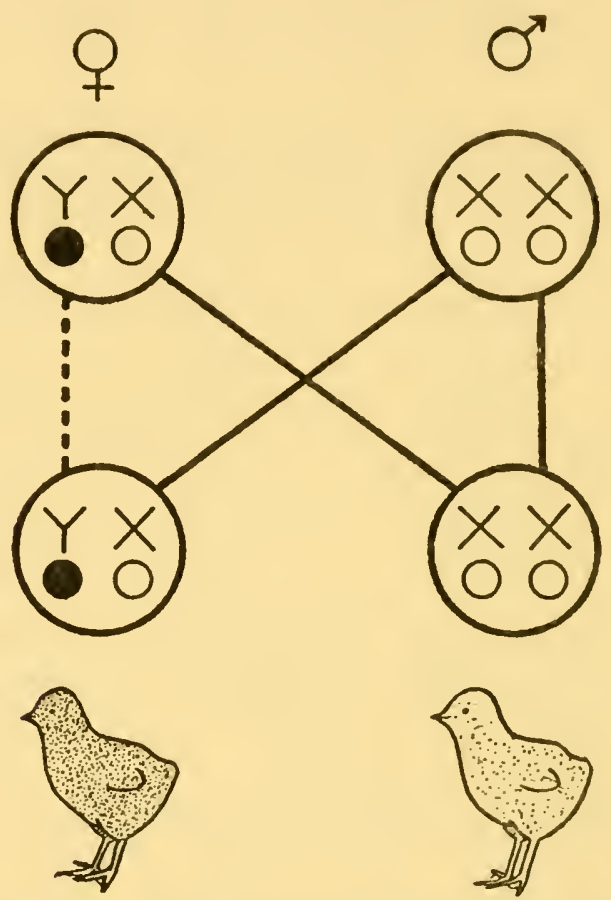

FIG. II.-The gene for barred feathers (open circle) in poultry expresses itself only partially in females; so suggesting that the $\mathrm{Y}$ chromosome carries its non-barred allelomorph (filled circle). Where the basic pigmentation of the birds is brown, rather than black, this effect of the Y chromosome is clearly detectable by deeper colouration in newly hatched chicks. The combination of the gene for brown pigmentation and that for barring thus gives a true breeding strain of birds in which the sexes are distinguishable at hatching by the decper intensity of down pigmentation, especially round the head, in females than in males. Such a breed is termed autosexing.

Sometimes, however, as in certain fishes such as Lebistes and in man, the $\mathrm{X}$ and the $\mathrm{Y}$ chromosomes not only carry the same genes, but also exchange them by crossing-over. These genes, therefore, no longer show complete sex-linkage. Their association with the sex chromosomes is recognizable by the partial, rather than the complete, restriction of a particular allelomorph to a particular sex in 
any family. Instead of all the Y-linked genes of the father passing to the son, a proportion pass to his daughters. The proportion depends on the crossing-over distance between the gene in question and the segment with complete sex linkage (Fig. I2).

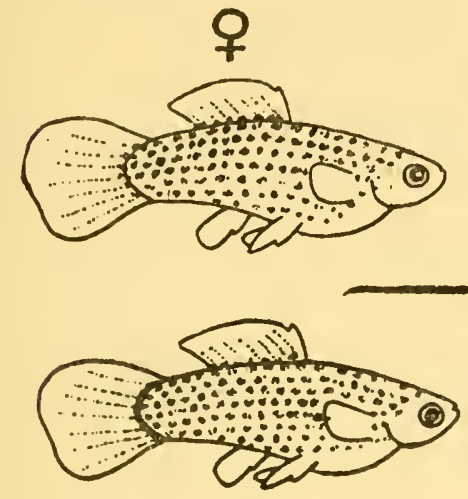

64,3

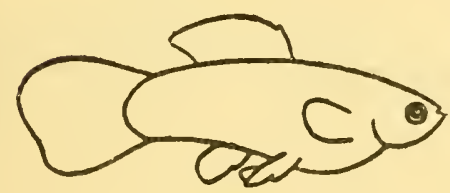

32,3
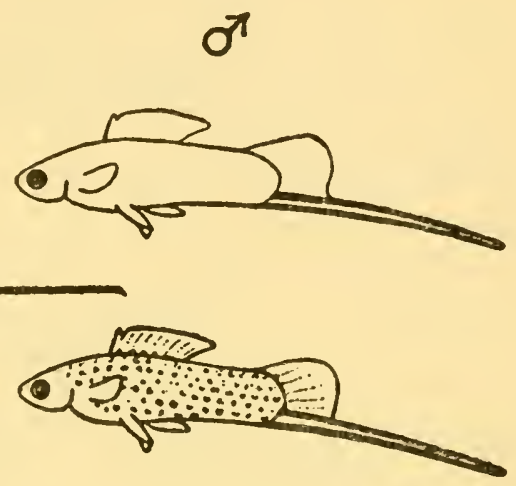

35,6

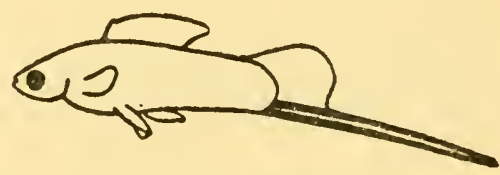

67,7

FIG. 12.- Segregation in a cross in the fish Xiphophorus helleri. Taken over the whole progeny the gene determining spotted $\nu$. spotless gives a good I : I ratio; but the two types are unequally distributed between the sexes. This is the type of segregation characteristic of partial sex-linkage, though the validity of this interpretation has not been fully established in the present case (from Kosswig, 1939).

Finally, in Drosophila and in man, there are completely Y-linked genes without any allelomorph in the $\mathrm{X}$. In man such genes have been recognized by their transmission only from father to son; in Drosophila by the effects of absence of the whole or part of the Y chromosome. Flies are sometimes hatched without any Y. They are perfect-looking males but their sperm cannot swim, so that they are sterile. The $\mathrm{Y}$ chromosome is therefore doing something, although what it does cannot be shown by its relationship with mendelian differences in the $\mathrm{X}$.

There are thus three kinds of parts in the sex chromosomes: 
X-linked, Y-linked and interchangeable. When we examine the sex chromosomes we see that they have similar parts which pair and cross-over; these are the pairing segments. They also have parts which are dissimilar and which do not pair; these are the differential segments belonging, one to $\mathrm{X}$, and the other to $\mathrm{Y}$. The differential segment of the $\mathrm{X}$ is usually larger than that of the $\mathrm{Y}$. It usually, as in man, lies at one end, the pairing segment at the other. The distribution of the chiasmata in the pairing segment relative to the centromere and the differential segments then decides whether the first division is reductional for these segments or equational. In the male Drosophila, where crossing-over occurs in the sex chromosomes, although nowhere else, the pairing segment is in the middle of the $\mathrm{Y}$ chromosome (separating two differential segments) and at the end of the X. Crossing-over occurs doubly and reciprocally, the second exchangc cancelling the first. It therefore keeps the two differential segments of the $\mathrm{Y}$ together and does not give any result detectable in ordinary breeding experiments (Fig. I3).

The same principles of sex determination apply generally not only to animals but also to plants with separate sexes. In the dioecious Campions (Melandrium dioica) as in Drosophila there arc distinct sex chromosomes, the male having XY, and sex-linked genes are found in both $\mathrm{X}$ and $\mathrm{Y}$. In different animals there are, however, many differences in detail. In grasshoppers (Orthoptera) and aphids (Hemiptera) there is no Y chromosome. Females have XX, males have one $\mathrm{X}$ only. The whole of the $\mathrm{X}$ is therefore differential. In some Hemiptera, and in spiders and Ostracoda the $\mathrm{X}$ is represented by several chromosomes which move and segregatc as a unit in meiosis, the females having twice as many as the males. In Lepidoptera, birds, and some fishes the female is the heterogametic or $\mathrm{XY}$ sex, the malc having XX. With these modifications in its chromosome basis, sex-linked inheritance is correspondingly modified.

Altogether these observations on mendelian breeding and chromosome behaviour show us the mechanism of heredity. They show us its common and universal principles. They also tell us something of the material structure and organization of heredity. They show it to be particulate and they show the units, or particles, or genes, into which it is separable to be smaller than the chromosomes. 


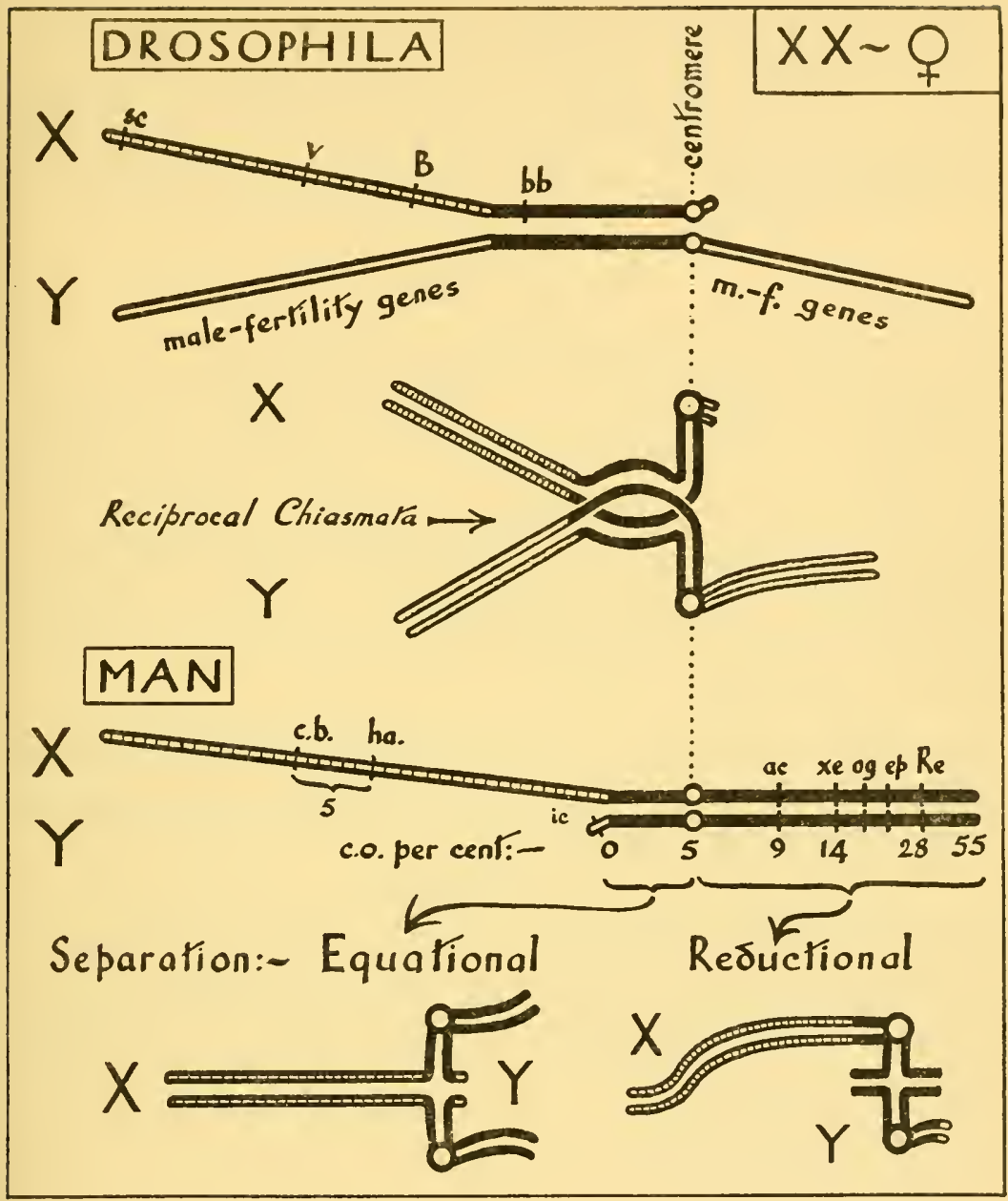

FIG. 13.-Crossing-over between the pairing segments of $\mathrm{X}$ and $\mathrm{Y}$ in heterogametic (male) sex of Drosophila and man showing the structure of the bivalent at pachytene and first metaphase of meiosis. The pachytene pairing gives a metaphase orientation and consequent separation at the first anaphase which depends on the position of crossing-over and chiasma formation. In Drosophila the genes $b b$, bobbed, and $B$, Bar, etc., and in man the genes c.b., colour blindness, $h a$, haemophilia, ic, ichthyosis, etc, are marked. The crossing-over distances in man are from Haldane. Black, pairing segment with partial sex linkage through crossing-over between $\mathrm{X}$ and $\mathrm{Y}$ (as well as between X's in females). Hatched, differential segment with complete sex linkage in $\mathrm{X}$ and with crossing-over between $\mathrm{X}$ 's in females. White, differential segment with complete sex linkage in $\mathrm{Y}$ and no crossing-over whatever. 
Evidently the chromosomes which we see as materials are composed of units which we can recognize by their differences. We shall carry our analysis further and consider the properties of these units. But before we do so we must find out how far, and above all how rigorously, the whole of hereditary differences can be expressed in terms of such units.

\section{REFERENCES}

Bateson, w. 1909. Mendel's Principles of Heredity. Cambridge.

DarLington, C. D. 1934. Anomalous chromosome pairing in the male Drosophila melanogaster. Genetics, 19: 95-118.

haldane, J. B. S. 1936. A search for incomplete sex-linkage in man. Am. Eugen., $7: 28-57$.

HeITZ, E. 1935. Chromosomenstruktur und Gene. Z.I.A.V., 70: 402-447.

KOLLER, P. C. 1937. The genetical and mechanical properties of sex chromosomes. III, Man. Proc. Roy. Soc. Edin. B., 57: 194-2I4.

коsSwig, С. 1939. Die Geschlechtsbestimmung bei Zahnkarpfen. Rev. Fac. Sci. Univ. Istambul, 4: I-32.

MATHER, K. 1938. Crossing-over. Biol. Revs., I3: 252-292.

MENDEL, G. I865. Experiments in plant hybridisation. (Translation in Bateson, 1909.)

MoEwUS, F. 1940. Die Analyse von 42 erblichen Eigenschaften des Chlamydomonas eugametos-Gruppe. Z.I.A.V., 78: 418-522.

MORGAN, T. H. I9I3. Heredity and Sex. New York.

MORGAN, T. H. I9I4. No crossing-over in the male of Drosophila of genes in the second and third pairs of chromosomes. Biol. Bull., 26: I95-204.

muller, H. J. I916. The mechanism of crossing over. Amer. Nat. 50: 193 et sqq. PICKFORD, R. W. I947. Frequencies of sex-linked red-green colour vision defects. Nature, 160: 335 .

Winge, $\varnothing$., and DITLEVSEN, E. 1947. Colour inheritance and sex deternination in Lebistes. Heredity, I : 65-83. 


\section{CONTINUOUS VARIATION}

The Limitations of Mendelism The Specification of Continuous Variation Heritable and Non-heritable Differences Cumulative Effects of Genes Polygenic Systems Linkage of Polygenes

MENDEL's BEQUeSt TO GENETICS was twofold. He gave us his principles of inheritance, and at the same time he gave us the experimental method by which he had been able to establish those principles and by which they could be tested and extended. This method, as we saw in the last chapter, has enabled us to show that Mendel's principles reflect the properties of inheritance through the nucleus. Wherever the hereditary determiners of variation are carried by the chromosomes we must therefore expect these principles to apply.

We cannot, however, expect the experimental method to be equally widely applicable. This depends for its success, as Mendel himself clearly realized, on the differences under investigation (tall v. short, round $v$. wrinkled, etc.) being so large compared with any residual or extraneous variation in the character, that individuals could be assigned unambiguously to one or other of the alternative classes. Thus Mendel's short peas ranged in height from 9 inches to I 8 inches, and his tall parents from 6 feet to 7 feet. The $F_{1}$ 's of tall and short even exceeded the tall parent with heights up to $7 \frac{1}{2}$ feet. But in spite of this variation in both talls and shorts, there was never any doubt that a plant, whether in the parental, $F_{1}, F_{2}$, or any other generation, was either the one or the other. The variation was in fact what came to be called discontinuous: all below the discontinuity in the distribution of heights were shorts and all above it, talls.

The residual variation, within the talls themselves and equally of course within the shorts, was evidently not of this kind. There was presumably a smooth, or at least an apparently smooth, gradation in height between the shortest and tallest talls and between the shortest and tallest shorts. The variation within these classes was 
continuous. In any family where the gene of major effect was not segregating, the variation must have been wholly of this continuous kind.

The variation that we normally see in stature in man, or indeed of size in any organism, is continuous in this way. Stature may vary between wide cxtremes, and every height between those extremes is represented. There is no discontinuity in the range of statures: the gradations are imperceptible. As a consequence we cannot classify people into talls and shorts in the way that Mcndel was able to do with his peas. It is therefore impossible cither to specify a family or population in respect of stature by a mendelian ratio of tall to short, or to investigate the inheritance of stature in man by the mendelian method. What method can we use to take its place?

\section{The Specification of Continuons Variation}

Where variation in a character is continuous, the number of classes into which individuals can be divided according to the manifestation of the character is limited only by the fineness of the means we possess for measuring it. Each observation is unique or potentially so. We may, however, define classes with arbitrarily chosen limits and describe any group of individuals by recording the numbers which fall into each of these classes. Thus we might describe a human population by recording the numbers of people with heights between 4 feet 6 inches and 4 feet 7 inches, 4 feet 7 inches and 4 feet 8 inches, 4 feet 8 inches and 4 fect 9 inches, and so on. The members of each class will not, of course, all have exactly the same height. The representation is therefore only approximate, and a closer approximation would be obtained by classifying into ranges of $\frac{1}{2}$ inch instead of I inch; for the frequency distribution of stature, as it is called, would then approach more closcly to the true state of continuity. The fincr the gradations we use, however, the fewer individuals will fall into cach class, and the more crratic will the distribution appear when limited numbers of individuals are available for measurement. To overcome this difficulty we need a means of specifying the frequency distribution as a whole. We need a means of representing it by a few constants (known as parameters), knowledge of whose values would cnable us to determine the proportion 
is, or how short a short one is, provided we can classify into the tall and short categories. Scaling is, however, of obvious importance in the analysis of continuous variation, since the scale will, in part, determine the shape and properties of the distribution which are all we have to base the genetical conclusions upon. We shall return to it later.

As we have seen, the type of grouped frequency distribution of Fig. I4 is to be regarded as an approximation to the underlying continuous distribution which we may seek to specify algebraically in terms of a few parameters. Where the distribution is symmetrical and the frequencies fall off on each side of the centre value, as in the figure, this ideal curve can be, and indeed usually is, taken as an example of the Gaussian or Normal Curve, whose general formula is

$$
\mathrm{df}=\frac{\mathrm{I}}{\sigma \sqrt{2 \pi}} \mathrm{e}^{-\frac{(\mathrm{x}-\mu)^{2}}{2 \sigma^{2}}} \mathrm{dx}
$$

where $\mathrm{df}$ is the frequency of individuals falling within the infinitesimal range, $d x$, of heights. Apart from $f$, the frequency, and $x$ the heights, four quantities appear in this formula $\pi, \mathrm{e}, \sigma$ and $\mu$. The first two of these, $\pi$ and $e$, are familiar mathematical constants, the ratio of the circumference of a circle to its diameter and the natural basc of logarithms respectively. These will be the same for all normal curves. The other two, $\sigma$ and $\mu$, are the parameters which specify the particular normal curve in question, and they must be found for each distribution individually. They are the constants by means of which we can specify the variation of the character in which we are interested.

The way in which $\mu$ and $\sigma$ specify the curve can be seen from Fig. I4. $\mu$ is the value of the character at the centre of the distribution and is estimated as the mean, or average, of the values of the character in all the individuals concerned. Thus where there are $\mathrm{n}$ individuals whose height has been measured, $\mu$ is estimated by $\bar{x}=\frac{S(x)}{n}$, $S$ standing for summation of the values of $\mathrm{x}$ from all individuals. $\mu$, or its estimate $\overline{\mathrm{x}}$, fixes the position of the curve of distribution.

The second parameter, $\sigma$, is the distance of the point of maximum slope of the curve from the mean, $\mu$. There are, of course, two 
such points, one on each side, but as the curve is symmetrical they must lie at equal distances from $\mu$. The estimate, $s$, of $\sigma$ obtained from any set of data is called the standard deviation and is found as

$$
s=\sqrt{\frac{\mathrm{I}}{\mathrm{n}-\mathrm{I}} \mathrm{S}(\mathrm{x}-\overline{\mathrm{x}})^{2}}
$$

the notation being as before. It is, however, more convenient for purposes of calculation to use the algebraically identical formula

$$
s=\sqrt{\frac{\mathrm{I}}{1 \mathrm{n}-\mathrm{I}}\left[S\left(\mathrm{x}^{2}\right)-\frac{\mathrm{S}^{2}(\mathrm{x})}{\mathrm{n}}\right]}
$$

where $S^{2}(x)$ stand for the square of the sum of all the $x^{\prime} s$.

It will be observed that $\mathrm{s}$ is found as the square root of $\mathrm{s}^{2}$, which is generally called the variance, and is denoted by $\mathrm{V}$. The variance is in fact of more use than the standard deviation in genetical work, because when variation is measured by the variance, the contributions made to it by independent causes of variation are additive. This obviously cannot be equally true when the variation is measured by the standard deviation. We shall, therefore, regard the normal curve as most conveniently specified by $\mu$ and $\sigma^{2}$; or rather by the estimates of these quantities, $\overline{\mathrm{x}}$ and $\mathrm{V}$, as obtained from a set of actual data.

Not all frequency distributions of this general shape, with the central values the most common, are normal curves. Some are asymmetrical. And even some symmetrical curves do not conform to the normal type. Such curves require further parameters, estimated from the values of $S(x-\bar{x})^{3}$ and $S(x-\bar{x})^{4}$, for their full specification; but even so, the mean and variance still retain their crucial importance for our understanding of these distributions. As we shall see, much can be learned of the genetical situation underlying continuous variation by the analysis of means and variances, and this still remains true even where we know the frequency distribution to depart from strict normality.

There is one further biometrical quantity which is of importance for the genetical study of continuous variation, since it helps to tell us directly the extent to which continuous differences are hereditary. Where we have simultaneous observations on pairs of relatives, say 
the heights of father and daughter, we can find the variance of fathers' heights and the variance of daughters' heights. Let us denote fathers' measurements by the suffix $f$ and daughters' by the suffix $d$. Then $V_{f}=\frac{I}{n-I} S\left(x_{f}-\bar{x}_{f}\right)^{2}$ and $V_{d}=\frac{I}{n-I} S\left(x_{d}-\bar{x}_{d}\right)^{2}$. n will be the same in both cases since for every father measured, a daughter was also measured. It is thereforc clear that we can find $S\left[\left(x_{f}-\bar{x}_{f}\right)\left(x_{d}-\bar{x}_{d}\right)\right]$ in a way parallel to that used for $S\left(x_{\mathrm{f}}-\bar{x}_{\mathrm{f}}\right)^{2}$ and $\mathrm{S}\left(\mathrm{x}_{\mathrm{d}}-\overline{\mathrm{x}}_{\mathrm{d}}\right)^{2}$, but multiplying the deviation of cach father's height from its mean, $\left(\mathrm{x}_{\mathrm{f}}-\bar{x}_{\mathrm{f}}\right)$, by the corresponding deviation of his daughter $\left(\mathrm{x}_{\mathrm{d}}-\overline{\mathrm{x}}_{\mathrm{d}}\right)$, instead of squaring the father's or the daughter's deviation, before, summation. We can then calculate the covariance, $\mathrm{W}_{\mathrm{fd}}$, of fathers' and daughters' height, as

$$
\mathrm{W}_{\mathrm{fd}}=\frac{\mathrm{I}}{\mathrm{n}-\mathrm{I}} \mathrm{S}\left[\left(\mathrm{x}_{\mathrm{f}}-\overline{\mathrm{x}}_{\mathrm{f}}\right)\left(\mathrm{x}_{\mathrm{d}}-\overline{\mathrm{x}}_{\mathrm{d}}\right)\right]
$$

One important point should be noted about these covariances. Variances must always be positive since they are derived from sums of squares. Covariances, on the other hand, are derived from sums of cross-products of deviations and so may be either positive or negative. A positive value indicates that the deviations from the mean in one distribution, say fathers' heights, are preponderantly accompanied by deviations in the other, say daughters' heights, in the same direction, positive or negative. A negative covariance on the other hand indicates that deviations in the two distributions are preponderantly in opposite directions. Where a deviation in the one distribution is equally likely to be accompanied by deviation of like or opposite sign in the other, the covariance, apart from crrors of random sampling, will be zero.

The importance of the covariance in genetics will now be obvious. If variation in height is under genetical control we should expect tall fathers generally to have tall daughters and short fathers generally to have short daughters. In other words we should expect them to have a positive covariance. Lack of genetic control would produce a covariance of zero. It was by this means that Galton first showed' stature in man to be under genetic control. He found that the covariance of parent and offspring, and also that of pairs of siblings, was positive. 
The size of the covariance relative to some standard gives a measure of the strength of the association between the relatives. The standard taken is that afforded by the variances of the two separate distributions, in our case of fathers' and daughters' heights. We may compare the covariance with either of these variances separately, and we do this by calculating regression coefficients which have the form, $\frac{W_{f d}}{V_{f}}$ (regression of daughters on fathers) or, less usefully, $\frac{W_{f d}}{V_{d}}$ (regression of fathers on daughters). We may also compare the covariance with the two variances at once in a correlation coefficient found as $\frac{W_{f d}}{\sqrt{V_{f} \cdot V_{d}}}$. Were the correlation coefficient to have its maximum value of $\mathrm{I}$, it would signify a complete association, a full determination of a daughter's height by her father. Independence of father's and daughter's height would be shown by a coefficient of $o$, because, of course, $\mathrm{W}_{\mathrm{fd}}$ would itself then be $\mathrm{o}$. Values between $O$ and I show partial determination of a daughter's height by her father. The coefficient actually observed in man by Galton was just under 0.5 , for reasons which we shall see later.

\section{Heritable and non-Heritable Differences}

In I900, when Mendel's results first received wide attention, Galton and his successor Pearson had already established by means of correlation studies the heritable nature of continuous variation in a variety of characters in man, in dogs and even in sweet peas. The results were used as the foundation of the so-called Law of Ancestral Heredity. Their approach suffered, however, from one weakness which we can now see to have been fatal to any theory of hereditary transmission. While Galton had an idea that the hereditary materials might be particulate, neither he nor his followers ever grasped the distinction between the determinant and the effect, the genotype and the phenotype.

Mendel supplied the missing principle of the determinant and demonstrated its validity in his peas. He was, however, dealing with discontinuous variation and it was held by the biometrical school that his principles would not apply to continuous variation. Indeed it was considered that the segregation of mendelian differences could 
not account for correlations between parent and offspring so high in value as those observed. It was also considered, in spite of Galton's earlier leanings towards a particulate theory, that discontinuity in segregation of the kind which the mendelian theory demanded was incompatible with continuous variation in the phenotype. This notion seens to have been shared by the early mendelian geneticists, for, just as Pearson and his school took the view that mendelian theory was limited in its application to such heritable variation as was not continuous, De Vries argued that the mendelian principles were of universal applicability because no continuous variation was ever heritable. This error was the only noticeable point of agreement between the two schools of thought.

The basis was laid for the interpretation of continuous variation on mendelian principles by Johannsen in Denmark and Nilsson-Ehle in Sweden. Johannsen took as his experimental material the dwarf bean (Phaseolus vulgaris) which shares with peas the property of regular natural self-pollination. Thus any bean which is not descended from a deliberate cross-pollination will be expected, on the basis of simple mendelism, to be homozygous for all or nearly all its genes. Its progeny, in their turn, will be to the same extent like their parent and like one another. They, with their descendants, will constitute what Johannsen called a pure line.

Johannsen started in 1900 with 19 beans, from which he derived I9 pure lines. In I90I he had $\$ 24$ beans whose individual weights he recorded in centigrams. These in turn yielded 5,494 beans in I902, and again he determined their weights. The essential results are shown in Table 4. If we compare the weights of the 5,494 beans with those of their 524 mothers we obtain the upper of the two tables. It is clear that the average weight of daughter beans is related to that of the mother, though the differences amongst the daughters' averages are smaller than those amongst the mothers. Thus the mothers range from the $20-\mathrm{cg}$. to the $70-\mathrm{cg}$. class, but the daughters' averages range only from $43 \cdot 8 \mathrm{cgs}$. to $56 \cdot 0 \mathrm{cgs}$.

The mothers can be subdivided further according to the pure lines to which they belong. The lower of the two tables shows the average weights of the daughters from the mothers in the various weight classes of 9 out of the Ig lines. These 9 were the most informative, but the remaining to lines tell the same story so far as they go. 
No matter what the weights of the mother beans from any one line were, the averages of their daughters are the same within the limits of sampling error. Even where the mother beans differed by as much

TABLE 4

A. FREQUENCIES OF BEANS ( $P H A S E O L U S$ VULGARIS) OF VARIOUSWEIGHTS, GROUPEDINTOCLASSES CENTRED ON THE WEIGHTS SHOWN (JOHANNSEN 1909)

\begin{tabular}{|c|c|c|c|c|c|c|c|c|c|c|}
\hline \multirow{2}{*}{$\begin{array}{l}\text { Weight of } \\
\text { mother beans }\end{array}$} & \multicolumn{9}{|c|}{ Weight of daughter beans } & \multirow{2}{*}{ Average } \\
\hline & 10 & 20 & 30 & 40 & 50 & 60 & 70 & 80 & 90 & \\
\hline 20 & - & 1 & 15 & 90 & 63 & 11 & - & - & - & $43 \cdot 8$ \\
\hline 30 & - & 15 & 95 & 322 & 310 & 91 & 2 & - & - & $44 \cdot 5$ \\
\hline 40 & 5 & 17 & 175 & 776 & 956 & 282 & 24 & 3 & 一 & $46 \cdot 2$ \\
\hline 50 & - & 4 & 57 & 305 & 521 & 196 & 51 & 4 & - & $48 \cdot 9$ \\
\hline 60 & - & 1 & 23 & 130 & 230 & 168 & 46 & 11 & 一 & $51 \cdot 2$ \\
\hline 70 & 一 & 一 & 5 & 53 & 175 & 180 & 64 & 15 & 2 & $56 \cdot 0$ \\
\hline Total .. & 5 & 38 & 370 & 1,676 & 2,255 & 928 & 187 & 33 & 2 & $47 \cdot 92$ \\
\hline
\end{tabular}

19 lines, 524 mother beans, 5,494 daughter beans.

B. AVERAGE WEIGHTS OF DAUGHTER BEANS FOR THE VARIOUS CLASSES OF MOTHER BEAN IN NINE OF THE NINETEEN PURE LINES (JOHANNSEN 1909)

\begin{tabular}{|c|c|c|c|c|c|c|c|c|c|c|}
\hline Line & . & I & II & V & VII & XII & XIII & $X V$ & XVIII & XIX \\
\hline ğ & 20 & - & - & - & $45 \cdot 9$ & $49 \cdot 6$ & - & $46 \cdot 9$ & $41 \cdot 0$ & - \\
\hline $\bar{\sigma}$ & 30 & - & - & - & - & - & $47 \cdot 5$ & - & $40 \cdot 7$ & $35 \cdot 8$ \\
\hline E & 40 & - & $57 \cdot 2$ & $52 \cdot 8$ & $49 \cdot 5$ & - & $45 \cdot 0$ & - & $40 \cdot 8$ & $34 \cdot 8$ \\
\hline 5 & 50 & - & $54 \cdot 9$ & $49 \cdot 2$ & - & $45 \cdot 1$ & $45 \cdot 1$ & $44 \cdot 6$ & - & - \\
\hline 해 & 60 & $63 \cdot 1$ & $56 \cdot 5$ & - & $48 \cdot 2$ & $44 \cdot 0$ & $45 \cdot 8$ & $45 \cdot 0$ & - & - \\
\hline$\sum^{\pi}$ & 70 & $64 \cdot 9$ & $55 \cdot 5$ & $50 \cdot 2$ & - & - & - & - & - & - \\
\hline \multicolumn{11}{|c|}{ Average } \\
\hline & line & $64 \cdot 2$ & $55 \cdot 8$ & $51 \cdot 2$ & $49 \cdot 2$ & $45 \cdot 5$ & $45 \cdot 4$ & $45 \cdot 0$ & $40 \cdot 8$ & $35 \cdot 1$ \\
\hline
\end{tabular}

as 40 cgs. this is still true. The lines differ, however, from one to another, the heaviest averaging $64 \cdot 2$ cgs. over all daughter beans and the lightest only $35^{\circ} \mathrm{I}$ cgs. Thus the variation in bean weight within a line was, as expected, non-heritable; the variation between lines being at least in part heritable. 
Four important conclusions follow from this experiment:

In the first place a character can (and in fact nearly always does) show heritable and non-heritable variation simultaneously.

Secondly, the two kinds of variation are indistinguishable by anything but a breeding test. Only experiment can tell us whether the bean is large because of its genotype or in spite of its genotype.

Thirdly, the effects of the environment can either reinforce or counter-balance genetical differences. Thus 70-cg. mother beans from line II are larger than $20-\mathrm{cg}$. mother beans from line XVIII, partly because of the genetical difference, which their offspring reveal as averaging I5-cg., and partly because of non-heritable or external effects. At the same time 6 of the 9 lines of Table 4 B included mother beans in the $60-\mathrm{cg}$. class, even though the genetical values of these beans nuust have differed by nearly $20 \mathrm{cgs}$. in the extreme case. These genetical differences were counter-balanced by non-heritable effects.

Finally, the genetical values of the lines show a graded series from $35 \cdot 1$ to 64.2 cgs., with 110 single great discontinuity. Indeed, the genetical differences that are here proved to exist between these lines are much smaller than the effects that environmental agencies are producing within each line. The genes that are acting must be genes of relatively slight effect.

\section{Cumulative Effects of Genes}

Johannsen's experiments with true-breeding lines, enabled him to demonstrate all these effects and properties of non-heritable variability. They also, however, tell us that there must be many genotypes having different effects on the character; for, even when separated from the non-heritable, the genetical differences are not simply due to a single gene.

Nilsson-Ehle showed by hybridization experiments how this could come about. He crossed varietics of wheat and of oats differing in various characters, which segregated clearly in $F_{2}$ and later generations; but not always into the familiar mendelian ratios. For example, in wheat, an $\mathrm{F}_{2}$ from crossing red- and white-grained varieties might segregate in a ratio of 63 plants with red grain to I 
with white grain. The white-grained plants bred true. So did some of the red-grained ones-37 out of the 63 of them, to be precise. But of the remaining red-grained plants, some gave 3 : I ratios in $F_{3}$, some Is : I ratios and some repeated the $63:$ I ratio. NilssonEhle realized that the parents must have differed by 3 genes (which we can now relate to their having six sets of chromosomes instead of the normal two), the red-producing allelomorph being dominant in each of them, and a single red-producing allelomorph being in itself sufficient, no matter to which gene it belonged, to give a redgrained plant. The genes were all like one another in their effects.

This similarity went even deeper. The reds, though all having red grains, did not all display quite the same degree of redness. The palest reds generally gave 3 : I segregations in the next generation. They had, so to speak, only one dose of red. The next palest might breed true, but give 3 : I segregations in $\mathrm{F}_{2}$ after crossing with white; or they might give a I5: I segregation immediately in the next generation. They had two doses of red, either by being homozygous for one of the genes or by being heterozygous for two of them. It was dosage of red-producing allelonıorphs that nuattered, not the particular genes involved. Thus genes with effects large enough for them to be followed individually by the mendelian method, could be shown to have similar effects, and effects which were cumulative, that is to say supplementing one another, on the phenotype.

It was realized by Nilsson-Ehle and independently by East that similar and supplementary action provided the basis for the graded genetical levels demanded by continuous variation. And, if the effects of the single gene differences were also snall compared with the non-heritable variation, as Johannsen's experiments had shown to be possible, no mendelian ratios would ever be obtained even though the genes segregated in the mendelian fashion. Mendelian inheritance was not limited to cases where it could be detected by the mendelian method. It might indeed be regarded as covering the whole of heritable variation.

We can thus picture a spectrum of variation. At the one extreme are gene differences of so large an expression in the phenotype that they stand out both from their fellows and from the non-heritable agencies which also cause variation in the character. These genes will, 
by their differences, cause discontinuities in the variation of the phenotype and they will therefore be traceable by the mendelian method. Included in this group are such genes as have a lethal effect when homozygous. At the other extreme are genes whose differences cause effects so slight that they are obscured by the remaining variation, both heritable and non-heritable. By itself, the effect of such a single gene might entirely escape detection, but a number reinforcing one another in action might produce quite a large difference between the extreme genotypes. This difference will not, however, create a discontinuity in variation, for two reasons. Between the two extremes there will lie a graded series of genotypes; and the environment will smooth out the small differences between the members of this series. These genes cannot, therefore, be followed individually in mendelian experiment; they must be handled as groups by the biometrical method.

Each character of the phenotype may show the effects of gene differences covering the whole range of the spectrum. Any example of variation is likely to involve several, if not many, of the gene differences of small effect, even where the major part of the variation is due to one or more genes of large effect. Non1-heritable variation is also ubiquitous in its occurrence. It may indeed be stated as a general rule that all characters can and do show variation due to genes of small effect, variation due to genes of large effect, and variation due to non-heritable agencies.

The susceptibility of a gene to detection by the mendelian method is thus dependent, not on its mode of inheritance, but on the size of its effect in relation to its fellows. We can in fact account for continuous variation on the basis of the simultaneous operation of a number of genes. These genes are inherited in the mendelian way, but their differences have effects which are small in relation to those of non-heritable agencies (or at least in relation to the total variation), similar to one another and supplementary to one another (Fig. Is). Such a set of genes constitutes a polygenic system, and its individual members may be conveniently termed polygenes.

Genes such as those with which Nilsson-Ehle was concerned have two of the properties of polygenes. They are of similar and supplementary action. But they cannot be described as polygenes because their effects are so large as to cause a sharp discontinuity in the 


\section{GENOTYPES}

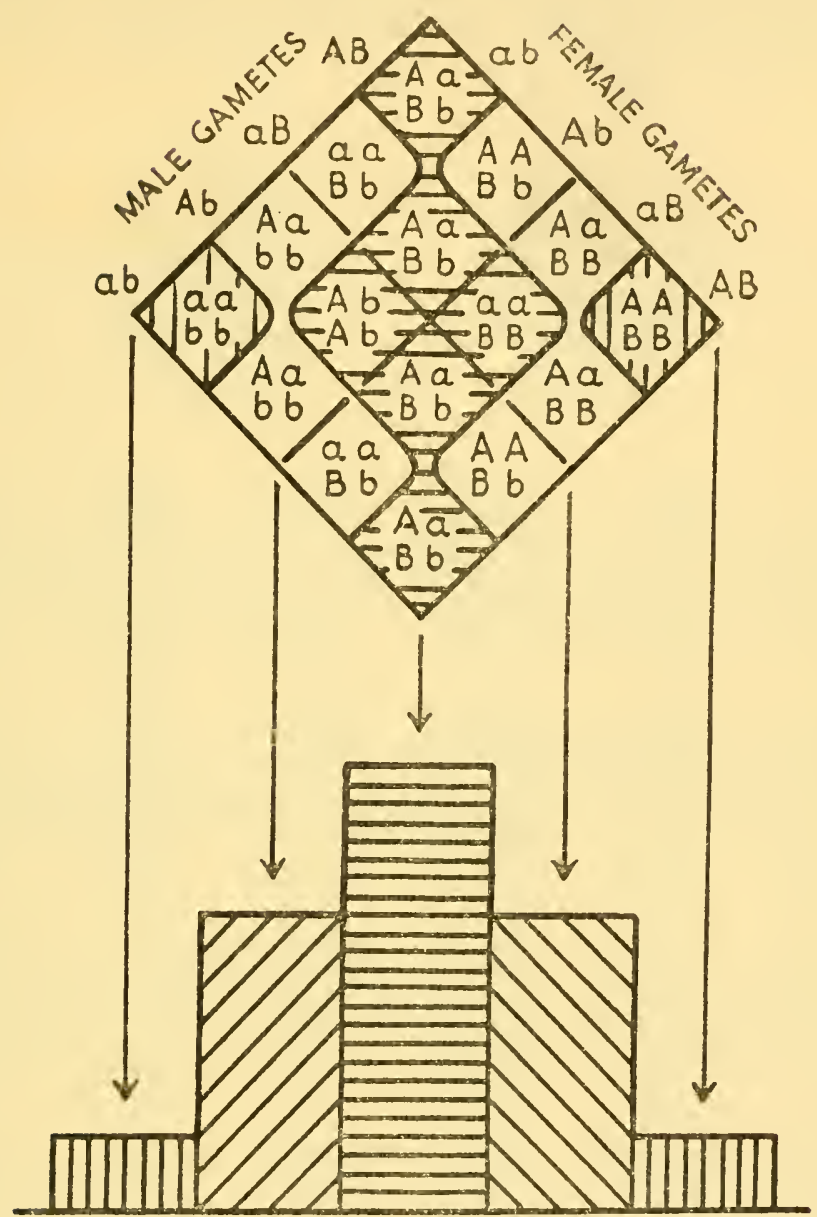

DISTRIBUTION OF PHENOTYPES

FIG. I5.-Diagram showing the relations between the genotypes and the frequency distribution of phenotypes where two genes of equal and additive effect are varying independently of one another and two allelomorphs of each gene are equally common. Dominance is assumed to be absent, so that the phenotypic expression is proportional to the number of capital letters in the genotype. Five phenotypic classes will be produced with two genes of equal effect, seven phenotypic classes with three genes, nine with four, and so on. As the number of classes increases, and as non-heritable influences exert their cffect, the distribution of phenotypes approximates more closely to a continuous curve. 
variation, a discontinuity which permits the analysis of the system by the mendelian method. Genes like these are often termed polymeric genes. All polygenes are therefore polymeric, but not all polymeric genes are polygenes.

\section{Polygenic Systems}

Two questions immediately arise about polygenic systems. First we see that such systems can explain continuous variation; but is continuous variation in fact to be ascribed solely to their operation ? Or to put it another way, we have been led to postulate systems of genes which cannot be analysed by the mendelian method. How then can we be sure that these genes are inherited in accordance with mendelian principles? Secondly, since we cannot follow the genes individually, we cannot describe the properties of these systens in terms of individual segregation ratios and linkage values. How then are we to understand and express and predict the behaviour of the systems? To the first of these questions we must now turn. The second will be discussed in the next chapter.

We have seen in the previous chapter that mendelian inheritance reflects the fact that the genes concerned are carried by the chromosomes. The cytological study of meiosis shows that two properties must mark this type of transmission. All the genes must show segregation, and those which are borne in the same chromosome must show linkage with one another. If, therefore, we can establish that the determinants of heritable continuous variation show both segregation and linkage, we cannot avoid the conclusion that they are nuclear genes and that as such they will conform to mendelian principles.

Where a stock or line of a species has long been inbred we must expect that its members will be homozygous for all or nearly all their genes and that differences between them will be non-heritable. Johamnsen's experience confirmed this with beans. If, now, we cross two such lines with one another, the $\mathrm{F}_{1}$ will be genetically as uniform as its parents. Though it will be heterozygous for any genes in which the parental lines differed, all the members of this generation will be alike in their heterozygosity. Thus variation in the $F_{1}$ will also be non-heritable. But in the $\mathrm{F}_{2}$ the individuals will not all be alike 
genetically, for segregation will have occurred during the formation of gametes from the $F_{1}$ zygotes. Heritable variation will have been added to the non-heritable, and we must expect therefore that the
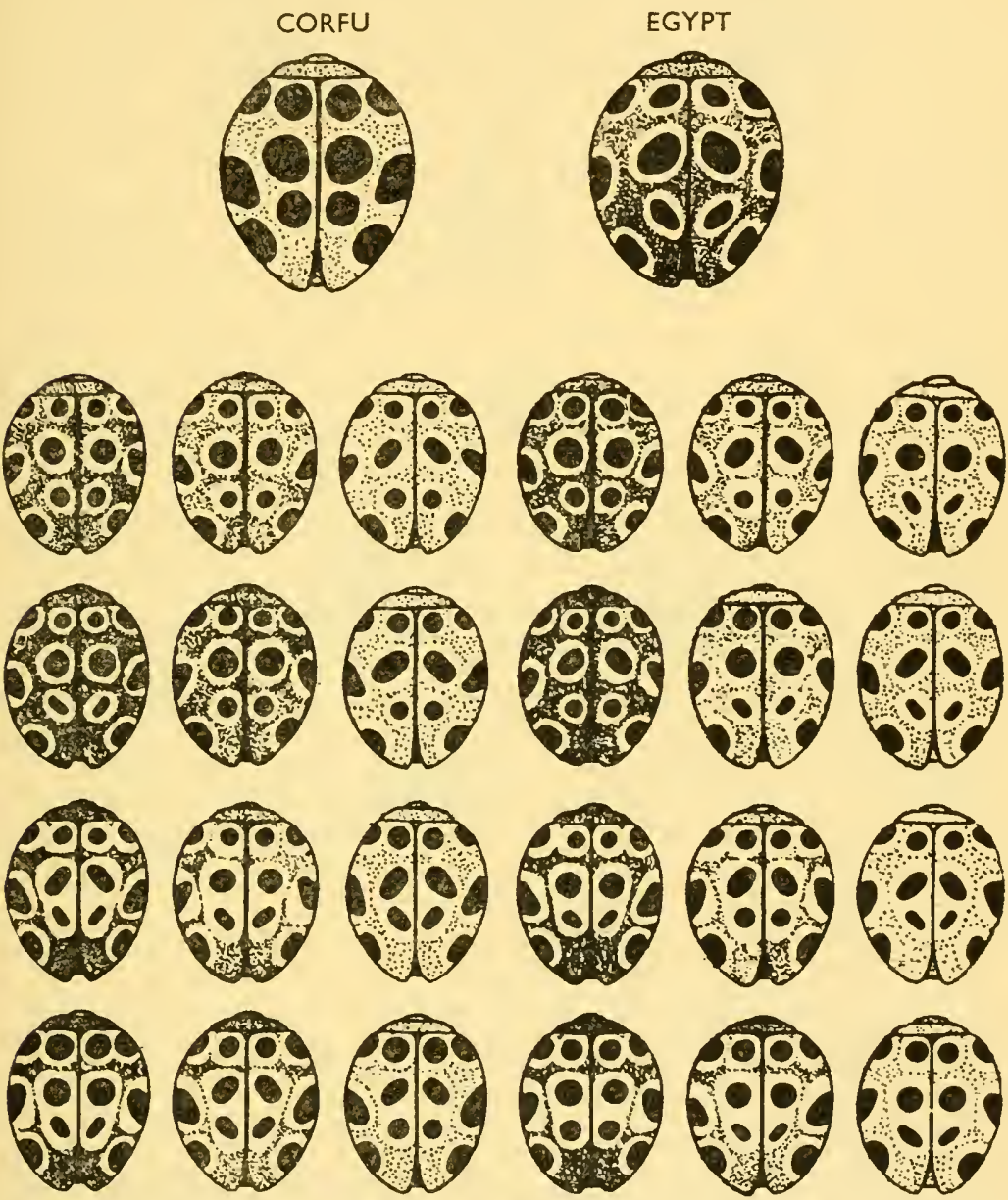

Fig. I6.-Parents and $F_{2}$ progeny of a cross between two races of the ladybird Epilachna chrysomelina showing polygenic segregation in respect of the black flecks and the dark colouring between them (after Bauer and Timoféeff-Ressovsky, 1943).

frequency distribution of the $\mathrm{F}_{2}$ will cover a wider range, have a larger spread, than those of the individual parent lines and $F_{1}$. In biometrical terms the $F_{2}$ should have a larger variance than parents or $\mathrm{F}_{1}$, because this variance will have a $1 \mathrm{cw}$, heritable component 
added to the non-heritable component which it shares with its predecessors.

Now any gene segregating in $\mathrm{F}_{2}$ will, by Mendel's principles, be homozygous in half the $\mathrm{F}_{2}$ individuals and heterozygous in the other half. If, therefore, we raise $F_{3}$ 's by inbreeding the $F_{2}$ individuals, half will show segregation for each gene and half will not. Taking all genes into account we shall expect that the average variance of all the $\mathrm{F}_{3}$ 's will lie between the variance of $\mathrm{F}_{2}$ on the one hand, and the variances of parents and $F_{1}$ on the other. For the average heritable component of the $\mathrm{F}_{3}$ variance will be smaller than that of $F_{2}$, but not, of course, so small as those of parents and $F_{1}$ where variation was solcly, or nearly solcly, non-heritable. But not all the $\mathrm{F}_{3}$ 's will be alike in either variances or means. The variances will reflect the different numbers of genes heterozygous in the different $\mathrm{F}_{2}$ mothers. Furthermore, for all genes in which the mother was homozygous, each $\mathrm{F}_{3}$ individual must carry the same allelomorphs. The mean expression of the character in each $\mathrm{F}_{3}$ will therefore be correlated with the expression in its $\mathrm{F}_{2}$ mother, just as Galton found the heights of fathers and daughters to be correlated in man.

So, even though we cannot follow segregation by ratios of types in the mendelian fashion, we can still hope to detect its occurrence by the biometrical properties of frequency distributions. And we can make so many predictions about these properties, that if all of them are borne out in experiment we can entertain no doubt that mendelian segregation is occurring.

A characteristic experiment of this kind is illustrated in Fig. I7. It shows the inheritance of corolla length in a cross between two lines of Nicotiana longiflora as recorded by East (19I5). The corolla lengths are expressed in millimetres and the data are grouped for convenience of presentation into classes each covering a range of $3 \mathrm{~mm}$. and centred on $34,37,40$, etc., mm. The frequencies are shown as percentages of the individuals of the various families which fell into the different classes. The variances, measuring the spreads of the distributions, are much the same for the two parents and the $F_{1}$, whose mean is just about midway between those of the parents. The $F_{2}$ has a much larger variance, while those of the four $F_{3}$ 's shown differ amongst themselves and lie between the values for $F_{2}$ on the one hand and parents and $F_{1}$ on the other. The arrows 


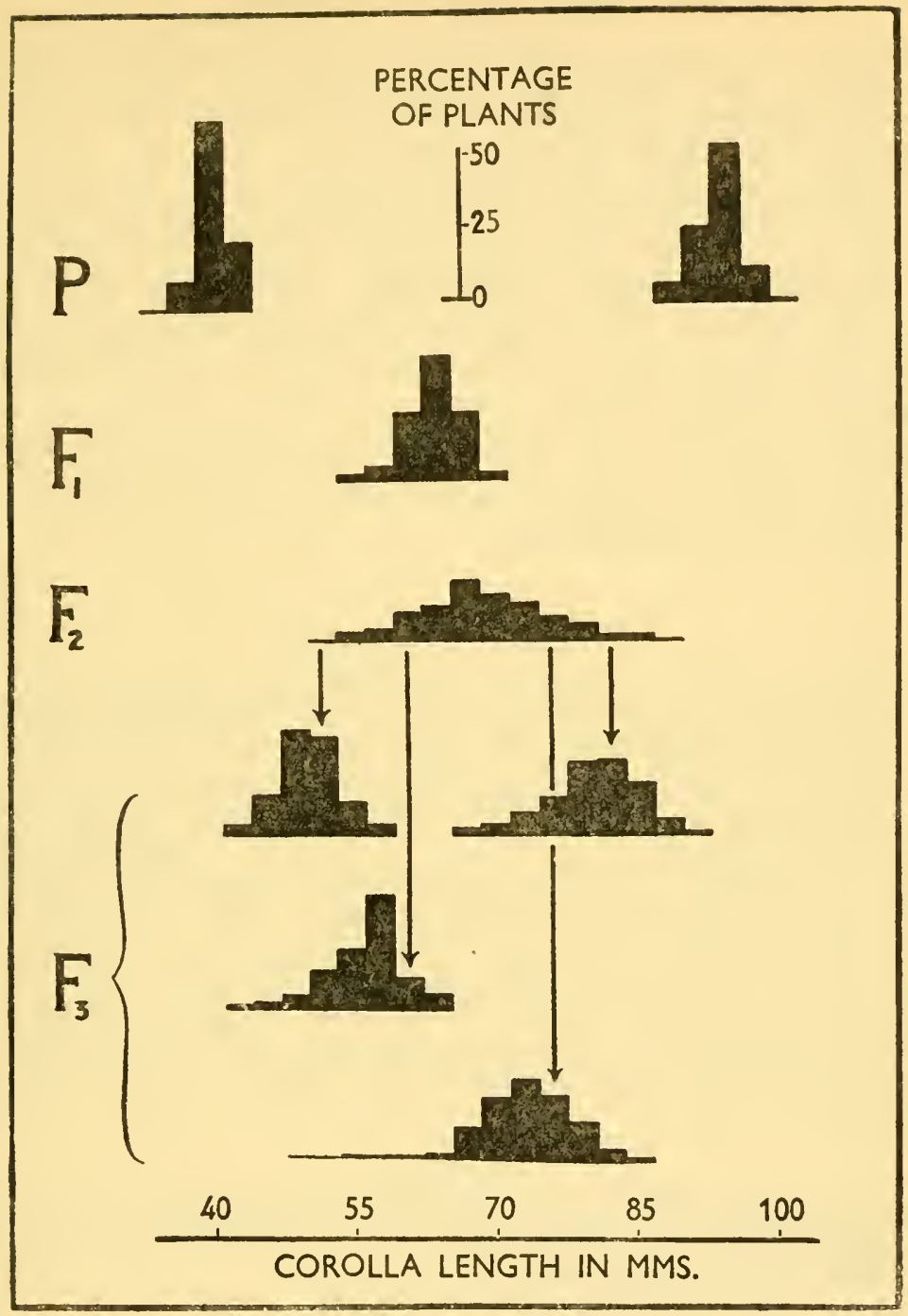

FIG. 17.-Frequency distribution of corolla length in families of Nicotiana longiflora, showing segregation for the polygenes controlling the differences between the twa parent strains $(\mathrm{P})$ in this character. Variation in $\mathrm{P}$ and $\mathrm{F}_{1}$ is wholly non-heritable. To this is added in $F_{2}$ the heritable component depending on segregation. The spread of the frequency distribution is thus higher in $F_{2}$ than in $F_{1}$ and $P$. In $F_{3}$ the average heritable component of variation is half that in $\mathrm{F}_{2}$, but it varies from family to family according to the number of genes for which the $F_{2}$ parent was heterozygous. The spread of the frequency distribution is thus variable in $\mathrm{F}_{3}$ though always lying between that of $F_{2}$ on the one hand, and of $F_{1}$ and $P$ on the other. The mean corolla length of each $F_{3}$ is related to the corolla length of its $F_{2}$ parent, indicated by the source of the relevant arrow (based on East, 1915). 
indicate the classes in which the $F_{2}$ plants fell, from which the $F_{3}$ 's came. It is clear that the $F_{3}$ mean is correlated with the value of the $\mathrm{F}_{2}$ individual from which it came.

In this experiment all our predictions are verified, and we cannot doubt that the polygenes controlling corolla length show segregation in the way to be expected from Mendel's principles. We may observe, too, that in this experiment the $\mathrm{F}_{1}$ 's from reciprocal crosses are alike. Male and female parents contribute equally to $F_{1}$ as would be expected with nuclear inheritance.

Many experiments of this kind have been performed and all have agreed, within the limitations imposed by their size and structure, with the behaviour expected.

\section{Linkage of Polygenes}

Not a few of the experiments which show polygenic segregation also reveal linkage of polygenes and major genes. The first case was that of Sax (1923) in dwarf beans. He made a cross between a strain with large coloured beans and another which had smaller white beans. In the $F_{2}$ there was a clear segregation into a ratio of 3 plants with coloured beans to I with white beans (Table 5). $F_{3}$ families were grown and by their aid the coloured plants were classified into homozygotes and heterozygotes. These appeared in the ratio of $\mathrm{I}: 2$. Thus seed colour was clearly under the control of a single gene which has an effect large enough to be followed by the mendelian method.

TABLE 5

AVERAGE WEIGHTS OF BEANS (IN CG.) FROM COLOURED AND WHITE MEMBERS OF AN F IN PHASEOLUS VULGARIS (SAX, 1923)

\begin{tabular}{|c|c|c|}
\hline Number of plants & Colour constitution & $\begin{array}{c}\text { Average bean } \\
\text { weight }\end{array}$ \\
\hline $\begin{array}{l}45 \\
80 \\
41\end{array}$ & $\begin{array}{l}\text { Coloured }\left\{\begin{array}{l}P P \\
P p\end{array}\right. \\
\text { White }\end{array}$ & $\begin{array}{l}30 \cdot 7 \\
28 \cdot 3 \\
26 \cdot 4\end{array}$ \\
\hline
\end{tabular}

Seed size, on the other hand, proved to be a continuously varying character. Segregation could be detected by the greater spread of 
the seed weight distribution in $\mathrm{F}_{2}$, but no major discontinuities appeared. The heritable variation is thus polygenic. But when Sax determined the average weights of the seeds in his three classes, distinguished by the seed colour gene, viz. homozygous coloured $(P P)$, heterozygous coloured $(P p)$ and white $(p p)$, he found that they differed. The $P P$ plants had the largest seeds and the $p p$ plants the smallest, just as the coloured parental strain had larger seeds than the white one.

Such an association between size and colour would be expected if some, at least, of the polygenes controlling seed size were linked with the major gene controlling colour. And since the major gene must be carried in the nucleus, so must the polygenes. On one point, however, the experiment is not decisive. That part of the difference in seed size which was associated with the colour difference could have been a secondary effect of the major gene which itself determined the colour difference. In other experiments, however, this possibility can be ruled out. We may take, as an example, an experiment done by Mather and Harrison on the polygenic system controlling variation in the number of hairs on the undersides of the 4th and sth abdominal segments of Drosophila melanogaster. With hair-number the class groupings to be used in the frequency distribution are of course decided for us, just as though we could not measure height except in whole inches.

Drosophila melanogaster, it will be recalled, has three large pairs of chromosomes and one small pair. This last is, in fact, so small that it can be neglected for most purposes. Most of the genes belonging to any polygenic system will be found on the three larger ones. Now those three large chromosomes can be marked by certain gene differences which are capable of being followed by the mendelian method, and which are particularly convenient because heterozygotes for each of them can be recognized phenotypically. The genes used in the experiment were Bar $(B)$ affecting the shape of the eye, Plum $(P m)$ affecting the colour of the eye and Stubble $(S b)$ affecting the shape of certain bristles (though not, it should be remarked, altering the number of abdominal hairs so far as is known). $B$ marks the X chromosomes, $P m$ the second chromosome (II) and $S b$ the third (III). These genes were combined with inverted pieces of chromosome, which as we shall see in Chapter 6 reduce, or in 
cxtreme cases suppress, recombination, so that the chromosomes tend to segregate as wholes at meiosis.

Two wild-type strains of Drosophila were used in the experiment. These showed no differences from one another which could be followed by the mendelian method, but they did differ from one another in their average numbers of abdominal hairs. The one, which we may denote as S, showed an average of $59^{\circ} 2$ hairs on females and the other $O$, only $43 \cdot 5$ hairs per femalc. These strains were each crossed to a third carrying the marker gencs, $B, P m$ and $S b$. The $F_{1}$ females, which showed the effects of $B, P m$ and $S b$ in their phenotypes, were crossed back to males from their respective wildtype parental strains. Eight classes could be distinguished by the simultancous segregation of $B, P m$ and $S b$ in the backcross progenies, and the hairs were counted on a number of female flics in each of these classes. The average hair numbers are shown in Table 6.

TABLE 6

A. AVERAGE NUMBERS OF HAIRS ON FLIES OF EIGHT CLASSES DISTINGUISHED BY THE ANCESTRY OF THEIR CHROMOSOMES

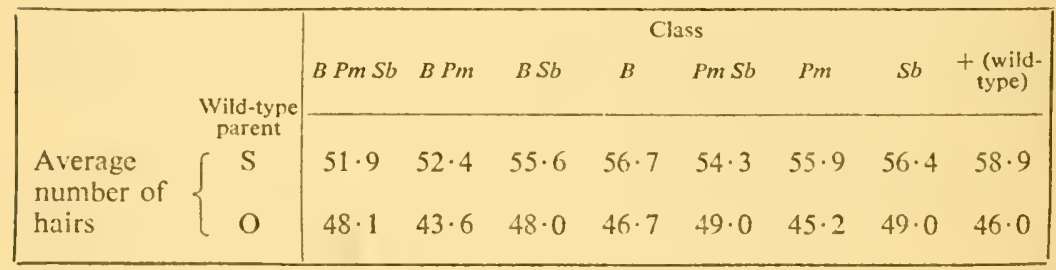

B. THE DIFFERENCES IN AVERAGE HAIR NUMBER ATTRIBUTABLE TO THE THREE LARGE CHROMOSOMES

\begin{tabular}{|c|ccc|c|}
\hline $\begin{array}{c}\text { Excess over tester in } \\
\text { chromosome }\end{array}$ & $\mathrm{X}$ & II & III & Tota\} \\
\hline $\mathrm{S}$ & 2.66 & $\begin{array}{l}3.35 \\
0.95\end{array}$ & $\begin{array}{r}1.64 \\
3.08\end{array}$ & $\begin{array}{c}7.65 \\
1.40\end{array}$ \\
\hline $\mathrm{O}$ & 0.73 & 2.40 & 4.72 & 9.05 \\
\hline
\end{tabular}

Now the $B P m S b$ class has one representative of cach of the thrce 
large chromosomes from the $B P m S b$ tester stock, and one representative of each from the wild-type strain under test. The $B P m$ class is in the same case for chromosomes X and II, but it has both its representatives of chromosome III from the wild-type strain. If, therefore, chromosome III from the wild strain differs from its mate, or homologue, from the tester stock in any genes affecting hair number, there must be a corresponding difference in the average numbers of hairs shown by the $B P m S b$ and $B P m$ classes in the backcross. The only limitation is that completely dominant genes from the wild-type strain will obviously have no effects which can be detected in a backcross of this kind. Similar estimates of the difference in chromosume III can be found by the comparisons between the average hair numbers of the $B$ and $B S b$ classes, the $P m$ and $P m S b$ classes and the + (wild-type) and $S b$ classes. The average difference in hair number due to genes in chromosome III can be found therefore as

$$
\frac{1}{4}\left(B P_{m}-B P_{m} S b+B-B S b+P_{m}-P_{m} S b+(+)-S b\right)
$$

where $B P m$ stands for the average hair number of the $B P m$ class and so on.

The effects of the other two chromosomes can be found similarly as:-

$\mathrm{X}$ chromosome $\frac{1}{4}\left(P_{m} S b-B P_{m} S b+P_{m}-B P_{m}+S b-B S b\right.$

$$
+(+)-B)
$$

II chromosome $\frac{1}{4}\left(B S b-B P_{m} S b+B-B P m+S b-P_{m} S b\right.$

$$
+(+)-P m) \text {. }
$$

The excesses of the three chromosomes of the two strains, $\mathrm{S}$ and $\mathrm{O}$, over the marked homologues from the tester, arrived at in this way, are shown in the lower part of the Tablc.

The differences between the chromosomes of either wild-type stock and those of the tester might be influenced by a secondary action of the marker genes, $B, P m$ and $S b$. Other obscrvations on the effects of these genes would not lead us to expect such secondary action, and indeed we can rule it out decisively in another way. The $\mathrm{S}$ stock has an unmarked $\mathrm{X}$ chromosome which, so far as this cxperiment goes, gives rise to the production of 2.66 more hairs than the tester $\mathrm{X}$ chromosome marked by the gene $B$. The $\mathrm{X}$ chromo- 
some of the $O$ stock shows a corresponding excess of only 0.73 over the same tester $\mathrm{X}$. Then the $\mathrm{X}$ from $\mathrm{S}$ gives rise to $2.66-0.73$ or $\mathrm{I} \cdot 93$ more hairs than does the $\mathrm{X}$ from $\mathrm{O}$; and this difference cannot be due to secondary action of the $\mathrm{B}$ gene, or indecd to any other major gene, for $\mathrm{O}$ and $\mathrm{S}$ differed in no gene of effect sufficiently large to be followed by the mendelian method. In the same way chromosome II from $\mathrm{S}$ exceeded its homologue from $\mathrm{O}$ by $3 \cdot 35-0.95$ or $2 \cdot 40$, and chromosome III by $1 \cdot 64+3.08$ or $4 \cdot 72$. Thus, we have observed a total excess of $\mathrm{I} \cdot 93+2 \cdot 40+4 \cdot 72$ or $9^{\circ}$ os hairs caused by the three large chromosomes of $S$ as compared with those of $\mathrm{O}$.

This method of assaying the action of the various chromosomes by comparison with the tester depends on comparing hair number in flics homozygous for the chromosome under test with that of flies heterozygous for that same chromosome and for the marked chromosome. As we have seen, this prevents us observing the full effect of any gene whose allelomorph in the wild strain is not fully recessive to that in the tester stock. Indeed, fully dominant allelomorphs must escape detection altogether by such a comparison. Now the $\mathrm{F}_{1}$ females in both crosses, $\mathrm{S} \times$ tester and $\mathrm{O} \times$ tester, differed in hair number from those in their parent wild strains. The genes from $\mathrm{S}$ and $\mathrm{O}$ were therefore not all, or not fully, dominant over their allelomorphs from the tester. These $F_{1}$ females also, however, differed from one another. So the genes from $\mathrm{S}$ and $\mathrm{O}$ were furthermore not all, or not fully, recessive to their allelomorphs from the tester. Wc, therefore, expect our survey to reveal only part of the difference which would be seen between flics homozygous for chromosomes from the wild strains on the one hand and from the tester stock on the other.

If we assume that dominance is absent or balanced in the two directions (an assumption agrecing well with our general experience of continuous variation), we should expect our tests to trace half the difference between each wild type strain and the tester. The comparison of the chromosomes from the two wild-type strains should then reveal half the effects of the genes by which $O$ and $S$ differ in their three large chromosomes. Now females within strain $\mathrm{S}$ have on the average i $5^{\circ} 7$ more hairs than those within $O$. We have found a grand difference of 9 hairs in our chromosome tests, and 
since 9 exceeds half of $15 \cdot 7$, we have no reason to doubt that the heritable difference between $\mathrm{S}$ and $\mathrm{O}$ is wholly due to differences in nuclear genes.

What position have we now reached? First, we see that continuous variation is capable of being caused by nuclear genes, which, to be sure, work in special systems. Furthermore, we see that the heritable part of this variation is wholly due to such genes. We see this both from the lack of differences between reciprocal crosses and from the balance-sheet of variation which the linkage experiments enable us to draw up. Finally, we have been able to infer the action of individual genes although these genes are not individually recognizable. We been able to do so simply because these individual genes are all transmitted in the same way, by the very mendelian inheritance whose principles they were at one time supposed to contradict.

\section{REFERENCES}

BAUR, H., and TIMOrÉETF-RESSOVSKY, N. W. 1942. Genetik und Evolutionsforschung bei Tieren. Die Evolutionen der Organismen (Ed. G. Heberer), 335-429. EAST, E. M. I9IS. Studies on size inheritance in Nicotiana. Genetics, I: I64-I76. GALTON, F. I889. Natural Inheritance. London. JOHANNSEN, W. I909. Elemente der exakten Erblichkeitslehre. Jena. MATHER, K. I943. Polygenic inheritance and natural selection. Biol. Revs. 18: 32-64. MATHER, K. I949. Biometrical Genetics. London.

Mather, K., and harrison, B. J. I949. The manifold effects of selection. Heredity (in the press).

NILSSON-EHLE, H. I909. Krenzingsuntersuchnngen an Hafer nnd Weizen. Lund. PEARSON and LEE, quoted by FISHER, R. A. I947. Statistical Methods for Research Workers. Ioth ed., Edinburgh.

SAX, K. I923. The association of size differences with seed-coat pattern and pigmentation in Phaseolus vulyaris. Genetics, 8: 552-560. 


\section{THE BIOMETRICAL ANALYSIS}

Additive Scales Constitution of the Statistics The Test of Dominance and Linkage Randonly Breeding Popnlations and Correlations between Relatives Effective Factors or Units The Union of Genetics and Biometry

THE KNOWLEDGE THAT THE POLYGENES are borne on the chromosomes provides us with a base from which to explore the genetical properties of continuous variation. We cannot follow these genes in their individual segregations by the mendelian method; we can nevertheless feel confident that they will segregate on mendelian principles. That is to say, the various types of family, raised by crossing and inbreeding, will contain the different homozygotes and heterozygotes in the proportions which mendelian experiments and a knowledge of chromosome numbers have led us to expect.

Before we turn, however, to the interpretation of the biometrical quantities, means, variances and covariances, in terms of mendelian segregations, we must first consider one other problem. This is the problem of the scale to be used in measuring or representing the degree of expression of the character. This problem does not arise with the mendelian method where we compare the frequencies of distinct types, but it is basic to all biometrical analysis, where we compare the magnitudes of individual expressions. An appropriate scale will go far to ensure the success of an analysis: an inappropriate scale may lead to serious misjudgment.

The scales that we use in measuring character expression are the ones which experience has shown to be most convenient. They do not bear any necessary relation to the ways in which genes act or supplement one another in action. They may be satisfactory for the purpose of genetic analysis or they may not. We need, in fact, some vvay of deciding whether a given scale, be it the one we customarily use for measuring length, weight or any other property, or be it some transformation of the customary scale, as for example into log measure, is satisfactory for our purpose.

Our task is that of interpreting the means, variances and 
covariances in terms of which we can express the genetical properties of our families. This is most easily done if the various agencies, genes and non-heritable factors, add together in their effects on the phenotype. That is to say, if the difference produced in the phenotype by substituting one allelomorph of a gene for another, or one environment for another, is the same no matter what the effects of the other genes or environmental factors may be. This may, of course, be merely an ideal, for it may be impossible to find a scale on which all genes supplement one another's action in an additive way. Since, however, we cannot isolate the genes and discover their individual properties we can measure only their average behaviour. A scale will, therefore, be satisfactory on which the gene and non-heritable effects are simply additive on the average.

Now, if we cross two true-breeding lines and then backcross the $F_{1}$ to one of them, mendelian theory shows us that the backcross will contain equal numbers of individuals homozygous, like the parent to which the backcross is made, and heterozygous, like the $F_{1}$, in regard to each gene by which the parents differedequal, that is, apart from sampling error. Then the average expression of the character in the backcross, will, in so far as any one of the genes is concerned, be mid-way between the parent and the $F_{1}$. If, and only if, the cffects of the genes are additive on the scale used, will this relation also hold between the parental miean, the $F_{1}$ mean, and the backcross mean, for all the genes taken together. In other words the two backcrosses provide us with two tests of adequacy of the scale. Letting $\overline{\mathrm{B}}_{1}$ be the mean measurement of the individuals in the backcross to the parent whose mean is $\overline{\mathrm{P}}_{1}$, the scale must be such that:-

$$
2 \overline{\mathrm{B}}_{1}=\overline{\mathrm{P}}_{1}+\overline{\mathrm{F}}_{1} \text { and } 2 \overline{\mathrm{B}}_{2}=\overline{\mathrm{P}}_{2}+\overline{\mathrm{F}}_{1}
$$

In the same way one quarter of the individuals in an $F_{2}$ are like each parent and half like the $F_{1}$ in respect of each genc, so that if the effects of the genes are additive $4 \overline{\mathrm{F}}_{2}=2 \overline{\mathrm{F}}_{1}+\overline{\mathrm{P}}_{1}+\overline{\mathrm{P}}_{2}=2 \overline{\mathrm{B}}_{1}+2 \overline{\mathrm{B}}_{2}$. Similar tests of the scale can be devised using $\overline{\mathrm{F}}_{3}$, the mean measurement of $F_{3}$, and so on.

The test of the additiveness of heritable and non-heritable contributions to the variation is somewhat different. The variation of 
true-breeding lines and of their $F_{1}$ 's is all non-heritable, but the lines and the $\mathrm{F}_{1}$ 's differ from one another in their genotypes. Then, if comparable environmental differences are to contribute to the variation (or rather to the quantities representing it) independently of the genetical differences, we must find a scale on which the variances of the frequency distributions of the lines and of their $\mathrm{F}_{1}$ 's are the same, even though their means differ for genetical reasons.

If the scale used in taking the measurements fails to satisfy these tests, the measurements may be transformed by taking logs, antilogs, square-roots, squares, or in any other consistent way, and the tests repeated on the new scalc. If, as may sometimes happen, no scale is found to be satisfactory on all counts, some suitable compromise has to be adopted.

\section{Constitution of the Statistics}

Mendelian theory thus helps us to cope with this first problem of finding a scale suitable for genetical analysis. It is equally essential for our analysis of the statistics which are obtained from the measurements represented on that scale.

Let us denote by $2 \mathrm{~d}_{\mathrm{a}}$ the difference in phenotype between two individuals raised in the same environment and differing genetically only by being homozygous for different allelomorphs of the gene $A-a$. Then, if neither allelomorph is dominant, their heterozygote will have a phenotype midway between the homozygotes. If there is dominance then let the heterozygote depart by $h_{a}$ from this mid point. The phenotypes corresponding to the three genotypes are then related in the following way:-

$\begin{array}{ccc}a a & A a & A A \\ -\mathrm{d}_{\mathrm{a}} & \mathrm{h}_{\mathrm{a}} & \mathrm{d}_{\mathrm{a}}\end{array}$

The effect of gene $B-b$ can be similarly measured in terms of $d_{b}$ and $h_{b}$, and so on, the convention being adopted that the capital letter denotes the allelomorph making for increased manifestation of the character in all genes. Thus $A$ is not of necessity dominant over $a: h_{a}$ may depart from the mid-point in either the positive or the negative direction. The same is true of $h_{b}$, and furthermore 
the sign of $h_{b}$ may not be the same as that of $h_{a}$. The dominances may be reinforcing or opposing one another.

Two true breeding lines raised in a comparable range of environments will have mean phenotypes differing by:-

$$
2\left[S\left(d_{+}\right)-S\left(d_{-}\right)\right]
$$

where $S\left(d_{+}\right)$is the sum of the $d$ increments added by all the genes represented by increasing allelomorphs $\left(\mathrm{A}, \mathrm{B}\right.$, etc.) and $\mathrm{S}\left(\mathrm{d}_{-}\right)$the sum of the increments added by all the genes represented by decreasing allelomorphs (a, b, etc.) in the parent with the larger manifestation of the character. The average of the two lines, the mid-parent as we may call it, will be the zero point from which the $\mathrm{h}$ increments can be measured. It is the natural origin of the scale.

The $F_{1}$ between the two lines will be uniformly heterozygous for all the genes in which its parents differed and so will differ from the mid-parent by $h_{a}+h_{b} \ldots=S(h)$, taking the signs of the various $h$ 's into account. Thus $S(h)$ can be zero even though each $h$ is not zero, because of the opposing signs of the h's, or, in genetical terms, because of the opposing dominances. In the same way the parental difference may be zero, i.e. $\left[S\left(\mathrm{~d}_{+}\right)-\mathrm{S}\left(\mathrm{d}_{-}\right)\right]=\mathrm{O}$, no matter what values $d_{a}, d_{b}$, etc., may have, because the increasing and decreasing allelomorphs of the different genes may be balanced in the parents.

The simple comparison of an $F_{1}$ with its parents is sufficient to establish the dominance relations of a gene in mendelian genetics. But, as we can now see, it is not sufficient to show even the average dominance relations of a polygenic system in biometrical genetics. For if we divide the departure of the $F_{1}$ mean from the mid-parent by half the parental difference, a ratio which is commonly used to represent the degree of dominance of single genes, we obtain:$\frac{S(h)}{s\left(d_{+}\right)-S\left(d_{-}\right)}$and this obviously bears no simple relation to the ratios $\frac{h_{a}}{d_{a}}, \frac{h_{b}}{d_{b}}$ and so on. It can vary between zero and an infinitely large value, according to the way in which the increasing and decreasing allelomorphs are distributed between the parents, and the way in which the h's reinforce or oppose one another. One thing 
it can show us, however. If this fraction departs significantly from zero, then $\mathrm{S}(\mathrm{h})$ cannot be zero and at least some of the genes are showing some dominance in the direction of the departure.

In $\mathrm{F}_{2}$ the genotypes $A A, A a$ and aa occur with the relative frequencies $\frac{1}{4}, \frac{1}{2}, \frac{1}{4}$, so that the mean measurement as affected by this gene will be $\frac{1}{4} \mathrm{~d}_{\mathrm{a}}+\frac{1}{2} \mathrm{~h}_{\mathrm{a}}-\frac{1}{4} \mathrm{~d}_{\mathrm{a}}$ or $\frac{1}{2} \mathrm{~h}_{\mathrm{a}}$. Then, taking all genes into account, the mean of $F_{2}$ will depart from the mid-parent by $\frac{1}{2} \mathrm{~S}(\mathrm{~h})$. The mean of $\mathrm{F}_{3}$ similarly departs from the mid-parent by $\frac{1}{4} \mathrm{~S}(\mathrm{~h})$. We can therefore learn no more about the genes' dominance relations from the $F_{2}$ and $F_{3}$ means than we can from the $\mathrm{F}_{1}$ mean.

The information to be gained merely from a study of the means is thus very limited. The different variances and covariances which we can calculate are, however, more helpful. The parental lines and the $F_{1}$ will show only non-heritable variation. We can consider their variances as including only one component, E. The variance of $\mathrm{F}_{2}$ will, however, contain a heritable portion, to which each gene will contribute. The contribution of gene $A-a$ to this variance can be ascertained by finding the deviations from the mean of the measurements of $A A, A a$ and $a a$ individuals, squaring them and adding them up. The $F_{2}$ mean in respect of this gene is $\frac{1}{2} h_{a}$, so that the deviation of an $A A$ individual will be $\mathrm{d}_{\mathrm{a}}-\frac{1}{2} \mathrm{~h}_{\mathrm{a}}$, the deviation of $A a, \mathrm{~h}_{\mathrm{a}}-\frac{1}{2} \mathrm{~h}_{\mathrm{a}}$, and that of $a a,-\mathrm{d}_{\mathrm{a}}-\frac{1}{2} \mathrm{~h}_{\mathrm{a}}$. Now one quarter of the individuals will be $A A$, one half $A a$ and one quarter $a a$, so that the contribution of gene $A-a$ to the variance of $\mathrm{F}_{2}$ inust be:-

$$
\begin{gathered}
\frac{1}{4}\left(d_{a}-\frac{1}{2} h_{a}\right)^{2}+\frac{1}{2}\left(h_{a}-\frac{1}{2} h_{a}\right)^{2}+\frac{1}{4}\left(-d_{d}-\frac{1}{2} h_{d}\right)^{2} \\
=\frac{1}{2} d_{a}{ }^{2}+\frac{1}{4} h_{a}{ }^{2}
\end{gathered}
$$

The remaining genes in which the parental lines differed will make similar contributions to the variance of $F_{2}$. Provided that the genes are unlinked, these contributions will be independent, and the total heritable variation will be the simple sum of the contributions made by the individual genes. Then if we write $D=d_{a}{ }^{2}+d_{b}{ }^{2}+$ $\mathrm{d}_{\mathrm{c}}{ }^{2} \ldots$ and $\mathrm{H}=\mathrm{h}_{\mathrm{a}}{ }^{2}+\mathrm{h}_{\mathrm{b}}{ }^{2}+\mathrm{h}_{\mathrm{c}}{ }^{2} \ldots$ the heritable variance of $\mathrm{F}_{2}$ becomes $\frac{1}{2} \mathrm{D}+\frac{1}{4} \mathrm{H}$. Since the variance will also contain a nonheritable component, the full formula must be $\frac{1}{2} \mathrm{D}+\frac{1}{4} \mathrm{H}+\mathrm{E}$.

Two important properties of the variation are revealed by this formula. In the first place, the effect of dominance, as measured 
by $\mathrm{H}$, is separable from the effect of differences between the homozygous phases of the genes, as measured by $\mathrm{D}$. In the second place, $D$ is compounded of items like $d_{a}{ }^{2}$, and $H$ of items like $h_{a}{ }^{2}$. The value of $\mathrm{D}$ will therefore be the same no matter how the increasing and decreasing allelomorphs of the various genes were associated with one another in the parents. And the value of $\mathrm{H}$ will be unaffected by the signs, whether positive or negative, of the $h$ increments, i.e. by the directions of dominance of the individual genes. $\mathrm{H}$ is, of course, expected to be $\mathrm{O}$ in the absence of dominance from all genes, and the ratio $\sqrt{\frac{\overline{\mathrm{H}}}{\overline{\mathrm{D}}}}$ provides us with a measure of the average dominance of the genes irrespective of whether dominance is in the same direction for all genes or not, and irrespective of the distribution of the genes between the parental lines. It provides us, in fact, with the information about dominance which could not be obtained by a simple comparison of the parental and $F_{1}$ means.

Were the variance of $F_{2}$ the only statistic available it would obviously be impossible to estimate the separate values of $\mathrm{D}$ and $\mathrm{H}$. Backcrosses and the $F_{3}$ generation, however, help us here. They supply us with additional information about $\mathrm{D}$ and $\mathrm{H}$, and they may be used in combination with $\mathrm{F}_{2}$ to effect the separation of $\mathrm{D}$ and $\mathrm{H}$ both from one another and from the non-heritable variation E. Taken separately, the variances of the backcrosses cannot be expressed in terms of $\mathrm{D}, \mathrm{H}$ and $\mathrm{E}$; but if we add together the variances of the two backcrosses, one to each parental line, they have the joint value of $\frac{1}{2} \mathrm{D}+\frac{1}{2} \mathrm{H}+2 \mathrm{E}$. The environmental component is, of course, $2 \mathrm{E}$ because each backcross must show as much non-heritable variation as the single $\mathrm{F}_{2}$ family of the same size.

It will be seen from Fig. I 7 that the $\mathrm{F}_{3}$ generation is able to provide us with three statistics. These will be derived from (I) the variation between the different $\mathrm{F}_{3}$ families taken as wholes; (2) the variation within the different $\mathrm{F}_{3}$ families; and (3) the relation between the expression of the character in the individual $\mathrm{F}_{3}$ families and their immediate parents in $\mathrm{F}_{2}$. The statistics which express these relations are:-

I. The variance of the means of $\mathrm{F}_{3}$ families, which has the value $\frac{1}{2} \mathrm{D}+\frac{1}{16} \mathrm{H}+\mathrm{E}$. 
2. The mean of the variances of different $F_{3}$ families, which has the value $\frac{1}{4} \mathrm{D}+\frac{1}{8} \mathrm{H}+\mathrm{E}$.

3. The covariance of means of $\mathrm{F}_{3}$ families with the measurements of the $\mathrm{F}_{2}$ parents, which has the value $\frac{1}{2} \mathrm{D}+\frac{1}{8} \mathrm{H}$.

In a properly designed experiment, the effects of the environment on average manifestation of the character in $F_{3}$ will bear no correlated relation to the effect of the environment on the character in the $\mathrm{F}_{2}$ parent. The covariance, therefore, has no $\mathrm{E}$ component. The $\mathrm{E}$ component of the variance of $\mathrm{F}_{3}$ means will in general differ from that of the other statistics since the variation of an average value will be influenced to a different extent than that of a single value by the vagaries of the environment. We must thus distinguish between $E_{1}$, the non-heritable component of variation of single individuals, and $E_{2}$, the non-heritable component of variation of family means which will usually, though not always, be smaller than $E_{1}$. The non-heritable component appropriate to individuals, $\mathrm{E}_{1}$, will appear in all the formulac except that for the variance of $\mathrm{F}_{3}$ means, which will contain $E_{2}$. $E_{1}$ and $E_{2}$ can be estimated directly from the variation within the true-breeding parent lines and $F_{1}, E_{1}$ as the variance of single individuals and $E_{2}$ as the variance of the means of groups containing the same number of individuals as the $F_{3}$ familics.

\section{The Test of Dominance and Linkage}

An example will illustrate the use of these results. The two species of Antirrhinum, majus and glutinosum, differ in, among other characters, their heights. In a true-breeding strain of majus the average length of the leading shoot was $2 \mathrm{I}^{\cdot} 45$ inches. No strain of glutinosum known to be truc-breeding was available, but one which was empirically observed to be no more variable phenotypically than the strain of majus, had leading shoots whose average length was 9.12 inches. The $F_{1}$ between the species gave a mean measurement of 17.08 inches.

An $F_{2}$ was raised which showed no segregation of genes with major effects on height, the variation being continuous and depending, presumably, on a polygenic system. The $F_{1}$ was also backcrossed to both parents, and $\mathrm{I}_{9} \mathrm{~F}_{3}$ families were raised by 
self-pollinating $\mathrm{F}_{2}$ plants. The values found for the various statistics are given in Table 7 . The estimate of $E_{2}$ obtained directly from the parents and $F_{1}$ is lower than that of $E_{1}$, in the way generally expected to be the case.

The seven observations provide us with seven equations for the estimation of the four quantities $\mathrm{D}, \mathrm{H}, \mathrm{E}_{1}$ and $\mathrm{E}_{2}$. The estimation is undertaken, therefore, by the method of least squares (Mather I949) and we find:-

$$
\begin{aligned}
& D=25 \cdot 708 \\
& H=-10.778 \\
& E_{1}=4.995 \\
& E_{2}=0.146
\end{aligned}
$$

These estimates can be substituted in the expectation formulae to give calculated values for the seven statistics as shown in column four of the Table.

Two points are inmmediately striking about the results of this analysis; a negative value has been obtained for $\mathrm{H}$ which, like $\mathrm{D}$, was defined as a sum of squares and therefore cannot be negative, except as a result of sampling variation; and the agreement of observation with expectation in the Table is rather poor, especially for the mean variance of $F_{3}$ where the discrepancy is nearly 25 per cent of expectation. The analysis has not achieved full success. The reasons for this partial failure become apparent when we consider the consequences of linkage between the polygenes.

When genes are unlinked their contributions to the various statistics are simply additive. This is not so when they are linked. If $\mathrm{p}$ is the frequency of recombination between the two genes $A-a$ and $B-b$, the contribution to $\mathrm{D}$ as it appears in the variance of $\mathrm{F}_{2}$ is $\mathrm{d}_{\mathrm{a}}{ }^{2}+\mathrm{d}_{\mathrm{b}}{ }^{2} \pm 2 \mathrm{~d}_{\mathrm{a}} \mathrm{d}_{\mathrm{b}}(\mathrm{r}-2 \mathrm{p})$, the third term being added when the genes are coupled and subtracted when they are repulsed. This reduces to $d_{a}{ }^{2}+d_{b}{ }^{2}$ when $p$ has its free value of $0 \cdot 5$. In the sanie way, the contribution to $\mathrm{H}$ in the variance of $\mathrm{F}_{2}$ is $\mathrm{h}_{\mathrm{a}}{ }^{2}+\mathrm{h}_{\mathrm{b}}{ }^{2}+$ $2 h_{a} h_{b}(I-2 p)^{2}$. Where more than two genes are involved we have as many terms of the kind $2 d_{a} d_{b}(r-2 p)$ in $D$ and $2 h_{d} h_{b}(r-2 p)^{2}$ in $\mathrm{H}$, as there are pairs of gencs. With three genes there are three such terms, with four genes six terms, and so on. All of these terms as they appear in D depend on $(\mathrm{r}-2 \mathrm{p})$ where $\mathrm{p}$ is the frequency 
of recombination betwcen the two genes concerned, and all in $\mathrm{H}$ similarly depend on $(\mathrm{r}-2 \mathrm{p})^{2}$. It will thus be noted that the effects of linkage of polygenes are expressible independently of the linear order.

Exactly the same linkage terms appear in $\mathrm{D}$ and $\mathrm{H}$ in variance of $F_{2}$, variance of $F_{3}$ mcans, and covariance of $F_{2}$ and $F_{3}$. But whilc the same number of linkage terms appears in the $D$ and $H$ of mean variance of $F_{3}$ 's, those in $D$ now depend on $(I-2 p)^{2}$ instead of

TABLE 7

THE INHERITANCE OF HEIGHT IN ANTIRRHINUM MAJUS $\times$ GLUTINOSUM

\begin{tabular}{|c|c|c|c|c|}
\hline \multirow[t]{2}{*}{ Statistic } & \multirow{2}{*}{ Expectation } & \multirow[t]{2}{*}{ Observed } & \multicolumn{2}{|c|}{ CaIculated } \\
\hline & & & No linkage & Linkage \\
\hline Variance of $F_{2}$ & $\frac{1}{2} \mathrm{D}+\frac{1}{4} \mathrm{H}+\mathrm{E}_{1}$ & $15 \cdot 838$ & $15 \cdot 155$ & $15 \cdot 663$ \\
\hline $\begin{array}{l}\text { Summed variances of back- } \\
\text { crosses }\end{array}$ & $+\mathrm{D}+2 \mathrm{H}+2 \mathrm{~F}$. & & 17.455 & $16.750 *$ \\
\hline Variance of $\mathrm{F}_{3}$ means & $\frac{1}{2} \mathrm{D}+\frac{1}{16} \mathrm{H}+\mathrm{E}_{2}$ & $11 \cdot 938$ & $12 \cdot 326$ & $11 \cdot 585$ \\
\hline Mean variance of $F_{3}$ 's & $\frac{1}{4} \mathrm{D}+\frac{1}{8} \mathrm{H}+\mathrm{E}_{1}$ & $12 \cdot 550$ & $10 \cdot 075$ & $12 \cdot 550^{*}$ \\
\hline Covariance of $F_{2}$ parent and & & & & \\
\hline $\mathrm{F}_{3}$ family $\ldots \quad \ldots \quad \ldots$ & $\frac{1}{2} \mathrm{D}+\frac{1}{8} \mathrm{H}$ & $10 \cdot 681$ & $11 \cdot 507$ & $11 \cdot 211$ \\
\hline Variance in parents and $F_{1}$ & & & & \\
\hline Single individuals .. & $E_{1}$ & $3 \cdot 250$ & $4 \cdot 995$ & $3 \cdot 426$ \\
\hline Means of groups .. & $\mathrm{E}_{2}$ & 0.534 & $0 \cdot 146$ & 0.887 \\
\hline
\end{tabular}

* The assumption of linkage permits a perfect fit.

on $(\mathrm{r}-2 \mathrm{p})$, and those in $\mathrm{H}$ on $(\mathrm{r}-2 \mathrm{p})^{2}\left(\mathrm{r}-2 \mathrm{p}+2 \mathrm{p}^{2}\right)$ instead of on $(1-2 p)^{2}$. Wc can thus see how linkage may explain the discrepancies in the analysis of the Antirrhinum results. Similarly, although the linkage terms in $\mathrm{D}$ of the summed backcross variances are just like those of $\mathrm{D}$ in $\mathrm{F}_{2}$ variance, the linkage terms in $\mathrm{H}$ of the backcross depend on $(\mathrm{I}-2 \mathrm{p})$ as compared with $(\mathrm{I}-2 \mathrm{p})^{2}$. When $p=0.5$ for frec recombination, all these linkage terms vanish from all statistics and $\mathrm{D}$ and $\mathrm{H}$ are constant over the whole range.

The test of linkage is, therefore, the test of differences in the values of $\mathrm{D}$ and $\mathrm{H}$ over the various types of family. In the experiment with shoot length in Antirrhinum, D and H should be constant, cven with linkage, over variance of $F_{2}$, variance of $F_{3}$ means and covariance 
of $F_{2}$ and $F_{3}$. The $D$ and $H$ of the summed variances of backcrosses and of the mean variance of $\mathrm{F}_{3}$ 's will, however, differ both from one another and from those of variance of $\mathrm{F}_{2}$ with linkage. If, therefore, we find the best estimates of $\mathrm{D}$ and $\mathrm{H}, \mathrm{E}_{1}$ and $\mathrm{E}_{2}$, omitting the

\section{ANTIRRHINUM}

VARIATION IN HEIGHT

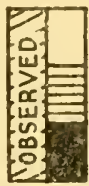

NON-HERITABLE

UNFIXABLE HERITABLE -H

FIXABLE HERITABLE -D

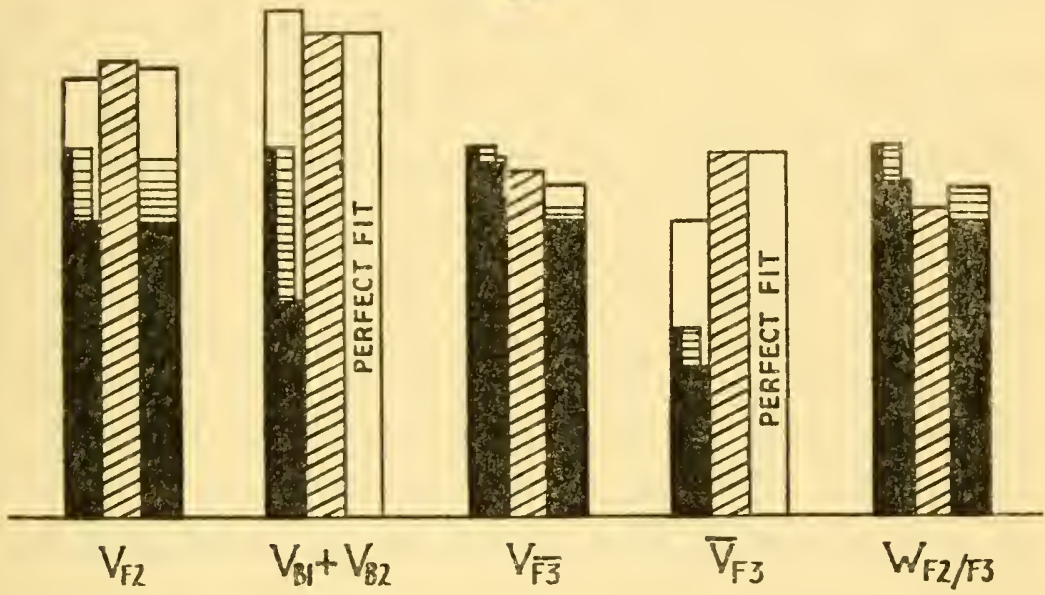

FIG. 18.-The components of variation in height of plants of Antirrhinum majus $\times$ glutinosum. For each statistic the centre column of the histogram shows the value observed, the left column that expected on the assumption of no linkage, and the right column that expected assuming the possibility of linkage. The compositions of the expected values are shown in terms of $\mathrm{D}, \mathrm{H}$, and $\mathrm{E}$. The best estimate of $\mathrm{H}$ is negative when linkage is assumed to be absent, so that the contribution of $\mathrm{H}$ is overlapped by the contributions of $\mathrm{D}$ and $\mathrm{E}$ in the left column. When the possibility of linkage is assumed, $V_{B 1}+V_{B 2}$ and $\bar{V}_{F 3}$ become perfect fits. $W_{F 2 / F 3}$ has no E component.

$$
\begin{aligned}
V_{\mathrm{F} 2} & =\text { variance of } F_{2} \\
V_{B 1}+V_{B 2} & =\text { summed variances of backcrosses } \\
V_{\bar{F} 3} & =\text { variance of } F_{3} \text { means } \\
\bar{V}_{\mathrm{F} 3} & =\text { mean variance of } F_{3} \text { families } \\
W_{\mathrm{F} / \mathrm{F} 3} & =\text { covariance of } \mathrm{F}_{2} \text { measurement and } \mathrm{F}_{3} \text { mean. }
\end{aligned}
$$

summed backcross variances and the mean variance of $F_{3}$ 's, these should give a better fit than do the values found for them using all the data, should linkage be interfering with the result. If the fit is not improved there can be no evidence of linkage. 
When we omit the summed variances of backcrosses and the mean variance of $\mathrm{F}_{3}$ 's from the Antirrhinum results, we find:-

$$
\begin{aligned}
& D=20.369 \\
& H=8.209 \\
& E_{1}=3.426 \\
& E_{2}=0.887,
\end{aligned}
$$

and again expected values can be calculated for the various statistics as shown in column five of the Table.

The analysis is now much more satisfactory (Fig. 18). In the first place a positive value has been obtained for $\mathrm{H}$, though it is too small to afford any reliable indication of dominance. Secondly, the discrepancies between observation and expectation have been very much reduced in Table 7. This is true, not merely for the summed variances of backcrosses and mean variance of $F_{3}$ 's, for which linkage allows us to assume a perfect fit, but also for the other statistics, notably $E_{1}$ and the variance of $F_{2}$. The results indicate that at least some of the polygenes are not recombining freely. Furthermore, the mean variance of $\mathrm{F}_{3}$ is larger than would be expected from the $\mathrm{F}_{2}$, so that the linkage must be preponderantly in the repulsion phase, i.e. of the kind $\frac{\mathrm{Ab}}{\mathrm{a}}$. Large, therefore, as was the phenotypic difference in height between the species, it did not represent the full difference between the genotypes, for some of the genes must have been counter-balancing one another's effects in these repulsion linkages.

\section{Randomly Breeding Populations and Correlations between Relatives}

The types of family which were used in this experiment on shoot length are not, of course, exhaustive. In particular, we can raise families of the third generation by intercrossing pairs of individuals taken at random from $F_{2}$. These biparental families will, in fact, be the only type available in the third generation of experiments with most animals, where true $\mathrm{F}_{3}$ 's are obviously impossible to obtain. The same range of statistics is available from biparentals as from $\mathrm{F}_{3}$ 's, viz. variance of biparental means, mean variance of biparentals, and covariance of $\mathrm{F}_{2}$ parents and their biparental 
progenies. The values of these statistics also depend on $\mathrm{D}$ and $\mathrm{H}$, thus:-

$\begin{array}{ll}\text { Variance of biparental means } & \frac{1}{4} \mathrm{D}+\frac{1}{16} \mathrm{H}+\mathrm{E}_{2} \\ \text { Mean variance of biparentals } & \frac{1}{4} \mathrm{D}+\frac{3}{16} \mathrm{H}+\mathrm{E}_{1} \\ \text { Covariance of } \mathrm{F}_{2} \text { and biparentals } & \frac{1}{4} \mathrm{D}\end{array}$

They may be used to supplement or to replace the statistics from $F_{3}$ families in the estimation of $\mathrm{D}$ and $\mathrm{H}$.

When we distinguish between the biparental families obtained by the intercrossing of different pairs of individuals from $F_{2}$, we break the heritable variance down into two parts: the variance of the means, which measures variation between families, and the mean variance, which measures variation within families. The total heritable variation of this generation is the sum of these two parts, viz. $\left(\frac{1}{4} \mathrm{D}+\frac{1}{16} \mathrm{H}\right)+\left(\frac{1}{4} \mathrm{D}+\frac{3}{16} \mathrm{H}\right)=\frac{1}{2} \mathrm{D}+\frac{1}{4} \mathrm{H}$. It is exactly the same as the heritable variance of $\mathrm{F}_{2}$ itself. And if we breed still another generation of biparental progenies, the pairs of parents being taken at random, without distinction of family, from the first biparental generation, we find that its heritable variance is once again $\frac{1}{2} \mathrm{D}+\frac{1}{4} \mathrm{H}$. This variance is, in fact, characteristic of the random mating system used. The variances will be the same from generation to generation provided that the system of random mating is continued, and provided also, of course, that the environmental variation, and with it the $\mathrm{E}$ component, is constant. The covariance of parent and offspring is also constantly $\frac{1}{4} \mathrm{D}$ under this system of mating, with no $\mathrm{E}$ component appearing in it.

In the particular case under discussion we commenced with the $\mathrm{F}_{2}$ of a cross between two true-breeding lines, so that the frequencies of the two allelomorphs of each gene by which the parents differed, $A-a, B-b$, etc., must be equal. The variance and the parent-offspring covariance still retain their values of $\frac{1}{2} \mathrm{D}+\frac{1}{4} \mathrm{H}+\mathrm{E}$ and $\frac{1}{4} \mathrm{D}$ even, however, where the frequencies of $A$ and $a, B$ and $b$, etc., are not equal in the group of individuals. The variation formulae are therefore characteristic of all randomly breeding groups.

The effect of variation in the gene frequencies appears in a different way, viz. in the contributions which the genes make to $\mathrm{D}$ and $\mathrm{H}$. With equal gene frequencies and no linkage we have $\mathrm{D}=\mathrm{S}\left(\mathrm{d}^{2}\right)$ and $\mathrm{H}=\mathrm{S}\left(\mathrm{h}^{2}\right)$; but when allelomorph $A$ has the frequency $u_{a}$, 
and a the frequency $v_{\mathrm{a}}\left(=\mathrm{I}-\mathrm{u}_{\mathrm{a}}\right)$, etc., we find that the contribution of the gुenc $A-a$ to $D$ is $4 \mathrm{u}_{\mathrm{a}} \mathrm{v}_{\mathrm{a}}\left[\mathrm{d}_{\mathrm{a}}+\mathrm{h}_{\mathrm{a}}\left(\mathrm{v}_{\mathrm{a}}-\mathrm{u}_{\mathrm{a}}\right)\right]^{2}$ and to $\mathrm{H}$ is $\mathrm{I} 6 \mathrm{u}_{\mathrm{d}}{ }^{2} \mathrm{v}_{\mathrm{a}}{ }^{2} \mathrm{~h}_{\mathrm{a}}{ }^{2}$ so that the general formulac are

$$
D=S\left\{4 u v[d+h(v-u)]^{2}\right\} \text { and } H=S\left(16 u^{2} v^{2} h^{2}\right)
$$

These reduce to the original forms when $\mathrm{u}=\mathrm{v}=0.5$ for all genes. The special virtue of equal gene frequencies for analysis now becomes clear. Unless $\mathrm{u}=\mathrm{v}=0.5$ for all genes, the full effects of all the dominance relations is not represented by $\mathrm{H}$; some of them appear in $\mathrm{D}$.

Using these general forms of $\mathrm{D}$ and $\mathrm{H}$, we can represent the relations between parents and offspring of any randomly brecding group or population in terms of the variance, $\frac{1}{2} \mathrm{D}+\frac{1}{4} \mathrm{H}+\mathrm{E}$, and the parent-offspring covariance, $\frac{1}{4} \mathrm{D}$.

The parent-offspring corrclation is given by $r_{p / o}=\frac{W_{p / o}}{\sqrt{V_{p} \cdot V_{o}}}$. We can now sec that $\mathrm{W}_{\mathrm{p} / \mathrm{o}}=\frac{1}{4} \mathrm{D}$, while $\mathrm{V}_{\mathrm{p}}=\mathrm{V}_{\mathrm{o}}=\frac{1}{2} \mathrm{D}+\frac{1}{4} \mathrm{H}+\mathrm{E}$, so that:-

$$
\mathrm{r}_{\mathrm{p} / \mathrm{o}}=\frac{\frac{1}{4} \mathrm{D}}{\frac{1}{2} \mathrm{D}+\frac{1}{4} \mathrm{H}+\mathrm{E}}
$$

$\mathrm{D}, \mathrm{H}$ and $\mathrm{E}$ are quadratic quantities and so must always be positive. The maximum correlation which parents and offspring can show for simple genetic reasons within a randomly breeding population must, thercfore, be $\mathrm{r}=0.5$ when $\mathrm{H}=\mathrm{E}=\mathrm{O}$.

We can find the covariances between other pairs of relatives, of which the most useful is that between pairs of siblings. This, too, can be represented in terms of our three components of variation as:-

$$
\mathrm{W}_{\mathrm{s} / \mathrm{s}}=\frac{1}{4} \mathrm{D}+\frac{1}{16} \mathrm{H}
$$

Then the fraternal corrclation will be:-

$$
\mathrm{r}_{\mathrm{s} / \mathrm{s}}=\frac{\frac{1}{4} \mathrm{D}+\frac{1}{16} \mathrm{H}}{\frac{1}{2} \mathrm{D}+\frac{1}{4} \mathrm{H}+\mathrm{E}}
$$

This, too, will have a maximum of 0.5 when $\mathrm{H}=\mathrm{E}=\mathrm{O}$. But when dominance is present, so that $\mathrm{H}>\mathrm{O}, \mathrm{r}_{\mathrm{s} / \mathrm{s}}$ must cxceed $\mathrm{r}_{\mathrm{p} / \mathrm{o}}$ becausc of the additional term $\frac{1}{16} \mathrm{H}$ in its numerator. 
Within human groups mating is not quite at random, but it is near enough to being so for the parental and fraternal correlations to show the relations we should expect. It has been found by Pearson and Lec, in respect of the human cubit measurement, that:-

$$
r_{p / 0}=0.4180 \text { and } r_{s / s}=0.4619
$$

The slight departure from random mating, and the likelihood that members of one family will develop in environments more alike than those of unrelated people, make detailed estimates of D, H and $\mathrm{E}$ somewhat untrustworthy. We can, however, draw some general conclusions from these correlations. They are both so near to their maximum value of 0.5 that the non-heritable component of variation must be small relative to the heritable. And since the fraternal correlation exceeds the parental we have evidence of dominance of the genes controlling variation in human cubit measurement.

\section{Effective Factors or Units}

We can now return to crosses between true-breeding lines. Where these lines differ in such a way that the increasing allelomorphs ( $A, B, C$, etc.) of all the genes by which they differ are assembled in one of them, and all the decreasing allelomorphs $(a, b, c$, etc.) in the other, the mean measurement of each will depart from the mid-parent by $\mathrm{S}(\mathrm{d})$. Now if we care to assume that all the genes have equally large effects, i.e. $\mathrm{d}_{\mathrm{a}}=\mathrm{d}_{\mathrm{b}}=\mathrm{d}_{\mathrm{c}} \ldots=\mathrm{d}, \mathrm{S}(\mathrm{d})=\mathrm{kd}$ where $k$ is the number of genes. Now we can estimate $D$ which, when the genes have equal effects and there is no linkage, will have the value $\mathrm{kd}^{2}$. Then the square of the departure of each line from the mid-parent, when divided by D, will give us an estimate of the number of genes, for it will be

$$
\frac{\mathrm{S}^{2}(\mathrm{~d})}{\mathrm{D}}=\frac{(\mathrm{kd})^{2}}{\mathrm{kd}^{2}}=\mathrm{k}
$$

The shortconings of this estimate are obvious from the assumptions which must be made to obtain it. In particular it will be an underestimate if the increasing and the decreasing allelomorphs are not concentrated in the opposite parents, or if the effects of the genes are not all equal. A second method of estimating $\mathrm{k}$ is based 
on the variances of $\mathrm{F}_{3}$ families. It overcomes the first difficulty because it demands no assumption about the distribution of allelomorphs between the parental lines; but it magnifies the second difficulty since differences between the effects of the individual genes reduce this estimate of $k$ to an even greater extent than the first one. Both estimates are also reduced by linkage.

When all these reservations have been made, the estimates of $k$ are still instructive. Our experience of the fine gradations which continuously varying characters show, would lead us to expect that many genes would be concerned in the polygenic system governing any particular variation. We find, however, that the estimate of $\mathrm{k}$ is never large and is commonly as low as 5 or even less. The value given by the Antirllinum data, using the first method of estimation, is, for cxample, $\mathrm{k}=\mathrm{I} \cdot 9$. The reason for this is that $\mathrm{k}$ is not truly an estimate of the number of genes, but of a different unit which we may call the effective factor.

To see what effective factors are, let us consider a hypothetical case when the parental lines differ by a large number of genes, scattered along the length of every chromosome. Genes which do not recombine must always appear as one in segregation. Now the number of chiasmata, upon the formation of which depends recombination of genes within a chromosome, is usually no more than one or two per bivalent. Not all the genes, then, will be separated from their neighbours in the chromosome by chiasmata. In fact, one chiasma will break the chromosome into two pieces, the genes within each of which will segregate together. Two chiasmata will give three such segments, and so on. These segments are the physical basis of the effective factors whose number is estimated by $\mathrm{k}$. At most this number cannot exceed the haploid number of chromosomes plus the mean number of chiasmata in the nucleus. It must generally be less, for some segments will be unmarked by segregating genes. Furthermore any variation, such as we know normally to occur, in the positions in which chiasmata form, will lead to the effective factors varying in their genic content and effect. This, too, as we have already secn, will reduce the estimate of $k$.

The effective factors which emerge from the segregation secn in an $F_{2}$ may be broken down further in an $F_{3}$ owing to the formation of chiasmata in new positions at meiosis in the $\mathrm{F}_{2}$ individuals. Thus 
the effective factors are not necessarily fixed in the course of an experiment and may increase in number. Their history will depend on the circumstances of localization of crossing-over which we have already noticed, and also, as we shall see later, on various restrictions of crossing-over which arise in hybrids.

When the individual genes have large effects unlike one another, we can follow them and count them as individuals by the mendelian method. A single recombination between them, however many tests must be made before it is found, is sufficient to show that two distinct genes are at work. Such a unique recombination can bc detected without ambiguity by the mendelian technique when the genes have different effects on the phenotype. But the members of a polygenic system cannot be followed individually in inheritance, nor their recombinations identified as individual events. In counting the units of polygenic inheritance we are therefore forced to use a coarser criterion, that of the occurrence of a particular rccombination frequency, usually so per cent. The effective factors whose number we estimate are thus, in the general case, not ultimate polygenes. Indeed we can have no certainty that any unit of polygenic inheritance, which we may find and whose properties we may deternine, is an ultimate unit.

We are debarred from studying polygenes as individuals by the inherent limitations of genetical method. Rather we must follow them as they are organized into effective factors. These units are not final in the way that individual major genes arc. They can be broken down into smaller parts by recombination and they can presumably be synthesized by bringing together their parts through recombination. Their properties must depend on the way the genes are put together to make the factor as much as on the genes themselves.

\section{The Union of Genetics and Biometry}

Biometrical genetics is built upon the foundation of mendelism. It could exist in no other way, and the relative failure of Galton and the early biometricians to achieve an understanding of inheritance was due to the lack of the foundation which Mendel, by an entircly different technique, was able to supply. There is thus no conflict between biometrics and mendelism. On the contrary, biometrical 
genetics extends mendelism to all, or nearly all, variation. In making this extension we learn to measure variation by new quantitics and to understand it in terms of new units: quantitics and units as appropriate to the study of continuous variation as the segregation ratios, recombination frequencies and individual genes are to the study of discontinuous variation.

The mendelian foundation upon which the biometrical method rests has been laid by the study of major differences. The geneticist has chosen the variants which he needed for this purpose from amongst the wealth of variation which living species offer him. He has not been concerned with the phenotype except in so far as its changes and differences marked for him changes and differences of the genotype whose understanding was his primary aim. No such choice is open to the breeder of crops and stock. His aim must always be to adjust and improve the performance of his plants and animals in respect of some character, yicld, quality, disease resistance or whatever it may be, which is chosen for him. He must be prepared to make good use of whatever heritable variation, continuous or discontinuous, his individuals may show. It is for this purpose that he, no less than the student of ultimate principles, needs the integration of the mendelian and biometrical techniques which we can now attain.

\section{REFERENCES}

FISUIER, R. A. I9I8. The correlation between relatives on the supposition of mendelian inheritance. Trans. Roy. Soc. Edin., 52: 399-433.

FISHER, R. A., IMMER, F. R., and TEDIN, O. I932. The genetical interpretation of statistics of the third degree in the study of quantitative inheritance. Genetics, I7: I07-I24.

MATHER, K. I949. Biometrical Genetics. London.

PANSF, V. G. I940. The application of genetics to plant brecding. II. The inheritance of quantitative characters and plant breeding. $J$. Genet., 40: 2 $\$_{3}-302$. 


\section{BASES OF CHANGE}

Polyploidy and Polysomy Strutural Change Misdivision of the Centromere

Deficiency and Balunce Intergenic and Intragenic Clange:

Presence and Absence Sontatic Mutation

The Gene as a Unit of Change

SO FAR WE HAVE CONSIDERED experiments in which genes have appeared as fixed in the chromosome, and chromosomes, apart from crossing-over, as fixed in the nucleus. In any large experiment, however, we find individuals which do not correspond to the predictions that we are justified in making on the basis of the simple rules of segregation and recombination. We find new and unexpected phenotypes. These variants, sports or mutants have changed in their heredity. What kinds of changes occur and how do they come about? To answer these questions we call on a variety of methods and observations.

\section{Polyploidy and Polysomy}

If we cut down a plant of the tomato, Lycopersicum esculentum, a proportion of the shoots which subsequently grow from the cut surface are of somewhat stouter growth. They have larger flowers, but the ripe fruits are smaller with fewer seeds. These shoots, which can be propagated as cuttings, and from their sceds, have 48 chromosomes instead of the usual 24. They are tetraploid with four sets of chromosomes $(2 n=4 x)$ instead of diploid $(2 n=2 x)$.

Doubling of the whole nucleus arises from a failure of mitosis to complete itself: the chromosomes have divided without the daughter nuclei scparating and without the cell dividing. This failure is very common in plants and can be readily induced by treatment of the seed, the growing point of the shoot, the young embryo, or the germ mother-cells with colchicine, with high or low temperatures, and in other ways. Tetraploids have larger cells and are generally larger and more robust than their diploid forbears. Their fertility is usually reduced. Otherwise their rescmblance to 
the diploids is close and they are consequently common in horticulture.

In a great many plants and a few animals triploid $(3 \mathrm{x})$ types occur. They arise by failure of meiosis in one of their parents. Gametes containing the diploid instead of the haploid number of chromosomes are produced, and by uniting with haploid mates give triploid progeny (Fig. 19). A cross between diploid and tetraploid will, of course, give the same result. Triploids are again more robust than their parents which they otherwise resemble closely except in being, as a rule, highly infertile. Triploid apples and pears, however, set just enough of the ten seeds in each fruit to provide a satisfactory crop without overburdening the tree and this places them amongst the most valuable varieties.

The immediate cause of the infertility of triploids is seen when we examine such of their progeny as survive. For the triploid rarely, if ever, produces triploid offspring. Its gametes contain all the possible combinations of the extra or odd chromosomes (Fig. 28); but most of these die either in the pollen or, if carried by the eggs, in the embryo-sac or young embryo, and the survivors have, as a rule, only one or two beyond the diploid number. These survivors are always of reduced vigour and abnormal form, but their abnormalities are different from those of triploids or tetraploids. They are no longer general in character, but rather specific. They affect different parts of the plant or animal, which consequently seems unbalanced. The type of its unbalance, we find, goes with the particular extra chromosomes which it gets from the extra chromosome set of the parent. In Datura stramonium and the tomato $(2 \mathrm{x}=24)$ there are 12 types of trisomic (with $24+$ I chromosomes) corresponding to each of the 12 chromosomes in the haploid set ( $f$. Fig. 67). The trisomics stand between the diploid and the triploid in fertility.

Similar off-types also come directly from the diploid in many plants and animals. In Drosophila melanogaster, triploid flies occasionally appear amongst the diploid males and females. And also some with the small fourth chromosome represented once or three times instead of twice. Such monosomic and trisomic flies are again distinct from the disomic type. They result, presumably, from the failure of the fourth chromosome to pair at meiosis, in which case the two partners behave independently and may pass to the same 

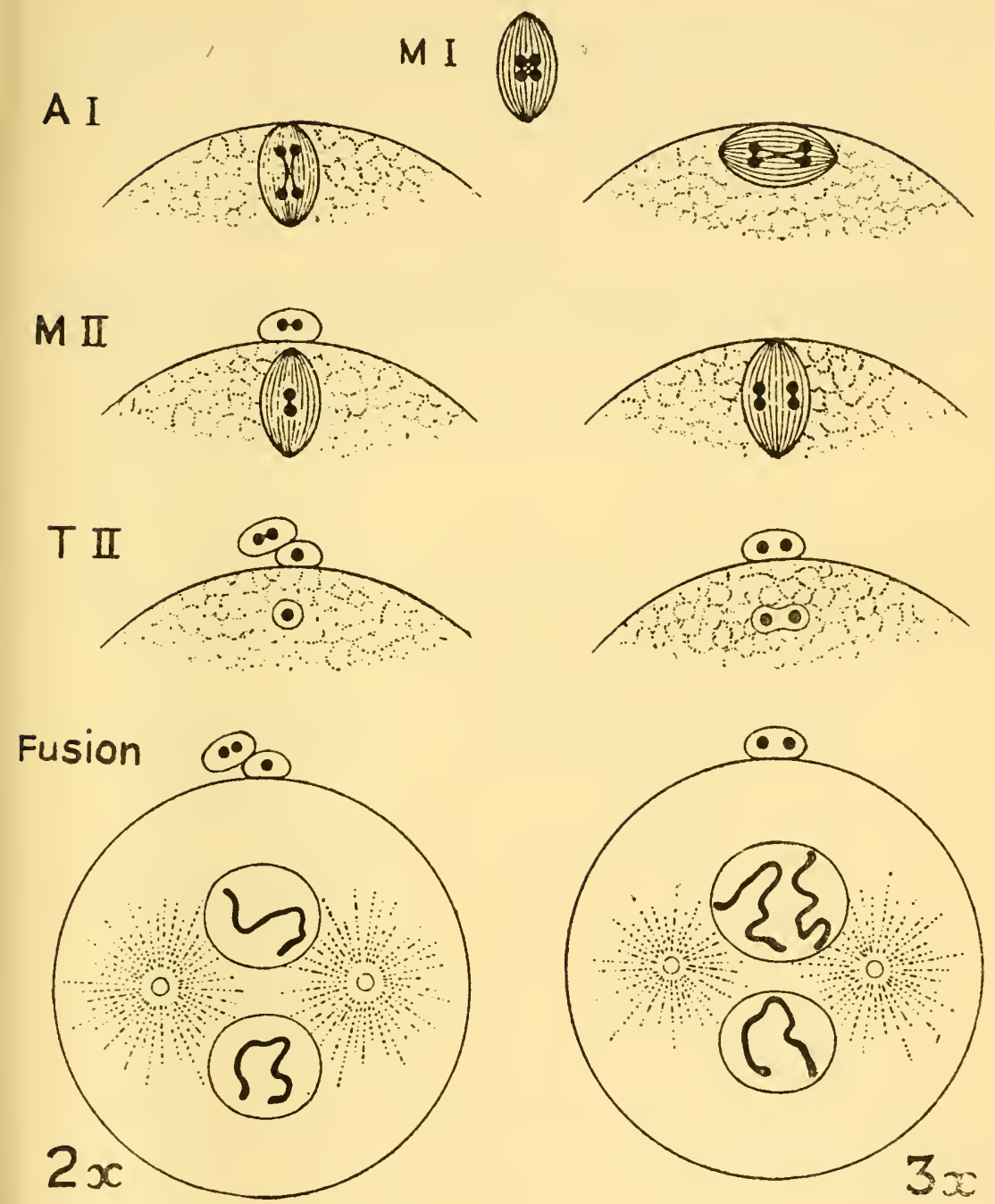

FIG. I9.-Meiosis in the egg of the horse thread worm Ascaris megalocephala, a race with two diploid chromosomes. Left: the normal series ending in fusion of single egg and sperm chromosomes. Right: the result of a misorientated first division spindle is the failure of expulsion of the first polar body which fuses with its sister nucleus. The second division leaves a diploid egg which is fertilized to give a triploid embryo. Fusion with a similarly exceptional diploid sperm would give a tetraploid of the race which is indeed found in nature (after Boveri, I904). 
gamete. The abnormality is not seen in respect of the larger chromosomes, whose unbalance presumably leads to too great a disturbance for the fly to live. Except, that is, for the Y. An extra Y does no harm and lack of the $Y$, as we saw, does not kill the fly, but produces a male with immobile sperm.

\section{TABLE 8}

THE TYPES OF BALANCED AND UNBALANCED CHROMOSOME COMPLEMENTS WHERE ABCD STAND FOR A SET OF FOUR

\begin{tabular}{|c|c|c|}
\hline \multirow{2}{*}{\multicolumn{2}{|c|}{$\begin{array}{l}\text { Haploid } \\
\qquad \times \quad A B C D\end{array}$}} & Deficient Gametc \\
\hline & & $x-1 \quad A B C-$ \\
\hline $\begin{array}{l}\text { Diploid } \\
2 x\end{array}$ & $\begin{array}{l}A B C D \\
A B C D\end{array}$ & $\begin{array}{l}\text { Monosomic Diploid } \\
\begin{array}{rl}2 \mathrm{x}-1 & \mathrm{ABCD} \\
& \mathrm{ABC}-\end{array}\end{array}$ \\
\hline $\begin{array}{l}\text { Triploid } \\
3 x\end{array}$ & $\begin{array}{l}A B C D \\
A B C D \\
A B C D\end{array}$ & $\begin{array}{l}\text { Trisonic Diploid } \\
\begin{array}{rl}2 \mathrm{x}+1 & \mathrm{ABCD} \\
& \mathrm{ABCD} \\
---D\end{array}\end{array}$ \\
\hline $\begin{array}{l}\text { Autotetr: } \\
\qquad 4 \mathrm{x}\end{array}$ & $\begin{array}{l}\text { ploid } \\
\text { ABCD } \\
\text { ABCD } \\
\text { ABCD } \\
\text { ABCD }\end{array}$ & $\begin{array}{l}\text { Tetrasomic Diploid } \\
\qquad \begin{array}{rl}2 \mathrm{x}+2 & \mathrm{ABCD} \\
& \mathrm{ABCD} \\
- & --\mathrm{D} \\
- & -\mathrm{D}\end{array}\end{array}$ \\
\hline Allotetra & ploid & Doubly Trisomic Diploid \\
\hline $4 x$ & $\begin{array}{l}A B C D \\
A B C D \\
A^{\prime} B^{\prime} C^{\prime} D^{\prime} \\
A^{\prime} B^{\prime} C^{\prime} D^{\prime}\end{array}$ & $\begin{array}{rl}2 \mathrm{x}+1+1 & \mathrm{ABCD} \\
& \mathrm{ABCD} \\
& \mathrm{A}--\mathrm{D} \\
\text { Unbalanced or Secondary } \\
\text { Polyploid } & \mathrm{ABCD} \\
& \mathrm{ABCD} \\
& \mathrm{A}^{\prime} \mathrm{B}^{\prime} \mathrm{C}^{\prime} \mathrm{D}^{\prime} \\
& \mathrm{A}^{\prime} \mathrm{B}^{\prime} \mathrm{C}^{\prime} \mathrm{D}^{\prime} \\
& \mathrm{A}^{\prime \prime} \mathrm{B}^{\prime \prime} \\
& \mathrm{A}^{\prime \prime} \mathrm{B}^{\prime \prime}\end{array}$ \\
\hline
\end{tabular}

No such change of balance occurs in another whole-nucleus variation, that due to the development of an egg without fertilization. Such parthenogenesis gives a haploid individual which, with inbreeding and therefore nearly homozygous stocks, often develops to maturity, though of reduced size. We have already seen one produced artificially in a sea-urchin (Fig. 2). At meiosis in haploids the chromosomes, lacking any regular partners, are scattered like the 
extra set in a triploid to give spores or gametes with, as a rule, every number up to the haploid. In consequence haploids are highly sterile and their rare progeny are diploids. Entirely homozygous diploids have been produced in this way in the tomato and in Datura.

In plants, and perhaps even more in animals, changes in chromosome numbers are a continual source of new variation which may be recognized by change in size, form or fertility of the variants. Their frequency will depend largely on the conditions of temperature at the time of formation of the parental germ cells and of their fertilization, so that the results recorded in Table 9 might no doubt be multiplied or divided by ten in special circumstances.

In plants the polyploid, at least the tetraploid, mutants may establish new races.

\section{TABLE 9}

WHOLE CHROMOSOME MUTANTS IN POPULATIONS OF PLANTS AND ANIMALS: LYCOPERSICUM FROM RICK (1945) AND TRITURUS FROM FANKHAUSER (1941)

\begin{tabular}{|c|c|c|}
\hline Type of mutant & $\begin{array}{l}55,000 \text { tomatoes: } \\
66 \text { unfruitful plants } \\
\text { examined }\end{array}$ & $\begin{array}{l}\text { 1,074 newts: all } \\
\text { examined }\end{array}$ \\
\hline Haploid: $x \quad$.. & 2 & 1 \\
\hline Trisomic: $2 \mathrm{x}+1 \quad \ldots$ & 2 & - \\
\hline $2 x+1+1$ & - & 1 \\
\hline Triploid: $3 \mathrm{x} \quad \ldots \quad \ldots$ & 45 & 10 \\
\hline Tetraploid: $4 \mathrm{x} \ldots$ & 3 & 1 \\
\hline Pentaploid: $5 \mathrm{x} .$. & - & 3 \\
\hline Chimaeras: $\mathrm{x} / 2 \mathrm{x} \quad \ldots$ & - & 1 \\
\hline $2 x / 3 x+\ldots$ & 一 & 1 \\
\hline Abnormal 2x Deformed & 3 & not recorded \\
\hline Sterile .. & 8 & not recorded \\
\hline Died & 3 & not recorded \\
\hline Total $\quad \ldots$ & 66 & 18 \\
\hline
\end{tabular}

Amongst animals on the other hand, polyploidy is of little account. Where the sexes are separate, obviously the first polyploid has no mate of its own kind. In experiment healthy and vigorous polyploids can be readily produced in Drosoplila, species of Triton and elsewhere. They cannot maintain themselves by reproduction. In nature, therefore, although they may occur in all species, they establish themselves only where sexual reproduction has been abandoned (as in 
the shrimp Artemia salina) or where sexual differentiation is replaced by hermaphroditism (as in nematodes and molluscs).

\section{Structural Change}

Apart from changes in the number of whole chromosomes, changes also occur within the chromosomes, structural changes. We have already an indication of such changes in the normal crossingover at meiosis; but of course crossing-over is reciprocal and can do no more than recombine differences that are already there. The results of new changes that have taken place in the resting nucleus we might see at the ensuing mitosis, especially at metaphase. But examination of hundreds of individuals and thousands of mitoses will fail to discover any spontaneous changes within chromosomes. Only in abnormal individuals or under abnormal conditions, as from sudden changes of temperature, do we find the chromosomes breaking up, and then the break-up is usually frequent and extensive. Its common characteristic is the breakage of the chromosomes or chromatids during the resting stage, with results that are seen at the following metaphase. Such spontaneous breakage has been described, for example, in the pollen grains of Tulipa fragrans. Breakage can also be induced by $\mathrm{X}$-rays or other ionizing radiations, or by chemical poisons such as mustard gas. The same description, however, can apply to all three, since they follow the same rules.

The simplest change, and the one which precedes all others, is breakage of the chromosome fibre into two or more parts called fragments. The breakage occurs in the resting nucleus. Now the chromosome reproduces, its single thread becoming doubled, in the resting nucleus. The breakage therefore affects either the whole chromosome before its reproduction $\left(\mathrm{B}^{\prime \prime}\right)$, or only one of its two daughter chromatids after its reproduction $\left(B^{\prime}\right)$. $B^{\prime \prime}$ always gives two kinds of fragments, one with the centromere and one or more without (Fig. 20).

Acentric fragments cannot move spontaneously on the spindle: they lag and are usually left out of the daughter nuclei, like unpaired chromosomes at meiosis. They degenerate in the cytoplasm. Evidently they cannot develop a new centromere. It seems, indeed, that we have to look upon the centromere as a specific and permanent 
self-propagating organ like any other gene or group of genes, concerned with the organization of proteins on the spindle just as others are in the nucleus.

Centric fragments on the other hand, move normally on the spindle. They are distributed to the daughter nuclei and appear in

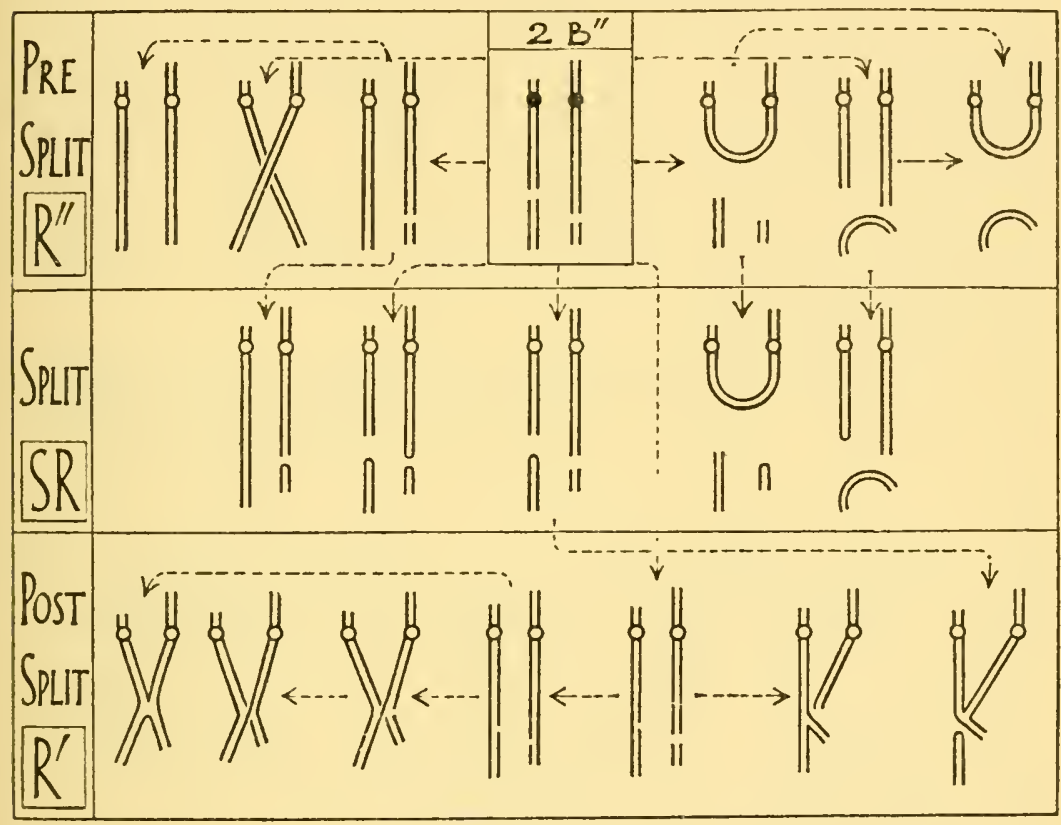

FrG. 20.-The chief successions of change that may follow two spontaneous or induced breaks $\left(\mathrm{B}^{\prime \prime}\right)$ in two chromosomes with restitution, chromosome reunion $\left(\mathrm{R}^{\prime \prime}\right)$, sister reunion $(\mathrm{SR})$ and chromatid reunion $\left(\mathrm{R}^{\prime}\right)$-in this order-at later stages. The pre-split stage is represented with the chromosomes divided for ease of comparison. Each step may be final and hence observable at the ensuing metaphase. SR is independent on the centric and acentric sides of a break (from Darlington and Koller, I947).

inheritance as chromosomes lacking particular blocks of genes. If too many genes are missing from a fragment the unbalance from this deficiency may be fatal even where its homologue is intact. Indeed X-ray experiments show that in diploid organisms most cells whose chromosomes are deficient, owing to breakage, never divide again after the mitosis where the fragment is lost.

A single break can lead to nothing but loss; two breaks begin to show us more fruitful possibilities. The broken ends can rejoin 
with one another to give two new combinations. Mechanically this reunion is just like that of crossing-over: physiologically, on the other hand, it is something quite new. Since the breaks can occur in any parts of any chromosomes, remion can give a prodigious variety of new combinations. It can occur between ends within one arm of one chromosome, between the two arms of one chromosome, or between the arms of two chromosones, homologous or nonhomologous. Some of these new combinations are unworkable: the new chromosomes have no centromere; or they have two centromeres which act independently and so cause yet another breakage by passing to opposite poles at anaphase. But other combinations have only single centromeres and these are workable and important (Fig. 20).

The workable combinations are of two kinds: an inversion happens where the two breaks are in the same chromosome, an interchange where they are in different chromosomes. Where they are in opposite arms of the same chromosome we may look upon the change either as an interchange or an inversion. These types of reunion are found wherever there is breakage. And, when the breakage is of chromatids, the association of the changed and unchanged chromatids remains at the following metaphase of mitosis to bear witness to the course that events have followed. For example an interchange of chromatids gives the appearance of a chiasma, and an inversion of a chromatid gives a loop in addition to a chiasma (Fig. 30).

Two chromosomes undergoing interchange may be represented as of two segments cach, $A B$ and $C D$, which acquire the new order, $A D$ and $B C$ or $A C$ and $B D$. For inversion we need four such segments to represent one chromosome, abed which changes into acbd. The homozygous types derived from these changes show new linkage relations. Experimental interchange in X-rayed Drosophila moves blocks of genes from one linkage group to another. Inversion leaves them in the same groups but their recombination frequencies correspond to a new order on the map.

Two breaks suffice to produce inversion or interchange. Threc will give more complicated changes. A piece may be taken out of a chromosome and inserted or translocated wherever else the same or another chromosome may be broken. Thus two chromosomes abc and def can become $a c$ and dbef. In a succeding generation this 
change can give homozygotes for the two new chromosome types. These are still balanced. Indeed all these primary structural changes, apart from simple loss, which eliminates itself, merely rearrange the materials in the chromosomes. New chromosomes are created but there is no change in their aggregate content. But by recombination with normal homologues at meiosis secondary changes arise. Our translocation will give heterozygotes and homozygotes for $a c$ and def which will be deficient in the segment $b$ and mect the fate of deficiencies. And it will give heterozygotes and homozygotes for $a b c$ and $d b e f$ which will have a duplication of $b$. Such individuals will usually be viable but they will be different from the original type.

Again, by translocation within itself abcde can become adbce. On crossing-over with the normal homologue in the heterozygote, such a changed chromosome will likewise give deficient (ade) and duplicated (abdbce) progeny from crossing-over in $d$, and similarly abce and adbcde from crossing-over in $b c$.

TABLE 10

THE CLASSIFICATION OF THE TWO ORDERS OF CHROMOSOME CHANGE IN THEIR PRIMARY AND, FOLLOWING RECOMBINATION, SECONDARY CONDITIONS

\begin{tabular}{|c|c|c|}
\hline Type of Change & $\begin{array}{c}\text { Primary: } \\
\text { Not changing balance }\end{array}$ & $\begin{array}{c}\text { Secondary: } \\
\text { Changing balance }\end{array}$ \\
\hline $\begin{array}{l}\text { NUMERICAL } \\
\text { heterozygous }\end{array}$ & $\begin{array}{l}\text { Nuclear } \\
\text { Triploid } \\
3 \mathrm{x}\end{array}$ & $\begin{array}{l}\text { Chromosonial } \\
\text { Trisonic, etc., } \\
2 \mathrm{x}+1,4 \mathrm{x} \pm 1\end{array}$ \\
\hline homozygous & $\begin{array}{l}\text { Tetraploid } \\
4 \mathrm{x}\end{array}$ & $\begin{array}{l}\text { Tetrasomic, etc., } \\
2 x+2,4 x \pm 2\end{array}$ \\
\hline $\begin{array}{l}\text { STRUCTURAL } \\
\text { (heterozygous or } \\
\text { homozygous) }\end{array}$ & $\begin{array}{l}\text { Inversion } \\
\text { Interchange } \\
\text { Translocation }\end{array}$ & 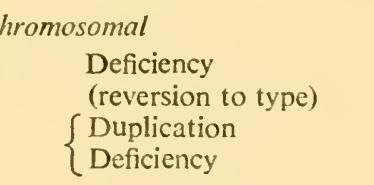 \\
\hline $\begin{array}{l}\text { NumERICAL }+ \\
\text { StRUCTURAL }\end{array}$ & $\begin{array}{l}\text { Misdivision of the centro- } \\
\text { mere and Formation of } \\
\text { two telocentric chromo- } \\
\text { somes }\end{array}$ & $\begin{array}{l}\text { Origin of an iso-chromosome } \\
\text { from a telocentric }\end{array}$ \\
\hline
\end{tabular}




\section{Misdivision of the Centromere}

There is one change in the hereditary mechanism, and one only, which affects both the number and the structure of the chromosomes at a single stroke. That change is the misdivision of the centromere.

The unpaired chromosome at the first anaphase of meiosis is in an equivocal position. Its centromere is unable to co-orientate with, or segregate from, a partner. Nor can it divide at the same time as the paired chromosomes segregate. It is therefore frequently lost. Sometimes, however, its difficulty is resolved by division within itself. Instead of separating lengthwise from the product of its own reproduction (which is not yet available) it separates crosswise into two components. It thus proves to be a compound or repetitive gene. Sometimes it explodes to give useless fragments, but often it divides into what must be two nearly equal halves, for each part successfully carries its chromosome arm to the pole and undergoes a second division of its new chromosome.

The new chromosome is telocentric and in some circumstances is stable. Both in Orthoptera and in the plant Campanula persicifolia races have been found where a particular chromosome is replaced by its two telocentric arms so as to add one more to the haploid number. But this is not always so. At the next mitosis (in the pollen grain of Fritillaria for example) reunion of the two daughter chromatids is found to have taken place within the centromere. The new chromosome, which passes to one pole without division, is then twice the size of the telocentric. It has a double-sized centromere and two identical arms. It is known as an iso-chronosome. A pair of isochomosomes are in effect tetrasomic. They have been found as part of the regular complement in Nicandra and as supernumeraries varying in number in different plants in Sorghum, Secale, and Datura.

Thus the primary changes of inversion, interchange, translocation and misdivision can never change the balance. But by recombination secondary changes occur, producing a new content for the nucleus as a wholc.

\section{Deficiency and Balance}

Deficiencies in parts of single chromosomes, if they are small enough, are not always fatal to diploid nuclei. But when such a 
deficient chromosome passes through meiosis and reaches the haploid generation it may still prove fatal. The danger is greater when this generation has to undergo several cell divisions: thus, as we shall see in detail in the next chapter, the pollen is more sensitive than the embryo-sacs, and both are more sensitive than the sperm in animals. The haploid egg nucleus in animals, since it is immediately fertilized, is never exposed to any trial of sensitivity. Sperm of Drosophila lacking whole chromosomes, even the large autosomes, can fertilize eggs. But pollen grains of lilies so lacking will never even pass through their vegetative mitosis. And, even where deficient gametes survive, a diploid homozygous for their deficiency cannot do so. Evidently, as we might expect, nearly all the genes are indispensable for the life of the cell in one dose and are most efficient in two doses.

The loss of a small part of a chromosome, and even of a whole chromosome, must be of some importance in the origin of natural variations as the sex chromosome differences show. The Y chromosome of mammals is little more than a deficient X. The male is therefore a deficiency heterozygote. The situation is even clearer in those insects and spiders with no $\mathrm{Y}$ chromosome at all. With them the male is heterozygous for the deficiency of the whole X. It is monosomic or XO.

Heterozygous deficiencies and duplications are, obviously, for segments what monosomics and trisomics are for whole chromosomes. Corresponding to the homozygous deficiencies and duplications are nullisomics $(2 \mathrm{x}-2)$ and tetrasomics $(2 x+2)$. Nullisomics cannot survive in diploids, but they appear in analogous forms in polyploids (e.g. $6 \mathrm{x}-2)$. Tetrasomics in Datura can survive, but are even poorer and more abnormal than the corresponding trisomics. The homozygous duplication, in Drosophila and maize, bears a similar relation to the heterozygote.

Two laws of gene balance emerge from these considerations. First, the larger the proportion of the chromosome outfit, up to a half, which departs from the numerical proportions of the rest, the greater the disturbance of development. A trisomic for two chromosomes, or double trisomic, is more abnormal than a single trisomic-except in Crepis capillaris $(\mathrm{x}=3)$ where $2 \mathrm{x}+\mathrm{I}+\mathrm{I}=$ $3 \mathrm{x}-\mathrm{I}$. Secondly, the larger the departure in proportion of the unbalanced element the greater the disturbance. A monosomic or 
tetrasomic diploid is more deranged than a trisomic, or, of course, than a monosomic or tetrasomic polyploid in a group of organisms working with the same chromosome series.

\section{Intergenic and Intragenic Change: Presence and Absence}

There is one other genetic effect of structural change. This is the physiological effect of change of position itself. Changes of order might be expected to produce no effect on the phenotype. Indeed in maize, where some two hundred have been produced by breeding with X-rayed pollen, it is doubtful whether they ever do so. In Drosophila and Oenothera, however, an effect often has to be inferred as the result of the mere change in order itself, a position effect.

The position effect is now known to be responsible for many changes which were described as gene-differences in breeding experiments with Drosophila before the chromosomes could be directly examined in polytene nuclei (that is, before 1933). Allelomorphs of the gene scute affect the number of bristles on the thorax and scutellum, and several of them are due to inversions of various lengths, following breakage by $\mathrm{X}$-rays. The Pale gene likewise gocs with a spontaneous translocation from the second to the third chromosome and is probably just due to the change of position. The scute mutations are simple recessives having no special effect on the heterozygote; the Palc gene is lethal when homozygous and varies in its effect on the heterozygote.

Another side of the picture is shown by the fact that a large proportion of inversions, which have arisen spontaneously as well as by X-raying in laboratory cultures of Drosoplita, are lethal in the homozygous state, while some of them are invariable concomitants of visible mutations like Curly and Plum. It thus seems that the action of one gene may be modified, directly or indirectly, by its neighbours. And, when it changes its neighbours, it does not necessarily work as it did before. Either the shapes of the chromosome constituents themselves, or at least of their products inside the nucleus depend on their linear arrangement. To this extent whole segments, or even whole chromosomes, must be considered as units of action in physiology just as they are units of structure, single giant molecules, in chemistry. 
Changes of balance likewise mimic gene differences in Drosophila. Notched wings and Minute bristles are produced by deficiencies. They can usually be recognized in two ways: by the absence of from one to ten bands from the polytene chromosomes, and by the absence of the allelomorphs of particular genes as shown by the failure to "cover" a recessive in breeding experiments. Some 70 varieties of Notch and 80 of Minute, both spontaneous and induced, have been described; all the Notches are at the end of the X chromosome while the Minutes may lie in any of the different chromosomes. Minutes seem to be lethal when homozygous; Notches are lethal to the male (which has no allelomorph to them in the $\mathrm{Y}$ ) and they cannot, therefore, be obtained homozygous in the female. All these deficiencies are dominant (for which reason they are given capital letters in the Drosophila usage*) and are known by the phenotype they give in the heterozygotc.

Thus a deficiency is recognizable in breeding by its drastic effect and its segregation as an allelomorph of a group of genes. In these two respects duplication is similar, but of course its effect is less drastic and its relation to other genes is the reverse. Deficiency "uncovers" a recessive allelomorph. Duplication "covers" even a homozygous recessive. Duplications also resemble deficiencies in usually affecting the bristles and the wings. The most interesting of them, however, is the narrow-eye or Bar factor in Drosophila (Fig. 2I).

For 25 years Bar was regarded as a simple gene-difference in the $\mathrm{X}$ chromosome. It was odd merely in its property of unequal crossing-over. Homozygous Bar females sometimes gave progeny in which the Bar gene was replaced either by the normal allelomorph, or by a new one of greater strength (giving still smaller eyes) called Double-Bar. Finally the polytene chromosomes showed Bar to have two like pieces side by side instead of one, as it were $A B B C$ instead of $A B C$. Then unequal crossing-over in the homozygote $A B B C / A B B C$ reconstructs the chromosomes, so as to give the types $A B C$ and $A B B B C$ detectable genetically and cytologically in the next generation.

A remarkable incidental observation in these experiments was that the heterozygous combination, $A B C / A B B B C$, although the same

* See Appendix z, on Symbols and Symbolism. 


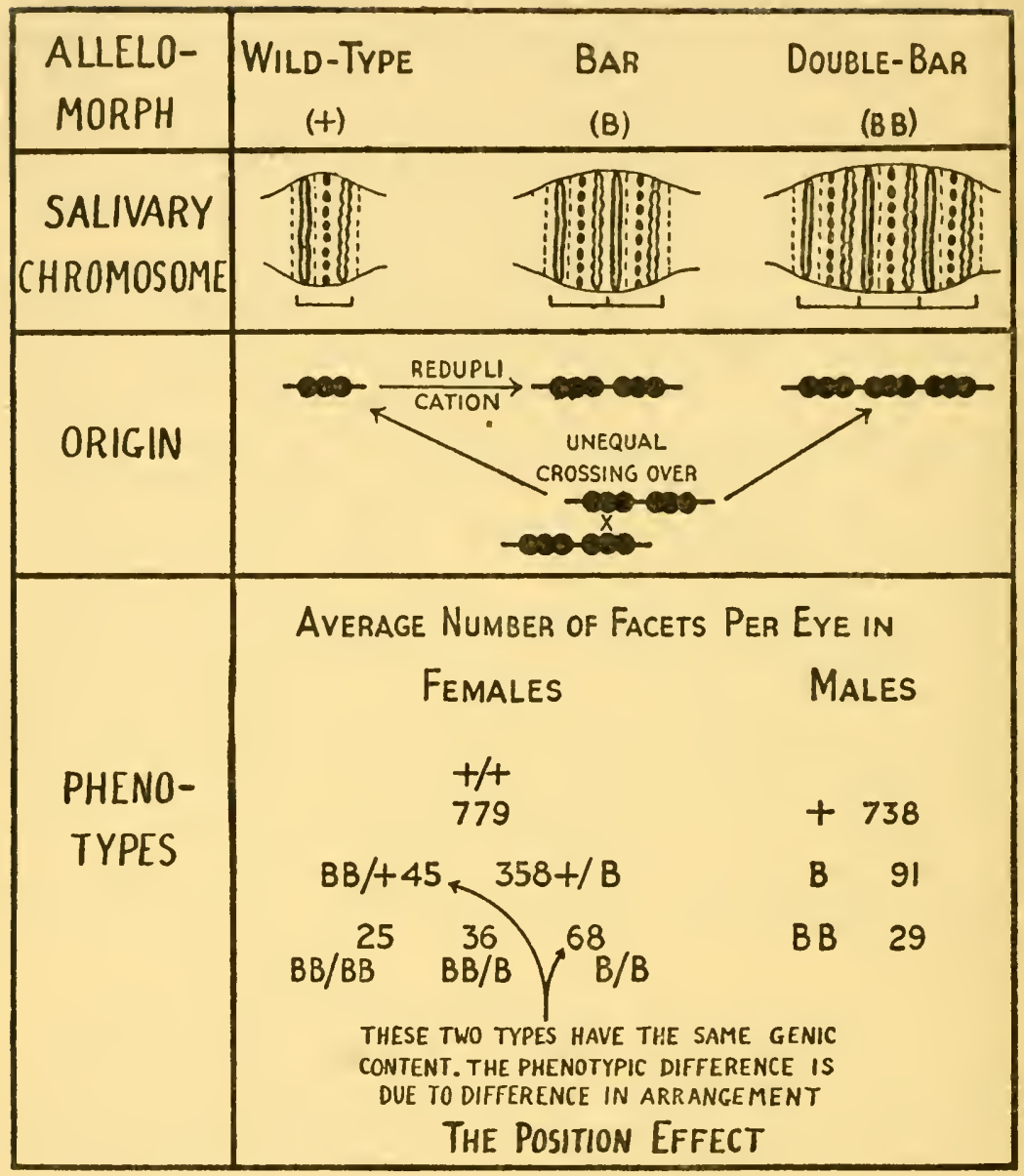

Fig. 2I.-The Bar gene, which reduces the size of the eye in Drosophila melanogaster, is itsclf a duplication for a segment of the $\mathrm{X}$ chromosome containing at least four distinct bands in the salivary chromosome. Unequal crossing-over in femaics homozygous for the Bar duplication leads to triplication of the segment, which thus gives the enhanced effect described as Double Bar. Females heterozygous for the wild type chromosome and Double Bar have the same total gene content as females homozygous for Bar, though the genes are distributed differcntly between the chromosomes. The eyes of these two classes of fly have different average sizes, showing that the arrangement of the genes in the chromosomes has its effect on their action. This is the Position Effect. 
in total $B$ content, has more Bar effect than does $A B B C / A B B C$. Its eyes have fewer facets. Evidently the $B$ 's are disproportionately effective when they are concentrated on one chromosome. The effect of their unbalance is not additive but multiplicative. This is another and special example of the position effect, and indeed it vas the first that could be proved, since it could be done and undone so readily by crossing-over.

Thus simple additions, subtractions, and dislocations of genes produce unit hereditary effects. At one time Bateson considered that plus and minus differences, due to mere presence and absence of determinants, were the essence of all hereditary change, the presence being dominant to the absence. This led to a difficulty because mere loss (or even perhaps gain) could not ultimately produce anything new. Unpacking requires a previous packing. Furthermore, crucial cvidence against Bateson's view is that the two allelomorphs of many genes (like white eye and red eye in Drosophila) can each mutate to the other. Change has often been observed in this way in both directions; and where it has been seen only in one, that is doubtless sonıetimes due to the frequency being low, and lower one way than the other. Yet the absence could hardly be expected to mutate to the presence. Unless, like the normal allelomorph of Bar, it is only half an absence. Finally, as we have seen, the absence, the deficiency, can be at least partly dominant.

It is not, therefore, surprising that when most mendelian heterozygotes are examined, for example, at pachytene in maize or at polytene in Drosophila, we find no visible differences between their pairing chromosomes. Both dominant and recessive thus prove to be present. There is evidently a residual class of differences which cannot be shown to be due to structural change. Ultimately, of course, we must assume that all changes are structural in the sense of being changes in number or arrangement of some constituents. Since a linear order has been established for the genes, changes between genes must always be longitudinal. We can still suppose, however, that genes have additional dimensions, and that lateral change occurs within them.

To put the argument in another way, the occurrence of a second kind of change other than one of mere arrangement is inevitable. Arrangement of units has no meaning unless there are differences 
between the units to rearrange, differences such as we actually see between the parts of the chromosomes. These differences between units cannot, therefore, have arisen by the changes of arrangement themselves.

Now crossing-over is a change in arrangement between genes. Changes within genes should, therefore, have no direct effects on their own crossing-over. Many genes, in fact, have an indirect or physiological effect on the process of chromosome pairing and on the observed recombination. For example, a so-called asynaptic gene often (in maize, pea, or Drosophila) produces a general reduction in the crossing-over of all the chromosome pairs when homozygous by causing incomplete pairing with results that we shall see later. But of direct effects produced in the heterozygote these residual intragenic mutations have none. They are apparently changes in indivisible points or loci on the linkage map, and are accordingly known as point mutations.

\section{Somatic Mutation}

Instances are known, in Antirrhinum and elsewhere, of two allelomorphic genes or two homologous chromosomes mutating in the cell simultaneously to the same new allelomorphs. Such events suggest a chemical determinacy in the mutation even if they arise in two steps. These instances are, however, very rare and it is a remarkable fact, or even principle, that allelomorphs are (with these few exceptions) independent in their mutations. The original form of any change or mutation in the body of a zygote must therefore be a heterozygote. And the original form of any heterozygote must be a mutation. Only later can the heterozygote appear as we characteristically find it, that is as a cross between two homozygotes, as a hybrid in the restricted pre-mendelian sense.

It is also worth noting that most mutations occur somatically, that is in the nuclei of a growing body. Hence the new type of cell is not merely heterozygous. It is also incomplete so far as the body is concerned: it is a patch. In plants where the germinal tissue is not separated early in development a change may or may not affect the 
germ layer. In any case only one layer is changed and hence gives rise to a chimaera with mixed tissues: a layer of mutant lies inside or outside a layer of the unchanged type.

Chimaeras can be produced artificially as graft-hybrids or by any treatment which alters the chromosomes. Most old vegetatively
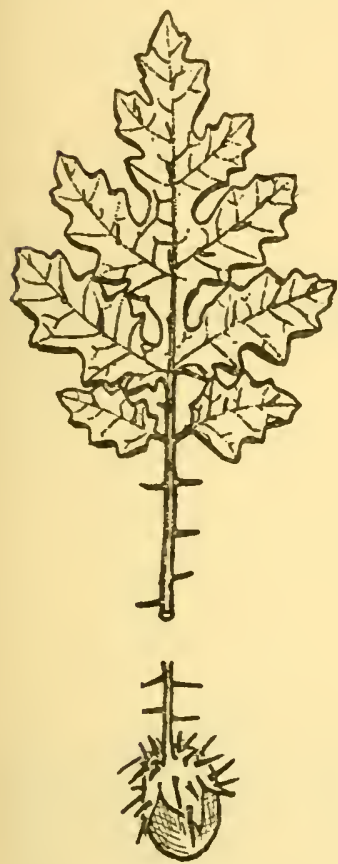

$s$
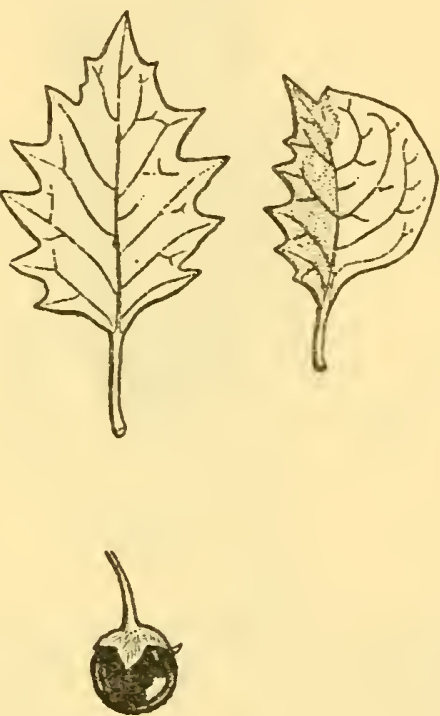

$\mathrm{s} / \mathrm{n}$
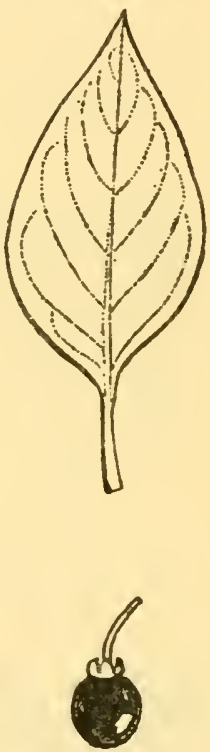

n

FIG. 22.-Leaves and fruits of $2 x$ Solanum sisymbrifolium (s), $6 x$ S. nigrum (n) and the graft-hybrid or chimaera with one layer of $s$ over $"$ which sorts out to give pure nigrum in parts of its leaves (after Jorgensen and Crane, 1927).

propagated plants such as pelargoniums and potatoes have become chimaeras owing to somatic mutation at some time in their history. When they are propagated from root-cuttings (or from disbudded tubers) shoots grow out of the concealed inner layers which reveal the chimerical structure and the ancient mutations of these plants. The elucidation of these properties by Baur, Bateson, Winkler and Crane has enabled us to understand that natural changes in the arrangement of tissues lead to the repeated appearance of "sports" 
in chimaeras which themselves have arisen from a single original mutation ( $c f$. Fig. 22).

In animals the effects of somatic mutation are slightly different. Development and life being limited, the mixture is not a permanent one. Development being less simple the mixture is also less regular. As a result, the changed cells give flakes and sectors instead of layers

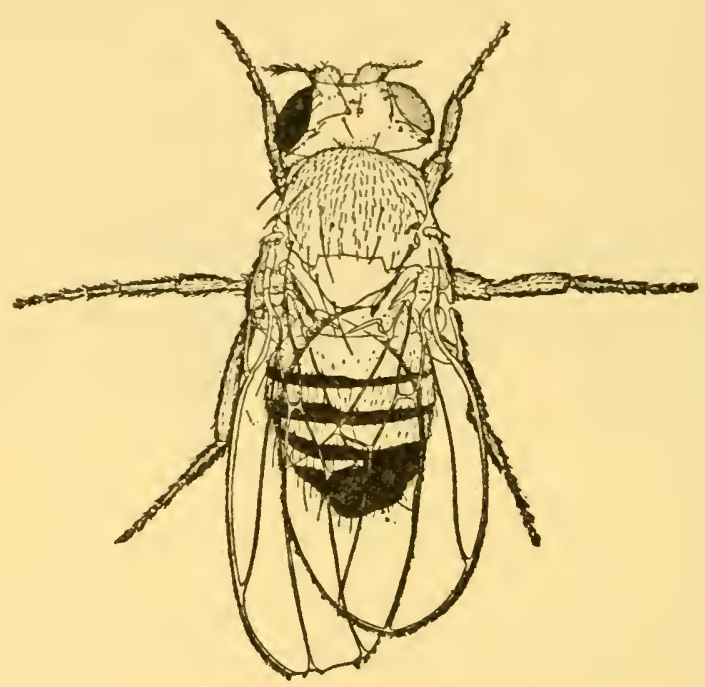

FIG. 23.-Gynandromorph of Drosophila melanogaster. The left side is $\mathrm{XX}$ and female. One X carrying the dominant gene Notch, whose effect appears in the left wing, has been lost on the right side which is therefore male and shows the effects of the recessive genes of the other X, viz. ruby (cye colour), scute (bristle reduction), broad (wing), and forked (bristle gnarling). The whole fly is slightly warped owing to the male side being shorter than the female (from Morgan, Bridges and Sturtevant, 1925).

and the product is known as a mosaic instead of a chimacra. There is, however, one regular type of mixture of particular interest and this is the gynandromorph. Such monsters, male in one part and female in the rest, arise from a genetic difference between a pair of nuclei produced at an early mitosis in the embryo. They give various mixtures according to the stages and relative positions of the mitoses, but perhaps the commonest is the half-sider of the type shown in Fig. 23. They come about in a varicty of ways as can be proved by generic as well as by cytological evidence (Fig. 24). 


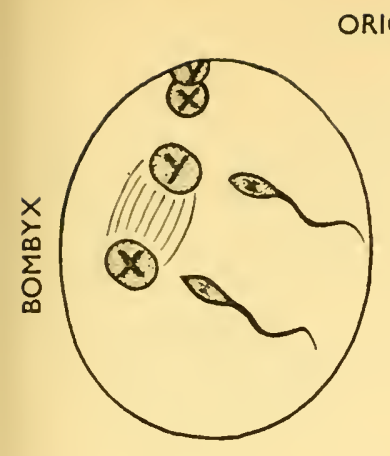

ORIGINS OF GYNANDROMORPHS
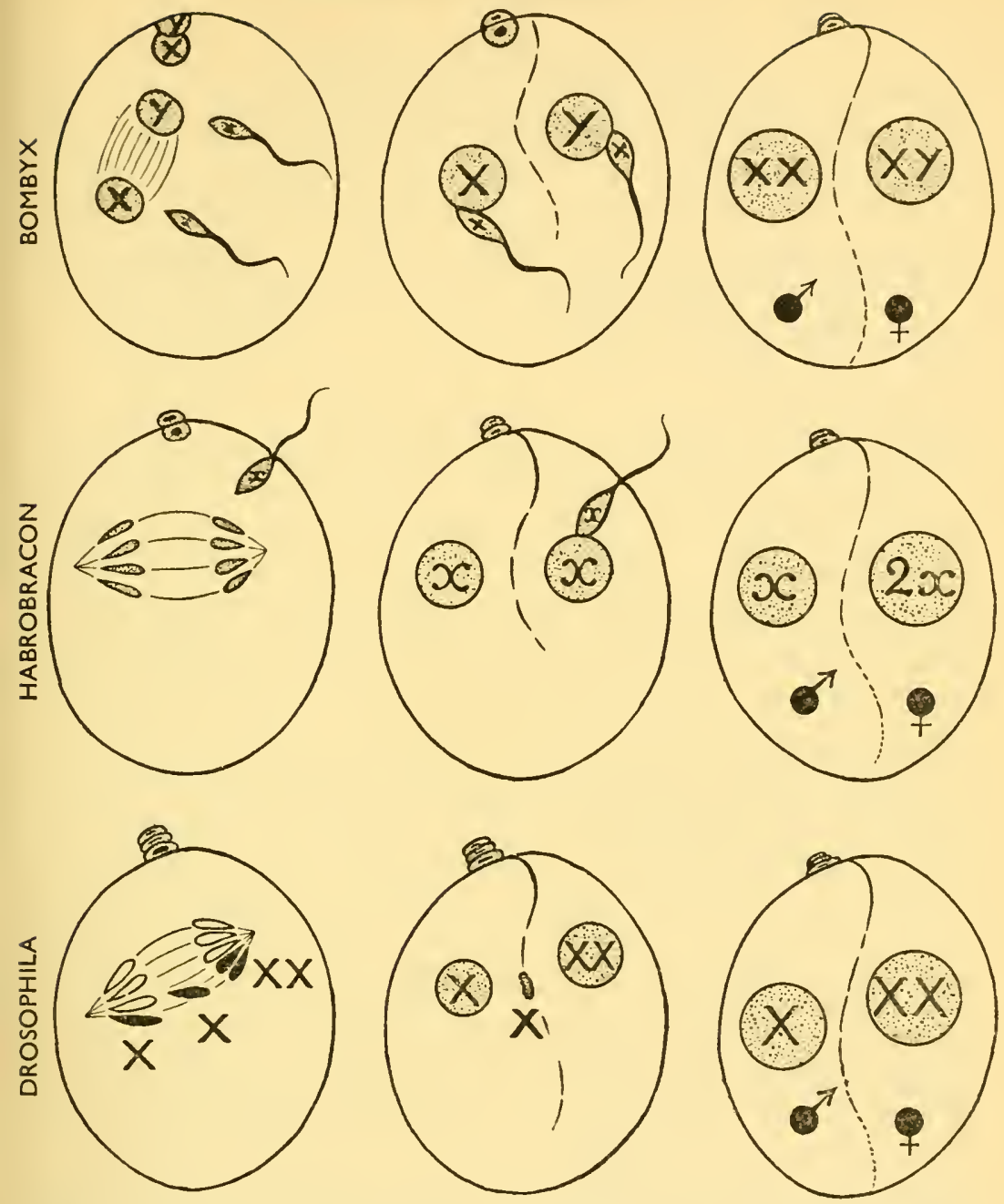

FIG. 24.-Diagram showing the modes of origin of gynandromorphs in different insects directly observed in the egg or inferred from the genetic character of the two sides of the body which are of opposite sex. In Bombyx (after Goldschmidt and Katsuki, 193I) two sperm may fertilize the two dissimilar products of the second meiotic division in the XY female. In Habrobracon (after Whiting, 1943) a sperm may fertilize one of the products of the second meiotic division to give a diploid female side, a haploid and parthenogenetic male side being derived from the other product. More rarely a second sperm may cleave side by side with the fertilized egg. In Drosophila one of the two X's in a fertilized female egg may be lost at the first cleavage division to give an $\mathrm{XO}$ side which is morphologically male (after Morgan, Bridges and Sturtevant, 1925). More rarely two sperms, one $\mathrm{X}$ - and the other Y-bearing, may fertilize the two first products of cleavage to give a perfect gynandromorph. Thus all the different irregularities occur which will give workable results in each organisn1. 


\section{The Gene as a Unit of Change}

How can we find out something about what is inside that point, the gene? The evidence of the visible or breakable structure of the chromosome, or of the linkage map inferred from the breeding experiments, clearly no longer helps us. We have gone beyond the limits of resolution by such means. Only by the study of action and of changes in action, that is of mutation, can we get any further light on the matter.

At most loci in the mapped chromosomes of maize or man, Drosophila or Oenothera, we have discovered only two allelomorphs of recognizably different effect. In addition to such simple allelomorphs, however, particular loci in scores of species show whole series of alternatives, multiple allelomorphs as they are called. In many such series the effect of the mutation of one allelomorph into another has been seen in experiment. Bar eye is an extreme example, where we have crossed the boundary into structural change. Most of the differences, however, are intra-genic.

Multiple allelomorphs are of particular importance when they control the breeding systems of plants. One set or series then have to be closely related in their actions, in order, as we shall see later, to control the growth of the pollen down the style. This physiological relationship is also seen in other series such as those at the whitecye locus in Drosophila. Here, a number of allelomorphs produce a range of intensity of pigmentation between none at all and the full red of the dominant wild type. A dozen have been recorded and there may well be more grades, and therefore more allelomorphs, than have yet been separated by eyc. Evidently all the allelomorphs are doing the same thing but to different degrees. One unit of action therefore suffices to account for the range of behaviour and mutation of this gene, although the range of its mutants shows the complexity of its parts.

Not all inultiple allelomorphs, however, are so simple. A series of four in Primula sinensis includes three which affect only the size of the greenish "eye" round the mouth of the corolla tube; the fourth, which is recessive to all the rest, has the same effect on the eye as the next most recessive but, in addition, shortens the style. The series may be arranged, therefore, as in Table II. 
TABLE 11

FOUR ALLELOMORPHS GOVERNING SIZE OF EYE IN FLOWERS OF PRIMULA SINENSIS

\begin{tabular}{|c|c|c|c|}
\hline Allelomorph & & Symbol & Effect \\
\hline Alexandra & .. & $A^{\prime}$ & No eye and normal style \\
\hline Original type .. & . & $A$ & Normal eye and normal style \\
\hline New type (1938) & .. & $a^{n}$ & Large eye and normal style \\
\hline Primrose Queen & .. & $a$ & Large eye and short style \\
\hline
\end{tabular}

Two interpretations are clearly possible. Either (i) $a$ has two effects independent from the beginning and one of them distinct from that shown by any of the others, even the otherwise indistinguishable $a^{n}$. This means two units of action, separable in their changes, but inseparable in recombination, so far as our experience gocs. For if short-style could occur with normal eye we have not yet recognized it. Or (ii) $a^{n}$ does the same thing as $a$ but does it more effectively, thereby passing a threshold which introduces a new or secondary ultimate effect, the visible change in style length. This means one unit of action as well as of recombination, but supposes a relation in development between eye and style of which we otherwise know nothing. The distinction between these alternatives is thus as follows:

\section{Complete Linkage}

Two genes having distinct initial effects but inseparable by recombination.

\section{Pleiotropic Action}

One gene having a single initial effect with manifold expression.

The choice between these alternatives is important wherever manifold expression is concerned, whether multiple allelomorphs are present or not. In every case it must depend on our knowledge of the whole series of steps from the gene to its expression. This knowledge is perhaps available in certain simple cases. In other more complicated cases we must turn to nerv ways of attacking the problen. If we can show that the series of steps that we do know can be traced back to one unit action, a single gene of manifold effect must be at work.

The tracing of gene action has been done in two ways. Embryo- 
logically, Grüneberg has shown, for example, the common and simple origin of a number of defects occurring together in the rat. Distortion of the blood system and lungs, blockage of the nostrils, imability to suckle and deformity of the bones of the forelegs, all

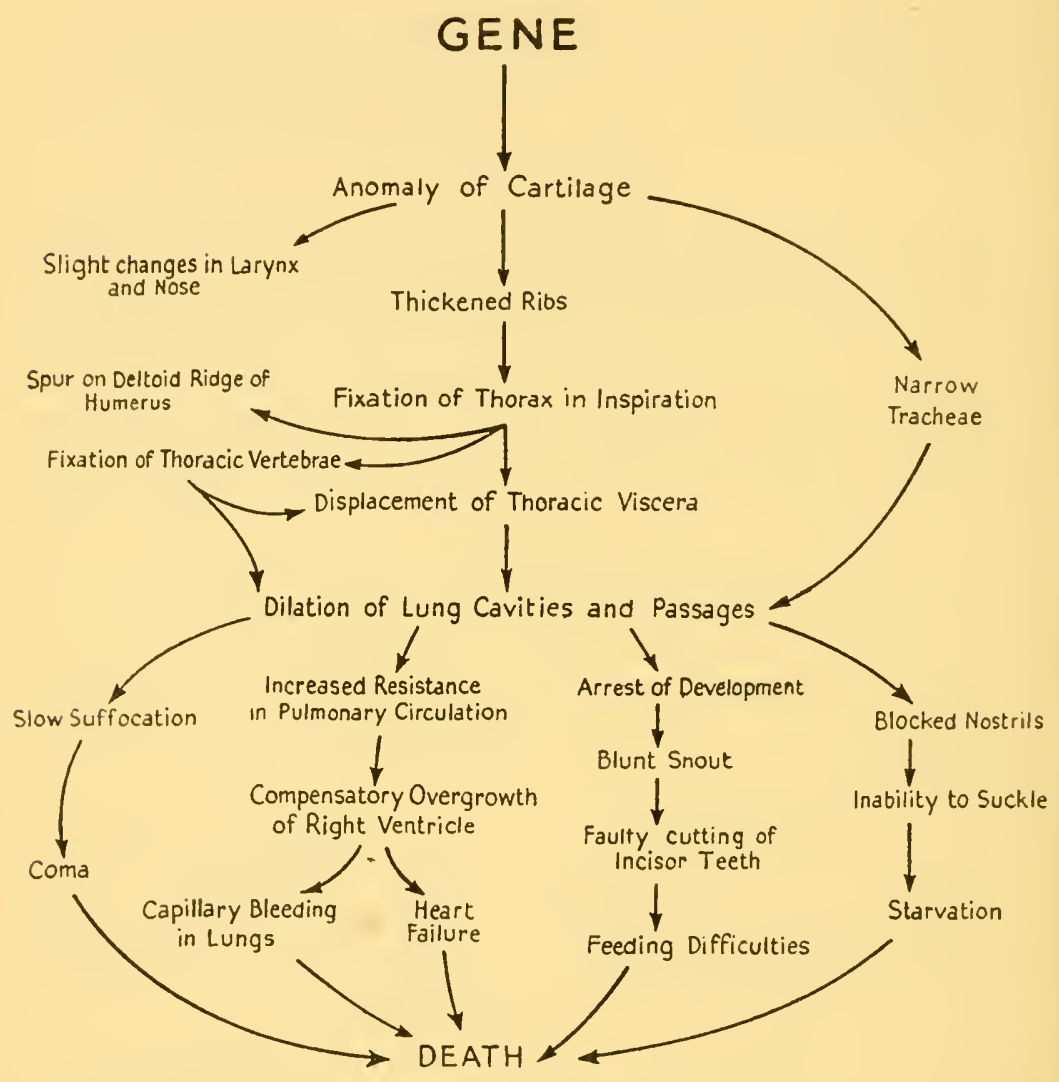

FIG. 25.-The pleiotropic effect of the grey-lethal gene in the rat (after Grïneberg, 1938).

these arise during devclopment from a single abnormality in the formation of the cartilage. The gene is single and plciotropic (Fig. 25). Genetically, we can get the evidence from the control of dominance and the effects of other gencs in modifying the action of the one we are chicfly concerned with. The agouti scries in the mouse is a case in point. 
The common type of wild mouse has entirely agouti fur; each hair is black with a yellow band near the tip. Some wild mice are agouti with light bellies, and in fancy mice there are corresponding types, a plain black and black with light belly (black and tan). Finally, there is an all-yellow type in the fancy. All these types behave as though governed by a multiple allelomorph series. Yellow stands by itself: the homozygote is never born alive, so that all yellow mice are heterozygous. It is like the Notch effect in Drosophila and, like it too, may well be due to a deficiency. The four other types evidently consist of combinations of light or dark belly with agouti or non-agouti (black). We might, therefore, again say that they were due to the combination of two gene differences completely linked in all known experiments.

The evidence for the two-gene view is better in the mouse than in Primula, for all four combinations are found. Furthermore, however they are combined, light belly is always dominant to dark belly, and agouti hair to non-agouti hair. Thus the mouse heterozygous for agouti and black-and-tan looks just the same as a light-bellied agouti. Finally, there is another gene which has the effect of reducing the dominance of agouti over non-agouti and this gene has no effect on the dominance of light over dark belly. Hence the dominance relations accord with the two-gene view.

A similar two-gene explanation may be given for the dumpy multiple allelomorph series affecting the development of wings and hairs in Drosophila. But here the two possibly independent components are affected in the same way by the same dominance modifiers.

How then are we to resolve the question of one gene or two? The answer is that it often cannot be resolved. What behave as two units of recombination under one set of conditions may, it secms, behave as one under others. Suppression or even reduction of the frequency of crossing-over, either general or local, may have this effect. Such a structural change as the inversion of a couple of genes, will, as we shall see, prevent crossing-over, or at least effective recombination, between them except when the two genes are homozygous and the recombination is, therefore, ineffective with respect to these two genes. The absence of crossing-over in the agouti group might be due merely to an inversion which constantly 
distinguishes all agouti from all non-agonti or all dark-bellies from all light-bellies. Fisher has suggested that an inversion is associated with the agouti difference (Fig. 26) and is putting this view to an experimental test.

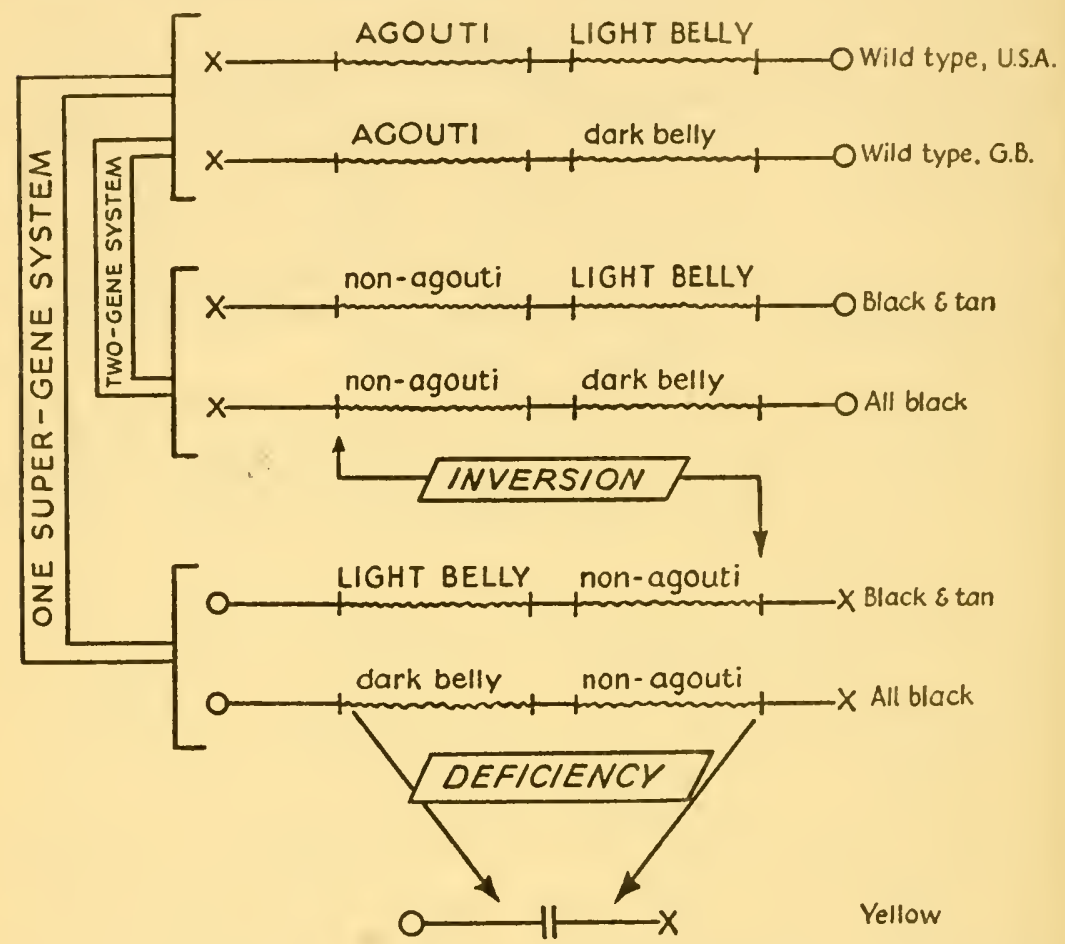

Fig. 26. - The possible relation of structural changes to gene recombination in the Agouti series of the mousc, where four allelomorphs represent all recombinations of two differences between which recombination has not, however, been observed. The differences could be interpreted as two gene differences so closely linked as never to recombine (two-gene system). Or one of them could be regarded as associated with an inversion inhibiting recombination (one super-gene system).

Further, it might be that what bchave as two units of action under one set of external conditions, or in one set of internal relations, behave as one unit of action under others. Indeed the position-effect has already shown us this in another way. Finally, it seems that recombination may be mistaken for mutation if it is rare enough. This we have seen already at the Bar locus and we shall sec it again in Oenothera. 
All these residual or minimal units of genetic structure are therefore conditional and there is no reason why they should be proved to correspond in regard to action and change in different experiments. Unless, that is, the unit of action is so built up that any change of its structure whatever has the same effect; then, of course, all its mutations would be alike and no recombination would be detectable within it.

The genetical unit into which we divide the chromosome, therefore, depends on our experimental technique or our theoretical purpose. The cytological unit must also depend on the wave-length of light used in the resolution of chromomeres. In ultra-violet photographs of polytene bands in Drosophila we must be near to an ultimate analysis, for Muller and Prokofieva have found them to agree with the limits of X-ray breakage. This correspondence, however, is not so important as the general principle that the chromosome consists of a linear arrangement of particles which are frequently, or cven usually, different from their neighbours in structure, in action, and in capacity for change. But though different, they are not wholly independent, for they have to move and to work together. The question of how they move and work together is on the border-line of chemistry and to answer it we shall have to get down to the chemical level of approach.

Meanwhile we can admit that the visible chromomeres of the cytologist, and the genes as units of crossing-over and mutation of the geneticist, agree to a first approximation. They are the ultimate units of structure, change, and action in the chromosome and in heredity.

\section{REFERENCES}

BAttaglia, E. I947. La "Senigamia," singolare comportamento del nucleo spermatico nelle uova diploidi delle specie apomittiche del genere Rudbeckia (Asteraceac) e consequente embriogenesi di tipo chimerico. Rendiconti Acad. Naz. Lincei, Ser. 8, 2:63-67.

BRIDGES, C. B. I936. The bar "gene" a duplication. Science, 83: 210-2II. DARLington, C. D. I940. The origin of iso-chromosomes. J. Genet., 39: 35I-36I. DARLINGTON, C. D., and KOLLER, P. C. I947. The chemical breakage of chromosomes. Heredity, I: I87-222.

DARIINGTON, C. D., and LA COUR, L. F. 1945. Chromosome breakage and the nucleic acid cycle. J. Genet., 46: I 80-267. 


\section{BASES OF CHANGE}

fankhausi:R, G. 1941. The frequency of polyploidy, cte., in the newt. Proc. Nat. Acad. Sci. Wash., 27: 507-512.

GOLDSCHMidt, R., and KATSUKI, K. I931. Vierte Mitteilung über erblichen Gynandromorphismus und somatische Mosaikbildung bei Bombyx mori L. Biol. Zent., $5 \mathrm{I}: 58-74$.

GRÜNEBERG, H. 1938. An analysis of the "pleiotropic" effects of a new lethal mutation in the rat (Mus norvegicus). Proc. Roy. Soc. B., I25: I23-I43.

JøRGENSEN, C. A., and CRANE, M. B. I927. Formation and morphology of Solanum chimacras. J. Genet., I 8: 247-273.

Morgan, T. H., BRIDGes, C. B., and StURTEVANT, A. H. I925. The genetics of Drosophila. Bibliogr. Gentet, 2: I-262.

MULLER, H. J., and PROKOFYEVA, A. A. I935. The individual gene in relation to the chromomere and the chromosome. Proc. Nat. Acad. Sci. Wash, 21: I6-26.

RICK, C. M. I945. A survey of cytogenetic causes of unfruitfulness in the tomato. Genetics, 30: 347-362.

STURTEVANT, A. H. I928. A further study of the so-called mutation at the bar locus of Drosophila. Genetics, I3: 40I-409.

whiting, P. w. I943. Androgenesis in the parasitic wasp Habrobracun. J. Hered., $34: 355-366$. 


\title{
CONSEQUENCES OF CHANGE
}

\author{
Meiosis and Fertility in Polyploids Structural Hybridity \\ Units of Recombination Allopolyploidy \\ The Integration of Differences
}

The DIFFERENT POLYPLOID AND POLYSOMIC TYPES, and such of the new structural types as are viable, behave normally at mitosis. Their vegetative life follows an even course. At meiosis, in their germ cell formation, however, we find new types of behaviour which lead to abnormalities of breeding and the loss of fertility.

\section{Meiosis and Fertility in Polyploids.}

Triploids, or trisomics which are merely triploid for one chromosome, show the principles governing all abnormalities of meiosis, and indeed governing meiosis itself. There are three chromosomes of each type capable of pairing with one another throughout their length at prophase. In the polytene nuclei of a triploid Drosophila this expectation is fulfilled. But in all triploids, at the prophase of meiosis, association is limited to two. One is left out, but this is usually a different one at different places; the chromosomes make contact in pairs at random either near the ends or near the centromeres; each association runs along the chromosomes until it meets another and they consequently exchange partners according to the chances of their original contacts. Crossing-over takes place and chiasmata are consequently formed which hold together the paired chromosomes at the points of crossing-over. If all three chromosomes have been paired, and if one has formed chiasmata with both the other two, an association of three, a trivalent, is held together until metaphase. If one fails to cross-over with either of the other two it is left out completely, a univalent, while the other two form a bivalent (Fig. 27).

At first metaphase in triploids the bivalents arrange themselves on the plate, and the trivalents also with their centromeres either 


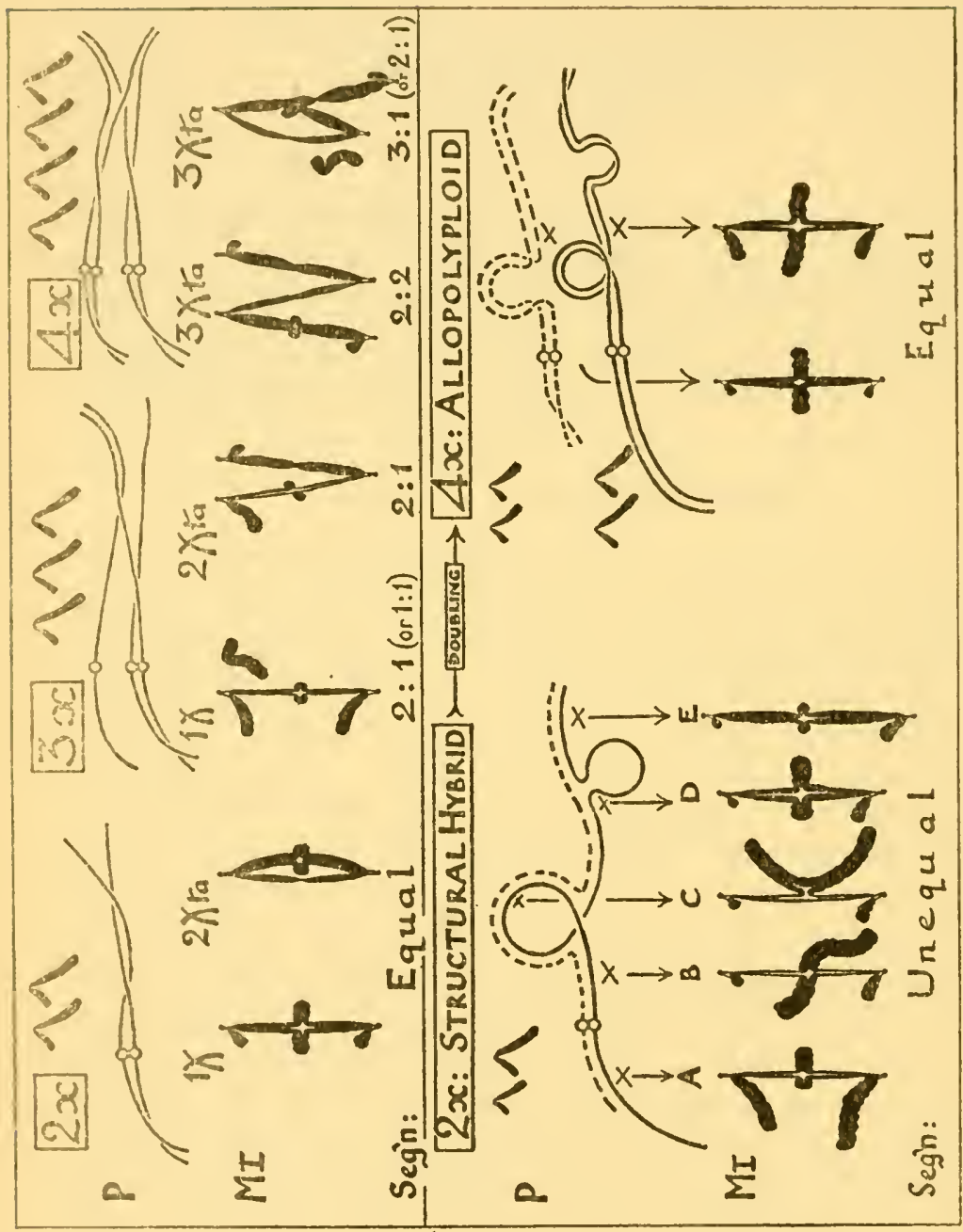

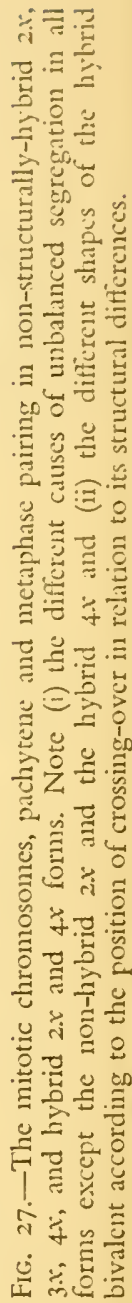


in one line or in a convergent $\mathrm{V}$. With convergence two chromosomes go to one pole and one to the other; with linearity the middle chromosome may go to either pole or it may be left behind and act as though it had been unpaired. Univalents either divide late at the first division and their daughters lag and are lost in the cytoplasm at the second; or they lag and are lost at the first; or again they are included without division in one of the daughter nuclei at the end of the furst division as though they werc daughter bivalents; or even, as we saw, they may misdivide.

TABLE 12

A. CHROMOSOMES OF POLLEN GRAINS IN SAMPLES TAKEN UNDER FAVOURABLE (F-502 GRAINS), AND LATE SEASON OR UNFAVOURABLE (U-116 GRAINS) CONDITIONS, FROM TRIPLOID CREPIS CAPILLARIS $(3 x=9)$. (FROM DATA OF CHUKSANOVA, 1939)

\begin{tabular}{|c|ccc|c|c|c|c|}
\hline $\begin{array}{c}\text { Percentage } \\
\text { of pollen } \\
\text { grains }\end{array}$ & \multicolumn{5}{|c|}{ Number of chromosomes } & Loss & $\begin{array}{c}\text { Percentage } \\
\text { of balanced } \\
\text { grains }\end{array}$ \\
\cline { 2 - 7 } & 3 & 4 & 5 & 6 & Mean & & \\
\hline Expected & $12 \cdot 5$ & $37 \cdot 5$ & $37 \cdot 5$ & $12 \cdot 5$ & $4 \cdot 50$ & - & $25 \cdot 0$ \\
F & $17 \cdot 2$ & $38 \cdot 6$ & $37 \cdot 8$ & $6 \cdot 4$ & $4 \cdot 33$ & $11 \cdot 1 \%$ & $23 \cdot 6$ \\
U & $32 \cdot 6$ & $23 \cdot 4$ & $28 \cdot 4$ & $15 \cdot 6$ & $4 \cdot 11$ & $15 \cdot 5 \%$ & $48 \cdot 2$ \\
\hline
\end{tabular}

B. PROPORTION OF THE EXTRA FREQUENCY PER GRAIN (0.5) ACTUALLY FOUND FOR THE THREE EXTRA CHROMOSOMES, A, C AND D

\begin{tabular}{|c|ccc|c|c|}
\hline Chromosome & $+\mathrm{D}$ & $+\mathrm{A}$ & $+\mathrm{C}$ & Mean & Loss \\
\hline $\mathrm{F}$ & 1.00 & 0.88 & 0.79 & 0.89 & $11 \%$ \\
$\mathrm{U}$ & 0.89 & 1.02 & 0.62 & 0.84 & $16 \%$ \\
\hline Weighted Mean & 0.98 & 0.90 & 0.76 & 0.88 & - \\
\hline Loss & $2 \%$ & $10 \%$ & $24 \%$ & $12 \%$ & - \\
\hline
\end{tabular}

Note: "Loss" is the deficiency of the mean with respect to the binomial expectation. As in Hyacinthus, it is highest for the shortest chromosome because this forms fewest chiasmata and therefore fewest trivalents.

As a rule the different trivalents orientate themselves at random with respect to one another on the first metaphase spindle. In con- 
sequence the distribution of the odd set is a binomial one with halt the triploid number expected to be the most frequent type. The mean and the mode, however, are usually below $3 x / 2$ on account of the loss of a varying number of chromosomes, usually to or 20 per cent. The loss is, of course, highest in species with the

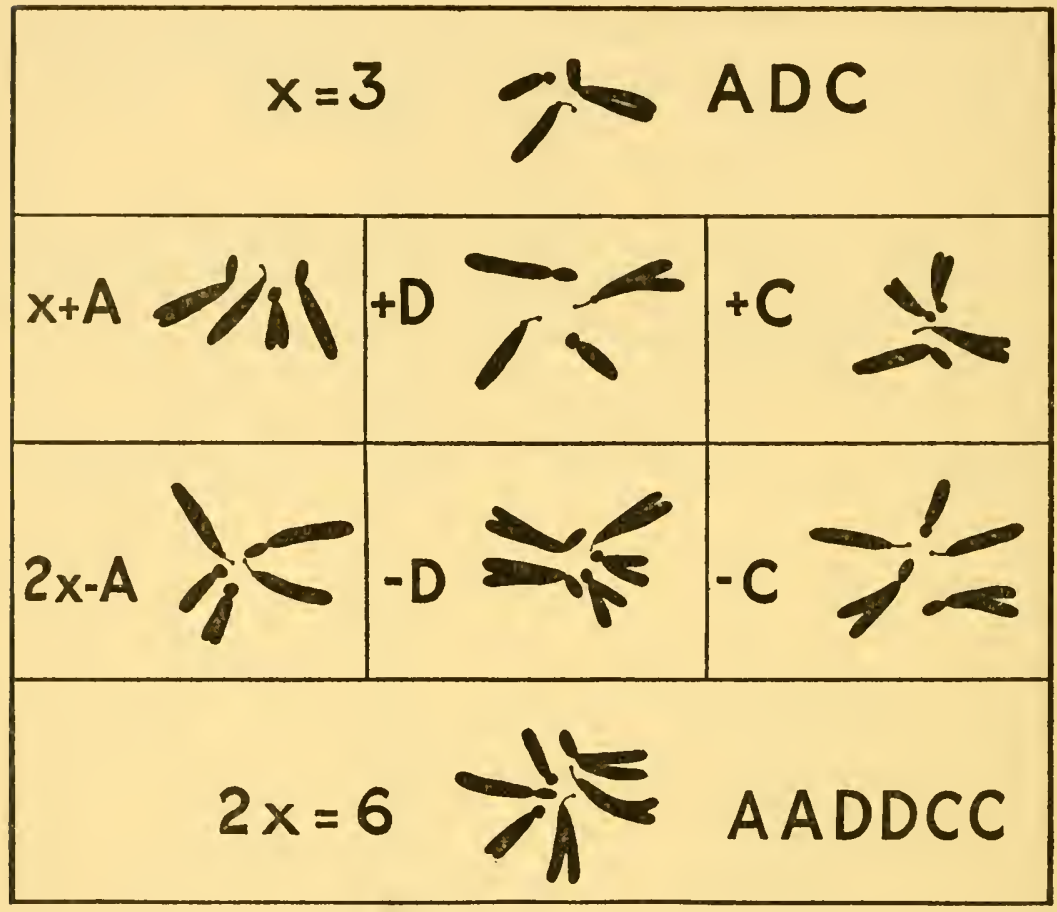

FIG. 28.-Mitosis in types of balanced and unbalanced pollen grain produced by a $3 x$ Crepis capillaris in the proportions shown in Table I2 (after Chuksanova, I939).

smallest chromosomes, and for the smallest chromosomes in a set, since these have the fewest changes of partner, the fewest chiasmata, and therefore the most failure of pairing. Loss is also higher in the larger cmbryo-sac mother-cell than in the smaller pollen mothercell.

The course of events at meiosis in triploids is reflected in the frequencies of the pollen grains having different chromosome numbers (Fig. 28 and Table I2). At mitosis in the pollen grains of Crepis capillaris each of the three chromosomes can be recognized. 
In the triploid the numbers of grains with an extra one of each kind can be recorded. The average number of chromosomes should be $4 \cdot 5$, but is always less owing to loss of unpaircd chromosomes at meiosis (Table I2A). Samples also differ owing to the fact that the balanced grains develop more quickly than the unbalanced oncs and some chromosomes even differ from others in their effect on balance and development (Tablc I2B).

\section{TABLE 13}

PERCENTAGE DISTRIBUTION OF EXTRA CHROMOSOMES AS SEEN IN THE POLLEN GRAINS (P.G.) AND IN THE PROGENY $(3 x \times 2 x$ AND $2 x \times 3 x)$ OF TULIPA, WITH LARGE, AND OF DATURA, WITH SMALL CHROMOSOMES. THE NUMBER OF OBSERVATIONS IS GIVEN IN PARENTHESIS IN THE FIRST COLUMN. (UPCOTT AND PHILP, 1939)

\begin{tabular}{|c|c|c|c|c|c|c|c|c|c|c|c|c|c|c|}
\hline No. of chromosomes & 0 & 1 & 2 & 3 & 4 & 5 & 6 & 7 & 8 & 9 & 10 & 11 & 12 & Mean \\
\hline Tulipa P.G. (100) & - & 一 & 3 & 12 & 9 & 14 & 19 & 16 & 10 & 9 & 6 & 2 & & $6 \cdot 17$ \\
\hline $3 x \times 2 x$ & - & - & 14 & 10 & 22 & 20 & 6 & 14 & 6 & 4 & 4 & 一 & 一 & $5 \cdot 04$ \\
\hline $2 x \times 3 x$ & 20 & 40 & 32 & - & - & - & 一 & - & 4 & - & - & 4 & 一 & $1 \cdot 90$ \\
\hline Datura P.G. (500) & 3 & 4 & 7 & 11 & 15 & 16 & 11 & 11 & 9 & 5 & 4 & 3 & 1 & $5 \cdot 35$ \\
\hline $3 x \times 2 x \quad(285)$ & 20 & 48 & 28 & 4 & - & - & - & 一 & - & - & 一 & - & 一 & $1 \cdot 15$ \\
\hline $2 x \times 3 x$ & 86 & 14 & - & 一 & - & - & 一 & - & 一 & - & - & - & - & $0 \cdot 14$ \\
\hline
\end{tabular}

Note: The basic number for both is 12 , so that the mean number of extra chromosomes without loss would be 6 .

What happens to the pollen and eggs with different numbers is shown by the frequencies of plants with correspondingly different numbers when these pollen grains and eggs are united with haploid gametes, that is to say, in reciprocal crosses with diploids (Table I3). We then find that there is a reduction of the types with the middlc numbers (which are the most unbalanced) and with the higher numbers (which are most unlike the gametes of the diploid parent).

The elimination of unfavoured types is much stronger when it is the triploid parent which is providing the pollen. Evidently the pollen is subjected to a stricter test than the eggs. The reason for this might be that the numbers of pollen grains on the stigma are alivays grcater than the number of ovules to be fertilized. Or it might bc 
that a proportion of unbalanced types which can survive as eggs if fertilized are unable to grow down the style at all as pollen grains. This is evidently the case, for the fertility of triploids is actually lower as pollen than as egg parents (Table I4).

\section{TABLE 14}

THE FERTILITY OF RECIPROCAL CROSSES OF DIPLOIDS AND TRIPLOIDS, SHOWING THAT IT IS LOWER WHEN THE TRIPLOID IS THE POLLEN PARENT, AND ALSO IN PLANTS WITH HIGHER BASIC NUMBERS OF CHROMOSOMES. THE DATA ARE EXPRESSED AS PERCENTAGES OF THE INFERRED NORMAL SEED SET. (UPCOTT AND PHILP, 1939)

\begin{tabular}{|c|c|c|c|c|c|c|c|}
\hline \multirow[b]{2}{*}{ Species } & \multirow[b]{2}{*}{$x$} & \multicolumn{3}{|c|}{$3 x \times 2 x$} & \multicolumn{3}{|c|}{$2 x \times 3 x$} \\
\hline & & $\begin{array}{l}\text { Ripe } \\
\text { seeds }\end{array}$ & $\begin{array}{l}\text { Germin- } \\
\text { ated }\end{array}$ & $\begin{array}{l}\text { Sur- } \\
\text { vived }\end{array}$ & $\begin{array}{l}\text { Ripe } \\
\text { seeds }\end{array}$ & $\begin{array}{l}\text { Germin- } \\
\text { ated }\end{array}$ & $\begin{array}{c}\text { Sur- } \\
\text { vived }\end{array}$ \\
\hline Campanula persicifolia & 8 & $23 \cdot 3$ & $2 \cdot 5$ & - & $9 \cdot 8$ & 1.8 & 一 \\
\hline Zea mays .. $\quad . . \quad \ldots$ & 10 & $14 \cdot 0$ & $5 \cdot 3$ & - & $6 \cdot 8$ & $4 \cdot 4$ & - \\
\hline Tulipa gesneriana $\quad$. & 12 & $19 \cdot 6$ & $7 \cdot 8$ & $2 \cdot 7$ & $5 \cdot 5$ & $2 \cdot 8$ & $1 \cdot 0$ \\
\hline Daturn stramonizm .. & 12 & $4 \cdot 4$ & $4 \cdot 1$ & $2 \cdot 9$ & - & - & $1 \cdot 2$ \\
\hline Primula sinensis $\quad$. & 12 & 0.06 & 0.03 & $0 \cdot 00$ & $0 \cdot 19$ & 0.00 & $0 \cdot 00$ \\
\hline Pyrus malus .. & 17 & $2 \cdot 15$ & $1 \cdot 40$ & $1 \cdot 25$ & $0 \cdot 29$ & $0 \cdot 19$ & $0 \cdot 13$ \\
\hline
\end{tabular}

— indicates that no data are available.

There is another interesting sequel to the random segregation of extra chromosomes and the selection of balanced types in triploids. As the basic number increases, the proportion of haploid gametes remains always about $\frac{1 \times}{2}$. Therefore fertility declines with increasing basic number. It is high in Drosophila or Crepis, low in Pyrus (Table I4).

Tetraploids behave as we should expect on these principles (Fig. 27). The chromosomes pair, that is each member of the set forms two pairs; but they pair differently at different points and thercfore have to change partners as they do in triploids. Their association at pachytene then resembles diplotene in a diploid. Where they are paired they may form chiasmata. Hence associations of four appear at metaphase wherever changes of partner and chiasmata permit. Failing such quadrivalents we find pairs of bivalents or trivalents and univalents.

The quadrivalents arrange themselves on the spindle at metaphase, like the trivalents, either in a line or zig-zag, that is 
convergently. Or, like two bivalents, they can lie parallel in a rectangle. Again, middle members of these configurations can lag and be lost so that separation may be two-and-two or three-and-one or two-and-one with one lost. The products of meiosis are thus sometimes equal in their numbers of cliromosomes, but perhaps equally often they show an error of one or two above or below. This error is what reduces the fertility of tetraploids, but the reduction is of the order of one quarter or less, not as with triploids of the order of one half, for each chromosome of the basic set.

We learn several new principles from these observations. In the first place, we see that the triploid is a numerical hybrid. It has two nuclear units from one side and only one from the other. Like other hybrids or heterozygotes it is incapable of breeding true and even its loss of fertility, as we shall find later, is characteristic of hybrids which arise from crossing species.

The genetic system of the iriploid is adjusted until it comes to the production of germ cells. It then breaks down. It does so because at meiosis new individuals, new self-propagating systems, with new and untried combinations of chromosomes are brought into being. Unbalance, which appears in mature plants and animals as the cause of abnormal forms, appears in immature individuals as the cause of death. The fertility of the parent is the inverse measure of the frequency of death of the offspring. The segregation of differences at meiosis is thus a prime cause of sterility.

Secondly, we see that development follows a standard course in organisms with the standard set of chromosomes equally muitiplied. But where the multiplication is unbalanced, development is unbalanced and disturbed. Evidently all the chromosomes are different in their effects on growth, just as they and their parts are different in their specific attractions for one another at meiosis. This disturbance accounts for the slightly reduced fertility of trisomics and of tetraploids, whose segregation is slightly irregular, and for the greatly reduced fertility of triploids, whose segregation is grossly irregular.

An exception proves this comnexion between balance and fertility. In triploid hyacinths, Hyacintluns orientalis, fertility is not greatly reduced, and plants with all intermediate numbers betreen diploid and tetraploid appear in their progeny. The development 
of these plants is not disturbed too much to prevent them providing some of our best garden varietics. Thus in this spccies the chromosomes are less sharply differentiated than usual. Or, to put it the other way round, each chromosome is balanced in much the same way as the chromosome set in the aggregate is balanced. The fertility of the triploid and the normality of the trisomic follow from this unusual condition.

\section{Structural Hybridity}

The product of structural change in the chromosomes, like the product of numerical change, reveals its peculiar properties at meiosis. We then see that it is a structural hybrid, an individual

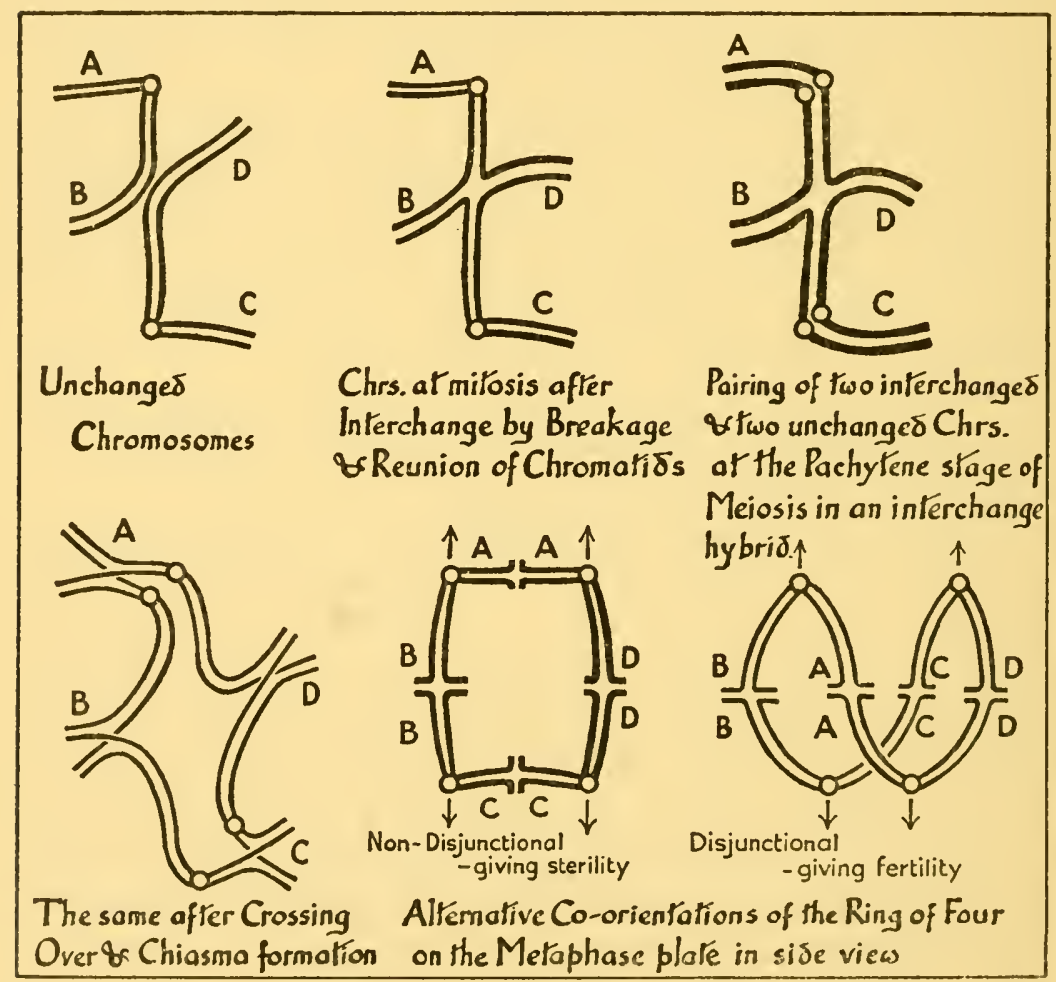

FIG. 29.-The consequences of interchange between two non-homologous chromosomes $\mathrm{AB}$ and $\mathrm{CD}$ at the succeeding mitosis and at meiosis where the zygote is in consequence heterozygous and forms a ring of four chromosomes which, in a proportion of cells, by failure of disjunction of alternate chromosomes, gives sterile haploid products. 
heterozygous for the new change. The simplest type is the interchange hybrid. Its movements resemble those of a tetraploid (or tetrasomic) although their genetic consequences are quite different. Interchange, by recombining segments, produces from two old chromosomes, $A B$ and $C D$, two new ones, $B C$ and $D A$. When the new and old types pair at pachytene in the hybrid they form a cross arrangement like that given, with one exchange of partners, in a tetraploid; but the position of the exchange of partner is fixed at, or near, the position of the interchange. There is no choice (Fig. 29).

\section{PACHYTENE PAIRING IN STRUCTURAL HYBRIDS}

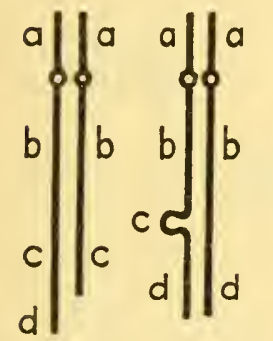

DEFICIENCY (Terminal)

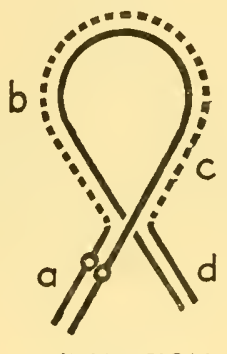

INVERSION

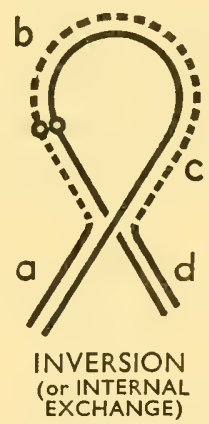

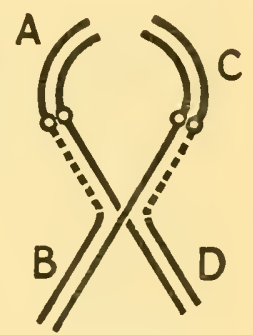

INTERCHANGE

Fig. 30.-The changes produced by loss, inversion and interchange illustrated by their effects at pachytene in the hybrid.

From the exchange system of pairing a ring of four, chain of four, two chains of two, or a chain of three with a univalent, can arise at metaphase. Again, as in the tetraploid, these different configurations arise according to the chance distributions of chiasmata and with frequencies depending on the lengths of the four pairing segments. Hence we have some, but not all, of the metaphase figures found in the tetraploid. What is more important, however, is that the results of segregation are balanced only in one particular combination, namely where $A B$ and $C D$ pass together to one pole, and $B C$ and $D A$ to the other. In a flowering plant such as Datura, therefore, whose spores have an independent life, $A B+B C$ or $C D+D A$, and $B C+C D$ or $A B+D A$ combinations die (Fig. 29). In Drosophila, on the other hand, where the genetic constitution does not affect the capacity of eggs or sperm, balanced 
zygotes can be produced by the chance fusion of complementary gametes, such as the first two or the last two, each of which was itself unbalanced. In both the plant and the animal a proportion of the eggs are sterile, but different classes of eggs in the two cases.

In breeding the simple interchange hybrid, such as has been found wild in Campanula (or produced by crossing in Datura) we are concerned only with those types of progeny which can live. We then find that it breeds like a simple mendelian hybrid with one difference: it is the hybrid which we distinguish and record as different from either of the two homozygous types of its parents or of its progeny. Sclfing yields I $A B . A B+C D . C D: 2 A B . C D /$ $B C . D A:$ I $B C . B C+D A . D A$; that is to say equal numbers are homozygous (with bivalents only) and heterozygous (with rings of four); provided, that is, that the homozygotes are as viable as the heterozygotes, which is not always the case.

The hybrid for two interchanges has potentially two rings of four chromosomes at meiosis, or where the interchanges involve a common chromosome, a ring of six. Thus, by interchange of $A$ and $E$ :-

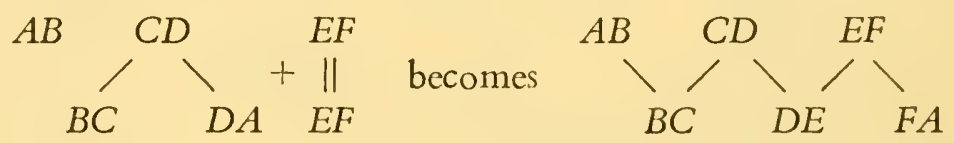

This double interchange hybrid again gives only three viable types of progeny and in the ratio of I : $2:$ I. Thus two unit changes in the chromosomes are giving one unit difference in segregation and in heredity. Not only this, but they are at the same time throwing three linkage groups into one. Here we have a first example of how structural change can, by combination, reduce the number of units of heredity: it restricts recombination, partly by directing segregation and partly by killing a selected portion of the recombinants.

Interchanges can successively increase the size of the ring, and the linkage group it represents, until they include all the chromosomes in the complement. This condition is found in many wild species such as Rhoeo discolor with I2, Oenothera muricata with I4, and Hypericum punctatum with I6, chromosomes. With this knowledge it was possible also, by crossing plants hybrid for different single 
interchanges in Campanula persicifolia with I6 chromosomes, to build up multiple hybrids with rings of twelve.

Inversions, though less obvious than interchanges, are a commoner and more important source of structural hybridity. They are found as heterozygotes in a proportion of individuals probably in all cross-breeding species. And in the homozygous condition they
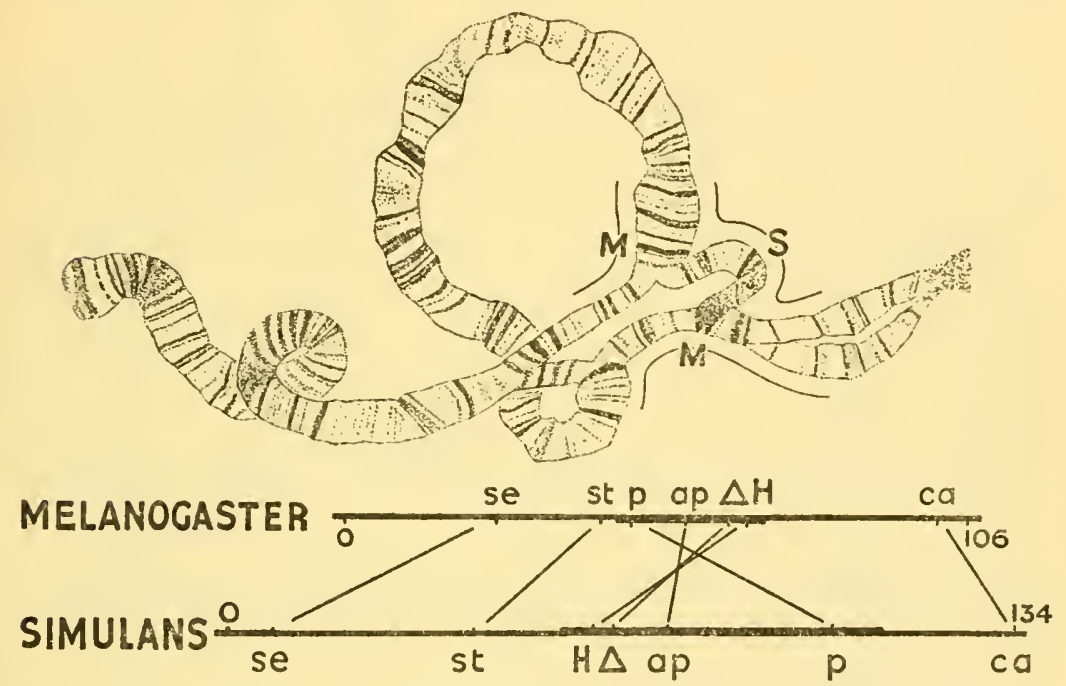

FIG. 31.-The right arm of chromosome III of Drosophila simulans has a segment which is inverted relative to the corresponding chromosome arm in D. melanogaster. The effect of this inversion may be seen cytologically in the pairing of the chromosome arm in salivary gland nuclei of the species hybrid (above) or genetically in the order of homologous genes when mapped by the crossing-over percentages occurring within each of the species (below). It will be observed also that crossingover is freer in chromosome III of simulans than melanogaster, the interval se-ca (which includes the whole of the inversion) being 127 units as compared with so (after Patäu, I935).

distinguish different species (Fig. 3I). A true inversion, one which does not include the centromere, leads to a pairing of the two relatively changed segments the wrong way round with respect to the centromere. The inverted segments pair in a loop (Fig. 30). Any single crossing-over within this loop gives two new chromatids, one with two centromeres attached to it, a dicentric, the other with none, an acentric. The consequences of this structure are seen at anaphase: the acentric chromatid is left helpless on the equator and 
is lost; the dicentric is stretched between the two poles and promptly broken, unless, indeed, it is long enough to join the two daughter nuclei. The two broken chromatids, even if the break is exactly midway, are bound to be deficient in the genes contained in the acentric fragment and the nuclei carrying them dic-at least in a diploid. The products of single crossing-over are therefore always lost.

\section{Units of Recombination}

We must now consider what relation these changes in number and structure of chromosomes have to the type of change ordinarily studied in mendelian experiments and to its mode of inheritance.

In triploids the unit of change, the whole nucleus, breaks up into its elements, the separate chromosomes, in the next generation. With single-chromosome and structural changes the unit of change may persist as a unit of heredity: in heterozygotes the abnormality may segregate from normality as a mendelian difference. But these changes have certain characteristics of their own by means of which they may be distinguished from the ordinary mendelian factor. In the homozygote, inversions and interchanges affect the linkages of blocks of genes in the ways we should expect; inversions, by setting the genes of a linkage group into a new order (see Fig. 3I); interchanges, by separating genes previously linked and bringing together genes previously in separate linkage groups. In the heterozygote, however, their effects are peculiar.

In inversion heterozygotes, single crossing-over within the inversion produces non-viable deficiencies. Only when double crossing-over occurs and chances to be reciprocal, i.e. between the same pair of chromatids and therefore genetically compensating, can there be recombination of genes within an inversion. Short inversions therefore suppress recombination within them. They establish a block of genes as a new single unit of recombination in all descendants which are heterozygous for the old and new arrangements. They work on the principle already suggested in our consideration of the agouti gene in mice.

In the light of these observations we find inversions appearing as "cross-over suppressors" when heterozygous in breeding experiments. Sturtevant found such "genes" in Drosophila which he 
interpreted as due to inversions, a view later confirmed by the observed formation of loops in the polytene chromosomes.
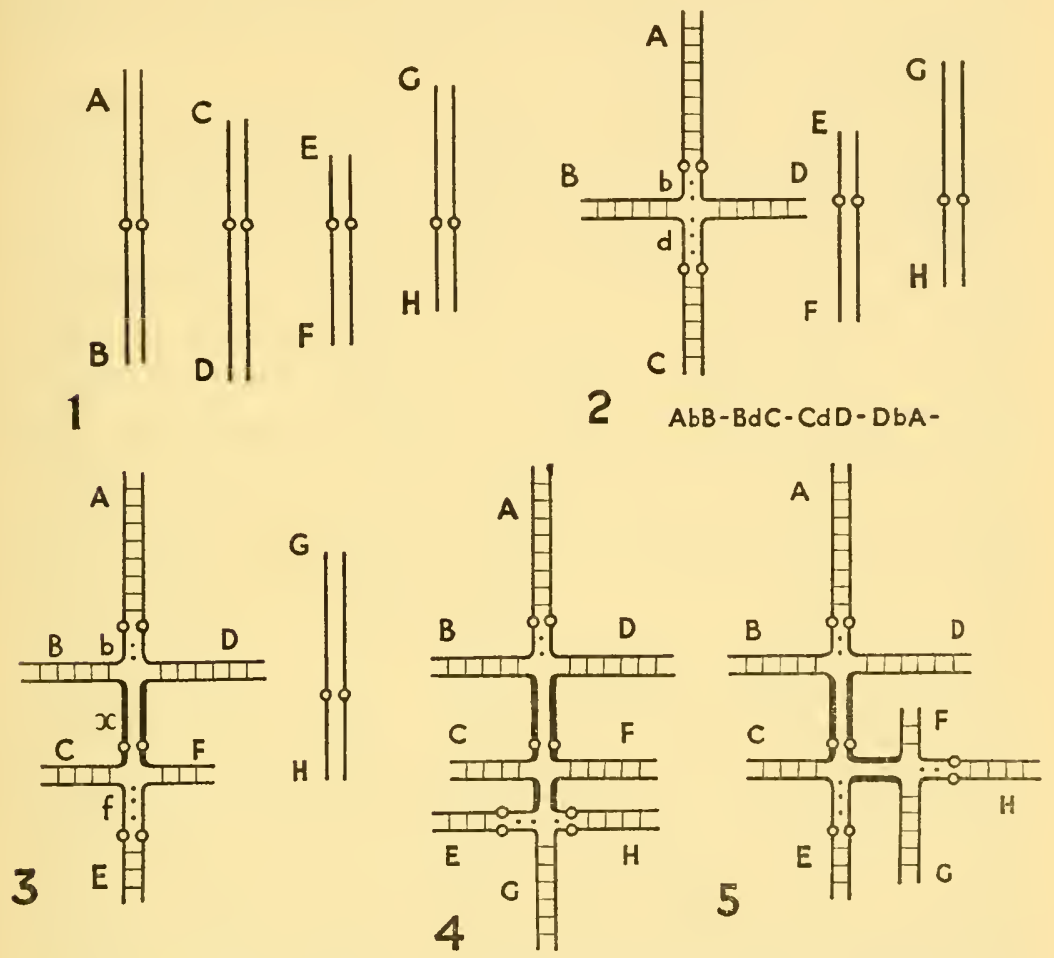

$A b B-B x C-C F E-E f F-F x D-D b A-$

$$
A B-B C-C E-E G-G H-H F-F D-D A
$$

FIG. 32.-Pachytene arrangement, showing how rings can be built up by simple interchange in various ways, but always giving interstitial segments as well as pairing terminal segments (united by cross lines). The centromeres are shown as circles; the terminal segments are marked by block capital letters: (I) four pairs; (2) ring of four (with two pairs of interstitial segments marked by small letters corresponding to the whole arms from which they are derived), and two pairs; (3) a ring of six, with two pairs of interstitial segments $(b$ and $f$ ) and a differential segment $(x)$ and one pair of chromosomes $\mathrm{GH}$; (4) and (5) two ways of producing a ring of eight by interchange with $G H$ from the ring of six. The two configurations have the same formula for pairing segments, but different arrangements and numbers of interstitial segments (from Darlington, 1935).

Inversion, indeed structural change in general, thus reduces the number of recombining units and creates, where the homozygotes are viable, a new super-unit-a super-gene giving a $\mathrm{I}: 2:$ I 
segregation. In the homozygotes, pairing and crossing-over being again normal, the super-gene breaks down into its separable units. The unit of heredity may therefore rise or fall from one generation to the next as hybridity rises or falls. To these flexible super-genes we shall have to return later.

Interchange heterozygotes likewise show a reduced recombination near the interchange. They also show a marked loss of fertility. Belling found such a "gene" in the bean Stizolobium which he interpreted as due to an interchange. In the heterozygote, interchange, again like inversion, reduces the distances in the recombination, linkage, or crossing-over map. In a diploid with $\mathrm{x}$ pairs of chromosomes, $\mathrm{x}$ separate lines make the crossing-over map and they correspond, as they must always do, with the $\mathrm{x}$ pachytene threads. The hybrid with a single interchange has two lines intersecting at the point where the interchange has occurred to make a cross; and with two interchanges it has a six-spoke figure just as it has six arms at pachytene. So each new interchange reduces by one the number of linkage groups in the hybrid.

The spokes of the interchange wheel would all radiate from one point if the interchanges all occurred at corresponding points; but in practice they occur at different points. The ring-of-six has six arms which radiate not strictly as spokes, but as the arms of a cross of Lorrainc. The middle piece of this cross is formed by median segments which are homologous and may pair, but have distal arms on both sides which are not homologous (Fig. 32). Crossing-over between these two median segments occurs in Campanula rings and gives gametes with only one of the two interchanges and consequently progeny with a ring of four. In other words the combination of chromosomes brought about by two interchanges can be broken down in the hybrid by crossing-over between them, just as the combination of two gene differences within one chromosome can be broken down in the hybrid by crossing-over between them.

\section{Allopolyploidy}

When a diploid plant or animal, as is usual with species hybrids, is heterozygous for numerous structural changes, their effect is less noticeable in distorting the pairing at the first metaphase of meiosis, 
than in reducing the total amount of it. The reason for this is mechanical. Chromosomes usually begin to pair at their ends and when they reach a place where homology changes, owing to a difference in the linear sequence of the two partners, they may fail to find their new partners. They may even go on pairing by torsion with the wrong partners. In either case there is a wastage of the time and energy needed for pairing. There is less crossing-over; fewer chiasmata are formed; these are more exclusively near the ends; and some chromosomes may altogether fail to be paired at metaphase. Hence, in crosses between distantly related species, there is generally a reduction, and sometimes a complete absence, of pairing. The structural differences between the homologous chromosomes have become so great as to prevent the formation of those chiasmata whose presence would enable us to define the differences. In such hybrids there can be no regular segregation. The gametes (or spores) receive unbalanced assortments of chromosomes in varying numbers, and sterility results.

The sterility of a hybrid with defective pairing need not, however, be final. Its case is much like that of the haploid. Separation of nuclei may fail at the first anaphase in some mother cells. Gametes are then formed with the unreduced and unsegregated number of chromosomes. Such diploid gametes are fertile and, if they fuse, they give tetraploid offspring. But tetraploids arising in this way are something new. They have four sets of chromosomes, but these chromosomes can associate only in pairs. They form bivalents which segregate regularly. An allotetraploid, as it is called, is therefore highly fertile. It is functionally a diploid, but it combines the characters and the chromosomes of two diploid species; it is amphidiploid. Such is the origin of the fertile true-breeding and giant radishcabbage hybrid, Raphano-brassica of Karpechenko (Fig. 33).

The allotetraploid stands at one extreme; the autotetraploid, the product of doubling a homozygous diploid such as a tomato, stands at the other. Between the two is a series derived from diploid parents and corresponding to every degree of differentiation of which diploid hybrids are capable. As a midway type we may take Primula kewensis. Its parents, P. floribunda from the Himalayas and $P$. verticillata from Southern Arabia, have an average of about 2 chiasmata in each of their nine bivalents. In the hybrid this is 

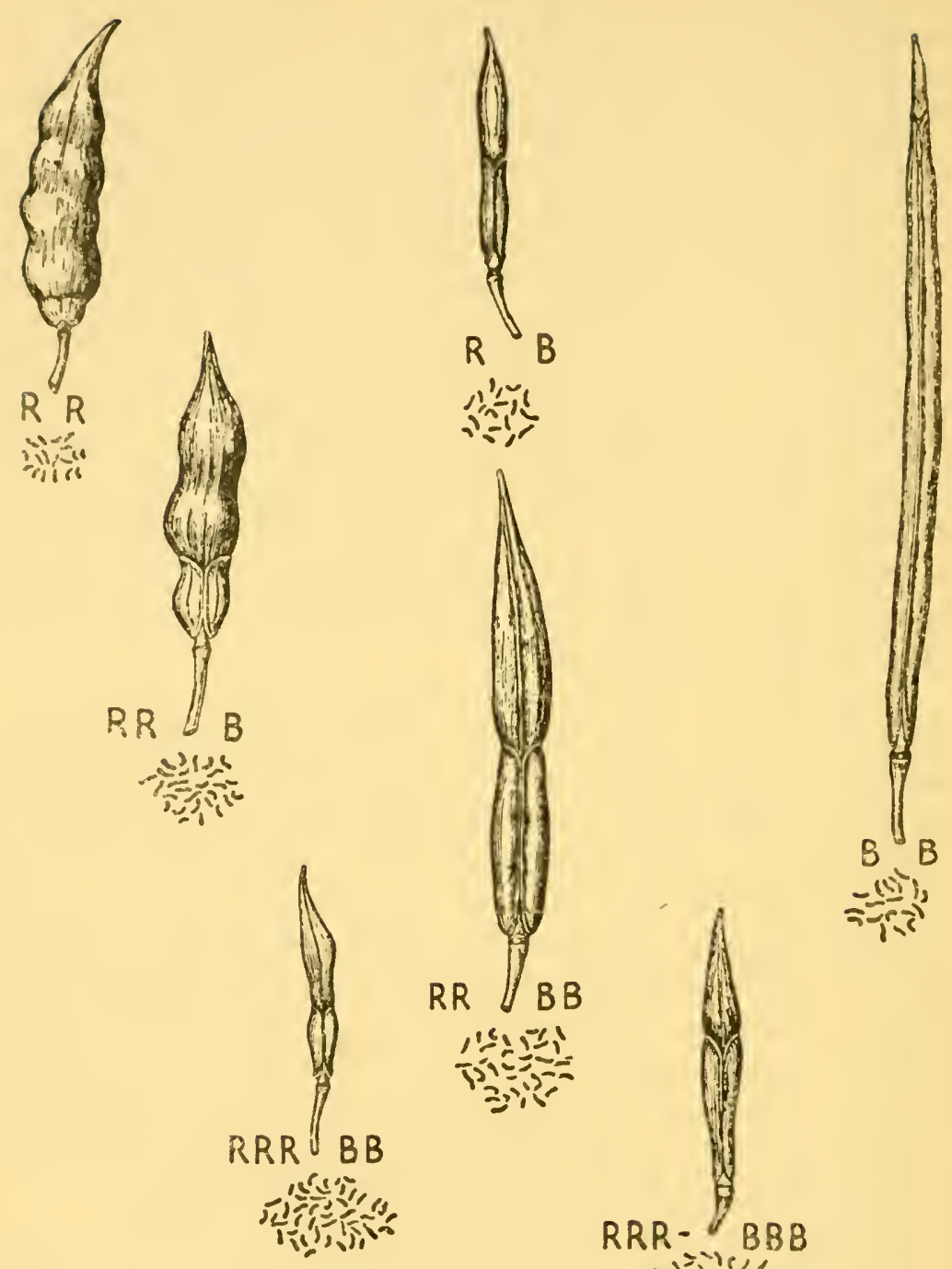

Fig. 33. -The pods (slightly reduced) and the chromosomes ( $\times$ I,000) of Raphanus (R), Brassica ( 13$)$, and their hybrids with $\mathrm{R}$ and $\mathrm{B}$ chromosomes in different numbers (influencing fertility and therefore size) and different proportions (influcncing shape) (after Karpechenko, 1927). 


\section{ALLOPOLYPLOIDY}

reduced to $\mathrm{I} \cdot 36$ and some have no chiasmata; that is to say metaphase pairing fails but the reduction of pairing is by no means so large as the reduction of chiasma frequency (Table I5).

TABLE 15

CHIASMA FREQUENCIES IN THE PARENTAL SPECIES AND IN THE DIPLOID AND TETRAPLOID HYBRID, PRIMULA KEWENSIS, $x=9$ (UPCOTT, 1939)

\begin{tabular}{|c|c|c|c|c|c|c|c|c|c|}
\hline & \multirow[t]{2}{*}{ No. of nuclei } & \multicolumn{5}{|c|}{$\begin{array}{l}\text { Configurations with } \\
\text { different nos. of } \\
\text { chiasmata }\end{array}$} & \multicolumn{3}{|c|}{ Chiasmata } \\
\hline & & 0 & 1 & 2 & 3 & 4 & Total & $\begin{array}{c}\text { per } \\
\text { nucleus }\end{array}$ & $\begin{array}{l}\text { per } \\
\text { bivalent }\end{array}$ \\
\hline P. floribunda .. & 10 & 0 & 10 & 70 & 10 & 0 & 180 & $18 \cdot 0$ & $2 \cdot 00$ \\
\hline P. verticillata .. & 10 & 0 & 11 & 58 & 18 & 3 & 193 & $19 \cdot 3$ & $2 \cdot 14$ \\
\hline P. kewensis $(2 \mathrm{x})$ & 20 & 17 & 82 & 80 & 1 & 0 & 245 & $12 \cdot 3$ & $1 \cdot 37$ \\
\hline \multirow[t]{5}{*}{ P. kewensis $(4 \mathrm{x})$} & $10 \int 1$ & 一 & 一 & - & - & - & 0 & 一 & - \\
\hline & $\{$ II & 0 & 19 & 95 & 10 & 0 & 239 & - & $1 \cdot 93$ \\
\hline & $\mathrm{III}+\mathrm{I}$ & 0 & 0 & 1 & 3 & 0 & 11 & - & $1 \cdot 38$ \\
\hline & IV & 0 & 0 & 0 & 4 & 20 & 92 & - & $1 \cdot 92$ \\
\hline & Total .. & 0 & 19 & 96 & 17 & 20 & 342 & $34 \cdot 2$ & $1 \cdot 90$ \\
\hline
\end{tabular}

I, pairs of univalents; II, bivalents; III + I, trivalents and univalents; IV, quadrivalents.

Structural differences are too small to show in this hybrid except by their interference with the formation of chiasmata. Segregation is therefore nearly regular. Nevertheless sterility is complete. Each gamete contains an apparently normal set of nine chromosomes, but some are from one parent and some from the other. These sets must be unbalanced and fail to work merely because they are mixed. How this might happen we can now see quite easily. If translocations had occurred between the chromosomes since the separation of the parental species then recombinations of their chromosomes would give duplications and deficiencies which would kill the gametes.

Now, the vegetatively propagated clone of the diploid Primula kewensis has produced tetraploid shoots on several occasions. Failure of mitosis has led to a doubling of the chromosomes in a cell of the growing point. The new tetraploid plant is fertile and true-breeding 
once more. But it is not quite so fertile or so true-breeding as the radish-cabbage tetraploid. Why is this?

At meiosis in the tetraploid $P$. kewensis the identical chromosome
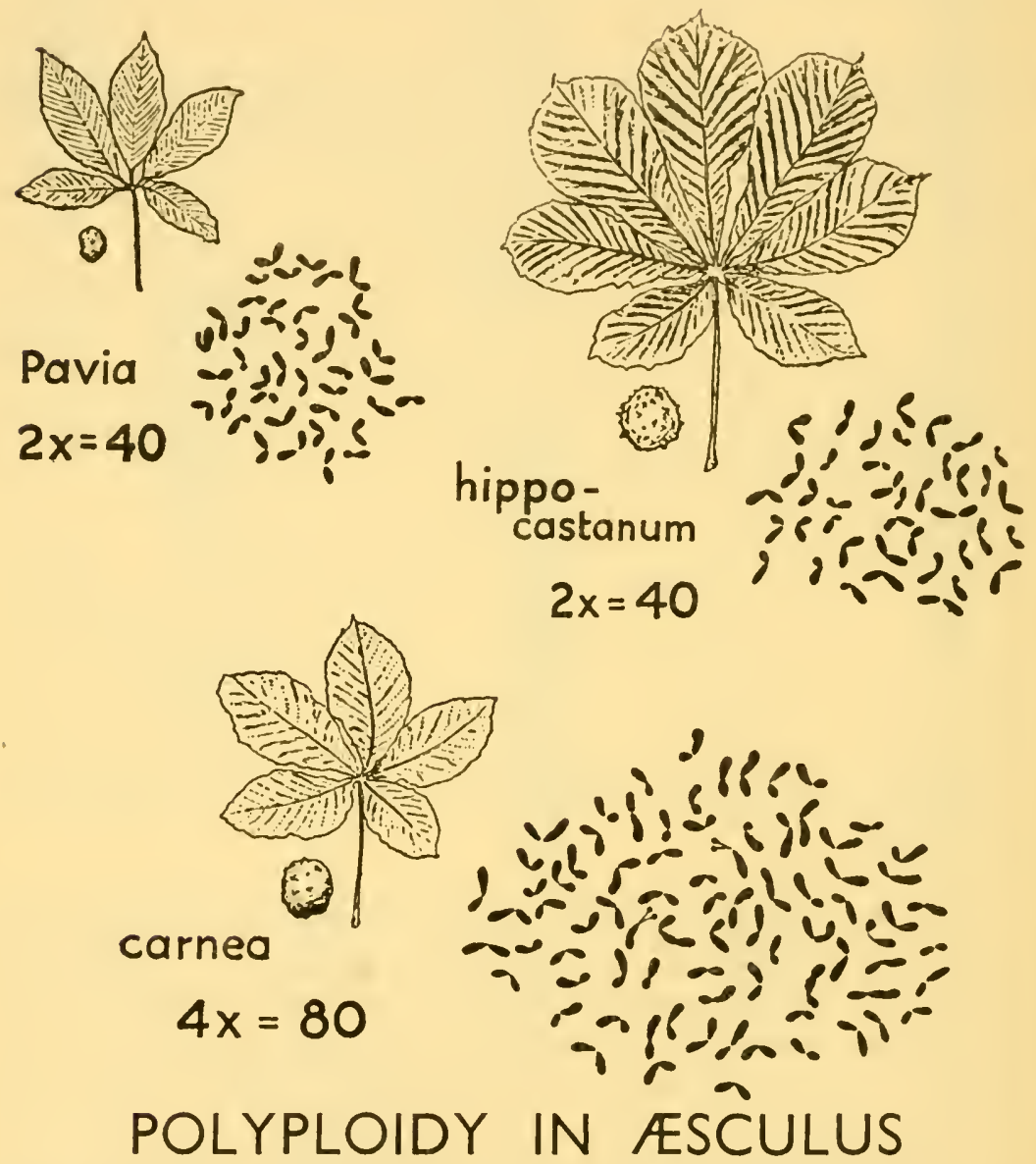

Fig. 34.-The leaves, fruits and chromosomes of Aesculus carnea, a tetraploid of the Primula kewensis type, and its diploid parents (after Upcott, 1936).

from one parental species pair with one another rather than with those of the other species. The frequency of chiasmata is almost as high as in the diploid species (Table IS). But some cross-pairing occurs and it can be seen in two ways. First, types that are no longer intermediate appear in the progeny. They resemble one of the 
parental species, for cxample in regard to the mealiness of the leaves. Secondly, one or two quadrivalents are seen at meiosis in each mother cell. These quadrivalents behave like those in autopolyploids; by their unequal three-and-one separation they give rise to gametes and to progeny with unbalanced chromosome sets. Some of these 35and 37-chromosome plants live and are seen to be deformed, while others evidently die and thereby reduce the fertility (Table I6).

\section{TABLE 16}

\section{DISTRIBUTION OF THE CHROMOSOMES AS SHOWN BY} THE FREQUENCIES OF POLLEN GRAINS WITH DIFFERENT NUMBERS IN THE TETRAPLOID PRIMULA KEWENSIS; $x=9$ AND $n=18$, THE MEAN EXPECTED WITHOUT LOSS (UPCOTT, 1939)

\begin{tabular}{|l|rrrrr|r|c|c|}
\hline Chromosomes & 16 & 17 & 18 & 19 & 20 & Total & Mean & Loss \\
Pollen grains .. & 2 & 44 & $\mathbf{8 1}$ & 22 & 1 & 150 & $17 \cdot 84$ & $0 \cdot 89 \%$ \\
\hline
\end{tabular}

Apart from the occasional quadrivalents of the Primula the same principle applies to both these allopolyploids. The differences which cause sterility in the diploid, whether with good pairing or with bad pairing, in the tetraploid cause a preferential pairing of identical partners, and this favours regular pairing (without quadrivalents or univalents), regular segregation, and in consequence a high fertility. The association of more than two chromosomes is the danger to the fertility of the polyploid. The association of less than two is the danger to the diploid. For this reason the more sterile is a diploid, the more fertile will be the tetraploid to which it gives rise. In fact completely fertile diploids like Primula sinensis, Datura stramonium or the tomato give rise to tetraploids of reduced fertility. And for this reason, too, when we find autopolyploid species of flowering plants it is because, as with the pentaploid Tulipa clusiana, seed production has ceased to matter or, as with the tetraploid T. turkestanica or octoploid Dahlia variabilis, no chromosome forms more than one chiasma and so quadrivalents are evaded (Fig. 35).

Between the two types of hybrid, in which structural hybridity is too slight or too great to be defined, lie a host of crosses between species, like those in Lilium or Drosophila, in which the chromosomes of the parents differ recognizably by inversions, interchanges, dupli- 
cated fragments or more complex changes. Allopolyploids are derived from hybrids of all such types and by successive additions appear as tetraploids, hexaploids and so on. Their increase of number gencrally increases their size. Their combinations of characters fit

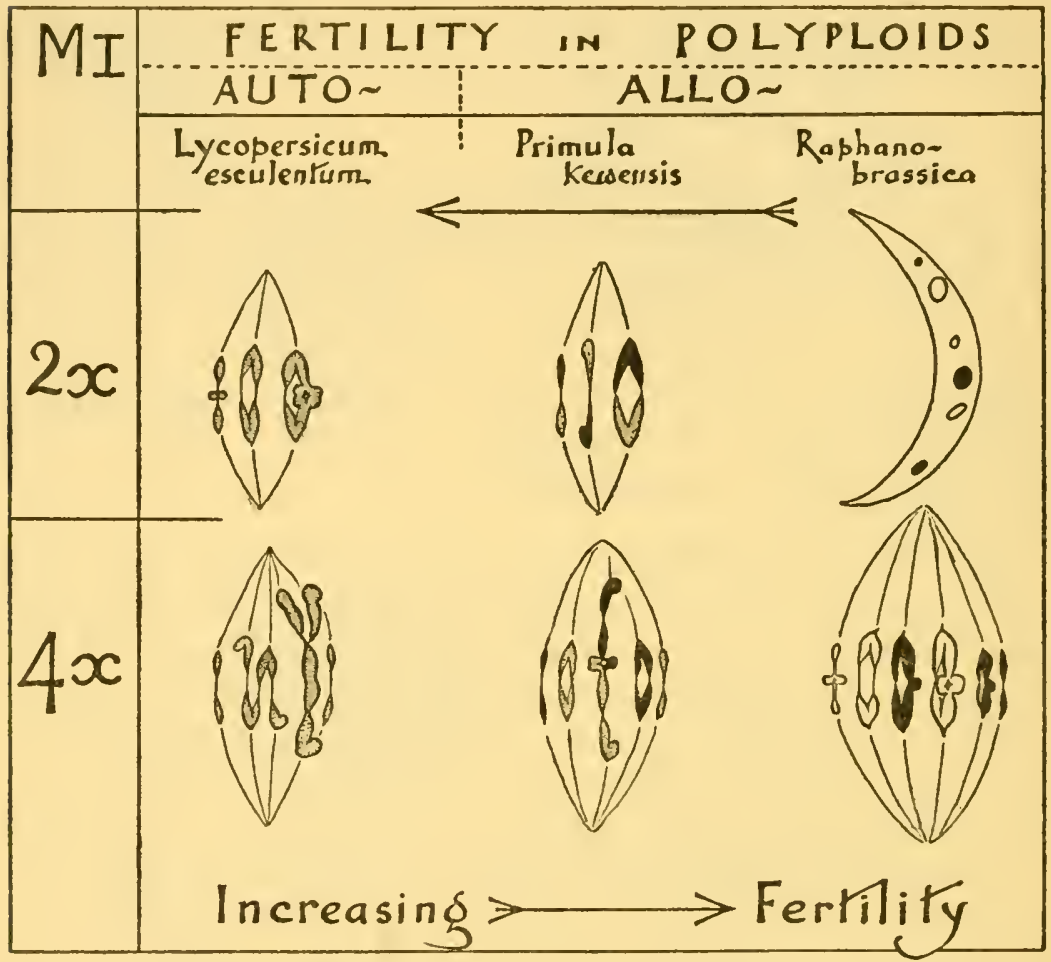

Fig. 35.-Pairing at meiosis in diploids of different degrees of hybridity and segregational sterility compared with that in the tetraploids derived from them: to illustrate the law of the negative correlation of fertility in diploid and tetraploid.

them for new conditions, fit them for colonization. Occasionally they segregate differences by accidental pairing of chromosomes from the ultimate diploid parents and this also gives them adaptability. But yet they remain highly fertile. It is not surprising, therefore, that a large proportion of the species of flowering plants owe their origin to alloploidy, and more especially a large proportion of the new types of plants that have covered the cultivated part of the earth in the last 10,000 years (cf., Fig. 82). 


\section{The Integration of Differences}

We have seen that the hereditary materials, the chromosome complement contained within the nucleus, can undergo changes of three types, in number, in structure, and in a residual class of point mutations. The distinction between the structural, or inter-genic, and the intra-genic changes follows inevitably in theory from the fact that the chromosome is a linear arrangement of dissimilar particles. But it cannot always be made in practice owing to the fact that the two types can be combined in one integrated difference.

We have seen that the combination of the dissimilar chromosomes and parts of chromosomes in a working set constitutes balance. The primary changes that occur in their numbers and in the arrangement of their parts do not upset this balance in vegetative life. But when meiosis takes place these primary changes are broken up and recombined so as to produce secondary changes with new kinds of balance. Many of these new combinations fail to work. The gametes and zygotes carrying them die and fertility is reduced.

We have also seen how structural hybridity will reduce chromosome pairing, crossing-over and recombination, or even abolish them altogether, and in so doing remedy the infertility of a polyploid on the one hand and of a gene hybrid on the other. Hence all three types of change, genic, structural and numerical are concerned with one another in permitting the principle of variation to operate in a system of heredity.

\section{REFERENCES}

Chuksanova, N. 1939. Karyotypes of pollen grains in triploid Crepis capillaris. Comp. Rends. (Doklady) Acad. Sci. U.S.S.R., 25: 232-235.

Darlington, C. D. I936. The limitation of crossing-over in Oenothera. J. Genet., 32: 343-352.

DARLington, C. D. I940. The prime variables of meiosis. Biol. Rev., IS: 307-322.

DARLINGTON, C. D. and GAIRDNER, A. E. I937. The variation system in Campanula. J. Genet., 35: 97-128.

DARLington, C. D., and MATHER, K. I944. Chromosome balance and interaction in Hyacinthus. J. Genet., 46: $52-61$.

KARPeChenko, G. D. 1927. Polyploid hybrids of Raphanus sativus L. $\times$ Brassica oleracea L. B. Ap. Botany, 17, 305-408. 


\section{CONSEQUENCES OF CHANGE}

PÄTAU, K. 1935. Chromosomenmorphologie bei Drosophila melanogaster und

Drosoplila simulans und ihre Bedeutung. Naturwiss., 23: 537-543.

UPCOTr, M. 1936. The parents and progeny of Aesculus carnea. J. Genet., 33: 135-149. UpCOT, M. 1939. The nature of tetraptoidy in Primula kewensis. J. Genet., 39: 79-100.

UPCOTT, M., and pHiLP, J. 1939. The genctic structure of Tulipa. IV Balance, selection and fertility. J. Genet., 38:9I-I23. 
PART II

CELLS 



\section{GENES, MOLECULES AND PROCESSES}

Chromosome Structure Chromomeres and Proteins Heterochromatin and Polygenes What Major Genes do The Interaction of Genes The Expression of Genes Synthetic Sequences: Supply and Demand

WE HAVE LEARNT SOMETHING of the resemblances and differences that occur between parents and their offspring, and of the materials and processes which are ultimately responsible for these relationships. But we also have to discover how these materials, lying in the cells of animals and plants, are so organized that they determine the effects we observe, and by what steps they do so. How does the cell nucleus with its outfit of genes and chromosomes act on the cell with the constancy that we recognize in heredity; repeating in each generation the elaborate processes of development which seem to contradict all possibility of a constant basis? What part does the cytoplasm play in this paradox? To these questions we must now address ourselves.

\section{Chromosome Structure}

The nucleus, as we have seen, is a body constituted of chromosomes. The chromosome is a body constituted of a protein fibre to which active groups or genes are attached. Pepsin disintegrates this fibre and it must therefore be based on a polypeptide chain. This chain may be single or multiple but it must be highly folded in the ordinary resting nucleus since, when the nucleus grows (as in the salivary glands), the chain itself stretches to many times its early prophase length.

During mitosis, nucleic acid is attached to the genes which, owing to the folding of the thread between them, form an almost continuous chain. The nucleic acid consists of an unlimited polymerization of desoxyribose nucleotides to form a column. The nucleotides are spaced at the same distance of $3 \cdot 3$ Ångström units apart as are the repeats in the stretched polypeptide chain. Desoxyribose nucleic 
acid is found only attached to chromosomes and in bacteria and certain animal and bacterial viruses. In all of these it must indicate the presence of genetically analogous structures.

At low temperatures in plants, or with low feeding in animals, the chromosomes can be starved of nucleic acid. Two consequences follow. First the chromosomes, especially their end genes, fail to reproduce, or at least to separate the products of their reproduction, in mitosis. The daughter chromosomes consequently sometimes stick together at anaphase. Secondly, certain short segments of the chromosomes are undercharged and therefore fail to stain. In extreme cases the coiling or spiralization of the chromosome thread fails. It remains uncoiled at metaphase. Such a failure of coiling is usual during rapid nitoses in certain protozoa but would be highly inconvenient in the specialized cells of higher organisms if it affected the whole chromosomes. Thus the attachment of nucleic acid regulates the reproduction of the chromosomes, possibly, as Astbury suggests, by acting as a template. It also locks them up in spiral bundles; and these are not merely neat bundles for movement and distribution. They are strong bundles protected against the dangers of extra-nuclear life, even, as experiment shows, against being broken by X-ray ionization or chemical poisons.

The uncoiling of the chromosomes at telophase depends on their throwing off this coat of nucleic acid. How do they do this ? Evidently the nucleic acid is depolymerized and broken down. At the same time the products of action of the genes, now uncovered, fill the nucleus; and the simple proteins produced, together with the ribose nucleic acid derived by reconstruction from the desoxyribose, are assembled by a particular gene or organizer to reconstitute a nucleolus. We now find, however, that in some cells of some organisms certain constant parts of the chromosomes do not throw off their coat. The nucleic acid becomes sticky, owing perhaps to depolymerization, but remains attached throughout the resting stagc. These parts are known as heterochromatin as opposed to the normally bchaving euchromatin. It is these parts or blocks which in some organisms, under the conditions we saw, are liable to be starved of nucleic acid at mitosis. 


\section{Chromomeres and Proteins}

There are, as we saw in the first chapter, two types of nucleus which reveal their chromosome structure while they are still active in producing proteins. They are the polytene and the pachytene nuclei. In both of these, chromomeres appear. The explanation of these chromomeres, advanced by Caspersson, is that the nucleic acid is attached to the active genes whose products stretch the normally folded thread between them. Their gradual emergence from a homogeneous thread at the beginning of the prophase of meiosis confirms this view (Fig. 36).

Thus chromomeres are genes making themselves visible as working units. This is not so in the diffuse resting nucleus where nucleic acid is not attached and where proteins are pushed straight outwards to give the appearance sometimes described as a lampbrush chromosome. In the polytene nuclei these products of gene (or chromomere) activity can be observed. Heterochromatin, which is active in these nuclei, produces simpler proteins of a histone type, while euchromatin produces larger, more complex and therefore, we must suppose, more specific proteins of a globulin type. The chromomeres of heterochromatin are accordingly smaller and less exactly paired than those of euchromatin.

There are chromosomes which contain little but heterochromatin, for example the $\mathrm{Y}$ chromosome in Drosophila, and supernumerary chromosomes in maize and other plants. These appear to have little specific effect on the organism. When extra ones are added, however, other things being equal, the size of the nucleolus and the quantity of nucleic acid in the cytoplasm are said to be increased. Such an increase within the organism can be shown to go with increased protein production. For example, ribose nucleic acid, which is absent from actively fermenting yeast in the absence of a source of nitrogen, immediately appears when nitrogen is made available and proteins begin to be produced.

Heterochromatin, ribose nucleotides, and protein production are thus closely bound up together. Ribose nucleotides are abundant, not only in embryonic and cancerous tissue, but also in the egg cells of plants and animals where protein production is not immediately taking place. But here, as Brachet has shown, it is 


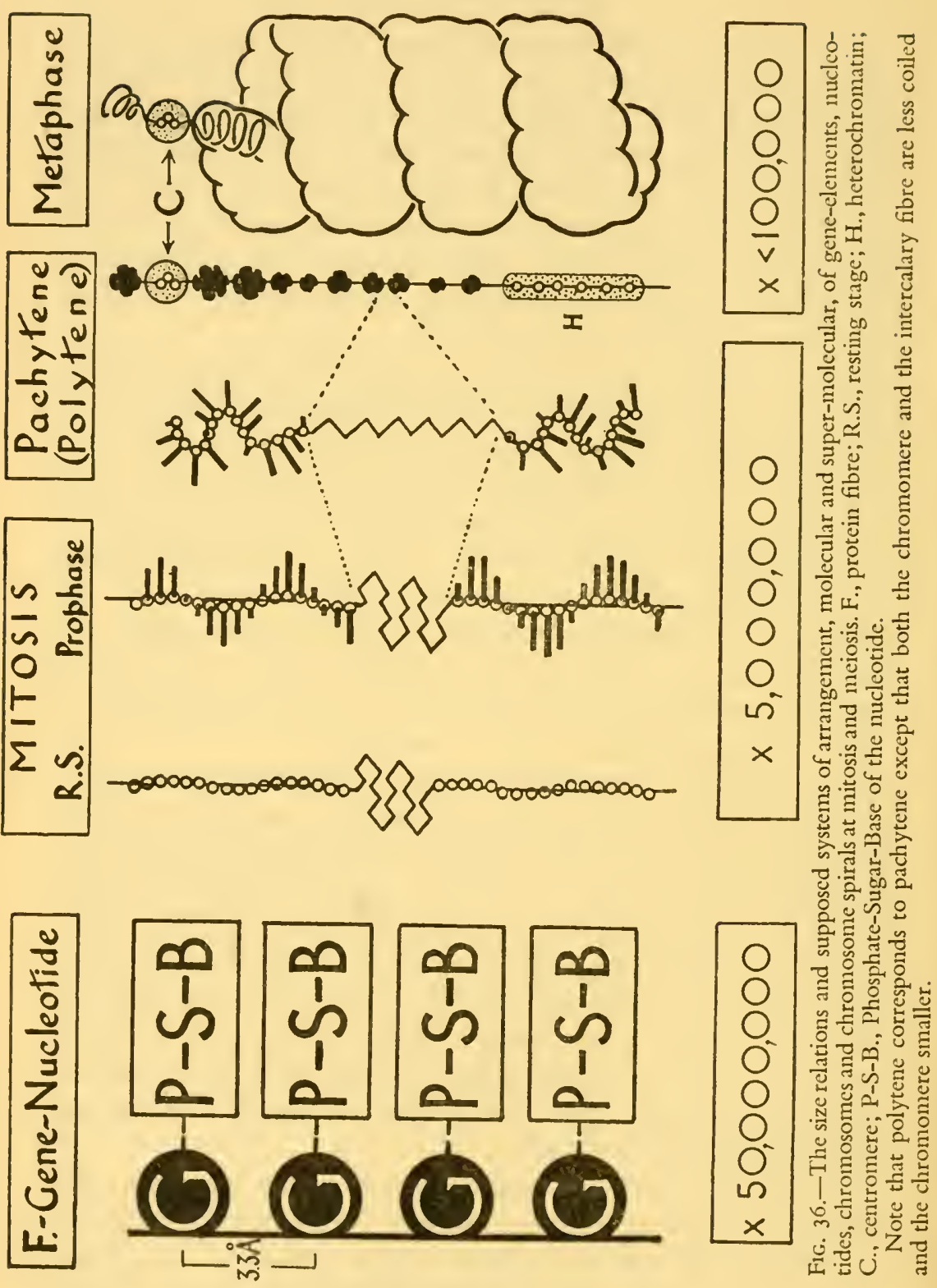


necessary for conversion into the desoxyribose form required for the rapid multiplication of nuclei which follows fertilization. Ribose nucleotides are capable of some polymerization. Their main work, however, seems to be less mechanical and more physiological than that of their desoxyribose relatives. They are concerned with protein reproduction. They are regularly found in the smaller viruses, in the plastids of plants and in other small protein bodies of the cytoplasm. The viruses and plastids are characteristically self-propagating bodies like the genes of the nucleus. Ribose nucleic acid, therefore, may well do for these proteins in a small way what the desoxyribose does for the more highly organized chromosome, namely, act as the agent of their reproduction.

What picture do we then get of the chemical relations of the nucleus and the cell? The nucleus is a differentiated and highly organized, mechanically stable and totally balanced system of protein-producing units. The cytoplasm has no mechanical stability to give it a permanent and total balance. Indeed, as we shall see, differentiation between cells depends on changes in its balance. It is not surprising, therefore, that the permanence of control in heredity and development so largely comes from the nucleus. At the same time the existence of self-propagating bodies in the cytoplasm would indicate that the control of the nucleus was not total, immediate and absolute. The cytoplasm must always feed the nucleus with the materials from which it builds up its specialized structures and with which it in turn feeds the cytoplasm. Each must feed the right materials to the other. When, as we saw, the nucleus of one species is put in the cytoplasm of another, breakdown often results from the failure of this reciprocal process. In development, therefore, we cannot argue for the greater importance of one or the other. It is only the long-term relations concerned with the permanence of heredity that enable us to take their interactions to pieces and show where precedence and authority lie.

This picture opens our eyes to a number of new possibilities. First, although there are many animals and plants in which the differentiation of the heterochromatin is not visible, it seems likely that the two kinds of genes are always present. The one kind gives the simpler products and should have less specific, and individually 
less pronounced, cffects. But these effects should include, as they do, an influence on the other kind which gives the more complex products and should have the more specific and elaborate effects. Secondly, we must expect the actions of genes to depend on each other and on the varying character of the cytoplasm as reflecting their earlier action. Differentiation will depend to some extent on the limitations of diffusion within the nucleus and the cell. Finally, we must expect that the cytoplasm as wcll as the nucleus will contain self-propagating particlcs, although they are likcly to bc subordinate to the nuclcus in gencral since they have not the same powers of orderly reproduction and transmission that are given by the giant chromosome molecule.

\section{Heterochromatin and Polygenes}

The difference between genes in heterochromatin and in euchromatin is in fact made clear in several ways. As we saw, the $\mathrm{Y}$ chromosome in Drosophila and the supernumeraries in plants, both heterochromatic, have few or no drastic effccts. The supernumeraries are not even indispensable. And none show mutations of the sharp and specific kind that are used in mendelian experiments. These principles apply whether the heterochromatin composes the whole chromosome, or, as in the X of Drosophila, a part of it. On these grounds, wherever it has been found, heterochromatin has been described as inert. Now, however, we have two means of demonstrating its peculiar activity. In maize many cultivated varieties regularly have supernumeraries which vary in number from plant to plant. Yct these chromosomes often have defective centromeres and so tend to be lost at meiosis or even at mitosis. Their maintenance must therefore depend on a sclection which favours increasc in number of chromosomes, and this can come about only if these chromosomes actively affect the character, the phenotype, of the plant. The same principle applies to supernumerary fragments of the $\mathrm{X}$ chromosome found up to the number of 14 in the bed bug, Cimex lectularius, as well as in many other Heteroptera.

The second means of approach is through the polygenic systems which we have scen to govern continuous variation. The members 
of such a system have small, similar and supplementary effects, because, we may presume, they have less differentiated products and less elaborate action.

The simpler action of these genes at once recalls the simpler products of heterochromatin as seen in the cell. The relationship is brought closer when we find that heterochromatic chromosomes, hitherto regarded as inert, affect quantitative variation.

In a diploid millet, supernumeraries, which are lost except in the stem, lead to extra mitoses in the pollen. The larger the number of supernumeraries, the larger the proportion of pollen which is killed in this way. Nor is this an isolated example, for supernumeraries are now known to affect the phenotype of a number of grasses and cereals in a quantitative way. Quantitative effects are also produced by the Y chromosome in Drosophila melanogaster. Y chromosomes from different strains vary in their effects on the numbers of bristles. But the greater part of this variation can be destroyed by association with a common $\mathrm{X}$ chromosome partner. It thus seems that genes in the $Y$, affecting bristle number, can be exchanged with corresponding genes in the $\mathrm{X}$. And, since only the heterochromatic portion of the $\mathrm{X}$ lies in the pairing segment and is homologous with part of the $\mathrm{Y}$, we can see that X-borne, as well as Y-borne, heterochromatin is active in this way. The crossing-over that would account for such exchange has, as we saw, been cytologically inferred from the occurrence of reciprocal chiasmata between their pairing segments.

The heterochromatin is thus active; it is variable in its activity; and this variability is divisible by crossing-over. In other words it is composed of genes similar in linear organization to the major genes, but differing in kind or order of physiological effect. Indeed the smallness of their individual effects implies the largeness of the number of these polygenes.

We can now recognize two types of gene and two types of chromatin. The major genes occur only in euchromatin and heterochromatin contains only polygenes. Euchromatin may well also contain polygenes, perhaps in the euchromatin proper or perhaps lying in segments not distinguishable as heterochromatin owing to their sniall size or for some other reason. This is all the more probable since, as we saw, many plants and animals have no 
recognizable heterochromatin. In the onion Allium cepa, for instance, some varieties have visible heterochromatin and others not. And in species with blocks of heterochromatin, these blocks are always more variable in size and number among individuals than are the parts of the cuchromatin.

\section{What Major Genes Do}

The mode of action of polygenes can, as yet, only be inferred from the activity of heterochromatin in producing small proteins and ribose nucleic acid. The modes of action of the major genes, on the other hand, are recognizable by other means. The most important of these is the dosage method of Muller. By X-raying sperm, Muller produced flies with broken chromosomes and, combining these with normal chromosomes, he was able to obtain particular genes or their mutant allelomorphs present in one, or three, or four doses instead of the proper two. He also obtained combinations of wild type and mutant in different doses. What he then found was that most mutant genes, whether themselves spontaneous or induced by X-rays, had the same action as their normal allelomorphs, only to a lesser degree.

Similarity of action of the normal and mutant gene could be shown in the following way. The mutant gene scute-I normally removes, or appears to remove, certain of the wild-type bristles. But, when an extra scute-I gene is added, the number of bristles is almost normal. With two extra scute-I genes the fly actually has more bristles than the normal. Thus, far from removing bristles, scute-I helps to produce them. It does the same job as the wild type, but less than half as effectively. Such a gene Muller calls a hypomorph or, in the extreme case, when it does nothing, an amorph. All deficiencies will, of course, appear as amorphs.

Other types of action are also shown by this method. The hypermorph is more efficient than the wild-type gene. The antimorph opposes the action of the wild-type gene. The neomorph does something new. Clearly these terms are comparative. The wild-type gene is hypomorphic to its hypermorphic mutant and amorphic to its ncomorphic mutant. Antimorphs and ncomorphs frequently, if not always, involve structural changes, though these may be only small duplications as in the neomorph Bar. It may well be, therefore, 
that they depend on a position effect, that is on the integrated action of more than one genic constituent.

Of these types, all but hypomorphs are rare. What does this

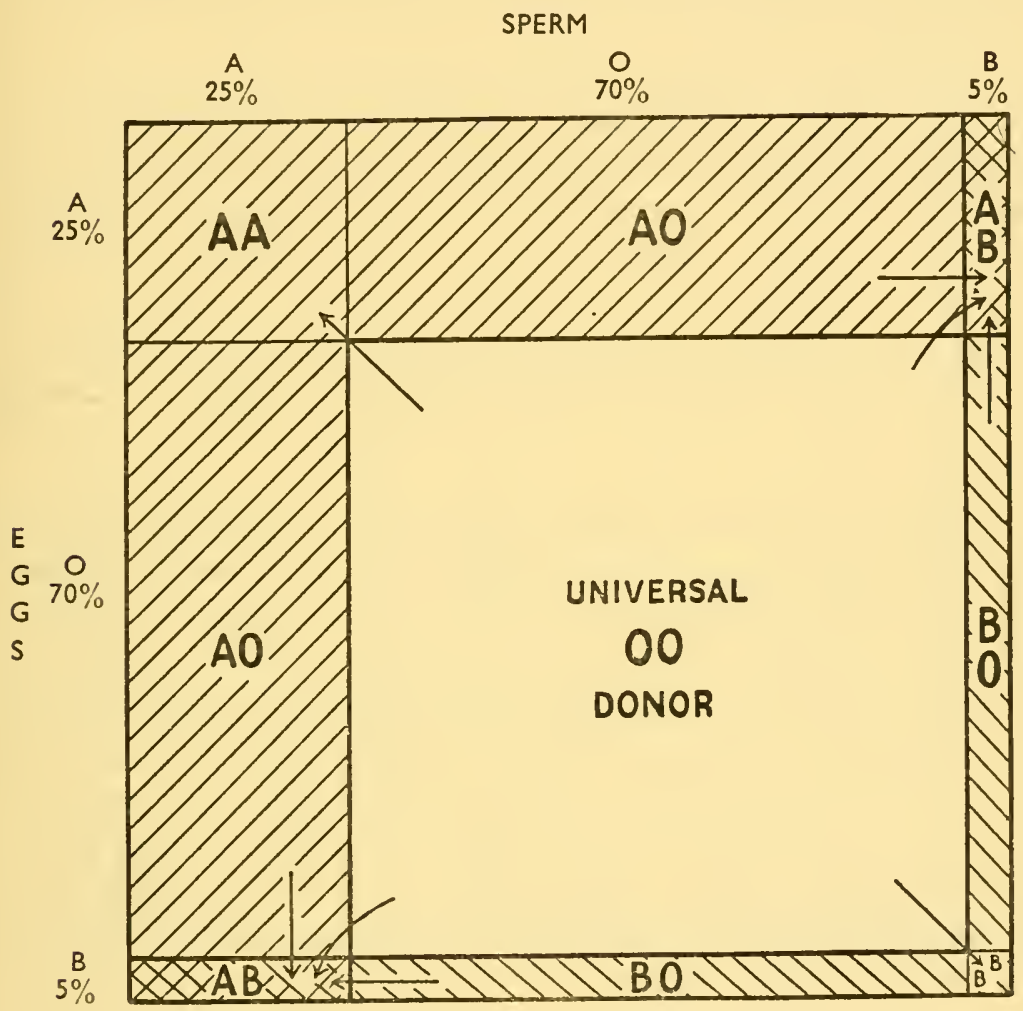

FIG. 37.-The A, B, O blood group system governing blood transfusion in man. The approximate frequencies of the three allelomorphs in the gametes of the English population are shown along the margins of the diagram. Six genetically different zygotes will then occur with frequencies proportional to the corresponding areas in the diagram. $\mathrm{A}$ and $\mathrm{B}$ are, however, each dominant to $\mathrm{O}$, though not to one another. Thus genotypes $A A$ and $A O$ are indistinguishable in the phenotypes they give, as are $\mathrm{BB}$ and $\mathrm{BO}$. There are then four groups distinguishable in transfusion behaviour, viz. $\mathrm{AB}, \mathrm{B}, \mathrm{A}$ and $\mathrm{O}$, as shown by the cross-hatching. The directions in which blood can be successfully transfused are shown by the arrows. Group $\mathrm{O}$ is the universal donor and $\mathrm{AB}$ the universal recipient.

mean? It means that mutation, in the major genes, is generally a loss of efficiency, a breakdown in the working of a complex mechanism, a pathological change in a healthy system. Thus, by a 
new method we have additional evidence that the elements of the euchromatin are producing complex proteins and therefore have a complex and delicately adjusted organization. In such a system undirected change is likely to break down the old rather than to build up the new.

Another way of getting at this problem is by examining what appears to be the closest to the gene of all the products of its activity. Antigen production is the most specific and unconditional activity of major genes. The chain of reactions from the gene in the nucleus to the antigen in the cell is therefore the shortest we can infer. All organisms contain antigens since any protein can behave as an antigen. But in the blood groups which govern the possibilities of transfusion in man and other animals, different antigens are directly associated with corresponding genes. If the gene is present so is the antigen.

There are many sets of blood-group allelomorphs such as $M-N$, and the Rhesus series. In a heterozygote of any of them the antigens of both allelomorphs are present in the cell-notably, of course, in the red blood cells where they are recognizable if the appropriate antibody is induced. In the important $A-B-O$ series in man and the apes, it happens, for a reason that is not yet understood, that the antibodies to $A$ and $B$ occur spontaneously, whereas $O$ cannot even induce one easily. Thus $O$ is recessive to $A$ and $B$ and serologically appears merely as their absence (Fig. 37). On the other hand, in certain species hybrids of doves new antigens appear to arise which are found in neither parent; thus the co-operation of more than one gene in their production is implied. Otherwise, so close is the connexion, that the distinction between gene and antigen is formally maintained only because of the necessity for distinguishing between determinant and product which genetics has taught us to respect. Now the blood antigens, and likewise the antibodies they call forth, are complex proteins; we therefore have the best evidence that such proteins can be the immediate products of gene activity.

\section{The Interaction of Genes}

The relation between the gene and the antigen it produces seems generally to be a simple one; as simple indeed as that between the 
gene and the daughter it produces in its own reproduction. Gene reproduction makes use of the special mechanism, we might almost say of the midwife molecule, of nucleic acid. But nucleic acid appears not to enter into the production of other molecules by the genes. We should not, therefore, expect the same simple correspondence between the gene and its effect in action, as between the gene and its daughter in reproduction. Furthermore it is hardly likely that genes will fail to interfere or react with one another, either in the consumption of materials or in the release of products. On the contrary, we should expect genes to interact with one another, both within the nucleus and outside it in the cytoplasm.

One type of interaction between genes in their work we have seen in the position effect. This effect not only shows interaction; it shows that the interaction can occur within the nucleus. Another interaction that is probably within the nucleus is that whereby one gene modifies the rate of mutation of another. In Drosoplila this happens at times as a result of a position effect. A gene of normal stability is moved close to the heterochromatin and becomes highly mutable, so much so that it regularly produces a mixture or mosaic of mutant and non-mutant tissues, such as that shown by Plum-eye. The control of mutability of one gene by others is common, and, though not generally acting by position effect, the modification of mutation rate may have something to do with the heterochromatin. For example, in maize the Dotted gene, which is recognized by its effect on the mutability of an anthocyanin gene, is shown by linkage tests to lie in or near the heterochromatic end "knob" of chromosome Io.

Mutability control being so widespread we should expect that an upset in the genetic organization or balance would be reflected in changed mutation rates. It is, in fact, often claimed that $F_{1}$ species crosses show a greater frequency of mutation-visible somaticallythan either parent.

The first types of genic interaction were recognized by Bateson in the course of the series of studies between 1900 and I9IO in which he established the general validity of mendelism. These interactions proved to be ones which cannot be traced back to the nucleus, and indeed probably arise very far away from it. They are the types which reveal themselves in the segregations of whole individuals in 


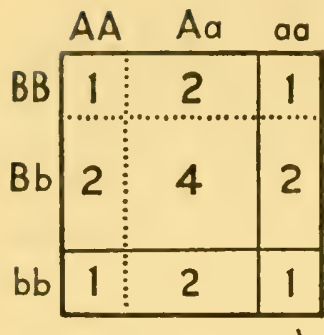

\section{$F_{2}$ RATIOS}

AS MODIFIED BY

GENE INTERACTION

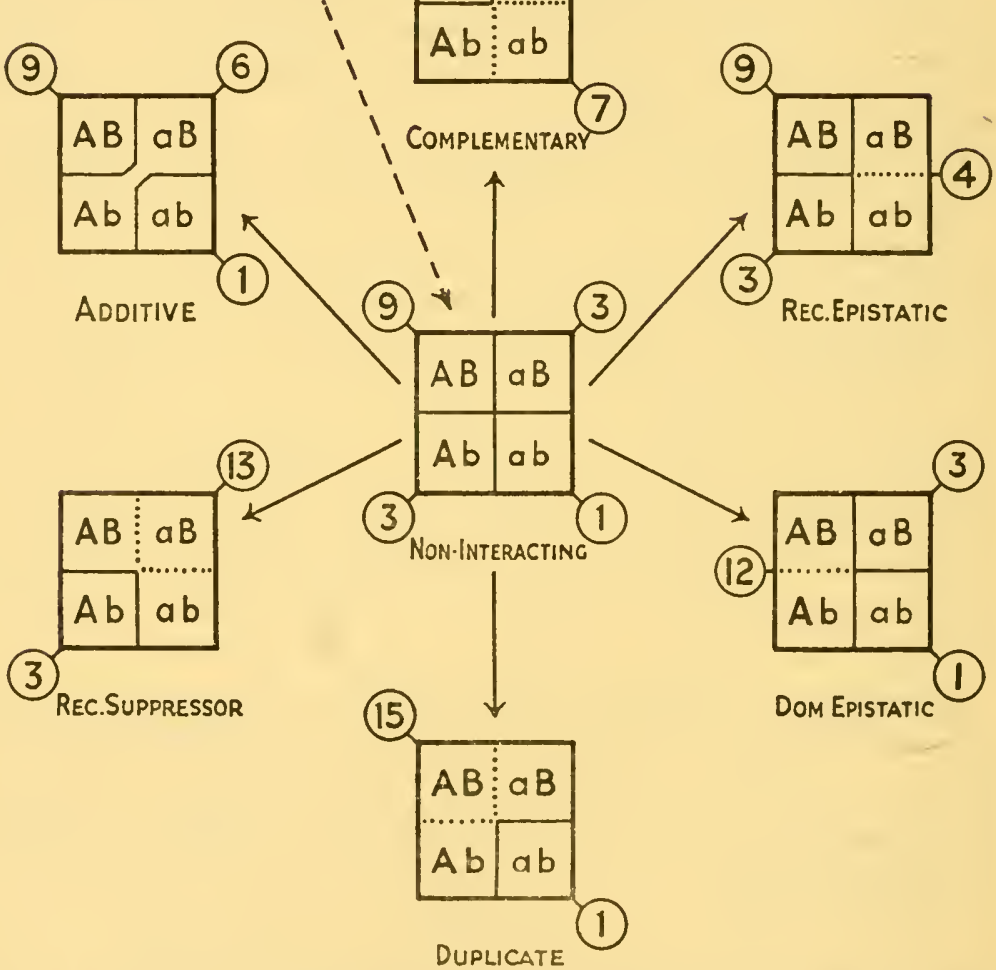

FIG. 38. - Where two genes are segregating in $\mathrm{F}_{2}$, ninc genotypes are expected with the frequencics shown at the top left of the diagram. With dominance of each gene they give four phenotypes in the characteristic $9: 3: 3:$ I ratio of the centre of the diagram. This ratio may be modified by genic interaction in the six ways shown. Three of these, complementary action, duplicate action and recessive suppressor 
$\mathrm{F}_{2}$ families. Where two genes which segregate independently do not interact, they give Mendel's familiar $9: 3: 3:$ I ratio. When they interact this ratio is simplified; classes are combined in most of the ways possible with complete dominance (Fig. 38).

Interaction in the simplest case consists of a mere anticipation: the effect of one gene may be cut out by that of another which is said to be epistatic to it.

Epistasy comes in at all stages of development, early and obvious or late and inferential. A gene removing life, a lethal gene, must obviously be epistatic to any other genes affecting the life it cuts short. A gene removing an organ is likewise obviously epistatic to one modifying that organ. A gene suppressing, or failing to produce, a substance, is obviously epistatic to one modifying that substance, or requiring it for its own activity. But the conclusion whether the substance is modified on the one hand, or is required on the other, is usually to be deduced only from the epistasy itself.

Epistasy expresses itself in two types of $\mathrm{F}_{2}$ ratio according to whether the epistatic allelomorph is dominant or recessive. Albinism in rodents is due to a gene which is recessive, and also of course epistatic to the various colour and pattern genes. The $9: 3: 4$ ratio is therefore characteristic of $F_{2}$ 's segregating for albinism. White leghorn fowls, on the other hand, owe their absence of colour to a gene, which is, again, epistatic to other colour and pattern genes just like the albino gene in rodents. But it is dominant. Thus they will give a I2:3 $:$ I ratio in $\mathrm{F}_{2}$.

Fig. 38.-Continued from page 156

action, give two phenotypic classes understandable on the assumption that the appearance of the character in question requires a particular allelomorph of each gene to be present. Failure in either or both genes results in absence of the character. These three cases differ only in the dominance relations of the effective allelomorphs, both being dominant in complementary action, both recessive in duplicate action, and one of each kind in recessive suppression.

Two other cases, dominant and recessive epistatic action, give three classes, one gene, said to be hypostatic, causing a difference in phenotype only in the presence of a particular allelomorph of the other gene, said to be epistatic. The cases differ only in the dominant or recessiveness of the effective allelomorph of the epistatic gene.

In the sixth case, of additive action, the two genes have individually indistinguishable, but cumulative, actions.

The classes which are confounded are separated only by dotted lines in the representation of their various interactions, and the relative frequencies of the pooled classes as they would appear in $\mathrm{F}_{2}$ segregations are shown in the small circles. 
In epistasy the expression of one gene difference conditions that of another. The relationship is not reciprocal. With reciprocal relations other $\mathrm{F}_{2}$ ratios are found. They are of three kinds according to whether it is the two dominants, the two recessives, or one of each which are needed for the combined effect. In any case only two classes are recovered instead of the three with epistasy. When two dominants are needed we have complementary genes. The classical case, indeed the first case, of any interaction to be described and understood, is the production of coloured sweet peas from a cross between two whites, the coloured-white ratio in $\mathrm{F}_{2}$ being $9: 7$. When two recessives are needed we have duplicate genes, in a number of characters such as ligule-liguleless in wheat and oats. The $\mathrm{F}_{1}$ may resemble both parents (when it is $A b \times a B$ ) but the $\mathrm{F}_{2}$ gives a IS : I segregation.

The duplicate genes are so-called because the allelomorphs appeared to be doing the same thing, whereas complementary genes, being themselves dominant, were supposed to be doing different things. In cereals the duplicate (or triplicate) genes are, no doubt, doing the same thing since these plants are polyploid. But, in diploid plants and animals, the I5 : I segregation may just as well be taken to indicate a complementary action of recessives. This interpretation is all the more plausible in view of the occurrence of yet a third type, the complementary action of dominant and recessive giving the $13: 3$ ratio which is shown by segregation for ivory and yellow flower colour in suitable Antirrhinum crosses. The recessive allelomorph of one gene is here called a suppressor of the recessive allelomorph of the other. The term suppressor is again, of course, merely an accidental tradition in this connexion. It might just as well be applied to the complementary and duplicate relationships, where it is the dominant allelomorph of one gene which, in effect, suppresses the recessive of the other. Since dominance is itself modifiable, the same kinds of interaction may underlie all three. The difference may depend merely on which combinations of dominants and recessives yield similar groups of phenotypes.

Distinct from the five types of interaction discussed so far is that where the $A b$ and $a B$ combinations are not distinguishable. We then have a $9: 6:$ I ratio and $A$ and $B$ are said to be additive. Simple additive interactions are rare, amongst major genes at least, 
but appear to account for the fact that two types of sandy pig have been seen in $\mathrm{F}_{2}$ 's from crosses between red and white. The genes governing red and white chaff in wheat show additive action but the ratios are complicated by incomplete dominance which leads, with two genes, to five distinguishable classes instead of three.

All these phenotypic groupings which give simplified ratios indicate gene interaction. The groups can, of course, be broken up into their genetic classes, in the same way as Mendel broke up his $\mathrm{F}_{2}$ classes, by appropriate breeding tests. They can also sometimes be broken up by closer microscopic examination or chemical tests. For example two races of Rudbeckia with yellow bud cones give purple bud cones on crossing and 9 purple to 7 yellow in $\mathrm{F}_{2}$. But of the 7,4 turn red and 3 turn black on treatment with caustic potash solution. At this new level of analysis, what had appeared to be complementary interaction is seen as recessive epistasy (see also Fig. 39). Clearly, therefore, classification of phenotypes in an $\mathrm{F}_{2}$, even aided by chemical analysis, can only give us a provisional account of the order and relationship of the individual processes at work. Further into this question we shall see later.

\section{The Expression of Genes}

So far we have considered the interactions of separably demonstrable genes, of major genes. The expression of all major genes, however, is affected by modifiers, presumably polygenes, which are otherwise not readily detectable. For example the gene "eyeless" reduces the eye in Drosophila melanogaster to a greater or less degree, characteristic of each laboratory stock. When flies from a particular eyeless stock are crossed with wild-type flies, the eyeless part of $\mathrm{F}_{2}$ shows a sharp increase in the range of reduction. Eyeless stocks can be selected from such a family with eyes differing in size from one another and from their eyeless ancestors. Thus, the wild-type fly must always carry genes capable of modifying, even of enhancing, the expression of "eyeless." The many grades of eyelessness in $\mathrm{F}_{2}$ show that many genes are concerned in this way in controlling what Timoféeff-Ressovsky calls the expressivity of the eyeless gene.

These comparisons, of course, apply to experiments under constant conditions of nutrition, temperature and so forth. Variations in the 
environment have often been shown to affect the expressivity of geries. In fact genotype and environment work in parallel.

Sometimes variation in the expression of a gene is such that it is not shown by all the individuals, even the homozygotes, carrying it; it does not, as it were, penetrate the whole population of individuals carrying it. The gene "antennaless," for example, studied in Drosophila by Gordon and Sang, removes either both or only one of the fly's antennae according to its degree of expressivity. A proportion of the antennaless flies even show no effect of the gene at all. Its penetrance is incomplete. But the degree of penetrance (and expressivity) varies with both genotype and environment, and both effects are shown in relation to sex. In the early emerging flies, penetrance is lower in males than in females; the reverse is true in the later part of the hatch. In both sexes the penetrance is at a minimum in a culture at about the fourth day of emergence and at a maximum at eight days.

These variations in expression are due to changes in the food of the larvae which arise from staling of the culture medium. This, in turn, is partly explained by the discovery that increase in the supply of vitamin $\mathrm{B}_{2}$ reduces penetrance. Indeed this vitamin enables all the flies to get over some of the difficulties, and some of the flies to get over all of the difficulties, presented by the shortcomings of the antennaless gene.

These experiments have had to do with genes in the homozygous conditions. When we examine heterozygotes we are concerned with dominance and we can regard dominance as a measure of the expressivity and penetrance of the heterozygous gene pair. We might, therefore, expect that dominance would also be subject to modification by genotype and environment. The effect of the genotype has indeed often been established-for example, in cotton, butterflies, and poultry. In the mouse, as we saw, agouti is normally dominant to non-agouti. The gene umbrous, however, shifts the expression of the agouti heterozygote towards non-agouti. The $\mathrm{F}_{2}$ ratio of 3 agouti to $\mathrm{I}$ non-agouti becomes $\mathrm{I}: 2: \mathrm{I}$. Umbrous thus modifies the dominance of agouti and, indeed, this is the only known effect of the gene.

The effect on dominance of the environment is, on the other hand, less widely known. The recessive gene cubitus interruptus in 
Drosophila melanogaster breaks a wing vein of the flies. At $19^{\circ} \mathrm{C}$. it affects all homozygotes but it is expressed in only half of them at $25^{\circ} \mathrm{C}$. In the heterozygote, according to Stern, it does not express itself at all at $25^{\circ} \mathrm{C}$. but at $13^{\circ} \mathrm{C}$. it is seen in about Io per cent of the flies. Another gene, Scutenick, which is lethal in the homozygote, produces multiple effects in the heterozygote at $25^{\circ} \mathrm{C}$. As the temperature rises its effect on the eye decreases, while its effect on the scutellum increases.

Both of these modifrable heterozygotes belong to genes in the small fourth chromosome near a block of heterochromatin, whose behaviour, as we saw, is sensitive to changes of temperature. They are, therefore, special cases. The reason for the general lack of outside effects on dominance is not far to seek. The normal or wildtype gene exists as part of a genotype long adapted to give a constant expression in varying environments. Now this normal and buffered gene is generally the dominant. Its heterozygote would, therefore, be expected to give a more stable result than would be given by its new untried allelomorph when homozygous. In fact, as Fisher has pointed out, the genotype, whose effects we have seen, must have been selected to give stable dominance.

Are there any general physiological principles that we may infer from these actions and interactions of genes? One most general principle is at once clear. Every gene depends, for its action, on that of others; of many others, possibly of all the others. The phenotype as a whole depends on the co-ordination of all genes, and this co-ordination, in the fit organism, is what we call balance. Hence we can see why polyploidy has a balanced effect on the genotype and why polysomy, duplication and single gene changes have unbalanced effects. Now, in the terms of materials and processes, co-ordination means that each gene is fed by others, and, in turn, feeds others. How, we must ask, is their feeding adjusted? The answer to this question is given by the precise chemistry of gene effects.

\section{Synthetic Sequences: Supply and Demand}

We can frequently see what the substitution of a particular gene by its mutant does to the chemical processes of the organism. A recessive dwarf in maize owes its dwarfness to a characteristic 
excessive ability to destroy auxin by oxidation. On the other hand the recessive alcaptonuric in man owes his peculiarity to a characteristic inability to destroy homogentisic acid by oxidation. Again the Dalmatian dog is distinguished from other breeds by a single recessive gene, which reduces the transformation of uric acid into allantoin, and so increases the concentration of uric acid in the urine about ten times.

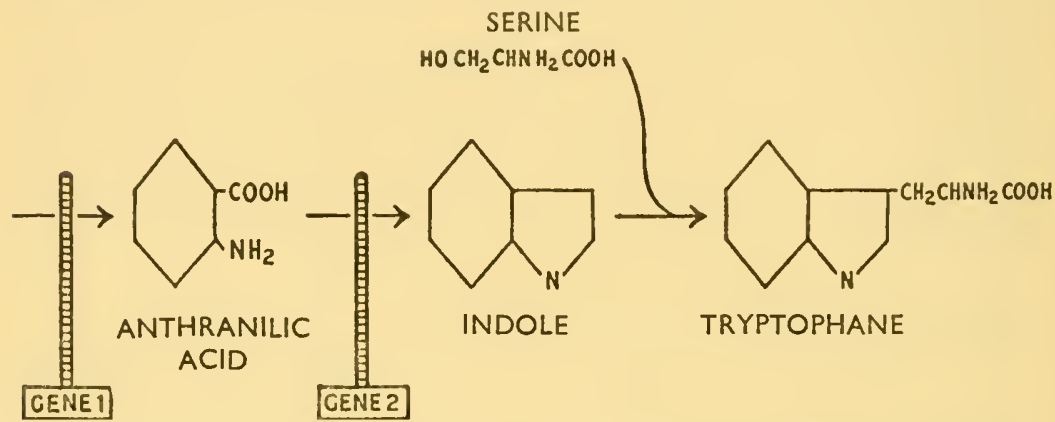

FIG. 39. - The bio-synthesis of tryptophane proceeds in three stages in Neurospora crassa which can be blocked by genes at two points at least. Mutation of Gene I blocks the first stages, that of production of the first precursor, anthranilic acid. Mutation of Gene 2 blocks the second stage, conversion of anthranilic acid into indole. Thus Gene I is considered to govern the production of an enzyme catalyzing the production of anthranilic acid, and Gene 2 the production of a second enzyme catalyzing the conversion into indole. Where only indole or tryptophane was recognizable, the genes would appear complementary in action, but where anthranilic acid was also detectable Gene I would appear in its true light, as epistatic to Gene 2 (see Fig. 38) (based on Beadle, 1945).

So much for single genes and simple processes. The combination of genes and processes can best be seen in simple organisms such as bacteria and fungi. By X-raying spores of Neurospora crassa Beadle, Tatum, Horowitz and others have produced single-gene recessive mutants, which differ from the wild type in their inability to grow, or at least to grow well, on media lacking certain substances, aminoacids, vitamins, pyrimidine bases and so on. Now the antennaless fly seems unable to make efficient use of vitamin $B_{2}$, and therefore requires it in greater quantity to produce antennac. In the same way these mutant moulds are unable to make use of precursors of these various substances, and so require a supply of them readymade in their food.

Different genes may, by mutation, lead to different kinds of 
failure in producing the same substance. As a consequence two mutant genes in Neurospora with the same apparent effect may be carried by two nuclei combined by vegetative fusion in one hyphal cell, a heterocaryon; they can then compensate for one another, each making good the other's deficiency, so that the hypha seems normal.

Many of these mutants appear complementary in their effects. Some, no doubt, act in parallel, but this is not true of all. Thus for two different mutant strains tryptophane is a necessary raw material; but it is apparently necessary in different ways, for one will grow on medium with indole, but not on medium with anthranilic acid, whereas the other will grow on either. Since both these are likely precursors of tryptophane, the final step in its synthesis is, we must suppose, unaffected by either mutant. Furthermore, the mutant gene in the strain which can utilize either substance must affect an earlier stage than the one which can utilize only the indole. In other words the chain of synthesis must follow the course shown in Fig. 39. And the one mutant must break the chain before anthranilic acid, while the other breaks it only before indole.

This inference of order is confirmed in a highly significant way. The mutant which grows only with indole accumulates anthranilic acid. New side chains of development may thus arise from blockage. For example, one mutant, failing to produce adenine, develops a new pigment, a polymerized purine. Here, then, we have our genes feeding one another, acting in succession, acting as we should say epistatically. They act not merely in fixed order in regard to the general sequence of development like the genes in Mendel's peas (Table I): they act in a fixed order in regard to particular other genes, wherever they take effect in development.

The effects of a mutation may be conditional. For example, as we saw, one mutation blocks the synthesis of adenine. But it does so only at temperatures over $32^{\circ} \mathrm{C}$. In such a case the mutant allelomorph may be merely hypomorphic or less efficient than the normal, or it may affect only an accessory process.

More complex series of relations are shown in the synthesis of pigments in higher organisms. The chemical structures of these pigments and of their probable precursors are well understood in the flowering plants, and are uniform throughout the group. The sap soluble pigments are of two main kinds, anthocyanins, which 
are the red and blue pigments, and anthoxanthins, which are the ivory and yellow flavones and flavonols. These two classes have the same basic structure and both are assumed on chemical grounds to be built up by parallel processes from sugars. What does genetics tell us of these processes?

In Pharbitis nil, two gene mutants, $c$ and $c a$, interfere with production of both anthocyanin and anthoxanthin. They must, therefore, act before the synthetic chains branch. Other mutants, $a$ and $r$, have a direct effect only on the anthocyanins. They act after the chains branch, as do most of the known pigment genes. Now if a gene acting on one branch sequence is reduced in activity we must suppose that an extra supply of materials will be available for the other sequence. Blockage should lead to diversion. This possibility has been tested in the autotetraploid Dahlia variabilis where every gene can be tested in five doses $\left(a_{4}, A a_{3}, A_{2} a_{2}, A_{3} a, A_{4}\right)$. The gene $B$, according to Lawrence and Price, produces anthocyanin, $I$ produces the anthoxanthin apigenin. $\mathrm{Bb}_{3} i_{4}$ has, of course, anthocyanin pigmentation as has $B b_{3} I_{3}$. But, still kecping $B b_{3}$, with $I_{3} i$ or $I_{4}$ the anthocyanin is much reduced. Thus $B$ and $I$ are evidently competing for a precursor. Further, $I$ shows the effect, even more strongly, with another anthocyanin gene $A ; A a_{3} I_{4}$ produces almost no anthocyanin at all. There is also evidence that cach of these genes $A, B, I$ and a fourth, $Y$, appears to contribute to a pool of common precursor. In fact, of course, they must increase the supply by increasing the demand: they act backwards, as well as forwards, in development.

When we say that the gene works backwards this is, of course, a figure of speech. It means that what is happening in the cytoplasm reacts on the nucleus. The utilization in the cytoplasn of materials produced by genes in the nucleus stimulates the activity of these genes. The reaction of nucleus and cytoplasm must be reciprocal, so far as the feeding of the nucleus is concerned. There seems little doubt that it must also be reciprocal so far as the feeding of the cytoplasm is concerned.

Yet another point is shown by the anthocyanin genes $A$ and $B$ in Dallia. In small doses they produce only cyanin, which is regarded as the primitive or precursor type of anthocyanin since it is commonest in the leaves, while its derivatives are commonest in the flowers, 
of flowering plants. The higher the dose of $A$ or $B$, the more the crimson cyanin is replaced by its reduced form the scarlet pelargonin. The quantity, or more strictly the proportion, of the determinant, lengthens the chain of production and changes the quality of the product. Thus we see how balance controls particular processes.

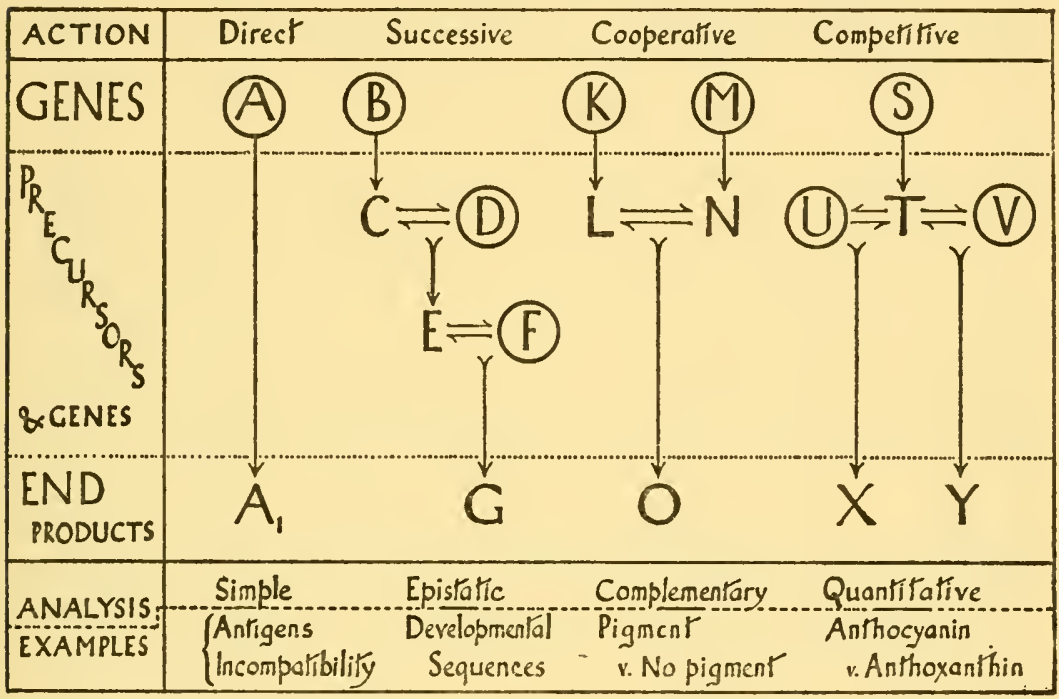

Fig. 40.- The four modes of gene action. In direct action, the end product must be very close to the gene itself. As the chain of processes lengthens the action of different genes combine in the synthesis of one end product. The product of one gene may be the raw material for a second, in successive action. Or the immediate products of two genes may interact directly to give a common end product, in complementary action, or two genes may draw on a limited quantity of a common raw material, itself perhaps the product of a third gene, in competitive action.

The appearances of the four types of action in genetical analysis are shown together with examples of characters in the production of which they have been recognized. More complex cases of gene interaction can be resolved into combinations of these four basic types.

Looking back over these types of action and interaction we see that they fall into four main systems (Fig. 40). These we may call direct, successive, co-operative, and competitive. The first three correspond roughly with simple action, and with epistatic and complementary interaction. The fourth is merely a modification of complementary interaction. In complex processes all these types may well be combined. Indeed they seem to be combined in the production of eye-pigment in Drosophila where, in addition, 
Ephrussi and Beadle werc able to show by transplantation cxperiments that some of the necessary substances are produced on the spot, and others are brought in from clscwhere.

Finally we must notice again that the majority of gene changes do not entirely stop or block a process; they merely slow it down. They are hypomorphs rather than amorphs. Indeed they often change a rate of activity in a way that can be exactly measured. The change $S-s$ in the shrimp Gammarus chevreuxi reduces to half the rate of deposition of melanin in the eyes of the young larva. The rate falls off with growth, but more rapidly in the quickly depositing type so that in the end the product is not very different.

A difference in rate may appear as a difference in time of expression. For example in some local races of the moth Lymantria dispar Goldschmidt found that the skin of the caterpillar has pigment throughout development; in others deposition begins half-way towards pupation, and even in these proceeds at different rates in different races. With the more highly integrated processes, for example, of sexual differentiation, all differences of races and hybrids become referable, as Goldschmidt has shown, to differences in rates of co-operating and competing processes common to all races and both sexes.

In these terms we begin to see the material and mechanical basis of differentiation. The actions of genes depend on the simultaneous actions of one another. But even more, and somewhat differently, they depend on what other genes have done earlier and have done elscwhere. Even if the immediate products of a gene's action were always constant in amount-which is unlikely-its derivative effects would depend on when and where it was acting, on conditions in the nucleus, and, of course, in the cytoplasm too.

These considerations bring us back to the cell as the sphere of action of the nucleus. If the cell feeds the nucleus and the nucleus fceds the cell, the separation of cells gives each of them its specific type of feeding relationship, its own pattern of reactions and development. Between the fertilized egg, and its mature development, the whole course is determined by the supply of raw materials, the rates of gene action and the rates of diffusion of its products. If the supply of raw materials never changed, or if the rates of gene action never varied, or if diffusion were instantaneous, differentiation 
could never occur. Before we come to grips with the problem of differentiation, however, we must look at the whole question from the other side. We must find out what part the cytoplasm plays in heredity.

\section{REFERENCES}

BEADLE, G. W. I945. Biochemical genetics. Chemical Revs., 37: I5-96.

BEADIE, G. W., AND COONRADT, v. L. I944. Heterocaryosis in Neurospora crassa.

Gentics, 29: 29I-308.

BEALE, G. H. I94I. Gene relations and synthetic processes. J. Genet., 42: 197-2I4. BRACHET, J. I944. Embryologie Chimique. Liége and Paris.

CASPERSSON, T. I947. The relations between nucleic acid and protein synthesis.

Symp. Soc. Exp. Biol., I: I27-I5I.

Darlington, C. D. 1947. Nucleic acid and the chromosomes. Symp. Soc. Exp.

Biol., I: 252-269.

DARLINGTON, C. D., AND UPCOTT, M. B. I94I. The activity of inert chromosomes in Zea mays. J. Genet., 4I: 275-296.

FISHER, R. A. I93 I. The evolution of dominance. Biol. Revs., 6: 345-368.

FORD, E. B., AND HUXley, J. S. I929. Genetic rate-factors in Gammarus. Arch. Ent.

Mech., I I7: 67.

GoldsChmidT, R. I938. Physiological Genetics. New York.

GORDON, C., AND SANG, J. H. I94I. The relation between nutrition and exhibition

of the gene Antennaless (Drosophila). Proc. Roy. Soc. B., I30: I5I-I 84 .

GULICK, A. 1944. The chemical formulation of gene structure and gene action.

Advs. Enzymology, 4: I-39.

HEITZ, E. I935. Chromosomenstruktur und Gene. Z.I.A.V., 70: 402-447.

HOROWITZ, N. H. et. al. 1945. Genic control of biochemical reactions in Neurospora. Am. Nat., 79: 304-317.

кÜHN, A. I94I. Über eine Gene-Wirkkette der Pigmentbildung bei Insekten.

Nachr. Akad. Wiss. Göttingen, Math.-Phys. Kl., 6: 23 I-26I.

LAWRENCE, W. J. C., AND PRICE, J. R. I940. The genetics and chemistry of flower colour variation. Biol. Revs., 15: 35-58.

Mather, K. I944. The genetical activity of heterochromatin. Proc. Roy. Soc. B., I32: 308-332.

MATHER, K., AND NORTH, S. B. I940. Umbrous: a case of dominance modification in mice. J. Genet., 40: 229-24I.

MULLER, H. J. 1932. Further studies on the nature and causes of gene mutations. Proc. 6th. Int. Cong. Genetics, I : 213-255.

ÖSTERGRen, G. I947. Heterochromatic B-chromosomes in Anthoxanthum. Hereditas, $33: 26 \mathrm{I}-296$.

STERN, C. I943. Genic action as studied by means of the effects of different doses and combinations of alleles. Genetics, 28:44I-475.

TIMOFÉEFF-RESSOVSKY, N. W. I93I. Gerichtetes Variieren in der phänotypischen Manifestierung einiger Genovariationen von Drosophila funebris. Naturwiss., I9: 493-497. 


\section{THE CYTOPLASM}

\section{Plastogenes Plasmagenes Cytoplasmic Equilibrium Cytoplasm, Nucleus and Environment Damermodification \\ Suppressiveness and Ambilinearity}

The CYTOPLASM MUST BE THE AGENT of the nucleus in development for it is only from the cytoplasm that the nucleus can obtain its raw materials, and it is only to the cytoplasm that it can pass on the products of gene action. But the cytoplasm might also act as an agent in its own right, by virtue of its own capacities of selfpropagation. Clearly the way of testing this possibility is by bringing together cytoplasm and nucleus in new associations.

There are two ways of effecting this re-association. The first is by grafting, whose consequences we have seen in Acetabularia. The second is by breeding. Sometimes the nucleus of one species can be associated with the cytoplasm of another. Thus an occasional seed borne on the lentil plant, Lens esculenta, in cultivation grows into a common vetch, Vicia sativa. Vetch pollen has entered the lentil embryo-sac. The male generative nucleus has supplanted the egg nucleus, doubled its chromosomes, and developed as a diploid vetch in a lentil seed coat. These intruders are entirely normal and show no effect of the cytoplasm in heredity. There are also instances of such exceptional behaviour in Fragaria and Nicotiana.

These rare examples do not, however, offer a sure basis for generalization, as we can see if we turn to animal merogons. Sometimes development is successful and, in the long run, patroclinal, as we saw earlier in sea-urchin crosses where the egg-nucleus has been artificially removed before fertilization (Fig. 2). In similar merogons between the newts Triton palmatus and $T$. cristatus the outcome is less successful. These develop under the control of the cytoplasm, that is maternally, for some time. But in the end they become malformed and development ceases. Evidently at the beginning, the cytoplasm had enough of the products of its past 
nucleus to carry it on. Development was harmonious because these products were harmonious. Later, when the new and foreign nucleus begins to pass its products into the cytoplasm, the mixture is no longer in harmony and development breaks down. In hybrids, too, such cases may be much more common than those of successful development as in Lens, for they will normally escape detection through their failure to survive. The rare exceptions which are successful enough to be found may give a false picture.

\section{Plastogenes}

The disharmony that we see between cytoplasm and nucleus in Triton shows that materials of the cytoplasm of the egg must have some permanency, but this need be nothing more than the persistence of already existing materials. The experiment is terminated by the death of the hybrid too soon to provide us with the evidence we are seeking of self-propagation in the cytoplasm. Rather than consider the cytoplasm as a whole, we must examine its parts, and the most obvious of these are the plastids which are responsible for the chlorophyll and carotinoid pigments of plants. The plastids are visibly self-propagating bodies in the lower plants and the same property is to be inferred in the higher plants, where they multiply as pro-plastids in a small and colourless stage. The plastids, moreover, contain ribose nucleic acid. Breeding experiments confirm the genetic character first attributed to them by Baur in 1909.

Primula sinensis, in common with hundreds or thousands of other species of green plants, has a chlorophyll-less or albino variant which is mendelian in inheritance. The homozygote is so pale that it dies; the heterozygote is yellower than the normal, but survives. Thus albinism is here due to something having gone wrong with a nuclear gene which supplies materials necessary for the production or stability of chlorophyll. In the same Primula, however, there is another yellow-leaved variant which passes on its character in a different way. All the offspring from such a yellow mother are themselves yellow, no matter what the character of the pollen parent. A yellow father, on the other hand, does not transmit this character to his offspring. Thus the colour of the offspring depends purely on that of the mother plant and the results of a cross depend 
on which way the cross is made, thus (putting the female parent first) :-

$$
\begin{aligned}
& \text { yellow } \times \text { green } \longrightarrow \text { yellow } \\
& \text { green } x \text { yellow } \longrightarrow \text { green }
\end{aligned}
$$

Evidently this inheritance is non-mendelian and therefore nonnuclear. The effective agent must be in the cytoplasm, though whether it is attached to the plastids or is free from them and, like the gene in the other case, acting on them, we cannot yet say.

Crosses between species of Oenothera answer this question. Two green species when crossed together sometimes give yellow offspring-but only one way. There are always differences between the reciprocal crosses. In Renner's experiments, for cxample:-

$$
\begin{aligned}
& \text { Oe. hookeri } \times \text { muricata }(\text { curvans }) \longrightarrow \text { yellow } \\
& \text { Oe. muricata (curvans) } \times \text { hooker } \longrightarrow \text { green }
\end{aligned}
$$

Thus the plastids of hookeri, which are green in the parent species, are yellow with the nucleus of the hybrid. But the plastids of muricata remain green in the hybrid. Now the nuclei in the reciprocal hybrids must be the same. It must, therefore, be the plastids or the cytoplasms that differ. One of them gets on with its parental nucleus but fails to get on with that of the hybrid. Thus both the nucleus and certain agents outside it play a part in the reaction.

This is not all. The yellow $F_{1}$ seedlings die but the green seedlings from the muricata mother occasionally develop flakes of yellow in their shoots. These are evidently derived from odd hookeri plastids which have come in with the pollen and sorted themselves out during development to make the full plastid complements of certain cells. Now, these yellow flakes can form the sub-epidermis of a shoot; they then produce the germ cells and can be used for breeding. When selfed, such a shoot gives two kinds of offspring; there is a simple segregation of the hookeri-muricata (curvans) nuclear difference, to give two types: one homozygous hookeri and the other heterozygous like the $\mathrm{F}_{1}$ hybrids; the homozygous muricata (curvans), dies in embryo. The two kinds are alike in having hookeri plastids and differ only in the nucleus. The heterozygote is again yellow; but the homozygote is green. Thus the hookeri plastids 
which turned yellow with the hybrid nucleus turn green again when restored to their own proper nucleus.

The sorting out of the colour determinant from mixed cells is a characteristic defect of cytoplasmic transmission. Now if the extranuclear determinant were of a molecular size it would scarcely be expected to sort out so quickly. It seems likely therefore, although mixed plastids have not so far been seen in the cells of variegated plants that it is the plastid itself which carries the determinant of its own character. This story shows that the plastids are permanent in their character. It also shows that this character is adjusted in the green plant to the character of the nucleus with which it has to do its work. The interaction of the two is that of two complementary genes, one mendelian, the other not mendelian.

To what does a plastid owe its permanent or self-governing genetic character? So far we have attributed all permanence to genes, tacitly assuming that they were all nuclear genes; an assumption, incidentally, which Johannsen refused to make when he proposed the term. Now, it seems, we must recognize the existence of determinants having the properties of genes but existing outside the nucleus, and even capable of surviving their disagreement with the nucleus. They are specialized by their attachment to the plastids and Imai has called them plastogenes.

Since, as we saw, the plastids of different species differ in genetic character, their plastogenes, like other genes, must be liable to mutation. Like ordinary gene mutation it is in most plants a rare event. In most Oenothera species a mutation is seen once in about 2,000 plants. But occasionally, as with gene mutation, it may become frequent, and it is then seen to be under a genetic control which is partly external to the plastid itself. The inheritance of certain kinds of variegation shows how this control works.

In barley, Hordeum vulgare, Imai found a variety in which the plastids are characteristically unstable. They often change during growth from green to white. Sorting out then gives green and white cells, and later green and white variegated tissues. Most of the eggs contain green plastids and most of the selfed seedlings, therefore, give rise once more to variegated plants. But some eggs contain only white plastids and these eggs give rise to albino seedlings which 
die. A few (too few to show in the diagram, Fig. 41) even contain plastids of both kinds and produce plants which are variegated already as seedlings. Evidently a white plastid never changes back to green.

The same proportions of white and variegated seedlings occur in
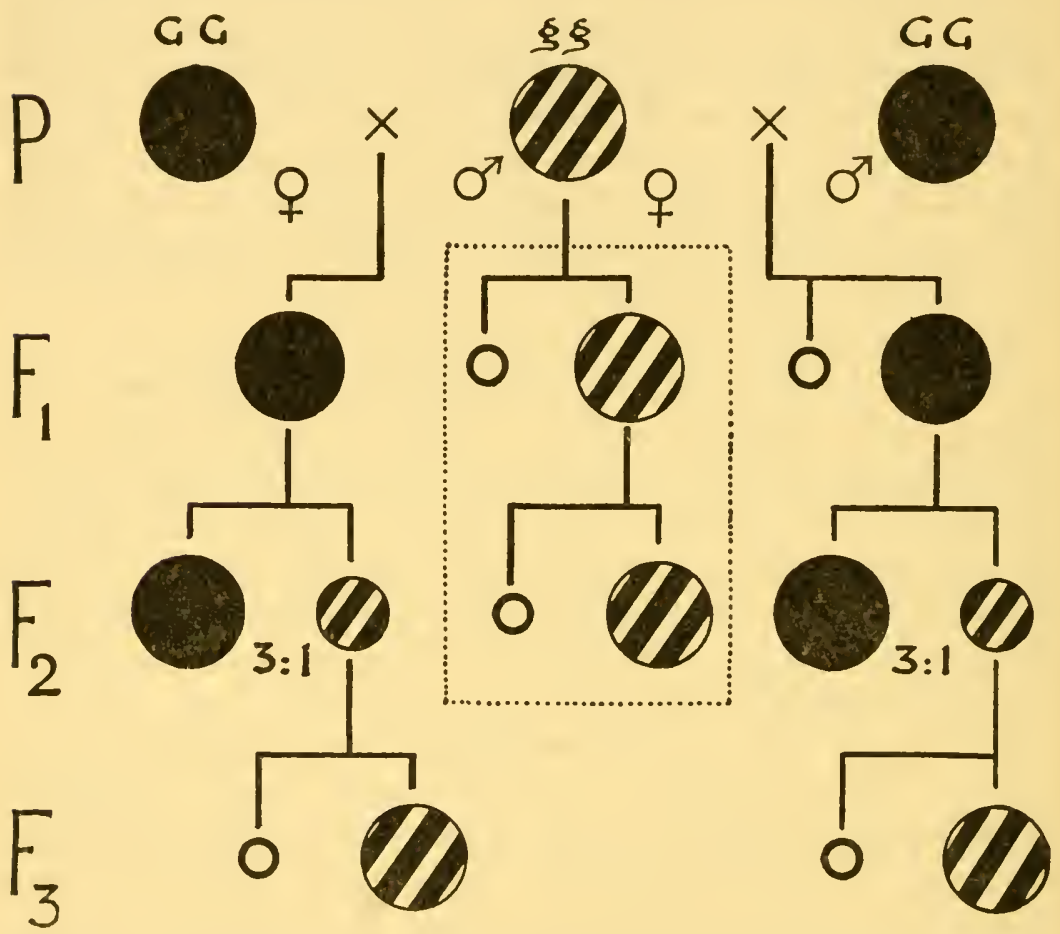

FIG. 4I.-The inheritance of variegation in barley, Hordeum vulgare, according to Imai (1928). Black circles are green plants, white circles white plants, and hatched circles variegated. Size of circle indicates frequency of the type amongst seedlings. Superimposed on mendelian segregation is the purely maternal inheritance of irrevocably white plastids in a small proportion of the progeny of mutating (variegated) plants whether selfed or crossed (after Darlington, I944).

the cross with pollen of a normal green barley as in the self of the variegated plant. The remaining plants from this cross, however, stay purcly green instead of developing variegation at a later stage. Plastid mutation has been suppressed. The suppressor must be a dominant nuclear gene, because in the $\mathrm{F}_{2}$ a quarter of the plants are variegated like their grandparent. Mutation begins again with the 
correct nucleus. The reciprocal cross, green by variegated, gives exactly the same result except that there are, of course, no white eggs from the green mother and no white seedlings in the $F_{1}$. In this cross the plastids which begin to mutate in the $F_{2}$ have no ancestry of mutability. The mutability of the plastids is the same, no matter what their ancestry may have been. It is determined by the nucleus, although their character before and after mutation is determined by themselves within the range of nuclear variation studied in this experiment.

This kind of situation (which is known also in maize and rice) puts control and independence in a new light. They are not so simple as they might seem to be. They exist in three spheres of action. The first is reproduction, and here each organ of the cell is controlled by the whole. The second is activity or production, and here again control is by the whole, but in this control the cytoplasm is the immediate, and the nuclear genes together with extra-nuclear genes the ultimate authority. The third is mutation, which is similar in principle to the second, although the detailed processes are entirely different and the predominance of the nucleus is even more obvious. We may indeed speak of certain genes inside the nucleus as being mutafacient with respect to particular other genes, either inside or outside the nucleus.

\section{Plasmagenes}

Not all extra-nuclear determinants are conveniently attached to markers so easy to pick out as plastids, or so clear in what they do. Their existence, however, is vouched for by the non-mendelian inheritance, of which reciprocal differences are the simplest evidence, appearing in a great number of crosses in flowering plants, ferns, mosses, and protozoa.

The moss Funaria provides an example of a reciprocal difference in crossing. Crosses made by Wettstein between F. mediterranea and $F$. hygrometrica differ in the shape of the diploid structure, the sporocarp, according to the direction of the cross. A diploid gametophyte can be produced from the sporocarp by regeneration following injury. Meiosis and segregation are thus avoided. Like the sporocarps whose unchanged nuclei they bear, these gametophytes differ in leaf-shape. Evidently the cytoplasms provided by the two species 
differ, and indecd they continue to show this difference after thirteen years of vegetative propagation.

The effect of the cytoplasm is maintained even through sexual reproduction. Ordinary haploid gametophytes obtained as segregates from the hybrid sporocarp can be back-crossed as females to the male parent. After several generations of such back-crossing, the nucleus, of course, becomes effectively identical with that of the male parent. Yet, after eight generations, Wettstein found that the plants were still different from the male parent. The cytoplasmic determinant was still unchanged, unimpaired by the continuous government of an alien nucleus. In other words it has the properties of a gene, a plasmagene.

The plasmagene, or plasmagenes, in Funaria are unconditional in their effect, so far as the experiment goes, but elsewhere the nucleus also plays a part. The $F_{1}$ cross between tall and procumbent flax, Linum usitatissimum, is normal both ways. But, when the procumbent variety is the female or cytoplasmic parent, one quarter of the $\mathrm{F}_{2}$ is male-sterile; the anthers abort. Here a nuclear gene of the tall flax is reacting with a plasmagene of the procumbent. Or perhaps it is failing to react because a plasmagene of the tall flax is missing in the procumbent. No other combination gives this defect. And it is constant over many generations back-crossed with pollen from the tall.

In Linum, just as with the plastids in Oenothera, the nucleus and the extra-nuclear component in heredity fit one another in their ancestral or habitual combinations, but fail to fit in some of the combinations or re-combinations that can be produced by crossing. The commonest symptom of this failure in the flowering plants appears at the last and most delicate stage of development, the formation of the pollen. Where, in Petunia and Nicotiana, selfcompatible and self-incompatible species (whose propertics, as we shall see, depend on pollen adjustment) are crossed, it is the nucleus of the self-incompatible parent whose activity fails in the cytoplasm of the self-compatible one. Thus it seems that the incompatibility mechanism depends on the adjusted properties of the two components, nucleus and cytoplasm. Changes in the cytoplasm can be related to constructive adaptation even though they may not be generally necessary for it. 
The first evidence of the particulate transmission of plasmagenes we find in Epilobium. Again, male-sterility appears when the nucleus of one species, E. hirsutum, lics in the cytoplasm of another, E. luteum, as a result of continued back-crossing of the hybrid $E$. luteum $\times$ hirsutum with pollen of hirsutum. But this pollen must occasionally carry over the operative particles of patcrnal cytoplasm in fertilization, for in the fourteenth generation of back-crossing about one plant in 400 has some shoots with fertile anthers. Self-pollination of these plants gives fewer male-sterile progeny when the male-fertile flowers are used, instead of the male-sterile flowers. The plasmagenes, therefore, sort themselves out in the growth of the plant, although they do so less rapidly than the plastids: they are probably more numerous.

\section{Cytoplasmic Equilibrium}

In the infusorian Paramecium aurelia, plasmagenes have revealed still more specific properties. Of the seven varieties which Sonneborn has described, four show nothing but mendelian inheritance of their differences. In the other three, however, there is always a cytoplasmic element in inheritance. This has been most fully investigated in the case of two antagonistic types: one of these, the Killer, poisons the other, the Sensitive, type when they are put together in the same water. They can, however, be crossed and it is sometimes found that the difference is wholly cytoplasmic in inheritance. In other cases a nuclear gene also comes into play.

Where this happens each of the two $F_{1}$ individuals from conjugation, in spite of their exchange of nuclei, resembles in character the cell from which it came, so revealing the cytoplasmic element in transmission. The offspring of the Sensitive individual continue to be entirely sensitive in the $\mathrm{F}_{2}$; but in the Killer line one quarter of the $\mathrm{F}_{2}$ goes back to the Sensitive character of the male grandparent (Fig. 42). Clearly the Killer character can be lost by virtue of a change in the nucleus as well as in the cytoplasm. Or to put it another way we have, as in flax, a character, Killer, which depends on the simultaneous presence of a nuclear gene $K$ and a plasmagene, termed kappa.

The relation between $K$ and kappa is not confined to their action, for it affects the reproduction of kappa. Where $K$ is introduced into 
a cytoplasm devoid of kappa, there is no development of Killer; but $K$, in the way characteristic of nuclear genes, continues to maintain itself indefinitely, so that, should conjugation or any other process restore kappa to the cytoplasm, Killer bchaviour would once more begin to appear.

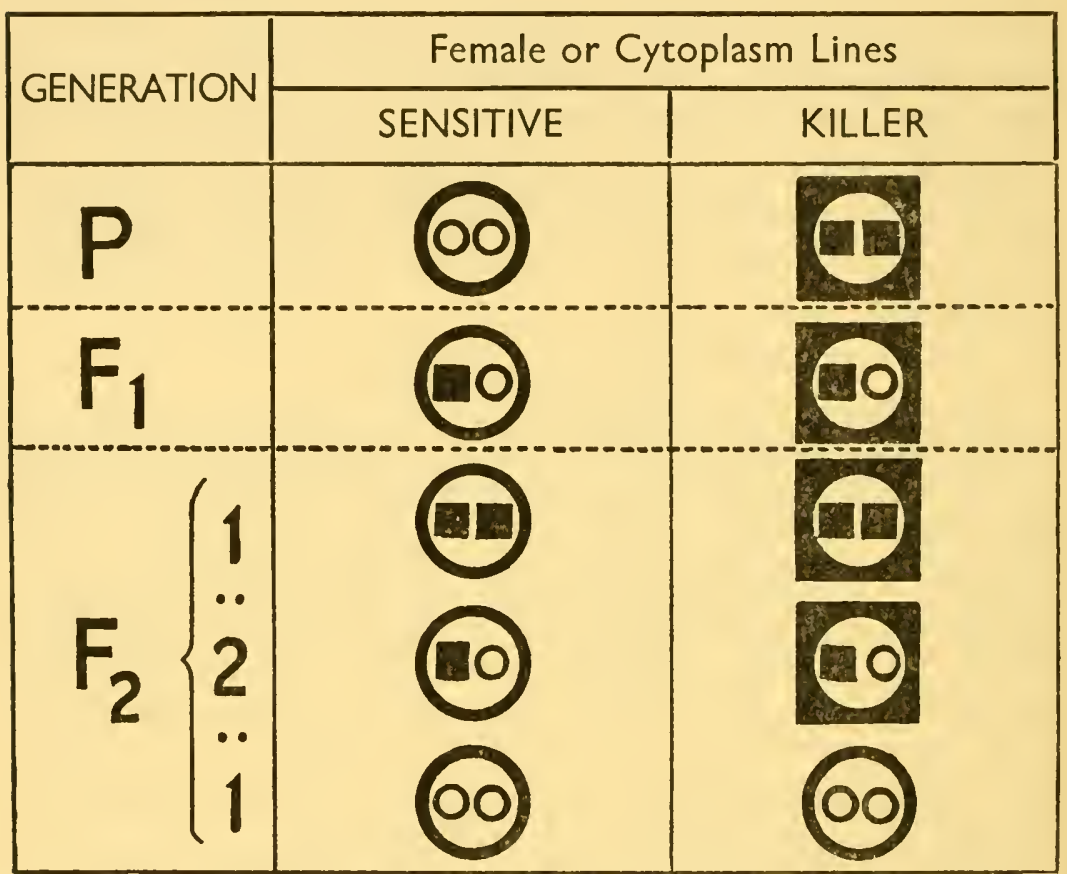

Fig. 42.-The inheritance of the Killer property as shown in certain crosses between strains of Paramecium aurelia by Someborn (I947). Within the circles is the square Killer gene dominant to its round allelomorph; outside them is the square cytoplasm with kappa which depends on both the action of the gene in the nucleus and the presence of its prototype plasmagene in the cytoplasm to maintain it (after Darlington, I944).

The reproduction of $K$ is independent of kappa, and indeed so far as we know of anything else in which the cytoplasms may differ. The reproduction of kappa is not, however, independent of $K$, for if $K$ is replaced by $k$ in the nucleus of an individual whose cytoplasm carries kappa, not only is there a loss of the Killer behaviour, but kappa also disappears after four or five fissions. Evidently kappa cannot reproduce in the absence of $K$, and so disappears as soon 
as the existing amount has been dissipated. Thus $K$, or rather some product of its activity, is necessary for the reproduction of kappa, but it cannot initiate the formation of kappa. The relevant product of $K$ is one, but only one, of the precursors of kappa, and these precursors can be welded together to give kappa only by kappa itself.

We have therefore to assume at least four substances in the cytoplasm concerned with the Killer behaviour, viz: (i) the diffusible Killer substance; (ii) the reproductive particle, kappa; (iii) the product of the nuclear gene $K$; and (iv) at least one other precursor substance. These substances must have the following minimal relation:-

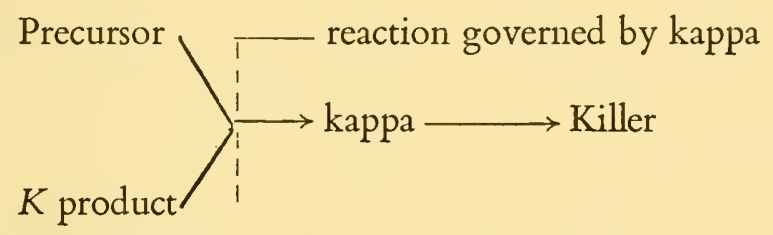

Nuclear genes are co-ordinated in reproduction both with one another and with the cell. Each gene appears, therefore, with the same dose in each nucleus, and, subject to the number of nuclei being constant, in each cell. For a plasmagene like kappa, whose reproduction is under chemical rather than mechanical control, this is no longer true. The amount of kappa is not constant from cell to cell, or even within a single cell. It depends on the rate of reproduction of kappa relative to cell fission.

In variety 2 of Paramecium aurelia, by raising the food supply, Preer was able to increase the rate of cell fission to 3.4 times a day, whereas kappa could double itself only $\mathbf{I} \cdot 9$ times a day. In these circumstances the concentration of kappa diminishes until it is finally lost. When the average concentration has become small, the proportion of cells devoid of kappa is found to be that expected from the random sorting out of particles. In this way Preer was able to demonstrate the particulate nature of kappa and to show that the concentration necessary for the Killer behaviour to be shown was 200-300 particles per cell. It was also evident that, so long as one particle remained in the cell, the full concentration, and with it Killer behaviour, would be restored when conditions supervened 
less favourable to fission, so that kappa reproduced more quickly than the cell. The concentration of kappa increases most rapidly when the cell is not undergoing any fission at all. This diminution of kappa by rapid fission proved impossible in variety 4 , where the reproduction of cells and of kappa remained in step even when each doubled itself six times a day.

The relation between cell multiplication and the concentration of kappa can be observed in another way. When the cells are heated up to $38.5^{\circ} \mathrm{C}$. kappa is destroyed before the cell is killed. Thus, by adjusting the time of exposure, the concentration of kappa can be reduced to a very few particles and the Killer behaviour lost. If the cell is restored to conditions favouring rapid fission, kappa will stay at this low level. But if the rate of fission is reduced by reduction of food, old age, or sexual processes kappa increases in concentration until it is back to the 200-300 particles per cell required for Killer behaviour.

When all the kappa particles are destroyed by heat treatment the ability even to develop Killer behaviour is irretrievably lost, just as it is when kappa is totally lost by sorting out in variety 2 . That the loss is due merely to the absence of kappa can be shown in another way. Normally conjugating cells separate in less than $3 \frac{1}{2}$ minutes and no cytoplasm is exchanged. If, however, the cells remain connected for 4 or more minutes (and this connection can be encouraged by heat treatment), cytoplasm may be exchanged. When this occurs between a Killer and a Sensitive, the Sensitive can develop into a Killer if its nucleus contains $K$. Its cytoplasm must have received kappa by exchange from the Killer, and once infected (as we may say) with kappa, Killer behaviour can ensuc. The time taken for it to develop depends on the relative reproduction rates of kappa and cell, in the way we have already seen. It also depends on the length of time the conjugants have remained connected. When this is for only 6 or 9 minutes, Killer bchaviour develops more slowly than when the connexion is maintained for a longer period. Evidently, as one might expect, fewer particles of kappa pass into the formerly Sensitive cell in the shorter space of time.

The extra-nuclear component in the heredity of the KillerSensitive difference can, as we have seen, be understood in terms of a single type of plasmagene, kappa, characteristically associated 
with Killer behaviour. There is no need to postulate a second type of plasmagene associated with Sensitive. This state is merely the absence of Killer and is associated consequently with absence, or at lcast over-low presence, of kappa. We must, however, recognize the more general possibility of two different plasmagenes, or alternative phases of the same plasmagene, being associated with the alternative characters. We should then be forced to interpret changes in plasmagene constitution as being, at least in part, due to the suppression of one plasmagene or phase by the successful competition of the other. Such suppressiveness might at first sight resemble the dominance of nuclear genes; but it must be different in that it will preclude, at least if complete, all sorting out of the particles or determinants that are suppressed. The suppressor must displace the suppressed.

Whether in competition with some alternative type of plasmagene in this way, or whether reproducing without any direct and immediate competition, the level or concentration of a plasmagene must depend on its rate of reproduction relative to those other constituents which determine the division of the cell itself. We have seen how the increase and decrease of kappa are governed in this way. In yeast we can go even further and see how the supply of a particular substance from outside can govern the concentration of a plasmagene.

Both Saccharomyces carlsbergensis and $S$. cerevisiae, when placed in a medium containing melibiose, are unable to ferment this sugar. But carlsbergensis soon becomes habituated or "adapted" to doing so, while cerevisiae is quite incapable of adaptation. When the two species are crossed, the diploid hybrid is found to be adaptable, and segregation into adaptable and unadaptable haploids occurs in its asci. It would appear that one, or possibly more, genes govern the difference in adaptability between the species.

The gene itself cannot, however, be responsible for the immediate production of all the enzyme in an adapted individual. Rather, as Spiegelmann points out, we should regard the allelomorph, which confers adaptability, as constantly seeding the cytoplasm with small quantities of the cnzyme, or of a precursor which, in conjunction with some substance available from the cytoplasm, can give rise to the enzymc. In the absence of melibiose, the enzyme or precursor 
breaks down as soon as it is formed so that the concentration at equilibrium is so low as to be indetectable by the fermentation it causes.

When melibiose is added, the concentration of enzyme rapidly

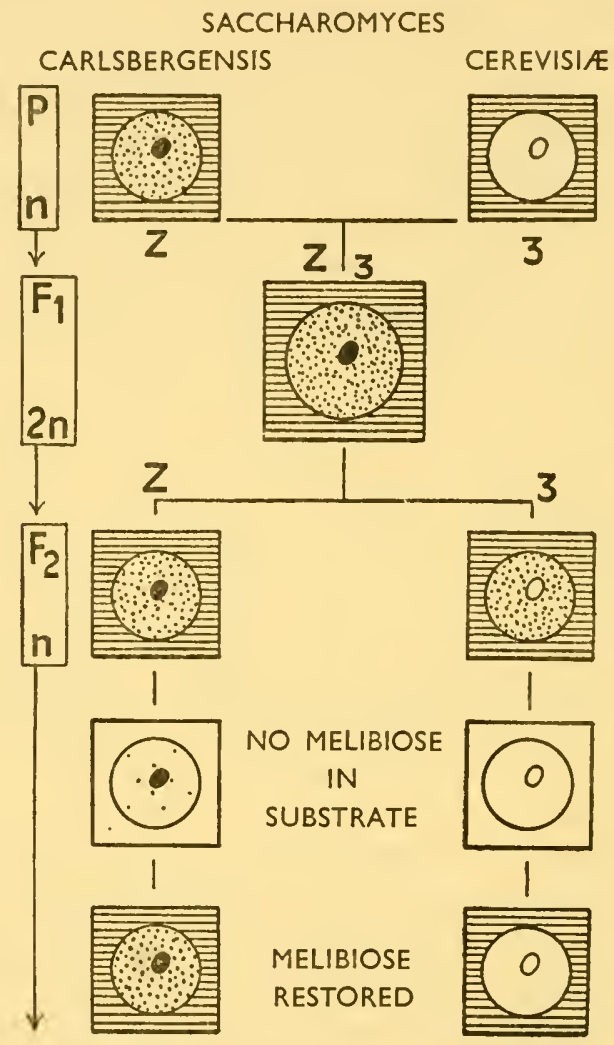

FIG. 43.-The inheritance of the capacity to ferment melibiose in yeast crosses according to Lindegren and Spiegelman (I944). The plasmagene responsible depends on the action of a nuclear gene for its origin, while for its survival in the absence of the nuclear gene it depends on the presence of melibiose in the substrate.

grows; so rapidly that it cannot be due to the mere accumulation of the output from the nucleus by removil of the counter-balancing destructive process. On the contrary the enzyme, or precursor, in the cytoplasm must take on the self-propagating properties of a plasmagene when melibiose is added. The melibiose itself conditions the plasmagenic properties of the enzyme which ferments it. 
We should expect, on this view, that the enzyme could persist and multiply in the cell eve away from the gene which was its ultimate origin, always provided that the supply of melibiose was maintained. That this is indeed the case is suggested by an experiment carried out by Spiegelmann, Lindegren and Lindegren (Fig. 43). They crossed the two species of yeast and allowed the diploid hybrid to form asci in the presence of melibiose. Instead of the ascospores segregating into ferm ntors and non-fermentors, all were fermentors so long as melibiose was supplied. When the melibiose was removed, however, so that the ability to ferment was lost, only half of the lines were able to readapt to its fermentation. The habit can persist with the plasmagene in the cytoplasm, in the correct chemical conditions; but once the plasmagene is lost it can be reinitiated only with the help of a nuclear gene. Thus, unless the experimental evidence is at fault, we must conclude that in yeasts a plasmagene which is produced or created by a nuclear gene can reproduce itself indefinitely without that nuclear gene.

\section{Cytoplasm, Nucleus and Environment}

The experiments with Paramecium and yeast together tell us a great deal about the properties of plasmagenes. Those with Paramecium are most comprehensive in showing us how anything which affects the rate of cell fission correspondingly affects the plasmagene concentration. In the yeast experiments a precise chemical footing has been achieved, and two new relationships revealed. One is that between the nuclear and cytoplasmic determinants. We have always supposed that the nucleus can pass out materials into the cytoplasm. We now find that those materials can sometimes cut adrift. They can propagate themselves independently, with this proviso: they seem to require more specific precursors or food materials than does the nucleus as a whole, which is assisted in this respect by the co-ordinated supply and demand of its constituent genes. Plasmagenes are therefore liable to extinction by changes either in the nuclear genes or (in micro-organisms) in the environment. The substance produced by a nuclear gene and necessary for the development of a particular plasmagene may be described as a specific precursor. It might be supposed that, in requiring such a 
precursor, plasmagenes differ from nuclear genes which multiply with even pace in all living conditions. This, however, would be a misconception. The nuclear gene also requires special precursors to live, but the only evidence we have of their absence is the breakdown of nuclear activity and reproduction, as a whole. This breakdown is always found in deficient, and often found in unbalanced, nuclei. The nuclear genes are specific in their food requirements. They are even specific in the balance of these requirements. They are non-specific only in the mechanical control of their reproduction, which compels them all to live and multiply in step with one another or all to die in step with one another.

The second relationship which we can now see is that between the plasmagene and the outside world. The plasmagene can adapt itself directly to change outside the unprotected simple cell. But it can do so only by suicide. The effect is Lamarckian, but it is an effect of disuse only and not of use.

In these circumstances we are bound to ask ourselves what the unicellular situation would look like in a multicellular organism undergoing differentiation. In flowering plants there are abnormalities which develop during differentiation and affect different parts of an individual, or of a divided clone, in different degrees. These abnormalities must depend in an unspecified way on environmental differences.

Plasmagenes, being dependent for their multiplication in the cell on its chemical equilibrium, should be subject to change in external conditions. For single cells in the multicellular organism these external conditions include development. A precise example is afforded by the heredity and development of rogues. These rogues are off-types which turn up unexpectedly as sports or mutants in inbred strains or clones of many cultivated plants, such as peas and tomatoes where they come from seed, and potatoes and tulips where they come from tubers and bulbs. In peas their mode of inheritance was made clear by the work of Bateson.

The rogue pea appears in most garden varieties, and has more pointed leaves and more curved pods than the type of the variety. It breeds true when selfed. It also breeds true, as a rule, when crossed either way with the type. There is no segregation in $F_{2}$, and we must suppose that a plasmagene is at work. It must clearly be sup- 
pressive of its normal alternative And in addition it must be carried by pollen as well as by eggs: it is ambilinear.

In one variety of rogue pea a difference shows itself between reciprocal $\mathrm{F}_{1}$ 's in the early stages of growth. In both crosses some of the plants begin, not as full rogues, but as intermediates. When the rogue character has been carried by the pollen there are more intermediates; the rogue effect is diluted and the dilution, as we might expect, is greater in the male contribution. Now, the intermediates turn into full rogues as they grow up, but the intermediate parts bear flowers which can be bred from. The progeny of these flowers includes both rogues and types and the proportion of rogues varies: it is correlated with the degree of expression of the rogue character at the node where the flower was borne. Thus the genetic properties of the plant must change and develop with its external character. For the first time we see heredity, unstable heredity, going hand in hand with differentiation, abnormal differentiation.

\section{Dauermodification}

Since the plasmagene system depends on a chemical equilibrium and is sometimes unstable in development it should be possible to change it by special chemical or physical treatments during development. Such treatments, inaugurated by Jollos, have in fact been successful in producing what he called Dauermodifications, changes in cytoplasmic heredity: with protista such as Paramecium, arsenious acid and heat shocks were used, with Phaseolus chloral hydrate, and with Drosophila merely high temperatures.

These changes are inherited in the female line but, even with selection of the individuals most strongly affected in each generation, the changed type has always hitherto been found to disappear, after a few sexual generations (see Table I7). The mutant plasmagene has not the hereditary staying power of the natural ones we have considered. Does this imply that it falls into another and inferior category? Probably not. These new effects are usually deleterious, at least when the determining conditions have been removed. This means that the conditions of chemical equilibrium governing the new plasmagene will favour its disappearance. And since it can scarcely be indispensable to the cell, those cells which have lost it 
by sorting out will inevitably enjoy a selective advantage during development. Alternatively we may say that the mutation is due, as with kappa, to a reduced concentration in a plasmagene that is
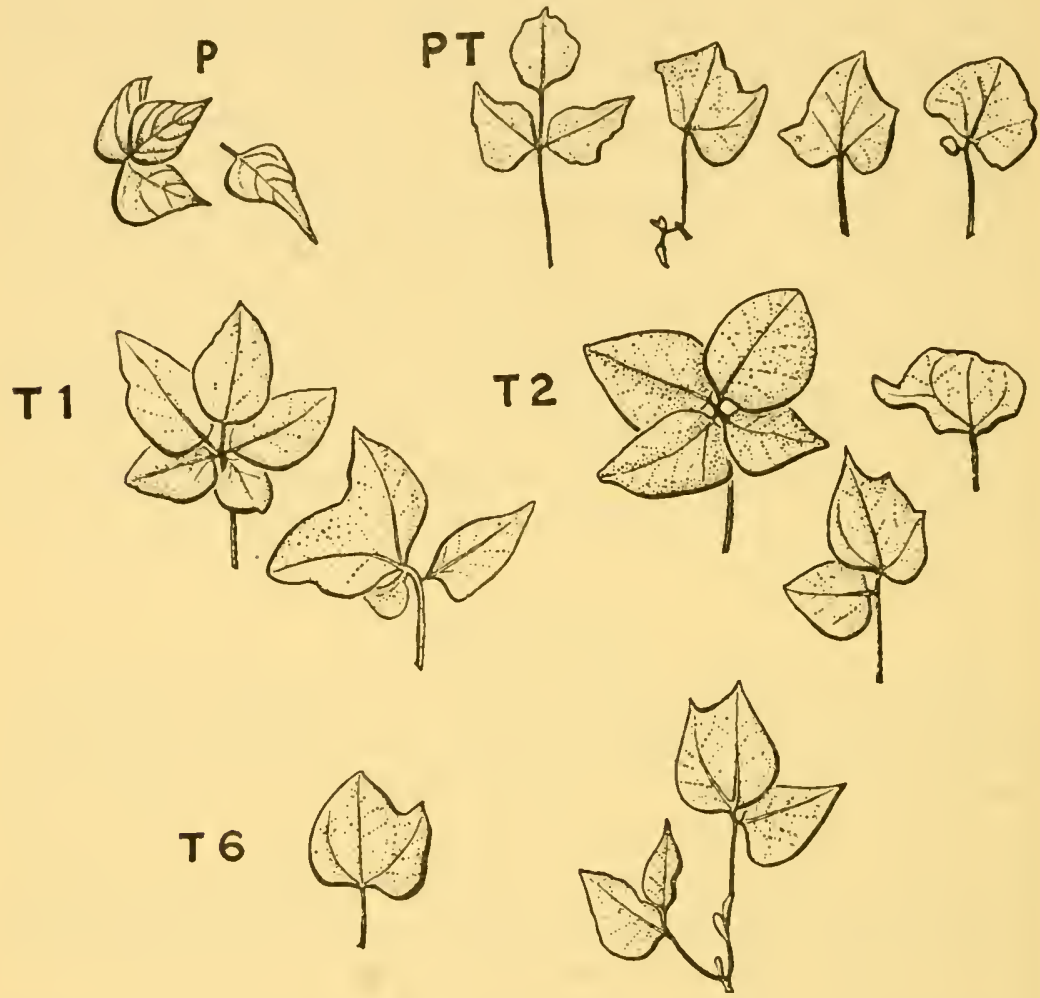

FIG. 44.-The origin and persistence of a defective capacity for development in Phaseolus vulgaris after treatment with chloral hydrate. P, parent; PT, treated parent; T I-6, derived generations (after Hoffinann, 1927).

normally present, its regular concentration being recovered, as Sonneborn has suggested, when sexual reproduction affords a respite to growth.

Severe external shocks may have an effect, similar to the dauermodification of plasmagenes, on self-propagating substances released into the cytoplasm by nuclear genes. Goldschmidt has found in Drosophila that it is possible in this way to imitate the effects of gene change by treatment of the pupae, producing what he has described 
as phenocopies. These, of course, differ from dauermodifications in not being heritable.

TABLE 17

DECAY OF THE DAUERMODIFICATION OF PHASEOLUS $V U L G A R I S$ AFTER TREATMENT WITH 0.75 PER CENT CHLORAL HYDRATE: 200 PLANTS IN EACH GENERATION, SELECTED FROM THE MOST ABNORMAL INDIVIDUALS (HOFFMANN, 1927)

\begin{tabular}{|c|c|}
\hline $\begin{array}{c}\text { Generation after } \\
\text { treatment }\end{array}$ & $\begin{array}{c}\text { Percentage of abnormal } \\
\text { progeny }\end{array}$ \\
\hline 1 & 73 \\
2 & 67 \\
3 & 47 \\
4 & 52 \\
5 & 8 \\
6 & 4 \\
7 & 0 \\
\hline
\end{tabular}

Suppressiveness and Ambilinearity

The working of the plasmagene system now begins to take shape. Intermediates show dilution and, in so doing, show that the system is divisible. There must be a number, a varying number, of like plasmagenes in each cell of the multicellular, as well as of the unicellular, organism. This number changes with development and its change appears as suppressiveness. The action of suppressiveness is revealed also in the origin of rogues. In one variety, where it happens that the mutation occurs later or more slowly than in others, the young rogue plants begin as intermediates. Evidently the rogue plasmagenes have not established themselves and reached equilibrium in the seedling. In crosses, provided that a necessary minimum number are carried over by the pollen, and in new rogues, provided that mutation is early enough, suppressiveness will ensure that the plant will ultimately be a rogue just as Killer admixture swamps non-Killer in Paramecium. The rate at which a plant becomes a rogue is a measure of suppressiveness. Thus suppressiveness implies ambilinearity even where, as with plasmagenes, inheritance cannot be equilinear. And if there were varieties having a nuclear genotype in whose company rogue plasmagenes were not suppressive, the change to rogue would never come about in them and the rogue mutation would never appear. 
The relationship between ambilinearity and suppressiveness is further clarified by the inheritance of one of the many types of variegation of the fern Scolopendrium vulgare studied by AndersonKottö. In this type of variegation the diploid sporophytes are pale green with dark green sectors, which arc produced by genetic change early in development. There is some determinant which bleaches or starves the chlorophyll in the plastids and this determinant is unstable; or perhaps, by sorting out, it fails to get into every cell of the rapidly growing young fern plant. Whole sporangia carrying this detcrminant give purcly palc haploid gametophytes. Other whole sporangia give purely green gametophytes. Since there is no segregation within sporangia where meiosis occurs, the determinant cannot be nuclear. Now the grecn gametophytes breed true, and when self-fertilized the pale gametophytes give variegated sporophytes once more. It is thus only the pale determinant which is unstable or is sorted out, and that is only in the young sporophyte. When the pale and the green gamctophytes are crossed, however, the sporophytes are variegated and the result is the same both ways; thus the scheme is as in Table 18.

TABLE 18

INHERITANCE OF VARIEGATION IN SCOLOPENDRIUM VULGARE

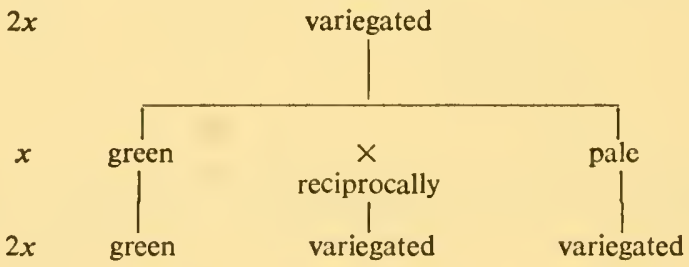

The unstable determinant, being carried with equal effect by cggs and spermatozoids, cannot be in the plastids. Nor, in the absence of segregation after meiosis, can it be in the nucleus. It must be a plasmagene, and one which is again both ambilinear and, in the fertilized egg, suppressive.

As a plasmagene the bleaching agent of Scolopendrium tells us two new things. A plasmagenc, like a nuclear gene, can control the plastids. It can also be unstable, but its instability is no doubt due 
merely to the negative aspect of suppressiveness already seen in Paramecium. In one stage of development it may fall as far behind the average rate of multiplication of their cells as, in another stage, it is in advance of it. And once it is sorted out and omitted from a cell, that cell and its posterity will be free of it.

Summing up: technically we recognize cytoplasmic heredity by its non-mendelian habits. Alternative conditions are found, but they are not alternatively inherited in the sense that their determinants are subject to segregation at meiosis. Further, their transmission is not equally by egg and sperm, but as a rule bears some relation to the volume of cytoplasm contributed. They are either matrilinear or ambilinear. A determinant that succeeds in being carried effectively by way of the pollen does so by virtue of being suppressive of its alternative.-Plastogenes obey the rules of plastid distribution: they are carried by the pollen in some plants and not in others. Plasmagenes, on the other hand, vary in effect in the same individual, that is to say, in their concentration in the cell during development. And when in low concentration, plasmagenes (like the plastids) are liable to uneven sorting out and consequent somatic segregation. At higher concentrations they are subject to the chemical equilibrium of the cell, an equilibrium imposed by the total reactions of genotype and environment.

It follows from these conditions that plasmagenes must be much easier to distinguish in one-celled organisms, whose environment can be changed experimentally than in the higher organisms, where the tissue is the invariable substrate of the cell. What we call plasmagenes in micro-organisms nuust have chemical counterparts in the cells of higher organisms. The reproduction of these counterparts must be under the control of the whole organism during development, and they themselves must be concerned in determining the processes distinguishing one kind of cell from another. The parts played by the cytoplasm and the nucleus must be different in development just as they are in heredity. What those parts are we must now examine.

\section{REFERENCES}

ANDERSSON-KOTTÖ, I. 1930. Variegation in three species of ferns. Z.I.A.V., 56: II7-20I. 
BAteson, w. 1926. Segregation. J. Genet., I6: 20I-235.

BATESON, W., and GAIRDNER, A. E. I921. Male sterility in flax, subject to two types of segregation. J. Gettet., II : 269-275.

BAUR, E. 1909. Das Wesen und die Erblichkeitsverhälmisse der "Varictates albomarginatac hort" von Pelargonimm zonale. Z.I.A.V., I: 330-35I.

BLEIER, H. I928. Karyologische Untersuchungen all Linsen-Wicken Bastarden. Gentica, II : I I I-II 8.

DARLINGTON, C. D. 1944. Heredity, development and infection. Nature, I $54:$ I64-I68.

HADORN, E. 1937. Die Entwicklungsphysiologische Auswirkung einer disharmonischen Kernkombination beim Bastardmerogon Triton palmatus $q \times$ Triton cristatus ô. Arch. f. Eutw. mech., 136.

HOFfmanN, F. W. 1927. Some attempts to modify the germ plasm of Phaseolus vulgaris. Genetics, I2: 284-294.

IMAI, Y. I937. The behaviour of the plastid as a hereditary unit: the theory of the plastogene. Cytologia, Fujii Jub. Vol., 934-947.

Jollos, v. 1938. Damermodifikation. Handb. Vererb., Berlin.

MICHAELIS, P. I937. Untersuchungen zum Problem der Plasmavererbung. Protoplasina, 27: 284-289.

PREER, J. R. 1946. Some properties of a genetic cytoplasmic factor in Paramecium. Proc. Nat. Acad. Sci., Wash., 32: 247-253.

RENNER, O. I934. Die pflanzlichen Plastiden als selbständige Elemente der genetischen Konstitution. Ber. Math.-Phys. Kl. Sächs. Akad., 86: 241-266.

SONNEBORn, T. M. 1947. Recent advances in the genetics of Paramecium and Euplotes. Advs. Genetics, I : 263-358.

SPIEGELMAN, s. 1946. Nuclear and cytoplasmic factors controlling enzymatic constitution. C.S.H. Symp. Quant. Biol., I I : 256-277.

WetTstein, F. V. I937. Die genetische und entwicklungsphysiologische Bedeutung des Cytoplasmas. Z.I.A.V., 73: 349-366. 


\title{
DEVELOPMENT AND DIFFERENTIATION
}

\author{
How the Nucleus Acts: Lag How the Cytoplasm Acts: Gradients \\ Co-operation and Competition The Sequence of Events
}

The DeVelopment of a MANY-CELled ORganism from an egg or spore does not consist merely of a multiplication of cells; it consists also of the development of differences in structures and properties between the cells making different tissues. The mature organism is differentiated. What is this differentiation due to? Two facts assure us that it is not due to changes in the nucleus. The first is the capacity of different parts of plants and animals to propagate or regenerate the whole character of the organism unchanged. The second is the corresponding capacity of the nucleus and its constituent chromosomes to propagate themselves unchanged by mitosis.

These two properties are opposite aspects of the principle of the genetic uniformity of the parts of an individual. It is a principle to which, as we have seen or shall see, there are many exceptions, due mostly to chromosome, gene, and plasmagene mutations; but they are rare exceptions which do not determine differentiation, and are not usually even connected with it. They merely prove the rule.

We are left, therefore, with the cytoplasm as the changing factor in differentiation. The power of the cytoplasm in guiding the course of events is very well shown by the alternation of gametophyte and sporophyte in the mosses, ferns and higher plants. Normally the one is haploid and the other diploid. But this momentous difference does not decide the line which development will take in the haploid spore or the diploid egg. For when the spore happens to be diploid (through the failure of meiosis) or the egg happens to be haploid (through the failure of fertilization) the alternation of generations follows its regular sequence undisturbed. Indeed, by injury, in certain mosses and ferns the gametophytic tissue can be made to develop directly or "regenerate" on the sporophyte (as we saw in the last chapter) without the intervention of a spore.

It is the ordinary process of development through the alternation 
of generations which seems to present the organism with more difficult problems than its suppression. In each pollen grain or embryo-sac or fertilized egg a nucleus of a new type produced by meiosis or fertilization is suddenly implanted in a cytoplasm accustomed to, associated with, or generated by, a previous and different nucleus. This situation requires, as we have already seen, that the cytoplasm has to take the lead in deciding the course of development, sporophytic or gametophytic. It also has the consequence that these cells are the most sensitive of all to upset in the relationship of nucleus and cytoplasm. In the case of embryo-sac and egg the large bulk of the cytoplasm, or the continued nursing by the mother tissue, relieves the crisis. But the pollen grain has to fend for itself. It is the pollen grain, therefore, which is the first to suffer for maladjustment. Male-sterility is the prevailing failure of plant hybrids and the prevailing outcome of cytoplasmic mutation. Of this principle we have seen some instances and we shall see more.

\section{How the Nucleus Acts: Lag}

The determination of gametophytic or sporophytic development is immediately cytoplasmic, but the observations of AnderssonKottö on Scolopendrium vulgare show that it is ultimately genic. A single dose of the gene "peculiar" turns some of the spores into sperm. A double dose has the still more drastic effect of shortcircuiting the reproductive cycle by causing the sporophyte to develop gametophytic sprouts. Similar transformations are, of course, a commonplace of genetics. Any number of genes are known which convert stamens into petals or carpels, petals into stamens or sepals and so on. In Drosophila one gene will restore the four wings of its ancestor, while another will turn the feelers, and another again the mouth parts, into legs. Thus, in the very process of proving that the cytoplasm is immediately responsible for differentiation, we are compelled to show that nuclear genes exercise the ultimate control. The cytoplasm is their agent.

The way in which the nucleus acts through the cytoplasm has been well displayed by Hämmerling's experiment with the singlecelled Acetabularia to which we have already had cause to refer. An individual deprived of its hat will grow a new one. It will still do 
so if deprived of its nucleus in the rhizoid, provided that no stem was taken away with the hat. In other words there is a hat-forming substance near the hat. If the hat is cut away with a large part of the stem, it can still be formed again provided that the nucleus remains present. But the regeneration then takes time-time for the nucleus to make some more hat substance.

This argument is clinched by species transplants. If the hat and stem of one species is grafted onto the rhizoid and nucleus of another the existing hat is unaffected. But, as we have already had occasion to observe, any new hat is modified in its development, taking more and more the form laid down by the nucleus as the length of its association with that nucleus increases.

Thus the nucleus is producing the materials, the proteins, which eventually decide the character of the cell; but time is needed for these proteins to accumulate and to become effective by replacing those produced by the previous nucleus.

The lag before a gene becomes effective is important in the ordinary life of an organism. There are some genes which become visibly effective within a single cell generation. In the pollen grains, following segregation of waxy from non-waxy at meiosis in a heterozygous maize, the two types are distinguished before the pollen is ripe by the presence or absence of starch. In Paramecium, on the other hand, 36 or more generations may elapse before a gene takes effect. Correspondingly, in the multicellular echinoderms, hybrids have been found to cleave at the maternal rate until the gastrula stage is reached, when the paternal genes make themselves felt. Their effects may then appear as a breakdown of development when the cross has been too wide, that is, when the hybrid genes act too dissimilarly from the maternal ones which have laid down the character of the cytoplasm.

Most of the characteristic genes of mendelian experiments, as we have seen, act later in development; not indeed until the individual is approaching maturity. Those which act more quickly act too strongly: they are lethal. A few act more slowly and do not manifest themselves until the next generation. The best known example is in the determination of the direction of coiling of the snail's shell which follows the mother's genotype whatever the character of the father (Fig. 45). 


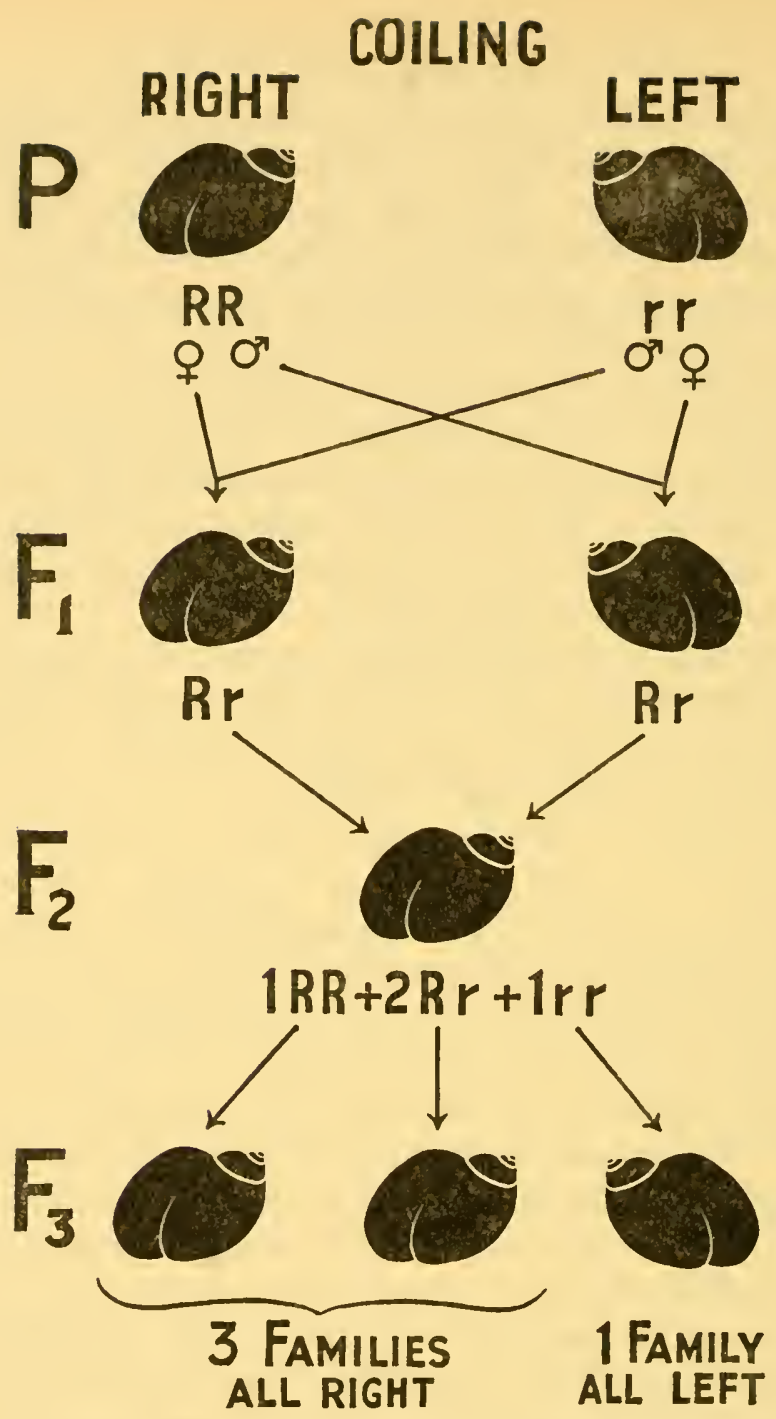

FIG. 45.-The direction of coiling of the shell in Limnen peregra is determined by a single gene difference, the allelomorph giving right-hand coiling being dominant to that giving left. The direction of coiling reflects, however, not the snail's own genotype but that of its mother. Thus all the offspring of a female have the same direction of coiling, even though she be a heterozygote. The segregation will appear in the phenotype as differences between the families of grandchildren.

In this way the reciprocal $F_{1}$ 's differ in the cross between true breeding strains of right and left coiled snails. The $\mathrm{F}_{2}$ 's from these reciprocals, however, are alike and are also uniform. The segregation among the genotypes of the $F_{2}$ 's shows itself only in the $\mathrm{F}_{3}$ gencration which consists of 3 families of uniformly right coiled snails to I of uniformly lcft coiled snails, no matter which way the original cross was made. 
The same principle applies to the sex determination of the midge Sciara. Some females produce only male offspring, and the rest only female. The females are again of the two types: those like the mother and those like the father's mother.

Sometimes we can see that the delay in the time at which a gene becomes apparent depends on the amount of store, or the degree of multiplication, of the substance it produces in the cytoplasm. The disappearance of the old gene's products and the accumulation of the new gene's products may be proportionately slow. Thus, with the moth Ephestia, Kühn (1937) found that, in the progeny of pigmentless a $a$ mothers (crossed with $A a$ ), the pigmentless a a eyes were at once distinguishable from the pigmented $A a$ eyes. But in the young progeny of pigmented $A a$ mothers the distinction was smothered. The pigment determinants in the cytoplasm of the egg provided full coloration until a late larval instar, so that only then did the segregation appear. These determinants, by the way, were diffusible, for transplanted $A a$ testes could darken the eyes of an aa host and of its young a offspring.

The extreme slowness in action, or in accumulation for action, is attained by the gene "grandchildless" in Drosophila subobscura. Females homozygous for this gene are fertile; but no matter to what male they are mated, their offspring are sterile. They can have children but no grandchildren. This gene's normal allelomorph must be producing something necessary for fertility, but producing it so slowly that its absence is not felt until a generation has elapsed.

Many genes will obviously depend for their manifestation not merely on a threshold value of their product, but also on the opportunity for its giving a different result from that of its allelomorph. The direction of coiling in the snail is determined at the second cleavage division of the fertilized egg. If this opportunity is missed, it will not recur for a whole lifetime. All we can know of the gene's lag, therefore, is that it is somewhere between two cell generations and a whole life-cycle in length. Since a gene's opportunity is itself determined by the totality of genes, it is easy to see how each gene depends for its expression on its fellows. 


\section{How the Cytoplasm Acts: Gradients}

The genes thus express themselves by what they give to the cytoplasm. But they vary in how quickly they give it; and it varies in how soon it finds something to react with. Genes express themselves consequently at different times in the course of development. We next have to see what the cytoplasm gives to the nucleus and

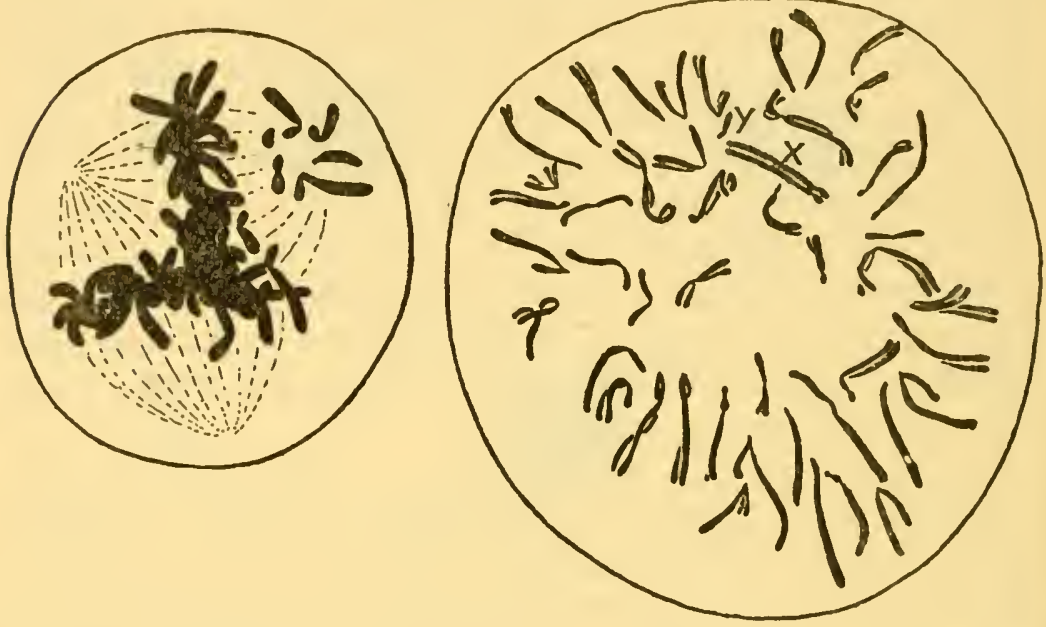

Fig. 46.-Blood precursor cells from the bone marrow of a man with pernicious anaemia, the normal contrast in nucleic acid charge between reds and whites being exaggerated. Left, over-charged red with thick chromosomes and over-developed and multipolar spindle which will give several non-viable hypo-diploid cells. Right, under-charged white with long thin chromosomes and under-developed open spindle which will give a single tetraploid cell by failure of anaphase. $\times 2,500$ (after La Cour, 1944).

how this varies with development. The control of the nucleus by the cytoplasm is most readily seen in the synchronization of mitosis (and meiosis) in groups of cells: Such a synchronization is found in all tissues where cell walls are poorly developed or absent. In the cleaving animal egg, and within follicles of the testis or anther, in the endosperm, in the twin diploidized cells of fungi, and even in the fecbly-walled pollen grains in the orchids, all the mitoses move in step. They do so, apparently, because the supplies of materials necessary for mitosis, especially proteins and nucleic acid precursors, are uniformly diffused in the cytoplasm of the whole group of cells. 
How this works has been shown by La Cour in the differentiation of the bone-marrow of mammals. Here two opposite types of celllineage are separated; one, with high nucleic acid content in the cytoplasm, has rapid divisions of its nuclei, a strongly developed spindle and chromosomes heavily charged and strongly spiralized. This type gives rise to the red blood corpuscles. The other, with low nucleic acid content, has slow divisions, a poor open spindle scarcely capable of executing the anaphase movement, and chromosomes feebly charged and weakly spiralized. This gives rise to the white blood corpuscles in their far smaller numbers. Thus two lines of development are set going in the same ancestral nuclei by tivo conditions of the cytoplasm, arising no doubt in different positions in the tissue (Fig. 46).

The differentiation of the embryo-sacs and pollen grains of flowering plants exactly parallels that of the blood precursors. In the pollen of Angiosperms one of the daughter nuclei is pressed by the first mitotic spindle against the wall, while the other is left in the middle of the cell. The peripheral, or generative, nucleus forms a small cell and is rich in nucleic acid: it divides again to give the two condensed sperm nuclei, often before the germination of the grain. The central, or vegetative, one forms a large cell and is poor in nucleic acid: it becomes large and diffuse and loses first its staining power, and then its coherence. It vanishes without further mitosis. (The nucleus of the ripe red blood corpuscle, similarly drained of nucleic acid, similarly dissolves.) This differentiation must depend on the fact that the distribution of materials in the pollen grain before mitosis is not uniform. There is a gradient and the position of the mitotic spindle is adjusted to lie along this gradient. If the axis of the spindle lies crosswise to the normal (following heat shock) cells and nuclei with similar properties are produced: differentiation fails (Fig. 47).

In a species of Sorghum extra heterochromatic chromosomes have been found to cause the vegetative, as well as the generative, nuclei to divide again. Extra nucleic acid, which the additional chromosomes make available in the cytoplasm, stimulates nuclear division in a way which recalls the plan of development of the gametophyte in the lower plants. In extreme cases the nuclear division may give four or five generative nuclei and thereby kill 
the pollen grain, much as the excessive mitosis of a tumour may kill an animal.

Pollen grains provide us, both internally and, as we saw earlicr, externally, with crucial tests of developmental principles simply on account of their unique property of being independent organisms limited as a rule to two or three cells. The different sequences of their development arise from the reactions of different types of

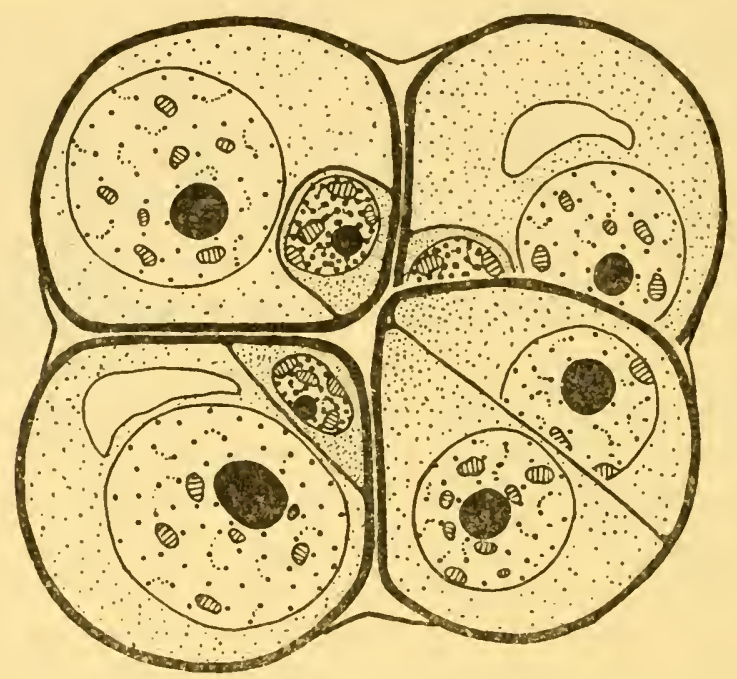

FIG. 47.-The four pollen grains of a tetrad in Scilla sibirica still exceptionally adhering after the first mitosis. As in Ascaris (Fig. 48), a radial spindle has given normal differentiation in thrce cells; an exceptional tangential spindlc has given no differentiation in the fourth. The concentration of generative-nucleus-forming substances, represented by stippling, is evidently central in the pollen mother cell (La Cour, original).

nuclei, symmetrically or asymmetrically placed with respect to the spatially differentiated cytoplasm of a single cell. The immense range of types of embryo-sacs in flowering plants depends on the same principles opcrating in more claborate settings, showing a range of behaviour depending, no doubt, on varying gradients in the distribution of nucleic acid in the mother cell (Fig. 50).

Similar to the differentiation in space in the blood and pollen is that in time, whereby the scries of mitoses in the development of a plant or animal is interrupted by meiosis, as a result, perhaps, of flooding of the nucleus with nucleic acid and the charging of the 

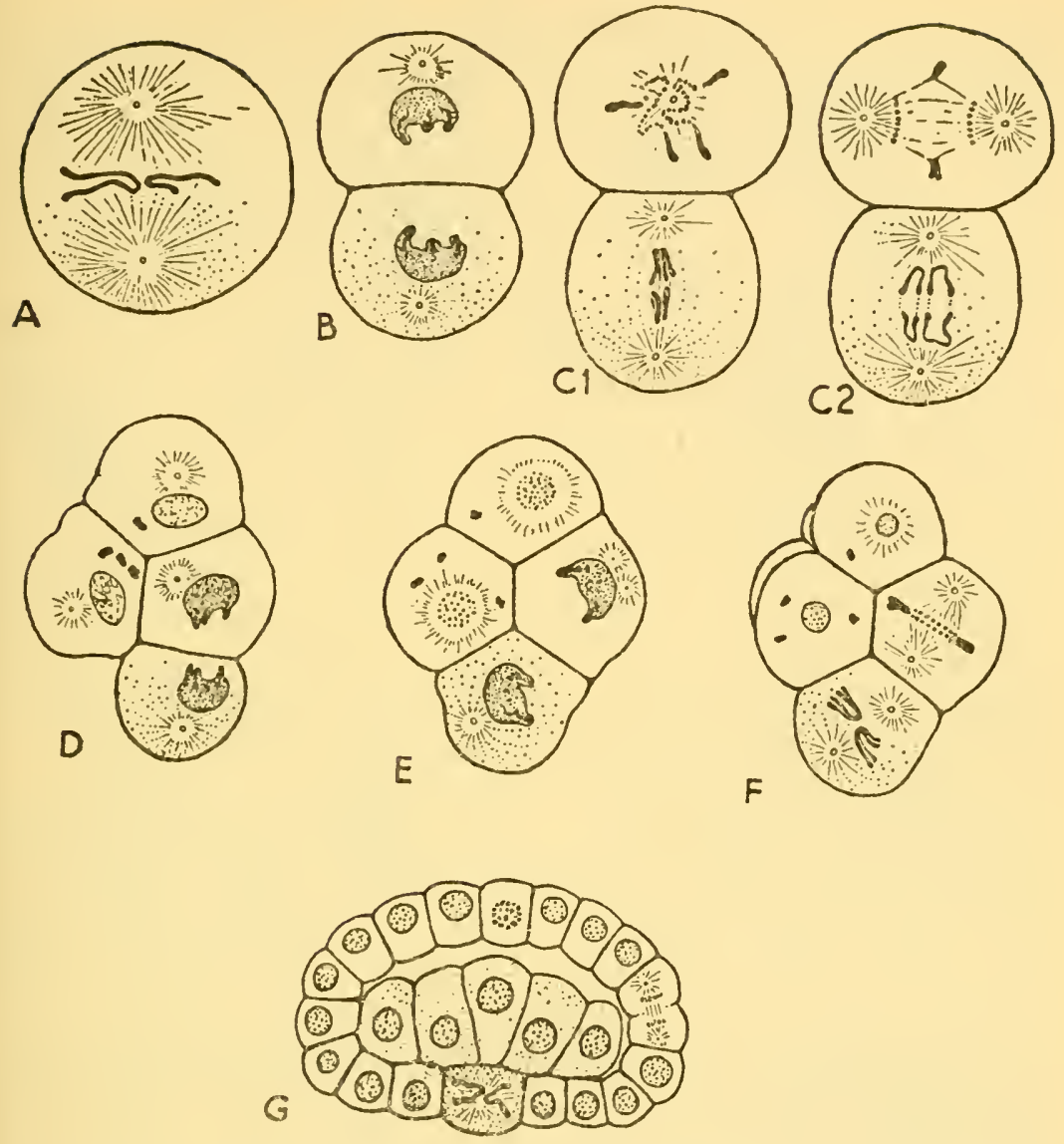

FIG. 48.-Cleavage, blastula and gastrula stages in the development of the egg of the thread worm Ascaris megalocephala (diploid race with one gametic chromosome). A, the maternal and paternal chromosomes meet on the first cleavage spindle. $\mathrm{B}$, formation of two cells, differentiated in cytoplasmic determinants, the dorsal with the potentiality for more rapid division, leading to fragmentation of its chromosomes at anaphase and loss of the distal heterochromatin, the ventral with the potentiality for regular mitosis without breakage. $C_{1}$ and $C_{2}$ views of the second divisions: the differentiation continues with progeny of the cell which divides in the same axis as that of the first mitosis. D, the T-shaped embryo rounded off to form the blastula. E, F, third cleavage division. G, the gastrula stage: 32 cells of which one only has the complete set of chromosomes and will give rise to the germ cells. The cytoplasmic germ-line determinants, which are directly visible in copepods and are here marked only by the presence of fewer vacuoles are shown here by stippling ( $c$. Bounure, 1939).

Note.-The difference in mitotic rates suggests a gradient in nucleic acid content which should be exaggerated by the absorption of lost heterochromatin in the cytoplasm (after Boveri's figures I9IO et al., but conflicting with the diagrains in Wilson, Iy00; Boveri, I904; Morgan, I9I 3 ; and Belar, I938). 
chromosomes before they have reproduced themselves. Differentiation in space as well as in time is responsible for the fragmentation of the chromosomes in particular embryonic cells in Ascaris. In those which arise from one region of the egg, and are predestined not to form the germ cells, the chromosomes break up at the anaphase of mitosis and leave some unessential pieces of heterochromatin (lacking centromeres) on the equator (Fig. 48).

In all these instances the cytoplasm of particular cells is telling the chromosomes what to do. But paradoxically enough, it can only be passing on what it has had from the nucleus. For in polymitotic maize, a recessive gene compels all the pollen grain nuclei produced by a plant homozygous for it to divide again as soon as they are formed, and to go on dividing until the whole nuclear organization is destroyed. In this case it is clear that the gene has acted on the cytoplasm (already in the mother plant, for the effect is not seen in the individual pollen grains of the heterozygote) and the cytoplasm has acted in turn on the nucleus, a different nucleus.

Finally, it should not be lost sight of that the cytoplasm is in immediate control of all the everyday details of mitosis. Each centromere and nucleolar organizer is a gene, simple or compound, and when all of them keep time in nuclear cycle they are illustrating the unity of reaction of any species of gene to the changes in the common substrate, the cytoplasm. Other genes whose activities are less minutely observable evidently behave in the same way to give the disciplined uniformity of nuclear propagation from which the authority of nuclear action derives.

\section{Co-operation and Competition}

The interaction of the cytoplasm and the genes in the nucleus, each modifying the behaviour and activities of the other, is now becoming clear. It expresses itself also in the relations of cells of different genetic character. When, as Barber found in Uvularia, two pollen grains are formed at meiosis, one having extra chromosomes, and the other lacking these same chromosomes, the first survives poorly and the second survives not at all. If, however, the cell-wall that should have separated them is inhibited by a heat-shock, so that the two complementary nuclei are lying in the same cytoplasm, 


\section{COOPERATION OF NUCLEI}

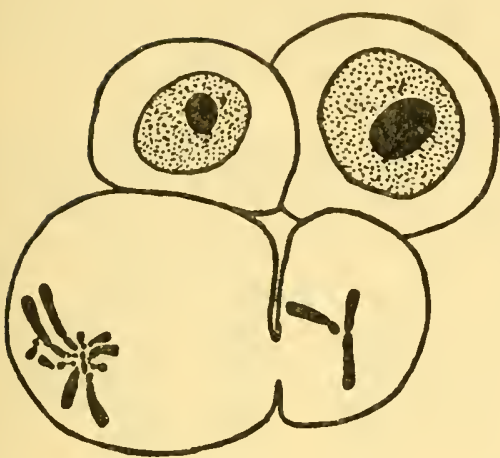

$12 / 2$

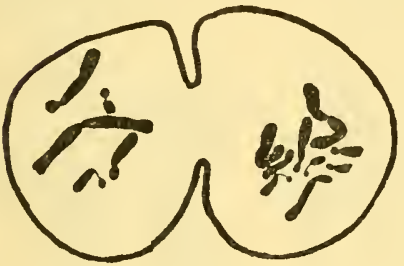

$5 / 9$

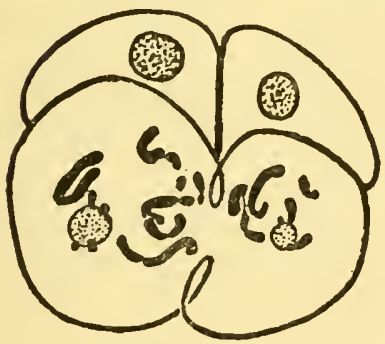

$8+1 / 5$

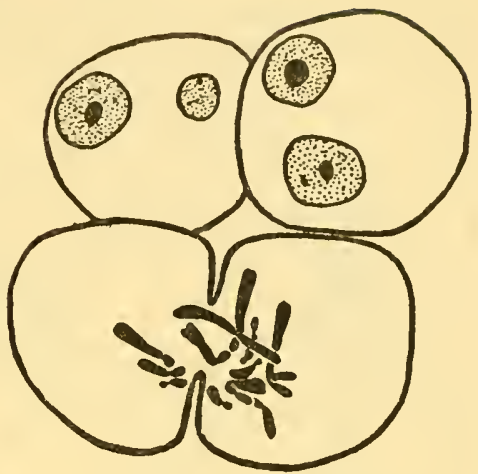

$3 / 11$
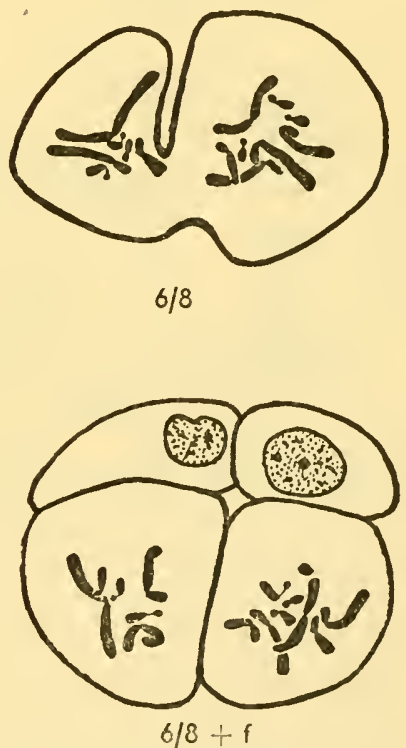

FIG. 49.-Pollen grains adhering in dyads and tetrads in Uvularia grandiflora $(x=7)$ after a heat shock which has deranged the second division spindle and consequently anaphase separation and wall-formation. The pairs of nuclei in the incompletely divided cells are unequal but complementary and therefore come into mitosis at the same time. In the two bottom cells an acentric fragment is present and the sum of the pair of nuclei is unbalanced, with less (in the prophase on the left) or more (in the metaphase on the right) thair a diploid complement. One of the resting cells shows double nuclei, but all have perfect wall formation and are not synchronized (after Barber, I94I). 
then both are entirely successful. More than that, the two are perfectly synchronized in mitosis. Thus each nucleus must be providing something necessary or useful to the other, and providing it by way of the cytoplasm (Fig. 49).

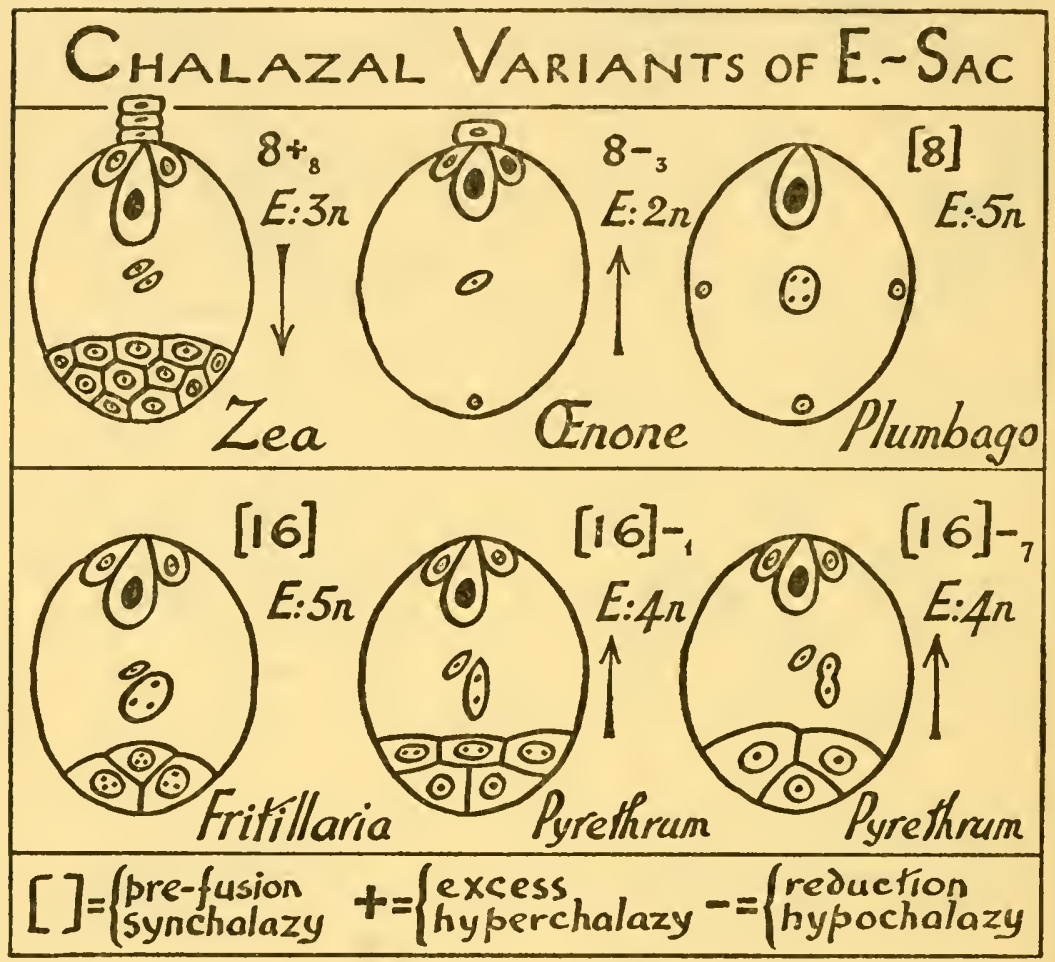

Fig. 5o.-Variations in the development of the embryo sac in Angiosperms disregarding the number of spores which take part in it. Egg cell nuclcus solid, other nuclei with one dot for each haploid set. 8 mcans 3 mitoses before egg cell formation, 16 means $4 .+$ and-refer to excess or deficiency in the number of mitoses at the chalazal end of the embryo sac and thus imply a gradient. E, the endosperm which varies in ploidy according to the number of nuclei fusing in the centre. Zea, Cooper, 1938. Oenone, Went-Maheshwari, 1936. Plumbago, Haupt, 1931. Fritillaria, Westfall, 1940. Pyrethrum, Martinoli, 1939 ( $f$. Darlington and La Cour, 194I).

In this question of life or death the part played by the cytoplasm can be as crucial as that of the nucleus. In certain hybrid species of Oenothera, whose organization we shall consider later, two genetically different types of germ cells are produced, say A and B, such that 
only the A pollen grains, and only the B embryo-sacs, germinate or grov. Thus the two kinds of cytoplasm, in pollen and embryo-sacs, favour the development of the available alternatives.

This reaction has another aspect. Not merely does the cytoplasm discriminate but, on the female side, the nuclei compete. Of the four potential megaspores, two B's at one end and two A's at the

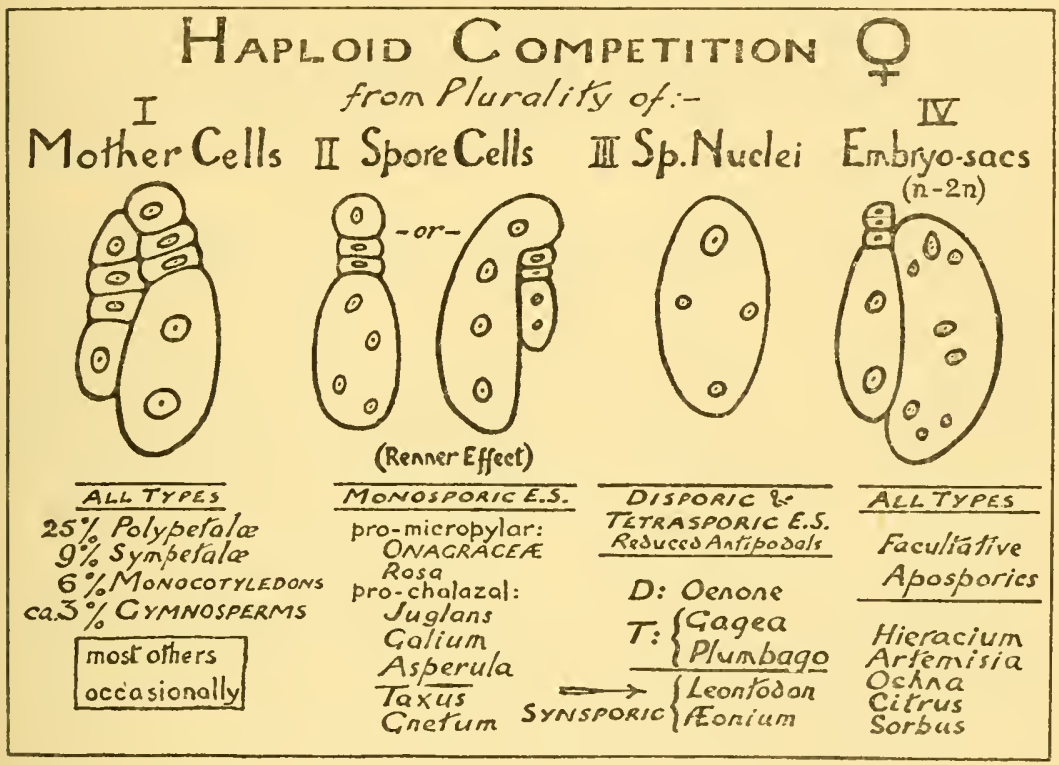

FrG. 5I.-Competition of cells depending on the interaction of the genetically different products of segregation with physiologically determined gradients at different stages in development of the embryo sac in the flowering plants. Rubiaceae, Fagerlind, 1937. Juglans, Nast, I935. Oenothera, Rudloff and Schmidt, 1932 ( $f$. Schnarf, I936; Darlington and La Cour, I94I).

other, the one at the micropylar end is favoured by its position in the gradient, that is by its cytoplasmic content. If a $B$ is at the more favourable end it always grows. If it is at the less favourable end there is conflict between the relative advantages of the cytoplasmic gradient and the nuclear genotype. After a short contest the nucleus wins and $A$ is strangled by $B$. Or vice versa. The proportion of victories one way or the other is characteristic of each species (Fig. SI).

This Renner Effect shows that nuclei and cells of different geno- 
type co-operate only where they are equally unfit, as in Uvularia, or equally fit, as in Ascaris, where presumably the complete and the diminished types of nucleus are, each of them, fitter for their own types of tissue. But where the nuclei are unequally fit, competition at once eliminates the weaker and establishes genetic uniformity. The Renner Effect shows also that one nucleus is not inherently fitter or stronger than another; it is merely better fitted to the cytoplasm in which it lies-A to that of the pollen, B to that of the eggs. But both cytoplasms have been produced under the control of the same $A B$ nucleus. They differ merely in their developmental history, in their cellular antecedents, in their chemical character as parts of different tissues.

Thus tissues, owing to their different relative positions in the organisms, come to differ in the character of their cells, as shown by the reactions of nuclei and cytoplasm. This is just the same principle that we have already seen at work in Grüneberg's lethal in the rat, whose manifold effects on different organs can be traced to a specific initial action on cartilage. The working of the nucleus carrying this mutant gene breaks down when it is faced with a specific job, that is when it lies in a specific cytoplasm.

Transplantation experiments show us another aspect of the genetic specificity of tissues. Hadorn transplanted into normal larvae organs of the slowly developing "lethal-giant" Drosophila, at a time when its imaginal discs were degenerating. He found that transplanted testes degenerated, but the ovaries continued to develop and even formed eggs. The cytoplasm thus determines whether and how the gene will express itself.

Further, Hadorn was able to select two lines throwing the homozygous recessive lethal-giants. These giants died, in the one early, and in the other late, in larval development. The lethal-giants thrown by reciprocal crosses between these two lines took after the mother line in the time of death. In other words the eggs differed in cytoplasmic constituents which reacted differently with the lethal gene and so determined the different times of death. These constituents must themselves have been slowly produced gene products, since the reciprocal difference was not continued in the grandchildren. 


\section{The Sequence of Events}

These experiments show something more than nuclear-cytoplasmic reaction. They show the cytoplasm as the field of reaction of products of different genes accumulated at different rates and over different periods. We may therefore ask, at this point, how it is that nuclear products accumulate in the cytoplasm over long periods. We have already seen that certain particles or determinants, the plasmagenes, can propagate themselves in the cytoplasm with a permanency and autonomy scarcely less than that of the nuclear genes. Wright has argued that growth could not proceed autocatalytically, nor could differentiated tissues retain their uniform character, without some similar propagation of other cytoplasmic particles.

Such particles would not necessarily continue permanently and independently. Indeed we have evidence of gradations between a high rate of propagation which leads to permanence and a low rate which leads to transience. These gradations are themselves under genetic control. Thus, as we have already seen, Preer found that in one variety of Paramecium the rate could be forced up to the point at which the kappa particles were unable to keep pace and were gradually lost. In another, the kappa particles reproduced so rapidly that they were never lost, even though the cells of this variety divided nearly twice as rapidly as those of the first one.

These differences in rates of propagation of cytoplasmic particles -some autonomous and some presumably nuclear products-show how the cell may, at one time, maintain itself and, at another, vary in constitution in the course of development, in the way that we observe it to do. The propagation of any one part of the cytoplasm is thus conditioned by the whole; and, since the whole is modified by the activity of the nucleus, the nucleus is in effect modifying its own activity (Fig. 52). It does so after a delay which must depend on such physico-chemical conditions as the permeability of cell and nuclear menbranes, the rates of diffusion of particles of different sizes, and the rates of propagation of nucleo-proteins of different shapes. These are the fundamental conditions of differentiation, and their essential consequence is the lag between determination and 
effect, or between one reaction and the next in the chain of processes.

We can now see how the reactions of nucleus and cytoplasm give a progression of events in the cell. But the progression must be an

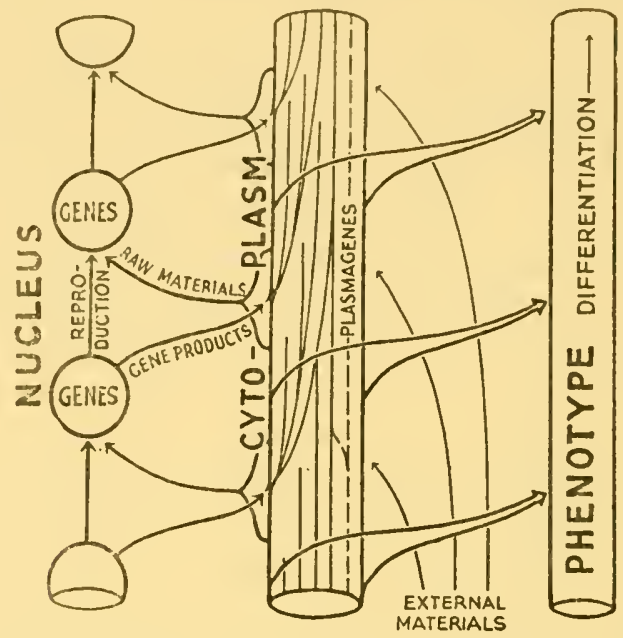

FiG. 52.- The genes of the nuclcus draw upon the cytoplasm for the raw materials of their own reproduction and action. The products of this action pass back into the cytoplasm, where these products may have only a short life or may further multiply themselves by self-reproduction, in association with nuclcic acid, according to whether the composition of the cytoplasm be favourable. In so doing they change the composition of the cytoplasm and thercby affect the fate of the further gene products which pass into the cytoplasm. In this way a constant nucleus can be associated with a changing cytoplasm, and hence with a differcntiating phenotype, for the cytoplasm is the agent of the nucleus in action.

In the diagram the gene products are represented by lines in the cytoplasm, and their varying fatcs by varying lengths of thesc lines. The products interact with onc another and with the plasmagenes (dotted line). The phenotype reflects the totality of the propertics of the cytoplasm as shown by the wide arrows. The cytoplasm is also the channel by which cxtcrnal materials and conditions reach the nucleus or affect its action (after Mather, 1948).

orderly one which passes through a regular life cycle, in the general case, with sexual reproduction. This orderliness is achieved by the adjustment of the hereditary materials, especially of the genes in the nucleus, and it is lost when the genic adjustment is destroyed. One cxample will illustrate this very general principle. In the morning and evening campions, sub-species of Melandrimm dioicum, the sexes are separated with $X X$ females and $X Y$ males. In the $F_{2}$ 
of a cross between them, however, some XY plants appear which bear female organs. From these Winge has been able to establish new hermaphrodite lines. Thus the regular progression of development must be determined by genes, for the ancestral order had been broken by the mixing of genes from the two stocks. The males were no longer clear males: they had lost some of their distinctive features. In each sub-species the genes had been balanced to give the same result, but they had been balanced in different ways. How this can happen is part of a much larger problem into which we shall have to inquire later.

Summing up: we may examine development and differentiation in plants or in animals or in micro-organisms, in individual cells or in vast aggregates; we may approach the problem by way of hybridization or of transplantation. But whichever way we go about it, we find that the centre of the control of development is the nucleus whose sphere of action is the cell. The nucleus, by way of the cell, controls both heredity and development. In development the nucleus provides the constant basis for the regular changes in the cytoplasm, changes which could not be regular unless they had that constant basis. The nucleus and the cytoplasm have long seemed to be the opposite poles of biological study; but their new unity need no longer surprise us. Heredity now appears as the repetition of the series of changes which constitutes development, and the study of each is bound to require, more and more, the understanding of the other.

\section{REFERENCES}

ANDERSSON-KOTTÖ, I., and GAIRDNER, A. E. I936. The inheritance of apospory in Scolopendrium vulgare. J. Genet., 32: I89-228.

BELAR, K. I928. Die Cytologischen Grundlagen der Vererbung. Berlin.

Bounure, L. I939. L'Origine des Cellules Reproductrices. Paris.

Boveri, T. I9Io. Die Potenzen der Ascaris-Blastomeren bei abgeänderten Forschung. Fest. R. Hertwig (Jena), 3: I33-2I 4.

BRACHET, J. I947. Nucleic acid in the cell and the embryo. Symp. Soc. Exp. Biol., I : 207-224.

BARBer, H. N. I94I. Chromosome behaviour in Uvularia. J. Gettet., 42: 223-257. BARBER, H. N. I942. The pollen-grain division in the Orchidaceac. J. Genet, 43:97-IO3.

DARLINGTON, C. D., and LA COUR, L. F. I94I. The genetics of embryo-sac development. Antt. Bot., N.S., s: 547-562. 
DARLNGTON, C. D., and THOMAS, P. T. 1941. Morbid mitosis and the activity of inert chromosomes in Sorghum. Proc. Roy. Soc. B., I30: 127-150.

GRUNEBERG, II. 1943. Congenital hydrocephalus in the mouse: a case of spurious pleiotropism. J. Genet., 45: I-2I.

HADORN, E. 1937. Transplantation of gonads from lethal to normal larvae in Drosopliila melanogaster. Proc. Soc. Exp. Biol. and Med., 36: $63=634$.

HAdORN, E. 1940. Prädetermination des Letalitätsgrades ciner Drosophila-Rasse durch den mütterlichen Genotypus. Rev. Suisse Zool., 47: 13-176.

hadorn, E. 1945. Zur Pleiotropie der Genwirkung. Archiv. d. Julius Klaus Stift., 20: $82-95$.

HALDANE, J. B. S. 1932. The time of action of genes, and its bearing on some evolutionary problems. Ati. Nat., 66: $5-24$.

кӥнN, A. 1937. Entwicklungsphysiologisch-genetische Ergebnisse an Ephestia kuhuiella. Z.I.A.V., 73: 419-455.

LA COUR, L. F. I944. Mitosis and cell differentiation in the blood. Proc. Roy. Soc. Editi. B., 62: 73-85.

MATHER, K. 1948. Nucleus and cytoplasm in differentiation. Symp. Soc. Exp. Biol., 2: $196-216$.

METZ, C. W. 1938. Chromosome behaviour, inheritance and sex determination in Sciara. Am. Nat., 72: 485-520.

RENNER, O. I940. Kurze Mitteilungen über Oenothera. IV Über die Beziehung zwischen Heterogamie und Embryosackentwicklung und über diplarrhene Verbindung. Flora, N.F., 34: 145-I 58.

SAX, K. I935. The effect of temperature on nuclear differentiation in microspore development. J. Arn. Arb., I 6: 30 I-3 I0.

SCHNARF, K. 1936. Contemporary understanding of embryo-sac development among angiosperms. Bot. Rev., 2: $565-585$.

SPURWAY, H. 1947. An extreme example of delay in gene action in Drosophila sub-obscura. Drosophila Information Service, 20: 91.

wilson, E. B. I925. The Cell in Development and Heredity. 3 rd ed. New York. WINGE, 9.193 I. X-and Y-linked inheritance in Melandrimm. Hereditas, I s: 1 27-165. wright, s. I945. Genes as physiological agents. Ait. Nat., 79: 289-303. 


\section{VIRUSES, PROVIRUSES AND THE CONFLICT OF SYSTEMS}

The Propagation of Viruses and their Genetics Animal Tumours

Neutral Hereditary Infection The Origin of Viruses

Nuclear and Cytoplasmic Systems

OUTSIDE THE NUCLEUS there are, as genetic evidence has shown us, reproductive bodies in the cell. The plastogenes we can distinguish as bodies, which must lie in the proplastids or the mitochondria. These bodies can be stained differentially and they are proteins with nucleic acid attached, sometimes combined also with phospholipids. The nucleic acid is the ribose form, not the desoxyribose of the chromosomes. Other bodies, "microsomes" 50-200 $\mathrm{m} \mu$ in diameter, Claude has separated by centrifuging lung, pancreas and plant cells. The ribose nucleic acid in the cell, whose quantity is correlated with the rate of protein production, is largely localized on these bodies. They, therefore, probably include both the plasmagenes and the other self-reproductive proteins important in development and differentiation.

\section{The Propagation of Viruses}

Bodies or particles also occur in cells which manifest heredity in themselves, but do not determine it in the organism which includes them; for, characteristically, they get where they are, not through the egg or the spore, but by infection or invasion or even the transplantation of tissues. The most obvious of these bodies are the recognized viruses, parasites of animals, plants and bacteria. The larger viruses are complex in organization. Vaccinia, for example, contains fat, carbohydrate and even copper, as well as nucleoprotein. It also contains desoxyribose nucleic acid. Its particles are about I $50 \mathrm{~m} \mu$ in diameter. The simpler forms are also proteins, but they usually have ribose nucleic acid attached to them. They exist in the cell as particles, either globular and $20-30 \mathrm{~m} \mu$ in diameter, or rodshaped, Is $\mathrm{m} \mu$ thick and I25-I,000 $\mathrm{m} \mu$ long. They may be separated 
from the cell and even precipitated as crystals or liquid crystals. But they cannot propagate outside the cclls of their host.

Now, particles transmitted in heredity as nuclear genes or plasmagenes are necessarily more or less suited to the organism of which they form a part. Any organism or race carrying such incscapable particles is likely to be elininated by natural selection if they are not so suited. Viruses are under no such compulsion. They infect their hosts through air or water, or through the body of a specialized insect carrier or vector. Their effects on a host are, therefore, within a wide range indifferent to the viruses themselves, and we find that these effects indeed cover a very wide range. At one extreme, perhaps, is the bacteriophage, which destroys its bacterial victim in a few hours. At the other are the viruses which cause variegation in Abutilon or "breaking" in tulips. They have existed in equilibrium in the cclls of the host for hundreds of ycars. If, as sometimes happens, they can be carried by the egg, they cither eliminate all susceptible stocks, or they will becomc, and may have become, universal in a species and indistinguishable from its plasmagenes.

The gencral effect of viruses consists in a distortion, great or small, of the metabolism of the host cell. The virus may act as a phosphatase or other enzyme, which pushes the cell processes of the host in a new direction. In doing this it multiplies itself and thereby greatly increases, for example, the protein content of an infected plant. Like the tryptophancless gene in Neurospora, as Rischkov found, it piles up its by-products beside it as a body of inert protein. Healthy processes, as we saw, are such as lead to the least accumulation of useless or inactive product. The virus starves or deforms the normal or healthy synthetic processes and replaces them by new ones. A bacterial infection may consequently benefit from the co-operation of a virus (as in Hog Flu). In green plants, we find that the plastids and pigments suffer most obviously.

With its specialized activity the virus naturally favours special tissues. Just as cholera is almost harmless when injected under the skin, so curly-top of tomatoes and sugar beet requires the phloën for its propagation. In animals which survive the crisis of a virus attack, diffusible anti-bodies are produced which restrain its multiplication and lead to recovery. In plants anti-bodies, perhaps owing to their lack of diffusion, are known only in one instance. But 
commonly the activity of the virus is starved by its own success, and equilibrium can often be restored to produce the kind of situation we have in the broken tulips. Plants can also be cured of viruses by growing away from them under conditions favouring the host at the expense of the parasite, just as the tissue of Scolopendrium or the stock of Paramecium gets rid of the plasmagene it has carried. In the extreme case, water at $45^{\circ} \mathrm{C}$. will kill a Vinca virus in a few hours without killing the plant, just as kappa can be killed in Paramecium.

The relations of viruses with one another, relations which are best seen in plants, are not without genetic interest. Many pairs of viruses have no effect on one another in the same host. They are neutral or independent. A second type of reaction is co-operation. For example, K. M. Smith has shown that Rosette Disease of tobacco is due to two viruses. These can be separated by inoculation, and also by the insect vector, which carries one of them only in the presence of the other; but they act together like two complementary genes in producing their effect, and in the self-multiplication on which this effect depends.

A third type of reaction is antagonism. This is commonly shown more strongly between related or mutant strains of the same virus. Thus an infection with a less virulent strain may enable a plant, which does not develop anti-bodies, to repel an infection by a more virulent strain. Such a situation is obviously due to the two strains needing the same food-or, in terms of gene physiology, to their having the same precursors. And finally, as Bawden and Kassanis found, Severe Etch actively replaces Potato virus $\mathrm{Y}$ and Hyoscyamus virus 3 in plants in which these viruses are already established. The one virus, as it were, digests the other. Here we see an analogy with suppressiveness as between plasmagenes, and also with the synthetic sequences in Neurospora.

\section{The Genetics of Viruses}

Two aspects of the behaviour of viruses are of genetic importance. The first is that of the virus itself. Viruses that have been most studied have been found, like all other pests and parasites, to exist in strains of varying virulence just as their hosts are of varying 
susceptibility. Many of these, like the so tested strains of Tobacco Masiac, have arisen under experiment and, we may say, by mutation analogous, as Muller first pointed out, to that of nuclear genes. The question arises as to whether this mutation is in some scnse frec and uncontrolled, or whether some control is exercised by the host over its frequency or direction such as we saw in the property of mutafacience. The answer scems to be that all degrees of control occur.

Bactcria, infected by viruses, mutate to become resistant to them. The viruses can then mutate, becoming adsorbable on the bacteria, so as to be once more successfully virulent. In this situation, described by Luria, we seem to have free mutation on both sides followed by the survival of the fittest mutailts. On the other hand there are many types of genctic change in which viruses, like bactcria, react so characteristically to a particular type of change of host or dict that control seems to be indicated. Of this kind is perhaps the wcakening or attenuation of rabies, sccurcd by Pastcur when he inoculated a scries of rabbits with the virus, passing it from onc to anothcr. This weakening varies in rate with the different strains of rabics. There is also the fortification of other viruses by rapid passage through highly susceptible hosts, as described by Holmes and Piric. Similar changes have lately been induced in plant viruses.

Certain recent studies of mutation in viruses and bacteria (which in this respect scem tó be anlologous) give us an opportunity of discovering the means of mutation itself. Two experiments have a parallel significance. Different strains of Pneumococcus differ in the specificity of their antigens, which in this case are polysaccharidcs carried in the capsulc. Avery and others found that if the capsule is lost, the spccificity of a strain A can be transferred to a strain B, by feeding it with dead cclls of $A$, or, cven more precisely, by feeding it with desoxyribose nucleic acid from the capsules of A. We have already scen that this nuclcic acid is necessary for the reproduction of nuclear gencs. We now see that it is neccssary for the specificity of this reproduction. It provides the pattern.

Similar transformations can be produced amongst the viruses causing different kinds of tumour in rabbits. Dcad myxoma virus, in a large excess, can be used to convert living fibroma virus into one producing myxoma of the same strain-type as the dead virus. 
In both bacterium and virus the living is copying the dead pattern, a pattern provided presumably, as in the chromosomes, by a nucleic acid template. But in the bacterium a gap is being filled in the structure, whereas in the virus a part is being taken down and replaced under the pressure of an excess of myxoma. Also the agent in the virus is presumably ribose and not desoxyribose nucleic acid. Thus at this molecular level we can again produce a pseudoLamarckian effect. We can control heredity from the outside and control it this time constructively.

The second aspect of virus genetics concerns the variation of the host. Variegated Abutilon striatum and the green $A$. indicum were both grafted by Baur onto different parts of a plant of a third species, $A$. arboreum. The virus passed from striatum through the arboreum, without seeming to affect it, and entered the indicum, turning it variegated. Thus the virus multiplied in arboreum enough to travel through it, but not enough to injure it. Indeed it behaved as though it were an ordinary cell protein of arboreum. But if $A$. arboreum is replaced by a species of Lavatera the virus cannot get across. Thus different species or varieties of host react in widely different ways with a particular virus, and in the intermediate case they may be "carriers" which show no effect. The difference between carrier and susceptible has been shown to segregate as a simple mendelian difference in the potato. The difference between carrier and noncarrier in the insect vector of Maize-Streak virus is likewise genetically determined. And in the Potato Yellow Dwarf virus there are strains fitted to two different insect vectors. There is thus a triple genetic adaptation of host, virus and vector, a relationship we shall examine later.

\section{Animal Tumours}

The connexions between viruses and other cytoplasmic determinants are revealed by the different modes of origin or causation of animal tumours. These tumours, we must recall, are all abnormalities of development inasmuch as they lead to some degree of return of differentiated or mature cells to an undifferentiated or embryonic condition with rapid mitoses, large nucleoli, and high nucleic acid and protein production. They are also abnormalities of heredity: they arise from genetic changes of particular cells, 
inasmuch as the specific properties of a tumour, fibrous, cpithelial, and so on, can be transmitted from one organism to another through a constant lineage of cells by transplantation.

In its physiological character of excessive growth and unlimited mitosis, the animal tumour is analogous to certain conditions in plants. But propertics of increased and even excessive growth can arise in plants by genetic changes of various kinds. In the higher plants development can be extended by extra mitosis in the pollen grains, through the action of particular "polymitotic" genes or of extra heterochromatic chromosomes. In Sorghum, as we have seen, the nuclei formed by polymitosis kill the pollen grains by mere exhaustion of materials. Here, then, a nuclear change leads to a cancerous condition, doubtless by favouring excessive ribose nucleic acid formation. In the yeast Torula utilis, on the other hand, treatment by camphor produces a permanent genetic change, not affecting the chromosomes, which, according to Thomas, increases the growth rate and doubles the size of the cell. The yeast, then, must undergo an induced plasmagene mutation. In a single-celled yeast or pollen grain, high mitotic rate and high growth rate correspond with tumour formation in many-celled animals, where changes from a normal to the potentially unlimited type of growth can arise, and be detected, only somatically.

In this unlimited growth there is a continuous transition from the encapsulated tumour or the wart, which is limited or merely cut off from further growth by its own effects, at one cxtreme, to the other extreme of the malignant tumour whose cells are sometimes capable of metastasis or migration to new sites. Correspondingly there is a transition in the type of cell division between the rapid mitosis like that of embryonic tissue and the hurricane mitosis like that found in the nucleic-acid-flooded nurse cells of plants and animals, where mitosis is rarely completed in order, and a jumble of haploid and polyploid cells is formed, strewn with fragments and micronuclei.

These graded differences probably do not depend on any genetic distinction in principle. There is another type of difference, however, which matters a great deal genctically: that is the mode of transmission. There are three orders or levels of transmission of tumours. 
The first is that where the tumour-promoting property can be transplanted only in or by whole cells. This condition arises in mammals both spontaneously and artificially. It can be induced by radium, ultra-violet and X-radiation, and also by the action of specific chemical agents. These carcinogens, apart from the sterols, produce their effects on particular tissues. Thus p-dimethylaminoazobenzene (or butter-yellow for short) acts only on the livers of rats or mice, and dibenzanthracene only on the skin of mice. Moreover, some carcinogens act with some strains of mice and not with others. Evidently, therefore, the change is a specific reaction between the carcinogen and some protein or protein-system. And in respect of this reaction cells differ as between tissues and as between races, just as they do with regard to spontaneous tumours. The carcinogen is a mutafacient agent, a highly specific one. The new particles produced in this way must be self-propagating, since the growth they induce is characteristic and unlimited and it persists when the carcinogen is removed. As particles, however, they have not the capacity of invasion. They spread by cell division and they are inherited by cell-lineage: they are transmitted only by transplantation, that is by grafts. Their spreading through the body is due simply to the migration of whole cells. In all these respects the spontaneous tumours of man and other mammals agree with the induced tumours. All of them have obviously arisen in the stocks in which they were found.

The second order of transmission is found chiefly in fowls. Several types of spontaneous tumours in fowls can be passed on by injection of filtrable particles of known properties. Thus the Rous Sarcoma particles are known to be about $70 \mathrm{~m} \mu$ in diameter, and to contain a specific antigen in addition to a normal fowl antigen. These particles have the properties of invasion of cells, although not of infection. They attack only damaged cells and they can be transmitted only artificially. They must, therefore, again have arisen where they were first found.

The third order of transmission is found in a type of breast cancer of the mouse, which is conditioned in its occurrence, as we might expect, by hormonal activity and by race, that is by nuclear genotype. Transmission is by the milk of the mother or foster-mother. It follows the milk line, not, like a plasmagene, the egg-line. Again 
this determinant must have arisen by the creation or mutation of a reproductive particle in the cell. But here we have just (only just) crossed the boundary from heredity to infection: we have a true virus.

Beyond the milk virus there are ordinary infectious principles such as those inducing benign cpidermal tumours or warts. The only difference between them is that it is difficult to imagine the milk virus arising otherwise than from its host's proteins whereas the wart, owing to its infection, might have done so.

In this last group the Shope papilloma of the cottontail rabbit, a known nuclcoprotein, descrves special credit. It serves to bind the whole series together. For on inoculation into the domestic rabbit it loses its capacity for infection and acquires malignancy: it is transmuted from a virus to a plasmagene.

In this series we are dealing almost entircly with modified cell protcins of the animals which develop the tumours. We begin with something not inherited through the cgg (which it would presumably kill) yet as close as possible to a plasmagenc. And we end with something infectious without limit and a true virus. In the middle stage we have something half-way between, something with all the properties of a virus save that of natural infection. We have a provirus.

The genetic conditions of cancer development can now be provisionally defined. Excessive growth, cither ncw growth or continuance of growth, may be dircctly determined by the genotype (nuclcus-cum-cytoplasm) of an individual at fertilization, and arise without any somatic mutation. This is truc particularly of plants. But the typical devclopment of tumour growth in animals is due, and could only be duc, to somatic mutation. This mutation is itself determined by the customary interaction of genotype and environment. Spontancous tumours are so called because the genotype is the predominant variable in determining the mutation; induced tumours because the environment is predominant. A first class of thesc tumours are capable of transplantation only as whole cells. A second class can be transmitted by cell-free filtrates capable of invasion, that is as proviruses. The possibility that nuclear mutation plays a part cannot be cxcluded in the first class. But all may have arisen, and the second class have certainly arisen, by change in self- 
propagating proteins in the cytoplasm. Since the proteins in question occur only in differentiated cells and are not transmitted in heredity, we may regard them as analogous to a substrate-conditioned plasmagene like the melibiozymase of yeast. And in their relation to the cell, their changes are analogous to the mutations of plasmagenes. This is the basis of origin of cancer cxpressed in genetic terms.

An interesting paradox now arises with regard to the treatment of cancer. The cancerous cells owe their condition to a change in their sclf-propagating cytoplasmic proteins. To break them of their vicious habit we can do nothing directly. They are too numerous and are, in any case, chemically self-adjusting. Reversion is unknown. Our sole remedy is to render their nuclei incompetent of supporting cell life. This wc do by breaking the chromosomes in the resting nucleus (whose rapid development makes them singularly susceptible) so that when it comes to divide its wrecked products immediately die. X-rays or radium are the means of brcakage. (Koller, I947.)

The genetic analysis of the origin and transmission of cancer puts us in a position for the first time to sec and understand the triangle plasmagene-cell-protein-virus, or (if you will) heredity-development-infection in its truc perspectivc. Tumour formation indubitably arises by mutation in the first instance, but its propertics are inherently untestable by the techniques of breeding. The distinction between the diffusible and the non-diffusible agent of tumour formation, on the other hand, throws into relief the distinction between the cell-protein which provides the basis of differentiation, and whose diffusion must be prohibited or at least controlled, and that which provides the basis of infection whose diffusion is inherently out of control. Thus cancer research has some fundamental importance: it lies at the meeting of the ways.

\section{Neutral Hereditary Infection}

The conflict between the requirements of heredity, development and infection becomes less as the particle or determinant becomes less decisive in its effect. L'Héritier and Teissier discovered in the cytoplasm of Drosophila the most neutral and indecisive of all particles, and it has proved to have the ambiguity of transmission that might be expected. 
Certain stocks of flies were found to die in the presence of moderate doses of carbon dioxide. This unique susceptibility was a merc biological freak which, in nature, neither helped nor hindered its possessors. The property was inherited largely in the female line. But it could be transmitted to a proportion of the offspring by the sperm of susceptible males fertilizing normal eggs. It was ambilinear although not equilinear. The susceptible offspring, however, could be cured by subjecting them to a temperature of $33^{\circ} \mathrm{C}$. for 24 hours either immediately on laying or during pupation, i.e. during the periods of most rapid cell-division. Thus the $\mathrm{CO}_{2}$-sensitive plasmagene failed to keep pace with the rest of the body proteins at this temperature, while at a normal temperature the reverse was the case: it was suppressive.

So far we see a close analogy with peas and Paramecium. But there is something more. Supernumerary sperm will carry the plasmagene over to the normal egg. And finally, transplanted ovaries, or even injected lymph, of susceptible flies will infect normal ones and the susceptibility will show in a part of their progeny. In other words the determinant is a provirus as well as a plasmagene. It must have arisen by mutation in the ancestors of the susceptible stock and its properties of infection and diffusion indicate that it is of no more consequence in differentiation than it is in heredity.

Neutrality again enables us to find an overlapping of the properties of heredity and infection in the case of the piebald guinea pig, a fancier's mutant. The black pigment is produced both in the hairs and in the skin of the coloured areas. Pieces of coloured skin grafted in white areas lead to pigmentation of the adjoining unpigmented cells and these will, in turn, infect more white cells after a second transplantation. Thus, as Billingham and Medawar have pointed out, there are cytoplasmic determinants, expressing hereditary characters and capable of indefinite self-propagation, which nevertheless are capable of diffusion, invasion or infection. It seems likely that in animals, at least, invasions of this kind, but of limited scope, are concerned in processes of normal differentiation.

\section{The Origin of Viruses}

In both animals and plants there are a number of diseases which have not arisen by infection although they can certainly be propa- 
gated afterwards by infection or by inoculation. Such diseases as virus III in rabbits and perhaps Herpes zoster in man, referred to by Holmes and Pirie, may appear spontaneously. Sometimes a nutritional defect or a bacterial infection seems to favour their appearance. In these cases the virus probably results from a distortion of protein metabolism in the individual in which it first appears. The new proteins may arisc from, or instead of, normal self-propagating cell proteins which need not be transmissible as such in heredity and need not, therefore, be called plasmagenes. But their replacement is most easily described as mutation since the result produced is indistinguishable from plasmagene mutation in a plant.

One step further on from these intrinsic viruses are those which are normal proteins in the individual of their origin, but become unfriendly in an alien cell. Such is, no doubt, the virus producing ascending myelitis in man after a bite by a healthy monkey, a monkey which can scarcely have been bitten by a man suffering from this disease. Here we have the origin of a virus by transplantation as opposed to mutation. Its fullest verification is in plants.

Plant viruses have, like tumour viruses, several orders of transmission. A few may be conveyed by contact of stems or roots: the important natural ones are carried by parasitic insects. There are others, however, like those causing the yellowing of Abutilon, privet, and laburnum, which can be passed on only by grafting. As diseases, therefore, they are again artificial, and their origin must be duc, either to a change in a cell protein, or more probably to the grafting itself, that is either to mutation or to invasion. They are not naturally infecting viruses, but proviruses. Their origin has been shown, in various groups, by grafting different varieties and species. Indced, though the possibility of the infection of scion by stock as opposed to the incompatibility of the two has long been overlooked, it was first pointed out by Patrick Blair in 1720 .

The types of abnormality resulting from the graft-creation of viruses are manifold.

Some instances of graft incompatibility are, it seems, due to the scion which produces substances poisonous to the stock, the effect being restricted even to particular localities as in Quick Decline or Tristeza of sweet oranges on sour stock, or Graft Blight of Lilac on privet stock. 
Other instances are due to the rclease and invasion of self-propagating particles. All plants of Lathyrus tingitanus, in the experiments of Johnson, produced infective symptoms when their sap was injected into the bean Phaseolus v'ulgaris. All plants of the potato clone King Edward, in experiments of Salaman and LePellcy, produced infective symptoms when grafted onto other clones. Similarly the varicty of apple, Lord Lambourne, when grafted on different stocks, according to Crane, develops different characteristic malformations, rubbery wood and stunted chat fruit, which spread through the whole tree and, like the Abutilon virus, will pass through wood of another variety.

The mode and degrec of infection varics in these various plants. The principle, however, is the same in all. Just as reciprocal crosses cnable us to test the reaction of a plasmagene in the cytoplasm of one species with the nucleus of another, so grafting cnables us to test the reaction of something reproductive in the cytoplasm of one species with the nuclcus of another, indeed of many others and over a range of many genera. A protein produced in the cclls of one organism can propagate itsclf injuriously in the cells of others. Invasion, a characteristic of true viruses, is a ncccssary means of demonstration of these agents. In the animal tumours, on the other hand, the principle is slightly different. A protein produced in one organism mutatcs so as to propagate itsclf injuriously in the same organism. Here invasion is no longer necessary for demonstration and docs not always occur.

We are bound to make the distinction between provirus and plasmagene on the basis of capacity for invasion following grafting. Yet this critcrion is, of course, a purcly practical one arising from diverse conditions. In some instances the difference between invasion and non-invasion may be simply one of the size of particles. In other instances the difference may be simply onc between presence and absence, as it sometimes is with nuclear gencs.

The case of Yellows in ever-bearing strawberrics demonstrates the great theoretical and practical importance-and difficulty-of the question of origin and transmission in making the distinction between plasmagenes and viruses. Some 30 clonal varieties of these strawberries are subject to this disease and many have been destroyed by it. The varicty Progressive, made in 1908, first showed symp- 
toms of Yellows in 1925. Other varieties have become affected as soon as three years, or as late as fifty years, after introduction. In symptoms of variegation it resembles those supposed virus diseases which lead to chlorophyll breakdown in other forms of Fragaria and Rubus. But it is not transmissible by grafts; and it is transmissible by seeds.

In inheritance Yellows resembles the rogues in peas. Thus 82 selfed seedlings from 5 healthy plants were all healthy at 27 months.

TABLE 19

THE THREE ORDERS OF TRANSMISSION OF PROTEINS AND THE TWO MODES OF ORIGIN OF VIRUSES

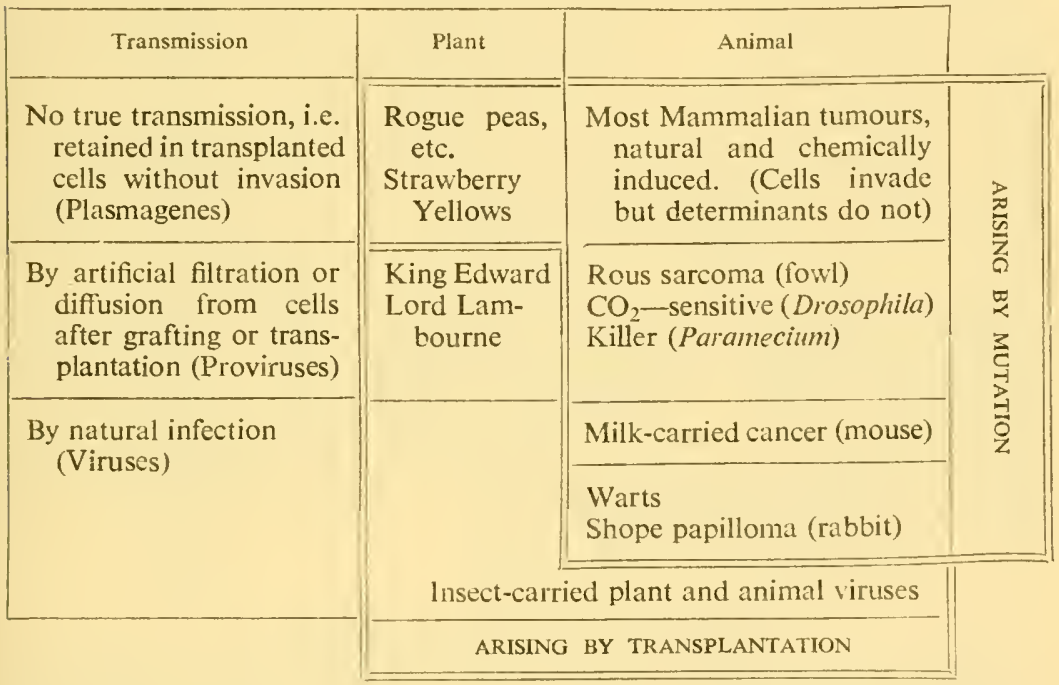

But of $4 \mathrm{I} 2$ seedlings of affected plants, 2I per cent showed symptoms at 4 months and 82 per cent at 27 months (Morris and Afanasiev). It seems that yellowed plants undergo an irreversible cytoplasmic change. Their chance of doing so must depend on the nuclear genotype, for some seedlings are more quickly affected than others. The character of their progeny also depends on whether the cytoplasm of their germ cells is healthy or diseased. Finally, external conditions, although none have been proved to influence the result, must account for the fact that some members of a clone succumb carlier than others. At a first glance we should say that the Yellows 
was a nucleus-controlled plasmagene mutation because it is not transmitted by grafting. Now, if it were an innovation or a presence, it might invade and be classifiable with the Abutilon principle as a provirus. But what if it is due to an absence, a deficiency in a necessary self-propagating protein which propagates itself too slowly? A deficiency could not well invade. Our classification for the time being must therefore be one of convenience.

And our difficulty is not so much to show the relationship of plasmagene and virus as to make a distinction between them of any validity beyond the requirements of their adaptation to heredity and infection (Table I9).

Taken together the two series of observations and experiments, animal and plant, show that all the general properties of the natural and noxious virus have arisen under experimental conditions. The special property of insect-carriage alone has not been demonstrated. Nor will it be readily demonstrated until the number of experimenters is comparable with the number of insects. We may say, therefore, that the properties of the reproductive particle, which are combined naturally in the plasmagene with heredity, and in the virus with infection, may, in these artificial conditions, be seen stripped of such accessories. Conditions produced by the $\mathrm{CO}_{2}$ test in Drosophila, by carcinogens in vertebrates, and by grafting and breeding in plants, demonstrate the two modes of origin of the natural virus: by mutation and by transplantation.

\section{Nuclear and Cytoplasmic Systems}

The close relationship between plasmagene and virus enables us to put the virus in its proper place in the scheme of things. But it does much more. It enables us to put the different agents of heredity, development and infection in their proper relationship. They all depend on self-propagating particles. These particles have different kinds of control over one another according to the systems in which they are organized and the methods by which they are propagated. These seem to be essentially of two kinds, the nuclear and the cytoplasmic, distinguished from one another in their intrinsic properties and separated from one another by a protective boundary. 
The nuclear system depends on the protein fibres of its chromosomes for its mechanical permanence and on desoxyribose nucleic acid for its propagation. It is protected from outside influences by the nuclear membrane in the resting stage, and, during mitosis, it is locked up by its spiralization and by its nucleic acid charge. It consists of linear arrangements of genes, which are of two kinds,

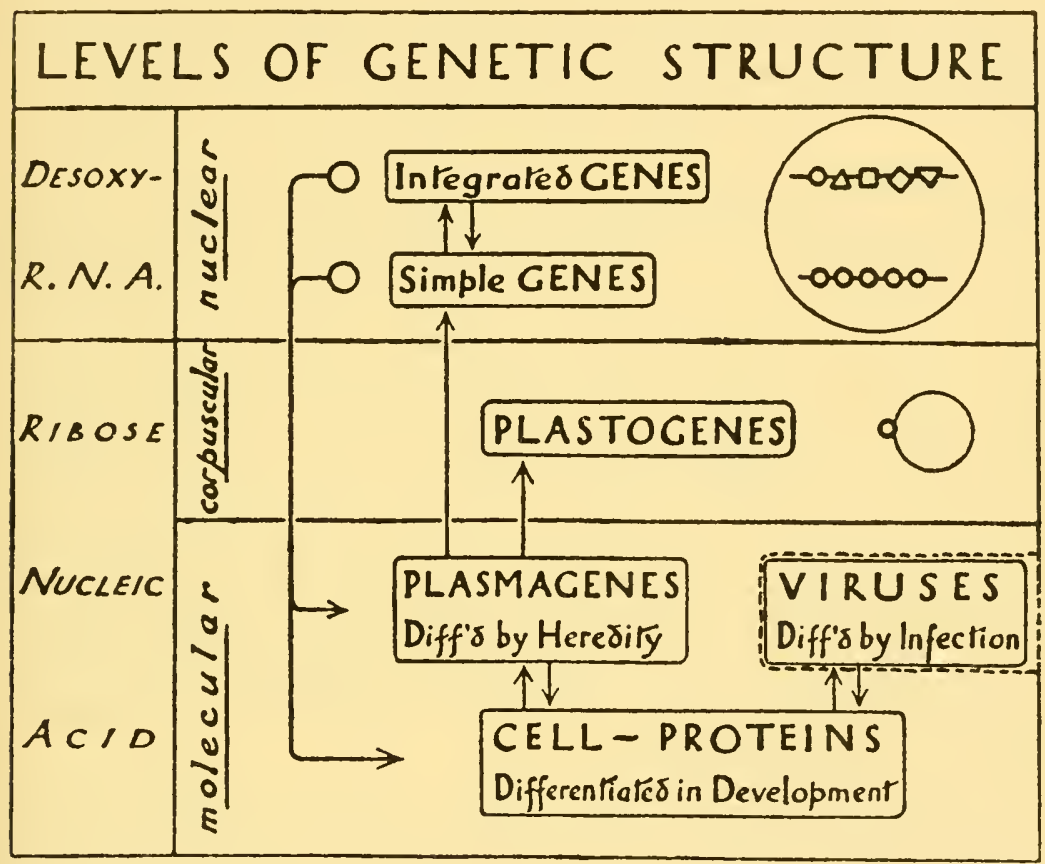

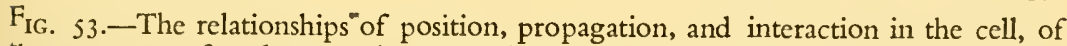
the two types of nucleo-protein responsible for heredity, development and infection. The downward arrows (except within the nucleus) are physiological, the upward evolutionary (after Darlington, 1944).

or at least lie between two extremes represented by heterochromatin and euchromatin in the cell, and by polygenes and major genes in heredity. The former are the small determinants with the simple products; the latter are the large determinants with the complex products. Between the two there are doubtless intermediates, transitional forms which are also transitory and, therefore, elusive. It is natural to suppose that the more complex is built up from the more simple, by integration of different simples (Fig. 53). Later we shall 
come across evidence of this integration on a larger scalc, at a higher level, when we come to the growth of genes in evolution.

The cytoplasmic system depends for its propagation largely (and originally) on ribose nucleic acid, which, not having the indefinite power of polymerization of its nuclear congener, cannot organize a differentiated fibrous structure such as a chromosome. The cytoplasmic units are, thereforc, limited in organization and size, corresponding in range to genes rather than to chromosomes. They depend, moreover, on chemical equilibrium, co-operation and competition, for the adjustment of their proportions and distribution. The larger and scarcer ones, or the oncs associated with larger and scarcer corpuscles like plastids, can therefore be accidentally sorted out. Again, the larger ones may be derived from smaller ones; and both, in the course of development, may be derived as cell-products from the nucleus; and both may in the end be lost. In these changes the cytoplasmic system is subject to the nucleus. It is also, to a greater extent than the nuclcus, exposed to the environment and, of course, to the effects of differentiation. Lastly, for their permanent transmission, cytoplasnic elements have another channel open to them than heredity: they can spread by infection. By this piracy they separate themselves, in adaptation and evolution, from the organism of which they form a part. And the same separation occurs when by mutation they come to multiply in such a way as to injurc or kill their own mother-body.

Comparing the two systems, we see why the cytoplasmic system has decayed as a mechanism of heredity step by step as development and differentiation grew stronger. Already in the higher plants, in the rogue, there is a conflict between the requirements of heredity and differentiation. And in the higher animals heredity has been reduced to completc, or almost complete, nuclear control. In this process the efficiency of the fibrous organization of chromosomes in the nucleus as a basis of heredity and of recombination has given it a long-term advantage over the cytoplasm. Only in the bacteria do we perhaps see an organization of life balanced between the cytoplasmic and the nuclear levels.

Within the individual there are thus systems of different kinds at work in determining heredity, development and infection. In their foundations, as self-propagating proteins in the cytoplasm, they are 
indistinguishable. But in their work at different levels of integration, each has its own rules of behaviour, and each is related to the others by yet other rules of conflict and co-operation. With all these the genetics of individuals is deeply concerned.

\section{REFERENCES}

AVERY, O. T. et. al. 1944. Studies on the chemical nature of the substance inducing transformation in Pneumococcus types. J. Exp. Med., 79: 137-I50.

BAUR, E. 1906. Weitere Mitteilungen über die infectiöse Chlorose der Malvaceen und über einige analoge Erscheinungen bei Ligustrmm und Laburnum. Ber. Deut. Bot. Gesel., 24: 416-428.

BAwDEN, F. C. 1943. Plant Viruses and Virus Diseases. 2nd ed. Waltham, Mass. BILlingham, R. E., and MEDAWAR, P. B. 1948. Pigment spread and cell heredity in the guinea pig's skin. Heredity, 2: I-20.

BURNET, F. M. 1946. Virns as Organism. Cambridge, Mass.

CLAUDE, A. 1943. The constitution of protoplasm. Science, 97: 45I-456.

CRANE, M. B. I945. Origin of virus. Nature, ISS: i I 5 .

DARLINGTON, C. D. 1944. Heredity, development and infection. Nature, I54: 164I68.

DARLINGTON, C. D. I949. The plasmagene theory of the origin of cancer. Brit. $J$. Cancer (in the press).

DUBUY, H. G., and wOODS, M. W. 1943. Evidence for the evolution of phytopathogenic viruses from mitochondria and their derivatives. II Chemical evidence. Pliytopatliology, 33: 766-777.

HADDOW, A. 1944. Transformation of cells and viruses. Nature, I54: I94-I99.

HADDOW, A., KON, G. A. R. et. al. I947. Chemical carcinogens. Br. Med. Bull., 4:309-426.

HESTON, W. E., DERINGER, M. K., and ANDERVOUT, H. B. I945. Gene-milk agent relationship in mammary tumour development. J. Nat. Cancer Inst., 5: 289-307. HOLMES, B., and PIRIE, A. I937. Biochemistry and the pathogenic viruses. Perspectives in Biochemistry. Cambridge.

Johnson, J. 1942. Studies on the viroplasm hypothesis. J. Agr. Res., 64: 443-454. KIDD, J. G. 1946. Distinctive constituents of tumors and their relation to autonomy, anaplasia and causation. C.S.H. Symp. Quant. Biol., II: 94-IIO.

KOLLer, P. C. 1947. Abnormal mitosis in tumours. Brit. J. Cancer, I: 38-47. l'Héritier, P., I948. $\mathrm{CO}_{2}$ sensitivity in Drusoplila. Heredity, 2: 300.

LURIA, S. E. I945. Mutations of bacterial viruses affecting their host range.

Genetics, 30: 84-99.

MORRIS, H. E., and AFANASIEV, M. M. 1944. Yellows, a 12on-infectious disease of the progressive everbearing strawberry in Montana. Muntana State Coll. Bulletin, 424 .

RISCHKOV, V. L. 1943. The nature of ultra-viruses and their biological activity. Plytopatl., 33: 950-955. 
VIRUSES, PROVIRUSES AND THE CONFLICT OF SYSTEMS

ROUS, p. 1936. Virus tumors and the tumor problem. Am. J. Cancer, 28: 233-272. SMITH, K. M. I945. Transmission by insects of a plant virus complex. Nature, I55: 174 .

тномаS, р. т. 1945. Experimental imitation of tumour conditions. Nafure, I $56: 738-740$. 
PART III

POPULATIONS 



\section{ADJUSTMENT AND BALANCE}

Levels of Adjustment-Haldane's Rule-Gernotypic and Segregational

Sterility-Inbreeding Depression and the Hybridity Optimum

\section{Levels of Adjustment}

Heredity, AS We have NOW COME To SEe IT, depends on an organization of chromosomes, genes and cytoplasmic determinants which are adjusted to one another in the development and reproduction of each individual. We have recognized this adjustment at three levels; the organismal adjustment of cells with one another; the cellular adjustment of the parts of single cells, in particular of nucleus and cytoplasm; and the nuclear adjustment, or balance as we have called it, of the genes within the nucleus. There is, of course, a further adjustment of the whole system with the environment.

Each level of adjustment has its own rules. The cells which work together as parts of the same body or soma normally have identical nuclear genotypes. This relation may, as we saw, be altered by gene or chromosome mutation in the body, with consequences ranging from a mere mosaicism of colour to a mixing of tissues normally confined to distinct individuals, as in gynandromorphs. The mixture of unlike cells may also lead to intersexuality and sterility.

The disparity of genotypes that can be associated in one soma has been investigated by grafting experiments. In mammals and birds, such grafts are successful only when the genotypes are very closely related. Otherwise antigenic differences come into play and the transplant is sloughed off. In other animals the tissues seem to be more nearly autonomous in their behaviour, for transplantation between genetically dissimilar individuals is more widely successful and in the insects, as we have seen, it has even led us to a clearer understanding of how genes act.

In plants grafting can be accomplished between still more widely separated forms, even between genera, to give more or less stable graft-hybrids. We can put the aerial parts of one species onto the 
roots of another. Or we can put a skin of one round a core of the other, as in Crataegomespilus, the graft hybrid of the hawthorn and the medlar. In this way, too, as we saw, the hexaploid and diploid species of Solanmm may be combined to give results whose appearance depends on which is inside and which is outside, and on how many layers of each there are.

These chimaeras are not always fertile, nor are they always stable. An irregularity at the meristem in Cytisus adami frequently exposes the yellow flowers of its Laburnum core; and less often the purple flowers of the Cytisus purpureus skin succed in displacing the mongrel colour. The chimaeras produced by somatic mutation may also be of these various kinds, and they show the same characteristic instabilities. Indeed many ornamental plants surprise the gardener when they reveal their chimerical condition, and its origin by mutation of gene or plastogene, in this way.

The normal adjustment of cell parts, of nucleus with cytoplasm, is not constant like that of cell with cell. On the contrary, it is regularly changing as the cytoplasm changes; and, as we have seen, $i$ is on these changes that the processes of devclopment and differentiation depend. Nevertheless, the changes must follow a fixed path if the outcome of differentiation is to be an organism that will work, and this is secured by the subordination of the changing cytoplasm to the constant nuclear genotype. If we put a nucleus into a cytoplasm which is not of its accustomed type, one of the two has to give way or the disharmony will lead to a failure of the normal sequence of changes and so to early death, as we saw in the various merogons with the cytoplasm of one species and the nucleus of another. When one gives way it always has to be the cytoplasm: the nucleus forces the cytoplasm into its own pattern, as in the Acetabularia grafts.

Disharmony of nucleus and cytoplasm can be produced by hybridization as well as by grafting. When made one way, a species cross in Epilobium or Streptocarpus may be successful in giving a hybrid capable of adequate development. Yet when made the other way the hybrid may be abnormal in greater or less degree. The hybrid nucleus can work with cytoplasm, or more precisely with the plasmagenes, from one parent, but not with those from the other. Such disharmony is not, however, the usual cause of abnor- 
mality, or failure in some vital function, in wide hybrids. Much more commonly these are to be traced to disharmony at the third and most fundamental level, that of the genes within the nucleus. The father's chromosomes and the mother's cannot work together properly in the hybrid.

\section{Haldane's Rule}

Disharmony, or unbalance of the genes, arising from hybridization affects every nucleus of the soma, from that of the fertilized egg onwards. It may therefore express itself in any stage of develop-

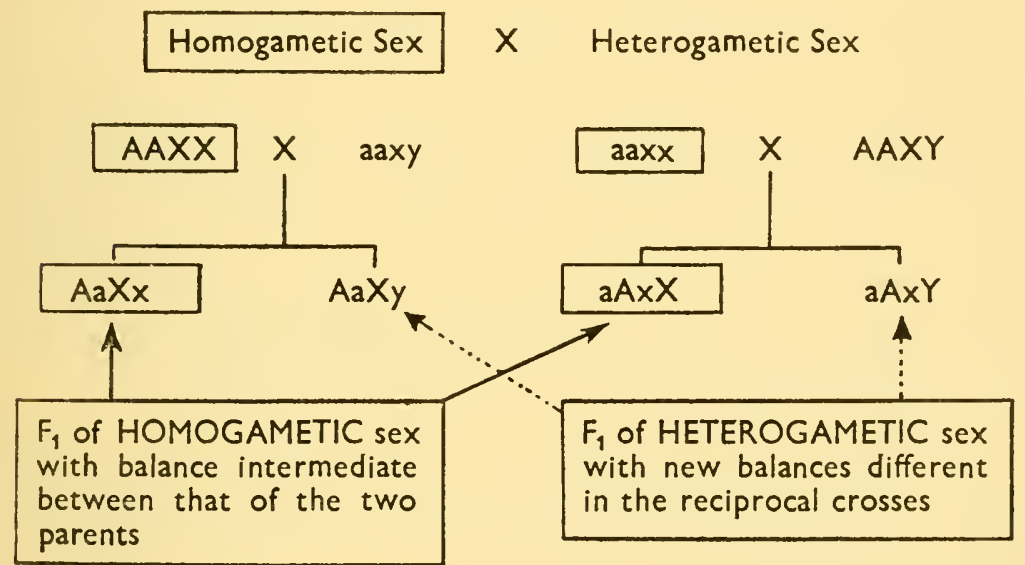

\section{FEMALE . . XX and MALE .. XY in Mammals and Flies MALE . . . XX and FEMALE .. XY in Moths and Birds}

Fig. 54-Diagram to show the relation of balance and fertility in reciprocal crosses between species in animals. Chromosomes of opposite species shown in large and small type.

ment, by untimely death or by loss of vigour; or in reproduction, by sterility of greater or less degree. The genic balance should be least disturbed where the contributions of father and mother are each complete and balanced, as in the case of a first cross between two races or species. And the greater the departure from this balanced 
completeness of the parental contributions, the greater should be the loss of vigour and fertility in the hybrid.

One consequence of this rule is to be seen in crosses between sexually differentiated species. In such cases the sexes very commonly differ in their vigour and fertility; and, as Haldane pointed out, it is the XY or heterogametic sex which is less vigorous and less fertile and even less frequent. The difference may be so extreme that only one sex survives to maturity, as in the moth crosses Chacrocampa elpenor $\times$ Metopsilus porcellus and Deileiphila cuphorbiae $\times D$.galli. Here Federley has found that although the males survive the heterogametic females die as pupae. Or the difference may not appear until germ cell formation, as in Drosophila pseudoobscura $\times D$. persimilis, where the females are fertile but the males, otherwise normal, are made sterile by a wholly irregular process of meiosis.

Why should disharmony affect the heterogametic sex more strongly? In the homogametic sex both autosomes and sex chromosomes are derived equally from the two parents. In the heterogametic sex they are derived unequally; indeed the sex chromosome contribution may come entirely from one parent. The sex-autosome balance can be neither that of one parent, nor intermediate between those of the two. It is much more likely, therefore, to be out of gear in the heterogametic than in the homogametic sex (Fig. 54).

The difference between the two is thus an expression of the same kind of balance between sex chromosomes and autosomes as that which is responsible for a Drosophila with two $\mathrm{X}$ chromosomes growing into a female if diploid, but into an intersex if triploid, for the autosomes (Fig. 55).

Now comparison shows that the composition of the sex chromosomes varies between species. One arm in the $\mathrm{X}$ chromosome of both $D$. pseudoobscura and D. persimilis, for example, corresponds to an arm of an autosome in $D$. melanogaster: there has been a translocation in the history of the species. Smaller structural changes and genic changes will be even more characteristic of the relations between the sex chromosomes of different species. The balance of sex chromosomes and autosomes must therefore often differ between species, and we might expect to find that reciprocal species crosses give similar results in regard to the homogametic sex, but differ in their heterogametic offspring (Fig. 54). This is in fact truc. In 
Federley's moths, the crosses reciprocal to those mentioned gave offspring of both sexes that survived to maturity. The males from the Drosephila cross were sterile, no matter which way it was made. But the testes, which were of normal size from psendoobscura $\times$ persimilis, were abnormally small in the reciprocal progeny.

\begin{tabular}{|c|c|c|c|}
\hline & $x$ & $x \times$ & $x \times X$ \\
\hline$A$ & $o$ & - & - \\
\hline AA & $\delta$ & $q$ & $i$ \\
\hline AAA & $\delta$ & $\phi$ & + \\
\hline
\end{tabular}

FIG. 55.-Diagram showing the relation of the X/Autosome balance to the sexual character in Drosophila. $\delta=$ supermale; $Q=$ superfemale; $\phi=$ intersex (all sterile).

Note.-(I) $\mathrm{Y}$ is without effect on sex-grading.

(2) Haploid is known as ovarian tissue.

(3) The homogametic balance can be produced in three ways, the heterogametic in only one.

\section{Genotypic and Segregational Sterility}

Sterility is the only outcome of hybridity in these male flies. Nevertheless it is to be traced to the same kind of disharmony that causes carly death of the female moths. It is a direct result of unbalance in the zygote's genotype, and we may therefore refer to it as genotypic sterility. The sterility of the diploid Raphano-brassica and of the diploid Primula kewensis might appear at first sight to be due to the same cause, but one significant observation shows that this cannot be so. Both of these plant hybrids recover fertility when the number of their chromosomes is doubled; whereas tetraploidy leads to no such recovery in the malc flies. Now tetraploidy does not cause any essential alteration of genic balance: it cannot therefore remedy genotypic sterility. It does, however, suppress segregation, and so it would appear that, in contrast to the 
sexually differentiated animals, the plants show segregational sterility.

In Raphano-brassica, pairing of the chromosomes is almost absent from meiosis, so that gametes may receive any number, as well as

TABLE 20

AVERAGE FREQUENCIES OF CHIASMATA IN THE BIVALENT CHROMOSOMES IN RELATION TO THE FAILURE OF METAPHASE PAIRING AND THE POLLEN FERTILITY OF DIPLOID $(x=7)$ GRASS SPECIES AND THEIR DIPLOID HYBRIDS (PETO, 1933)

\begin{tabular}{|c|c|c|c|c|}
\hline \multirow[t]{2}{*}{ Plants } & \multicolumn{2}{|c|}{ Cross I $(L . p . \uparrow)$} & \multicolumn{2}{|c|}{ Cross $11(F . p . q)$} \\
\hline & Xta.p.biv. & Good pollen $\%$ & Xta.p.biv. & Good pollen \% \\
\hline Lolium perenne & $1 \cdot 81$ & 92 & $1 \cdot 59$ & $25^{*}$ \\
\hline Festuca pratensis . & $1 \cdot 89$ & 81 & $1 \cdot 59$ & 87 \\
\hline$F_{1}$ (all male-sterile) . & $1 \cdot 71$ & 13 & $\begin{array}{l}1.62 \dagger \\
1.62(2 \%) \\
1.61(2 \%) \\
1.57\end{array}$ & $\begin{array}{l}0 \\
0 \\
0 \\
0\end{array}$ \\
\hline Backcross to L.p. ${ }^{\hat{0}}$. & $\begin{array}{l}1 \cdot 80 \\
1.66 \\
1.66 \\
1.66 \\
1 \cdot 57 \\
0.80(35 \%) \\
0.62(48 \%)\end{array}$ & $\begin{array}{r}85 \\
92 \\
90 \\
77 \\
84 \\
40 \\
0\end{array}$ & $\begin{array}{l}1 \cdot 76 \\
1 \cdot 50\end{array}$ & $\begin{array}{l}74 \\
61\end{array}$ \\
\hline Backcross to F.p. $\hat{o}$. & & & $\begin{array}{l}1.43 \\
0.88(33 \%)\end{array}$ & $\begin{array}{l}37 \\
23\end{array}$ \\
\hline
\end{tabular}

Note: $\mathrm{Xta}=$ chiasmata. The percentage figures are univalents where these occur.

* The Lolium parent in this cross was itself a varietal cross, hence the low pollen fertility.

$\dagger$ Used for backcrossing.

any combination, of chromosomes. In Primula kewensis pairing is sufficiently regular for the great majority of the gametes to receive the customary 9 chromosomes, one from each pair, but these 9 may represent any combination of the maternal and paternal sets. Evidently the property of harmonious working, which is shown by the complete paternal and maternal sets, is not shared by their individual parts. Owing to structural changes in their ancestry these 
parts are differently distributed in the two species. So segregation leads to unbalance, either numerical or genic, in the gametes of these hybrids, just as it does in those of triploids and interchange hybrids. The unbalanced gametes cannot survive, and sterility is the resultuntil segregation is suppressed by tetraploidy.

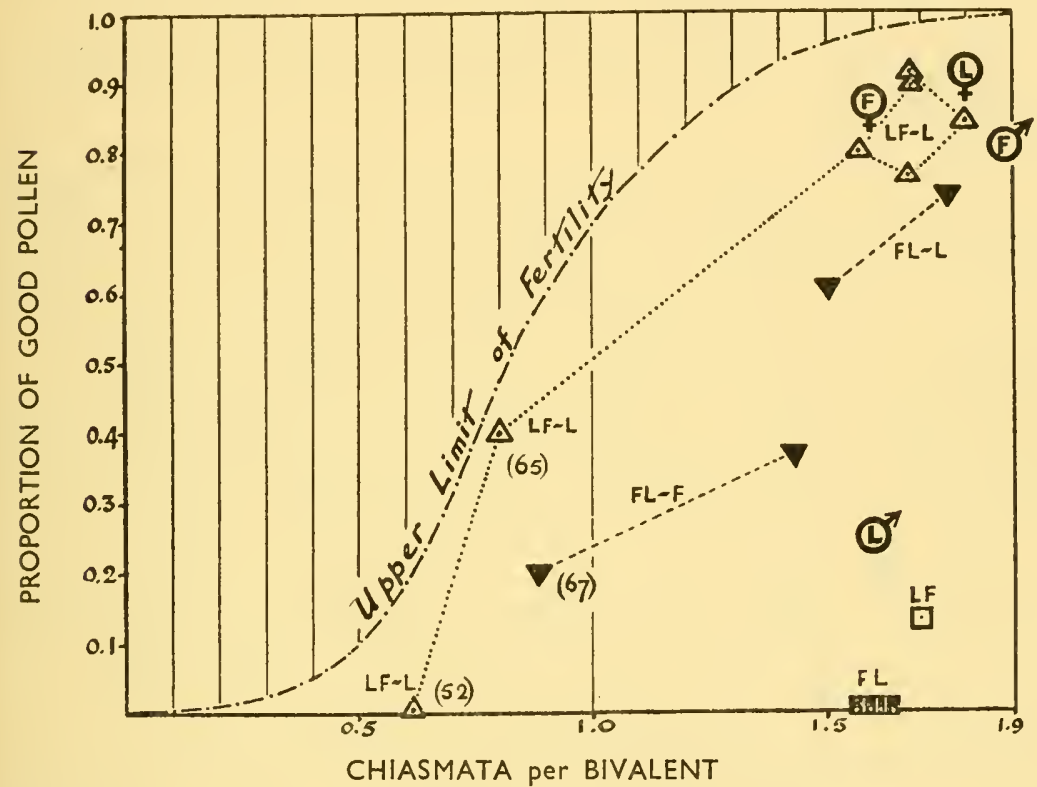

Frc. 56.-Relationship of chiasma frequency, chromosome pairing and pollen fertility in Lolium-Festuca hybrids. The graph shows that: (i) The upper limit is set by chiasmata to pairing and by pairing to fertility. (ii) The $F_{1}$ is male-sterile probably owing to a defective reaction of the hybrid nucleus with both parental cytoplasms, in respect Festuca is regularly worse than Lolium. (iii) The backcrosses show segregation controlling chiasma frequency and hence fertility. Each point represents an individual: circles for plants used as male and female parents; F, Festuca pratensis; $\mathrm{L}$, Lolitm pereme; squares for $\mathrm{F}_{1}$; triangles for backcrosses; numbers in brackets for percentage of potential bivalents formed where first metaphase pairing is incomplete (based on Peto, 1933).

The distinction between segregational and genotypic sterility is thus seen to lie in the time of taking effect. Genotypic sterility is due to relative unbalance of the contributions of the two parents as wholes, which gives absolute unbalance of the diploid $F_{1}$ hybrid. This is especially noticeable when sex chromosomes are concerned, for in them the $F_{1}$ has something of the nature of a backcross. 
Segregational sterility, on the other hand, results where the contributions of the two parents are relatively balanced as wholes, but balanced in different ways, so that segregation and recombination of their parts give unbalance. This unbalance expresses itsclf genotypically in plants in the haploid generation, in the gametes that will give the $\mathrm{F}_{2}$ or backcross; but in animals where, as we have secn, unbalanced gametes regularly survive and function, segregational sterility appears only as the death of the zygotes of the $F_{2}$ or backcross.

When the unbalance due to segregation is not so drastic as to lead to death, it may still cause a developmental upset in the individuals obtained in a backcross or $\mathrm{F}_{2}$. This upset may, of course, express itself at germ cell formation so that it leads to a reduction in fertility. In this way segregation in the $F_{1}$ may lead to genotypic sterility in the next generation (Fig. 77). These relationships are well shown by the comparison of the $F_{1}$ and the backcross generations in the cross between the two grasses Lolium perenne and Festuca pratensis examined by Peto (Table 20 and Fig. 56).

Reciprocal crosses were made. The parents were different individuals with slightly different chiasma frequencics but they were all highly fertile except onc, which was itself a varietal hybrid and doubtless owed its bad pollen to segregation. Both of the reciprocal $\mathrm{F}_{1}$ 's had chiasma frequencies similar to the parents, and showed no evidence of structural hybridity, apart from 2 per cent of potential bivalents unpaired in two plants. There was, however, a great reduction in pollen fertility, again presumably due to segregation.

The results of this segregation appeared clearly, though in a different way, in the next gencration, which was the first backcross to Lolium perenne. Here 5 plants had normal chiasma frequencies, no failure of pairing and high fertility; but 2 plants had chiasma frequencics greatly reduced, in fact below the level necessary for regular pairing. Their fertility was therefore greatly reduced. But this reduction in fertility, in contrast to that of $F_{1}$, was presumably due in a great part to the failure of pairing. Now the dissimilarity of the pairing chromosomes must have been at its greatest in $F_{1}$. The decrease of chiasmata and pairing cannot therefore itsclf have been due to an increase in dissimilarity. Rather the failure of pairing, and with it the sterility, must have arisen from a genic upset 
following segregation in the gametes of the $F_{1}$ hybrids. In other words the sterility of the backeross is largely genotypic, but it is a consequence of the segregation which caused an even greater sterility in $F_{1}$. It is also, we may notice, superimposed on a small amount of segregational sterility in the backcross plants themsclves.

A single mendelian difference, which might be supergenic and distributed over most of one chromosome, would account for the segregation scen in this backcross of the $\mathrm{F}_{1}$ from the Lolium mother. In the second cross, where Festuca was used as the mother of the $\mathrm{F}_{1}$, segregation also appears, but it is not so clear as in the first. Probably a larger number of differences are being recombined. For this reason, however, the corrclation between chiasma and univalent frequency and pollen fertility are all the more clearly manifested (Fig. 56).

There is also a small overall difference between the two reciprocal crosses and their progenies. The $\mathrm{F}_{1}$ from the Lolium mother has a little good pollen, while that from the Festuca mother has none. In the same way the progeny whose chromosomes show full pairing arc more pollen-fertile in the backcross to Lolitm of the $F_{1}$ with Lolium cytoplasm than in the backcross of the $F_{1}$ with Festuca cytoplasm. This difference may well be due to failure of the other type of adjustment, that between nucleus and cytoplasm. If so, however, it merely serves to emphasize the point that disharmonies at the cellular level generally cause less disturbance than do disharmonies within the nucleus, genic disharmonics such as are revealed by segregation in both these backcrosses.

\section{Inbreeding Depression and the Hybridity Optimum}

Hybrid sterility may thus be duc, either immediately to genotypic unbalance of the hybrid, or less immediately to the inability of such wide hybrids to avoid the segregation of unbalanced gametes. Such segregation must, of course, be peculiar to hybrids. But genotypic sterility, and equally loss of vigour, is not the property of hybrid nuclei alone. If we inbreed maize by self-fertilization, the result is invariably a loss of vigour, or of fertility, or of both, in succecding gencrations of the inbred lincs. This inbreeding depression, as it is called, is rapid at first but soon begins to slow down (Fig. 57). After five or six generations no great increase of the depression is 
to be observed, no matter how long the inbreeding may be continued: something like the maximum depression has been reached.

At the same time that vigour and fertility are decreasing, so is the variation between the plants of any line in each generation. This decrease also virtually ceases after the first five or six generations. Evidently the increasing depression is due to increasing homo-

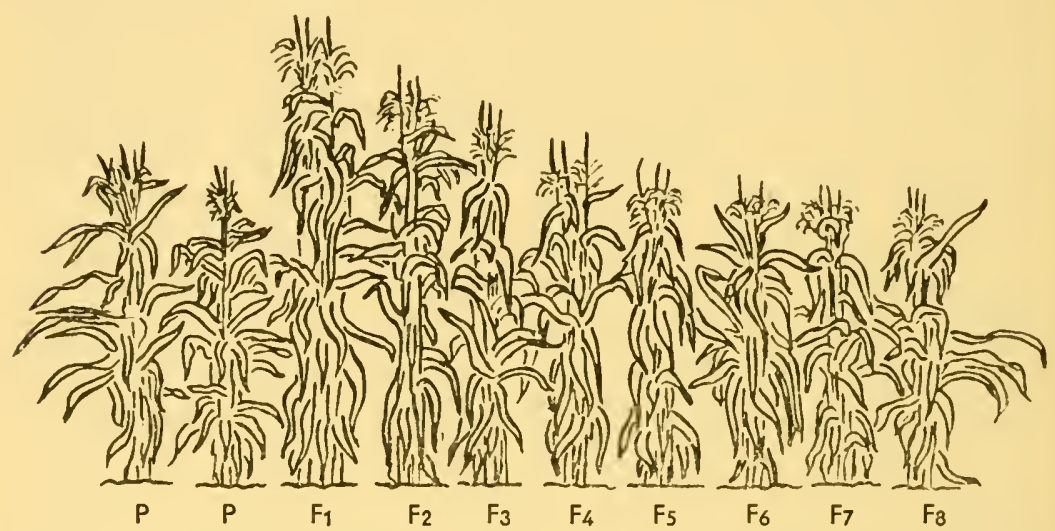

Fig. 57.-Characteristic plants to show the relative heights of two inbred lines (P) of maize, of the $F_{1}$ obtained by crossing them, and of the subsequent generations up to $\mathrm{F}_{8}$ obtained by continued selfing. The maximum hybridity, following crossing, is associated with maximum height, and the reduction in hybridity by selfing is paralleled by a reduction in height. In $F_{7}$ and $F_{8}$, when homozygosis in almost complete, height is reduced once more to the minimum. The increase with crossing is termed heterosis, and the decrease is termed inbreeding depression (based on Jones, in Sinnot and Dumn, I939).

zygosity and to decreasing heterozygosity. The heritable variation will then decrease until full, or nearly full, homozygosity is reached and only non-heritable variation remains. Other things being equal, an average of only I part in 32 of the heterozygosity will remain after five generations of selfing, and only I in $\sigma_{4}$ after six generations.

As we should expect, the vigour of the original parents is restored, even exceeded, by crossing inbred lines derived from different varietics, or, if from the same varicty, scparated carly in the inbreeding programme (Fig. 57). Though each line is homozygous, or nearly so, different lines are homozygous for different allelomorphs of many genes, with consequent heterozygosity in the $F_{1}$ 's 
between them. By the same token, crossing plants within the same inbred line has no effect in restoring vigour. The vigour is hybrid vigour, or heterosis as it is sometimes called.

In maize, therefore, it is possible to show that full vigour and fertility are the result of a hybrid condition: loss of hybridity leads to loss of vigour and fertility. The same principle applies in rye and sugar beet, in pigs and flies, indeed, as we shall see later, in nearly all cross-fertilizing species. In this way we are led to see that both undue hybridity, that is unaccustomed hybridity, and unaccustomed lack of it, may lead to genotypic unbalance. Hybridity is dangerous only where its kind or its degree is unaccustomed. Each species has, it seems, an optimum degree of hybridity to which it is accustomed. Any departure from this optimum, no matter in which direction, is followed by unbalance.

Now, with rare exceptions, species can maintain hybridity only by the crossing of different individuals in every generation. Equally, of course, excessively wide crossing will lead to undue hybridity. Thus the adjustment of the genes within the nucleus, the ultimate one of the three adjustments we have been led to recognize, itself demands a fourth, and one not between parts of the cell or even of cell with cell, but of individual with individual. There must be a relationship between the hereditary materials, and their behaviour, throughout the whole group or species; and it is on this relationship that the genetic system depends for its character. Hybridity optimum, segregation, and recombination must all be related throughout the group. Furthermore, they must be related not merely amongst themselves, but also to the mating habits of the individuals which compose the group, and to the barriers which make that group by separating or isolating it from others.

If we are to understand the nature of heredity and variation, all these relations require our consideration. We shall commence with the breeding system, the habit of mating within the group, in its simplest and most general characteristics.

\section{REFERENCES}

BRIDGES, C. B. 1922. The origin of variation in sexual and sex-limited characters. Am. Nat., 56: 5i-63.

dobzhansky, т. I941. Genetics aind the Origin of Species. 2nd ed. New York. 


\section{ADJUSTMENT AND BALANCE}

EAST, E. M., and JONES, D. F. 1919. Inbreeding and Outbreeding. Philadelphia. FEDERLEY, H. I929. Über subletale und disharmonische Chromosomenkombinationen. Hercditas, $12: 27 \mathrm{I}-293$.

HALDANE, J. B. S. I922. Sex ratio and unisexual sterility in hybrid animals. J. Genet., I2: IOI-IO9.

PETO, F. II. 1933. The cytology of certain intergeneric hybrids between Festuca and Lolium. J. Genet., 28: I I3-I 56.

sinnor, E. W., and Dunn, L. C. 1939. Principles of Genetics. 3 rd ed. New York. 


\title{
BREEDING SYSTEMS
}

\begin{abstract}
Breeding Systems Inbreeding and Ontbreeding Devices Incompatibility Heterostyly Mating Discrimination The Breakdown of Control Stratification The Inbreeding Hybrid Apomixis
\end{abstract}

The GAMETES Which PAIR AND fUSE IN SEXUAL UNION can differ from one another in two ways; in form and in origin. In regard to form, there is a complete range, from the equality of some algae, fungi and protista, to the vast inequality between male and female germ cells reached in birds and reptiles. This extreme of differentiation represents a division of labour, for it enables the organism to combine the mobility of the sperm, which makes fertilization possible, with the food storage of the egg, which gives the embryo a start in life. Like other differentiation, this difference between the male and female gametes is not to be traced to any corresponding difference in the genes which the cells carry. Gametic differentiation can be as complete when the gametes are genetically alike as when they are genetically unlike; when they are produced by the same individual as when they are produced by different ones.

Of greater genetical significance is the origin of the gametes. Whether they are equal or unequal, the two which fuse may come from the same, or from different, diploid individuals. And if, as in most animals, from different individuals, these may have different degrees of relationship with one another. Where, as in some plants like Johannsen's beans, they regularly come from the same individual, generation after gencration, the product of fusion will be homozygous, or nearly so. Where, on the other hand, they come from different individuals the product will be in some measure heterozygous or hybrid.

Now, in particular populations or mating groups of plants and animals there will be an average degree of hybridity. This will follow from the average relationships of the parents. And this in turn will depend on a variety of conditions of which the most obvious and most important is the relative frequency of self- and 
cross-fertilization. This habit is the breeding system of the population, or group, or species. We now want to know what breeding systems exist, how they are controlled, and what are their cffects.

\section{Breeding Systems}

Brecding systems work at different levels with different methods of reproduction. In some fungi, such as the phycomycete Allomyces javanicus, two cells of a single haploid hypha fuse in sexual reproduction. This is called homothally and we can sec that its result is haploid self-fertilization. All the haploid gametes produced by a haploid are identical and, mutations apart, this type of mating must therefore give homozygosis, complete and immediate. In other fungi, such as Mucor, there is a special device, heterothally, which prevents self-fertilization, and ensures that every fusion shall be the result of a cross between different haploid individuals. We shall examine heterothally in more detail later.

In the higher plants and animals, all sexual reproduction demands the crossing of different haploid individuals, since each haploid individual produces, or even consists of, only one germ cell. But these may or may not come from the same diploid individual. In peas and barley they usually do: diploid self-fertilization is the rule, even if the parent is heterozygous. This process, like haploid self-fertilization, leads to homozygosis; but it does not do so inmmediately. On mating at random, the haploid gametes produced after mciosis by a heterozygous diploid give zygotes half of which are heterozygous, and half homozygous, in respect of each gene pair which is heterozygous in the parent diploid. They give $\mathrm{F}_{2}$ proportions of a half $A A$ and $a a$ taken together, and a half $A a$. Diploid self-fertilization, therefore, does not give immediate homozygosis. Instead it moves (on the average) half-way towards it in each generation.

In the flowering plants devices exist which encourage selffertilization. Far more exist, in both plants and animals, which discourage or prohibit it. Of these the most striking, especially in animals, is sexual differentiation, or dioecy, of the diploid organism. Even in these conditions, however, with regular brother-sister mating, heterozygotes will give place to homozygotes just as they do when self-fertilized, though again not so rapidly. For example, 
in the grass-mite, Pediculopsis, copulation of brothers and sisters occurs in the uterus of the mother with the same regularity as it can do in experimental breeding. Further, in man, where brothersister mating is normally prohibited by social conventions, the same conventions in some societies ensure a regular first-cousin marriage. This, too, when starting with heterozygotes, will bring about homozygosis, though it will do so only very slowly.

In short, hetcrozygosity will at once follow any lapse from haploid or diploid self-fertilization, brother-sister mating or cousin marriage. Homozygosity will be gradually recovered when the system of inbreeding is restored. But it will be recovered at different rates, each characteristic of its own system. Hence, where a proportion of sexual unions habitually depart from the strict inbreeding habit (as they do even in peas and barley) homozygosity and heterozygosity will always exist side by side: they will be in equilibrium. This hybridity equilibrium will depend on (i) the rate of recovery of homozygosity, which, as we have seen, depends in turn on the type of inbreeding, (ii) the frequency of lapses from inbreeding, and (iii) the amount of heterozygosity produced by lapses, which depends in turn on (iv) the amount of genetical variation in the breeding group, which of course is influenced by mutation. These varying factors jointly constitute what has been called the closeness of inbreeding of the group.

\section{Inbreeding and Outbreeding Devices}

The hybridity equilibrium can be controlled in a great variety of ways, so great a variety that their common effect is usually overlooked. This is particularly true of the flowering plants. To begin with, the primary function of the pollen mechanism is that it allows crossing; other secondary functions we shall see later. Superimposed on this mechanism we find devices favouring both selfing and crossing.

Mechanisms favouring close inbreeding generally depend on the development and structure of the flowers. The failure of flowers to open (cleistogamy), best known in Viola, is videspread, and obviously favours self-fertilization. In other cases (like our peas and barley) the flower opens, but only after the pollen has been shed 
on the stigma. This device is therefore almost as effective as cleistogamy in securing inbreeding. Again, in the tomato, pollination follows the opening of the flower, but the stigma is so enclosed in the cone of stamens that it always receives pollen from the same flower. That is, execpt in stocks where the cnclosure is incomplete (or in countries, like its native Peru, where certain insects specialize in cross-pollinating this flower). Some cross-pollination then occurs. British glasshouse commercial varictics are mostly of a rigorously inbreeding type; American ficld-grown varictics of the occasionally crossing typc.

Slight genctic variations in the growth of the style (in tomatocs) or time of pollen-shedding (in the cereals) alter the regularity of sclf-fertilization and thus must control the amount of inbrecding. A more extreme effect has been described by Rick in the tomato. He discovered a recessive gene which partially removes the hairs from the plant and, since the cone of anthers is held together by hairs, thereby deprives the flower of its means of regular selffertilization, and so of some of its fertility. The absolute rate of outcrossing is unaffected so that, of the reduced amount of seed produced, a greater proportion is crossed; in fact nearly so per cent instead of the usual I or 2 per cent.

Regular cross-brecding, or outbrecding as it is perhaps better called, is more difficult to secure than inbrecding, and is in fact sccured by more claborate devices which act at every stage of the reproductive cycle. The most obvious of these is the formation of unisexual flowers, with the sexes borne on different plants, known as dioccy, or on the same plant, known as monoccy. Dioccy cnsures outbreeding. Monoecy only favours it; but its effect may be reinforced by a timing difference in the production of male and femalc flowers, as in maizc. This timing difference is also common in hermaphrodite flowers. The pollen is shed before (protandry) or after (protogyny) the stigma of the same flower is receptive. Self-fertilization is thus prevented in the place where, with insect pollination, it is most likely to occur-in the same flower. This timing difference does not, of course, prevent pollination between different flowers on the same plant. In genetic effect this is still, as Darwin showed, self-fertilization, and the outbrecting mechanism is therefore not fully efficient. 


\section{Incompatibility}

Secondary systems favouring either self- or cross-pollination in bisexual flowers depend on control of a habit which is uniform for all individuals, and therefore adapted to the use of the species very much as are the shape and colour of petals. We now have to deal with another system which depends, like dioccy, on genetic differences, not genetic uniformity, for its maintenance, but which operates in biscxual flowers. This system operates through incompatibility of pollen and style.

Separation of pollen from the styles of the same flowersscparation either in space or time-can only hinder, not preclude, self-fertilization. Incompatibility acts later and can be absolute in its effect, as we can see if we consider an example.

The Sweet Cherry, Prmmus avium, is a diploid species. It consists wholly of individuals, wild or cultivated, which are incapable of setting fruit or seed when self-pollinated (with which we include, of course, pollination with trees of the same vegetatively propagated variety). When cross-pollinated one of two things may happen. The seed set is either complete or is as much a failure as with selfing. For cxample, Bedford Prolific is completely successful with Napoleon or Waterloo, but it fails with Early Rivers. With reciprocal crosses the success or the failure is always the same. Moreover there are groups of varieties (one of them includes 13 names) which are mutually unsuccessful or incompatible, whilc being compatible with all other groups of varieties of which some 16 are known (Fig. 58). Thus to ensure a crop it is necessary to mix together compatible varieties from different groups when planting the orchard.

How does this incompatibility work: It is clearly due to like things failing to agree in pollen and style. When we look into the matter we find that with incompatible pollination the pollen grows too slowly down the style ever to complete its journey to the egg. With compatible pollinations, we find one of two situations. Sometimes, as in Bedford Prolific by Napolcon or vice versa, all the pollen grows quickly and well; and sometimes, as in Bedford Prolific by Waterloo and vice versa, half of it grows well, and the other half behaves as though it is incompatible. The good half, however, 
is cnough to fertilize every egg and so give a full crop of fruit. This sccond type, half-and-half, is always found in the relationship of parent and offspring.

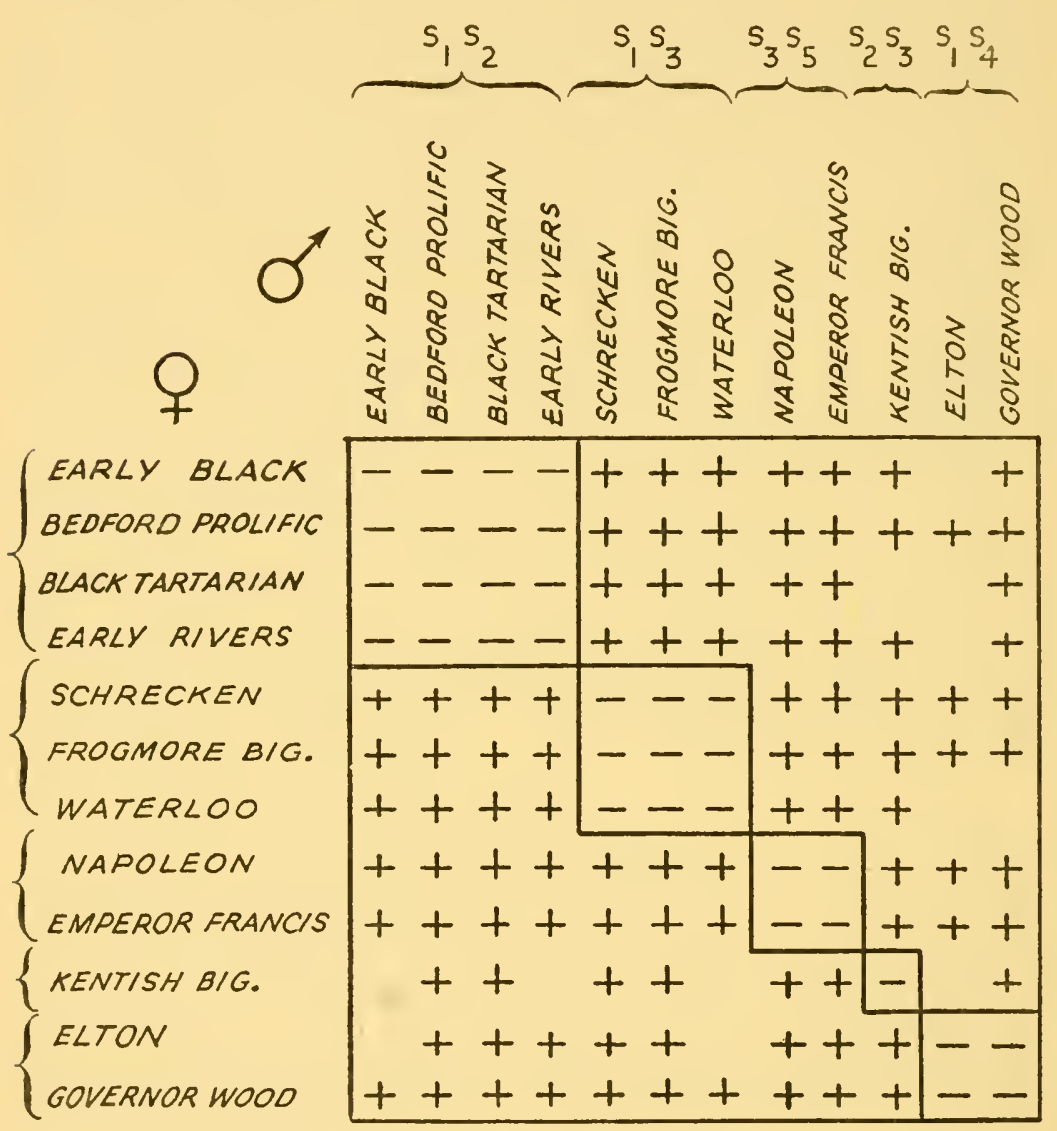

Frg. 58.-The compatibility relations of 12 varieties of sweet cherry, falling into 5 incompatibility groups. + indicates successful pollination, - unsuccessful pollination, and a blank that the pollination has not been tried. The compositions of the varieties relative to the $\mathrm{S}$ gene are shown above (after Crane and Lawrence, 1947).

These results give us the clue to the genetic problem. The halfand-half types of pollen are obviously produced by single gene heterozygotes. Since the parent's style rejects all its own pollen and only half that of its offspring, the rejected pollen must be that which 
carries a gene, an incompatibility gene, also present in the style. Completely incompatible pairs of plants will then always be such as have both allelomorphs the same. Compatible ones will be such as differ in respect of one or both (Fig. 59).

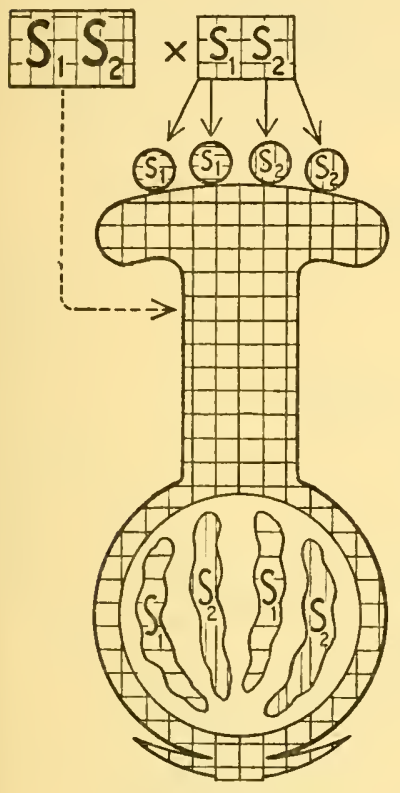

NO SEED
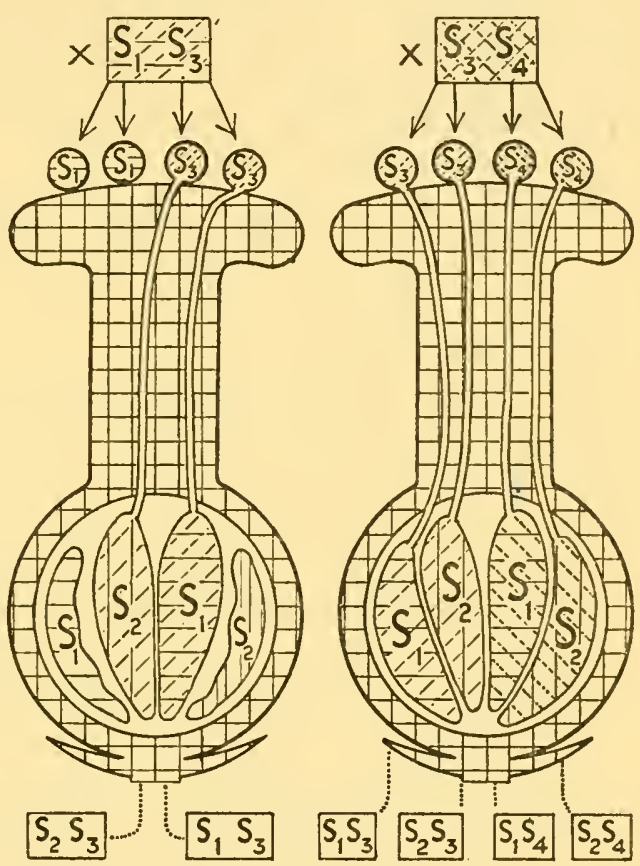

FIG. 59.-Diagram showing the physiological relationship between pollen and style and the genetic relationship between parent and offspring with homomorphic incompatibility. $\mathrm{S}_{1} \mathrm{~S}_{2}$ styles receive, (i) self or similar pollen on the left and their ovules remain unfertilized, (ii) in the centre mixed pollen part of which, if in sufficient quantity, can fertilize all ovules, and (iii) on the right wholly compatible pollen all of which can fertilize the ovules.

Note.-No progeny can be incompatible with their mother, but in the middle case a half will be incompatible with their father.

This explanation suggested by Prell in I92 I was confirmed, a few years later, by East and Mangelsdorf with Nicotiana alata and by Filzer with Veronica syriaca. On its basis the inheritance of incompatibility has been successfully predicted in a large number of diploid plants. Indeed the mechanism probably occurs in some stage of development or decay in about half the species of flowering plants. 
The controlling incompatibility gene, it will be noticed, takes us a new step in genetical inference. We recognize its allclomorphs as distinct, not by a difference in behaviour of the various genctical kinds of pollen in styles carrying the same allclomorphs, for these are alike in that none will grow; but by the difference between such bchaviour, on the one hand, and the growth of pollen carrying any allelomorph in a style carrying different allclomorphs, on the other. It is just the same as the way in which we sec the cffects of inversion

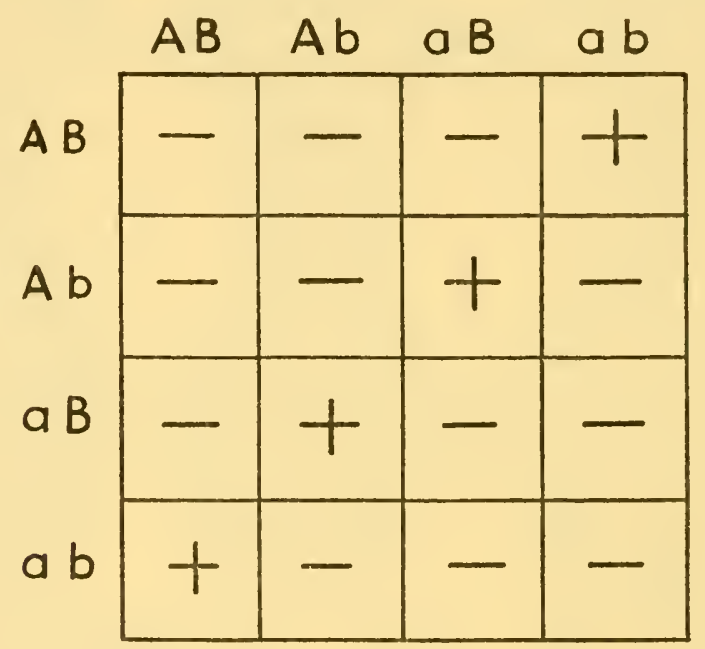

Fig. 60.-Hetcrothally detcrmined by two loci in fungi. Successful fusion, indicated by + , occurs only where the two hyphae differ at both loci. All other combinations, whether alike at both or only one of the loci, fail to achicve fusion, indicated by - .

or interchange at meiosis; not in homozygotes, which show normal bchaviour, but in the structural hetcrozygotes.

With suitable brecding experiments the linkage of this gene, $S$ as it is called, with other genes can be established and its mutations recorded. Of course in a fully operative system the number of allelomorphs of the $S$ gene can never be less than three and is always in fact nuch more numerous. In the small species Ocnothera organensis, with a total wild population of perhaps no more than 500 plants, 35 allelomorphs have been identified. In the large species of Red Clover, Trifolium pratense, one group of 24 plants had $4 \mathrm{I}$ different allelomorphs and another group of 20 plants had 37 
allelomorphs. And, although closely related, the 60 cultivated varieties of Sweet Cherry examined must have over 20 allelomorphs.

The $S$ gene tells us many things of importance. Its large number of allelomorphs is without parallel. The specificity of their action

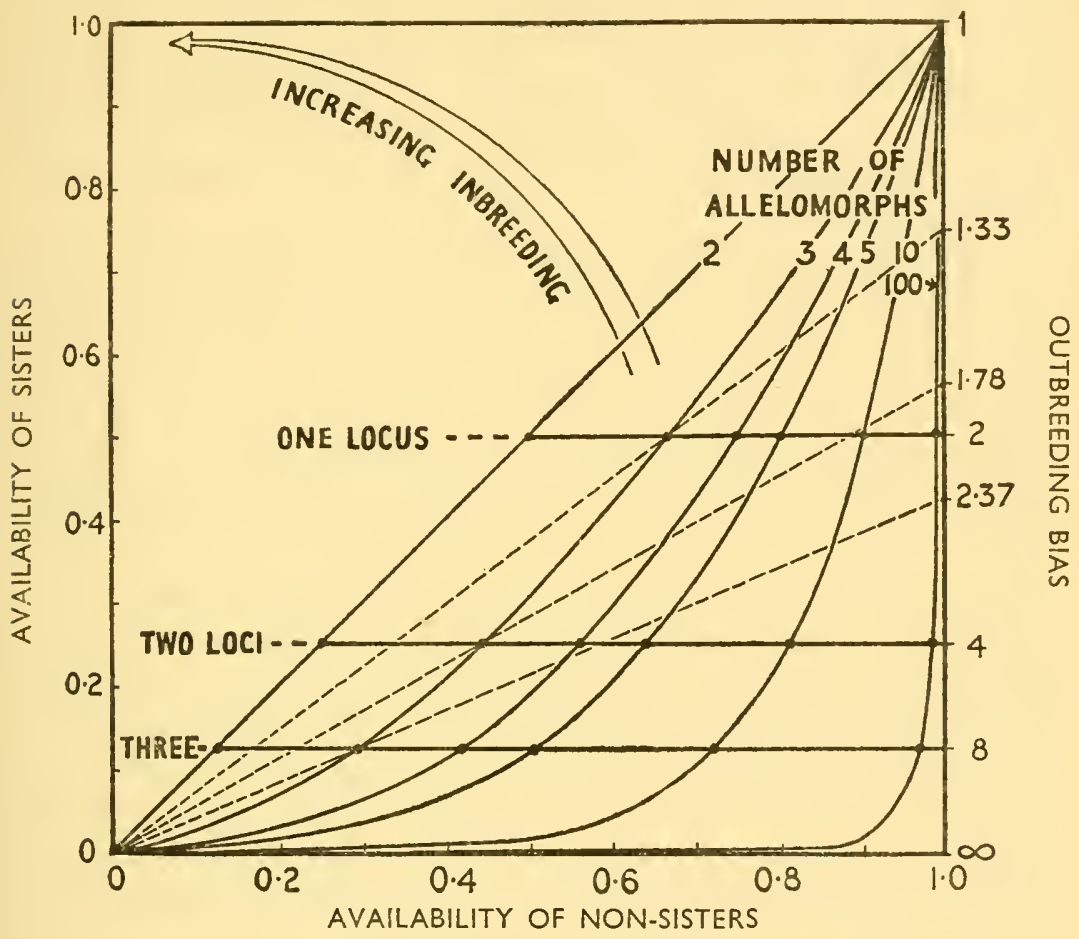

FIG. 61.-Heterothally is an outbreeding mechanism in fungi. The proportion of sister haploids (i.e. from the same diploid parent) available for mating is plotted against the proportion of non-sister haploids available for mating. Thus with two loci each of three allelomorphs, $\frac{1}{4}$ of the sisters will be available but $\frac{4}{9}$ of 11on-sisters. A line projected from the origin through this point shows that the outbreeding bias, found as the ratio of availability of non-sisters to availability of sisters, is $\frac{t}{4} \div \frac{1}{4}$ or $\mathrm{I} \cdot 78$. The maximum outbreeding bias with one locus is 2 , with two loci is 4 , and with three loci is 8 . Nothing short of intervention of diploid tissue, as in incompatibility in flower plants, can give an outbreeding bias of $\infty$.

in the style is nearly always complete, since $S_{x}$ pollen fails on a style carrying $S_{x}$ no matter what the other allelomorph may be. In some cases this specificity cxtends to the strength of action, for some allclomorphs are stronger than others whatever others are present, though in other cases the allelomorphs can strengthen one another's 
action. Morcover the propertics of the pollen itself are detcrmined by the single allelomorph carried in its own nucleus after segregation: there is no delayed effect from the other allelomorph present in its diploid parent (and in its sister pollen). This rapid and specific action puts one in mind of the relation between gene and antigen in the determination of blood groups. The analogy is still more evident from the effect of a rise of temperature which, so Lewis found, speeds up the growth of compatible pollen, yet slows down the growth of the incompatible. Incompatibility is thus duc to a positive blocking reaction.

In the haploid fungi, heterothally secms to work in the same way as incompatibility in the flowering plants. In Coprinus rostrupianus, for example, multiple allelomorphs exist within the species which prevent the fusion of likes. In Coprinus lagopus and elsewhere there arc two serics of allelomorphs, similarity in cither of which is sufficient to prevent fusion (Fig. 60). This double series in the haploid plants, as opposed to the universal single series of allelomorphs in the diploid plants, is significant. In the diploid plants the action of the $S$ gene in the style prevents any fusion of ganctes from the same diploid mothcr. In the haploid plants such fusions can never be wholly prevented (Fig. 6I). Indeed, with a single series of allelomorphs, sister gametes can fuse in half the cases. A double series is needed to reduce this chance to a quarter. A third series, of which no case is known, would reduce it only to an eighth. Thus the different systems of incompatibility genes are clearly related, we may say adapted, to their effect in reducing inbreeding, or enforcing outbrecding, in organisms with different systems of reproduction.

\section{Heterostyly}

Not all systems of incompatibility depend on the genetically autonomous behaviour of the haploid pollen grains. There is another type in which the properties of the pollen are controlled by the diploid plant bearing it. The control is excrcised by way of the differentiation of the flower. The morphological aspects of this system have long been recognized under the name of heterostyly proposed by Hildebrand in I 864. Its physiology was first described in detail by Darwin in his "Forms of Flowers" in 1877. 
Heterostyly takes various forms. The simplest and best known is that found in most of the diploid species of Primula. Here there are two types of plant distinguished by the shape of their flowers: the thrum with a short style has the neck of the corolla filled with a thrum of anthers; the pin with a long style has the anthers half way down the corolla (Fig. 62). In pin and thrum types opposite organs are in corresponding positions, so that a pollinating insect naturally transfers the pollen of one to the style of the other.

This simple mechanical means of encouraging cross-pollination is not, however, all. The pin flower has a rougher stigma and smaller pollen grains than the thrum. Thus there is evidently some physiological difference beneath the morphological one. This difference was revealed by Darwin's experiment of comparing the amount of seed set when pin and thrum plants are crossed and when each is bred with its own kind. The one, the "legitimate" mating gives high fertility; the other, the "illegitimate" mating gives lower fertility (Table 2r).

TABLE 21

SEED SETTING IN THE PRIMROSE (DARWIN, 1877)

\begin{tabular}{|lll|r|r|c|}
\hline & Mating & & $\begin{array}{c}\text { Flowers } \\
\text { pollinated }\end{array}$ & Capsules set & $\begin{array}{c}\text { Average seeds } \\
\text { per flower } \\
\text { polinated }\end{array}$ \\
\hline Legitimate & Thrum $\times$ Pin . & $\ldots$ & 8 & 7 & $56 \cdot 9$ \\
& Pin $\times$ Thrum .. & $\ldots$ & 12 & 11 & $61 \cdot 3$ \\
Illegitimate & Thrum $\times$ Thrum & $\ldots$ & 18 & 7 & $7 \cdot 3$ \\
& Pin $\times$ Pin $\ldots$ & $\ldots$ & 21 & 14 & $34 \cdot 8$ \\
\hline
\end{tabular}

We now know that this effect is due to a difference of growth rate of pollen tubes, as with ordinary incompatibility. The illegitimate is slower and, if the two are mixed together, the legitimate gets there first. But there is one striking difference from ordinary incompatibility: all the pollen of one plant behaves in the same way. Yet the pollen (and eggs) of one of the types must be of two kinds in the heredity it carries, because crossing pins and thrums gives equal numbers of pins and thrums. Intercrossing pins gives only pins. Intercrossing thrums, as found in nature, gives 3 thrums to I pin. Thus thrum, in nature, is always heterozygous, and the thrum 
allelomorph $(S)$ is dominant over the pin allelomorph $(s)$. But $s$, as much as $S$, pollen grains of $S s$ plants behave in a thrum way, while $s$ pollen grains of $s s$ plants behave in a pin way.

Thus, either of two different mechanisms of gene action with which we are familiar elsewhere can determine incompatibility. One acts directly through the haploid pollen grain; the other is imposed upon the pollen through the cytoplasm of its diploid parent.

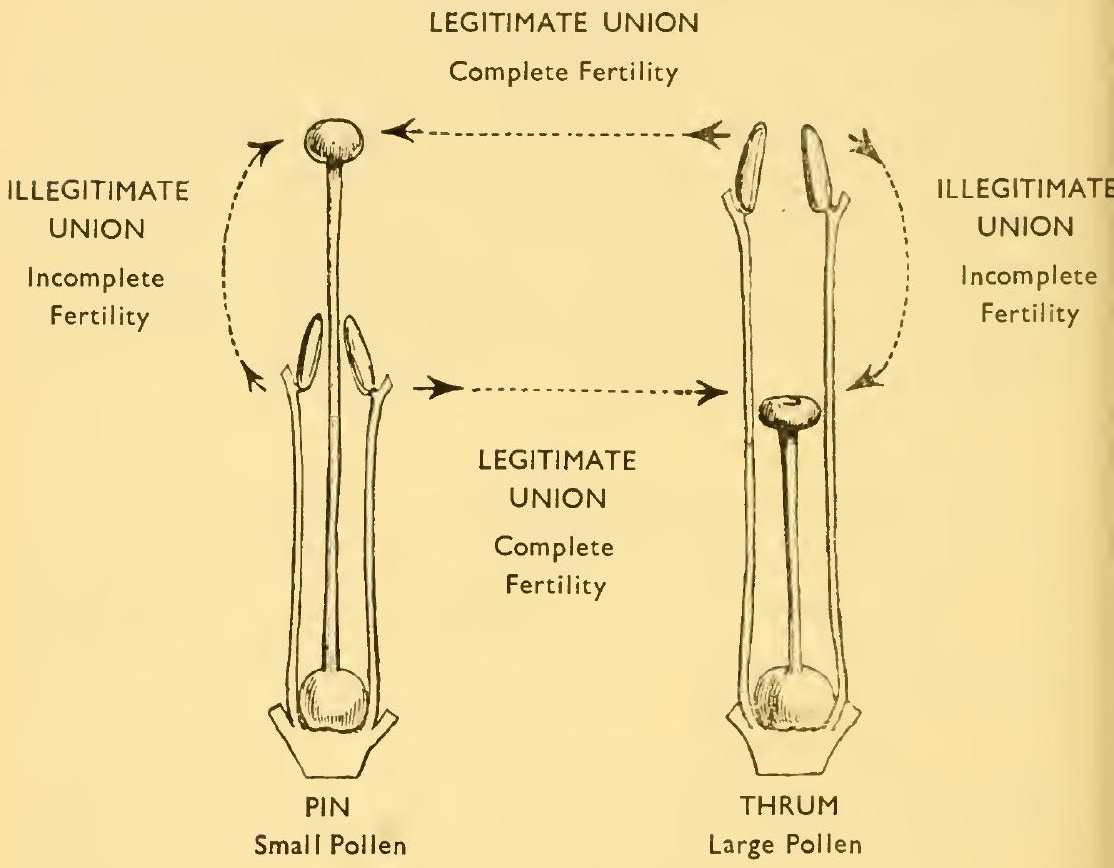

Fig. 62.-Distyly in Primula, showing the incompatibility associated with it. The illegitimate unions, of pin $\times$ pin and thrum $\times$ thrum, are incompatible whether the pollination is made within a flower, as shown in the diagram, or between flowers. whether from the same or different plants (after Darwin, 1877).

This delayed action is made still clearcr by a further claboration in the Purple Loosestrife, Lythrum salicaria, also studied by Darwin. Here there are three types of plants, each with its own form of flower. There are three levels for the sexual organs within each flower, two of which are occupied by anthers and one by the stigma, so that the three types have long, mid, and short styles. Legitimate, that is fertile, unions are always those between anthers and stigmata of 
different plants borne on the same level (Fig. 63). All others are sterile or nearly so. Thus a short stigma accepts pollen from short stamens of both long and mid-styled flowers. It will not accept pollen from the other stamens (mid or long) of these same flowers, any more than from its own or any other mid and long stamens.

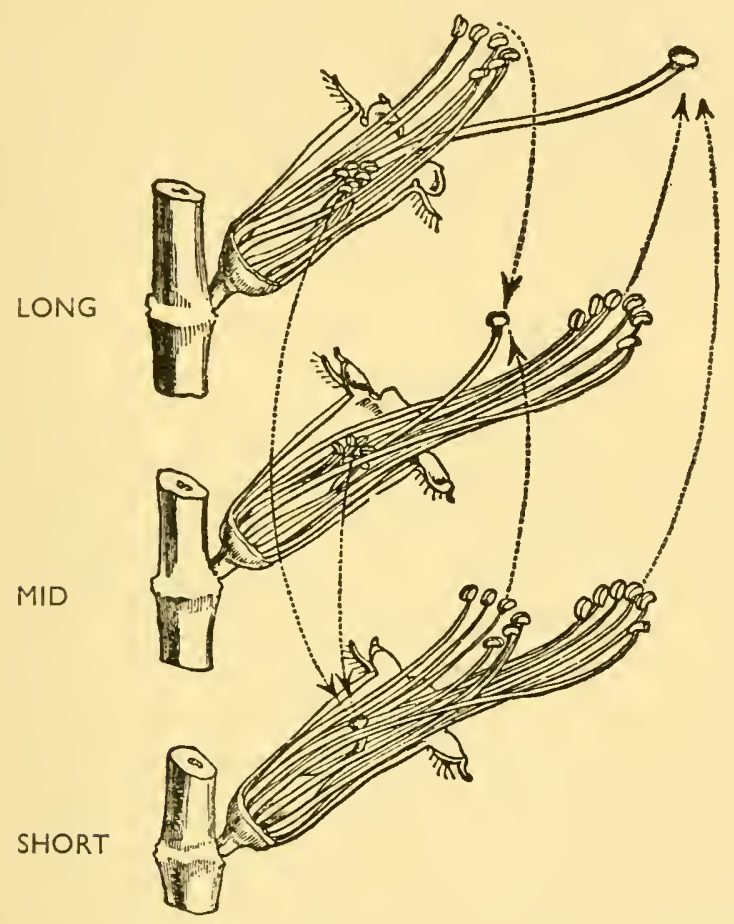

FiG. 63.--Tristyly in Lythrum salicaria. Only the pollinations indicated by arrows are compatible. All others fail or virtually fail. Thus the two tiers of anthers in one flower give pollen having different properties in incompatibility, while anthers from tiers at the same level in different flowers give pollen having the same properties in incompatibility (after Darwin, 1877).

Before we return to our general genetic problem we must note three highly instructive physiological properties of this system. First, cach parental genotype has two paths along which it can drive its own pollen. Secondly, pollen from parents of different genotypes may be driven along the same path. And thirdly, the genotype of the pollen itself never comes into the question.

Tristyly in Lythrum (or in Oxalis or Narcissus) could scarcely be 
worked on the basis of a scries of multiple allclomorphs, and in fact it depends on two unlinked genes. One decides the difference between the short-style and the not-short-style. The other has no action on short-style, but decides whether a not-short-style shall be mid- or long-style. It is a case of epistasy.

The same genetic and physiological organization as with heterostyly is revealed by Capsella grandiflora. The pollen depends for its behaviour on its diploid parent, and two genc differences work the device as in Lythrum. But morphologically the flowers of the different types are all alike. The heterostyly is cryptic. Thus the physiological difference, which in the other instances goes with a difference of position, can be achicved without the help of such a difference.

\section{Mating Discrimination}

The diversity of outbrecding mechanism in plants, dioccy, monoccy, protandry, incompatibility in its various forms, is not matched in animals. With them dioccy, scxual differentiation of the diploid organism, is the basic device almost without exception. Even the hermaphrodite oyster can separate its sexes in time by the cyclical succession of male and female phases, and thereby become effectively dioecious. Dioecy cxcludes the extreme inbrecding mechanism of self-fertilization so common in plants. But, as we saw, dioecy can be determined by many sex-chromosome and other mechanisms, and on it can be superimposed a number of devices working both ways, favouring either inbreeding or outbreeding. We have already noticed intra-uterine copulation and first cousin marriage as favouring inbreeding. We can now consider the outbreeding mechanisms.

Animals move about, but their movement is limited. Dioccy forbids sclf-fertilization, but it does not forbid brother-sister mating such as is most likcly where eggs are laid in clutches. In some insects, e.g. Sciara, the dung-fly, this incest is avoided by the uniscxual brood. By delayed action, as with heterostyly, the mother directly determines the sex of her offspring, which may be all male or all female. The brood of one sex is thus excluded from incest by the genetic properties of the mother.

The habits of individuals or the laws of society may have the same effect on the breeding system as delayed gene action. Where 
flies are offered a choice of mate they may exercise that choice in the avoidance of extreme inbreeding and extreme outbreeding. Courtship behaviour in fishes, birds and mammals elaborately testifies to the same exercise of discrimination. In man the prejudice of individuals against incest and bestiality has become hardened and generalized into social tabu and legal prohibition.

Looking back on all these different systems we see that in a hundred different ways the same end is achieved, the end being the control of the mating system. The means adopted reflect the circumstances and capacities of the species. Plants make use especially of the diploid style as a sieve for sorting the pollen delivered to it by a pollinating agency, over which it can exercise no direct control. Animals have powers of perception which they use in accepting or rejecting mates. And lastly man uses his unique power of social coercion for the same acknowledged purpose.

The control of the mating system is evidently such as will give a controlled degree of hybridity generally intermediate between homozygosity and that extreme heterozygosity which we see to be disastrous in crosses between species. In other words it is capable of giving the kind of hybridity optimum we were led to expect. Two consequences of this state of things may now be seen.

First, bars to crossing between species are, in effect, inbreeding devices and should therefore show a dependence on the mechanisms which control mating within the species. This is clearly seen in discriminative mating which is as strong in flies as it is in mammals. When in Drosophila a male of melanogaster is presented with females of simulans and of his own species (which are alike to us) he never makes a mistake. Similarly, an experiment with Petunia shows how the plant style makes its choice. Pollen of the $F_{1}$ between the species axillaris and violacea, put on the style of either parental species, is sorted out so that the grains carrying the chromosomes preponderantly of the same parent get through to the egg more often. This effect is even clearer when mixed pollen of two distinct types is used in Streptocarpus. Self-pollen then has an advantage over other species' pollen (an advantage proportional to the remoteness of the other species): but cousin-pollen has an advantage over both. 
TABLE 22

RESULTS OF POLLINATING THE TYPE VARIETY (FROM KNYSNA) OF STREPTOCARPUS REXII WITH EQUAL MIXTURES OF ITS OWN POLLEN AND THAT OF OTHER VARIETIES AND SPECIES IN ORDER OF INCREASING DISTANCE OF RELATIONSHIP (LAWRENCE, UNPUBLISHED)

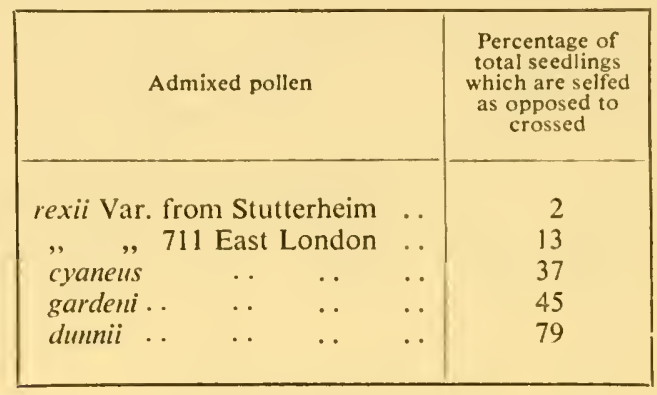

\section{The Breakdown of Control}

The second consequence of the relationship of the mating system to a hybridity optimum is that, in so far as we expect the hybridity optimum to change, so we must expect the mating system to change. Mating systems must be built up and broken down with changing conditions. Of thesc the conditions of cultivation in plants are the most easily verifiable. The wild tomato growing in Pcru is, as we saw, regularly cross-pollinated. In England there is no pollinating insect-a common result of acclimatization. In consequence the cultivation of tomatoes, especially in glasshouses, has been contingent on the acquircment of a property of automatic self-pollination: a property which has thercfore been developed by the prefcrence of the grower for plants setting most fruit, and of nature for plants setting most sced. Hence the property we saw of modem glasshouse tomatocs, which have flowers whose structures ensurc self-pollination, at least with a little shaking. Outbrecding has been replaced by inbreeding.

Primula sinensis has been cultivated in glasshouses since I 824 . Being heterostyled, it presumably had, at first, a strong outbreeding mechanism, more rigorous than the tomato. Now the sced which the glasshouse grower saves is always from pin plants because, in dropping off, the corolla self-pollinates the pin flowers, and even 
a small seed-set obtained in this way is easicr than a large seed-set obtained from legitimate pollinations by hand. This process of unconscious selection for self-compatibility has had its results recorded at intervals over nearly half the $\mathrm{I} 20$ years it has been at work. They show a steady increase in the success of self-pollination of the pin plants, a steady decay in the effectiveness of incompatibility (Table 23).

TABLE 23

THE BREAKDOWN OF INCOMPATIBILITY IN PRIMULA $S I N E N S I S$ (MATHER AND DE WINTON, 1941)

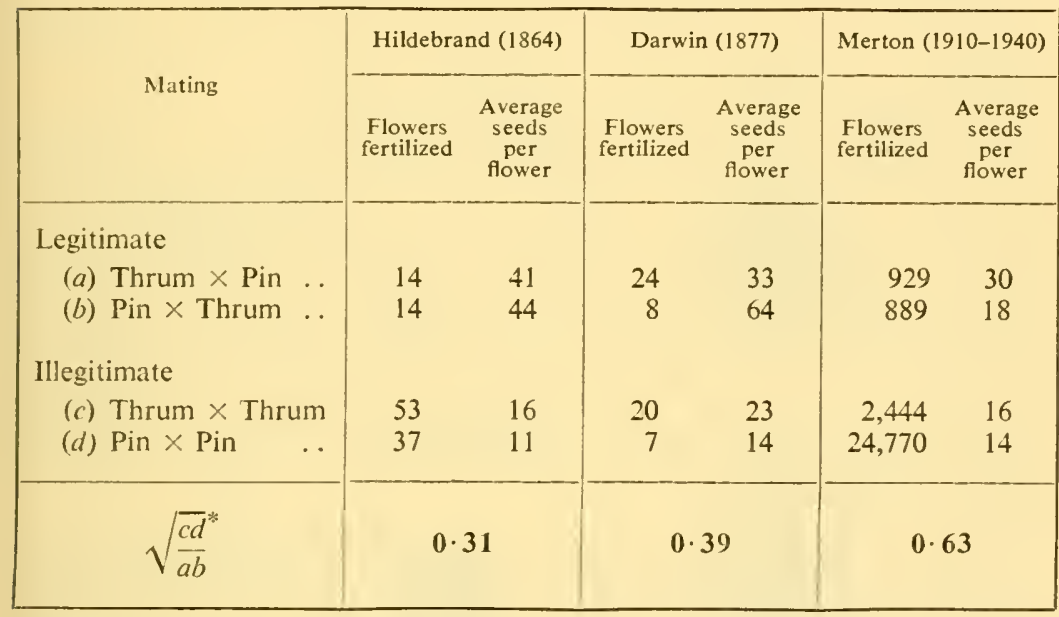

* Measure of fertility of illegitimate pollinations relative to legitimate ones, used in order to correct for the decline in fertility introduced into the later results by the accumulation of recessives genes in material used for genetical experiments.

In spite of this partial breakdown of the incompatibility mechanism, its action is still controlled by the pin-thrum gene, $S-s$. This gene can therefore control incompatibilities of different strengths, whose differences are governed by other genes. In other words the $S-s$ gene acts as a switch directing development into one of two alternative paths, of which the precise course depends on other genes, or rather polygenic systems, whose variation is not normally detectable.

Ordinary incompatibility is controlled in the same way: it has the same genetic structure as heterostyly. For cxample, Petunia violacen has the usual multiple allelomorph system of pollen-style 
relationships: self-pollination rarely succeeds. $P$. axillaris, on the other hand, shows no trace of incompatibility: selfing and crossing succeed equally well. In the $F_{1}$ different $S$ allelomorphs from violacea vary in effectiveness and plants differ in the degree of self-compatibility. By crossing together $F_{1}$ plants with different $S$ allelomorphs, or by backcrossing them to their violacea parent, we can get plants with the same $S$ constitution as violacea but with other genes, half in the $\mathrm{F}_{2}$ and a quarter, or nearly a quarter, in the backcross, from axillaris. Although alike in regard to $S$ these three types of plants show incompatibility relations differing in two ways (Fig. 64).

First, the amount of seed set on self-fertilization increases with the proportion of axillaris genes. These genes must therefore be undermining the operation which the $S$ genes control.

Sccondly, the amount of seed set on pollination of backcross or $\mathrm{F}_{2}$ plants by violacea is greater than any produced by self-pollination, although the same $S$ genes are at work. Thus the axillaris genes not merely weaken the operation of the $S$ genes; they shift their operation so as to put it out of step in plants with different proportions of axillaris genes. Or, the other way round, we may say the same $S$ genes can not mercly control systems of different strengths, but systems which are less efficient with one another than each is within itself.

Now, we may ask, what happens when we cross two self-incompatible species, each with the $S$ genes? This has been done for Nicotiana alata and $N$. forgetiana in the production of the garden form $N$. sanderae. Pseudo-compatibility, that is successful fertilization with pollen having an $S$ allelomorph the same as in the style and therefore not legitimately capable of growth, is unknown in forgetiana. It occurs only rarely in alata. In their derivative it is common, especially with certain of the weaker allelomorphs. Thus the recombination between the general gene systems of two species has robbed each of its efficiency as a basis for the action of the $S$ series.

The best known example of all switch genes, or gene complexes, is of course that determining sex. We have already seen how on crossing the two sub-species of Melaudrium dioicum Winge was able to obtain in $\mathrm{F}_{2}$ plants male in regard to the sex chromosomes but bearing female as well as male organs. As with Petmmia and Nicotiana 


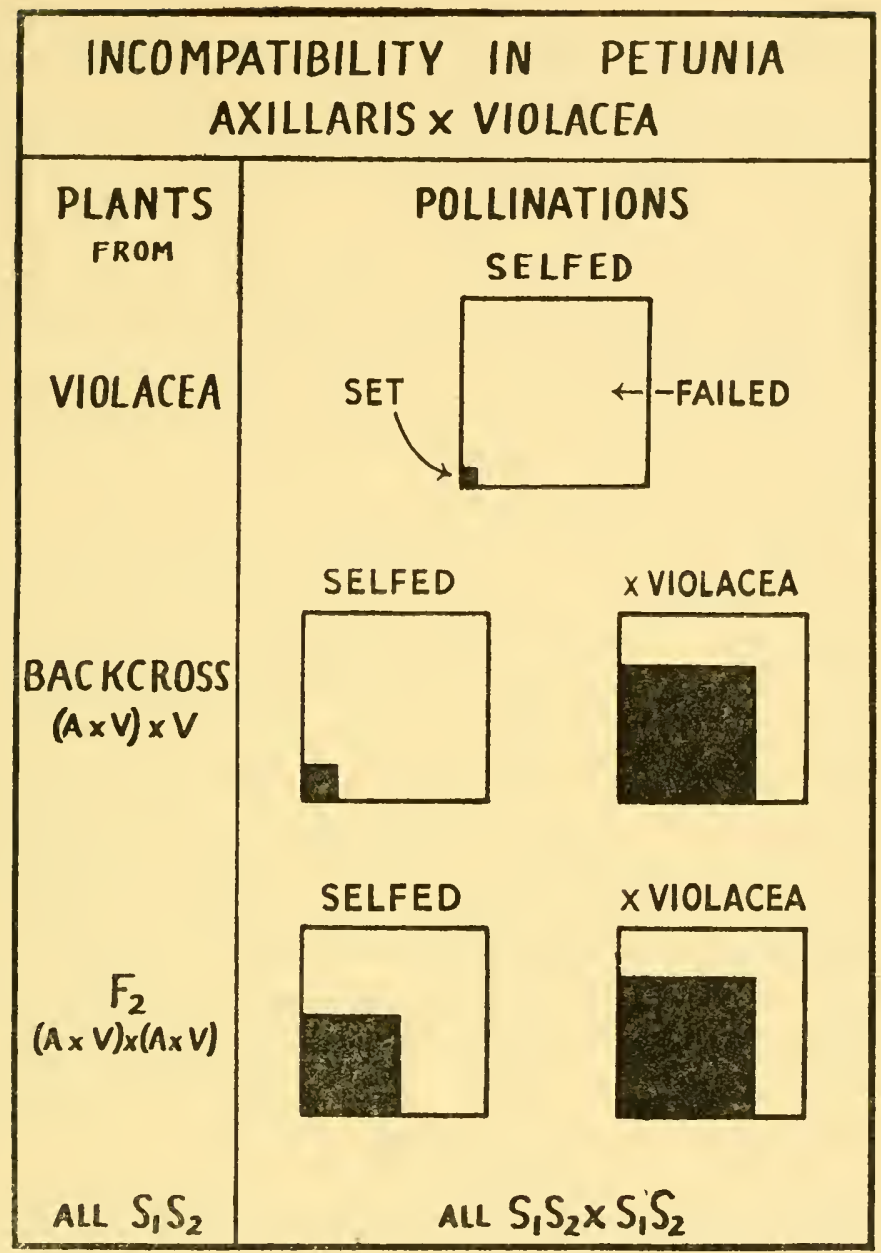

FIG. 64.-Breakdown of the genetic determination of incompatibility in Pctunia. $P$. violacea plants of the constitution $\mathrm{S}_{1} \mathrm{~S}_{2}$ set seed from only about I per cent of flowers which have been self-fertilized (indicated by size of black square relative to the white one containing it). $S_{1} S_{2}$ plants recovered in the $F_{2}$ of the cross with the selfcompatible $P$. axillaris set seed from about 25 per cent of flowers after self-pollination and $\mathrm{S}_{1} \mathrm{~S}_{2}$ plants recovered in the backcross to $P$. violacea are intermediate in behaviour. Thus the addition of genes from $P$. axillaris causes progressive breakdown of the incompatibility mechanism of $P$. violacea. It also causes some change in the operation of the mechanism which is left, as pollination of the backcross or $F_{2}$ plants by $P$. violacea gives a greater set of seed than does selfing, with which the cross is identical in respect of $S_{1}$ and $S_{2}$ (based on Mather, 1943). 
certain recombinations amongst the general genes had broken down the action of the switch gene. They had in fact prevented the XY combination from inhibiting the development of the ovary. They had thus turned males back into hermaphrodites, the condition from which maleness and femaleness must have recently arisen in both the parent plants.

In animals with long-established dioccy, the same breakdown occurs in hybrids, but it produces sterility. Race crosses in the moth Lymantria dispar, for example, give intersexes. In these, female development is superseded by male, or vice versa, in the course of growth, the time of the change determining the grade of the intersexuality, as we saw in Chapter 7.

In Drosophila we can go two steps further. Intersexes can be produced by altering the numbers of chromosomes present. As we have already secn, an extra set of autosomes with the ordinary two X's of the female turns it into an intersex. The presence or absence of $Y$ makes no difference. Evidently, therefore, the switch gene effect of $\mathrm{X}-\mathrm{Y}$ segregation depends on the differences in proportion of autosomes and $\mathrm{X}$ chromosomes in $\mathrm{XX}$ and $\mathrm{XY}$ flies (Fig. 55). Again, as in Petunia and Nicotiana, genes whose differences are not segregating are making the switch mechanism work.

The second step in Drosophila is in showing that the switch gene itself is compound. When the X chromosome is broken by X-rays, and its fragments are lost, its effect is diminished: females are turned into intersexes. The $\mathrm{X}$ chromosome, or at least its differential segment, is thus a super-gene in respect of sex determination, although an aggregate of genes in other respects.

In genetic terms, breeding systems are now seen to be of two types. First, all those giving inbreeding and some giving outbreeding, like protandry and cyclical hermaphroditism, are controlled by general gene systems which do not show any special variation within the species. Their operation does not depend on diversity, that is on segregation. Secondly, the chief cross-breeding systems, sex and incompatibility, on the other hand, require segregation and therefore depend on the action of switch genes. But the existence of the mechanism which these genes switch, depends on a general gene system which does not need to show segregation.

On this basis new systenus can come into existence by adjustment, 
through selection, of the general gene system; the switch gene, where it exists, is thereby given a progressively stronger effect. The evidence that this is at least sometimes the case arises from the method of breakdown seen already in Primula sinensis. Sudden breakdown can, of course, occur in the switch gene itself. Various species of Primula, such as the common primrose, sometimes have the pin-thrum type replaced, in nature, by a homostyle type with anthers and stigma at the same level. This change was found by Ernst in one case to be due to a change of the switch gene to a third allelomorph. The new type must inbreed with a regularity which is equal to the outbreeding of its predecessor, because, of course, the genetic background remains the same. Similarly, in ordinary incompatibility, the usual $S$ allelomorphs can be replaced by a so-called fertility allelomorph, $S$, which no longer inhibits self-pollination or even any kind of cross-pollination.

Most fertility allelomorphs have been found in self-compatible species related to other species which are uniformly self-incompatible. But mutations to $S_{f}$ have appeared in the otherwise regularly incompatible red clover. Moreover, in Antirrhinum majus, but nowhere else so far as is known, a fertility gene exists, overriding the $S$ system, but not allelomorphic to it. These various cases of breakdown show that a system which is built up gradually of many elements including an operator, the switch gene, can be knocked down by removing any one of them, especially, of course, by removing the operator.

\section{Stratification}

All these facts and arguments relate to changes in the breeding system at one level, or at one time of operation. But, as we know, control occurs at many levels. How do they interact?

One system can be superimposed on another, while leaving all the evidence of the other plainly revealed to us. We then have a stratified system. For example consider wheat, any species of Triticum. The anthers and stigmata are thrust out into the wind to allow of cross-pollination, or so one would suppose; but in fact the anthers burst and pollinate the stigma inside the flower before it opens. Inbreeding is superimposed on outbreeding: a low hybridity optimum can replace a high one. This sequence of systems 
is confirmed when we look at a closely related cereal. Rye pushes out its anthers and stigma in just the same way; but instead of vitiating the crossing mechanisms by premature bursting, it reinforces it by incompatibility. Wheat has developed one way and rye the other, after their ancestors diverged. Of peas the same story can be told. To be sure, the cross-pollination was to have been by insects, but its vitiation is still by premature bursting of the anthers. And when we return to the mitc Pediculopsis, premature mating is again the means by which outbrecding is suppressed whilc the sex systcm adapted to outbreeding is retained.

Outbreeding, so far as we know, never supervencs on inbreeding. Always it is the reverse. Complete inbreeding is evidently a dead end. The reasons for this we shall touch on later. But there are a variety of ways in which the effect of cross-breeding, the high degree of hybridity, may be maintained while the ancient system of crossing is allowed to lapse. One of these we have already scen in the allopolyploid. A sccond method is that of the trucbreeding hybrid or complex heterozygote.

\section{The Inbreeding Hybrid}

The true-breeding hybrid is known by the exact breeding analysis of Renner, as well as by chromosome study, in a hundred or so species of the American Evening Primrose, Oenothera. These species are characterized by having $6,8, \mathbf{1 0}, \mathrm{I} 2$, or all 14 of their chromosomes linked in a ring at meiosis. They are interchange hybrids. They arc hybrid not for onc only, but for two, three, four, five or, in the extreme case of the complete ring, six, interchanges. Take a ring of 6 resulting from two interchanges. We can represent its chromosomes as follows:-

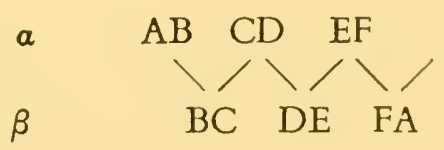

The ring, in fact, usually arranges itself on the spindle in this alternating way, so that two kinds of workable germ cell are formed, both in eggs and pollen. They bear the two complexes $\alpha$, or $\mathrm{AB}+\mathrm{CD}+\mathrm{EF}$, and $\beta$, or $\mathrm{BC}+\mathrm{DE}+\mathrm{FA}$. When a complex 
heterozygous Oenothera is selfed, however, it does not produce a mendelian combination of $\mathrm{I}: 2: \mathrm{I}$. The two classes of homozygotes fail to appear. The hybrid breeds true. Indeed it is only when a heterozygous species is crossed with another, heterozygous or homozygous, that its heterozygosity is shown by the mixture in the progeny, by the production of twin hybrids. Thus if the second species is homozygous for a set $\gamma$ with the pairs: $\mathrm{AB} C \mathrm{CE} D \mathrm{DF}$ $\mathrm{AB} \quad \mathrm{CE} \quad \mathrm{DF}$ then the crossed seedlings will be of two kinds:-

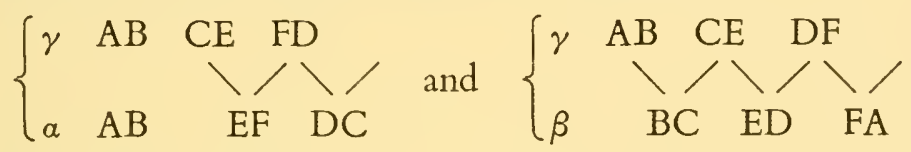

These two kinds will be recognizable by the differences in the size of the ring and in their external appearance.

These observations present us with two problems. Why do sets or complexes, with different arrangements of chromosomes produced by interchange, differ in their phenotypic effects? And why do their homozygous combinations so often fail to appear, or at least to live? Renner showed that sometimes, as we have already seen, one complex fails in the pollen and the other in the egg. He also showed that, in many other cases, homozygotes were often formed, but quickly died. Evidently each set is defective in some way or other, and its defect is covered by its partner. If we can find out what this defect is we shall have explained why the different sets have different effects.

A moment's consideration will show that our symbols $\mathrm{AB}, \mathrm{BC}$ and so on make an unjustifiable assumption. Chromosomes, as we saw, can and do break and rejoin at various points, indeed between any two genes. They are not each composed of two unbreakable arms exchangeable only at the centromere. There are segments therefore (as we saw earlier in Fig. 32) which are omitted from our diagram between $\mathrm{A}$ and $\mathrm{B}$, between $\mathrm{B}$ and $\mathrm{C}$, and so on. The sum of these segments in one complex would be equal originally to the sum of those in its partner complex, provided that no superfluous breaks or mutations had ever occurred. But it is of the nature of chromosomes that superfluous breaks and mutations do occur. When they occur in the complex heterozygote their consequences 
are something quite different from what arises with ordinary sexual reproduction. The whole set acts as a single linkage group, which is broken into two parts if crossing-over occurs at any point between its middle segments. A new type of gamete is then produced which is only half-heterozygous. Thus crossing-over in the middle of differential segments must have been suppressed in all the ancestors of a complex heterozygote during its building up. The differential segments of opposite complexes in the heterozygous Oenothera species have been separated from one another, isolated, during this evolutionary process, just as much as the chromosomes of different species.

Since the Oenothera species have incomplete chromosome pairing beginning near the ends, crossing-over is localized near the ends. The situation is therefore prepared for the development of the complex heterozygote before any interchange takes place.

The complexes of Oenothera are differently adapted. Soine fail as pollen. Others fail as embryosacs, and are displaced by their more female-vigorous sister segregates. So different, indeed, are the complexes that a triploid endosperm with a 2 : I balance would probably have been an embarrassment in building up the system; and we find in fact, that the Oenothera family is remarkable for having a diploid endosperm.

The complex heterozygous species of Oenothera are, in the aggregate differences between their complexes, at least the equivalent of hybrids between pairs of species. But the differences are mechanically concentrated in the differential segments and physiologically adjusted to balance one another. They cannot, therefore, have arisen by hybridization in the ordinary sense. Crossing different stocks cnables us to build up by steps a stock of Campanula persicifolia with a ring of twelve chromosomes. But the two sets of six into which this ring segregates do not constitute two mutually adapted complexes. The complexes of Oenothera have evidently been built up gradually by internal change. Thousands of generations of almost uninterrupted self-fertilization in species which, as we shall see, were previously cross-fertilized, accompanied by the continual elimination of homozygotes, have resulted in the production of hybrid species. Hybridity in Oenothera is a matter of crossing different gametes, not different zygotes; a distinction pointed out by Mendel but still not under- 
stood by all mendelians; a distinction to which we shall have to return.

It was the mutations, of course, which first attracted De Vries' attention to Oenothera lamarckiana in 1886 and led him to propound his Mutation Theory of Evolution. What part do the famous mutations in fact play in this genetic system? They represent the breakdown in the mechanism of the true-breeding hybrid. The ring of chromosomes sometimes fails to arrange itself alternatingly on the spindle, and germ cells are then produced with 8 and 6 instead of only 7 chromosomes. Hence the series of trisomics with is chromosomes of which 68 types are to be expected in Oenothera lanarckiana, whose chromosomes normally form a ring of $\mathrm{I} 2$ and one pair. Again, segregation may fail altogether and unreduced germ cells will then give triploids and tetraploids. And finally the ring may break down by crossing-over, as we saw, to give new halfheterozygous forms. The mutants of Oenothera are therefore nothing more than symptoms of its peculiar hybridity and as such are of little significance in evolution. Later we shall see the ways in which the Oenothera system is significant.

\section{Apomixis}

The third way in which a high degree of hybridity may be maintained while the ancient system of crossing is allowed to lapse, is by apomixis, the suppression of sexual reproduction. Apomixis comes about in two ways. Some organisms achieve apomixis, others have it thrust upon them by the circumstances of their birth. The first are of less importance to us. Many organisms, in producing their female spores or eggs under certain external conditions, are compelled to forgo meiosis. A single mitotic division replaces meiosis and gives a diploid egg which can develop without fertilization by a male germ cell.

So is it with many blackberries and other plants. So it is also with the summer-brood females of aphides, which thereby continue to multiply vegetatively like a plant propagating itself by suckers. When the colder weather comes, a first symptom of meiosis begins to appear in some females. One of the two sex chromosomes is lost at the single maturation division of the egg, so that diploid $\mathrm{XO}$ eggs 
develop. They are sexual males. At the same time females are produced whose eggs undergo regular meiosis and are therefore sexual. Both types undergo perfect reduction, but only the X-bearing sperm of the males function. The haploid eggs after fertilization, therefore, give only females, which re-establish the parthenogenetic summer series (cf. Fig. 65).
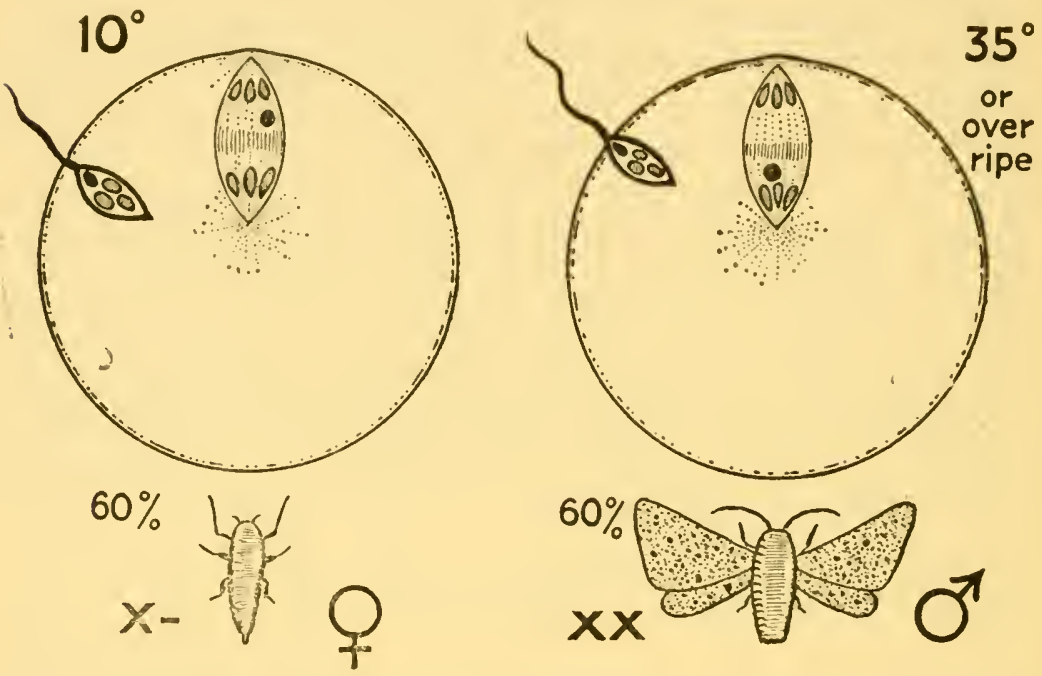

FIG. 65.-The effect of temperature on sex determination in the egg of the psychid moth Talaeporia tubulosa where the female with one $\mathrm{X}$ is heterogametic according to Seiler, 1920. At the higher temperature or when over-ripe the unpaired $X$ chromosome passes more often to the egg than to the polar body at the first meiotic division and therefore gives a preponderance of males. An adaptable sex-ratio is important in these moths where the female is immobile.

This whole process of cyclical parthenogenesis is a means of economizing on sexual reproduction during the summer period of rapid expansion of numbers. If, of course, such a species moves south into a region of perpetual summer, as seems to happen in the United States, then the species will not enjoy its sexual season. It had achieved facultative apomixis but it will have had obligatory apomixis thrust upon it.

Quite a different story is it with those plants and animals which find themselves suddenly deprived of means of regular sexual reproduction by the circumstances of their birth. A triploid of the 
crustacean Trichoniscus, or of the dandelion Taraxacum, arises suddenly owing to the fertilization of an egg with the unreduced I6 chromosomes by a normal sperm with 8 (or vice versa). Such a triploid, although only a number-hybrid, suffers all the misfortunes of the authentic fruit of cross-fertilization. The lagging of unpaired

ARTEMIA SALINA
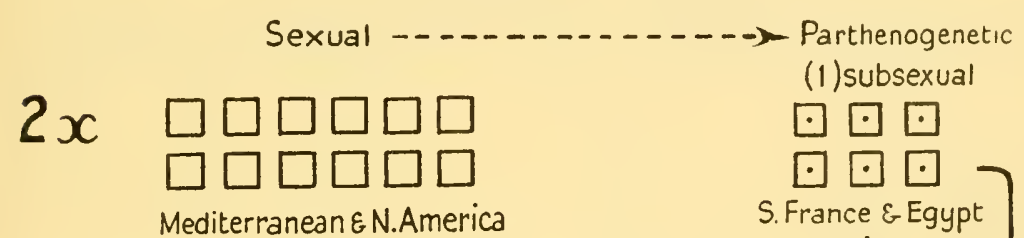

(1) subsexual

$\bullet \square \square$
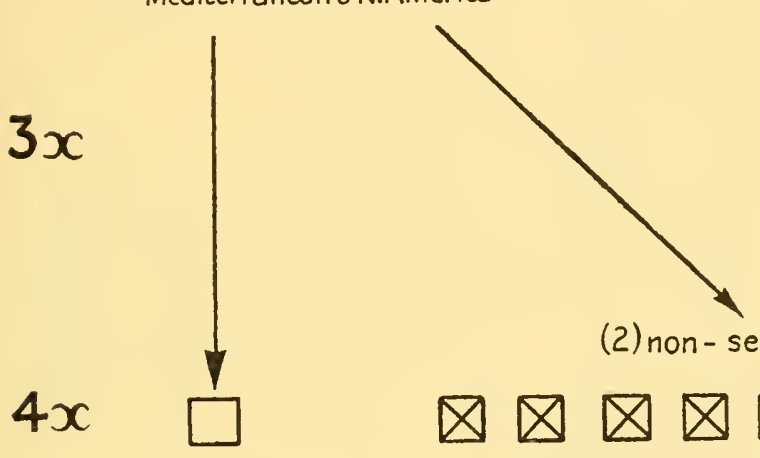

France E Egypt

Odessa

(2) non-sexual

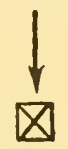

Portorose

\section{$8 x$}

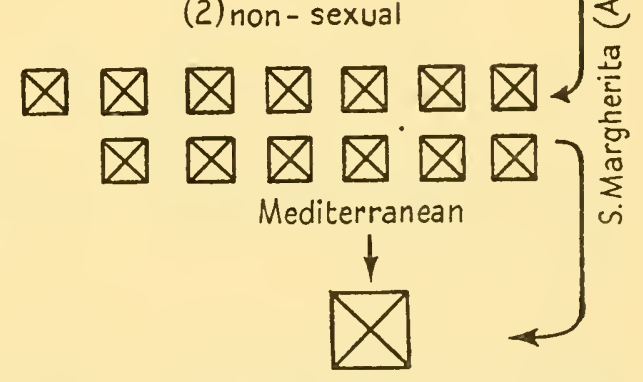

Frg. 66.-Evolution of sexual, sub-sexual and non-sexual forms in the brine shrimp Artemia salina. The sub-sexual forms have meiosis followed by fusion of its products. The non-sexual ones suppress both meiosis and fusion: the unreduced egg develops. Each square represents a tested location (after Barigozzi, 1946).

chromosomes at meiosis in its mother cells prevents the separation of the two daughter nuclei. A single restitution nucleus is reformed with the whole triploid complement. The second division then follows its normal course and two triploid nuclei are formed. In other words the reduction of chromosome number is evaded and a female germ cell formed in this way, and developing as it often can, especially if unreduced, without fertilization, reproduces its 
female parent and continues to do so generation after generation. Such is the undoubted origin of triploid apomictic "species" of plants and animals.

There is, however, an omission in this account which would lead to an error if it were to go uncorrected. The pairing chromosomes in a triploid pair because they have crossed over and formed chiasmata. The sister chromatids at the second division, in a triploid as in a diploid, are therefore in part derived from partner chromo-

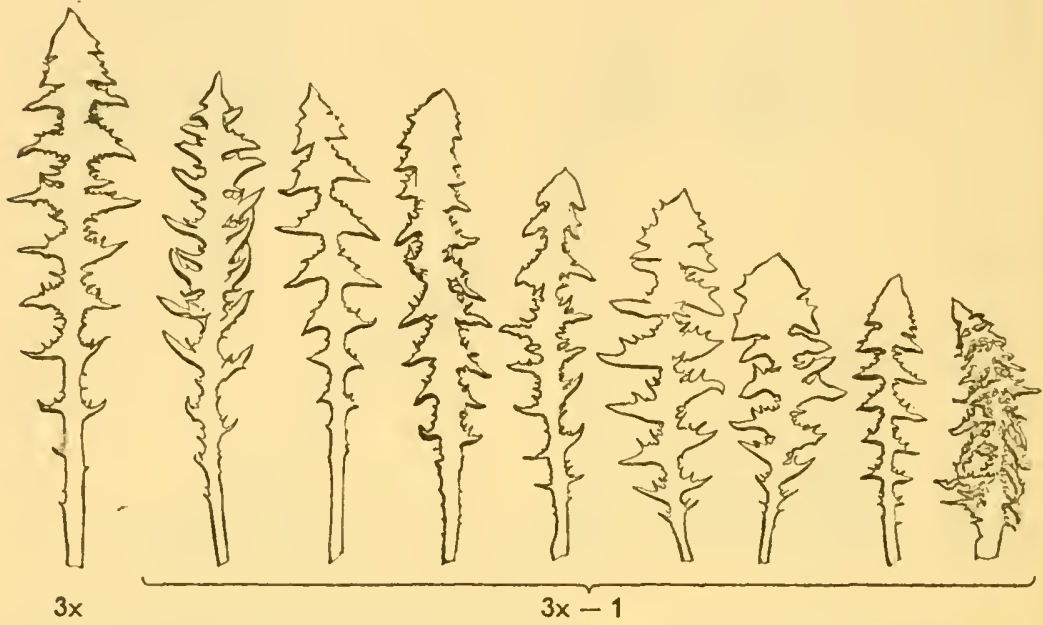

Fig. 67.-Leaves of the normal triploid Taraxacum polyodon and its eight types of disomic mutants occurring as apomictic seedlings in nature. $\times \frac{1}{4}$ (after Gudjonsson, 1946).

somes, i.e. they are dissimilar; their separation thus leads to the segregation of differences. The triploid egg cells of the original triploid apomict are not therefore genetically identical with it, or with one another. As compared with sexual species variation is much reduced, but it still occurs. The new apomictic species is thus often subsexual (Fig. 66).

The adaptation of apomicts for fertility in Taraxacum has evidently taken the form of making the suppression of meiosis more complete and therefore more regular. Efficicnt meiosis so desirable in a diploid has become a mere nuisance in a triploid. Triploid aponicts, in fact, show almost complete suppression of chromosome pairing and of the consequent reduction of number. 
The evidence of variation is worth returning to. It has been revealed most comprehensively in some apomictic stocks of common descent-for we must not give them the name of race or species intended for biparental groups-in Taraxacum. The range is not unlike that in Oenothera. Some of the variation is attributable to the

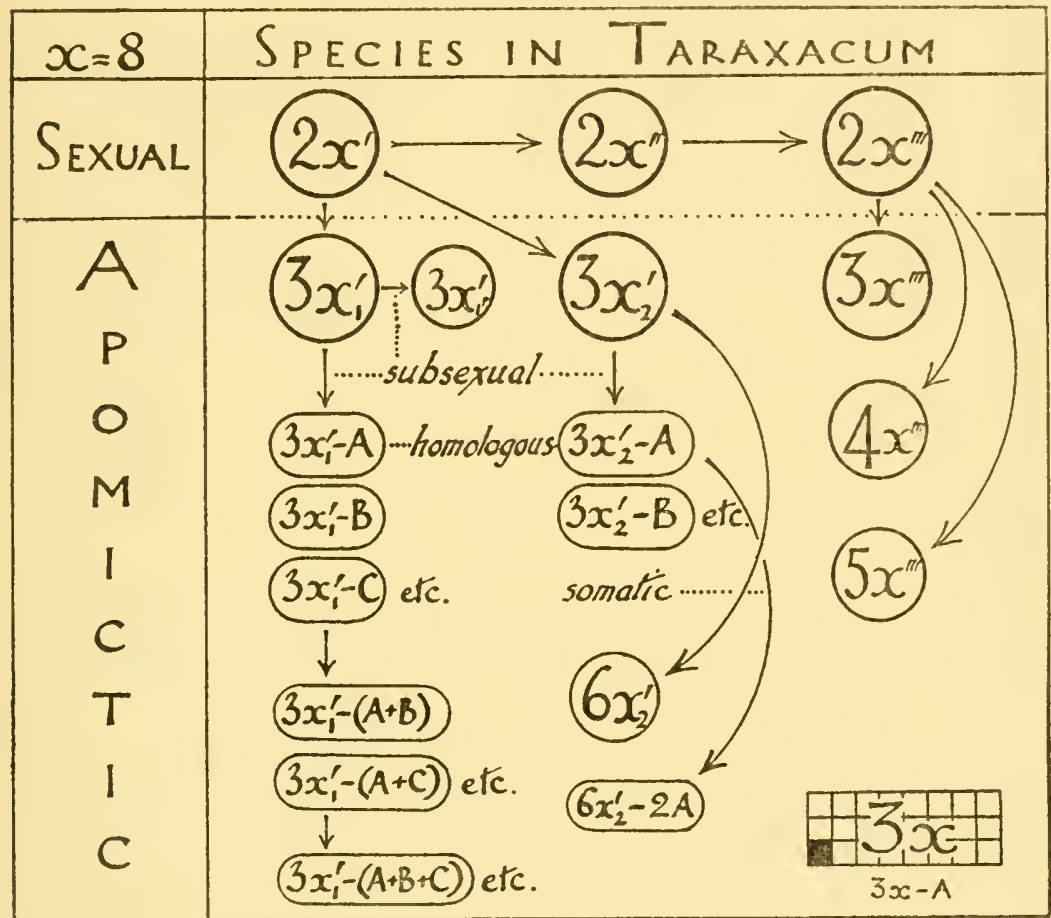

FIG. 68.-The origin of apomictic from sexual species in Taraxacum and their subsequent limited and irreversible evolution under conditions of sub-sexual reproduction, i.e. by recombination, loss and polyploidy at the suppressed meiosis. The unbalanced forms are homologous in different triploid series and are increasingly dwarfed with increasing unbalance (based on Sørensen and Gudjonsson, 1946).

mere effects of the crossing-over without reduction seen at the suppressed meiosis: the variants have the normal triploid complement of 24. Others are due to grosser aberrations. Chromosomes are lost to give types with 23 , i.e. one short. And these are recognizably of the 8 expected kinds, thus giving 8 parallel variants in each group (Fig. 67). Or again both meiotic divisions may fail so that the chromosome number is doubled and plants with 48 
(or 46) chromosomes arise. Again there are parallcl variants in the different stocks. Thus each stock of apomicts is a complex and sclf-sustaining system of variation (and adaptation) outsidc the ordinary sexual system, but neverthcless owing its character to an imperfect suppression of meiosis, whose imperfection inherently provides the means of perfecting itsclf (Fig. 68).

The breakdown of sexual reproduction comes about in many other ways. Onc further cxample will illustrate certain uscful principles. In the flowering plants, as a rulc, evcry embryonic secd or ovule contains only a single cmbryo-sac mother-cell. This cell gives by meiosis four haploid cells or spores. Only one of these sporcs can, as a rulc, devclop into an cmbryo-sac and, as we have scen, the sporcs may compctc for this opportunity, thereby giving the Renner effect. The cmbryo-sac in its turn contains a number of cells, only one of which can, as a rule, devclop into an egg capable of fertilization. The choice of the egg cell therefore depends on a series of choices during the development of the ovule (Figs. 50 and $S \mathrm{I}$ ).

Many and entertaining volumes have been written in a language of their own to describe variations and exceptions in this predestined course of differcntiation, even in the circumstances of normal scxual reproduction. We have already had cause to relatc the choicc of spore in embryo-sac formation to protein and nucleic acid gradients, and the variations and cxccptions no doubt also depend on the variable concentrations and distributions of proteins and nucleic acid available in the ovule. But from our present point of vicw what is important is that the issuc is not always cxclusively decided and sevcral cells are not uncommonly available for fertilization within one or more cmbryo-sacs; and at the same time some of thesc, and others outside the embryo-sacs, whether derived from meiosis or not, are often available for development without fertilization. Between these potential cggs and potential embryos therc must be competition for devclopment; competition whose outcome will depend on the two interacting factors of genotype and of position within the ovule. The Renner effect is thus but a special case of a morc gencral phenomenon.

The consequences of this free enterprise in development are found in many apomictic plants. Perhaps they appear best in Poa pratensis. 
Here a plant with 42 chromosomes can give progeny with 63 and 84 as well as 42 ; and a plant with 84 can give progeny with 42 and 63 and nearly 126 as well as with its own number. One plant with 72 even gave a vigorous seedling with $\mathrm{I} 8$. It is not surprising, therefore, that a world collection of 56 plants of Poa pratensis revealed such a distribution as that shown in Table 24. It follows a normal curve, produced evidently by continual variation from a mode between 60 and 70 .

TAB LE 24

THE WORLD FREQUENCY DISTRIBUTION OF CHROMOSOME NUMBERS IN POA PRATENSIS (HARTUNG, 1946)

\begin{tabular}{|l|l|l|l|l|l|l|l|l|}
\hline Chromosome number & 40 & 50 & 60 & 70 & 80 & 90 \\
\hline Frequency & & 3 & 13 & 25 & 12 & 3 & \\
\hline
\end{tabular}

This is an extreme instance of versatile reproduction, but great numbers of normally sexual species are known in which exceptional seeds reveal the same principle, since they contain twin embryos. One of these embryos is usually a normal sexual diploid, while the second is usually a haploid or a triploid which has arisen illegitimately in competition with its lawful twin (Fig. 69). These examples show that many plants, perhaps indeed individuals in nearly all species of flowering plants, have the capacity for evading meiosis or fertilization or both, and thereby giving rise, in the right circumstances of sexual sterility, to the obligatory apomict which cannot do otherwise.

In the normal sexual system, the hybridity optimum is related to the mode of breeding. The inbreeder has low hybridity, the outbreeder high hybridity, and the breeding system is genetically adjusted to secure these ends. This relation between hybridity and breeding system can be broken only by such extreme devices as complex hybridity or the abandonment of sexual reproduction. Complex hybridity and apomixis must have been derived from the normal sexual system, within which we can also see that the inbreeding system may replace outbreeding. What is it that determines these changes? What advantages do inbreeding and 
outbreeding confer on their possessors: Indeed, what is the advantage of the normal sexual system? These questions we shall examine in the next two chapters.

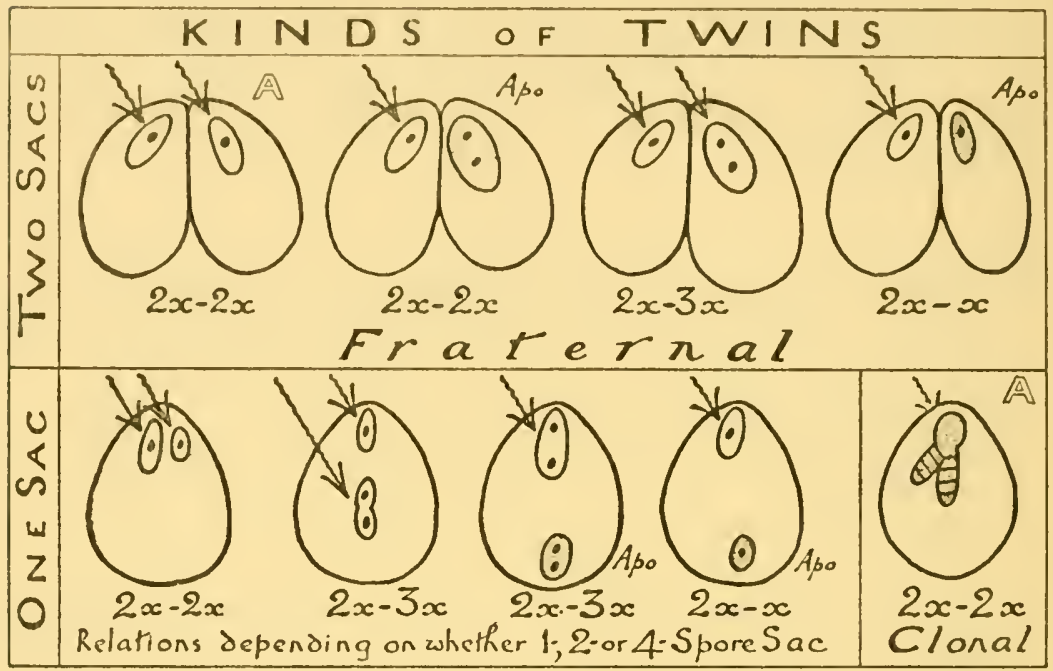

Fig. 69.-Twins produced in flowering plants classified according to their genetic relationship. One body in the nucleus stands for one haploid set. Arrows represent sperm which are assumed to be haploid (but can be diploid). Apomictic nuclei or embryos (which will develop without fertilization) are stippled. A, the two types formed in animals except that the type giving identical twins is not clonal but the result of fertilization. The lower group are fraternal or semi-fraternal, i.e. identical on the female side only (based on Linum, Kappert, r933; Poa, Secale, etc., Müntzing, 1933; Gossypium, Trifolium, etc., of. Skovsted, 1939).

\section{REFERENCES}

BARRIGOZZI, C. 1946. Über die geographische Verbreitung der Mutanten von Artemia salina Leach. Archiv. d. Julius Klaus-Stift., 2 I : 479-482.

BRIEGER, F. 1930. Selbsterilität und Kreuzungsterilität in Pflanzentreich und Tierreich. Berlin.

COOPER, K. W. 1937. Reproductive behaviour and haploid parthenogenesis in the grass mite, Pediculopsis graminum. Proc. Nat. Acad. Sci. Wash., 23: 4I-44.

CRANe, M. B., and LAwrence, w. J. C. 1947. The Genetics of Garden Plants. 3rd ed. London.

CRANE, M. B., and THOMAS, P. T. I940. Reproductive versatility in Rubus. J. Genet., 40: $109-128$.

DARIINGTON, C. D. I93I. The cytological theory of inheritance in Oenothera. J. Genet., 24: 40s-474. 
DARWIn, C. 1877. The Different Forms of Flowers on Plants of the Same Species. London.

DAVIDSON, J. 1927. The biological and ecological aspect of migration in aphides.

Sci. Prog., 85 .

EAST, E. M. 1929. Self-sterility. Bibliogr. Genet., 5: 33 I-370.

EMERSON, S. 1939. A preliminary survey of the Oenothera organensis population.

Genetics, 24: 524-537.

FISHER, R. A., and MATHER, K. 1943. The inheritance of style length in Lythrum salicaria. Ann. Eugen., London, 12: 1-23.

GOLDSCHMidT, R. 1934. Lymantria. Bibliogr. Gentet, II : I-I86.

hartung, M. E. 1946. Chromosome numbers in Poa, Agropyron and Elymus. Am. J. Bot., 33: 516-531.

LEWIS, D. I944. Incompatibility in plants: its genetical and physiological synthesis. Nature, 153: 575-578.

mather, K. I943. Specific differences in Petunia. I. Incompatibility. J. Genet., 45: $215-235$.

MATHER, K., I944. Genetical control of incompatibility in Angiosperms and Fungi. Nature, I 53: 392-394.

MATHER, K., and DE WINTON, D. I94I. Adaptation and counter-adaptation of the breeding system in Primula. Anm. Bot., N.S., 5: 297-31 I.

MULLER, H. J. I9I8. Genetic variability, twin hybrids and constant hybrids in a case of balanced lethal factors. Genetics, 3: 422-499.

RENNER, O. 1946. Artbildung in der Gattung Oenothera. Naturwiss., 33: 2 I I-2 I 8.

RICK, C. M. 1947. Partial suppression of hair development indirectly affecring fruitfulness and the proportion of cross-pollination in a tomato mutant. Am. Nat., $81: 185-202$.

SEILER, J. I920. Geschlechtschromosomen-Untersuchungen an Psychiden I. Arch. Zf., I5: 249-268.

SKovsted, A. 1939. Cytological studies in twin plants. Comp. Rend. Lab. Carlsberg, 22: $427-446$.

SøRENSEN, T., and GUDJONSSON, G. 1946. Spontaneous chromosome aberrants in apomictic Taraxaca. Kong. Dansk Vid. Selsk. Biol. Skr., 4 (2).

whitehouse, H. L. K. 1948. Sex and heterothallism in the fungi. Biol. Revs. (in the press).

wiLliamS, R. D. 1939. Incompatibility alleles in Trifolium pratense L; their frequency and linkage relationships. Proc. 7th. Int. Cong. Genetics, 316. 


\section{SELECTION AND VARIABILITY}

Selection Darwinism and Genetics The States of Variability Fitness and Flexibility

The Effect of Linkage The Control of Recombination Balance in Homozygotes and Heterozygotes Selection and the Reservoir of Variability

Change of Genetic Systems: Inertia Correlated Response:

Capital and Subordinate Characters

Almost from the beginning of Genetics we are bound to examine and discuss variation. New forms of plants and animals are the materials for the study of heredity. But what happens to these forms in nature? It is obvious that a lethal mutation extinguishes the individual in which it expresses itself. Those which cannot live must die. It is less obvious, but no less certain, that many mutations which are not lethal make their possessor's survival doubtful, or at least reduce his chance of leaving progeny.

Drosophila subobscura, for example, uses its power of sight in courtship, and Rendel finds that flies made blind by the absence or abnormality of eyes are unable to mate. The genes producing this effect are not lethal somatically; but they are lethal genetically. They must therefore be selected against, or as we may say, they have a negative survival value. Again this is an extreme case; but any mutation which impairs the faculties of its possessor must have a survival value reduced by an amount proportional to the impairment.

\section{Selection}

The principle of selection can now be seen in much greater breadth and depth than would have been possible at the beginning of this book. In the first place we can see it operating in different stages of development and at different levels of organization. The defective cell which arises by gain or loss of a chromosome is eliminated as readily as the defective individual. This cell also may be a spore or a fertilized egg. In the Renner effect we see the defective spore removed. Also we notice that it is removed owing to a defect 
which is by no means lethal in an absolute sense, but is fatal to

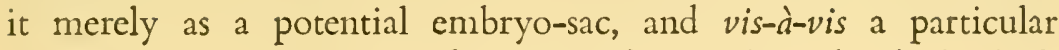
competitor. Selection, therefore, must favour the individual which fits the conditions of its environment better at all stages of development.

Thus we can establish two important generalizations; first, that heritable differences occur between different individuals of any group; and secondly, that, as a consequence of these differences, the individuals enjoy different, yet characteristic, chances of surviving and leaving offspring under the conditions of incomplete survival, arising from both internal failure and external competition, that exist in nature. As Darwin and Wallace pointed out, natural selection will work to eliminate some variants and to perpetuate others. The theory of evolution by natural selection relates the whole of evolution to this process. As conditions change and as new variants occur new types will arise, better fitted, or adapted, than their predecessors to survival in the reigning environment. By virtue of these new adaptations evolutionary change will constantly be going on: cvolution is the sum of adaptation.

\section{Darwinism and Genetics}

Genetics has strengthened and amplified Darwin's theory in both of its basic relations: to variation and to selection. Darwin was able to show that hereditary differences occurred between individuals on the scale required by his views, but he was never in possession of a theory of heredity capable of relating them to the action of natural selection.

This lack of knowledge of the mechanism of inheritance placed Darwin in a dilemma about variation, to the solution of which the whole of his work on Animals and Plants under Domestication was unsuccessfully devoted. He thought that inheritance was blending: that the contributions made by two parents to the hereditary endowment of their individual offspring blended like ink and water. Throughout posterity no separation was then possible of the maternal and paternal elements. Thus variation was always being lost, nay destroyed, to the extent of a half in every generation of a randomly breeding population. Either evolution 
must come to a standstill, or the loss must be made good. Only by the production of new hereditary variation on a vast scale could this come about. It was to the means by which this new variation was produced that Darwin devoted his discussion. He supposed that the effects of domestication in altering plants and animals were direct effects of the changed environment. His difficulties, in fact, ultimately led him to adopt the ancient hypothesis of direct adaptation, the Lamarckian inheritance of acquired characters, which he called pangenesis.

While Darwin was wrestling with this problem, Mendel had already solved it: or rather had shown that it did not really exist. On the mendelian, or indeed any particulate theory of inheritance, as Fisher has pointed out, crossing does not destroy variation. When a tall pea is crossed with a short one, the parental difference vanishes in the $F_{1}$. But the disappearance is only temporary: both parental types appear once more, side by side, in the $F_{2}$. Whatever differences disappear into a hybrid by crossing, reappear in its progeny by segregation. And since there is no permanent loss of variation by crossing there need be no production of new variation on a correspondingly large scale. Nor is there any large scale production of new variation, as Johannsen proved when he established his pure lines. Mendel's peas removed the need for postulating the rise of variation on a grand scale: Johannsen's beans showed that it did not occur. Darwin had been misled by not knowing enough about heredity.

Mendel's experiments thus gave Darwinism the foundation it needed. Later experiments in genetics, by developing its particulate theory in the way we have seen, add to the strength of the joint structure. They show us that the most important changes in conditions, which lead to selective adjustment, are not, as was supposed, changes in the inanimate world: they are changes in heredity. One class of these, which we may mention in passing, are such as occur in other species, especially where the species live in the host-parasite relationship. All organisms are either hosts or parasites or both. Any genetic change in either is liable to affect the other, and a continual succession of inutual adaptations is the result. Each mutation to greater virulence by the parasite leads to selection of a mutation for greater resistance in the host. The system is showir 
diagrammatically in the relations of bacterium and bacteriophage described by Delbrück and Luria, and is also very simply demonstrated by the recent history of a wide range of crop plants.

A second class of changes leading to selective adjustment are those in other genes of the same individual or race. Selection is a means of fitting the genes to one another. If one gene in a population is changed, all the others are exposed to new conditions of selection. Thus, as we saw, a culture of "eyeless" Drosophila becomes modified after a few generations. Polygenic modifications are selected which prevent the eyeless gene expressing itself so drastically. The environment for any one gene includes, therefore, the other genes.

In this light we can see that the genic balance we have been discussing must always be the product of selection. Good balance will be one which has been exposed to selection under the established conditions and is consequently adapted to those conditions. Bad balance will be one which is not adapted to those conditions. Thus, while genetical principles enable us to see the answers to many questions of evolution, it is equally true that Darwin's principle of natural selection shows us how the genetical property of balance comes about. In the same way it shows us both the meaning and the origin of genetic systems. We have seen how the breeding system is genetically adjusted, and indeed Darwin was himself aware that the individuals of a species stood in a special adaptive relation to one another in reproduction. He was the first to show that the cnforcement of inbreeding on plants which naturally outbreed resulted in a decline of vigour and fertility; but he was baffled by the general problem of these adaptations. He saw that the sex-ratio of a species must be selectively adjusted, but was unable to see how the selection could be achieved; for he was unable to make out how adjustment of the sex-ratio, or indeed of any other property of the breeding system, could benefit the individual-which indeed it does not.

This is the problem which we must now consider in order to see why some species outbreed and some inbreed; why there is sometimes a change from one to the other, or even the adoption of some more drastic device. We must compare these systems 
through their effects on Darwinian fitness, the competitive power of the individuals comprising the species. How will inbred and outbred populations differ in the genotypes and phenotypes of their individuals?

To answer this question we must look at the implications of clementary Mendelism, not now for experimental families, but for massed populations. The static must be translated into the dynamic. And in doing this, to understand crossbreeding and inbreeding, we shall find that we coine to understand the organization of heredity in the wider sense of the interdependence of the relations between the genes in the nucleus and between the individuals in the population.

\section{The States of Variability}

Homozygotes and heterozygotes always differ in one respect, the respect by which they are recognized, viz. that heterozygotes give segregation in their progeny while homozygotes do not. If we breed a homozygote, or a group of like homozygotes, the offspring will be genetically and, apart from non-heritable fluctuations, phenotypically identical both with their parents and with one another. If, however, we breed heterozygotes (whether for one gene or for many), the offspring are neither all alike nor all like their parents, genetically or somatically. Variation has appeared as a result of segregation. Now the germ of this variation must have existed in the heterozygotes, even though they themselves showed no variation. Thus we may distinguish between the latent or potential variability which heterozygotes themselves contain, and the variation or free variability which will make itsclf apparent in the phenotypes of their offspring when segregation has occurred.

Let us consider a simple theoretical situation on a mendelian basis and, in the first place, in the absence of selection. A continued programme of inbrecding, by self-pollination or the mating of close relatives, when applied to the offspring of heterozygotes, will cventually result in the establishment of a population of homozygotes. Half of these will carry one $(A A)$ and half the other $(a a)$ of the two allelomorphs of any gene for which the original ancestors were heterozygotes $(A a)$. The variability due to such a gene will then have changed from being entirely potential in the heterozygous 
ancestors, to being entirely free in the homozygous descendants. Crossing two unlike homozygotes among these descendants $(A A \times a a)$ will restore the ancestral condition: the variability will once more be entircly potential. Variability is thus set free by segregation, and locked up by crossing unlike types.

An inbrecding population consists completely of homozygotes, apart from the effects of mutation; but a crossbreeding population cannot consist only of heterozygotes, except in the special case where the gene happens itself to determine the breeding system. A gene such as that controlling incompatibility in cherrics must always be in a heterozygous condition, because individual gametes alike in respect of it cannot be brought together. But a gene without this effect on the breeding system is in a different case. Maximum outbreeding in a large population can do no more than maintain such a gene in the states and frequencies given by random mating. With random mating, where the relative frequencies of the two allelomorphs ( $A$ and $a$ ) are $\mathrm{u}$ and $\mathrm{v}$, the frequencies of individuals of the types $A A, A a$ and a $a$ will clearly be:

$$
A A \mathrm{u}^{2}: A a \text { 2uv : } a a \mathrm{v}^{2}
$$

and the maximum proportion of heterozygotes will be 0.5 , achieved when $\mathrm{u}=\mathrm{v}$. With more than two allelomorphs the proportion of hetcrozygotes may be higher.

Now, potential variability in respect of a single gene can be carried only by hetcrozygotes. A crossbreeding population will therefore have some of its variability free and some potential. With two allelomorphs of equal frequencics, half the population will be $A a$ and half the variability must consequently be potential. Segregation will release half this potential variability in each gencration, but at the same time inter-crossing of $A A$ and a will be returning half the free variability to the potential state. These two processes thus balance one another, and the proportion of free to potential variability will remain constant. Neverthcless, because of the continual interchange of variability between the free and potential states, the situation cannot be a fixed one. There is, in fact, a flow of variability: any particular variant, although present in the same proportions in different generations, appears in different families or lineages. The population may be, in a certain sense, 
fixed or stable; but the genes are still moving and their combinations are changing.

The flow of variability can be altered by selection; that is by the choice of certain types of individual as parents of the next generation. For example, we (or nature) can take as parents only the heterozygotes and one of the two types of homozygote $(A a$ and $A A)$. We then effectively cut off the return of free variability to the potential state, by rendering impossible the cross $A A \times$ aa, through which this return comes about. At the same time we do not interfere with the reverse change, from potential to free, which depends on segregation amongst the progeny of heterozygotes. The net result of such a selection of parents is thus to lower the potential and to raise the free variability. The proportion of heterozygotes falls in the next generation; that of the homozygotes, of the two types taken together, increases. The joint increase is, however, due to increase of only one of these homozygous types, $A A$.

In this process of selection the gene frequency has been changed. One allelomorph $(A)$ is now more, and the other $(a)$ less, common than formerly. The average phenotype will have moved in the direction of the more common type. If the selection is continued indefinitely the population will ultimately come to consist wholly of the one homozygote $(A A)$, the other allelomorph of the gene having been eliminated. No variability will be left: it will have been expended on the cliange in average phenotype of the population. Thus selection of particular parents in each generation changes the mean phenotype, and this change is brought about by an alteration of the flow of variability in such a way that ultimately it will all pass into a fixed state. Selection must then cease to produce any further change, because all the individuals have become genetically alike. Any differences they may show will be nonheritable. In a word, selection cannot produce changes unless free variability exists in the population. At the same time selection fixes, we may even in one sense say destroys, the variability on which the change depended. The alteration is permanent.

A further aspect of this relation between variability and selection is seen when it is the heterozygotes which are used as the sole parents of the next generation. The flow from potential to free variability must go on, since (with the exception we have seen in 
the complex heterozygote) segregation cannot be prevented. This segregation will be repeated regularly, and since the homozygotes are eliminated as parents in each generation, the population will have the same composition each time after the first selection. It will always be $\frac{1}{4} A A: \frac{1}{2} A a: \frac{1}{4}$ aa. Selection is not lowering the potential variability. No variability is being fixed, and selection is therefore ineffective in changing the average phenotype.

With one gene difference in operation, as in the case we have been considering, there are only two states of variability. The heterozygotes contain all the potential variability, while the difference between the phenotypes of the homozygotes always expresses the full action of the gene. There are, however, more possibilities where, as in polygenic variation, the genes have similar and supplementary effects on the phenotype. Even in the absence of dominance, different genotypes can, in such a case, give phenotypes differing only to the extent by which the genes do not correspond in effect. With genes of equal effect the same phenotype can be produced by a number of genotypes. In particular, genetically dissimilar homozygotes may, as we saw in Chapter 3, show like phenotypes.

Thus with only two genes of equal effect, the homozygotes $A A b b$ and $a a B B$ will be alike, and will be intermediate between $A A B B$ and $a a b b$ in their phenotype (Fig. Is). Yet these two intermediate homozygotes contain between them all the genetical material necessary for the production of the whole range of variation to which the two genes can give rise. They contain a new kind of variability, the potential variability of homozygotes, as opposed to that of heterozygotes. This new potential variability depends for its existence on the genes $A$ and $b$, or $a$ and $B$, balancing one another in action.

Unlike the potential variability of heterozygotes, which not only can, but must, be partly freed by segregation in the next generation, the homozygotic potential will remain as such so long as crossbreeding is absent or at least restricted to like homozygotes. Thus, with close inbreeding, a population could be maintained which consisted of pure lines uniformly of the same phenotype, yet genetically of several kinds. The potential variability would be, as it were, frozen in such a population. Only by the crossing of unlike 
homozygotes could it be freed, and even then not immediately (Fig. 70). The first effect of crossing must be to produce heterozygotes in which the variability is still potential. Only in the second, segregating, gencration derived from these heterozygotes will the

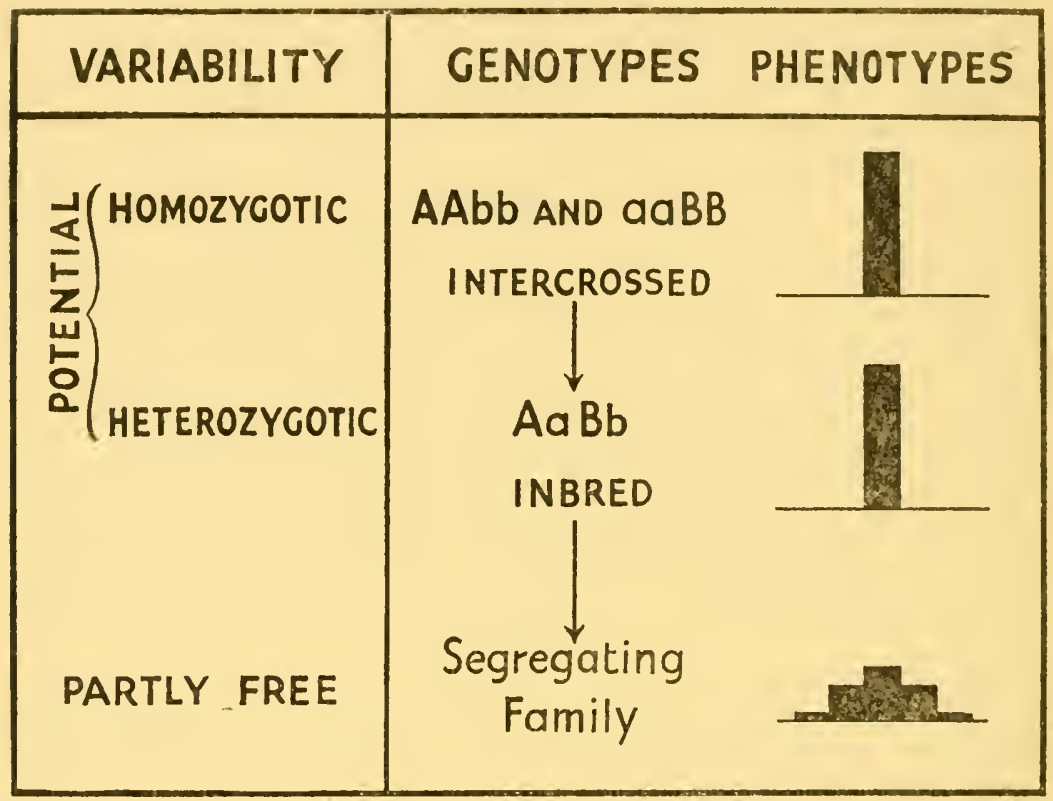

FIG. 70.-Part of the potential polygenic variability of heterozygotes is released, so that it shows as free variation in the phenotypes, by segregation in one generation. The potential variability existing in the differences between homozygotes, which are genetically unlike but having a similar balance and hence similar phenotypes, cannot be released in one generation. It must first be converted into heterozygotic potential by intercrossing. For the purpose of exposition in the diagram the genes $A-a$ and $B-b$ are assumed to be alike and additive in their action, and also to show no dominance. Thus $A A b b, a a B B$ and $A a B b$ have the same phenotype (neglecting non-heritable variation), but the segregating fanily includes five phenotypes, in the frequencies shown also by Fig. Is (after Mather, 1943).

more divergent phenotypes be produced and the variability become partly frec-only partly free because the balanced homozygotes will themselves reappear as part of the segregating generation.

The heterozygotic potential state is therefore an essential intermediate step in the freeing of homozygotic potential variability. But on the other hand, the crossing of the phenotypically extreme $A A B B$ and $a a b b$ gives $A a B b$ from which $A A b b$ and $a \cap B B$ must 
segregate in the second generation. The heterozygotic potential is thus equally the intermediate step in the opposite process-the transference of free variability to the homozygotic store.

Where more than two genes are concerned the same principles apply: there is merely a greater range of possible balanced homozygotes. In the case of four genes having equal and supplementary

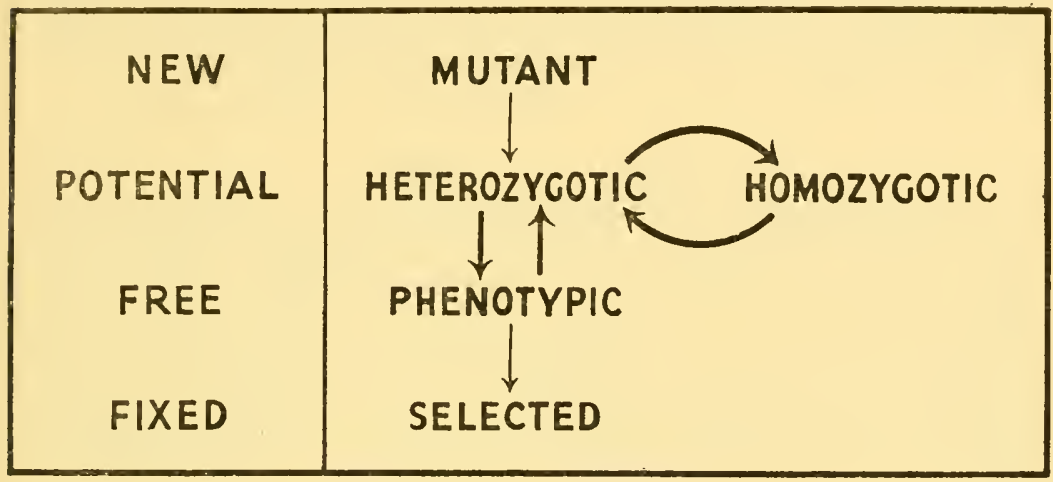

FIG. 7r.-The states of polygenic variability. There is a constant flow between the free and potential states, the heterozygotic being distributed by segregation to the free and homozygotic states in proportions depending on the linkage relations of the polygenic system, and the free and homozygotic variability passing into the heterozygotic state by crossing. There is no direct flow between free and homozygotic potential. Free variability is the raw material for selection, which is constantly using up or fixing a portion of it. This loss will be balanced over long periods of time by the rise of new variability through mutation; but the balance will not generally be struck accurately over short periods of time. The thinner arrows indicate that the loss by selection and gain from mutation are slower than the flow between the other three states (after Mather, I943).

effects, for example, six homozygotes exist of the general type $A A B B c c d d$, and four each of the less balanced general types $A A B B C C d d$ and $A A b b c c d d$. The more the genes, the greater the variety, and hence importance, of the homozygotic potential state.

Since the variability must all go through the heterozygotic potential state before it can be redistributed between the homozygotic states, free and potential, the proportion of heterozygotes in a population will govern the rate of flow of variability from one state to another (Fig. 7I). Heterozygotes can, of course, arise from the inbreeding of pre-existing heterozygotes, but under such a system they will be present in a proportion which decreases from 
generation to generation. A fixed proportion of heterozygotes in the population can be maintained only by means of crossing or outbreding, and any given amount of outbreeding will maintain a corresponding proportion of heterozygotes. The brecding system, in determining the proportion of heterozygotes, determines the rate of flow of variability between the different states. Random mating gives virtually the maximum outbreeding and the maximum flow, while close inbreeding gives no flow at all. Outbreeding means genctical lability, inbreeding genetical fixity.

\section{Fitness and Flexibility}

The importance of the difference in effect between inbrecding and crossbrecding bccomes clear when we consider what natural selection is doing. If we obscrve a character in which a population is freely and contimuously variablc, as for cxamplc stature in man, we find that most individuals show it to an intermediate degree and the extreme expressions arc rarc. As Galton put it, the majority arc mediocre.

The significance of this is shown by Bumpus' obscrvations. He found that a number of characters, bone lengths, wing spread and skull conformation, varied in a sample of sparrows in the same way as stature in man, the mediocre again bcing the most common. Now these sparrows had been disabled in a rainstorm, but some of them later recovered and flew away. The birds which thus overcame the ill cffects of the storm proved to be those which approximated most closely to the form characterized by means of the various measurements. Those that died were the more extreme individuals, regardless of the direction, positive or negative, in which they were extremc. Natural selection was operating in favour of the mcan, and against departure, whatcver its dircction, from the common typc. So far as the variation of these sparrows was heritable (and while there is no cvidence on this point in regard to the sparrows, it is the general rule derived from a wide variety of organisms that a portion of such continuous variation is polygenically determined), the effect of selection was to favour the morc balanced combinations of genes.

Natural selection has been observed in operation in this way on 
very few occasions, but the favouring of the mean type is in agreement with expectation. If one extreme were favoured consistently, the mean of the population would move in that direction, in so far, of course, as the variation was heritable. And it would continue to move as rapidly as the available variability permitted until it approximated to the optimum phenotype, at least sufficiently well for the environment not to favour departure preponderantly in one particular direction. In the third case, where both extremes are favoured at the expense of the mean, a new state of affairs arises, one we shall consider in the next chapter.

A character such as fertility might be regarded as being in a different situation, for one might expect greater fertility to be favoured almost without limit. It must be remembered, however, that fertility is itself the expression of a number of sub-characters, and these must be balanced against one another. To take an example, man has a smaller number of offspring at a birth, or in a lifetime, than the pig. But we could hardly regard an increase to the pig's litter size as likely to increase the expectation of posterity in man, because the success of each child requires an expenditure of parental care and training which would thereby be rendered impossible. Too many offspring would be as bad, though in a different way, as too few.

The adverse effect of too large a litter can be seen even in the pig itself. The mortality between birth and the age of three weeks becomes so great in litters of $\mathrm{I} 4$ and more that the number of pigs surviving to this age is somewhat lower in the bigger litters than it is in those of I4 and I5. At six weeks the disadvantage of too large a litter is still more striking and the size of litter which gives the maximum average number of survivors is even lower than at three weeks (Fig. 72). In the same way, other things being equal, the excessive production of eggs or seed by any animal or plant would mean a crippling reduction in the food supply with which each was endowed. Thus with fertility, too, the principle of the optimum must apply.

What will be the effect of this principle that the average is favoured at the expense of the extremes? With the inbreeding system, where the population consists entirely or very largely of homozygotes, the effect of selection in favour of the intermediate 
classes must be mainly to favour the more balanced homozygotes at the expense of the less balanced. The favoured individuals will produce offspring genetically like themselves; and in so far as the environment is stable, the population must show high agrecment with the optimum phenotype. Should the environment change

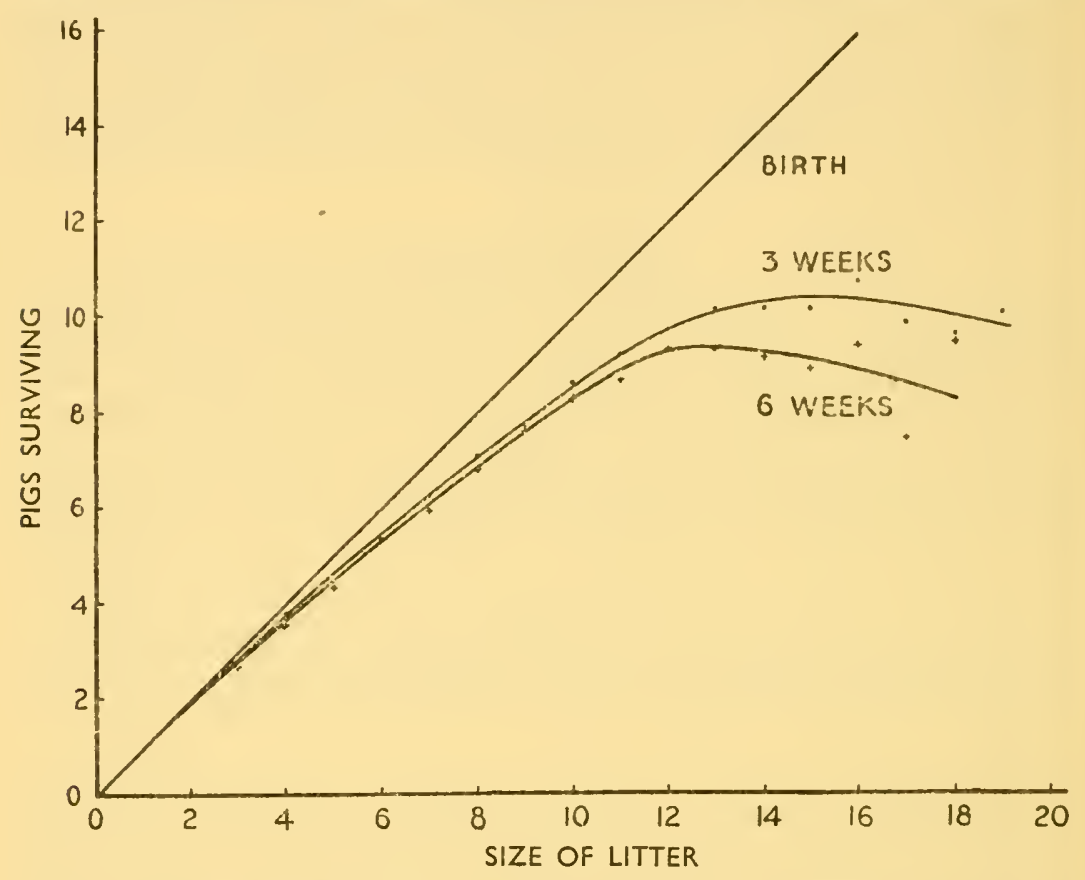

FIG. 72.-The average numbers of young surviving for 3 and 6 weeks from litters of various sizes at birth in pigs. The straight "birth" line is the line of no loss. The loss becomes disproportionately greater as the size of litter increases. The maximum survival to 3 or 6 weeks old is given by litters of intermediate size at birth. (Data for 3 weeks (as dots) from Johansson, I93 I, and for 6 weeks (as crosses) from Menzies-Kitchen, 1937.)

permanently, however (as sooner or later it presumably must) the population cannot change genetically in the way necessary to give a new adjustment: the variability is frozen in the homozygotic potential form. Such a population, therefore, shows high immediate fitness but no flexibility. The inbreeding system, advantagcous so long as the environment is stable, becomes a handicap when the cnvironment changes.

A population with an outbreeding system is in the other case. 
Balanced genotypes of intermediate phenotype are favoured; but owing to the flow of variability, which arises from the outbreeding, the extreme types can never be lost. They will constantly reappear by segregation. Thus the population must always vary around the optimum, and fitness can never be so high as in an inbreeding group. The flow of variability ensures, however, that should the environment change, and thereby shift the optimum phenotype, the population will be able to achieve a corresponding adjustment by utilization of the free variability which is always available. Individuals approximating to the new optimum will be present and, being favoured by the new conditions, will contribute more to the next generation. Thus the genetical constitution of the population can, and will, change to meet the demands of the new conditions. The system does not maintain the maximum immediate fitness, but it is flexible. As compared with inbreeding it is certainly at a disadvantage for the moment; but it confers on an organism a much greater prospect of leaving descendants in a changing world.

\section{The Effect o, Linkage}

The disadvantage of high variability under the outbreeding system may be mitigated, and the opposing needs of fitness and flexibility be partly reconciled, in a way which has been revealed by selection experiments. One of these, described by Sismanidis, was concerned with the number of bristles borne on the scutellum of Drosophila melanogaster. This number is almost always 4, but in some mass-bred stocks occasional flies, mainly fenrales, are found with s. Commencing with such females mated to normal males, Sismanidis endeavoured to raise the average bristle number by selective breeding. In this he was successful. Two of his selection lines, maintained by brother-sister mating, are shown in Fig. 73 . After 23 generations of selection the average was raised in females from below $4 \cdot 1$ to over $5 \cdot 2$, but the advance had not been smooth. There was a large change from just below $4 \cdot 2$ to over $4 \cdot 5$ between generations 2 and 3 in one line, and between generations 6 and 7 in the other. In the next II generations in the one and 7 in the other the advance was only $0 \cdot 1$, but between generations $I_{4}$ and 17 a 
second quick change of nearly 0.6 occurred in both, to be followed by another period of near stability until the end of the experiment. The responscs to the selection shown by the males werc smaller, but conformed to the same pattern.

Tests were made to determine the effects of the three major chromosomes on bristle number at gencrations $0, I_{3}$ and 2 I,

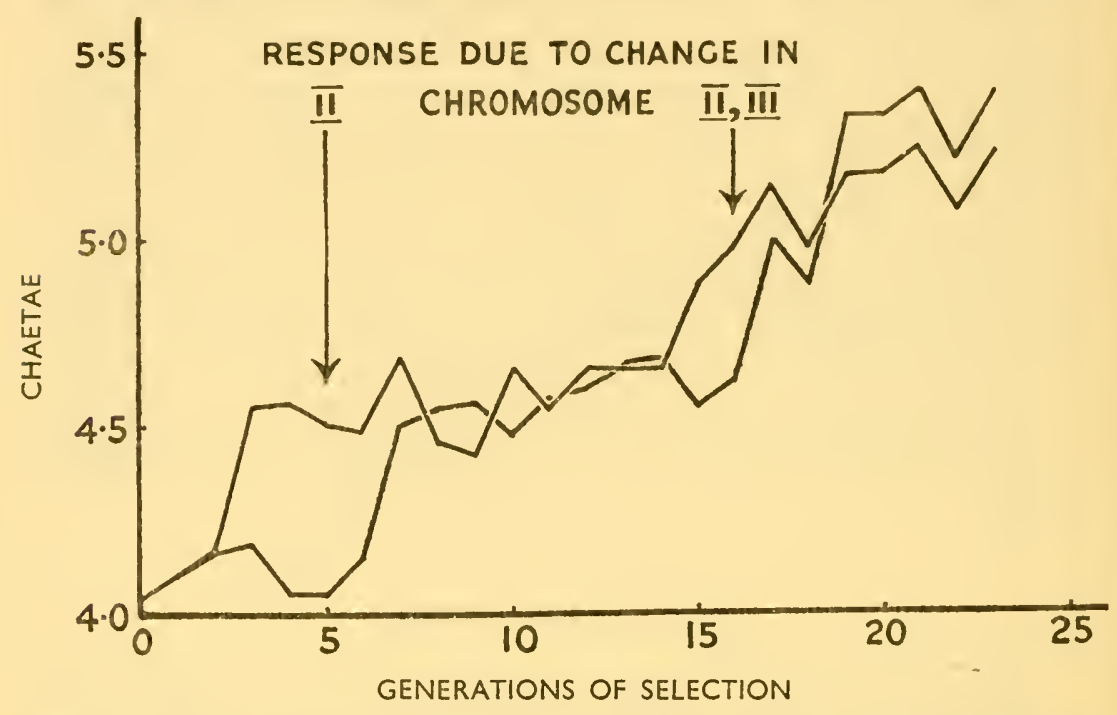

FIG. 73.-The effects of selection for increased number of scutellar bristles in Drosophila melangastcr. The average number of scutellar chaetae is plotted against generations of selection, all matings being of brothers and sisters. The two selection lines came from the same parents in generation $\mathrm{I}$, and gave parallel results except that the first major response occurred four generations later in one than the other, and the second major response perhaps one generation later. The responses were nevertheless of equal or nearly equal size, and due to change in the same chromosomes, in the two lines (based on Sismanidis, 1942).

i.e. before selection commenced, after the first chief advance, and after the second. They showed that the first advance in both lines was due entirely to an increase in bristle-producing power of chromosome II; while again in both lines the later advance also involved chromosome III. The X chromosome appeared not to have changed in either line during the experiment. Such regular changes can hardly be attributed to new variability arising by mutation. To what are thcy due?

Sclection, as we have scen, is effcctive in changing the mean 
phenotype only in so far as there is free phenotypic variability, which becomes fixed as a result of the selection. Since the method of selection was constant throughout Sismanidis' experiment, we must suppose that free variability was low or absent during the periods of little advance, but that it was present when the two chief responses to selection occurred. That one of these came after generation I4 shows that variability was present during the preceding generations; but since the response was delayed, the variability must have been largely in the potential state.

Now potential variability is freed by segregation and recombination. In the present case the chromosome tests showed that the changes occurred within whole chromosomes. In other words, the effective recombination must have been of linked genes, as a result of crossing-over between them. Originally in the form of balanced linked combinations of polygenes, crossing-over must have led to the production of less well balanced genotypes, giving more extreme phenotypes, i.e. to the freeing of variability which must have been present in the group of parents with which the experiment began.

Linkage must have the general effect of tending to maintain genes in the same combinations even in cross-bred and heterozygous populations. If we cross $A A B B$ and $a a b b$, where the genes are linked, the combinations $a B$ and $A b$ will be produced in the segregating generation only so far as crossing-over gives rise to recombination. If the linkage is close, they will be uncommon for some time. The combinations $A B$ and $a b$ will be released equally slowly where the parents are $A A b b$ and $a a B B$. Thus, while the proportion of heterozygotes, and hence of heterozygotic potential variability, will depend solely on the breeding system, the rate of flow of free variability to the homozygotic potential state and back will be governed by linkage as well. The flow will be at its maximum when recombination is free, for a double heterozygote then produces equal numbers of $A B, a b, A b$ and $a B$ combinations no matter what its origin may have been. But at the other extreme, with close linkage, the interchange of variability between the free and homozygotic potential states will be small: after passing into the heterozygotic pool, free variability will mostly emerge as free variability, and homozygotic potential as homozygotic potential. 
Where natural selection is penalizing the less well balanced combinations of genes in a chromosome, genes of opposing or balancing effects must tend to become tied together by the linkage. The population will then show a smaller spread round the optimum phenotype than it would in the absence of linkage. Fitness will consequently be higher, as it is with an inbreeding system. But the more extreme combinations will still be produced on occasion by crossing-over; and, even though less common, they will still afford the material for selective adjustment of the genotype to changing environment. In fact they will be just as effective in this way as if they were released with the unlinked frequencies. Flexibility will therefore be fully maintained at the same time that fitness is increased. In a large measure, linkage can reconcile their rival needs.

\section{The Control of Recombination}

We may now ask oursclves: how tight is linkage in practice? How much crossing-over actually takes place between paired chromosomes: The chiasmata have shown us that all chromosomes in all species of sexually reproducing organism undergo crossingover, and that the position of crossing-over in any particular pair varies from cell to cell and therefore, obviously, from generation to generation. It sometimes happens, for example in Vicia faba and in many species of lilies, that cach pair of chromosomes forms an average of five or more chiasmata-far more than the bare minimum number needed to hold them together at the first metaphase of meiosis. But this is rare. In gencral, what we find is a close restriction of the number of chiasmata to this mechanical need. Some variation in number is doubtless required if variation in position is to be maintained, and two-armed chromosome pairs frequently form two chiasmata.

The adjustment of chiasmata to a minimum has been neatly shown in maize. An "asynaptic" gene, in the homozygous state, reduces the frequency of chiasmata at meiosis. Indeed in extreme cells of extreme plants it causes the chromosomes to fall apart at the end of pachytene without any formation of chiasmata at all, so that they are all unpaired at diakinesis and metaphase. Typical samples are shown in Table 25. 
TABLE 25

CHIASMA FORMATION AND CHROMOSOME PAIRING IN NORMAL AND ASYNAPTIC MAIZE (BEADLE, 1933)

\begin{tabular}{|c|c|c|c|c|c|c|c|c|}
\hline & & \multirow[t]{2}{*}{$\begin{array}{l}\text { Number } \\
\text { of cells }\end{array}$} & \multicolumn{4}{|c|}{$\begin{array}{l}\text { Numbers of bivalents with } \\
\text { different numbers of } \\
\text { chiasmata }\end{array}$} & \multirow[t]{2}{*}{$\begin{array}{l}\text { Average } \\
\text { bivalents } \\
\text { per cell }\end{array}$} & \multirow[t]{2}{*}{$\begin{array}{c}\text { Average } \\
\text { chiasmata } \\
\text { per } \\
\text { bivalent }\end{array}$} \\
\hline & & & 0 & 1 & 2 & 3 & & \\
\hline Normal . . .. & . & 10 & 0 & 27 & 72 & 1 & $10 \cdot 0$ & $1 \cdot 7$ \\
\hline Asynaptic, moderate & . & 27 & 50 & 49 & 171 & 0 & $8 \cdot 1$ & $1 \cdot 4$ \\
\hline Asynaptic, extreme & . & 58 & 552 & 28 & 0 & 0 & 0.5 & $0 \cdot 05$ \\
\hline
\end{tabular}

When the asynaptic plants are bred, one might expect their progeny to show a great reduction in the frequency of crossing-over. The reduction, however, is not uniform. On the female side, in fact, there is no reduction at all. The explanation is instructive in several ways. Either the action of the gene in upsetting meiosis is different on the female side. Or the selected sample of the products of meiosis is different. The germ cells which are effective in breeding are those with a complete set of chromosomes. Complete sets are produced only when the mother cell, at least in the large female cell where unpaired chromosomes are always lost, has had complete pairing. And complete pairing has been attained in those cells only in which the chromosomes had a normal, or nearly normal, frequency of chiasmata. In the male cells incomplete pairing will sometimes give complete sets by chance. And on the male side some reduction of crossing-over is found. The results are: normal, 24 per cent recombination between the genes shrunken and waxy; female asynaptic, 24 per cent; male asynaptic, I3 per cent.

Apart from low-frequency adjustment there are two other methods of reducing the effective frequency of recombination. One is by localization. In a greater or less degree all organisms have some localization of chiasmata. This localization seems to be produced by a delay in pairing, so that the later pairing produces no coiling strain or is even totally inhibited. Crossing-over is then confined to the region where pairing began. In some species, such as the grasshopper Mecostetlus grossus or the lily, Fritillaria meleagris, the crossing-over is localized near the centromere. In others, a more numerous congregation, it is localized near the ends, for example in 
Tradescantia virginiana, the newt Triton palmatus and, as we saw, in the species of Oenothera. Localization of cither kind of course, does something more than merely reduce recombination. It divides the genes of each chromosome into two regions, one with high and the other with low recombination or none at all. It is interesting, if disappointing, to discover that although the heterochromatin falls in the low group in Paris it occurs in Fritillaria chicfly in species without localization and in regions where crossing-over occurs.

The third method of reducing recombination is found only in animals. It consists in nothing less than the abolition of crossing-over in the heterozygous sex. A new method of holding the chromosomes together at meiosis is found in the males of certain Diptera and Orthoptera. The attraction between chromatids is extended from twos to fours, and the early repulsion of centromeres is suppressed. Crossing-over is retained with a normal meiosis in the homozygous sex. This means that a chromosome passing, for example, from one male Drosophila to another may avoid crossing-over generation after gencration. But this may also happen with an ordinary low frequency of crossing-over in plants. For, with a single chiasma, only two of the chromatids are cross-overs, and from each bivalent two of the four germ cells have unchanged chromosomes. So far as the species is concerned, therefore, the genetical result of the abolition of crossing-over in one sex is quite indistinguishable from a reduction of chiasma-frequency to one half in both sexcs, itself an unworkable arrangement since, as we saw, that frequency is usually already at a working minimum.

These variations show that, in the development of the genetic systcms of plants and animals, natural selection has paid a great deal of attention to this problem of recombination. Close linkage is nearly always encouraged. This we can understand if the tendency is for variability to exist in the form of balanced linked combinations, genetically unlike but of similar effect on the phenotype. In such cases, the amount of recombination favoured over any period will depend on the balance of advantage of fitness and flexibility, and hence indirectly on the rate of change of the environment during that period. Since only occasional recombination is required to maintain flexibility, the frequency of crossing-over which is favoured will gencrally be low. 


\section{Balance in Homozygotes and Heterozygotes}

While inbreeding can maintain a state of uniform homozygosity apart from the effects of mutation, outbreeding, as we have seen' cannot of itself maintain a state of uniform and high heterozygosity, except for such genes, or super-genes, or differential segments, as control the breeding system itself. The maximum proportion of heterozygotes for any one gene with two allelomorphs will not exceed one half in a large population. Yet, when all the genes carried by a zygote are taken into account, clearly the chance of its being completely homozygous is remote. Each genotype will be a mixture of the heterozygous and the homozygous. The average proportions of the mixture will depend on three factors, the breeding system, the number of allelomorphs of each gene, and the relative frequencies of these allelomorphs.

This dual condition of the genotype has two consequences. At any given time some of the combinations of genes are being exposed to the test of natural selection in the homozygous condition. Linkage will therefore be favoured in the way that we have already seen. The whole set of genes, on the other hand, even those of one chromosome, will virtually never be homozygous simultaneously. The combinations of genes will therefore be adjusted or balanced to perform their task always in a partly heterozygous condition.

Now where more than one gene is involved, and each gene shows dominance, the phenotype of a heterozygote will show no predictable relation to those of the corresponding homozygotes. Even with only two genes, the phenotype of $A a B b$ may fall between those of $A A b b$ and $a a B B$ or may transgress their range in either direction, according to the directions and strengths of the dominance properties of $A-a$ and $B-b$. Thus, where a polygenic system is concerned, the favouring and balancing of partly heterozygous genotypes by natural selection can offer no guarantee that the corresponding homozygotes will be similarly balanced. Indeed in view of the multiplicity of genotypes, all more homozygous than the heterozygote from which they arise by segregation and recombination, there is no reason why any one of them should be automatically balanced. Smaller gene combinations forming parts of the genotype may themselves be balanced as a result of 
previous exposure to natural selection in the honozygous state; but the wholes, made up of these parts, are unlikely to be adjusted. This is the more so because cven the parts themselves must tend to change in the flow of variability.

Thus, forcing an umatural inbreeding on an outbreeding specics will lead to the exposure of genotypes which, through the rarity of their occurrence under the original system, will not have been subjected to adjustment, by natural sclection, in their action on the phenotype. The result is maladjustment, or inbreeding depression. It is contingent on inbreeding and must vanish when outbrceding is resumed.

Even where a heterozygote was made up from gene combinations which were balanced when fully homozygous, inbrecding depression must soon become a property of its descendants. Segregation and recombination would ensure that, of the homozygotes which could be extracted from such a hetcrozygote, those which went to its making, or indeed any balanced combinations, would form but a small fraction. The greater the number of variable genes concerned, the sooner and the more completely would this scrambling of the genotype lead to the loss of the property of balance in homozygous derivatives.

Heterozygosity must obviously shelter recessive genes from natural selcction. Not only docs this lead to the lack of balance of polygenic combinations, liable to segregate as homozygotes, in the way we have seen; delcterious mutant allelomorphs of major genes, genes of drastic effect, should also survive under this shelter. Plants and animals collected in the wild have remarkably often proved to carry such genes when tested in the laboratory. Indeed, on the basis of tests with Drosophila pseudoobscura, Dobzhansky estimates that at least one wild fly in four carries a mutation causing lethality, or near lethality, when homozygous.

The segregation of such sheltered recessive allcloniorphs of major genes will always follow inbreeding, but it is not, as has often been supposed, the cause of the characteristic inbreeding depression. Inbreeding docs not producc a rising proportion of the more deleterious of two alternative phenotypes in succecding generations, so giving an average decline only when viewed over the whole gencration. Rather it gives a steady and progressive decline of all 
members of the population. Such a steady decline is to be expected from the increasing homozygosity for numerous polygenic combinations within each individual. The averaging process is within the individual rather than within the population.

Inbreeding an outbreeder consistently leads to inbreeding depression: complete homozygotes are poor. Outbreeding an inbreeder has no such effect. The heterozygotes are seldom very different in vigour and fertility from their homozygous parents. Indeed, crossing different lines has not uncommonly given heterozygotes of somewhat greater vigour than either parent, for example, in the diploid cultivated tomato, and in the tetraploid wild Galeopsis tetrahit.

The reason for this difference is apparent immediately we consider the flow of variability. In an outbreeder this flow is strong and, even were a heterozygote to be made from combinations balanced in the homozygous condition, these would soon be destroyed by the constant crossing, segregation and recombination. In an inbreeder, on the other hand, the flow of variability is weak or non-existent. Combinations, once established, remain in being, apart, of course, from mutation. If, when they were established, these combinations gave an adequately balanced heterozygote, as well as adequately balanced homozygotes, they would retain this property.

Now the tomato is known to be derived from outbreeding ancestors in Peru. Inbreeding is similarly a recent imnovation in Galeopsis tetrahit, whose diploid ancestors were outbreeders. It is therefore to be expected that the combinations, now homozygous in the tomato and in G. tetrahit, will have retained the capacity for giving balanced heterozygotes. There is a reduction of advantage in vigour and fertility of heterozygote over homozygote. But this is due to the grading up of the homozygotes, by the selection of balanced homozygous combinations, not to the grading down of heterozygotes, by loss of balance through shelter from selection. Heterozygous or relational balance can only be produced by the action of selection in an outbreeder. It is nevertheless retained in the absence of selection when inbreeding freezes, as we may say, the flow of variability. Balance presumably can be lost in such a case through mutation; but loss by mutation must be a slow process 
compared with loss by redistribution of variability, as we can see from observations such as those on the tomato and Galeopsis.

\section{Selection and the Reservoir of Variability}

The sheltering effect of heterozygosity and dominance depends on the fact that selection discriminates primarily between phenotypes, and hence between genotypes only to the extent that they give different phenotypes. The phenotype is, as it were, the organ by which the genotype is sclected. Yet it is upon the favouring of particular genotypes that response to sclection, as it is expressed in the phenotypes of succeeding generations, must depend. A lag of at least one generation must consequently intervene between the action of sclection and the response which it produces. Thus, changes under selection can be adjustive only to the extent that the changes in cnvironment, which produce them, are permanent.

Not all changes in the cnvironments to which succecding generations are subjected can be of this kind. In an ephemeral organism, such as a fruit fly or a chickweed, the different generations live at different times of the year, and hence are subjected to a series of cnvironments whose main changes are cyclical rather than permanent. The changes of environment in which successive generations of annual organisms find themselves are, if not cyclical, at least erratic rather than permanent. Only with longer-lived species will the vagaries of environmental change tend to even themselves out.

Non-permanent fluctuations of environment must always be occurring, though their significance to the organism may not be constant. It will depend on the relative magnitude of any permanent changes which may be going on at the same time and which may be more rapid at one period than another. When, for example, the first birds evolved, they had a whole new field to thenselves. As they multiplied and filled it, competition must have increased rapidly, and with it the environment of each bird must have changed in the direction of becoming permanently more exacting. With the attainment of something approaching naximum numbers the environment, as determined by this competition, must have become more stable, though non-permanent changes would still 
occur according to chance fluctuations in numbers and other contributory circumstances. The non-permanent changes, originally of lesser importance, must have come to mask the permanent.

Now the organism has no means of distinguishing permanent from non-permanent changes in its circumstances. If it is sufficiently flexible genetically to respond to permanent changes, it must also respond to the non-permanent ones. Cyclical changes in the environment may be accommodated by cyclical changes in the

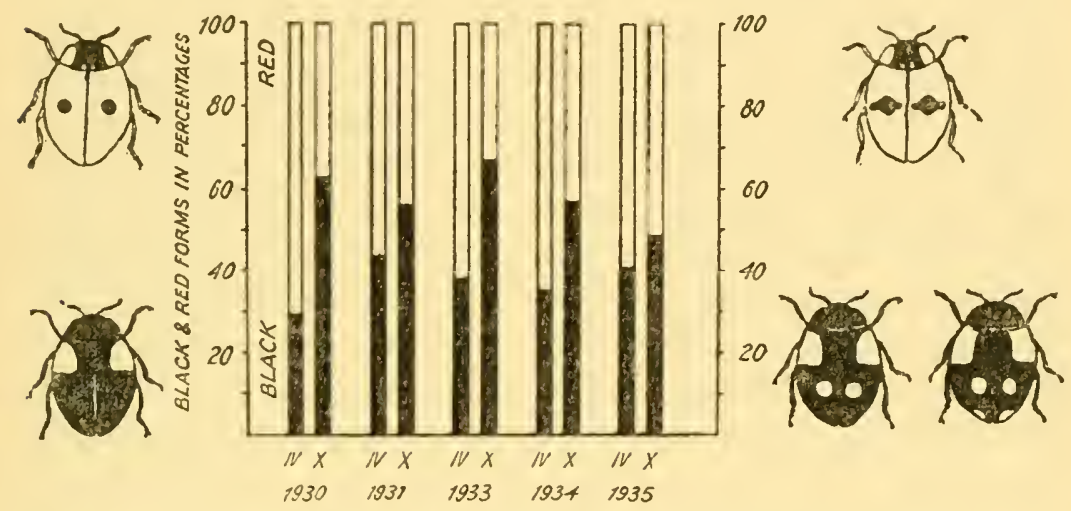

FIG. 74.-The percentages of the population of the ladybird Adalia punctata with black and red ground colours in April (IV) and October (X) over a period of six years in Buch, Germany. There is a regular seasonal change in the frequencies of these genetically controlled alternatives, red being commoner in spring than it is in autumn (after Timoféeff-Ressovsky, 1940).

relative frequencies of allelomorphs of genes or super-genes governing polymorphism in an ephemeral (Fig. 74); but this is a special case. Permanent adaptation must generally demand the irreversible fixation of genes, and if this can be brought about by permanent changes, so it can by temporary ones too. In responding thus to non-permanent changes, the stock will gain nothing in fitness, and will lose some of the variability on which prospective adaptation must depend, by the profitless fixation of free variation under the erratic selection. This loss must be made good in the long run by new variability from mutation (Fig. 7I), as indeed must any reduction in variability whatever its cause, if the species is to survive. In fact the amount of variability, when at equilibrium in a population, will be such that the loss, itself proportional to the free and 
hence to the total variability, is equal to the increase by mutation, an increase which is obviously independent equally of both kinds of variability. When loss exceeds gain, the total variability will diminish until an equilibrium is reached, and vice versa .

Loss and gain of variability must balance in the long run, but they are unlikely to do so over short periods. Mutation might be expected to over-compensate for loss when this arises solely from erratic changes in environment, but to fall short of doing so when rapid permanent change is also causing the expenditure of variability. The importance of the reservoir of potential variability lies in its smoothing effect, in its ability to permit compensation of rapid expenditure over short periods by a steady trickle of gains over a longer time. All selective changes must ultimately depend upon mutation; and the capacity for mutation, itself apparently under some selective control, represents the final reserve of variability. But it is a reserve which is released at a more nearly constant rate. The immediate reserve of potential variability represents the storage of these mutations by the genotype, in such a way as to permit more rapid response to selection than mutation could give directly.

\section{Change of Genetic Systems: Inertio}

We can now consider the properties to be expected of genetic systems in the light of these principles. The genetic system seen in any stock or race of organisms today, with its special propertics of balance, storage and release of variability, is that which has enabled the ancestral line of the organisms concerned not merely to become, but to be continue to be, adequately adapted to its changing environment throughout the course of its history. The stock has survived, and with it the genetic system upon which survival has depended. Continuation of this system must depend on the extent to which the genetic system enables its possessor to meet the exigencies of future change. So long as the advantage lies with the maintenance of a highly uniform and constant phenotype, the inbreeder will be well endowed by its genetic system for success. But whenever the advantage lies less with high uniformity than with easy change of phenotype, the outbreeder will be the more successful. If the environment changes in its demands, the genetic system will cease 
to be advantageous to its possessor except in so far as it can change too. Since the genetic system must always be under its own control, an outbreeding system will be able to change in the direction of inbreeding by virtue of the flexibility which is its special property. The more rigid inbreeding system will be less likely to show change towards outbreeding. Evidently inbreeding systems must generally be in evolutionary dead ends, doomed by inflexibility to extinction when a crisis arises, but always being thrown off by the continuous stream of outbrecding systems. We have already seen that the evidence from stratification of breeding systems fully accords with this expectation.

This evidence of stratification in the breeding systems of, for example, wheat and Pediculopsis, can leave no doubt that changes of system do occur. Nor can the comparison of the breeding systems of relatives such as wheat and rye, or the Galeopsis species. Yet there are obstacles to change inherent in the systems themselves: obstacles which must slow the change-over even where they do not prevent it. With the inbreeding type the obstacle, a fatal one, is, as we have seen, its own inherent rigidity. With the outbreeding system it is the less serious one of lack of balance of the more homozygous genotypes which increased inbreeding produces in greater numbers. Each system is internally consistent: change introduces a measure of discordance. They show what we must describe as genctic inertia.

\section{Correlated Response: Capital and Subordinate Characters}

Genetic inertia is due to integration, the building up of an adaptive system providing the very properties which have been responsible for its past success. This inertia of adaptation can also be seen at work in other ways than in the retardation of change in the breeding system. In his experiment on the scutellar bristles of Drosophila, Sismanidis records that of seven selection lines with brother-sister mating, three died out-through infertility. This may, as we saw earlier, have been the simple effect of inbreeding. But in other such experiments with flies, and also with fishes, a similar loss of fertility has regularly been found to accompany the change of a somatic character under selection. It has done so even where outbreeding was kept at a maximum. Furthermore, in an experiment 
with flies, selection for change in chacta number not merely robbed, the stock of its fertility: it also changed the pattern of pigmentation, the mating discrimination and the number of spermathecac. This last change was especially marked: instead of the normal two, as in the parents, selected females had from none to five spermathecac.

Correlated responses to selection such as these may be due, in part, to one gene affecting several characters, the pleiotropy which we discussed carlier. But there is also another agency at work. Chacta numbers showing the effects of selection and at first associated with a lower fertility have, later in the same experiment, become associated with a higher fertility. The two effects nust therefore be due to different genes, and the cause of the correlated response must bc linkage. Crossing-over within a chromosome will bring about recombination, not merely within the polygenic combination affecting the character in which we are interested, but also within the other polygenic combinations which are intermingled with it along the chromosome. So in selecting for changed combinations of one set of genes, change in the combinations of sets affecting other characters, for which no sclection is practised, will also be brought about (Fig. 75). And if thesc changes cause a sufficiently large unbalance, their consequences will appear as an alteration in the expression of the character they control; an alteration for which there is no direct selective causc. When this has happened, however, continued recombination of the gene sets can eventually lead to a reassociation of the characters, of the kind we see when the flies with changed chacta number regain their fertility. Even pleiotropy may, as we saw, merely express completc linkage. But, with pleiotropy, the reassociation of characters would have been out of the question.

Although the response must go in a fixed direction for the character on which selection is primarily acting, the correlated responses in other characters need not do so. The correlated changes will therefore often be deletcrious. Correlated response should in consequence act as a brake on the primary response to selection, both in nature and in experiment. Decline and death is characteristic of selection lines in experiment and bears witness to the truth of this principle. The better adjustment in one character must be paid for by the worse adjustment, at least for a time, of others. The 
price of linkage is therefore that, apart from the primary character, the immediate overall response to selection is poorer. Time is

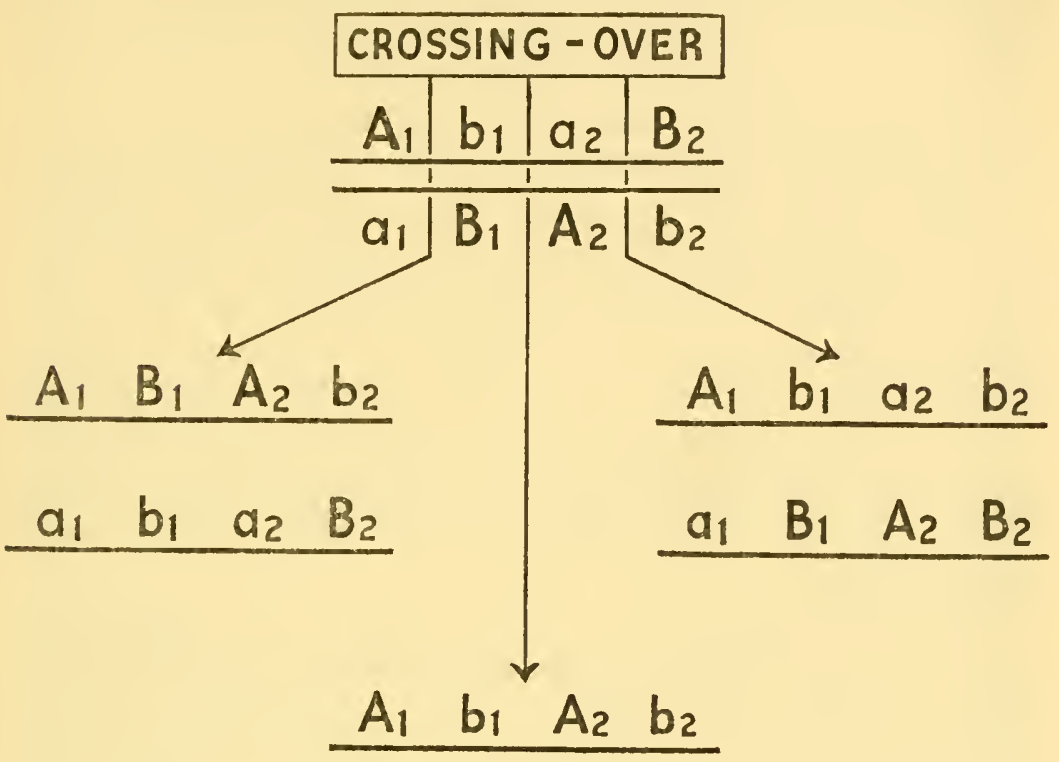

\section{$\begin{array}{llll}a_{1} & B_{1} & a_{2} & B_{2}\end{array}$}

FIG. 75.-The mechanism of correlated response to selection. The two $A$ - $a$ genes represent members of one polygenic systen and the two $B-b$ genes of another all carried on the same chromosome.

Both systems are balanced in the sense that each of the original chromosomes carry genes of opposing tendencies, as indicated by capital and small letters. Selection will pick out the chromosomes which have lost this balance by recombination bringing together two genes of reinforcing tendency either in the direction indicated by capitals or that by small letters. According to the position of the crossing-over, upon which recombination depends, (i) the A system may be unbalanced, the B system remains balanced (left); (ii) the B system niay be unbalanced, the A system remaining balanced (right), or (iii) both systems may be unbalanced (centre). Then in (i) selection can be effectively practised for the character controlled by the A system without that controlled by $B$ changing, or (ii) selection can be effective for the $\mathrm{B}$ character without the $\mathrm{A}$ character changing. But in (iii) effective selection for either character must also result in change in the other, solely by virtue of the linkage of the genes. This is correlated response, and it may be in either direction for the subordinate character, again according to the precise linkage arrangement.

needed for recombination to permit the attainment of a full new adjustment in the primary character without deleterious effects on 
others. Again we sec that, although a change can be achicved, there are obstacles to be overconc first. The very linkage which is so powerful in reconciling the needs of flexibility and fitness when displayed between genes of similar action, results in an inertia when displayed between genes of divergent action.

The extent of this inertia will be dependent on the degrec of selective disadvantage resulting from the correlated response as compared with advantage arising from the primary response. If the correlated change should occur in a character of minor importance to the organism's fitness, it could result in the fixation of a new expression of this character under the action of an unrelated selective force. In other words, subordinate characters may be pushed about by the selective forces acting on capital characters. Sclection can in this way produce a change from which no selective advantages could arise, and which therefore will show no trace of the agency which caused it.

Subordinate selection is scen where the character in question was once of great moment for the organism but has lost all its importance. Such a character, for example, is sight in cave animals. Once the selective advantage of adequate sight is removed by the adoption of cave life, selcction for other characters, such as scnse of touch, is able, through correlated responses, to bring about the brcakdown and atrophy of the visual mechanism which has becn degraded to the rank of a subordinate character.

We are now in a position to look back at the situation as it was left by Darwin. To Darwin, variation was what he saw in the members of a living specics. He discovered the great problems of inbreeding and outbreeding, of adaptation and selection; but, in terms of the variation that he saw, these problems could not be solved. Now we realise that underlying the visible variation are organisations of genes and chromosomes which exist largely as means of suppressing the appearance of variation. They contain the variability and reveal it in ways which the principles of Mendelism and Morganism enable us to understand. The operation of these principles is, of coursc, complex and in a sense abstract. But it is capable at cvery stage of being subnitted to experimental test, and of yiclding the predictions that are necessary both to the theory of evolution and the practice of plant and animal breeding. 


\section{REFERENCES}

BEADLE, G. W. I933. Further studies of asynaptic maize. Cytologia, 4: 269-287. BUMPUS, H. C. I 899. The climination of the unfit as illustrated by the introduced sparrow. Biol. Lect. Woods Hole, 1898: 209.

DARWIn, C. I 868. The Variation of Animals and Plants under Domestication. London. DARWIn, C. I87I. The Descent of Man and Selection in Relation to Sex. London. DARWIN. C. I876. The Effects of Cross and Self-Fertilization in the Vegetable Kingdom. London.

Dobzhansky, T. 194I. Genetics and the Origin of Species. 2nd ed. New York. FISHER, R. A. I930. The Genetical Theory of Natural Selection. Oxford. Johansson, I. 1931. Breeding pigs for high prolificacy. Pig Breeder's Ammal, II : $80-87$.

LURIA, S. E. 1945. Mutation of bacterial viruses affecting their host range. Genetics, 30: $84-99$.

MATHER, K. I943. Polygenic inheritance and natural selection. Biol. Revs., I 8: 32-64. mather, K. I943. Polygenic balance and the canalization of development. Nature, I5I: 68-7I.

mather, K., and harrison, B. J. I949. The manifold effects of selection. Heredity (in the press).

MENZIES-KITCHEN, A. W. 1937. Fertility, mortality and growth rate in pigs. J. Agr. Sci., 27: 61 I-625.

MUNTZING, A. 1945. Hybrid vigour in crosses between pure lines of Galeopsis tetrahit. Hereditas, 3I : 391-398.

RENDEL, J. M. 1945. Genetics and cytology of Drosoplita subobscura. II Normal and selective mating in Drosoplila subobscura. J. Genet., 46: 287-302.

SISMANidis, A. 1942. Selection for an almost invariable character in Drosophila, J. Genet., 44: 204-215.

timofíefr-ressovsky, N. W. 1940. Zur Analyse des Polymorphismus bei Adalia bipunctata. Biol. Zbl., 60: I30.

vavilov, N. I. I9I4. Immunity to fungous diseases as a physiological test in genetics and systematics, exemplified in cereals. J. Genet., 4: 49-65 


\section{THE BREAKDOWN OF CONTINUITY}

The Mating Contimum Restrictive Practices The Internal Origins of Isolation Floating and Fixed Discontinuity Restriction and Flexibility

The Traces of Ancestry

Selection, AS We Have SEen, distinguishes only between phenotypic differences. It is, therefore, effective in changing the genetic constitution of a population, or group of individuals, only in so far as the genetic differences of the group are, or can becomc, cxpressed in the phenotypes, whether of cells, individuals or populations. Now the release of potential variability into the frec state depends on segregation from heterozygotes. Hence, as we have scen, the potential variability of an inbreeding specics is excluded from providing the material for sclective changc. In such species, change under selection will be confined to the sorting out of such differences as appear immediately in the phenotype: it will soon be over and will be small in effect.

Only groups with outbreeding systems can respond by extensive change to the continued action of selection, whether natural or artificial. And this is as true of the production of change in the genetic system itself, a property of the species, as it is of change in the phenotype of the individual, since the one as much as the other depends on change in the genotype. It is, therefore, to the propertics of outbreeding that we must turn in order to sec how changes in genetic systems come about, and in particular how groups initially unitary in their breeding come to break up.

\section{The Mating Continuum}

Under an outbreeding system an individual receives its genes from two parents which are always, or at least nearly always, genetically different. The same will have been true of the two parents in their turn. Each individual can therefore be regarded as representing the fusion of a number of lines of descent. In the same 
way, each individual will pass on some of its genes to, on the average, two genetically distinct offspring and so may be regarded as initiating a number of lines of descent. The lines of descent, of which any individual represents either the culmination or the initiation, are obviously not independent of those which are represented by other individuals of the same generation. Sooner or later, if we trace the ancestry of the members of a population at one time, or if we trace their descendants, we shall find the lines merging. In this sense an outbreeding group represents a mating continuum: a continuum within which any member may have received genetic materials from over the whole area which the group occupies. Looked at in another way, from the standpoint of the distribution of the group, the various sub-groups into which it may be divided spatially, or perhaps in other ways, can be regarded as exchanging chromosomes or exchanging genes. The individuals in one sub-group will have lines of ancestry tracing to other sub-groups, and will in turn leave lines of descent in these others; though the number of lines joining sub-groups will be less than those staying within any one of them.

In so far as the members of a continuum cover an area of territory, they must be occupying a variety of ecological slots or niches, distinguished by altitude, nutrition, moisture and so on. Each individual, or each local sub-group of individuals, must be under the action of selective forces tending to adjust its genotype to the local environment (Fig. 76); and to the extent that these adjustments are to different environments, exchange of genes between the local sub-groups must lead to maladjustment. A mating continuum, therefore, is genetically flexible, but it does not permit the best local adaptation where differences occur within the common environment.

Local maladjustments of an outbreeding species in a differentiated environment will always tend to increase, for the relations between environment and genotype are not constant. No environment can be stable in the long run, and almost any change must be for the worse in respect of an existing organism. Furthermore the environment will not only be worsening for each individual: it will be worsening in different ways for individuals in different ecological circumstances. The need for local adjustment will not only be growing: it will be growing in different directions in different parts 
of the mating continuum and nust be met by different genetical adjustments. The mating continuum of any group in a differentiated enviromment therefore has an inherent instability. How is this instability resolved ?

The instability arises from the exchange of genes between individuals and groups requiring different local adaptations. As fast as the local conditions select and separate more favourable combinations of genes, these will be broken down and brought back towards

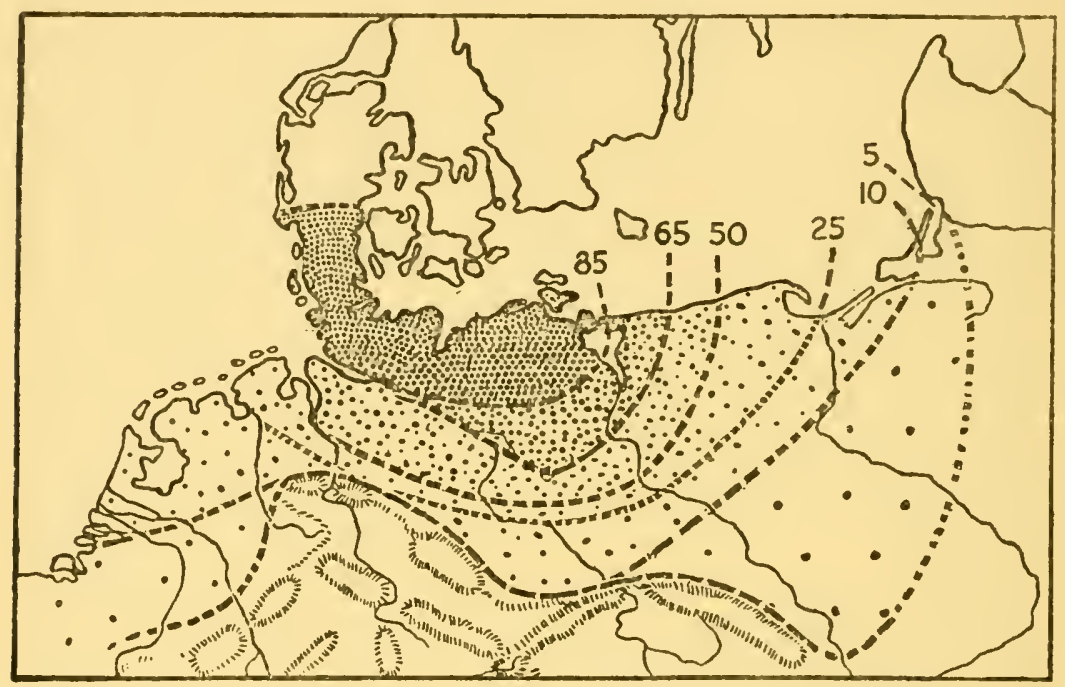

FIG. 76.-The distribution of a recessive mutant determining "simplex" tecth in the field mouse Microtus arvalis, according to the percentage of homozygous individuals (after Zimmermann, 1937).

the common level by the intrusion, from some other part of the continuum, of other combinations which have been selected under different conditions and hence adjusted in a different way. As fast as advantageous combinations of genes arise by segregation and recombination, they will break down by the same processes. The flow of variability, which arises from segregation and recombination, is necessary for the production of new and supcrior combinations of genes. Once, however, these combinations have been achicved, the means of their origin immediately becomes not merely unnecessary; it becomes actively harmful. The value of recombination lies, in fact, in bringing together combinations of 
genes which are so desirable as to make further recombination undesirable - at least for the time being.

Thus, wherever there exists an advantageous combination of genes, selection must favour any device which restricts the effective recombination of its constituents. These devices are of various kinds, and they do their work in various ways.

\section{Restrictive Practices}

Recombination is effective only to the extent that the individual is heterozygous. The evil effects of recombination will not therefore be felt if heterozygosity can be eliminated, or at least reduced so that it does not endanger the basic advantageous combinations. We have already seen a great deal of restriction of inbreeding: in fact outbreeding itself is, in one sense, the result of restricting inbreeding. We now have to meet the opposite-a restriction of outbreeding. Restriction of outbreeding helps to preserve favourable combinations of genes. It may arise from either environmental or genetic causes, and it may be either partial or complete.

The partial restriction of an individual's freedom of outbreeding is described as isolation. Mating is unhampered within the group but prevented between isolated groups. This restriction may be imposed by geographical means, as in the case of the different species of finch which Darwin found on each of the Galapagos islands. Or it may be by genetic means, that is to say by the development of gene or chromosome differences preventing mating, pollination, or fertilization, such as we have already seen at work in Petunia species. Geographical isolation cannot, of course, be under the control of the organism, and it is therefore in a sense accidental. Nevertheless such accidental isolation, particularly where it is incomplete may, as we shall see, favour the rise of genetic isolation.

In the last chapter we have seen that the balance of gene combinations is brought about by the action of selection. In wild populations the poor combinations are eliminated and the advantageous combinations favoured. Now, in outbreeding organisms, many combinations are possible for every one which is advantageous, and only the test of natural selection can lead to the differential elimination of those that are less favourable. If they are 
not exposed to this test they will not be eliminated. One consequence of this we have seen. When we inbreed a normally outbreeding stock, combinations become exposed in the homozygous condition for the first time. Their internal balances have never been tested and refined by natural selection, and in consequence they are mainly poor. Inbreeding depression is the result.

In just the same way, by crossing individuals which would not normally interbreed, we can obtain combinations in an untried heterozygous relation. If they have been free to change since the lines of descent diverged to give the individuals that carry them, these combinations will generally not be capable of working adequately together. Their joint action will not have been kept up to an adequate level by natual selection: balance is not stable, except in inbreeders. It decays if it is not being constantly adjusted by selection.

The complete prevention of crossbreeding by some geographical obstacle will therefore mean that the genic combinations of the separated groups will gradually lose their relational balance, provided that within at least one of them sufficient outbreeding occurs not to freeze the flow of variability and so prevent all genetic change. As time goes on, the hybrid made by artificial intercrossing of the groups will come to be inadequate genetically. It will show an abnormal character or reduced fertility, more often the latter. The groups will ultimately become separated by the bar of genetical intersterility. They will be separate species. The same effect may, of course, also be brought about by ecological or seasonal isolation. The isolation, and not the means by which it is brought about, is the cause of the decay in relational balance.

Where isolation by environmental agencies is incomplete, the decay of relational balance will be slower until genetical bars to crossing also arise. Soon after crossing becomes restricted between groups, the product of such crossing will begin to show the effects of the restriction in the form of some measure of hybrid incapacity, some failure in growth or reproduction. Such progeny are a waste of reproductive effort, and the less the number that an individual produces, the greater its chance of contributing to posterity. Any gene, therefore, which further restricts the rate of crossing between groups in individuals which bear it, will confer a selective advantage 


\section{EXTERNAL and ENVIRONMENTAL}

1. GEOGRAPHICAL - separation in range of occurrence

2. ECOLOGICAL-separation in habitat occupied

3. SEASONAL - separation in time of occurrence or breeding

INTERNAL and GENETIC

1. PREVENTION OF MATING OF ZYGOTES bY

(i) Discrimination in Mating

(ii) Mechanical Inability

(iii) Failure of an Essential Intermediary (as where insects fail to carry pollen between two species of plants)

(iv) Prevention of all Crossing in Hermaphrodites (by devices securing self-mating)

2. INCOMPATIBILITY of

(i) Gametes

(ii) Male Gamete and Female Soma (owing to genetical unlikeness) FERTILIZATION

\section{UNBALANCE OF}

1. $F_{1}$ ZYGOTE-leading to Inviability and Genotypic sterility

2. $F_{1}$ GAMETES - resulting from Genotypic effect of $F_{1}$ zygote or, in plants, from Segregational sterility

3. $F_{2}$ ZYGOTES - resulting, in animals, from Segregational sterility

FIG. 77.- Isolating mechanisms preventing the effective exchange of genetic materials between populations. They fall into two groups: Bars to Crossing acting before fertilization and capable (in so far as they are genetic) of being produced by the direct action of selection; and Hybrid Incapacity occurring after fertilization, and incapable of being produced by the direct action of selection. The two groups are not mutually exclusive: they tend to encourage one another's occurrence. 
on its possessors and so will increase in frequency. The environmental isolation will be reinforced by genetic means. And as the restriction on crossing becomes greater, the relational balance will get even worse. Hybrid incapacity will increase, and with it the advantage of genetical bars to crossing. The system must therefore be selfstimulating, and once started, isolation will sooner or later become complete. Furthermore, the bars to crossing which are thus stimulated indirectly by loss of relational balance, may be reinforced by the direct expression of differences in form and function which arise as expressions of the increasing divergence of the two groups (Fig. 77).

Where divergence has followed the course just described, the bars to crossing will generally prevent, or at least make difficult, crossing by artificial means. This is so, for example, with the Antirrhimum species, majus and orontium. One of the bars to crossing between them is the prevention of growth in the style of one species, of pollen from the other. Such a bar operates against artificial as well as natural cross-pollination. The two species, majus and glutinosum, however, show us that the bar to natural crossing need not prevent artificial hybridization. When grown together and allowed to pollinate naturally under English conditions, they show less than 3 per cent crossing. They can, nevertheless, be crossed readily by artificial means. There appears to be no barrier to crossfertilization once cross-pollination has been achicved. Here the isolating mechanism is found to consist in the failure of crosspollination, consequent on the failure of pollinating insects, mainly bees, to visit flowers of the two species alternately when making their working flights on a mixed stand of plants.

In these species of Antirrhinum the plants have, so to speak, taken advantage of the insects' power to discriminate between different flowers, and of their predilection for confining their visits on one run to flowers of the same type. We have, therefore, the remarkable, but possibly frequent, situation of isolation between two species depending on the habits of a third. Flowers are, of course, generally adapted in form and colour to the insect which pollinates them. Here, in turn, not only the form but the discontinuity in form of the flower depends on the habits of the insect. 


\section{The Internal Origins of Isolation}

Isolation, as we have been discussing it, arises from the external genetical relations of one species with others. It can also arise from purely internal causes in a variety of ways. Of these the simplest is provided by the mere habit of inbreeding itself. Isolation is complete, or virtually so, while artificial crossing is readily achieved, in many inbreeding organisms. In wheat or oats, for example, all the species, even the diploids, can be readily crossed. Yet, when grown together, the species show no natural crossing. Here, however, in contrast to the Antirrhinum species, the lack of crossing between plants of different species is only a special case of the general failure of the individuals to outcross even with others of their own species. They are habitual inbreeders, and their isolation is a concomitant of their inbreeding.

Where crossing of any kind is absent, restrictions on crossing between groups, as opposed to crossing within them, are obviously unnecessary. Indeed, since inbreeding freezes the flow of variability, the production by recombination of groups of genes producing bars to crossing will be impossible: so likewise will be the divergence of genic combinations from which hybrid incapacity arises. It is not surprising, therefore, that there is an absence of all genetic bars to crossing other than the normal inbreeding mechanism. Nor is it surprising that the species hybrids, when artificially produced, are both fully vigorous and fully fertile; apart, of course, from the effects of the numerical hybridity which follows the crossing of species having different chromosome numbers.

These principles are seen in their simple form in wheat, oats and tobacco. They are illustrated even more critically in the species of Oenothera, which happen by their own special device to be highly heterozygous. They are habitual inbreeders, and they have an unlimited capacity for crossing within the genus.

Isolation and inbreeding represent restriction on crossing of different degrees and with different consequences. They are both achieved, however, by means within the limits of the sexual mechanism. Apomixis, on the other hand, resolves the instability of a mating continuum by the abolition of the normal sexual cycle. Fertilization is done away with, and meiosis either does not intervene, 
or does not run its normal course. Segregation, even from a hybrid, is thus either avoided completely, so that reproduction is clonal in all but appearance, or is so severely restricted as to give something near the genetical uniformity of a clone.

Apomixis, as we have already seen, is shown very commonly by individuals so hybrid, even in regard to chromosome number, that only by side-stcpping scxual reproduction call they propagate thenselves; but it is not confined to such hybrids. In the aphides it is shown by species which under other circumstances are sexual. The sexual phase is to be seen at those seasons of the year which keep their numbers small, and the parthenogenctic phase in the summer when populations are rapidly increasing in size. As we have carlicr observed, the use of parthenogenesis during a stage of rapid expansion of the stock is a reproductive cconony. We can now sec that it is also something more, for it avoids the deterioration which would result from sexual recombination at a period of prosperity when natural selection is dangerously relaxed.

Isolation and inbreeding on the one hand, and apomixis on the other, though differing sharply in their mechanisms, agree in avoiding recombination by the abolition of segregation. Isolation and inbreeding do so by avoiding heterozygosity for at least the critical gene combinations; apomixis does so by accepting heterozygosity but avoiding sexual reproduction altogether. Polyploidy offers a device which accepts both hetcrozygosity and sexual reproduction, yet still avoids segregation of the old differences.

In an allopolyploid, or amphidiploid $\left(A_{1} A_{1} A_{2} A_{2}\right)$, the individual is heterozygous for the differences between the sets of chromosomes of the original parents $\left(A_{1} A_{1}\right.$ and $\left.A_{2} A_{2}\right)$. Yet in so far as the two representatives of each set segregate from one another on the scheme of Primula kewensis $\left(A_{1}\right.$ from $A_{1}$ and $A_{2}$ from $A_{2}$ ), rather than from the sets of the opposite parent $\left(A_{1}\right.$ from $\left.A_{2}\right)$ thesc parental differences are passed on as wholes through each gamete to each offspring. They never segregate and their parts never recombine, so that a polyploid is, as we saw, potentially a truc breeding hybrid. So even when descended from cross-breeders, a polyploid species can be an inbreeder without becoming homozygous and hence without serious inbrecding depression. It offers, in fact, a short cut from the flexibility of outbreeding to the fitness of 
inbreeding, besides combining the bases of variation from the two parents.

Polyploidy may also offer other advantages. An autopolyploid, derived by the simple doubling of the chromosome number of an existing species, can show physiological properties different from those of its parent, while yet maintaining the balance, the capacity for working as an integrated whole, of that parent. The drawback of autopolyploidy, especially in annuals, where seed production is needed for maintenance, is the reduced fertility which follows multivalent formation at meiosis. This may, however, be avoided by restriction of pachytene pairing or of chiasma formation, so that only bivalents occur, as is seen in the autotetraploids Lotus corniculatus and Tulipa chrysantha.

Allopolyploidy avoids recombination within whole sets of chromosomes. Interchange, in its turn, offers a means of avoiding recombination of different individual chromosomes within the set, so that two pairs have one linkage group. As we have already seen, the recombinant gametes from interchange heterozygotes are lethal in plants, because they show simultaneous duplication and deficiency. In animals the zygotes to which such gametes give rise are lethal for the same reason, except where, either by a rare chance or by deliberate experimentation, fusion is with another gamete showing complementary unbalance. Those gametes which, on the other hand, carry a parental combination of chromosomes do not suffer from this handicap, and consequently enjoy a greater chance of success. Thus recombination of the chromosomes involved in the interchange is rarely or never effected.

In one sense the suppression of recombination in interchange heterozygotes may be regarded as due to the exaggeration of the ill-effects of all recombination. At the same time, in Oenothera species and their like, we can see how, by the additions of differentially balanced gametic lethals and of that competition between potential embryo-sacs which we call the Renner effect, the loss of fertility consequent on this suppression may be overcome. The mechanism is then adjusted to give a situation resembling that in polyploids, except that interchange abolishes recombination between any number of chromosomes from two up to the whole set. Thus interchange, like polyploidy, affords a means of attaining the 
uniformity which inbreeding can give without its depression of vigour and fertility.

Interchange and polyploidy avoid gross recombination between chromosomes, and it may be, therefore, that their chief advantage lies in the short cut each offers to inbreeding. Inversions, on the other hand, limit recombination within chromosomes. They can tie together, or peg, the very combinations of genes which will have been built up by the normal process of linkage in the way we have discussed in the previous chapter. It is also known that inversion heterozygosity need not lead to any marked sterility on the female side, for the inviable cross-over chromatids may be confined to those products of meiosis (spores or polar bodies) from which the egg never develops.

\section{Floating and Fixed Discontinuity}

Inversion, interchange and polyploidy have one property in common. They abolish the recombination of genes which would otherwise recombine more or less freely, and in so doing they give rise to super-genes, units of transmission greater than the gene itself. They peg the combinations of genes which have been successful in leading to high adaptation in the past. This pegging is not, however, a final process since the avoidance of recombination is seldom quite complete. In polyploids, aberrant pairing occasionally leads to aberrant recombination, while rare double crossing-over in interchange and inversion heterozygotes can lead to some redistribution of the constituents of the super-gene and so to the origin of spurious mutations. The result is that some genic heterogeneity may occur within one structural type. And even without this recombination a similar result would come about in time by virtue of true mutation alone.

The consequences of this development have been followed by Dobzhansky in Drosophila pseudoobscura. In this fly the third chromosome (to a much greater extent than its fellows) exists in a number of sequences derived from one another by inversions as revealed by polytene chromosomes. These inversions overlap one another so that they cannot recombine as units, and it has consequently been possible to work out the phylogenetic tree of 
the sequences themselves. This is shown in Fig. 78, from which it will be further seen that not merely are the inversions of this species relatable to one another, but that they must be descended from an ancestral type, now lost from pseudoobscura so far as is known,

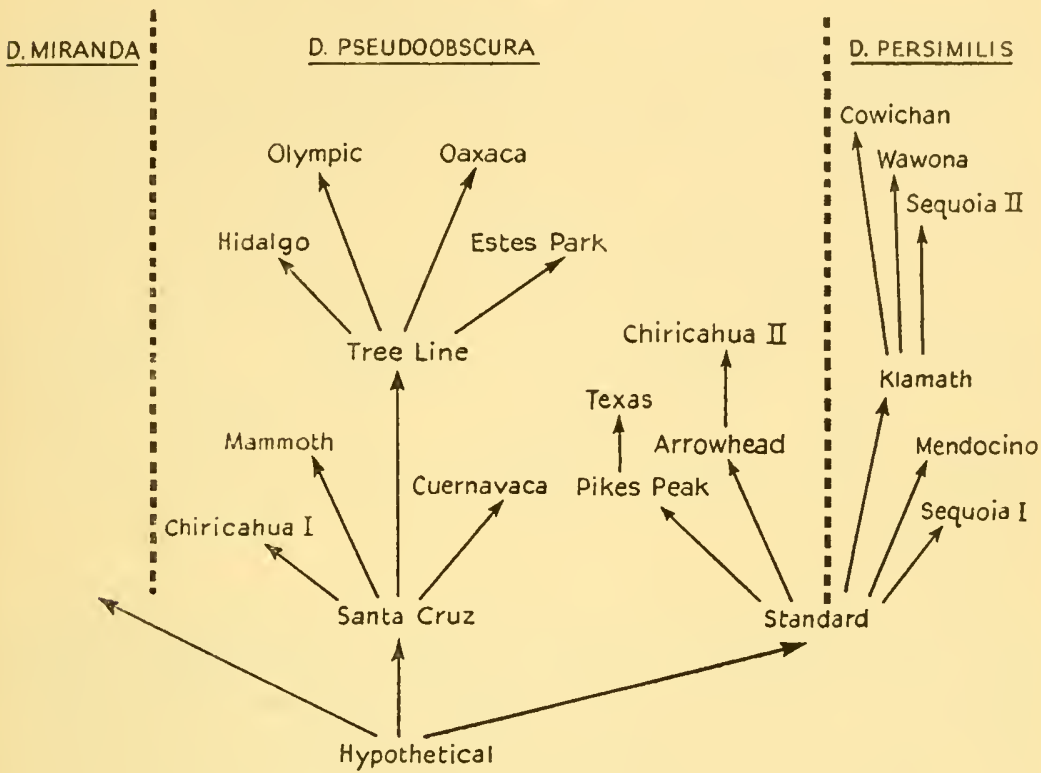

FIG. 78.-The phylogenetic relationships of the third chromosome sequences of the Drosophila species pseudoobscura, persimilis and miranda. The hypothetical sequence is closely resembled by that found in miranda. Standard is the only sequence which occurs in two species: with its derivatives it floats in pseudoobscura but is fixed in persimilis.

The regional names associated with the sequences relate chiefly to the places along the Pacific coast of North America where they were first found (after Dobzhansky and Sturtevant, I938).

which was common also to the related species persimilis (once called D. pseudoobscura, race B) and miranda.

Each of these sequences in pseudoobscura is itself heterogenic, that is to say it exists in genically diverse forms in nature, and the different combinations within the sequence display relational balance with respect to each other. Thus any one chromosome may give rise to a poor, even a lethal, individual when homozygous; but when heterozygous with another chromosome of the same structural type, the resulting individual will be perfectly viable 
and fertile. Yet there must be an overall super-balance between sequences, because selection tests have shown that flies heterozygous for two alternative sequences have an advantage over others homozygous in this respect, but heterozygous for at least part of the genic differences that occur within the same sequence in a strain or in the species at large. This balance of advantage between sequences changes with temperature.

In psendoobscura the various sequences float in the population, partly in a heterozygous and partly in a homozygous condition. A number of them may be picked up at any time in any group of individuals. Evidently their super-balance in respect to one another is still more effective in leading to the adjustment of the phenotype, than is the relational balance of the combinations within any one sequence. This is to be expected in a cross-breeding population in the early history of sequential variation, for the inversions will be pegging the combinations which as wholes work well in the heterozygous condition. A further development may, however, be envisaged.

When sufficient variation is present within sequences to give a good relational balance without sequential heterozygosity, genic adjustment to any permanent change in environmental circumstances will go on within sequential types rather than between them. Within a sequential type the adjustment of combinations can go on by the recombination of their genes; but different sequences are genetically isolated from one another (Fig. 79). Thus each sequence may become better fitted than its alternatives for a particular environment. Any further pressure of selection will therefore tend to establish a sequence in one or more populations. The sequence will cease to be floating in these populations, and will become, with its derivatives, fixed, uniformly and exclusively homozygous; though at the same time it may continue to float in still other populations. This process appears to be in progress today in pseudoobscura, for although several sequences may be found in any one population, different populations are characterized by particular groups of sequences.

The three species we have mentioned show the last stage of the process. All three have third chromosomes descended from a common type, but the line of development of miranda differs from 
that of the other two. Two sequences, the so-called Santa Cruz and Standard types, have floated (together, possibly, with others of which all trace has been lost) in the common ancestor of pseudoobscura and persimilis. They and their derivatives still float within pseudoobscura, but persimilis has neither Santa Cruz nor any of its derivates. The Standard type has been fixed in the populations ancestral to this species, even though it has remained floating in the populations ancestral to pseudoobscura. Since the time of this fixation, the lines of variation of Standard have, of course, separated in the

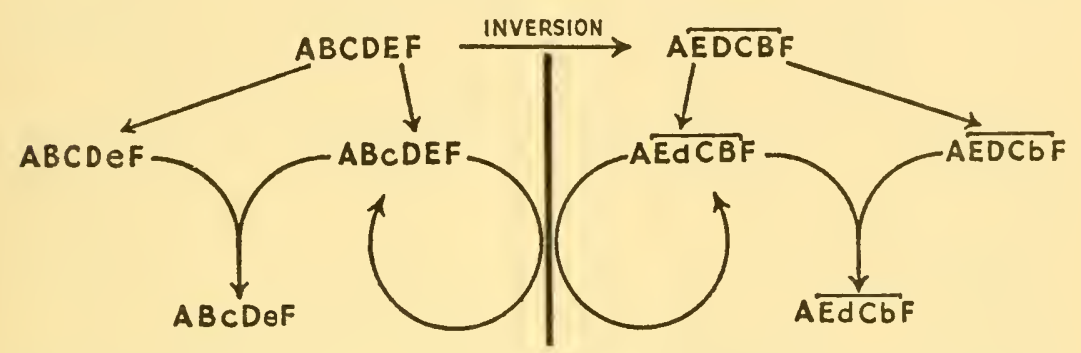

Fig. 79.-The occurrence of an inversion divides the affected chromosomes into two lines, the old and the new, genetically isolated from one another in that recombination is prevented in those segments affected by the inversion. Genic heterozygotes which are also structural heterozygotes can give only the parental types; but genic heterozygotes which are structural homozygotes can give rise to new types by recombination. Thus progressive genetical adjustment must proceed independently in the two lines of chromosome descent distinguished by the inversion.

two species, so that although this sequence is itself common to them, each of its various derivatives occurs in only one of them (Fig. 78).

The transition from the floating to the fixed condition is seen also in interchanges, and the plants which show us this transition enable us also to compare its progress in inbreeding and outbreeding forms. For example, in the inbreeding species, Datura stramonium, different interchange types have quickly become fixed, that is to say homogeneous and homozygous, in different parts of the world (Table 26).

When plants from various places are crossed, rings of 4 and 6 chromosomes are produced at meiosis in the hybrids. The different frequencies of the several interchange types in different parts of the world where more than one type is found indicates that selection is favouring one type in one region and another in another. It is 
sorting them out in such a way as to produce the differentiation between races in respect of interchange which already occurs between the species stramonium and meteloides, for example.

TABLE 26

DISTRIBUTION OF INTERCHANGE TYPES IN NATURAL STRAINS OF DATURA STRAMONIUM AS REVEALED BY CROSSING WITH A STANDARD TYPE (PT2) (BLAKESLEE, et al., 1937)

\begin{tabular}{|c|c|c|c|c|c|c|c|c|c|c|}
\hline \multirow[b]{2}{*}{ Region } & \multirow[b]{2}{*}{$\begin{array}{l}\text { Basic } \\
\text { type } \\
\text { (PT2) }\end{array}$} & \multicolumn{7}{|c|}{ Interchanges } & \multicolumn{2}{|c|}{ Total } \\
\hline & & $\begin{array}{c}\text { A } \\
\text { (PT1) }\end{array}$ & $\begin{array}{c}\text { B } \\
\text { (PT3) }\end{array}$ & $\begin{array}{c}\mathrm{C} \\
(\mathrm{PT} 4)\end{array}$ & $\begin{array}{c}\text { D } \\
\text { (PT7) }\end{array}$ & $C+D$ & $\begin{array}{c}\text { E } \\
\text { (PT87) }\end{array}$ & $\begin{array}{c}\mathrm{F} \\
\text { (PT88) }\end{array}$ & Strains & $\begin{array}{l}\text { Locali } \\
\text { ties }\end{array}$ \\
\hline C. and S. & & & & & & & & & & \\
\hline America & 20 & 15 & 54 & - & - & - & 1 & 1 & 91 & 65 \\
\hline Asia $\quad .$. & 26 & 7 & - & - & - & - & - & - & 33 & 23 \\
\hline Africa and & & & & & & & & & & \\
\hline Australia & 13 & 4 & - & - & - & 14 & - & - & 31 & 23 \\
\hline Hawaii .. & - & - & - & - & 3 & 1 & - & - & 4 & 2 \\
\hline $\begin{array}{l}\text { N. America } \\
\text { (general) }\end{array}$ & - & 39 & - & - & - & - & - & - & 39 & 31 \\
\hline E. U.S.A. & 9 & 163 & - & 30 & 13 & 2 & - & - & 217 & 126 \\
\hline Europe .. & 124 & 8 & 1 & 23 & 8 & - & - & - & 164 & 82 \\
\hline Total .. & 192 & 236 & 55 & 53 & 24 & 17 & 1 & 1 & 579 & 352 \\
\hline
\end{tabular}

$\mathrm{PT}=$ Prime Type.

In the cross-pollinating Campanula persicifolia, on the other hand, one basic type occurs throughout Europe, and the numerous interchanges occur singly as heterozygotes in individuals. By crossing six of these successively a ring of $\mathrm{I} 2$ chromosomes can be built up step by step. Hence it seems that interchanges which are immediately fixed in an inbreeder (or by inbreeding) can be preserved floating for a great length of time in an outbreeder.

With this comparison in mind we can understand what happened to Oenothera, for there is a transition from the outbreeding western species with floating interchanges, such as organensis of Arizona, to the inbreeding eastern species, such as novae-scotiae, in which the interchanges are fixed, but fixed as heterozygotes. It is evident that the transition from outbreeding to inbreeding has been combined with the fixation of the interchanges in the heterozygous condition. 
And the result has been to maintain the same degree of heterozygosity at the end as there was at the beginning. Inbreeding has fixed the interchanges, but only by virtue of this fixation has the move towards inbreeding been successful. Moreover the inter-

(1)

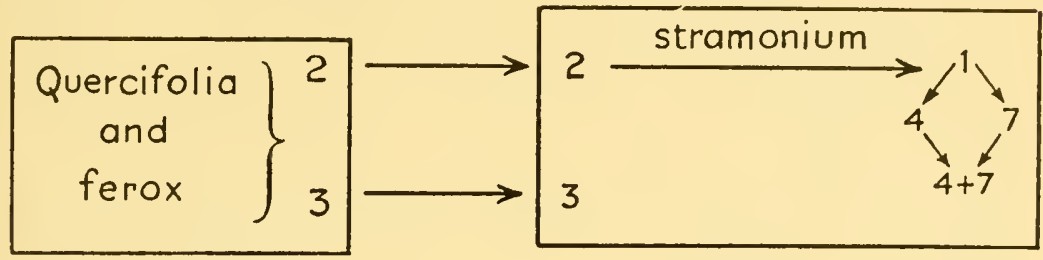

(2)

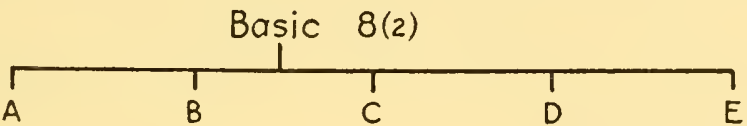

(3)

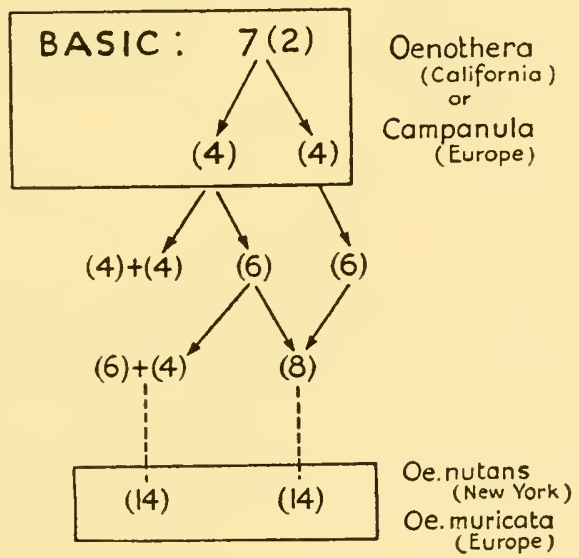

FIG. 80.-Three types of interchange evolution, as seen in (I) Datura, (2) Campanula, and (3) Oenothera.

mediate stages of incomplete ring formation are unstable. There are as many species with a ring of 14 chromosomes as there are with all the lower sizes of ring (Fig. 80). In Hypericum and Rhoeo this process has gone further and the incomplete ring types have already disappeared. It only remains to add that in animals fixed ringformation is hindered by the difficulty of establishing fixed inbreeding. 


\section{Restriction and Flexibility}

Recombination is important in discovering combinations of genes which remove the need for further recombination under existing conditions. The restriction of recombination is important, thercfore, in stabilizing present fitness. Yet in the same paradoxical way, these restrictions reduce the recombination which is necessary for prospective readjustment when conditions change, as sooner or later they presumably must. Devices restricting recombination must thercfore be viewed not mercly in the light of the immediate fitness which they will preserve. They must also be examined for their effects on genetic flexibility, for on these effects will depend the long-range success of the stocks or races which carry them; and simultancously, of coursc, the success of the devices themselves in evolutionary history.

Inbreeding and apomixis restrict recombination by the abolition of heterozygosity and of the sexual cycle respectively. They abolish the very means on which genetic adjustment depends, and as we have seen they are dead ends in cvolution.

The isolation of races, whether by geographical or genetic means, restricts recombination only between those genes which are of immediate importance in giving good local adaptation. Crossbreeding still occurs within each isolated group and the use of any residual heterogeneity, or any new heterogeneity arising by mutation, for adaptive adjustment is not precluded.

The same principle of checking recombination while leaving the way open for later expansion applies with inversion, as we have seen, and also with interchange, provided that the system has not, as in Oenothera, become adapted to inbreeding. When an Oenothera stock has reached the top of the ladder with its ring of 14 chronosomes it can go no further; but this need not be true of the floating interchange system of Campanula which has not become tied down by inbrceding.

Polyploidy is in a similar case. If it has arisen as a short cut to a rigid inbreeding system, the polyploid must be doomed by this rigidity. Probably most polyploids have a relatively short life for this very reason. But, in so far as any crossbrecding has been retained, and in so far as there occurs mutation or recombination 
between the genes of the parental sets, genetical flexibility will be maintained and prospective readaptation will remain a possibility. Even a new inbreeding polyploid can for many generations retain some flexibility, through the gradual unloading of the differences between its diploid ancestors.

We can thus see that complete restriction of recombination is

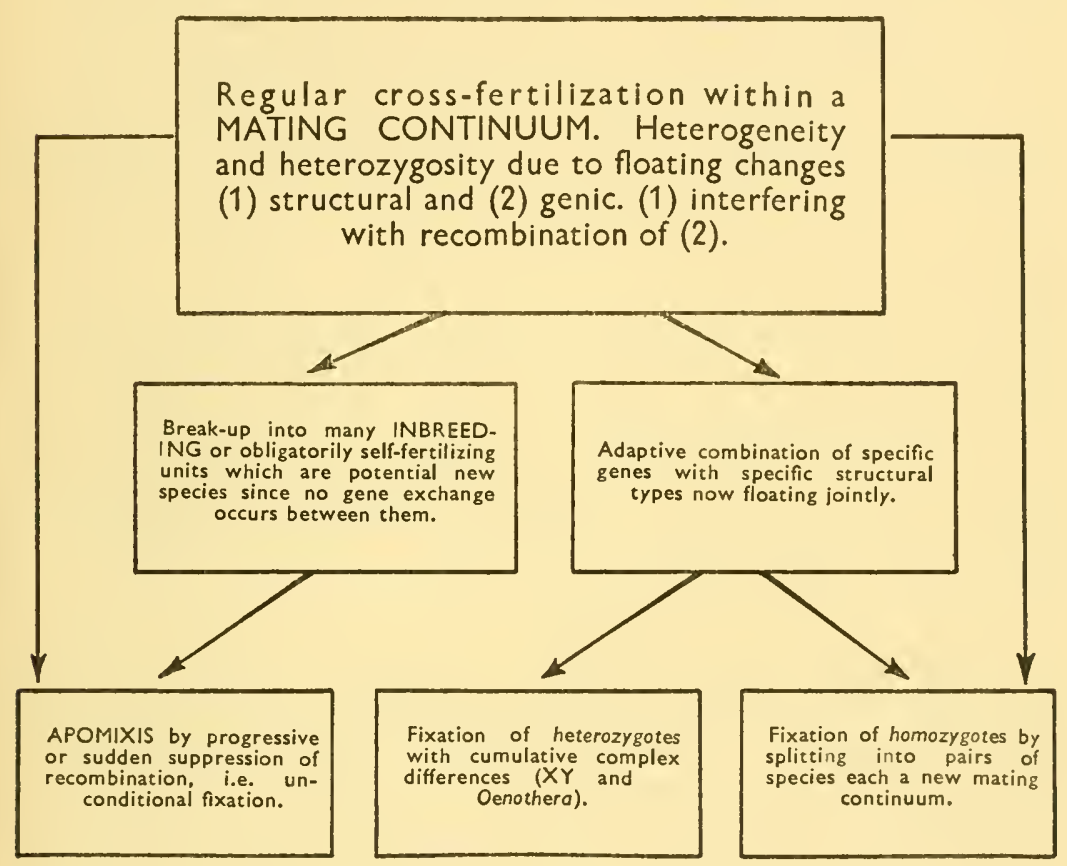

FIG. 81.-Scheme of genetic changes in relation to species formation.

final and suicidal. As usual, it is the compromise solution, the partial and adjustable restriction, which is ultimately the most successful. The desirable genic combinations are held together sufficiently well for present needs by the abolition, or near abolition, of breeding between populations and of recombination between structural types; but the possibility is retained of further adjustment either within the population or within the structural type. Each population or each structural type is separated from its fellows, but remains itself as defining a new and reduced mating continuum. This must start 
small. In so far as it is successful, however, it will grow until its own inherent instability once again becomes too great and new restrictions on recombination arise within it to give a further break-up of the continuum (Fig. 8I).

This course of events is well illustrated by the three Drosophila species which we have already had cause to discuss. These are distinguished simultaneously by differences in chromosome sequence and in breeding behaviour, the differences being less between the more recently separated pseudoobscura and persimilis than between these taken together and miranda. Although they will cross, they prefer to mate within the species. Their hybrids are both cytologically erratic and genetically inadequate. Evidently their genetic architectures are different and the exchange of genes is at least largely prevented between them. In consequence they can overlap in territory without loss either of fitness or of specific distinction. It would appear that miranda has been the least successful, and pseudoobscura the most successful, derivative of their ancestral mating continuum. D. pseudoobscura, in particular, has become widespread and is now showing signs suggestive of instability. Itself one of the products of splitting in past continua, it appears likely to be on the verge of another splitting.

Summing up, it appears that the only universal and permanent type of species is that which allows of regular outbreeding. This type of species is a group of individuals held together in a continuum by genetic recombination. Any change leading to the restriction or breakdown of this recombination leads to discontinuity, or the origin of new species. Whether the first step in the restriction or breakdown is external, e.g. geographical or ecological, or whether it is internal, e.g. structural or numerical change of the chromosomes, a bar to crossing will be established which will bring all other forces favouring isolation into action. There is yet another type of internal change, a gene or chromosome change which establishes obligatory inbreeding or apoinixis, which in itself breaks up the continuum into its individual items. In so doing it produces species which, however, are unlike the parental outbreeding type and have no evolutionary future. 


\section{The Traces of Ancestry}

All forms of discontinuity give us a clue to the relationships of organisms through descent, in other words phylogeny. In so doing they both utilize and confirm the conclusions we have reached on the origins of discontinuity. The chromosomes remain to mark many of the steps by which species, genera, and even families, have diverged. The changes that we have to use for the larger groups are no longer the inversions and interchanges that serve to trace relationships within a small and inter-fertile group. We have to use changes of number arising from polyploidy, reduplication, and the fragmentation and fusion of chromosomes.

Polyploidy is in a special position. It can take place effectively only in one direction: the diploid must nearly always be the parent of the polyploid. Moreover, even as simple doubling, it deternines a genetic change at the same time as it establishes a discontinuity by creating a new form which will not cross with the old. Finally, a single colonizing polyploid individual can unload a degree of variation that would be far beyond the reach of a solitary diploid. For this reason when sudden changes are needed and a sudden opportunity for colonization created, as after the retreat of the ice, the new polyploid steps in and quickly acquires a large range from which its diploid parent is excluded. In Paeonia the Mediterranean seems to have created a barrier that has favoured the appearance of tetraploids in Europe such as are found only once in Asia and not at all in America. An external discontinuity has called forth an internal one (Table 27).

Changes of single numbers also require a word of explanation. Fragmentation can be effective in adding to the number of chromosomes only when the centromere itself is split to give two new chromosomes with terminal centromeres. The result has been found as a fragmentation heterozygote in Campanula persicifolia. Each fragment pairs with the arm of the old chromosome with which it is homologous, so that a chain of three chromosomes is formed at meiosis $(A-A B-B)$. Homozygotes with $8(A B-A B)$ and with $9(A-A, B-B)$ pairs have been derived by segregation from the heterozygote (Darlington and La Cour, unpub.).

Changes ascribed to fusion, and doubtless preceded by breakage, 
THE BREAKDOWN OF CONTINUITY

TABLE 27

SPECIES IN PAEONIA (BARBER 1941, STERN 1946)

\begin{tabular}{|c|c|}
\hline Region & $4 x$ \\
\hline EUROPE & 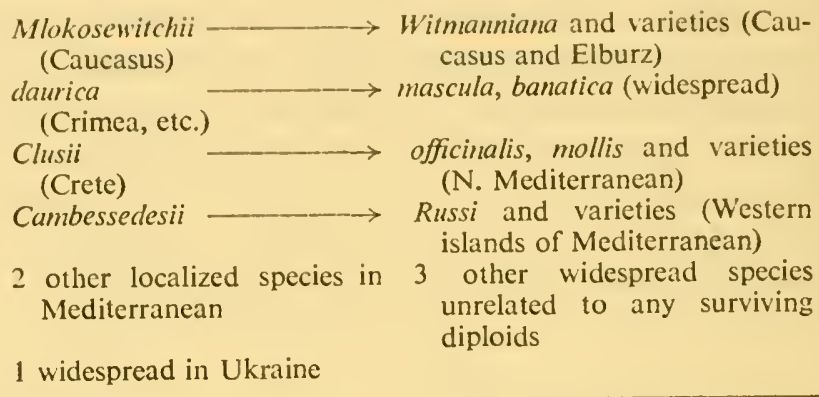 \\
\hline ASIA & $\begin{array}{l}\text { japonica } \longrightarrow \text { obovata and varieties (E. Asia) } \\
\text { (Japan) } \\
8 \begin{array}{l}\text { other widespread } \\
\text { diploids }\end{array} \\
\text { No other tetraploids }\end{array}$ \\
\hline N. AMERICA & No tetraploids \\
\hline
\end{tabular}

have been inferred in grasshoppers. They likewise give novel configurations at meiosis. When any such new types arise as fragmentation or fusion in heterozygotes there is some loss of fertility through irregularity at meiosis. This will favour the segregation and separation of homozygotes of the two types: so soon, at least, as their genic homozygosity docs not conflict too strongly with the hybridity optimum of the species. In other words a chromosome change of fusion or fragmentation may be expected to act, like one of inversion and interchange, as a focus of discontinuity in the species. This expectation is borne out when we discover that in making inferences from changes in chromosome numbers in the larger groups, we are able to apply the rules we derive from what happens to the smaller structural changes within the smaller groups, the races and species.

In the first place the basis of adaptation of races and species lies in their genes. Changes of structure and number of chromosomes are, as a rule, merely the means of directly or indirectly preventing recombination of these genes. The chromosome discontinuities, 
such as inversions, interchanges, and fragmentations, float in the species until they happen to combine favourably with the gene discontinuities. Two consequences follow from this accidental character in the relationship. In the first place changes may take place in chromosome structure or number, or in gene content, without any obvious change in the phenotype. Two complementary instances are offered by Drosophila and the bug Thyanta. D. simulans and melanogaster are externally almost indistinguishable except to one another. Genetically they differ by an inversion. In a species of Thyanta, Wilson in I9I I found two races, one with 7 and the other with 12 pairs of chromosomes. Obviously these two races, not previously distinguished, would be intersterile and they were indeed described (after the event) as distinct species.

Thus great structural and polygenic changes can go on under the surface, as cryptic variation, without any differentiation of external form. It follows that similarity of chromosomes is sometimes a worse guide to similarity of descent than is external form. It also follows that it is a matter of pure chance whether the newer type of gene effect is combined with the new kind of chromosome character or not. It may as well be that the primitive morphology is combined with the new chromosome number as the reverse. In the second place a particular structural difference that we observe now as distinguishing two groups may, as we saw in Drosophila, not be the one which actually helped to separate them. It may still have been present in both at the time they were separated by some other agency of which we can no longer uncover the traces.

Let us not, however, suppose that the systematist may safely proceed unguided by breeding experiment or chromosome analysis. Too often he feels compelled on the one hand to regard as a hybrid an individual or a type which is intermediate between two other arbitrary types from a heterogeneous mating continuum. And, on the other hand, he feels compelled to take as a variety an individual or a type which represents merely a marker gene, whether freely floating in such a continuum, or attached to a super-gene or a chromosome variation or an ecological situation. These crrors he can avoid by combining the rules to be derived from external form and geographical distribution with those to be derived from breeding behaviour and chromosome variation. This combination has yet to 
be brought about but even now we can reach a number of plausible conclusions about ancestry from chromosome numbers.

When we come to apply these rules we find that an enormous variation exists in the stability of chromosome numbers in relation to the external form in different groups of plants and animals. In the flowering plants, where we can compare the numbers of some Io,000 species, the chief source of this variation becomes clear. In the shorter-lived herbaceous plants chromosome numbers usually vary within gencra; in Crepis and Crocus, for example, every haploid number occurs between 3 and I 8 . In the longer-lived woody plants, on the contrary, they remain constant sometimes for whole tribes and families. Take the Pomoidex with their constant 17 chromosomes. This group presumably arose by an uneven or secondary polyploidy from a section of the Rosacex with 7 chromosomes in the Eocene period, since when no change save a renewed polyploidy has occurred and established itself, and very little even of that. It is, therefore, to the woody flowering plants (which themselves must have arisen at different times from herbaceous plants) that we can make the most far-reaching conjectures from chromosome numbers with regard to descent and relationship.

The diagram (Fig. 82) shows how these principles work, in the first degree by polyploidy, in the second by losses and gains in a polyploid, and in the third by losses and gains in diploids. It shows 7 as the common ancestral chromosome number of flowering plants. From this origin 8, 9 and an increasing series have arisen on only a few occasions, whereas $\mathrm{I} 4$, with its diminishing series, has arisen very frequently. In this series 12 has often been stabilized and from its addition to 7, 19 has appeared several times.

One remarkable instance of this last step in the Magnoliales requires special comment. If, as is customary, we divide the genera with 19 chromosomes among 3 families we are implying that this number arose from the union of $\mathrm{I} 2$ and 7 on three occasions. Thus the morphologist's subdivisions of this group seem to be in conflict with the probabilities of chromosome evolution, a conflict which further study will readily resolve.

These conclusions are at the moment necessarily conjectural. But they enable us, by means of genetic theory and cytological technique, to join hands with the equally conjectural limits of systematics 


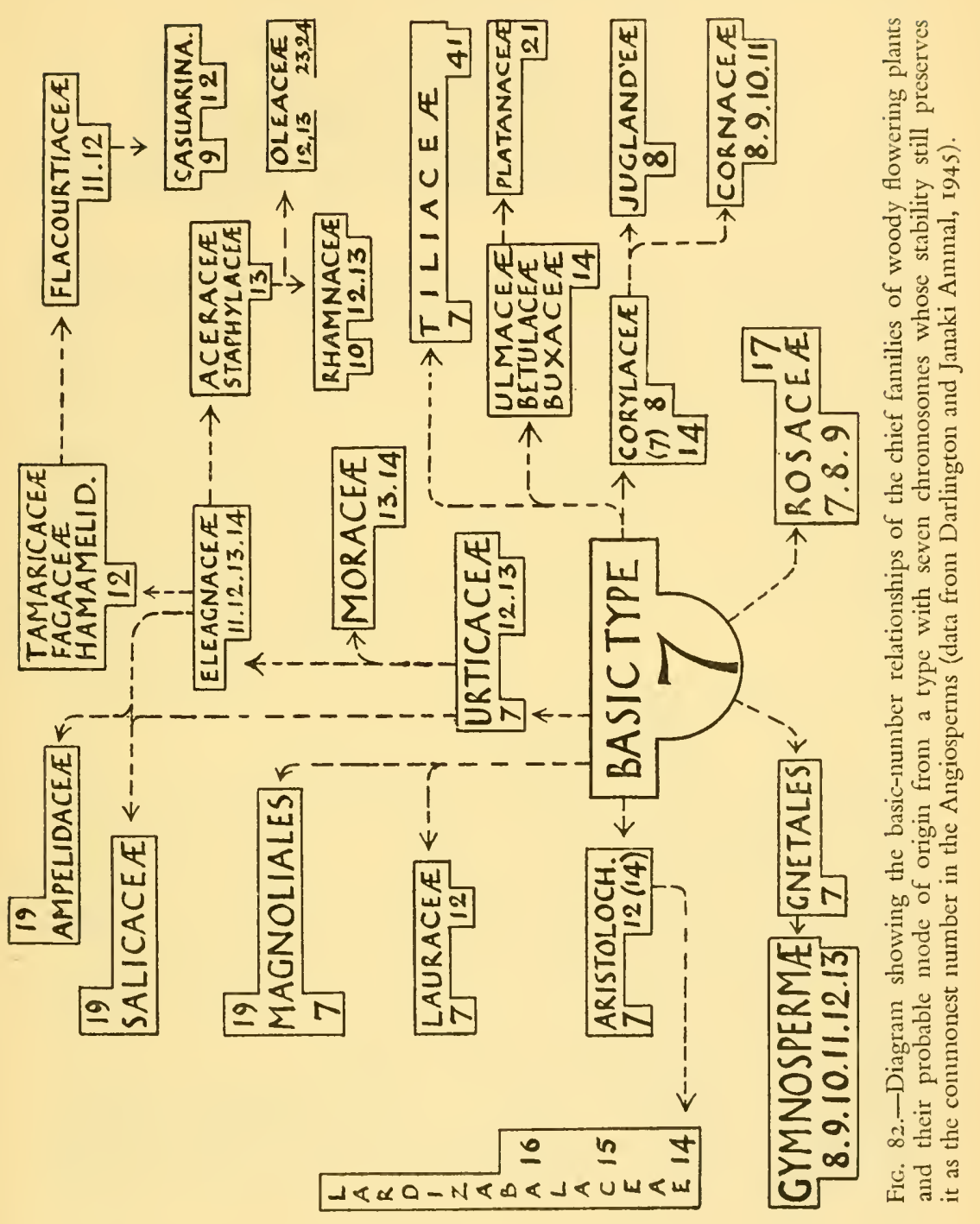


and paleaontology. And in doing so we discover agreements and regularities far beyond the possibilities of coincidence. Conjectures conjoined become verifrable predictions.

\section{REFERENCES}

BARber, H. N. 194I. Evolution in the genus Paeonia. Nature, I48: 227.

BLAKESLEE, A. F., BERGNER, A. D., and AVERY, A. G. 1937. Geographical distribution of chromosomal prime types in Datura stramonium. Cytologia, Fujii Jub. Vol., 1070-1093.

CLELAND, R. E. I936. Some aspects of the cyto-genetics of Oenothera. Bot. Rev., 2: 3 I6-348.

Cleland, R. E. I948. Phylogenetic relationships in Oenothera. Proc. 8th Int. Congr. Genetics (in the press).

Darlington, C. D. I940. Taxonomic species and genetic systems. New Systematics (ed. J. S. Huxley), Oxford.

DARLington, C. D., and GairdneR, A. E. I937. The variation system in Campanula persicifolia. J. Genet., 35:97-128.

DARlington, C. D., and JANAKI-AMmaL, E. K. I945. Chromosome Atlas of Cultivated Plants. London.

DAwson, C. D. R. I94I. Tetrasomic inheritance in Lotus corniculatus L. J. Gentet, 42: 49-72.

DOBZHANSKY, T., 1947. Genetics of natural populations. XIV A response of certain gene arrangements in the third chromosome of Drosophila psendoobscura to natural selection. Genetics, $32: 142-160$.

DOBZHANSKY, т., I948. Observations and experiments on natural selection in Drosophila. Proc. 8th Int. Congr. Gentics (in the press).

DOBZHANSKY, T., and STURTEVANT, A. H. I938. Inversions in the chromosomes of Drosophila psendoobscura. Genetics, 23: 28-64.

HUXLEy, J. S. 1942. Evolution: The Modern Synthesis. London.

Mather, K. I947. Species crosses in Antirrhinum. I Genetic isolation of the species majus, glutinosum and orontium. Heredity, I : I75-I 86.

mather, K., and edwardes, P. M. J. I943. Specific differences in Petunia. III Flower colour and genetic isolation. J. Genet., 45: 243-260.

Stern, F. C. 1946. A Study of the Genus Paeonia. London.

wilson, E. B. 1925. The Cell in Development and Heredity. $3 \mathrm{rd}$ ed. New York.

WRIGHT, S. I93I. Evolution in mendelian populations. Gentics, I6: 97-I 59.

zimmermanN, K. 1935. Zur Rassenanalyse der mittel Europäischen Feldmäuse.

Arch. Naturgesch. (N.F.), 4: 258. 


\section{THE GROWTH OF GENES}

Constructive and Destructive Changes Kinds of Chrontomeres and Genes

The Position Effect Complex Genes Tying Genes Together

Super-Genes The Sex Mechanism

The Limits of the Super-Gene

IN ORDER TO UNDERSTAND THE WAY in which genes becomes adjusted to one another within the nucleus, it was necessary for us to look at the ways in which individuals were adjusted to one another within the population. We found that balance could be understood in its entirety only in terms of the breeding system.

We must now take a reverse step in regard to selection. Having seen how selection operates on the phenotype and, through this channel, on the inter-relations of the genes, we must turn back to examine its effects on the genes themselves, in their dual capacity as units of recombination and of action.

\section{Constructive and Destructive Changes}

In general, plant and animal breeding and evolution suggest that the changes which are constructive in the sense of being new adaptations are of polygenic origin. The major discontinuities used in mendelian experiment, on the other hand, are largely destructive. The overwhelming majority of mutants in Drosophila and Autirrhinum, especially those produced by X-rays, are hypomorphic and selectively negative, or at best selectively nearly neutral.

One or two apparent exceptions are worth noting. In the liverwort Marchantia, Burgeff produced a mutant, blastophora, in which the single three-sided apical cell was replaced by a many-celled meristem, a change which first appears in the Pteridophyta. Similarly there is the mutation found by Andersson and Gairdner in Scolopendrium, by which the spores are converted into spermatozoids and the fern promoted in its life cycle, as it were, into an animal. A third mutation is the hemiradialis of Antirrhinum which restores radial 
symmetry and five stamens to the flower. This looks an even more constructive change, although it is definitely backward to the simpler ancestral type of Verbascum.

Two things, however, need to be said about these mutations. One is that the changes, whether backward or forward in evolution, may nevertheless be physiologically negative. The other is that each mutation by itself leads to a less satisfactory total system: it requires other subordinate mutations to make it work well. We must ask how well-adjusted is meristematic behaviour in Bergeff's liverwort. And how well adjusted is the system to which the sporangial spermatozoids give rise in the fern ? The answers are, very poorly.

Thus these mutations do not affect the general rule. The steady advance of evolution through a series of types, each adjusted and indeed better adjusted than the predecessors which it ousts, cannot proceed by large jumps no matter how potentially constructive these might be. Rather it must proceed (as Darwin imagined) slowly, by a number of small steps, accumulating until in total they give the big changes. But once an advance has been achieved it may be lost by a large jump backwards to some ancestral, and perhaps more flexible, type as in the Antirrhinum. The adjustment of the new type is not a new onc in such a case, but is achieved by taking advantage of gene complexes adjusted by selection long ago, though perhaps more recently put to other work. In a word, it is possible to go downhill by big steps, but to go uphill only by small ones.

How then, we must ask, do the genes, whose mutations produce such drastic changes, come into being? Now, as we saw, most of the changes that we use in studies of inheritance represent decreases or defects in the activities of the gene. These changes imply that the gene itself is something built up and hence capable of degradation. They imply that other changes must also occur having the effect of building genes up. And they imply that genes of different degrees of building up will occur within the same organism. Otherwise we should have to take the view (which was formerly taken) that evolution is a mere unpacking of original units, which themsclves alone are specially created and frec from the consequences of evolution.

The beginnings of any gene must consist of the simplest structure which is capable (with the help of nucleic acid) of reproducing 
itself, that is to say, of collecting together and organizing materials in an arrangement like its own. This activity is scarcely conceivable except as involving the organizing of related arrangements which will pass into the cytoplasm. A more complex gene would be a larger structure, its essential unity depending on the insufficiency of its parts to produce fully organized products, although these parts themselves might be simple genes in a reproductive sense. The minimum qualification for a gene, in this complex sense, then shifts from reproduction to action.

On this view, simpler genes might be the source of materials for more complex genes, which would arise through their components being brought together by structural change in an order or arrangement suited for efficient action in building something useful for the cell and for the organism.

\section{Kinds of Chromomeres and Genes}

What is the evidence for an organization of this kind? Let us begin on the cytological side. Chromomeres, which appear according to Caspersson to be units of activity, units of protein production, are of different sizes. Those in the heterochromatin (when they are free) are smaller and are engaged in organizing smaller proteins of histone type. Those in the euchromatin are larger and are producing larger proteins of globulin type. The smaller chromomeres are less specific and less powerful in their attractions, both in the polytene and the pachytene stages. This may even be the cause as well as the consequence of extensive reduplication of identical genes. Such reduplication, in turn, will scarcely have deleterious effects since the action of heterochromatin is of a lower specificity than that of euchromatin. An extra Y chromosome has little specificity of effect in Drosophila, one of its most noticeable consequences being, it is said, the presence of extra ribose nucleic acid in the egg. Similarly, supernumerary chromosomes in maize, which are heterochromatic, may be present to the number of 20 or 30 without producing any specific abnormality. One or two such chromosomes, if cuchromatic, would on the other hand cause a visible disturbance of growth. Heterochromatin and euchromatin therefore appear to represent two levels of gene integration, two visible stages in the growth of the gene. 
There are attached to the chromosomes two special types of structure which fall into a class by themsclves, whether they are regarded as chromomeres or as genes. The nucleolar organizer and the centromere can both be broken into parts less effective than the whole but nevertheless having the same kind of effect. The organizer, which McClintock broke by X-rays in maize, gives two organizers each with a smaller nucleolus. The centromere, which splits into two crosswise by misdivision in univalent chromosomes, gives two daughter centromeres, each terminal and each less effective than the whole though usually capable of carrying its single arm to the pole. The broken centromeres show no other defect than the customary non-division of a broken end gene which leads to the formation of isochromosomes. Both the centromere and the organizer, therefore, depend for their integration on the mere multiplying of small simple elements.

The genetic counterpart of the cytological evidence lies in the distinction between the different degrees of strength and specificity in the actions of differences in polygenes and in major genes. Experiments with the $\mathrm{X}$ and $\mathrm{Y}$ chromosomes in Drosophila have now shown that the heterochromatin contains only polygenes, while the euchronatin most likely contains both. This is only to be expected on the evolutionary view. The heterochromatin contains only simple elements and can act only in a simple way. The cuchromatin contains complex elements which can act, at least in some stages of development or in some relationships, as integrated wholes, although in others they may act as disintegrated parts. Further, in this latter capacity the genes of the euchromatin influence the same processes of devclopment in the same way as the genes of the heterochromatin: they act as members of the same polygenic systems. We are bound, therefore, to suppose that they are similar elements, the difference being that in the euchromatin they are organized so that complexes of dissimilar ones can also act together in the integrated way by which we recognize major genes.

\section{The Position Effect}

The process of integration of genes is indicated by the position effect. Two genes near one another on the chromosome can influence one another's effects. This influence could be due to an interaction 
of their products, since diffusion camnot be instantaneous. Ephrussi, however, has shown that the distances are too great for this explanation to serve all cases.

The unit of physiological action as shown by the position effect can be compared with the unit of X-ray breakage but not with the unit of crossing-over. Thus the scute gene of Drosophila melanogaster undergoes many natural and induced mutations. They all affect the number of bristles on the thorax and head. They are all due to rearrangements (as Muller and Prokofieva found) in a series of six chromomeres or polytene bands at the distal end of the $\mathrm{X}$ chromosome. The rearrangements involve inversion, sometimes of the greater part of the chromosome, and sometimes of segments within the gene. The distinction between gene mutation and structural change is thus arbitrary in the last resort. The whole set of six segments being concerned with bristle development, the two ends of the gene cannot be marked and crossing-over between thein cannot therefore be detected. It is unlikely to occur, however, since none is found between scute and its neighbour yellow except in structurally hybrid or triploid flies. Thus, in the scute complex, the unit of crossing-over is larger than the unit of action, and this in turn is larger than the unit of breakage and of self-reproduction, which last corresponds to the units of visible discontinuity in the chromosomes.

On the view that the genes are integrated in themselves the breakage of the chromosome should be inherently liable to disintegrate them and so upset, for some distance on either side of the break, the joint and adaptive action of the genes and their parts. Like most other mutations, position effects are, in fact, nearly always deleterious and indeed often lethal in the homozygous state. They should, therefore, usually be lethal to the haploid gametophyte and it is perhaps for this reason that position effects have rarely been found in plants: cells having breakages that would yield position effects presumably die.

The disintegrating effect of chromosome breakage on complex genes is reflected perhaps in the cytological effects of breakage. The normal nucleic acid attachment cannot be correctly arranged at the mitosis following breakage by irradiation (or by misdivision of the centromere). Consequently, in a proportion of cells, the repro- 
duction of the broken end gene is interfered with as much as its action, and by sister reunion or non-division one continuous chromatid loop is formed at metaphase and a bridge at anaphase instead of two sister chromatids.

\section{Complex Genes}

On these general grounds we may approach the question of certain special lines of development that genes may take. One of the clearest is shown by the self-incompatibility allelomorphs in plants. These are of outstanding importance for our purpose, because on no view can they be regarded as produced by degradation from an ancestral type: each is unique and all are fit. When, on the other hand, these natural genes are damaged by $\mathrm{X}$-rays they might be made to mutate to give degradation products revealing their structure and organization.

The degradation of an $S$ allelomorph has been brought about by Lewis in Oenothera organensis. The mutant derived by irradiation from $S_{6}$ was unable to produce an incompatibility reaction by itself in the pollen: it would grow down styles carrying $S_{6}$. But on the diploid female side, when either homozygous or combined in the style with $S_{6}$ or any other allelomorph, it gave the $S_{6}$ reaction: it inhibited $S_{6}$ pollen. Thus the mutation evidently depended on the knocking out of enough of the power to produce incompatibility substance to prevent the gene being effective in the haploid pollen, but not enough to prevent it being effective in the diploid style. At the same time it has not impaired power to stamp its own specific shape or quality on that substance when once produced. Evidently the $S$ gene has two parts. The larger one, the carrier or primer, makes the key blanks; the smaller, the specifying group, gives them their specific shape, their unique character.

The same principle is expressed in certain special reactions of the natural $S$ allelomorphs in the wild Petunia violacea. $S_{2}$ pollen will grow in $S_{2} S_{2}$ styles more readily than in $S_{1} S_{2}$. Evidently $S_{2}$ is a less efficient producer of the carrier than $S_{1}$, but an equally efficient stamp for an excess of materials produced by $S_{1}$. And $S_{1}$ is doing two different things which can be separated by its interaction with $S_{2}$. 
The $S$ gene shows us just how the parts of a gene and their actions must be fitted together. The evolutionary steps in building up a gene must consist in replacing old parts or in putting on new ones in such a way as to give the whole gene a new, and advantageously new, action at each step.

\begin{tabular}{|c|c|c|c|c|c|c|c|c|c|}
\hline \multirow{2}{*}{\multicolumn{2}{|c|}{ ALLELOMORPHS }} & \multicolumn{4}{|c|}{ RHESUS $+(=D)$} & \multicolumn{4}{|c|}{ RHESUS $-(=d)$} \\
\hline & & $R_{z}$ & $R_{1}$ & $\mathrm{R}_{2}$ & $R_{0}$ & $R_{y}$ & $R^{\prime}$ & $\mathrm{R}^{\prime \prime}$ & $r$ \\
\hline \multicolumn{2}{|c|}{ CONSTITUTION } & CDE & CDe & CDE & CDe & $C d E$ & Cole & CdE & cde \\
\hline \multirow{6}{*}{$\frac{\varangle}{w}$} & Anti-C & + & + & - & - & + & + & - & - \\
\hline & Anti-c & - & - & + & + & $\longrightarrow$ & - & $t$ & + \\
\hline & $\underset{\text { (common anti-Rh) }}{\text { Anti-D }}$ & + & $t$ & + & + & - & - & - & - \\
\hline & Anti-d & - & - & - & 一 & + & + & + & + \\
\hline & Anti- $E$ & + & - & + & 一 & + & - & + & - \\
\hline & Anti-e & - & + & - & + & 一 & + & - & + \\
\hline \multicolumn{2}{|c|}{$\begin{array}{l}\text { PERCENTAGE } \\
\text { FREQUENCY IN } \\
\text { ENGLAND }\end{array}$} & 0.24 & 42.05 & $14 \cdot 11$ & $2 \cdot 57$ & $\begin{array}{l}\text { NOT YET } \\
\text { OISCOVERED }\end{array}$ & 0.98 & $1 \cdot 19$ & $38 \cdot 86$ \\
\hline
\end{tabular}

FIG. 83.-The Rhesus blood groups in man. Each allelomorph reacts with three of the six sera and each serum with four allelomorphs, so indicating that the allelomorphs represent the combinations of three primary elements each of which can exist in two alternative forms, viz. $C-c, D-d$, and $E-e$. Of the eight combinations, the one which is expected to be rarest has been discovered in Belgium since this diagram was drawn: it is still unknown elsewhere. Two others occur in England with frequencies of less than I per cent of gametes and only three with frequencies of over Io per cent. The common Rhesus + re reaction is determined by $\mathrm{D}$, Rhesus - ve being $\mathrm{d}$, and the anti-Rh. serum being anti-D or $\Delta$ as it is sometimes called.

The constructive series formed by the natural allelomorphs of the $S$ gene help us to understand the variations in other genes. Take, for example, the Rhesus blood-group gene, an ancient gene which man shares with several monkeys. This gene has been represented as having seven main allelomorphs. With each of these, as predicted by Fisher and proved by Race, there is not one, as with the $A B O$ and $M N$ series, but three antigenic reactions (Fig. 83).

One of these antigens, $D$, carried by four allelomorphs, is of 
predominant importance in determining the agglutination of the blood in late embryos of $D$ type. The mode of action is that, after the mating of a $D$ father with a $d$ mother, a $D$ cmbryo "immunizes" its mother against $D$ by causing her to produce the $\triangle$ antibody and so jcopardizes for many years to come any $D$ cmbryo that may follow it. The other antigens act less often because they are less efficient immunizers.

Thus the Rhesus gene can be looked upon as built up of three genes generally transmitted as a unit but always independent of one another in action. Each of these three can exist in two common forms: $C-c D-d$ and $E-e$. Each of the forms yields a single antigen and hence can induce a single antibody. Thus there should be eight combinations (seven of which are now known) each yielding three antigens and so reacting with three sera. Equally there should be six sera (five of which are now known) each reacting with four combinations or, from the point of view of the Rhesus gene as a whole, four allelomorphs.

We have said that the three parts of Rhesus are independent in action. But, on the basis of what we know of the $S$ gene, we may well suppose that they have a unity of action based on a carrier, and that the allelomorphs of $C, D$ and $E$, are merely stamping products of this common carrier with specificity. It is indeed already certain that the stamps used are different in man and the chimpanzee. On this view the number of allelomorphs will be limited only by the number of specifying groups that can attach themselves to the carricr.

The Rhesus and incompatibility genes agree in showing us natural integration. They also agree in showing us, what we could by no means have expected, that the carrier and the key have to travel next to one another on the chromosome. Co-operation requires juxtaposition, and the two underlie integration.

\section{Tying Genes Together}

These observations clarify the growth of genes as units of action. Such a development presupposes their corresponding growth as units of transmission. But the converse, as we saw earlier, is not necessarily true. Holding together a group of genes will in the first instance give us, not a more integrated unit, but a multifarious or 
pleiotropic one; not a single new effect but a mechanical union of old ones.

Mechanical union can be achieved in two ways which we have discussed separately. The first is by localization of crossing-over, either terminally or near the centromere. This method ties up large segments of all the longer chromosomes: it ties them up perhaps for good and at least for so long as the character of the race persists. The second method is by inversions or interchanges. This is effective only in the structural heterozygotes (where alone it is important) and only for the segment concerned. In other words it is a specific pegging device. In all species inversions are continually occurring. They are of all lengths and in all parts of all chromosomes. They are therefore always available for the task of super-gene construction.

The flexibility of inversions as the building materials of supergenes is well shown by Drosophila pseudoobscura. The third chromosome exists, as we saw, in a number of structural forms differing by inversions. Some flies are heterozygous for sequences differing by several inversions in this chromosome alone. Within these inversions all effective recombination is suppressed in the female, just as much as it is in the male, where there is no crossing-over in any case. For such flies the whole third chromosome is thus a single super-gene. Other flies again are hybrid for fewer or smaller inversions, and some for none at all, so that every gradation can occur within one group of individuals in the unit of recombination.

If there were a dominant and visible gene to mark each of these inversions, or characteristic sequences, in the fly, the population would present us with the appearance of a polymorphic species with a stable (or in some cases changing) equilibrium in the frequencies of the different phenotypes. Such a situation has, in fact, been revealed in a significant series of species of grouse-locusts by the work of Nabours. The first, Acridium arenosum, is polymorphic in regard to colours and patterns controlled by some thirteen genes which recombine easily although in the same chromosome. The second is Apotettix eurycephalus, in which corresponding genes show about 7 per cent crossing-over between two tightly linked groups. The last is Paratettix texamis with a complete suppression of crossingover between 24 out of the 25 colour pattern genes, which, however, remain individually recognizable both by their correspondence with 
those in the other species and by their individual dominance relations. There is, therefore, little room for doubt that in the cvolution of Paratettix the group of genes responsible for its important property of polymorphism has been gradually aggregated into a more convenient single unit. Whether it has been done by inversions or by some other method of restriction we do not yet know.

Polymorphism can exist only because the heterozygote has some special valuc. With heterostyly and sex, for example, this value consists in their property of segregation which is essential for their use in the control of breeding. With the patterns of grouse-locusts it has been shown by Fisher to consist in their greater viability. But the greater viability cannot well depend on the colour genes themselves. It must depend on accessory and invisible differences as it does with the inversion heterozygotes in Drosophila. Thus some of the complexity of the developing gene complex, or super-gene, is cryptic or submerged.

\section{Super-Genes}

The gradual submergence of the variability determined by supergenes may be illustrated by a series of examples. In many animals one sex alone is polymorphic. In the guppy fish, Lebistes reticulatus, there are a great number of gene differences on the sex chromosomes. Some of these genes lie in the pairing segments; others lie in the differential segment of the $\mathrm{X}$ chromosome and mostly show complete linkage with one another and of course with the sexdetermining complex. These gene differences are expressed only in the male, which is consequently polymorphic like the grouselocust in colour and pattern. In the female they are submerged.

The super-genes of Paratettix and Lebistes give no indication of their origin beyond the fact that their components lic in the same chromosomes. In Drosophila we have a super-gene, which is similar in some respects, but which must be more ancient for it is common to a group of species which have a second arm to their $\mathrm{X}$ chromosomes. And in all of the species it is held together by a visible inversion in the second arm of this chromosome. This super-gene is Sex-Ratio. It is a gene, like those we have earlier examined in plants, having a controlling influence on the breeding system and subordinate only to the $\mathrm{X}-\mathrm{Y}$ segregation itself. 
The Sex-Ratio super-gene is again sex-limited: in females it is submerged, like the super-gene of Lebistes. Males carrying it in their $\mathrm{X}$ chromosomes have an abnormal meiosis. Their X and Y chromosomes suffer from a nucleic acid surfeit and one which is greater at a lower temperature. In consequence of the surfeit they fail to pair. The $\mathrm{X}$ chromosome reproduces twice. The $\mathrm{Y}$ chromosome, which, as we know, is still more largely heterochromatic than the $\mathrm{X}$, is so heavily flooded with nucleic acid that it fails to divide at all

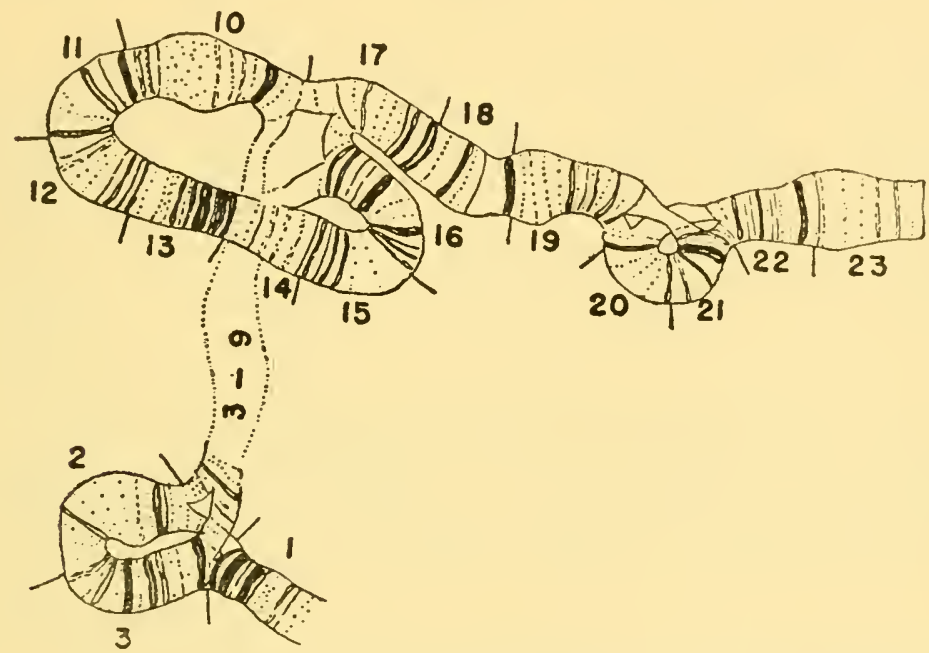

FIG. 84. - The $\mathrm{X}$ chromosomes in the salivary glands of a female Drosophila azteca heterozygous for the sex-ratio super-gene, to show the three inversions associated with sex-ratio in this species (after Dobzhansky, 1939).

and is lost. This course of events results in almost all sperm containing an $\mathrm{X}$ chromosome. The progeny of Sex-Ratio males consist of 94 per cent of females at $25^{\circ} \mathrm{C}$. and 99 per cent at $16 \cdot 5^{\circ} \mathrm{C}$. Owing to the lower efficiency of the gene at higher temperatures, it is not surprising to find that it exists in a higher proportion of the population at the warmer end of its habitat. The supergene, as it floats in the population, is evidently working to modify the sex-ratio as determined by simple $\mathrm{X}-\mathrm{Y}$ segregation in such a way as to economize in the reproduction of the species. No doubt its frequency is raised above the optimum for this effect by the general advantage of hybridity in respect of an inverted segment. 
For the Sex-Ratio super-gene an inversion is indispensable. But this by no means implies stability. The inverted sequence in which it is found in D. persimilis is the normal sequence for pseudoobscurn, with regard to which the Sex-Ratio lies in an inverted sequence. It has escaped from an inverted sequence into a normal one (presumably by double crossing-over); but to be preserved it has required its normal allelomorph to be inverted. Indeed, in this species as well as in D. azteca, Sex-Ratio has picked up three inversions with which it is now combined-or rather locked, since escape from multiple inversions is impossible (Fig. 84).

These apparently random variations show us that the same principle of chance which applies to the fixation of a sequence floating in a sequentially mixed species, extends to the fixation of a gene floating in a genically mixed sequence. They also show us that Sex-Ratio is a super-genc but not always the same onc. This polymorphism, preserving the heterozygous condition and visible both genetically and cytologically, enables us to see the changes that Sex-Ratio has undergone, and that all super-genes can undergo, in the course of a long evolutionary progress.

The last and oldest stage in the submergence of a super-gene is shown by a group of elcments determining the cultivated character of the two hexaploid cereals, wheat and oats. In these, as in other grain crops, the cultivated species have, by involuntary selection, partly or wholly lost three properties that existed in their wild ancestors: shattering of the ear, bearding, and toughness of the chaff. Loss of a pair of chromosomes from one set exposes a group of these characters determined by the corresponding chromosomes of a second set, and results in the appearance of a speltoid or fatuoid mutant (Fig. 85). These mutants, resembling the wild species Triticum spelta and Aveur fatua, are useless in cultivation. The characters concerned are all related to the reproductive system of the plant, yet they seem to have no inherent relationship in devclopment and they have therefore been assumed to be determined by a group of genes. But this group never recombines and has been found by Nishiyama to be housed entirely in the short arm of the chromosome that bears it. It is, in other words, a super-gene.

The speltoid case recalls attention to the two opposed principles concerned in building up genes. In the $S$ allelomorphs there is an 
integration of action within the cell to produce a single phenotypic result. In speltoids and also in Scx-Ratio and in Lebistes there is merely a combination of effects to produce several results that are mutually compatible in whole individuals. The first are complex genes; the second are gene-complexes or super-genes. We have now to see how, in systems we have already described, the two methods of building are in fact combined.

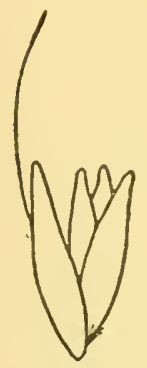

N

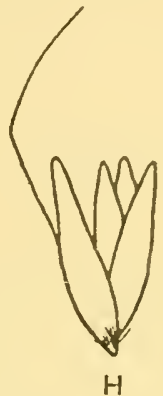

H

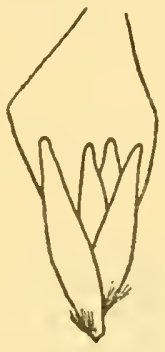

ST

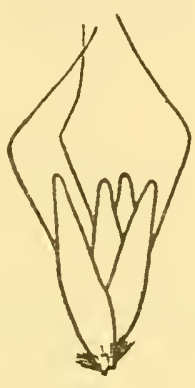

SF

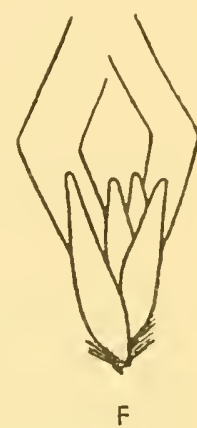

F

Fig. 85.-Spikelets of normal (N) and fatuoid (F) and various intermediate oats. The fatuoids are distinguished by the simultaneous occurrence of an awn on every flower of the spikelet and by the oval or sucker-mouth articulation surface surrounded by dense pubescence. These characters are inherited as a unit and they vary simultaneously, the heterozygote $(\mathrm{H})$ and intermediate types, steriloid (ST) and sub-fatuoid (SF), showing intermediate expressions of all the characters (after Huskins, 1946).

\section{The Sex Mechanism}

Sex determination in most animals and plants, even in the haploid liverworts, depends on the segregation of a difference at meiosis. In the simplest cases this difference is indistinguishable from that of the simplest gene. In mosquitocs and in the amphibia no recognizable difference separates any pair of possible sex chromosomes. But in mosquitoes partial sex linkage is found, so that there must be an $\mathrm{X}-\mathrm{Y}$ pair. How can we imagine a single gene setting off the differentiation of males and females? In maize the system has been constructed experimentally.

Maize has male and female inflorescences on different parts of the same plant. Jones was able to make a dioecious plant out of it. $\mathrm{He}$ began with a homozygous silkless stock, $s_{k} s_{k}$, in which the female inflorescence fails to devclop. Introducing a second gene for 
tassel-seed, $t_{s} t_{s}$, he turned the male flowers into females (and at the same time restored fertility to the female flowers). The segregation $T_{s}-t_{s}$ in the silkless stock then gave equal numbers of males and females in the progeny crossed with $t_{s} t_{s}$, as follows:

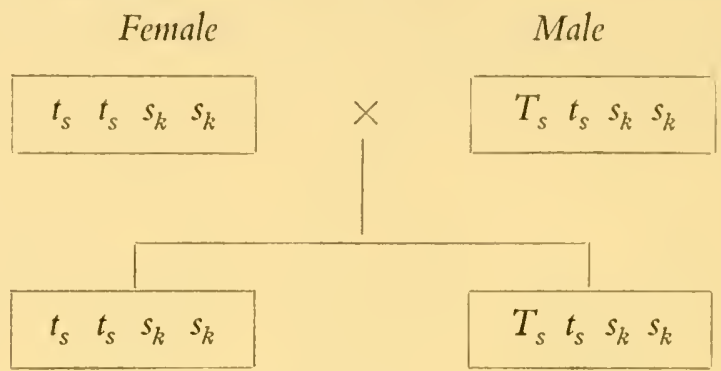

Thus a single gene segregating acts as a trigger or switch for sex differentiation or determination. But it does so only against a selected genetic background. In Jones' experiment this background was maintained by keeping the silkless stock away from the ordinary stock. In nature the same result could be attained correspondingly by isolation, geographical or genetical, from the older stock. It could also be attained, even where crossbreeding is not prevented, by a suppression of recombination between the two genes arising in any of the various ways we have seen. The two dioecious types and the old monoecious type would then work as though expressing a multiple allelomorphic series.

In the fish Lebistes, with the simplest known mechanism, a single gene pair (making an $\mathrm{X}-\mathrm{Y}$ pair of chromosomes) does in fact seem to act as the switch. Again it acts against a correct background provided by the autosomes. The switch gene, as we saw, is associated with additional polymorphic colour genes. It is a super-gene in process of birth. But it is not yet stabilized, for it can still be replaced by a new pair of genes in another pair of chromosomes. Winge has found two such alternative systems. Both of these provide autosomal switch actions. One of them works only in combination with $X Y$. This type necessarily segregates $X X$ and Y Y types and the double segregation of course means that half the progeny are wasted in each generation. The other works in combination with $\mathrm{X} X$ and provides a stable new system. The $\mathrm{X} X$ 
males are completely fertile and have the male-limited polymorphism shown normally only by X Y. The male coloration, which had been sex-linked as well as sex-limited, is now only sex-limited.

The easy substitution of the Lebistes switch mechanism argues a simple gene structure. The sex determiner is part of the super-gene, but sex-determination is not finally bound up with it. Quite a different situation meets us in a species with an ancient system of sex differentiation such as we find in Drosophila. Three differences are outstanding. In the first place, large differential segments in the two sex-chromosomes fail to pair or of course to cross-over: and one of these contains many major genes. Secondly, sex is determined by a more completely and inelastically adjusted balance between the $\mathrm{X}$ chromosome and the autosomes. Thirdly, new switch genes such as have been found several times in the third chromosome of $D$. melanogaster fail to achieve full success, for the new sex genotype, the XX male, is always sterile.

The advanced sex switch gene is therefore an adaptive complex, a super-gene. And we know very well how this super-gene has been sheltered and protected from recombination: it is by the localization of crossing-over at meiosis in the heterozygous sex. Such localization is indicated elsewhere. A difference in the frequencies of crossing-over of the two sexes is characteristic of the advanced systems of sex-determination. In some, to be sure, as in the grasshoppers, it probably does not occur. But in them it would in fact have no meaning, for their $\mathrm{Y}$ has completely disappeared. The $\mathrm{X}$ chromosome is then in the final stage of its development. So far as the heterozygous male is concerned, and so far as sex-determination is concerned, it constitutes a single inviolable super-gene.

\section{The Limits of the Super-Gene}

The next step in the consideration of the super-gene must be Oenothera. Elsewhere super-genes make only one side of each system: some individuals are homozygous for them, and selection can then cause readjustment of their components. In the complex heterozygote of Oenothera, on the other hand, two complexes develop. Each is a super-gene including large differential segments of each chromosome and representing, in fact, almost the whole 
of a haploid set or seven chromosomes. Between the two complementary super-genes of one species no crossing-over can take place without giving rise to a mutant with smaller complexes and so pulling the system to pieccs. We therefore have the remarkable situation in which all adjustment of each super-gene must be relative to its partner. If, in Oe. muricata, the rigens complex becomes less viable or effective as a pollen genotype, then adjustment is restored and seed fertility is increased by the curvans complex becoming less viable as an embryo-sac genotype. And this is what has been happening, as shown in the Renner effect, whereby rigens spores replace their partners as embryo-sacs. With such a limited recombination as is possible in a ring of I4 chromosomes it may well be asked how such an elaborate adjustment can come about. The answer is that it probably takes place through the expansion of the complex, break by break, at the expense of the distal segments of the chromosomes in which crossing-over can still take place. In other words by a growth of the super-gene.

The limit to the growth of the super-gene is reached only when it comprises the whole of the nucleus. This may happen in two ways.

The first is in the fungal heterocaryon. This arises in heterothallic fungi where an illegitinate fusion has occurred between the multinucleatc cells of hyphac of like mating type. As a result each cell contains a number of different nuclei (Fig. 86). Each nucleus divides by mitosis; but division is not co-ordinated, so that different cells at one time, and the same cell at different times, have different numbers of nuclei present in different proportions. Presumably the need for a nucleus of a particular kind stimulates its division relative to that of others. The nuclei, being incompatible, never fuse and there is no meiosis. All the recombinations effected mechanically between genes in ordinary sexual reproduction, are here effected physiologically between nuclei by the changing chemical equilibrium dependent on the changing reactions of nuclci, cytoplasm and cnvironment. The gene is still the unit of mutation, as we saw in Neurospora, but if all but one of the mating types has becn lost so that the heterocaryon is obligatory the nucleus beconics the unit of transmission. Each nuclcus has become a single super-gene. Inasmuch as this super-gene is under general cell control, it is 


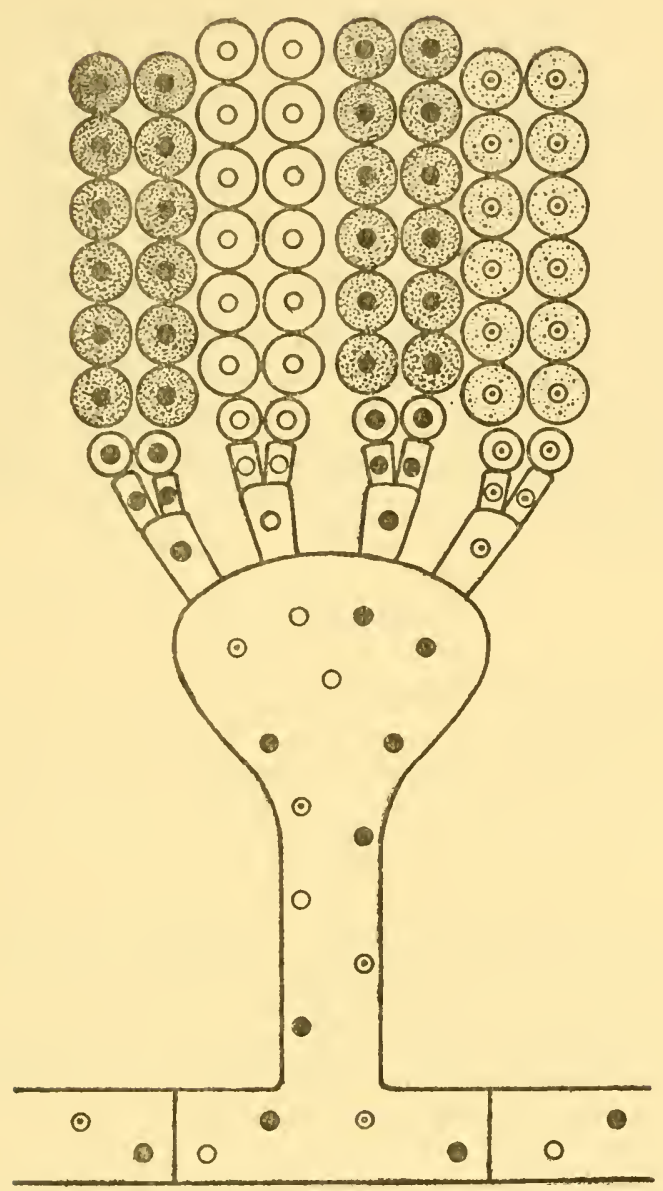

FIG. 86.-Heterocaryosis in fungi. The heterocaryon contains nuclei of more than one kind, with relative frequencies determined by external conditions. These nuclei co-operate in action to produce a phenotype depending on the balance of all their genes simultaneously. The conidia contain only one nucleus each, all in one row having the same nucleus, and the relative frequencies of the rows with the different nuclei affords a measure of the relative frequencies of the nuclei in the heterocaryon. Three types of nuclei are illustrated, their ratio being $2:$ I : I in both heterocaryon and conidia. In Aspergillus the colour of the conidium is determined by its own nucleus (as illustrated), but in Penicillium it is determined by the genetic constitution of the heterocaryon (based on Pontecorvo, I946). 
physiologically analogous with a plasmagene and therefore represents a relapse to the kind of organization that must have preceded the invention of sexual reproduction.

The second limit to the growth of the super-gene is that which is reached by the suppression of sexual reproduction in any apomictic species of higher organism. Where meiosis and fertilization fail, as they do in triploid species of beetles and dandelions, heredity may vary, as we saw, by the occasional loss or gain of chromosomes; but the mitotic nucleus is the working unit of heredity. Recombination has been abandoned. The new type, unlike the heterocaryon, can no longer adapt itself to changing conditions. In attaining its maximum size of super-gene, the whole nuclear outfit, evolution has run its course.

\section{REFERENCES}

BURgefF, H. I94I. Progressive Mutationen bei der Lebermoos Gattung Marchantia. Biol. Zbl., 61: 337-360.

Caspersson, T. 1941. Studien über der Eiweissumsatz der Zelle. Naturuiss., 29: $33-43$

DARLINGTON, C. D. 1942. Chromosome chemistry and gene action. Nature, I49: 66-69.

Darlington, C. D., and DobZhansky, T. 1942. Temperature and "sex-ratio" in Drosophila pseudoobscura. Proc. Nat. Acad. Sci., Wash., 28:45-48.

Dobzhansky, т. I939. Fatti e problemi della condizione "rapporto-sesse" (sexratio) in Drosophila. Scientia Gentet., I: 68-75.

DOBZHANSky, T., and EPLING, C. I944. Contributions to the genetics, taxonomy and ecology of Drosophila psendoobscura and its relatives. Carti. Inst. Wash. Publ., 554 .

EPHRUSSI, B. and SUTTON, E. I944. A reconsideration of the mechanism of position effect. Proc. Nat. Acad. Sci., Wash., 30: 183-I97.

Fisher, R. A. 1947. The Rhesus factor: a study in scientific method. Am. Scieutist., 35: 95-103.

FORD, F. B. I945. Polymorphism. Biol. Revs., 20: 73-88.

GILCHRIST, B. M., and HALDANE, J. B. S. I947. Sex linkage and sex-deternination in a mosquito, Culex molestus. Hereditas, 33: 175-190.

IIUSKINS, C. L. I946. Fatuoid, speltoid and related mutations of oats and wheat.

Bot. Rev., I2: 457-5I4.

LEWIS, D. 1946. Useful X-ray mutations in plants. Nature, I58: 519-520.

LURIA, S. E. 1947. Recent advances in bacterial genetics. Bact. Rel's., I I : I-40.

MCCLINTOCK, B. 1934. The relation of a particular chromosomal element to the development of the nucleoli in Zea mays. Zeit. Zellf., 21 : 294-328. 
mather, K. I9.43. Specific differences in Petmia. I. Incompatibility. J. Genet., $45: 215-235$.

Matuer, K. 1946. Genes. Sci.J. Roy. Coll. Sci., I6: 64-71.

mulier, H. J. I947. The gene. Proc. Roy. Soc. B., I34: I-35.

NABOURS, R. K., LARSON, I., and HARTWIG, N. I933. Inheritance of colour patterns in the grouse locust Acrydiun arenosum Burmeister (Tettigidae). Genetics, I8: I59-I72.

pontecorvo, G. 1946. Genetic systems based on heterocaryosis. C.S.H. Symp. Quant. Biol., II: 193-201.

StUBbe, H., and wetTStein, F. v. I94I. Über die bedeutung von Klein- und Grossmutationen in der Evolutionen. Biol. Zbl., 6r : 264-297.

sturteVant, A. H. I945. A gene in Drosophila melanogaster that transforms males into females. Geutics, 30: 297-299.

SuOMAlainen, E. I945. Zu den Chromosomenverhältnissen und dem Artbildungsproblem bei parthenogenetischen Tieren. Sitz. d. Finll. Akad. Wiss.

TATUM, E. L., and IEDERBERG, J. I947. Gene recombination in the bacterium Esclerichia coli. J. Bacter., 53:673-684. 


\section{MAN AND MANKIND}

Commnon Principles The Special Uses of Man Race Theory Breeding Systems Culture and Language Clines, Tribes and Classes

THE GENETIC PROPERTIES OF MAN are at once less known and better known than those of any creature. On the one hand, his uncontrolled breeding puts him outside the pale of experiments in heredity. On the other hand, the prodigious refinements of historical, cultural, linguistic and medical studies give us the most exact and most prolonged account available of variation in any plant or animal. And not only of variation, but of something else to which we have learnt to attach great genetic importance, namcly, mating habit. Man therefore poses a special problem, but it is one that can be resolved by applying the laws of heredity discovered elsewhere and discussed in our earlier chapters. To do this we must demonstrate the relations between his heredity, his mating system and his variation.

\section{Common Principles}

The mendelian foundations we have already glanced at. From the study of pedigrees many hundreds of segregating gene differences have been discovered in man. These are sharp differences, mostly concerned (as in Drosophila) with abnormalities or defects. They are due to mutant major genes of high penetrance and expressivity, of the type which conveniently show mendelian segregation.

The chromosomes of man we have also seen something of. They number 48 (Fig. 46) including an XY pair in male, an XX in the femalc.

Some genes in man, as we saw, are sex-linked and are therefore locatable in the differential segments of $\mathrm{X}$ and $\mathrm{Y}$. Some also are partially sex-linked and are therefore locatable in the pairing segments of $\mathrm{X}$ and $\mathrm{Y}$ (Fig. 13), a situation otherwise known only in Culcx, Lebistes, Drosophila, and perhaps the cat. The rest, showing no 
evident sex-linkage, are assumed to lie in one of the 23 pairs of autosomes. Of these a few are sex limited. Like most of the colour genes of Lebistes (both sex-linked and autosomal) they are expressed only in the male. Thus it seems that early baldness in men is the expression of a heterozygote, which appears only in the homozygote in women. A greater number show the more remarkable property of incomplete sex limitation. They have greater penetrance in the males. Cleft-palate-with-hair-lip, for example, shows in a higher proportion of homozygous males than females. Many hereditary abnormalities are determined by dominant genes,

TABLE 28

THE FREQUENCIES OF FIRST-COUSIN MARRIAGES IN THE PARENTS OF AFFECTED INDIVIDUALS (HOG BEN，1931; COCKAYNE，1933；DAHLBERG，1942)

\begin{tabular}{|c|c|c|}
\hline \multicolumn{2}{|r|}{ Class } & $\begin{array}{l}\text { Percentage of marriages } \\
\text { which are between } \\
\text { first cousins }\end{array}$ \\
\hline \multirow{5}{*}{ 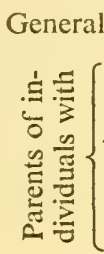 } & 1 population & $0 \cdot 5-1 \cdot 0$ \\
\hline & Friedreich's ataxia & 9 \\
\hline & juvenile amaurotic idiocy.. & 15 \\
\hline & retinitis pigmentosa & 17 \\
\hline & alcaptonuria & ca. 30 \\
\hline
\end{tabular}

although in some the study is complicated by incomplete dominance (or penctrance).

Already in 1902 Garrod pointed out that the incidence of abnormalities and defects determined by recessive allelomorphs should be higher in the progeny of first-cousin marriages than in the general population. The frequency of cousin marriages in the parents of homozygous segregants should increase with the increasing rarity of the disease. If I in I0,000 of the population show the recessive character, 5 per cent of their parents should be first cousins. If I in $1,000,000$, then the frequency should be 35 per cent. Estimates have in fact been made, as given in Table 28, which correspond roughly with the frequency of the disease.

Polygenic action gives a plausible genetic basis for the apparently 
continuous variation in such characters as height, pigmentation and left-handedness. It cnables us to present a picture of human inlicritance with much firmer outlines and in a totality that was lacking with merely mendelian cxamples. This is especially true since, while the major gene differences are largely destructive, the polygenes are all potentially constructive in their cffects, and can thus account for much of the genuine diversity of man depending on characteristically neutral variation.

The independent and combined effects of the actions of chromiosome breakage, major-gene mutation and polygenic recombination arc capable of covering a more complex pattern of variation than formal mendelian statements allowed for. Monstrous births, which are never advertised in any community but occur in all, are probably often due to deficiencies of chromosome parts such as follow the breakage of bridges obscrved by Koller at meiosis in man as elscwhere. Unique and complex syndromes probably often have the same origin. Combinations of gene mutations with other chromosome changes are difficult to demonstrate but must certainly occur. Many common variations, in cye or hair colour or tonguc curling, for cxample, suggest combination of major gene with polygenic effects. And finally, somatic mutation is revealed both by asymmetrics of shape resulting from chromosome derangements at mitosis, and by flecks of colour variation, especially in the iris, resulting from genc-change.

These foundations cnable us to say with confidence that the first principles of genetics, depending on the detcrmination of heredity by the chromosomes, apply to man. Further, cytology agrees in showing that the chromosomes actually cross-over at meiosis in the normal way as the rules of inheritance suggest. And the malc, the sex with the higher mortality before and after birth, is the heterozygous scx. Genctically, the two sexes differ, perhaps, much as two species differ. They are mutually adapted for purposes of reproduction. But each is also adapted to its special cultural activitics. In these the two sexes are complementary but differently adapted in different races, classes and stages of evolution, matriarchal or patriarchal, monogamous or polygamous, slave or free, agricultural, nomadic or predatory. 


\section{The Special Uses of Man}

There are four respects in which heredity in man, in spite of its drawbacks, has stepped ahead of the experimental flies and flowers and has been able to advance the theory of genetics. One is in the study of twins.

In man multiple births vary in frequency from 0.3 to $\mathbf{I} \cdot 8$ per cent according to race. The bulk of these are derived from the fertilization of several eggs at the same time. But about a quarter

TABLE 29

COMPARISON OF THE PERCENTAGES OF SIMILARITY OF REACTION, OR CONCORDANCE, TO DIFFERENT TESTS OF ONE-EGG AND TWO-EGG TWINS IN MAN (BOYD, 1939; DIEHL AND VERSCHUER, 1936 ; ROSANOFF, et. al., 1941)

\begin{tabular}{|c|c|c|c|c|c|c|c|}
\hline \multirow[t]{2}{*}{ Test } & & \multirow[b]{2}{*}{ Concordance } & \multirow{2}{*}{$\begin{array}{c}\text { A or B blood } \\
\text { group } \\
\text { (Agglutination) } \\
\text { Concordance }\end{array}$} & \multicolumn{2}{|c|}{$\begin{array}{l}\text { Tuberculosis } \\
\text { (Susceptibility) }\end{array}$} & \multicolumn{2}{|c|}{$\begin{array}{l}\text { Delinquency } \\
\text { (Institution } \\
\text { Records) }\end{array}$} \\
\hline & & & & Total & $\begin{array}{l}\text { Concord- } \\
\text { ance }\end{array}$ & Total & $\begin{array}{c}\text { Concord- } \\
\text { ance }\end{array}$ \\
\hline One-egg & . & $100 \%$ & $100 \%$ & 80 & $60 \%$ & 137 & $87 \%$ \\
\hline Two-egg & . . & $50-52 \%$ & $40-70 \%$ & 125 & $22 \%$ & 272 & $37 \%$ \\
\hline
\end{tabular}

arise by the splitting of single eggs into two, four, or eight after the first, second or third cleavage divisions. One-egg, monozygotic or identical twins make it possible to show even more clearly than in other animals such as cattle, which are less elaborately variable, the relative effects of differences of genotype and environment on development. One-egg twins are not merely always identical in sex, blood groups, hair-whorls, taste faculties and allergies (Table 29). Their finger-prints are as alike as those of the two hands of one individual. They are also closely parallel in psychology, intellect and susceptibility to delinquency and disease (Tables 29 and 30).

It is the last which gives the most accurate discrimination and the most unequivocal testimony. Of all diseases tuberculosis is perhaps the most suitable for the test of genetic susceptibility, since infection does not lead to immunization and in some regions it is almost 
universal. Dichl and Verschuer compared susceptibility in onc-cgg and in two-cgg (or fraternal) twins, one or both of which had tuberculosis (Table 29).

They found that the identical twins do not always show the same susceptibilitics. This is due to their environmental differences. But they show much more similar susceptibilitics than the fraternals. This is due to their genetic identity. The difference between identical and fraternal twins is thus equally significant in showing that environmental differences on the one hand, and genetic differences on the other, affect susceptibility. This principle has been carried further by Kallmann and Reisner in showing the gradual reduction

TABLE 30

CONCORDANCE IN INCIDENCE OF TUMOURS, TIME OF ONSET, AND TYPE OF TUMOUR IN ONE-EGG AND TWO-EGG TWINS IN MAN (MACKLIN, 1940)

\begin{tabular}{|c|c|c|c|c|c|}
\hline Type of twin & $\begin{array}{l}\text { 1. Number } \\
\text { of pairs }\end{array}$ & $\begin{array}{l}\text { 2. Both } \\
\text { affected }\end{array}$ & $\begin{array}{l}\text { 3. Concord- } \\
\text { ance of type }\end{array}$ & $\begin{array}{l}\text { 4. Aggregate, } \\
2 \text { and } 3\end{array}$ & $\begin{array}{l}\text { 5. Interval } \\
\text { between onset } \\
\text { in } 2\end{array}$ \\
\hline One-egg ... $\ldots$ & 62 & $62 \%$ & $95 \%$ & $58 \%$ & $7 \cdot 5$ yrs. \\
\hline Two-egg $\left\{\begin{array}{l}\text { All } \quad \ldots \\
\text { Like sex }\end{array}\right.$ & $\begin{array}{l}43 \\
27\end{array}$ & $\begin{array}{l}35 \% \\
44 \%\end{array}$ & $\begin{array}{l}54 \% \\
58 \%\end{array}$ & $\begin{array}{l}19 \% \\
26 \%\end{array}$ & $\begin{array}{l}11.7 \text { yrs. } \\
10.8 \text { yrs. }\end{array}$ \\
\hline
\end{tabular}

in correlation between individual susceptibilitics to tuberculosis as genetic relationship decreases. Thus where one member of a pair is affected the other member is also affected in the proportions of cases shown in Table $3 \mathrm{I}$.

The difference between the proportion of full siblings and of parents and offspring, where the same degree of genctic relationship is involved, is due to the fact that different generations obviously must differ in environment more than the same generation. The gradation, therefore, again neatly reflects the reaction of differences in both heredity and environment.

The second special development of heredity in man is in the study of blood groups whose physiological and evolutionary interest we have already cxamined (Fig. 37). This study was called into being by the needs of transfusion, since bloods containing $A$ and $B$ cannot be mixed. It developed a sccondary interest as a means of proving 
paternity since (apart from mutation) a child with an $A$ or a $B$ must have an $A$ or a $B$ in one of its parents. The determination of blood groups on a large scale has led to the unforeseen discovery of further new blood groups. Amongst these are the Rhesus series which we have already discussed as showing the unsuspected antigen reactions between mother and embryo where the two differ.

A last consequence of the study of blood groups has been derived from their frequency distributions in human populations. Comparison has gradually exposed the existence in respect of the blood groups of those clines which are well known for genes in equilibrium

TABLE 31

THE FREQUENCY OF AFFECTION IN VARIOUS RELATIVES OF INDIVIDUALS AFFECTED WITH TUBERCULOSIS (KALLMANN AND REISNER, 1943)

\begin{tabular}{|c|c|c|}
\hline \multicolumn{2}{|c|}{ Relation } & Percentage affected \\
\hline Identical twins & $\ldots$ & $87 \cdot 3$ \\
\hline Fraternal twins & $\ldots$ & $25 \cdot 6$ \\
\hline Full siblings .. & .. & $25 \cdot 5$ \\
\hline Half siblings. & $\cdots$ & $11 \cdot 9$ \\
\hline Parents and offs & ng & $16 \cdot 9$ \\
\hline Husbands and $\mathrm{n}$ & es... & $7 \cdot 1$ \\
\hline General populat & (over 14) & $1 \cdot 4$ \\
\hline
\end{tabular}

in other variable or polymorphic animals (Fig 76). The individuals with $B$ decrease in frequency from 60 per cent in parts of India to Io per cent in Ireland. This gradual transition overrides the divisions of language, skin-colour and skull-shape laid down by earlier social and physical studies; and it shows the genetic gradations underlying racial differences in man which knowledge of his propensities for migration and hybridization, and of his variation in all large groups, would lead us to expect. To this question we shall return later.

A third respect in which the study of man has shown the way is in cancer research. The importance of cancer has led to its most extensive study primarily in man, but also elsewhere, with fundamental results, some of which we have already seen in relation to plasmagenes and viruses. Cytologically, moreover, it has been possible to show that these particles act in the development of 
cancer by a derangement of the nucleic acid cycle. The mechanism is like that found with the development of pernicious anacmia, which leads to upset of mitosis and the formation of both polyploid and sub-diploid nuclei. The defective nuclei survive through existing in groups whose members, having no differentiated cells or spheres of action, support one another. Sufficient breakage of chromosomes by irradiation, however, can give rise to defective nuclei which are unable to support onc another. A tumour whose growth has presumably becn started by the cytoplasm can thus be stopped by the nuclcus. Finally, it has becn possible to show in mice as well as in man that cancer is determined by heredity, although the penctrance of the genotype is often low, the growth of a tumour being subject to cnvironmental conditioning. At cvery point of causation, diagnosis, and treatment, the techniques of genetics or cytology are thus concerned with cancer.

The fourth respect in which human heredity is pre-eminent is that in which it is obviously unique, namely in the study of the mind, or of the body by way of the mind. The simple propertics of taste and allergy, which must obviously be hereditary in all animals, can be readily shown to be so only in man: the ape can mercly confirm what the man has discovered. And when we come to the still more delicate question of instincts, it is only in man and his domesticated dog that the individual character shows the hereditary diversity of the species.

These foundations are important for their direct assistance to education and medicine, but they are still more important for their potential assistance to research. They provide us with a programme of future rescarch on a broader basis. They show the importance, for diagnosis and for treatment, of accurate medical rccording of family historics, and especially of those of identical twins. They show that the extended study of blood groups is bound to yield fruit of value in three different ficlds. They show that our practical knowledge of the rclations of host to discase, gained in agriculture, can be applied with advantage to the study of immunity and epidemics in man. They show that the chromosomes can be used in the study of human development and disease with a profit that can as yet hardly be cstimated. 


\section{Race Theory}

In an altogether wider field, that of the study of the evolution of society, of culture, and of language, genetics is important in enabling us to apply rigorous methods to the study of races, classes and individuals, in rooting out dangerous superstitions and in replacing them with sounder judgments. One example of this replacement may be taken, that of the racial theory which has lately been used as a political instrument in several countries. The theory has three parts:-

(i) That mankind is made up of groups, called races, which are separable by religion or language.

(ii) That these races are sufficiently homogeneous to be placed in fixed order on a scale of unconditional merit.

(iii) That crossing between higher and lower races on this scale always produces offspring inferior to the higher.

Consideration, equally of the history of mankind and his present state, shows that he resembles the crossbreeding species which we have discussed earlier in being adapted to some degree of hybridity. Groups fluctuate widely in the degree of inbreeding or outbreeding they practise and in the tenacity with which they hold to religion and language, neither of which proves an insurmountable barrier to mating. Consequently they may well differ in their optimum degrees of hybridity. We can only predict that the sudden succession of inbreeding to outbreeding (as on Tristan da Cunha or Pitcainn Island) as well as the reverse, are likely to be hazardous in their immediate effects.

Nor can we suppose that, so far as racial groups uniformly differ, their differences are unconditionally advantageous to one or the other. The negro benefits in the tropics from his greater resistance to a variety of tropical diseases. The European benefits in temperate climates from his greater (although very variable) resistance to tuberculosis. In other words races in man, as in all other animals, are adaptive. Crossing between them, therefore, reduces the immediate fitness of the progeny while increasing the long-range flexibility of the stock. In man, again as in all other organisms, neither the fitness nor the flexibility is the highest good but the balance between the 
two or the periodic change from one to the other. And where one has failed it is time to choose the other.

Our genetic principles, therefore, favour neither the extreme advocate of racial purity nor the equally extreme anthropologists, philosophers and historians (whether liberal, Marxist or Catholic) who dogmatically assert what they desire to belicve, namely, that genetic and racial differences in man are trivial or temporary products of an all-powerful environment, "not a cause but a consequence," and so on. Genetic analysis resolves the conflict between "race" and "cnvironment." And it does so by assuming an entire determinacy of individual differences, physical and mental; a determinacy in the reaction of genotype and cnvironment; a determinacy which is likely to prove more important in explaining the past and present structure of our species than of any other.

\section{Breeding Systems}

Ever since, in I865, McLennan first used the terms endogamy and exogamy the importance of the brecding system has becn understood in man. Risley, for example, in I9Is pointed out its genetic significance as follows (p. I 54): "Amongst the various causes which contribute to the growth of a race or the making of a nation by far the most effective and persistent is the jus conmubii-the body of rules and conventions governing intermarriagc. The influence of these rules penetrates every family; it abides from generation to gencration, and gathers force as time gocs on. The more eccentric the system the more marked are the consequences it tends to produce."

The first principle of importance in measuring the effect of breeding systcms is that the heterogencity, the frequency of gencdifferences, within any group is reduced with the rise of inbreeding and is reduced the more rapidly the smaller the group which the inbreeding delimits.

In man the two restrictions of breeding which are characteristic of crossbrecding organisms are both maintained. Extreme outbrecding and extreme inbreeding are both discouraged, but the direct genetic control is, as usual in man, replaced by social and 
intellectual control. Thus the whole of mankind consists of groups which are separated by race, language, general culture and economic status. Between these groups mating is discouraged. Within them, therefore, inbreeding is encouraged. At the same time extreme inbreeding is discouraged almost universally by the prohibition of incest. The rare exceptions to this rule are of great interest and are of many different kinds and degrees according to whether they occur in a homogencous group, as in the Royal Families where inbreeding was sometimes but not usually cumulative, or in an extremely heterogeneous group like the populations of the islands already mentioned.

Inbreeding, to the degree described elsewhere as incest, and existing as a continuous social habit, probably survives only in a few primitive tribes of southern Asia. One of its most interesting forms was in the Ernadan tribe of Malabar in which the father of a family habitually took his eldest daughter as his second wife. Such a system probably represents a dying vestige of earlier inbreeding systems which have been killed wherever, as in Australia, migration and conquest are easy, by the diffusion of more successful systems of outbreeding, often carrying with them diseases which inbred populations, as we shall see, are not flexible enough to resist.

Here we are assuming that natural selection will operate on breeding systems which are culturally determined and not therefore dircctly under genetic control. This is an assumption required only in the field of human genetics, but it has serious implications for the evolution of man.

The succession of outbreeding by inbreeding causes human society to be continually breaking up into small groups, races and tribes, castes and classes. These groups resemble the races of animals and plants except in one important particular: they are consciously made by man himself and not by external or genetic accidents. Throughout history these groups are as continually being broken down by man's own activities, by conquest, slavery, conversion, and mere migration. There is, therefore, an alternation of inbreeding and outbreeding of a rapidity unknown in any other organism and successively favouring fitness and flexibility, the rises and falls of races and classes.

Owing to the rapid changes in his cultural capacity and economic 
power, the equilibria of races and classes in man have always been unstable throughout his history. Secondarily, too, these changes have brought logarithmic increase in the total population of the world, which has approximately multiplied itself by ten in each of the last four periods of a hundred generations. It is, however, not merely these cultural and numerical changes (leading to conquests and migrations) that have upset the equilibria of races and classes in human history. All such groups are inherently impermanent owing to the increasing homogeneity and consequent lack of flexibility produced by inbreeding within them.

The largest inbreeding experiment ever carried out is that of the caste system of India. The caste system breaks up the Hindu community, according to the Indian census, into some 2,300 mating groups. These are of sizes varying from a few hundreds to a few hundred thousands, and of ages varying from $\mathrm{I}$ to 50 generations. They differ also in exclusiveness, which in general is increased by the operation of the census itself, and through the imitation of Hindus by Muslims. The remarkable position, however, is often reached that, when all the persons are excluded who are too closely related to marry and also all those who are too remotely related, that is outside the caste, no one but a first cousin is left, and for a woman sometimes only a fraction of that. This must give a very great stability to the flow of variability discussed in our earlier chapters. The situation is the opposite of that where outbreeding is suddenly followed by inbreeding.

With Hinduism, such diffusion as occurs between castes is chiefly by social demotion or out-casting. The social promotion which is characteristic of European systems is available only for women. The whole system can be maintained only by religious conviction, and it shows its effect in reducing the flexibility and initiative of the peoples concerned and of their culture (Fig. 87).

Where inbreeding is combined with geographical isolation another effect is to produce a homogeneous susceptibility to diseases from which the community has been sheltered. The sudden breakdown of this sheltering has been characteristic of the opening up of backward countries since the great navigations. Its consequences are well known. Large groups, like the old world population which received syphilis from America in 1492, recovered from the first 


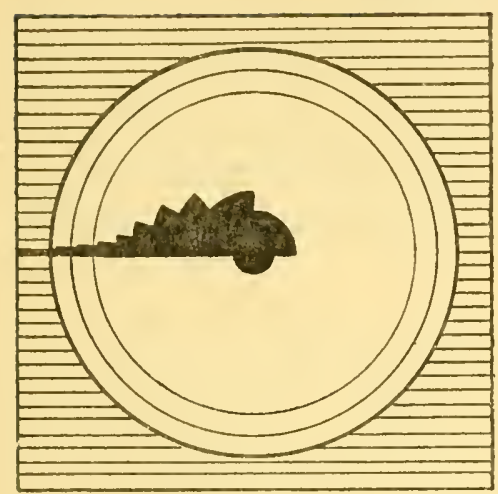

PLANTS

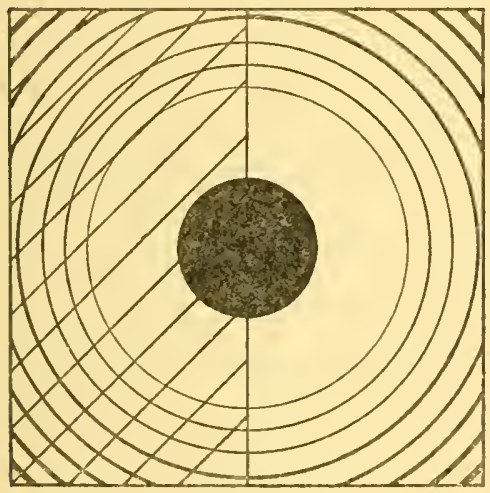

EUROPEANS

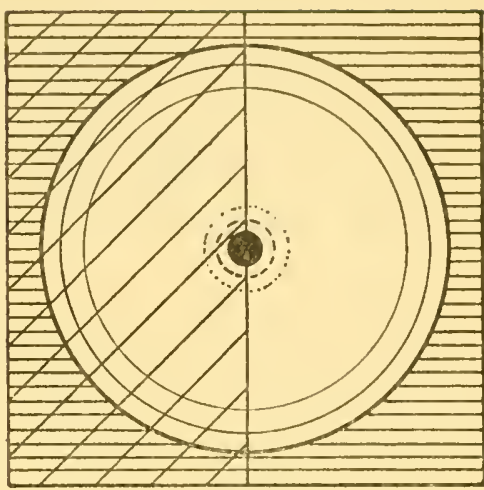

ANIMALS

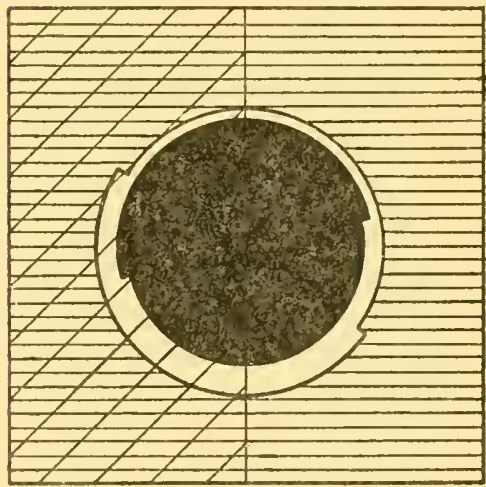

HINDUS

FIG. 87.--Simplified diagrans showing the comparative non-spatial restrictions of mating in relation to divergence of descent with the various systems of control found in man and other organisms. Black represents exclusion from mating by too close relationship (the inmost circles being self-fertilization). Circles represent obstruction of mating; broken circles by discrimination against inbrceding, unbrokc1 by selection against remote crossing. Horizontal hatching represents exclusion from remotc crossing, diagonal from crossing with the like sex. In PLANTs incompatibility of the too-like and the too-unlike are the agents; in ANIMALs mating discrimination against the too-like and the too-unlike; in EuropeAns taboos and laws against incest and mating preferences; in Hindus the cxample illustrated represents the working of high caste rules in North India: the prohibition of inbreeding extends to sapindas, i.e. the 6th cousin on the male side and $4^{\text {th }}$ on the female; outbreeding is also more restricted for females than for males by the hypergany rules which prevent women but not men marrying beneath then so that the possible mates for a fenale may be reduced to a small fraction of unity. 
shock and gradually increased by sclection their capacity for resistance. Small groups, like the South Sea and Andaman islanders, rapidly succumbed to diseases like measles, to which larger and more exposed populations had become resistant. Such small populations are often wiped out in this way. Highly fitted to their previous conditions, they are not flexible cnough to survive the change.

The great alternations of inbreeding and outbreeding, which destroy old races and their empires, and establish new ones, have the same effect on the smaller groups within them. Every nation is subdivided into economic and cultural groups which we call classes. The classes adapt themselves continually to special functions in a changing society. The most important of these (whose function is also changing with changes in the means of production) is the governing class. All governing classes have the aim and the ability to remove themsclves from the pressure of natural sclection. But the mating systems they favour lic between the usual extremes. Governing classes which adhere to the most exclusive inbreeding are always overthrown through lack of genetic flexibility when conditions change, either from inside or outside their empire. Governing classes which allow of a social osmosis, a not too limited diffusion by intermarriage like that in Rome under the Empire, often succced in surviving, with appropriate dilution, very drastic cultural changes.

Those who favour the oppositc extreme to an Indian caste system, an indifferent, classless unadapted heterogencity of the whole of the community may look to polygamous socictics for an cxample of how it works. One of these appears as a convenient control in the Mohammedan population of India. Polygamy can never be effective except for a wealthy governing or conquering group. But such a group cannot be a class in a strict hereditary sense since polygany implies recruitment or dilution from below, or from the outside, on a vast scale in cvery gencration. Moreover the governing group propagates itself from women selected for their value as individuals in a harem, and not for their parentage or their position in socicty. It seems to be this method which permitted the explosive growth of Islam by a series of umprecedented conquests over a period of I,O00 years. But each explosion has becn followed by a somewhat 
rapid decay, a decay which we may associate with the absence of a stablc and continually selected governing class.

In India the recruitment of Islam from Hindu lower castes has in addition hampered any possible growth of an intellectual class. Caste has therefore the appearance of triumphing over non-castc, although neither could compete with a west European system of an clastic type.

\section{Culture and Language}

While noting the readiness with which genetic principles may be combined with simple obscrvations of mating practice to explain the chief characteristics of human socicty, we must also note certain peculiarly human properties of human genetics. Genetic changes in all animals and plants change their environment and thus react on themselves. Man, howvever, from his beginning has been creating his own environment, or rather men have been creating their own (increasingly diversified) environments, more rapidly than any other organism and with continually increasing speed and power. All races and classes and individuals in their activities are concerned with their own survival. But these activities, since they so often lead to innovations of theory and practice, increase the chance of survival, not merely of the innovators, but of their imitators who may be of unlike race and class.

Thus the basic principle, whereby natural selection in other organisms depends on the action of varying environments on varying genotypes, becomes distorted in man by the predominant long-range effect of the genotype and of reactions between different genotypes whose aggregate we describe as culturc. Man's control of his environment has given an exponentially increased valuc to the genotype. This genotype reacts with an environment which it has earlicr modified, just as in devclopment the nucleus acts on a cytoplasm which it has carlier modificd, and so becomes the predominant partner. Not only this, but man's social and cultural organization, depending on the integrated effects of the genotypes of groups of individuals, often with a few of predominant importance, is responsible for the weighted, or exaggerated, or indecd catastrophic, fluctuations which are so evident in human history or 
cvolution. The outstanding individual, that is the individual having an outstanding reaction with his cnvironment, is exccedingly important in human history even though his emergence can be roughly predicted from the flexibility of the mass. The adaptations and fluctuations are, in turn, responsible for the increasing tempo of humian change and the increasing contrasts of genctic capacity for culture between different genetic groups.

Gencral propositions concerning the genetic bases of culture need to be stated first, but for a practical and specific example we can as yet foreshadow their probable effects only in one ficld, that of language.

The rapid development of language from emotional cries, postulated by Darwin, is the outstanding example of the self-propagating or cumulative properties of human culture. We have already secn that races of man largely depend on the barricrs of language for their maintenance. We must note also that the recognition of the unity of the Aryan languages by Sir William Jones in I 784 was one of the mainsprings of evolutionary theory (as well as of racial superstition) to which genetics owes so much. It is a debt which genetics can now begin to repay.

The existence of a genetic component or racial basis for culture has long been obvious enough to have been worth denying. The genetic component of language, on the other hand, has been assumed with very little controversy to be either everything in it, or nothing at all. Let us consider the evidence.

The precise genetic control which we now know to exist over the development of all bodily structures, as we can see from the strength of racial and family resemblances, extends, as Duckworth and others have shown in great detail, to the organs of speech. This genetic control must limit, not so much the ability, as the ease with which races and individuals are capable of uttering the various sounds within the range of the human voice.

Certain racial, family, and individual differences in tongue shape and tongue movement are so sharp as to be obviously hereditary. Their racial significance was first noted in the division of the Jews according to those who could, and those, numbering 42,000 , who "could not frame to pronounce" the word "shibboleth" right (Judges $12: 4-6$ ). The widely differing command that individuals 
have over the sounds both of their own and of foreign languages affords evidence of this limitation. Education and booklearning do their utmost in civilized countries to suppress the vagaries of dialect and the impediments of individual speech. The general effect of education, as we see it today, is not, as it might be, to discover and exploit individual genetic properties, but to obliterate them in uniformity. For this reason the unlettered yokel or infant provide us with the best evidence, and it is very clear evidence, of the natural and uncorrupted genetic differences between the speech capacitics of individuals and populations.

Yet another type of evidence is available for the genetic control of language. A phonetic map of any area of the world revcals gradients or clines parallel with those we saw in the blood groups and like them sometimes agreeing with, but more usually overriding, the ordinary linguistic or rather verbal barricrs. For example the fricative dental ( $\mathrm{TH}$ and $\mathrm{DH}$ ) has arisen only in the Eurasian region. It is most strongly developed in Iceland, Spain, Greece, India and Burma, that is, peripherally. Such an orderly distribution seems to indicate relationship going back beyond neolithic times. And it extends from Iceland to Ireland and England, from Spain to the Basque country, and from Greece to Albania, from Southern India to Burma, in each case uniting what philologists (and those anthropologists who take their lead from language) regard as divergent stocks. Moreover, there is a steady diminution as we pass from Ireland to Wales, Wales to England, England to West Jutland and so on to Zeeland and South Sweden where the TH sounds disappeared 500 years ago. We thus have a cline which may be expressed in both space and time.

It is, indeed, possible to draw maps showing the isogenic lines both for blood-gene frequencies and for phonetic capacities or preferences. Both of these characters are as nearly neutral in regard to natural selection as one can imagine, and we might, therefore, expect them to reffect equally well the long-standing genetic properties of the population. When we do so we find a remarkable agreement in Europe between $64^{\circ} .5$ per cent line for the $O$ chromosomes (in the $A-B-O$ scries) and the boundary between those who use TH in speech today and those who do not. We also find an agreement between both of them and our knowledge of the historical 
pressure of centrifugal migrations from castern into western Europe (Figs. $88 \mathrm{~A}$ and $\mathrm{B}$ ).

Students of language have often held the view that some inherent

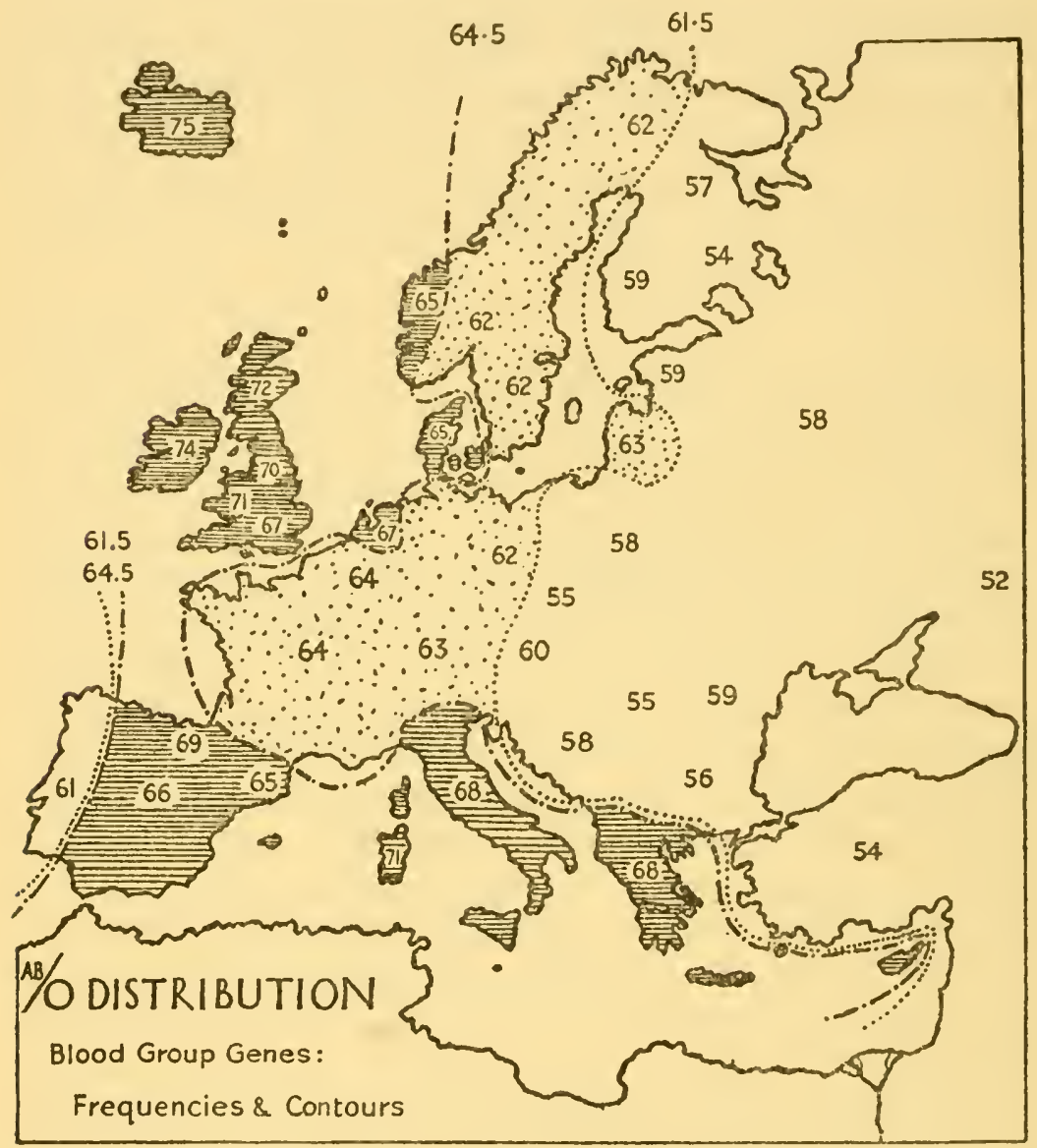

Fig. 884.--Genetic contour map of Europe to show the geographic gradicnts in O blood group chromosomes in man. Note that the peripheral distribution of the high populations is as though determined by migration from the cast across the ccutral plains.

property of peoples influcnces the sounds they use and the changes this use undergoes. We now see that the undefined "Substratum" assumed in linguistics is the aggregate genotypic action of the community. But it may be asked why such action should change sounds, 
shifting some and eliminating others. The answer is that conquering peoples, such as the Danubian Aryans and the later Huns and Mongols, who spread east, west, north and south, could suppress the

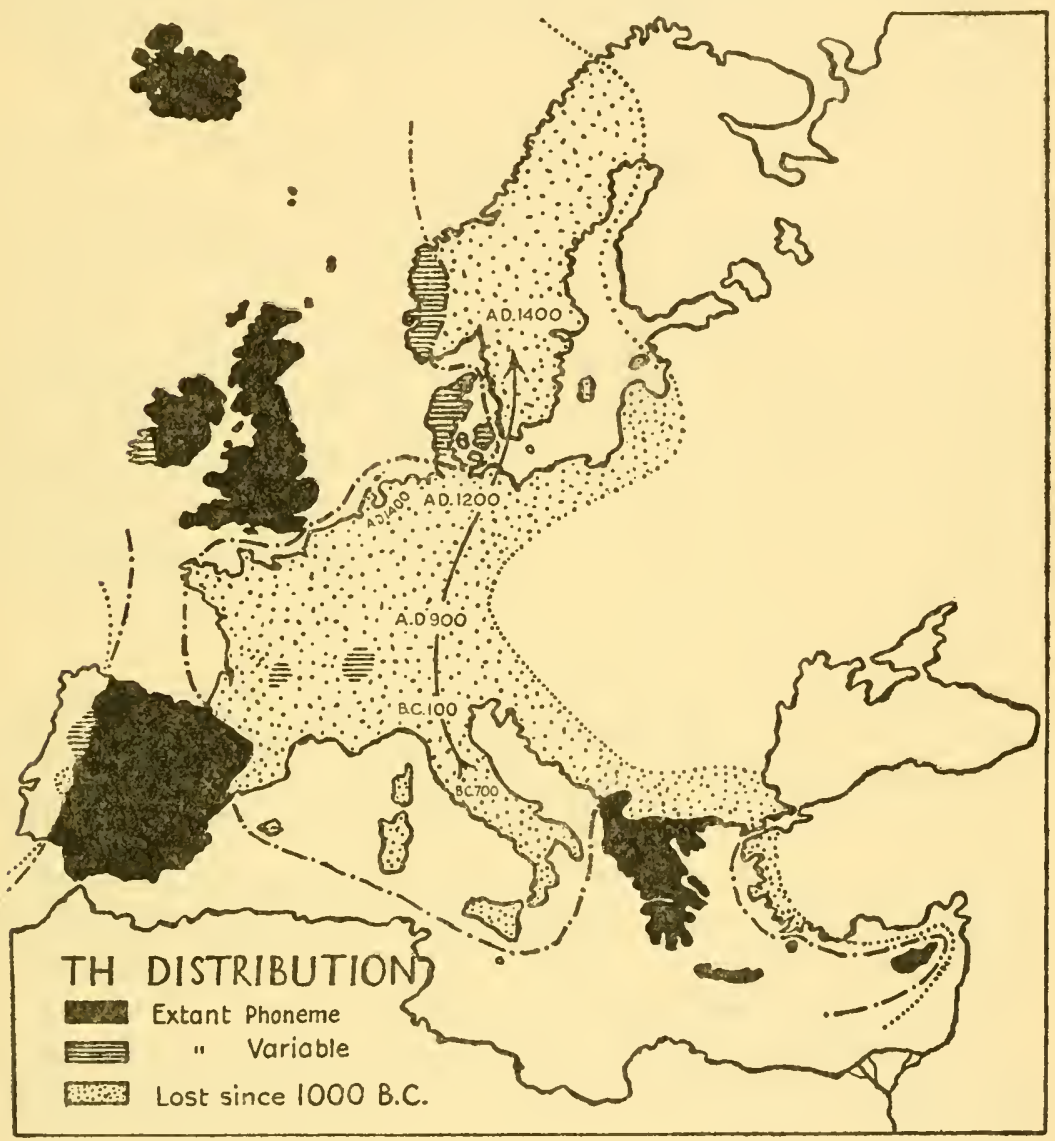

FIG. 88B.-Contour map to show the historic changes in the distribution of the TH sound in Europe since IO00 B.C. In the intermediate zone of loss the order of loss has followed the direction of the arrow with complete regularity in independent languages. Note the resemblance to the blood group contours (from Darlington 1947).

aboriginal languages so far as words and arrangements of words (used in ordinary language classification) were concerned. They could not, however, except by their slight genetic contribution, ultimately modify the limited capacity for sound production of the 
conquered peoples. Hence the peripheral similaritics of certain sounds would seem to depend on their aboriginal origin.

Thus the study of phonetic preference and its historical changes and geographical distribution may provide us with a key for a new and genctic approach to one of the simplest but most significant aspects of the history of culture.

\section{Clines, Tribes and Classes}

Three ways of classifying man and defining his genetic variation now become apparent.

The first is clinal and depends on migrations and conquests operating over great periods of time. These establish large-scale gradients in genetic character which, in the Old World, show great stability. The $\mathrm{O}$ blood-group gradient is an cxamplc. It runs across Britain from north-west to south-cast. A colour-vision defect gradient on the other hand runs from north-east to south-west. Hundreds of such gradients would be necessary to give even a superficial description of the clinal variation of Europe.

Superimposed on this primary type of human variation is another working on an entircly different scalc. Where mating groups have been stabilized either by geographical or cultural barriers they establish what we may call a tribal subdivision. This subdivision must have a genetic importance proportionate to the duration of stability and to the smallness of the group. For this reason the tribal subdivision varicgates the primary pattern of clinal variation to different extents in different regions and periods.

And finally, superimposed on the tribal subdivision, is the functional one in respect of the classes and castes we have already discussed.

The relative importance of the three modes of variation in man will depend on a number of circumstances. It will obviously depend on the time and place under examination. During the Dark Ages in Europe or after an Islamic conquest, clines would have submerged tribes just as they are doing with African tribes now. In the United States and other new countries, on the contrary, owing to the freedom of movement, classes and castes rather than tribes or clines are the predominant method of division. In Europe we find that subdivision 
by class, cline and tribe are all made to contribute to a local culturai and national adaptation. Finally we may notice that, to slow-moving observers on the earth, compelled to examine human variation at close quarters, the broad clinal slopes have naturally been obscured in the past by the narrow tribal ridges and ravines.

Clearly to reap the full harvest in the field of human heredity we shall have long to wait. But it is necessary for the student of genetics to recognize the possibilitics of his method. Mendelian interpretation of a few physical defects provides their foundation. But on this foundation can be built a structure which will unite in a coherent system of analysis the most varied aspects of the study of man, medical and social, cultural and political.

The methods of Mendel and Darwin have to be used and combined. The techniques of chromosome study and statistical analysis have to be fully exploited. And it would be as foolish to deny these cnquiries thcir proper scope as it would be to admit their conclusions without continued trial.

\section{REFERENCES}

BONNIER, G. I948. Identical twin genetics in cattle. Heredity, 2 (I-27).

BOYD, w. C. I939. Blood Groups. Tab. Biol., I 7: I13-240.

COCKAyne, E. A. I933. Inherited Abnormalities of the Skin and its Appendages.

London.

DAhlberG, G. 1942. Race, Reason and Rubbisli. London.

DARLington, C. D. I943. Race, class and mating in the evolution of man. Nature, I52: 3I $15-3$ I 7 .

DARLINGTON, C. D. I947. The genetic component of language. Heredity, I : 269-286. Dieht, K., and verschuer, O. v. I936. Der Erbeinfluss bei Tuberkulose. Jena., Duckworth, w. L. H. I947. Some Complexities of Human Structure. Huxley Mem.

Lect. (R. Anthrop. Inst.).

GARROD, A. E. I923. Inborn Errors of Metabolism. 2nd ed. London.

rtansson, A. 1946. Studies on monozygous cattle twins. IV Twin studies on the

predisposition for diseases. Acta Agr. Suc., I : I63-169.

Hodson, T. C. I937. Indian Census Etlunograpliy, I901-I93 I. New Delhi. hogben, L. I931. Genetic Principles in Medicine and Social Science. London. KALLMAN, F. J., and REISNER, D. I943. Twin studies on genetic variation in resistance and susceptibility to tuberculosis. J. Hered., 34: 269-276, 293-30I.

KOLLER, P. C. 1937. The genetical and mechanical properties of the sex chromosomes. III Man. Proc. Roy. Soc. Ediu. B., 57: 194-2I4.

KOlLer, P. C. I943. Origin of malignant tumour cells. Nature, I 5 I : 244-246. 


\section{MAN AND MANKIND}

Llvit, S. G., ct. al. 1936. Proc. Maxim Gorky Medico-Genetical Research Inst., Moscow.

$$
4: 1-543
$$

MACKLIN, M. T. 1940. An analysis of tumours in monozygous and dizygous twins, with report of fiftecn unpublished cases. J. Hered., 31: 277-295.

MUleer, H. J. 1935. Human genetics in Russia. J. Hered., 26: 193-196.

RISLEY, 11. H. I9I5. The People of India. 2nd ed. London.

rosanOFF, A. A., et. al. I94I. The etiology of child behaviour difficulties, etc., in twins. Psychinitric Monographs I, Calif. Dept. of Inst.

thurston, E., and RANGachari, K. I909. Castes and Tribes of Southeru India (Vol. II). Madras (Gov. Press). 


\section{THE FUTURE OF GENETICS}

The Technical Synthesis The Three-Level Analysis

The Three-Level Synthesis

A FINAL TASK REMAINS FOR us in completing our work: to show what connexion its parts have with one another, how they arise out of the past of the subject, and how they may be used to develop its future. We must for this purpose remind the reader in the first place of the combinations of method which have gone to build up genetics. Secondly, we must recall the consequences of the manylevelled structure we have built up. And finally we must attempt to pick out the central principles which support its framework.

\section{The Technical Synthesis}

The first combination is, of course, that of chromosome studies and experimental breeding. Genetics might have been built without the chromosomes ever having been seen (just as chemistry was built without the atoms having been seen), but it could not have been built without their having been imagined. The double foundation, while giving the subject a technical duality, has also given it a theoretical coherence and unity which expresses itself in all its later developments. Above all, the connexion it establishes between the determinant and its expression gives us a pattern of prediction which covers the organism from conception to death.

This first combination gives us genetics in the narrowest sense. It takes us down to the molecular level and invites us to make the next combination, that with chemistry. Chemistry enables us to define our system in more precise terms and expands it at each level. The chromosomes themselves, their movements, their products, their changes and, bencath everything else, their capacity for self-propagation, are seen in terms of protein chemistry. And this chemistry is of a uniquely biological character owing to the vast size and adaptive organization of the molecules concerned. 
The chemical point of view leads us naturally to the third combination, that by which heredity and devclopment are brought together as aspects of the same problem of propagation and organization in two types of structure, the nucleus and the cytoplasm.

These relations between nuclear genes and cytoplasmic protcins prepare the way for yet another combination, namely that between heredity and infection. By comparing the organization of the nuclcus and the chromosomes with that of the less coherent or smaller systems outside or beneath them, we can put these different disciplines into their correct relationships for the first time.

The range of relationships of cytoplasmic determinants with the nucleus and with one another, its parallelism with the relationships of viruses, and finally the gradation that exists between viruses, plasmagenes and other proteins, have shown that the distinctions between these types of body are conditional rather than fundamentally genctic or chemical. The legitimate inheritance of the plasmagene, and the infection of the piratical protcin which could become a virus, can be combined, as we saw, in one body. The dichotomy cxists rather between different types of viruses. Here, then, genetics combined with chemistry has provided the means of combination of plant, animal and human pathology and has even shown what is significant in the different types of origin and transmission of cancer.

Finally, there is that great union which has resolved the conflict of biometry and mendelism, of continuity and discontinuity. This union depends on the modern refinement of statistical theory. But it is again related to the activitics of the chromosomes, putting, as it does, the difference between the appearances of continuous and discontinuous variation into terms of differences in size and specificity of the genes and of the proteins they produce. At the same time this combination resolves the long-standing conflict between the supposedly uscless methods of mendelian analysis and the practical methods of improvement of plants and animals by brceding.

Around these central combinations are several peripheral ones of great interest. Two are notable. First, the union of systematics and the theory of descent with the study of chromosomes has already, as we have seen, borne great fruit in flowering plants. Later it will 
no doubt be equally productive in the study of animals, especially the important mammals and their most dangerous enemies and parasites, and in the study of the lower plants. Secondly, the application of genetics to man is beginning to show results which are as important for man as was expected, but more important for genetics than could have been expected, owing to the great extension which man's special character gives to the visible spectrum of variation. We are only beginning to acquire confidence in the application of genetics to pathology, psychology, and sociology. As we do so, however, the new social science that will develop will increase in power more than in proportion to its continually increasing predictive capacity.

\section{The Three-Level Analysis}

Looking back at our account we can now see why it was that so many false starts were made with genetics. Many very different kinds of study have contributed to our conclusions. We have observed structures at many different levels of organization, but we have not been able to ascend in simple order from the gene to the chromosome, from the chromosome to the nucleus and so on to the cell, the organism, the family, and the population, the species and the whole world of evolution. Or even to descend in opposite order. The first two parts of our account were, to be sure, concerned with the family and smaller units, and the third part with larger units. Yet our beginning had to be made in the middle, with individuals and families. It was Mendel who discovered that heredity, a property of the family, had to be studied from the relationships of individuals in familics, and it was with Mendel that we had to begin working.

From the individual we have to proceed downwards largely with the aid of the microscope to the cell, the nucleus, the chromosome, the gene and eventually the protein molecule. At this lower level heredity and variation remain our basic notions, but they acquire new senses. For they begin to depend on the capacity of selfpropagation of nucleo-proteins, a capacity which we find exists both inside and outside the nucleus. The differentiation between the nucleus and the cytoplasm then appears as a differentiation in method 
of propagation, from which a hicrarchy in control is inevitably derived. For the mechanically stable strings of genes with their potentially permanent organization, their co-ordinated reproduction, their controlled mutation, and their regulated recombination by crossing-over, acquire a sensitiveness to selection, and hence a capacity for adaptation, such as is bound to give them control of the unorganized proteins of the cytoplasm, which depend on their individual chemistry for their proportions. The nucleus, no doubt, has step by step acquired the property of putting together, after due delay, the materials given it by the cytoplasm, to make larger protein molecules. In these circumstances the cytoplasm is bound to appear as the weaker partner. On the other hand, the variable and controllable cytoplasm provides the neccssary vehicle for a differentiation which could not be directly related to the permanent and rigid organ of heredity. For separating the two controlling instruments, for stabilizing the government of each, for regulating differentiation and for delaying interaction, the nuclear membrane is the king pin of the whole mechanism.

We cannot, however, pursue our investigations downwards to the limit without considering the results of carrying them to the higher level. It was at the higher level of populations, races, species and the larger classes of animals and plants, and their changes over the greater spaces of time, that Darwin was able to see principles which are more obscure within the individual with its short span of life. These principles we are now able to see with a new clarity in the light of genetics. We are able to see the interlocking of the heredity of the individual with the variation of the group. We are able to sce genetic systems as composed of mating habits, reproductive mechanisms, hereditary materials, and in man of traditional cultures as well, which are adapted to the needs of groups large or small, as the case may bc. These adaptations arise from selection which acts, most obviously on individuals, but in its long-range effects on larger and longer-lived units, and in its shortrange effects on smaller and shorter-lived units such as single cells.

The threc levels of integration of cclls, individuals and populations correspond as we thus see not only to differences in size of units but also of their duration in time. And heredity, variation, and selection act at all three levels in respect of both size and duration, 
each level interacting with the others. The simplest example of this interaction of levels is seen in the principle that the chromosomes are controlled by the genotype. They themselves are as near to the genotype as materials can be, yet, at the cell-level, they are in fact part of the phenotype: they are subject to the reaction of heredity and environment. In conscquence the heredity which controls evolution is itself subject to evolution. Its mechanism varies, is selected, and is adapted, sometimes for the short-term advantage of immediate progeny, as with apomixis, and sometimes for the longterm advantage of an indefinite posterity, as in the evolution of crossing-over.

There is an abiding conflict in the operation of selection between long-and short-term advantages. It is a conflict which is represented by the opposite advantages of the fitness of the individual and the flexibility of the population. This again is a two-level affair. What fitness is for the fleeting individual, flexibility is for the enduring population. Two-level equilibria can never be stable and the see-saw of fitness and flexibility is probably one of the major factors of instability in the races, stocks, and strains of all living communitics.

\section{The Three-Level Synthesis}

Our three-level analysis is made possible only by a rigorous application of the principle of determinacy. From the determinacy of genetics proceeds its rigour and coherence as a basis of prediction. This assumption of determinacy is itself justifiable by prior consideration as well as by practical success.

In passing from one level to another we change the laws of behaviour. We also regulate at a higher level the disorderliness of the lowver level. Apparent indeterminacy is reduced to determinacy, as Schrödinger has pointed out, when the gene is incorporated in the chromosome. But it is also similarly reduced when the cell is incorporated in the body and again when the body of the apparently indeterminate individual is incorporated in the population.

It would be idle to pretend that the application of prediction in a many-levelled system is a simple one, or that its principles are well understood. But it is clear that they demand a reconstruction of single-level notions. In the course of this book some examples will 
have already intruded themselves. The theory of selection is one, and the theory of human society is another. We may call attention to yet a third, which we may call the genc-recombination complex.

The mating continuum depends for its function, as much as the nucleus depends for its predominance, on recombination. Discontinuity between permanent chromosomes arises when recombination is obstructed. But recombination again depends on activities at a higher and a lower level. In the first place, there must be crossing in the population between individuals which differ in heredity. In the second place, there must be segregation and crossing-over in the cells of hetcrozygotes produced by this crossing. The segregation and crossing-over system in turn presents us with its own conflicts; for crossing-over in all organisms is a condition of pairing and segregation (at least in one sex). And crossing-over is restricted both in its frequency and in the size of the units it recombines by the extent of the differences between mating chromosomes. This is due to the existence of two modes of differentiation of chromosomes, the axial or structural and the lateral or genic. The greater the structural differentiation, the smaller the crossing-over. The result of this system is to buffer the amount of effective recombination, which is a function of the product of the amount of difference and the frequency of crossing-over within, and random assortment between, chromosomes.

These interactions take effect in a wider field. We have seen them in some variety determining how discontinuities of different sizes may float in the flow of variability or become stranded in the formation of races and species, the gene being trapped by the inversion and the inversion trapped by the environmental discontinuity. We have also seen them in a different way determining, under the guidance of selection, the growth, and the organization of gencs over periods of time much greater than those responsible for the origin of species.

The examination of the growth of genes has indeed given us a glimpse of a new world of which as yet we know little, except that it is probably as complex as that of populations. We can see, in progenies, in chromosomes, and in cell products, the broad outlines of the distinction which separates the polygenes, the major genes and the super-genes. We can also see how the polygene can be 
changed, shifted or destroyed without danger and with the possibility of creative adaptation, while the larger units can be broken down only with disastrous effect. In other words we can sec dimly the delicate framework of the evolution of genes on whose handling, often rough handling, we depend for our experimental effects.

We also see, in place of the abstract gene of Johannsen, a matcrial particle; a particle, however, which is no longer the billiard ball of Morgan, but a unit whose size is functional and depends on the nucleus in which it lies, the activities in which it is engaged, and the linear correspondence of the partner with which the chances of the mating system have given it the opportunity of crossing-over.

On this view-and no simpler view will comprehend the evidence -the gene, like the species, is a growing and diversifying and, of coursc, unstable structure continually adapting itself under the action of selection. Amongst self-propagating units the one barrier which secms almost irremovable is that between those inside and outside the nucleus. Outside, the absence of fibrous structure maintained by chromosome nucleic acid limits the unit to something comparable to the smallest genes. These may multiply, as viruses do, to form large molecules, but not to permit the internal differentiation of a chromosome, still less of several chromosomes. The fibrous organization of chromosomes has given them an advantage over the rest of the cell, which they have clearly never lost since cellular differentiation was first established. Indeed, we may say that sexual reproduction, with its crossing-over, is necessary to make the chromosome work, and the chromosome is necessary to make the cell work.

Thus the principles of life are not subdivisible according to the same rules as those for subdividing the techniques used for discovering them. The old barriers are now breaking down, and for the first time we can see biology as a whole. We can see a system working subject to four primary laws (of heredity, variation, selection and adaptation), at the three levels of integration, between the molecule and the species, otherwise subject to different laws at different levels, but no longer differentiated in regard to these laws as between plants and animals, or even at the lowest level as between heredity and development.

We began our study of genetics with the modest intention of 
finding out why offspring were like, or were not like, their parents. We have gone much further aficld than we might have expected or than some may have thought necessary. We believe, however, that the unity of biology, or indeed the unity of science, which has been gradually emerging from what we have said, is of just as great importance to those who breed animals and plants or those who treat the discases of man as it is to the academic teacher, to the student of society or to the philosopher of life. 


\section{GLOSSARY OF GENETICAL TERMS}

I intended to augment our English tongue, whereby men should express more abundantly the thing that they conceived in their hearts (wherefore language was ordained) having words apt for the purpose.

THOMAS ELYOT (Knowledge which maketh a Man Wise, I 533)

WORDs are the weapons of science. They serve for defence and for attack. They tell us what we know. But, if we use them aright, they also tell us what we don't know, and this is what we especially need to be told if we are to discover something new. Since Genetics is now in the full flood of its discovery a precise definition of its words and their meanings seems to be a prime necessity.

We have prepared this list, not therefore as a record of final achievement, but as an instrument for new advances which will call for its own reconstruction. We have attached the first importance to consistency, the second to convenience, and the third to the opposing claims of fitness and flexibility.

For the first purpose our main task has been to reconcile the technical habits of plant and animal workers. For the second we have had to abandon archaic forms, leaving merely obituary notices of many obsolete expressions and adapting others to entirely new purposes. And for the last purpose we have had to recognize in many cases two stages in the ontogeny of the words: the empirical or popular, and the analytical or explanatory.

This transition is nowhere better illustrated than by the word Heredity itself, which was formerly applied to a Force or a Law and has now come to be applied to a Process or a Substance, as the study has passed first from the empirical to the abstract and then from the abstract to the mechanical and the chemical.

The terminology of our subject began with what was needed for describing the relations of parents and progeny. It now extends to Cytology and Chemistry, Embryology and Physiology, Anatomy and Taxonomy, Statistics and Biometry. Indeed it obviously covers the whole structure of biology, although it is concerned only with providing the analytical framework of this structure.

Initial Capital Letters are used in the text for terms defined elsewhere under their own names. The Author references are to the first use of the term in approximately the same sense as that given.

Finally, we are indebted to the following earlier works (some of which also contain bibliographies and sources) for certain definitions:-

Wilson, E. B. I925. The Cell in Development and Heredity, 3 rd ed. New York. Darlington, C. D. I937. Recent Advances in Cytology, and ed. London. mather, к. 1946. Statistical Analysis in Biology, 2nd ed. London.

Also J. Heredity, 28: 7I-80. I937. 


\section{GLOSSARY}

Accissory Chromosome, Sex Chromosome. McCling 1902.

ACENTRIC, of a detached part of a chromatid or chromosome lacking a Centromere. Darlington 1937.

ACQUiRed Character, variously used for modification of phenotype or genotype, adaptive or non-adaptive, induced by one environment and not by another.

Acquired Characters, Theory of Inheritance of, that acquired characters are inherited and adaptive, and thus account for evolutionary change. Lamarck i $80 \mathrm{I}$.

ADAPTATION, the process, and the result of the process, by which members of a genetic group, either as individuals or as parts of individuals or as groups are fitted to past or present changes in their environment. Adaptation is understood to occur in form and function, and the enviromment to operate both ecologically and biologically.

AgAmosPeCIES, apomictic species. Turesson I 929.

Age And Area, Theory of, that the areas of distribution of the members of a group of allied species are correlated with their ages as species in any given region. Willis 1922 .

Allelomorph (Allele), one of two or more dissimilar Factors or Genes which on account of their corresponding position in corresponding chromosomes are subject to alternative (mendelian) inheritance. Two factors producing unrelated physiological effects, but apparently inseparable in inheritance, may be taken provisionally as nonallelomorphic, e.g. yellow and scute in Drosophila melanogaster. Bateson 1902.

Multiple Allelomorph, a member of a series of more than two allelomorphs of one gene in one species. Morgan I9I4.

Allogamy, Cross Fertilization.

Allopolyploid, a polyploid whose chromosomes form at meiosis not multivalents, but, so far as their homologies permit, bivalents always in the same way, e.g. a tetraploid with $2 x$ bivalents or a triploid with $x$ bivalents and $x$ univalents. In a broad sense one approaching this condition as opposed to an Autopolyploid. Special classes are Allotetraploid, Allohexaploid, etc. Kihara and Ono 1926.

Allosome, as opposed to Autosome. Sex Chromosome. Montgomery 1906. Allosyndesis. I. The pairing in a cross between two polyploids of chromosomes derived from opposite parents.

2. Pairing in an Allopolyploid between chromosomes derived from different ultimate diploid ancestors. Opposed to Autosyndesis. 
Alternation of Generations, the occurrence of two phases in the life cycle corresponding to, or derived from, the haploid and diploid phases of sexual reproduction. Various forms of Apomixis can equalize the chromosome numbers of the two phases.

Alternative Inheritance, $v$. Allelomorph.

Ameiosis, the occurrence of one division of the nucleus in place of the two of a normal Meiosis, leading to Non-Reduction of the mothercell.

Amitosis, the division of a nucleus without the appearance of chromosomes. Originally a misinterpretation. Flemming $\mathrm{I} 882$.

AMORPH, a recessive mutant gene which has no detectable effect on the characters affected by the non-mutant allelomorph and is indeed equivalent to a mere absence of it. Muller 1932.

Similarly for other types of mutant gene:-

ANTIMORPH, having an effect contrary to that of the non-mutant allemomorph.

HYPERMORPH, having an effect similar to but greater than that of the non-mutant allelomorph.

HYPOMORPH, having an effect similar to but less than that of the nonmutant allelomorph.

NEOMORPH, having an effect apparently unrelated to that of the nonmutant allelomorph.

Amphidiploid, Allotetraploid. v. Allopolyploid. Navashin 1927.

Амтніміхіs, reproduction by the fusion of two gametes in fertilization. As opposed to Apomixis. Weismann I89I.

ANAPHASE, the stage at which daughter centromeres move apart in mitosis and at which co-orientated centromeres move apart in the first division of meiosis. Strasburger I 884 .

ANDRODIOECY, the condition of plant populations or species in which both male (i.e. female sterile) and hermaphrodite individuals are found. Cf. Andromonoecy, Gynodioecy. Darwin I 877.

Androgenesis, Male Parthenogenesis. Wilson 1928.

ANDROMONOECY, the condition of an individual plant or of a species having individuals which bear both male (i.e. female sterile) and hermaphrodite flowers. Cf. Androdioecy, Gynomonoecy. Darwin $\mathrm{I} 877$.

ANEUPLOID, having different chromosomes of the set present in different numbers, through purely numerical aberration and therefore an Unbalanced Polyploid. $C f$. Euploid. Täckholm I922.

ANISOGAMY, Heterogamy. 
ANISOgENy, the property of having different inheritance in reciprocal crosses. Now known to be due to Cytoplasmic Inheritance, Complex Heterozygosity, or lack of Balance. Bateson 1925.

Antimorpil, v. Amorph.

Apogamy, Apomixis in which the reproductive function of the gametes is taken over by unspecialized cells. De Bary I 878 .

APOMIXIs, the occurrence of the external form of sexual reproduction with the omission of fertilization and usually of meiosis as well. As opposed to Amphimixis. Subdivided into Natural, which may be Facultative or Obligatory, and Artificial. In animals it always takes the form of Parthenogenesis. Winkler 1908.

APOSPORY, Apomixis in which the reproductive function of the spores is taken over by unspecialized cells. Bower 1887 .

Asexual Reproduction, $v$. Reproduction.

ASYNAPSIS, non-pairing of chromosomes at pachytene, or a failure of

chiasmata supposedly derived from non-pairing. Beadle I93 I.

ATAVisM, resemblance to more remote ancestors, rather than to parents.

Now understood as duc to Segregation, Recombination, incomplete

Penctrance or Mutation.

Attachment, v. Spindle Attachment and Attached X Chromosomes. Autogamy, Sclf-Fertilization, especially in Infusoria.

Auto-Orientation, $v$. Oricntation.

Autopolyploid, a polyploid with identical sets of chromosomes, or approaching this condition. Kihara and Ono 1926.

Autosome, a chromosome whose segregation from its partner at meiosis does not normally affect the determination of sex. Montgomery I906. Autosyndesis. I. The pairing in a cross between two polyploids of chromosomes derived from the same parent.

2. Pairing in an allopolyploid between chromosomes derived from the same ultimate diploid ancestor. As opposed to Allosyndesis. Ljungdahl 1924 .

Azygote, individual arising by haploid Parthenogenesis. Whiting and Benkert 1934.

BACKcross, the cross of a hybrid with one of its parents or a genetically equivalent cross. N.B. not to include Test-Cross.

ACTERIOPHAGE, a Virus acting on bacteria.

ALANCE, a fit adjustment of the genetic components of an individual. Inter-Chromosome Balance, the condition in which wholc chromosomes are adjusted in proportions that give satisfactory development of the organism. 
BALANCE (continued)

Intra-Chromosome Balance, such a condition produced by the adjustment of genes within one chromosome.

Genic Balance, such a condition produced by adjustment of the proportions of genes.

Polygenic Balance, such a condition produced by adjustment of the proportions of polygenes having opposite effects. A balanced set of polygenes within a chromosome is a Balanced Polygenic Combination. Where the balance is achieved within a single representative of the chromosome, and hence is displayed by a homozygote, it is InTernal BALANCE; and where the balance is achieved by two homologous chromosomes acting together, and hence is displayed by a heterozygote, it is Relational Balance. Mather I94I.

Secondary Balance, a new balance derived by change in the proportion of genes, as in a secondary polyploid, from an old balance, and capable of competing with it. Darlington and Moffett 1930.

Balanced Lethals, $v$. Lethal Gene.

BASIC Number, the number of chromosomes in a Set, represented by $x$. v. Chromosome Set.

BASIC TYPE, a chromosome complement of characteristic structure and number which exists throughout a species, variants occurring only as heterozygotes. $v$. Prime Type. Darlington and Gairdner 1937.

BIAs, a consistent departure of an observed quantity from its proper value owing to error of observation or estimation.

BIMODAL, of a frequency distribution having two Modes.

Binomial Series, the series obtained by expanding the sum of two quantities to any power. $(a+b)^{n}$.

BIOTYPE, a group of organisms occurring in nature assumed to be genotypically almost identical. Johannsen 1903.

Bivalent (Geminus), v. also Univalent. A pair of homologous chromosomes (partners) united at the first division of meiosis, usually by chiasmata.

Ring Bivalent, one with terminal or nearly terminal chiasmata at both ends of the chromosomes.

Rod Bivalent, one with such a chiasma at only one end.

UNequal Bivalent, with the two partners of unequal size.

Bouquet Stage, zygotene and pachytene in those organisms in which the chromosomes lie in loops with their ends near one part of the wall of the nucleus. Cf. Polarization. Eisen 1900.

BREED, Varicty in domestic animals. 
BRFEDING SYSTEM, the organization of mating in a species or smaller group which determines the degrees of similarity or difference between the gametes which are effective in fertilization. Mather 1940.

BRIDGe, $v$. Chromatid.

BUd SPORT, of a plant, Somatic Mutation. $v$. also Sport.

BURDO, an individual of the higher plants formerly supposed to be produced by the fusion of two genotypically different somatic cells and their nuclei. Winkler I9I2.

CANCER, a potentially unlimited renewal of growth in differentiated cells, accompanied by their de-differentiation, and liable to be followed by their migration (metastasis) which chiefly constitutes malignancy.

CARrier, an individual whose genotype allows it to carry an infective organism without showing the symptoms to which individuals of susceptible genotype of the same or another species are liable.

CASTE, a group of a species in social organisms distinct from other such groups in mating habit and social function. In insects caste distinction depends on food and sex differentiation, in man on endogamous grouping.

CELL (in the structure of animals and plants), the sphere of action of one nucleus or of a group of nuclei (usually derived from one nucleus). Also perhaps an enucleate body derived from such a unit. Hooke I68I.

Cell Competition, Renner Effect.

Cell Selection, $v$. Selection.

Cell Theory, that all organisms are wholly constituted of cells. Conventionally attributed to Schleiden and Schwann I 840 .

Centre of Origin, Theory of, that the centre of origin of a wild or cultivated species is found in the region of its greatest genetic variation. Vavilov 1926.

Centrogene, one of the similar and self-propagating units into which the Centromere can be broken by X-rays or can break itself by Misdivision. Darlington 1939.

Centromere, a self-propagating particle in the chromosome thread whose cyclical activity of protein organization, polarization, division and repulsion determines certain movements of the chromosomes, viz. terminalization, congression, orientation and separation: spindle attachment, kinetochore, insertion region, etc. $v$. Acentric, Dicentric, Misdivision, Polycentric, Structural Change, Telocentric. Darlington 1937. 
Centrosome, the self-propagating body which, during mitosis in many organisms, lies at the two poles of the spindle and appears to deternine its formation. Boveri I 885 .

Certation, competition between pollen tubes, having different genotypes, leading to their having unequal chances of accomplishing fertilization. Heribert Nilsson 1920.

Character, a property of an organism in regard to which (genetic) similarities or differences of individuals are recorded. May be either a Complex or Unit Character.

$\chi^{2}\left(\mathrm{chi}^{2}\right)$, the ratio of an observed sum of squares to the appropriate or corresponding variance as fixed by hypothesis.

SIMPLE $\chi^{2}$, one in which the observed sum of squares is based on a single comparison (i.e. one Degree of Freedom). The square of a Normal Deviate.

Compound $\chi^{2}$, one in which the observed sum of squares is based on several independent comparisons (several Degrees of Freedom), and which can therefore be resolved into two or more simple $\chi^{2}$ 's.

Chiasma, an exchange of partners in a system of paired chromatids, observed between diplotene and the beginning of the first anaphase in meiosis. Janssens 1909.

Classes of single chiasmata (Darlington 1929) are:-

I. Interstitial, where there is a length of chromatid on each side of the chiasma.

2. LAteral, where the chiasma is terminal as to two chromatids and interstitial as to two others. Of two kinds, symmetrical and asymmetrical.

3. Multiple, where a terminal chiasma engages three or four pairs of chromatids.

4. Terminal, where an exchange occurs amongst the end particles of the chromatids, following terminalization.

Classes of pairs of chiasmata (Darlington 1937) are:-

Comparate and Disparate, where pairs of successive chiasmata compensate and do not compensate, respectively, for one another in regard to the changes of partner which produce them.

Comparate chiasmata, according to the Crossing-Over which determines them, may be:-

RECIPROCAL, 2-strand crossing-over, regressive.

COMPlementary, 4-strand crossing-over, digressive.

Disparate chiasmata, 3-strand crossing-over, progressive, diagonal. 
Cinasma Tineory of Pairing, that whenever two chromosomes which have been paired at pachytene remain associated until metaphase, they do so by virtue of the formation of one or more chiasmata. Does not apply to the heterogametic sex in certain animals. Darlington 1929.

Chiasmatype Theory, that only sister chromatids are associated after chiasma formation and that every chiasma therefore results from crossing-over between two chromatids. Darlington I930. Derived from Jansscns' (1909) theory that crossing-over and chiasmata were causally related in various ways.

Chimaera, a plant composed of tissues of two or more genetically distinct types, as a result of mutation, irregular mitosis, plastid segregation, or artificial fusion by grafting. Divisible into two kinds. Baur I909:-

Periclinal, in which the distinct tissues are arranged in concentric layers.

SeCtorial, in which the distinct tissues are arranged in cross-sections as sectors of a circle.

A compound Sectorial-Periclinal is also termed Mericuinal. Jergensen I928.

Chondriosomes, bodies in the cytoplasm which are believed to be selfpropagating, including Mitochondria and Golgi-Bodies. Benda I904.

Chromatid, a half chromosome between early prophase and metaphase of mitosis, and between diplotene and second metaphase of meiosis. After these stages, i.e. during anaphase, it is called a DAughter Chromosome. The scparating chromosomes at first meiotic anaphase are known as daughter-bivalents, or, if single chromatids derived from the division of univalents, daughter-univalents. McClung I900. Chromatid Bridge, a dicentric chromatid with centromeres passing to opposite poles at anaphase. S. G. Smith I935.

I.oop Chromatid, a chromosome arm whose chromatids show sisterreunion $v$. X-ray.

Sister Chromatids, those derived from division of one and the same chromosome, as opposed to non-sister chromatids which are derived from partner chromosomes at pachytene, or from any distinct pair of chromosomes at mitosis. Sister Strands of Bridges and Anderson I925.

Chromatin, Chromosome Thread to which nucleic acid is attached. Fleming I879, Boveri 1904. 
Chromatin (contilued)

Classifiable into (Heitz 1929):-

Euchromatin, part of the chromatin having its maximum nucleic acid attachment on the mitotic spindle.

Heterochromatin, any chromatin differing from euchromatin in its nucleic acid cycle.

Chromocentre, a body produced by fusion of the heterochromatin of the chromosomes in a resting nucleus. Especially applied to the Polytene chromosomes of Diptera. Painter I935.

Chromomeres, the smallest particles identifiable by their characteristic size and position in the chromosome thread between leptotene and pachytene and in polytene nuclei. Wilson I 896.

Chromonema, the Chromosome Thread. Similarly leptonema, pachynema, etc., are the chromosome threads at leptotene, pachytene, etc. Wilson 1896.

Снromosome. I. One of a group of bodies in the cell undergoing a co-ordinated cycle of reproduction, Spiralization and charging with Desoxyribose Nucleic Acid.

2. One of the bodies into which the nucleus resolves itself at the beginning of mitosis and from which it is derived at the end of mitosis. Waldeyer 1888 .

Chromosome Arm, one of the two parts into which an intercalary centromere divides a chromosome.

Chromosome Complement, the group of chromosomes derived from a particular nucleus in gamete or zygote, composed of one, two or more sets. Darlington 1932.

Chromosome Diminution, the loss or expulsion of a part of the chromosome complement at meiosis or mitosis so that a daughter nucleus is formed lacking this part. Herla I 895 .

Diplo-Chromosome, a chromosome which has divided twice instead of once since the preceding effective mitosis, its centromere being undivided. White I935.

Chromosome Elimination, diminution which occurs at meiosis alone. Seiler and Haniel I92I.

Chromosome Fragmentation, the breakage of chromosomes. $v$. X-Ray.

Chromosome Fusion, the permanent linear attachment of two chromosomes.

Limited Chromosomes, those found only in certain tissues, including the Germ Track, and eliminated in other tissues. Metz 1938. v. Chromosome Diminution. 
Chromosome (continued)

Chromosome MaP, a diagram portraying the Linear Order or arrangement of the genes within the chromosome. The distances between the points representing genes are made proportional to the frequency of recombination (usually), lethal mutation, or breakage observed between the genes, or to their physical distances apart as measured in mitotic or polytene chromosomes.

Chromosome Set, a minimum or basic complement of chromosomes derived from the supposed gametic complement of an ancestor. $v$. Polyploid.

Supernumerary or B Chromosomes, such as are unessential to development.

Chromosome Theory, that the chromosomes are the material basis of inheritance.

Chromosome Thread, the protein framework of the chromosome, bearing the genes.

Cline, a gradient within a continuous population in the frequencies in different localities of different genotypes (Genocline) or phenotypes (Phenocune). J. S. Huxley 1939.

Clone, a group of organisms descended by mitosis from a common ancestor, i.e. multiplied by Asexual Reproduction. Webber 1903.

Coenospecies, $v$. Species.

Coll, one complete revolution of an Internal Spiral.

Coincidence, Coffricient of, the proportion which the numbers of double cross-overs observed bears to the number expected from the random combination of single cross-overs between three or four linked genes. $v$. Interference. Muller I9I6.

Collateral-s, individuals, in a family, not related by direct descent.

COllateral INHFritance, the appearance of characters in some but not all the collateral members of a family.

Complementary Genes, genes which together produce an effect qualitatively distinct from the effects of any of them separately. As Complementary Elements, Bateson, Saunders and Punnett 1906.

CompleX, $v$. Heterozygote.

Complex Character, one whose difference from an alternative is not transmitted as a single unit in heredity. $C f$. Character.

Condensation or Contraction (of the chromosome), the spiralization and charging of a chromosome with nucleic acid.

Configuration, an association of two or more chromosomes at meiosis segregating independently of other associations at anaphase. Darlington 1929. 
CONGENITAL, of a character of an individual recorded at birth or referable to constitution at birth. Either of genetic or environmental origin.

CONGRESSION, the movement of chromosomes onto the metaphase plate, especially at the first meiotic division. Darlington 1937.

Conjugation, the pairing of gametes or zygotes, or the fusion of pairs of nuclei.

Conjugation of Chromosomes, side-by-side association of chromosomes in the early prophase of meiosis ( $v$. Pairing, Zygotene).

Constriction, an unspiralized segment of fixed position in the metaphase chromosome. Agar I9II.

Nucleolar Constriction, a secondary constriction determined by the organization of a Nucleolus. Darlington I937.

Primary (or Centric) Constriction, one associated with, and determined by, the Centromere. Darlington 1937.

Secondary Constriction, any Acentric constriction, either Nucleolar or Heterochromatic. Darlington 1926.

Contact Points, places where the chromosomes first come together in pairing at Zygotene. $v$. Localization, Polarization. Darlington I940.

CONTINGENCY TABLE, a table of frequencies showing two classifications simultaneously, and used in testing their independence.

Continuity, YATES' CORRECTION FOR, a correction applied in the calculation of Normal Deviates or $\chi^{2}$ 's to allow for the discrepancy arising by the observations being discontinuous while tables of the Normal Deviate and $\chi^{2}$ are calculated on the supposition of continuity in the variate.

Convergent Improvement, the simultaneous improvement of two inbred strains by recurrent back-crosses of their hybrid to each strain in separate lines, selection being practised for the desirable features of each strain in that line of which it is not the recurrent parent.

Co-Orientation, $v$. Orientation.

Correlated Response, change in one character due to selection for another. Due either to pleiotropy of one gene or to linkage of several genes concerned. Wigan and Mather 1942.

Correlation, the interdependence of variates. The opposite of independence. Applied also to the analysis of such interdependence by methods involving the two variates symmetrically. Cf. Regression.

Correlation Coefficient, the ratio of the covariance of two variates to the geometrical mean of their variances. Ranges from + I to $-I$. Partial Correlation, the correlation of certain variates when due allowance is made for the effects of other uncontrolled variates. 
Coupling, the presence of two given genes in the same chromosome in a double heterozygote, as opposed to Repulsion, where they are in different homologous chromosomes. Butesou, Saunders and Pumnett I 906.

Covariance, the mean of the products of corresponding deviation of two variates from their individual means. Estimated as the ratio of the sum of Cross Products to the corresponding number of Degrees of Frecdom.

Criss-Cross Inheritance, $v$. Inheritance.

Cross, an act or product of cross fertilization.

Crossbreeding, Outbreeding. $v$. Inbreeding.

Cross-Fertilization (Crossing), v. Fertilization.

Cross Products, Sum of, sum of the products of corresponding deviations of two variates from their individual means.

Cross Sterility, $v$. Sterility.

Crossing-Over, the exchange of corresponding segments betwecn chromatids of homologous chromosomes, by breakage and rcunion following pairing; a process inferred, genetically, from the recombination of linked factors in the progeny of heterozygotes, and, cytologically, from the formation of chiasmata between homologous chromosomes. Morgan I9II.

Crossing-over Value, the frequency of crossing-over between two genes or markers. Often loosely used for Recombination Value to which it may however be equated when small.

Double Crossing-over, the production of a chromatid in which crossing-over has occurred twice (for further classification v. Chiasma). Sturtevant i9i4.

Effective Crossing-over, that which is detectable in breeding experiments. Darlington 1937.

Illegitimate Crossing-over, crossing-over, in a haploid or polyploid which is not a structural hybrid, betwcen homologous and reduplicated segments of two chromosomes, which, being structurally dissimilar as a whole, do not normally pair. Determines Sccondary Structural Change. Darlington 1932.

Somatic Crossing-over, crossing-over at mitosis as opposed to meiosis (either in somatic or germinal tissue). Stern I935.

UNEQUAL CROSSING-Over, such as produces one chromatid containing a gene twice and another lacking that gene. Due to inaccuratc pairing. e.g. of the Bar gene in Drosophila melanogaster.

Cumulative Factors, $v$. Polymeric Genes. Nilsson-Ehle igi I. 
Cytoplasm, the Protoplasm other than the Nucleus. Strasburger r 882. Cytoplasmic Inheritance, $v$. Inheritance.

DAUERMODIFCATION, a lasting heritable change, presumably cytoplasmic, produced by treatment. Jollos I9r 3 .

Daughter Chromosome, v. Chromatid.

Deficiency, loss of a terminal acentric segment of a chromosome. Often used to include Deletion. $v$. Structural Change. Bridges I9I7.

DegREE OF FREEDOM, a comparison between items within a given body of data, independent of other comparisons used in the analysis.

Number of DegreEs of Freedom, the number of independent comparisons that can be made within the body of data.

Delayed Inheritance, $v$. Inheritance.

Deletion, loss of an intercalary acentric segment of a chromosome. v. Structural Change. Painter and Muller 1929.

Descendance, all the individuals descended by sexual reproduction from a single individual or particular mating pair.

Determinant, an element in the cell postulated to control heredity and development. The precursor of the gene concept. Weismann I89I.

Developmental Genetics, the study of the operation of genes during development.

Deviation, departure of a quantity (derived from one or more observations) from its expected value.

Standard Deviation, the distance on the abscissa of the point of inflection (maximum slope) from the mean in a normal curve. Generalized as the square root of the Variance. Called the STANDARD ERror when the deviation can be regarded as an error, e.g. with estimates of parameters.

DiAkinesis, the last stage in the prophase of Meiosis, immediately before the disappearance of the nuclear membrane. Hacker I 897 .

Diallel Crossing, the system where each of a number of males is mated to each of a number of females.

Dicentric, of a chromatid or chromosome having two Centromeres.

Differential Afrinity, the failure of two chromosomes to pair at meiosis in the presence of a third, although they pair in its absence. Darlington $\mathrm{I} 928$.

Differential Segment, a block of genes in respect of which two pairing chromosomes differ, in a permanent hybrid (Sex and Complex Heterozygote) in contrast to a PAIRING SEgment where they pair and cross-over, and are therefore homologous. Darlington I93 I. 
Difrerentiation, the process whereby a cell, through mitosis and not through meiosis, produces two daughter cells of immediately or ultimately dissimilar form or function with or without genetic dissinilarity; hence the process by which dissimilar tissues arise within the same individual. The process of differentiation is repeated by heredity and is therefore genetically determined.

Digenic, $v$. Monogenic.

Dihybid (Diheterozygote), heterozygote in respect of two genes.

Diminution, $v$. Chromosome.

Dioecy, Haploid or Diploid, the condition in which male and female gametes are borne on separate haploid or diploid individuals respectively. Has usually been termed Unisexuality in animals. $v$. also Hetcrothally, Sex.

Diplochromosome, $v$. Chromosome.

Diplogenotypic Sex-Determination, causing Diploid Dioccy. Hartmam I9I8.

Diploid, I. The zygotic number of chromosomes $(2 n)$ as opposed to the gametic or HaploID number ( $n$ ). Strasburger ig05.

2. An organism having two sets of chromosomes $(2 x)$ as opposed to organisms having one (HAPLOID), three (TRIPLOID) or more sets $(x, 3 x$, etc. $)$.

DiploidizATION, process of fusion of hyphae followed by division of nuclei in pairs in fungi, whereby haploid cells or mycelia become diploid. Buller 1930.

Diplont, organism at the diploid stage of the life-cycle, as opposed to HAPLONT at the haploid stage.

Diplophase, the diploid stage (as opposed to the haploid, Haplophase) of the life history in plants with an Alternation of Gencrations, especially in Basidomycetes and higher Ascomycetes where the nuclei do not fuse when the hyphae become diploid, Vuillemin, Goebel.

Diplotene, the stage of mciosis which follows division of the chromosomes and chiasma formation at the pachytene stage. von Winiwarter 1900.

Discriminant Function, linear compound of a series of variates, obtained by giving the different variates individual coefficients such as will maximize the differences between the classes, relative to the variation within classes, of objects of which the variates are measurcments.

Disjunction, the separation of chromosomes at anaphase, particularly of the first meiotic division. $v$. Non-Disjunction. 
Dislocated Segments, homologous pairs of segments differing in their linear sequence with other segments. Darlington 1937.

From Dislocation. Navashin 1932.

Disome, Bivalent. Blakeslee i92I.

DisOMIC INHERITANCE, arising from the determinate association of chromosomes in bivalents at meiosis.

DisPERMIC, of an egg fertilized by two male gametes.

Distal, of a part of a chromosome arm, which is further from the centromere than another (Proximal) part.

Dizygotic, from two fertilized eggs, or from the halves of one egg fertilized by two sperm, in contrast to Monozygotic (of Twins then called Fraternal, etc.).

Dominance, the relationship of two allelomorphs where the single gene heterozygote resembles one of the two homozygous parents (said to carry the Dominant allelomorph) rather than the other (said to carry the Recessive allelomorph) on an arbitrary scale distinguishing the two phenotypes. Mendel i 865 .

Conditioned Dominance, I. Dominance affected by the presence of other genes.

2. Dominance affected by environmental variation.

Degree of Dominance, the extent to which the heterozygote resembles the homozygote for the allelomorph in question.

Dominance Modifier, a gene which modifies the degree of dominance of another gene. Fisher 1928.

Partial Dominance, incomplete dominance.

Dominant Mutant, term applied in Drosophila to any mutant whose effect is detectable when heterozygous with its wild-type allelomorph.

Double Heterozygote, heterozygote in respect of two genes.

Double Reduction, $v$. Non-Disjunction.

DRIFT, changes in the aggregate of genotypes in a small population resulting from the random (non-selective) extinction of allelomorphs of genes, in regard to which the population was heterogenic. Wright I93I.

Duplex, $v$. Nulliplex.

Duplicate Genes, $v$. Polymeric Genes.

Duplication, Reduplication.

DYAD, a pair of cells formed by a meiosis instead of a Tetrad.

DysGENIC, opposite of Eugenic.

ECOPHENE, the range of phenotypes produced by one genotype within the limits of habitat under which it is found in nature. One genotype must be taken to mean a class of closely similar genotypes. Turesson 1922. 
ECOSPECIES, $v$. Specics.

Ecotype, $v$. Species.

EFriency of a Method of Estimation, the quantity of Information extracted from the data by the method, expressed as a fraction of that extracted by maximizing the likelihood. $v$. Likelihood.

EGG, a gamete specialized for the storage of food, as opposed to a SPERM which is specialized for mobility and which fertilizes the egg. The Female gamete.

Elimination, v. Chromosome.

EMBRYO SAC, the female gametophyte in the angiosperms.

Endogamous Group, Mating Group.

ENDOGAMY, Inbreeding. McLennan I865

ENDOMIXIS, replacement of the macronucleus by a product of the micronucleus, in Infusoria. Woodruff I917. Now believed to occur only at Autogamy. Sonneborn 1947.

ENDOSPERM, nutritive tissue developed within the embryo-sac of flowering plants, from the fusion of one female nucleus with one or more others, or with a male nucleus, or with both (hence $2 x, 3 x, 4 x$, or $5 x$ ). Environment, those conditions external, or antecedent, to an organism which are related to its development. Its reaction with the genotype determines the phenotype.

Genotypic EnVironment, the aggregate of all the genes considered as acting on one or more of them.

EPIGENESIS, the theory that new structures arise in the course of development, as opposed to Preformation. Wolff i 759.

EPISTASY (EPISTASIS), the property of non-reciprocal conditioning of the manifestation of one gene difference, said to be Hypostatic, by the action of another, said to be EPISTATIC. Bateson 1907.

Equational Exceptions, v. Exceptions.

Equational Separation, v. Reductional Separation.

Equatorial Plate, Metaphase Plate.

ERror, SAMPLING, the variance of a statistic arising from, and a function of, the limited size of samples.

ERROR VARIANCE, that arising from agents unrecognized or uncontrolled in the experiment with which the apparent cffect of any recognized agent, controlled or uncontrolled, must be compared.

Standard Error, $v$. Deviation.

Estimation, Combined, the calculation of a statistic or statistics from several scts of unlike data (e.g. backcross and $\mathrm{F}_{2}$ data) taken together.

Simultaneous Estimation, the calculation of two or more statistics from data simultancously. 
Euchromatin, $v$. Chromatin.

EugENIC, that which tends to increase the fitness of the race.

Eugenics, the study of the means of producing eugenic changes, or avoiding dysgenic changes, in man. Galton I 883 .

EuploId, having all Chromosomes of the Set present in the same number. Cf. Aneuploid. Täckholn 1922.

Ever-SPORTING, producing frequent Sports. Especially applicd to a heterozygote which segregates homozygous recessives, but not homozygous dominants, in every generation, e.g. doubleness in Stocks. v. Unfixable. Bateson, Punnett and Saunders I906.

EXAGGERATION, of the expression of a hypomorphic gene placed opposite a deficiency. Mohr I923.

Exceptions, Primary, Secondary and Equational, individuals showing by their character the consequences of Primary, Secondary, and Equational Non-Disjunction in their parents. Bridges I9I6.

Exogamy, Outbreeding. MacLennan 1865 .

EXPRESSIVITY (of a gene), the degree of manifestation of a genetic effect in those individuals in which it is detectable. Timoféeff-Ressovsky I93I.

EXTENSION FACTORS, genes which increase the expressivity of other genes. Applied to area of pigmentation.

$\mathrm{F}_{1}$, the first generation of the cross between two individuals homozygous for the particular genes which distinguished them. Mendel 1865 .

$\mathrm{F}_{2}$, the second filial generation obtained by self-fertilizing or crossing inter se individuals of an $F_{1}$. Mendel I 865 .

$\mathrm{F}_{3}$, progeny obtained by self-fertilization of an $\mathrm{F}_{2}$ individual. (Intercrossing two $\mathrm{F}_{2}$ individuals gives a biparental progeny of the third generation, not an $F_{3}$.)

FACTOR, that which is responsible for the independent inheritance of a mendelian difference. $v$. Gene. Mendel 1865 .

Factorial Experument, one in which all the treatments or agents under investigation are varied simultaneously, and combined in such a way that any derived effect of one or a group of them may be isolated and separately evaluated.

Female, $v$. Sex.

Fertilization, the fusion of male and female gametes and of their nuclei, without which their later devclopment is usually impossible.

Cross-Fertilization (Crossing), the fusion of male and female gametes from different haploid or diploid individuals. 
Fertilization (continued)

Double Fertilization (in Angiosperms), the fusion of one male gamete with the egg nucleus at the same time as a second male gamete fuses with a second female gamete to beget nutritive tissuc. v. Endosperm.

Selective Fertilization, the fusion of germ-cells of different types from one or both sexes in combinations having non-random frequencics.

Self-Fertilization (Selfing), the fusion of male and female gametes from the same haploid or diploid individual.

First Division, the first of two divisions of Meiosis, formerly known as the heterotypic or reduction division.

Fission, division of a one-celled organism into two equal parts in ascxual reproduction, i.e. by mitosis.

Floating, of structual or gene changes for which a Mating Group is not uniform. Darlington and Gairdner 1937.

Fragment, I. A new acentric product of chromosome breakage.

2. A small Supernumerary Chromosome.

Freemartin (in cattle), a female which is sterile through having had a male twin.

Frequency Distribution, the distribution of the frequencies of observation with respect to the classes into which the observations are subdivided.

Fusion, of chromosomes, v. X-Ray Breakage, Chromosome Fusion.

Fusion Nucieus, product of fusion of nuclei in embryo-sac, parent of Endosperm.

GAMETE, cell which is specialized for fertilization and does not usually develop without it. v. Egg. Strasburger I 877.

UNREDUCED GAmEte, having the unreduced or somatic number of chromosomes.

Gametogenesis, the formation of gametes, including meiosis where meiosis immediately precedes the formation of gametes.

GAMETOPHYTE, an individual of the haploid generation in plants, normally producing the gametes.

Geminus, Bivalent.

Gene, I. Any particle to which the properties of a mendelian factor may be attributed. Johannsen I909.

2. Any partiele in a chromosome which is distinguishable from other particles by cither crossing-over or mutation. Morgan I925.

3. A minimum particle of the chromosome which can be separated from other particles by X-ray breakage. Muller and Prokoficva 1935. 
Gene (continued)

4. A unit of physiological action (not rigorously applicable in all cases).

Its changes may be inter-genic and structural or intra-genic and molecular. Darlington I932. v. Mutation.

Gene Dosage, the number of times a gene is present in the nucleus of a given cell or organism.

Gene Dosage Compensation, the equal physiological effectiveness of one gene in the $\mathrm{X}$ chromosome of the male to two genes in the X chromosomes of the female. Muller 1932.

Gene FreQuency, the proportion of the total chromosomes of a population which contain a given allelomorph of a gene.

Gene Mutation, $v$. Mutation.

Mutable (Unstable) Gene, one liable to frequent mutation.

Super-Gene, a group of geneś acting as a mechanical unit in particular allelomorphic combinations.

Genetrc, pertaining to or analogous with Heredity. Bateson I906.

GeNeTIC EqUilibrium, the condition of a population in which successive generations consist of the same genotypes with the same frequencies, in respect of particular genes or arrangements of genes.

Genetic System, the reproductive and hereditary organization of mating group. Darlington 1940 .

Genetrcs, the science of heredity including the study of its chemical foundation, its developmental expression and its bearings on variation, selection, adaptation, evolution, animal and plant breeding and the activities of man. Bateson 1906.

Genome, Chromosome Set, especially as considered genetically. Winkler I9I6.

Genotype, I. The kind or type of the hereditary properties of an individual. Johannsen 1909.

2. The hereditary materials considered as a unit.

Genotypic Control, the control of chromosome behaviour by the genotype, in contrast to Structural Control, especially at meiosis through the effects of dissimilarity of the pairing chromosomes in Hybrids. Darlington I932.

Genotypic Environment, v. Environment.

Germ Cell, Gamete.

Germ Plasm (Keimplasma), the hereditary materials, i.e. the aggregate of self-propagating particles, which are transmitted to the offspring through the germ cells. $v$. Gene, Protoplasm, Virus. Weismann I892. 
Germ Track (Keimbahn), the lineage of cells in the development of an organism, particularly in animals, which are potential ancestors of germ cells, as opposed to somatic cells.

Golgi Bodies, v. Chondriosomes.

GONAD, an organ (testis or ovary) producing gametes in animals.

GRADING-UP, improvement of an animal stock by using in successive generations males from a supcrior stock only.

Graft Hybrid, a Chimacra produced by grafting dissimilar plants.

Grouping, the process of arranging measurements (for the purpose of calculation) in such a way that those falling within a given range are replaced by an equal number of hypothetical measurements at the centre of that range. Sheppard's correction for grouping must then be applied in calculating variances, to correct for the inflationary effect of grouping.

Gynandromorph, an individual of a dioccious species containing both genetically male and genetically female tissues (not yet described in plants). Goldschmidt I9I5.

GyNodioecy, the condition of plant populations or species in which both female (i.e. male sterile) and hermaphrodite individuals are found. Cf. Androdioecy, Gynomonoecy. Darwin I 877.

Gynogenesis, Pseudogamy in animals. Cf. Androgenesis. Wilson i925.

Gynomonoecy, the condition of plant individuals, or of species having individuals, which bear both female (i.e. male sterile) and hermaphrodite flowers. Cf. Andromonoccy, Gynodioccy. Darwin I 877 .

Half-Mutation, $v$. Mutation.

Haplogenotypic SeX-Determination, causing Haploid Dioecy. Hartmann I9I8.

HAPLOID, v. Diploid. Strasburger I905.

Haplophase, $v$. Diplophase.

Haplo-Polyploid, a plant derived by Haploid Parthenogenesis from a Polyploid, therefore having half its chromosome number.

Hemizygous, of genes which can occur in the diploid, when they are occurring in a haploid condition, as in male becs or the differential segments of an X chromosome. Muller 1932.

Heredity, the process by which like begets like (chiefly in sexual reproduction). By extcnsion the law or substance governing this process. v. Inheritance. Herbert Spencer 1863.

Pseudo-Heredity, rescmblance between parent and offspring caused by external conditions, e.g. congenital syphylis. 
Hermaphroditism, the bearing of effective male and female gametes by the same flower in Phanerogams, by the same prothallus in Cryptogams and by the same individual in animals. Can be simultaneous or consecutive in animals. Sometimes incorrectively used for Intcrsexuality.

Heterocaryosis (fungi), the property of having cells with two or more dissimilar nuclei.

Heterochromatin, $v$. Chromatin.

Heteroecy, I. Dioecy. Correns 1928. Subdivided by Hartmann i9i 8 into Diplo-Heteroecy, Diploid dioecy and

Haplo-Heteroecy, Haploid dioecy.

2. (fungi) having alternate unrelated hosts in different stages of the life cycle.

Hetero-Fertilization, the fertilization of the endosperm- and embryoforming nuclei by gametes of different genetic constitution.

Heterogametic Sex, producing gametes of two kinds in regard to their properties of sex-determination. Heterozygous Sex. Wilson I9IO.

HeTEROGAMY, the property by which male gametes of a hybrid transmit a genotype or genotypes different from those of the female gametes. $\nu$. Renner Effect. Applied to Complex Heterozygotes in Oenothera. De Vries I9I I.

Heterogeneity, lack of agreement of different bodies of data in regard to the value of one or more parameters of which they are all capable of yielding estimates. The opposite of Homogeneity.

Heterogenic, of $I$. a population, or 2. a gamete containing more than one allelomorph of a particular gene or genes. Cf. Homogenic. Fisher, of. Lewis 1947.

Heteromorphic Chromosomes, homologous chromosomes which are different in size or form, especially at meiosis. Carothers I9I7.

Heteroploid, Aneuploid. Winkler i9i6.

Heteropycnosis, excessive charging of heterochromatin with nucleic acid in meiotic and premeiotic divisions. Gutherz 1906.

Negative Heteropycnosis, deficient charging of heterochromatin with nucleic acid in meiotic and premeiotic divisions. White 1936. The nucleic acid starvation of Darlington and La Cour 1940.

Heterosis, the property of a $F_{1}$ hybrid of falling outside the range delimited by its parents in regard to one or more characters. Assumed to be due to special combinations of dominant and recessive genes. Usually applied to increase in vigour, when it may be termed Hybrid Vigour. Cf. Transgressive Segregation. Shull igi I. 
Heterostyly, the division of a species into individuals of two (distylic) or three (tristylic) kinds, distinguished by the relative positions of stigma(ta) and anthers. The expression of Heteromorphic Incompatibility. In distylic species the long-styled form is PIN and the short-styled form is THRUM.

Heterothally, haploid Incompatibility in Thallophyta. Blakeslee I904. Heterozygote, a zygote derived from the union of gametes dissimilar in respect of the quality, quantity or arrangement of their genes. Usually used in respect of particular gene differences. Bateson I9O2. Complex Heterozygote, one whose gametes have numerous differences which segregate as a unit. Renner I9I 7.

Sex Heterozygote, a zygote of the Heterogametic Sex. A form of Complex Heterozygote.

Heterozygous, of a heterozygote. Bateson I902.

Heterozygous Sex, Heterogametic Sex.

Hexaploid, $v$. Polyploid.

Hexasomic, $v$. Polysomic.

Homoeosis (Heteromorphosis), the alteration of one organ of a segmental or homologous series from its own characteristic forms to that of some other member of the series. Bateson I 894 .

Homogametic Sex, producing gametes of one kind in regard to their properties of sex determination. Wilson i9io.

Homogenic, of I. a population or 2. a gamete, containing only one allelomorph of a particular gene or genes, or for all genes. $C f$. Heterogenic.

Homologous Variation, LAw of, that related species show similar variation owing to mutation in homologous. genes. Vavilov 1922.

Homology, the similarity of structures in the same or different organisms which they owe to relationship of development or descent. Owen I 840 .

Homology of Chromosomes, applied to whole chromosomes or parts of chromosomes so far as their pairing at meiosis or their visible structure permits them to be regarded as units. Recessive variants enable us to use $F_{1}$ as the test of homology in action of the chromosome, as reflecting its constituent genes, while they enable us to use the $F_{2}$ as a test of homology in position of these genes.

Homothally, the absence of Incompatibility within species of the Thallophyta. Cf. Heterothally. Blakeslee igo4.

Homozygote, a zygote derived from the union of gametes identical in respect of the quality, quantity and arrangement of their genes, or of certain of them, Bateson 1902. 
Homozygous, of a homozygote. Bateson 1902.

Homozygous Sex, Homogametic Sex.

Hybrid, Heterozygote or Heterozygous. Usually used where chromosome or multiple gene differences are concerned. Cf. Heterokaryosis.

INVERSION HYBRID, hybrid for inversion of a segment not including the centromere. Darlington I937.

Numerical Hybrid, one whose parental gametes differed in respect of the number of chromosomes. Darlington 1932.

Structural Hybrid, one whose parental gametes differed in respect of the arrangement of their genes, Darlington I929.

HyBridization, the production of a hybrid by crossing two individuals of unlike genetic constitution. Usually applied to wide crosses.

Hybrid Vigour, v. Heterosis.

HyPERMorph, v. Amorph.

Hyperploids, diploids with an extra piece or pieces of chromosome included in their complement. Muller 1932.

HYPERPOLYPLOIDS, polyploids in which one or more chromosomes are added to the complete chromosome sets (hypertriploids, etc.). Winkler i9ı6.

HүромоRPH, $v$. Amorph.

Hypoploids, diploids lacking a piece or pieces of chromosomes from their complement. Muller I932.

HyPopolyploids, polyploids in which one or more chromosomes are subtracted from the complete chromosome sets (hypotriploid, etc.). Winkler I9ा6.

Hysteresis, a lag in the movement at one level in response to stress at another level, e.g. in the adjustment of the external form of a chromosome to its internal stresses during the spiralization cycle. Darlington 1935 .

Identical Twins, Monozygotic Twins.

Idioplasm, Germ Plasm. Nägeli 1884 .

INBREEDING, the raising of progeny by the mating of two more closely related gametes or zygotes, as opposed to OutbreEDing from the mating of two less related gametes or zygotes.

Inbreeding Coefficient, a measure of the intensity of inbreeding. Pearl I9I 7.

INCEST (in man), breeding between individuals related in degrees prohibited by custom, tabu or canonical injunction. 
INCOMPATIBILITY, the failure of self- or cross-fertilization by reason of genetic similarity, within an otherwise frecly interbrecding group; especially limited to the effects of hindrances which act between mating or pollination and fertilization. Stout 1918.

Classifiable into (Mather 1943):-

HeTERomorphic IncompatibiLITy, which is associated with, and dependent on, morphological variation including Hetcrostyly.

HomomorpHic Incompatibility, which is not dependent for its action on morphological variation (includes the types found in Nicotiana and Capsella and Heterothally-sometimes erroncously referred to as sexuality-in fungi).

INDEPENDENCE, the rclation between two variates, the variation of each of which is uninfluenced by that of the other. The opposite of dependence and Correlation.

INDEPENDENT COMPARISONS, comparisons between observations whose values are uninfluenced by changes in each other. Such comparisons are termed Orthogonal and the functions from which they are calculated are Orthogonal Functions (such as are used in manipulating genetical data for the detection of linkage).

INDIVIDUAL, a unit of life considered either physiologically or genctically. A genetic individual comprises all the derivatives of one fertilization up to the succeeding meiosis, or all the derivatives of one of the four spores produced by meiosis up to the succceding fertilization. v. Clone.

INERT GENE, a gene (definition 3) which, through apparent lack of mutation or effect in disturbing balance, has been assumed to be inactive. Often associated with Heterochromatin.

INFORMATION, AMOUNT of, the quantity which characterizes a body of data with respect to a parameter independently of the method of estimating that parameter. Generalized as the reciprocal of the Variance of an efficient Statistic.

INHERITANCE, Hercdity.

Blending InHeritance, the suppression of heritable differences by the fusion of heredity in the hybrid offspring of a cross. (The Darwinian assumption as opposed to particulate or Mendelian Inheritance.) Sometimes wrongly used for Quantitative Inheritance.

Criss-Cross InHERITANCE, the resemblance of offspring to the parent of opposite sex, owing to sex linkage on the $\mathrm{X}$ chromosome $\nu$. Linkage. Bridges I9I3. 
INHERITANCE (continued)

Cytoplasmic Inheritance, the occurrence of heritable differences determined by the transmission of the cytoplasm. Detccted by the difference in contribution of the male and female parents or by the absence of segregation referable to chromosome movement. $v$. Plasmagene.

Delayed InHeritance, that whereby each generation manifests, with regard to particular characters, the genotype of the female parent. Boycott et. al. 1923.

MATERNAL INHERTANCE, the occurrence of heritable differences, referable to materials transmitted by way of the egg, but not by way of the sperm, owing to Cytoplasmic Inheritance. $v$. Delayed Inheritance.

MAtrilinear INHERITANCE, of determinants (in the cytoplasm) which are transmitted only in the female line.

Matroclinal InHeritance, where the offspring resembles the mother more closely than the father. As opposed to Patroclinal InHerITANCE.

Mendelian Inheritance, obeying Mendel's laws. Particulate as opposed to Blending.

Plastid Inheritance, determined by Plastogenes.

Quantitative InHerutancE, of characters where the variation is continuous. $v$. Polygenes.

UNILATERAL INHERITANCE, the resemblance of offspring to the parent of the same sex owing to sex linkage in the $\mathrm{Y}$ chromosome. $v$. Linkage. Winge 1927.

INTERACTION OF GENES, the process by which one gene-difference affects the expression of another gene-difference.

INTERCHANGE, an exchange of non-homologous terminal segments of chromosomes. May be symmetrical or asymmetrical (giving acentric and dicentric products) with respect to the centromere. The term "segmental interchange" was used by Belling to include crossingover, but the two are conveniently distinguished. $v$. Structural Change. Belling 1927.

INTERFERENCE, the property by which one cross-over interferes with the the occurrence of another cross-over in its neighbourhood.

I. Genetical Interference, as measured from the frequencies of recombinations in the progeny of a multiple Heterozygote. $v$. Coincidence, Coefficient of. Muller I916.

2. Cytological Interference, as measured by the variance of the frequency distribution of Chiasmata in a bivalent. Haldane $193 \mathrm{I}$. 
INTERFERENCE (continued)

3. Chromatid Interierence, as measured by the relations of chromatids exchanging partuers in successive Chiasmata, i.e. whether complementary, reciprocal or disparate. Haldane $193 \mathrm{I}$.

INTERPHASE (INTERKINESIS), the resting stage that may occur between the first and second division of meiosis. Should not be applied to the resting stage in general. Lundegard I9I 5 .

INTERSEX, an individual of a dioecious species in which the reproductive organs are partly of one sex and partly of the other. Goldschmidt I9Is. INVARIANCE, the reciprocal of the Variance.

INVERSION, the reversal of the linear sequence of the genes in a segment of a chromosome especially in relation to the centromere. Sturtevant I926.

An inversion including the centromere (Pericentric, Muller) is equivalent to an Interchange of the two arms of the chromosome. v. Structural Change.

Isochromosome, a chromosome with two homologous arms derived from sister chromatids by sister reunion within a terminal centromere. Darlington 1940

Isogamy, I. The absence of differentiation of the gametes into male and female.

2. Absence of Heterogamy.

Isolating Mechanism, any agency which results in isolation. Dobzhansky I937.

Can be classified (Muller I942) into: r. Bars to crossing. 2. Hybrid incapacity.

Isolation, I. The condition in which individuals of common ancestry are separated into two mating groups. Dartvin 1859 . Of two kinds:GeOgraphic, depending merely on position.

Genetic, depending merely on genotype. Darlington 1933 .

These may arise successively and occur simultaneously to give combined geographic and genetic, which includes ecological, isolation.

2. The condition in which chromosomes or parts of chromosomes of common ancestry (as in Complex Heterozygotes) are prevented from undergoing effective recombination.

Karyokinesis, Mitosis. Schleicher I878.

KarYology, nuclear cytology. Troul I 895 .

KARYOTYPE, the character of a nucleus as defined by the size, shape and number of the mitotic chromosomes. 
Kinetochore, Centromere. Schrader 1938.

KuRTOSIs, the departure of a symmetrical frequency distribution from the normal by excess (PlatyKurtosis) or deficiency (Leptokurtosis) in its shoulders as opposed to tails and centre.

LePtotene, the undivided and unpaired chromosome threads at the earliest stage of the prophase of meiosis, and, by extension, the stage itself. Winiwarter 1900.

Lethal Gene, a gene whose substitution for its normal allelomorph converts a viable into an inviable gamete or zygote. May be dominant or recessive. The expression of the gene may be modified by genotype or environment, and when incomplete in effect the gene is said to be Sub- or SemI- lethal.

Balanced Lethal Genes, non-homologous, lethal genes in chromosomes from opposite gametes. The chromosomes concerned must be homologous except in ring-forming interchange heterozygotes. Muller I9IS.

LikeliHood FunCtion, the function relating an unknown parameter to observations from which it can be estimated. Cf. Probability Function.

Method of Maximum Likelihood, the method of estimation depending on the maximization of the log likelihood (and hence of the likelihood) function. Always leads to an Efficient Statistic, and also to a Sufficient Statistic if one exists.

LINEAGE, in man a section of a tribe or mating group forming a unit of common descent, with a name inherited in either the male or female line, within which mating is tabu, i.e. forbidden by custom or law.

Linear Order, $v$. Chromosome Map.

LINE BREEDING, the mating in successive generations of individuals having the same known common ancestor, excluding the closest relationships.

LINKAGE, the combination in the cell products of meiosis (spores or gametes) of pairs of segregating genes in non-random frequencies owing to their presence in the same chromosome or in chromosomes associated at meiosis. Morgan I9II.

LINKAGE MAP, Chromosome Map, determined by recombination relations.

Partial Sex Linkagf, where the linkage is incomplete, i.e. where the gene lies in the Pairing Segment. Darlington, Haldane and Koller 1934.

SeX Linkage, the segregation of a gene in association with the sex inequality. Morgan I9I4. 
Localization, the genotypic property of restriction of crossing-over and chiasma formation to certain corresponding parts of all the chromosomes. It may be procentric or proterminal according to whether the Contact Points are near the centromere or near the ends. Darlington I93 I.

Locus, the position occupied by a gene in a chromosome, with regard to its linear order.

M I and MII, Metaphase of first and second division of Mciosis. Similarly A I, A II, T I and T II for Anaphase and Tclophase.

Macronucleus, $v$. Micronucleus.

Macrospore, Megaspore.

MAJOR GENES, genes whose differences or mutations are large enough to be identified by their individual effects in individuals, and which are therefore presumed to have a large effect on the sclective advantage of the individuals. $C f$. Polygenes.

Male, $v$. Sex.

Malthusian Parameter (of Population Increase), a measurc of the relative rate of increase or decrease of a population when in the appropriate steady state. Fisher 1930.

Maternal Effect, Delayed Inheritance.

Maternal Inheritance, $v$. Inheritance.

MATING, the assortment of individuals, usually zygotes, in couples leading to sexual reproduction; by extension includes self-mating.

Mating ContinuUm, an aggregate of individuals whose genes systematically recombine.

ILLEGITIMATE MATING, any mating normally precluded by the breeding system of the organism (e.g. self-pollination in incompatible or heterostyled plants, or the crossing of species).

Mating Group, a group of individuals, haploid or diploid, within which mating is favoured at the expense of mating outside the group, by genetic or environmental conditions characteristic of the group.

Matroclinal Inheritance, $v$. Inheritance.

Maturation, the formation of gametes or spores by meiosis.

Maturation Divisions, Meiosis.

MeAn (ARITHMetic), the arithmetic average of a scries of observations, or quantities.

Weighted Mean, a mean obtained when different classes of observations or quantities are given different weights in the calculation. 
MEAN (continued)

Working MEAN, a value approximating to the mean used for the purpose of lightening the calculations of the mean, the sum of squares and other quantities.

MEDIAN, the value of a variate on each side of which lie equal numbers of observations.

Megaspore, a spore which develops into a gametophyte bearing only female gametes, as opposed to Microspore.

MeIosis, a double Mitosis in which the nucleus divides twice but the chromosome only once. The prophase of meiosis is the prophase of the first of the two divisions. v. Mother Cell. Farmer and Moore I905.

Mendel's Laws of Inheritance. I. The Law of Segregation, that the gametes produced by a hybrid or heterozygote contain unchanged either one or the other of any two factors determining alternative unit characters in respect of which its parental gametes differed.

2. The Law of Recombination, that the factors determining different unit characters are recombined at random in the gametes of an individual heterozygous in respect to these factors. Mendel 1865.

Merogony, development of part of an egg with the sperm nucleus, but without the egg nucleus. Genetically equivalent to Male Parthenogenesis. Delage I 899.

Metabolic Stage, Resting Stage.

Metaphase, the stage of mitosis or meiosis at which the undivided centromeres of the chromosomes lie on the spindle. Strasburger I884.

Metaphase Plate, the group of chromosomes arranged in the equatorial plane of the spindle.

Metaxenia, $v$. Xenia.

Microchromosomes, small chromosomes which pair at meiosis only at metaphase and therefore without chiasma formation (in the Hemiptera-Heteroptera). Wilson I905.

Micronucleus. I. The small permanent reproductive nucleus of the Infusoria, as opposed to the short-lived multiple MACronUCLEUS.

2. A nucleus separate from the main nucleus formed at telophase by one or more lagging chromosomes or fragments.

Microsome, largely submicroscopic particles in the cytoplasm characterized by the presence of ribose Nucleic Acid and phospholipids. Claude i943.

Microspore, a spore which develops into a gametophyte bearing only male gametes, as opposed to Megaspore. $v$. Pollen Grain. 
Misdivision, spontancous crosswise (instead of lengthwise) division of the centromere on the spindle especially of univalents at M I and daughter univalents at M II. $v$. Telocentric. Darlington 1939.

Mitochondria, v. Chondriosomes. Benda I 897.

Mrtosis, the process by which division of the nucleus is accomplished

by that of its constituent chromosomes and usually accompanied

by that of its containing cell. Flemming I 882.

Mixoploid, a diploid-polyploid Chimaera. Nemec i9ro.

MODE, the value of a variate shown by the class most frequently observed.

Modifcation, a non-heritable change (caused by difference in the environment). v. Daucrmodification. Baur I9II.

Modifying Gene, Modifier, a gene whose differences are revealed by their effects on the expression of another genc. $v$. Polygene.

MoNoECY, in plants the condition in which male and female gametes are borne by different flowers of the same individual. $v$. Dioecy.

MONOGENIC, of an hereditary difference determined by one gene difference as opposed to two (Digenic), three (Trigenic) or many (PolyGENIC).

MONOHYBRID, heterozygote in respect of one gene.

MONOPLOID, having the Basic Number of chromosomes.

Monosomic, an otherwise diploid organism lacking one chromosome of its proper complement (2x-I). v. also Polysomic. Blakeslee I92 I. Monozygotic, derived from one fertilized egg (of Twins or multiplets then called Identical).

MORPHOGENESIS, the differentiation of structures during development.

MosAic. I. In animals, the equivalent of a Chimaera.

2. In plants a Chimaera produced by recurrent mutation.

3. A Virus disease producing the appearance of a mosaic in plants.

Mother Cell, a cell with a diploid nucleus which, by meiosis, gives four haploid nuclei, e.g. the spore mother-cell in Bryophyta and Pteridophyta, the Microspore or Pollen mother-cell and the Megaspore or Embryo-Sac mother-cell in Flowering Plants. In animals the sperm mother-cell is known as the SPERMatocyte and the egg mother-cell as the Ö̈cYTe.

Multinominal Series, the series obtained by expanding, to any power, the sum of three or more quantities $(a+b+c \ldots)^{n}$.

Multiple Allelomorph. $v$. Allelomorph.

Multiple Chromosome, the product of fusion of two chromosomes. McClung 1917.

Multiple Factors, Polymeric Genes.

Multivalent, $v$. Univalent. 
Mutable Gene, $\nu$. Gene.

Mutafacient, of one gene or genetic element which determines, or increases, the chance of mutation of another. Darlington 1944.

Mutant, aberrant individual, cell or gene produced by Mutation.

Mutation, a change of heredity not ascribable to segregation or recombination. De Vries I 890 . Classifiable as follows:-

Gene Mutation, intragenic change, i.e. from one gene to an allelomorph. A residual category after the exclusion of identifiable structural changes.

Structural Mutation, intergenic change in the linear arrangement of genes, sometimes producing a Position Effect. $v$. Structural Change.

Numerical Mutation, a change in the number of chromosomes, either balanced to give Polyploidy or unbalanced to give Aneuploidy. Plastid Mutation, a change in a chloroplast affecting its capacity to produce chlorophyll. $v$. Plastogene.

Cytoplasmic Mutation, a change in cytoplasmic heredity. $v$. Plasmagene.

InDuced Mutation, determined by externally controlled conditions.

MUtATION RATE, the proportion of individuals or cells in a given group which show mutation for a given gene under given conditions in one generation, or other stated unit of time.

Somatic Mutation, a change observed or inferred in somatic tissue. Its nature is assumed from analogy with mutation in the germ track.

Mutation Pressure, the measure of the action of mutation in tending to alter the frequency of a gene in a given population. Cf. Selection Pressure. Wright I92I.

Mutation Theory, that new species arise by single mutation. De Vries I890.

Certain consequences of crossing-over and aberrant segregation in hybrids have been attributed to mutation, e.g. Half-Mutation (De Vries 1918) and Mass-Mutation (Bartlett I9I5) in Oenothera and Pseudo-Mutation in apomictics with sub-sexual Reproduction. Gustafsson i934. Attenuation and Fortification are the consequences of mutation in viruses.

$n$, gametic as opposed to $2 n$, the zygotic, number of chromosomes.

Nature and Nurture, the antithesis between Genotype and Environment.

NeOMORPH, $v$. Amorph.

NON-Disjunction, Bridges i9i4.

Cytological (Numerical) Non-Disjunction, the failure of separation of paired chromosomes at meiosis and their passage to the same pole. 
Non-Disjunction (continued)

Genetical Non-Disjunction, any result that might be imputed to cytological non-disjunction, although usually arising from the failure of pairing, or from inultivalent formation. Bridges 19i6. Includes:Chromatid Non-Disjunction, the passage of the parts of sister chromatids distal to a chiasma to the same gamete at meiosis. Where this occurs in a polyploid it leads to Double Reduction. $E$. $R$. Sansome 1933 .

In an XX-XY systems of sex determination ( $v$. Exceptions):-

Primary Non-Disjunction, the production of eggs with two or no $\mathrm{X}$ 's by an XX individual.

SECONDARY Non-Disjunction, the production of eggs with two X's or a $\mathrm{Y}$ by an XXY individual.

Equational Non-Disjunction, is chromatid non-disjunction in a $\mathrm{XXY}$ individual.

Non-Homologous Pairing, v. Pairing.

Non-Reduction, failure of Reduction, $v$. Ameiosis.

Normal Deviate, the ratio of an observed deviation to the appropriate or corresponding Standard Deviation as fixed by hypothesis.

Normal Distribution (Normal Curve of Errors), the limit which is reached either by the Binomial or the Multinomial series where the power is large and none of the summed quantities very small in relation to the power and to one another. This frequency distribution is expected from a series of observations on a variate whose magnitude is affected by a large number of agents having small independent effects.

Nucellar Embryony, a form of Apomixis where the embryo arises directly from the nucellus.

NuCLEAR SAP, the fluid which is lost by the chromosomes as they contract during prophase and which fills the space of the nucleus.

NuCleIC ACID, the product of polymerization of Nucleotides which have either all ribose sugars giving yeast or RuBO-NUCLEIC ACID, or desoxyribose sugars, giving thymus (or chromosome) DesoxyriBoNuCLEIC ACID.

NuCleolus, a body not containing desoxyribose nucleotides and secreted by a specific organizer, gene or super-gene, in the resting nucleus. McClintock 1934 .

Nucleoprotein, a protein which is combined, or combinable, with a nucleic acid.

NUCLEOTIDE, a chemical group consisting of purine or pyrimidine base, ribose or desoxyribose sugar, and phosphoric acid. 
NuCleus, a cell body constituted by chromosomes, i.e. arising by mitosis. R. Brown I83I.

Null Hypothesis, the hypothesis from which the expectations are formulated for the purpose of a test of significance. $v$. Significance.

Nulliplex, condition of a polyploid in which all the chromosomes of one homologous type carry the recessive allelomorph of a particular gene, as opposed to Simplex, Duplex, Triplex, Quadruplex, etc., in which the dominant allelomorph is represented one, two, three, four, etc., times. Belling, Blakeslee and Farnham 1923.

OgIve, the integral of a frequency distribution. Galton 1883.

ONTOGENY, the developmental history of an organism.

Oöcyte, $v$. Mother-Cell. Boveri I89I.

OöGENESIS, development of the egg from the oöcyte.

Ö̈GONIUM, a cell giving rise to oöcytes directly or by mitosis. Boveri I 891 .

Orientation, the movement of centromeres so that they lie axially with respect to the spindle, either as to their potential halves at mitosis (Auto-Orientation) or as to members of a pair or higher configuration at meiosis (Co-Orientation). Determines Congression. Co-orientation or multiple configurations may be Linear, ConverGeNT or ParalLFi.

Orthogonal, $v$. Independent Comparisons.

OUtBREEDING, $v$. Inbreeding.

Ovum, Egg.

$P$, the parental generation of an $F_{1}$.

Pachytene, the double thread, and by extension the stage at which it occurs, produced by pairing of the chromosomes in the prophase of meiosis. Winiwarter 1900.

PAEDOGENESIS, the asexual multiplication of sexually immature animals.

Pairing (of Chromosomes), the coming together of chromosomes at zygotene (active) or the continuance of their association at the first metaphase (passive) of meiosis.

Non-Homologous Pairing, the association of non-homologous segments of one or two chromosomes at pachytene. McClintock 1933.

Pairing Segment, $v$. Differential Segment.

SeCondary Pairing, the lying of homologous pairs of bivalents especially close to one another at meiosis. Darlington 1928.

Somatic Pairing, the lying of homologous chromosomes especially close to one another at metaphase of mitosis. 
Pairing (cantimued)

Torsion PAIRING, non-homologous association at pachytene which releases a torsion without satisfying an attraction, when it occurs in continuance of homologous association. Darlington 1935 .

Toucil-and-Go PAIRING, momentary pairing, especially of sex chromosomes, at second division of meiosis in Hemiptera of the heterogametic sex.

PANGENESIS, the theory that the heredity of organisms is determined by the summation of influences from an indefinite number of particles (pangenes) derived from all parts of the body tissue and variably affected by variations in the environment. Darwin 1868 .

Panmixis, unrestricted Random Mating. Properly excluded by any restriction, but sometimes used where random mating is assumed within the operation of a restriction, especially with dioccy in animals. Weismanm I 895 .

Parameter, a quantity necessary for the specification of a Population.

PARASYNAPSIS, the association of chromosomes side by side observed at zygotene and pachytene, as opposed to their alleged (I905-1935) end-to-end association at this stage, or Telosynapsis. Wilson i9i2.

Parthenocarpy, the development of fruit without seed as the result of (a) lack of pollination, with or without artificial stimulation, (b) lack of fertilization, i.e. by incompatibility or gametic sterility, or (c) lack of embryo development, i.e. by zygotic sterility. Noll 1902 . Parthenogenesis, Apomixis in which the egg cell develops into an embryo. May be facultative, obligatory, or cyclical. Owenl I 849.

Arrhenotocous Parthenogenesis, male-producing (and facultative) haploid parthenogenesis as in Haplo-Diploid Sex determination.

Diploid (Somatic) Parthenogenesis, in which mciosis fails as well as fertilization.

Haploid Parthenogenesis, in which meiosis is successful and the embryo haploid.

Male Parthenogenesis, that in which the male nucleus entering the egg cell gives rise to the embryo, without the egg nucleus.

Thelytocous Parthenogenesis, female-producing diploid parthenogenesis.

Patroclinal Inheritance, $v$. Inheritance.

Patrogenesis, Male Parthenogenesis.

Pedigree, table of ancestry or of posterity.

Penetrance (of a gene), the proportion of individuals of a given genetical constitution in a given population in which the effect of the gene concerned phenotypically distinguishes them from those bearing its allelomorph. Timoféeff-Ressovsky 193 I. 
Pentaploid, v. Polyploid.

Periclinal Chimaera, $v$. Chimaera.

Phenocopy, the phenotype of a given genotype changed by external conditions to resemble the phenotype of a different genotype. Goldschmidt 1938.

Phenogenetics, Developmental Genetics. Haecker I9I8.

Phenotype, the kind or type of organism produced by the reaction of a given Genotype with a given Environment. Johannscn 1909.

PhYLOGENY, the evolutionary history of any related group of organisms. Pin and Thrum, $v$. Heterostyly.

Plasmagene, a particle whose reproduction in the cell, but outside the nucleus, and transmission by the egg (and sometimes by the pollen or sperm) determines a likeness of daughter cells and individuals to their parents. $C f$. Virus. May be taken to include Plastogene. Darlington 1939.

Plasmon, the cytoplasm of an individual considered as a single hereditary agent; the sum of the plasmagenes. Wettstein 1924.

Plasmosome, Nucleolus.

Plastid, a self-propagating body in the plant cytoplasm associated with pigment production (chloroplast, chromoplast) or assumed to be capable of being associated with it (leucoplast, proplastid). Schimper I885.

Plastid Inheritance, $v$. Inheritance.

Plastogene, a particle attached to a plastid whose reproduction determines a likeness of daughter to parent plastid. Cf. Plasmagene. Imai 1937.

Pleiotropy, the production of physiologically uncorrelated effects by a mechanically unitary, i.e. single, gene. Ascribed to one gene initiating two or more chains of reactions. Attributable to complete linkage of two or more physiological units. Plate I9Io.

False Plemotropy, where the lack of correlation is spurious and the various effects are traceable to one initial reaction chain. Grineberg I943.

P.M.C., Pollen Mother Cell.

Point Mutation, Gene Mutation. Bridges I923.

PolAR Bodies, the expelled products of the two divisions of the oöcytes in animals. Robin I 862.

Polarization. I. Of chromosomes at telophase of mitosis and, later, the maintenance of their proximal parts on the polar side of the nucleus.

2. Of chromosomes at zygotene, the movements of their ends towards the part or parts of the nuclcar surface where the centrosomes lie. 
Polarizatiun (continued)

3. Of centromeres, the initiation of orientated division during mitotic metaphase. Darlington 1937.

Pollen Grain, the male gametophyte in the flowering plants.

Pollination, the placing of pollen on the receptive stigma of a flower.

Polycentric, of a chromosome or chromatid having several Centromeres. Darlington 1937.

POLYembryony, the production of more than one embryo within the testa of one seed in a flowering plant, cither from one or several zygotes, extra zygotes being sexual or parthenogenetic, from reduced or unreduced eggs. The extra eggs may be derived from vegetative nucellar cells, sister mother-cells, sister spores or sister nuclei within one embryo-sac. Marchal I904.

Polygamy, the system of mating where one male unites with several females (polygyny) or vice versa (polyandry).

Polygenes. I. Members of a Polygenic System.

2. Genes whose differences or mutations are too slight to be identified by their individual effects in individuals, and which are therefore presumed to have a small cffect on the selective advantage of the individuals. Cf. Major Genes.

Polygenic Combination, v. Balance. Mather i94I.

Polygenic System, of genes having effects similar and supplementary to one another, and small in comparison with the total variation. The members of such a system are replaceable in their effects.

Polyhaploid, Haplo-polyploid. Katayama i935.

Polymeric Genes, non-allelomorphic genes of apparently identical and cumulative action. Undefined with regard to individually recognizable effect. The Cumulatrve Factors of Nilsson-Ehle. Characteristic of allopolyploids. $C f$. Polygenes. Two genes of identical but non-cumulative effect are said to be Duplicate. Lang I9I I.

Polymitosis, the intercalation of rapid supernumerary mitoses in the life cycle immediately after meiosis, without division of the chromosomes, Beadle 1933, or with division of the chromosomes. Darlington and Thomas I94I.

Polymorphism, the occurrence in the same habitat of two or more distinct forms of a species in such proportions that the rarest of them cannot be supposed to be maintained by recurrent mutation from any other. Ford I940. In practice the genetic diversity associated with the control of mating is excluded, but Polymorphism may be confuned to one sex, e.g. Lebistes. 
Polymorphism (continued)

Cryptic Polymorphism, such as is not distinguishable by external morphology.

Polyploid, an individual having three (Triploid), four (Tetraploid), five (Pentaploid), six (Hexaploid) or more complete sets of chromosomes instead of two as in the Diploid ( $v$. Allo-, Autopolyploid). Winkler г9г6.

Di-, Tri-, Poly-Basic Polyploid, Allopolyploid derived by the addition of diploid complements with two, three or more basic numbers. Darlington and Janaki Ammal i945.

Secondary Polyploid, a homozygous allopolyploid in which some of the types of chromosomes in the basic set are represented more frequently than others. Darlington and Moffett I930.

Unbalanced Polyploid, $v$. Aneuploid.

Polysomic, an otherwise diploid individual having one chromosome represented three (TRISOMIC) or four (TETRASOMIC or doubly Trisomic) times instead of twice $(2 x+\mathrm{I}, 2 x+2)$. Blakeslee I92 I.

Polysomic InHERItANCE, that which arises where any chromosome in a polyploid or polysomic individual has more than one possible partner at meiosis. By analogy Tetrasomic InHeri'tance, HeXasomic InHeritance, etc. Blakeslee, Belling and Farnham 1923.

POLYSPERMy, the entrance into the egg of more than one sperm, under normal and abnormal conditions, whether effective or ineffective in fertilization.

Polytene Nucleus, one in which protein production and gene reproduction go on side by side. A permanent stage in which the chromosomes are represented by many linearly stretched and paired threads in the Diptera, especially in the salivary glands. Koller 1935 .

Population. I. (genetical), a Mating Group limited for special consideration either by environment or breeding system.

2. (statistical), the hypothetical infinitely large series of potential observations or individuals, of which those observations or individuals actually obtained form a sample.

Position EFFECT, the difference in effect of two or more genes according to their spatial relations in the chromosomes. An effect of intergenic structural change. v. Gene, Mutation. Sturtevant 1926.

Post-Reduction, as opposed to Pre-Reduction. Korschelt and Heider I903.

I. The alleged reduction of the number of chromosomes at the second, as opposed to the first, meiotic division (obsolete). 
Post-Reduction (continued)

2. The segregation of differences between partners at the second, as opposed to the first, meiotic division.

Potence, the property of a group of Polygencs corresponding to the degrec of Dominance of a Major Gene. Wigan I944.

PRECOCITY, the property in the nuclcus at meiosis of beginning prophase before the chromosomes have divided. Darlington I93I.

Precocity Theory, that mciosis is primarily distinguished from mitosis by showing a precocity of the prophase, which successively determines pairing, crossing-over, chiasma formation, non-division of the centromeres and insertion of a second division, together with the reduction of chromosome number and the segregation of differences. Darlington I930.

Differential Precocity, the property of some chromosomes, or of their parts, condensing, dividing or pairing in advance of the rest of the complement during prophase. Shown by some heterochromatic chromosomes. v. Heteropycnosis. Darlington 1937.

Preformation, $v$. Epigenesis.

Prepotency, the capacity of an individual to produce offspring more like itself and less like the other parent. Practical breeder's term for a property depending (where genuine) on superior homozygosity, dominance and potence.

Pre-Reduction, v. Post-reduction.

Presence And Absence Theory, that the dominance of one allelomorph is due to the presence of something in it which is absent from its alternative. Bateson 1909.

Prime Type (in Datura), one of the homozygous types of chromosome structure distinguished from other such types by interchange and used as a basis of reference. $v$. Basic Type. Blakeslee 1928.

Probability, the proportion of an infinite (and hypothetical) scries of cases in which an event, possible in any one of them, actually occurs.

Probability Function, the function rclating observations to the parameter from whose value their frequencies may be predicted. $C f$. Likelihood Function.

Simple Probability Function, that relating to the occurrence of a single event.

Compound Probability Function, that relating to more than one event.

Proband, Propositus.

Prochromosome, body of Heterochromatin seen in the resting stage.

Overton I909. 
Progeny Test, the method of assessing the genetic character of an individual by the performance of its progeny. First used systematically by Mendel.

Pro-Metaphase, stage between the dissolution of the nuclear membrane, and the congression of the chromosomes on the Metaphase Plate, especially at the first meiotic division. Lawrence I93I.

Prophase, the stage in mitosis or meiosis from the appearance of the chromosomes to Metaphase. Strasburger I 884.

Propositus, the individual through whom a pedigree is ascertained.

Prothallium (Prothallus), the gametophyte in the Pteridophyta.

PROTOPLASM, the aggregate of self-propagating structures and materials within the Cell. Purkinje I840.

Protoplast, the protoplasm of one cell. Hanstein 1880.

PROXIMAL, of a part of a chromosome arm which is nearer to the centromere than another (Distal) part.

Pseudo-Compatibility, the occurrence under exceptional conditions of fertilization such as would normally be excluded by Incompatibility. Pseudogamy, apomictic development of an embryo from a female gamete or cell under the stimulus of male gametes but without fertilization. Focke I88I.

Pure Line. I. The descendants obtained from self-fertilization of a single homozygous parent. Johannsen I903.

2. In modern usage, an inbred homogenic strain.

Quadrivalent, $v$. Univalent.

Quadruplex, $v$. Nulliplex.

RACE, a genetically, and as a rule geographically, distinct Mating Group within a Species.

RANDOM MATrNG, the situation within a population in which, subject to known restrictions of determinable effect, such as Dioecy, Incompatibility and intra-uterine copulation, each individual has an equal chance of mating with any individual, including itself. $v$. Panmixis.

RANDOMIZATION, the process of arriving at a random combination of types of event.

RANDOMIZED BLOCK, an experimental design involving one Restraint.

RANDOMNESS, that combination of types of event in which the distribution in classes of one has no causal relation with the distribution in classes of the others, i.e. arrived at by chance without discrimination or choice.

ReCapitulation, Theory of, that the developmental history of organisms repeats their evolutionary history. Kielmeyer 1793 . 
ReCessive, $v$. Dominance. Mendel i 865 .

RECIProcal, of crosses where the sources of male and female gametes are reversed. Mendel 1865 .

Reciprocal Translocation, Interchange.

RECOMBINATION, the formation by crossing-over or segregation at meiosis of new combinations of genes with respect to exther (i) individual chromosomes or (ii) whole gametes.

REDUCTION, the halving of the chromosome number at meiosis and, by extension, its genetical concomitant of segregation. Weismann I 887 .

DOUbLE REDUCTION, the occurrence of a reductional separation at both divisions of meiosis in regard to particular parts of chromosomes. Possible in some hybrids and polyploids. Hence Equational Exceptions. Darlington 1929.

GAMETic Reduction, meiosis immediately before fertilization.

Reduction Divisions, Meiosis.

Zygotic Reduction, meiosis immediately after fertilization.

ReDUCtional Separation, separation of homologous parts of non-sister chromatids at anaphase of one or other meiotic division, as opposed to Equational Separation of sister parts of chromatids.

REDUPLICATION, the occurrence of a segment of chromosome twice in the same haploid set. $v$. Structural Change.

REGENERATION, the growing again of a part of an organ lost by injury. In Bryophyta, the growth of a diploid gametophyte direct from a sporophyte after injury.

REGRESSION. I. Galton's Law, that in any population of families the deviation of the mean of the offspring from that of the population is less than the deviation of one specified parent, adjustments being made for sex differences. Galton 1889.

2. The dependence of one variate, termed the dependent, on another, termed the independent, variate.

Regression Coefficient, the rate of change of the dependent variate on the independent, variate.

REgRESSION LINE, the straight line or curve showing a regression in a co-ordinate representation.

LinEAR REgression, that involving the independent variate to the first power.

Multiple Regression, regression, usually linear, on two or more independent variates, which may themselves be correlated (also called Partial Regression).

Simple Regression, linear regression on one independent variate. 
Relative Sexuality, the ability of a gamete to act as female when mated to one gamete and as male when mated to another. Hartmann 1923. RENNER EFFECT, competition among the four genetically different spores formed by one meiosis in regard to which shall form the embryo sac. Renner I921, Darlington 1932.

REPLICATION, the equal incorporation of all combinations two or more times in an experimental design, which is then said to be replicated. Reproduction (Self-Propagation), the production of an organism, a cell, a chromosome, or any other cell component, by one like itself either as parent or sister.

Asexual Reproduction (of individuals), that which does not require meiosis or fertilization for its completion. v. Apomixis, Clone, Vegetative Propagation.

Sexual Reproduction, that which requires meiosis and fertilization for its completion.

Subsexual Reproduction, Parthenogenesis following Ameiosis in which segregation occurs owing to crossing-over but without reduction. Darlington 1937.

Versatile Reproduction, capacity for either sexual or apomictic development of an embryo according to the kind of male gametes, owing to variation in pollination, or of female gametes, owing to variation in the products of meiosis. Crane and Thomas I94I.

Reproduction Rate, v. Malthusian Parameter.

Repulsion, v. Coupling. Bateson 1908.

REsting StAge of the nucleus, that in which the linear structure of the chromosomes is not visible.

Restitution, $v$. X-ray Breakage.

Restitution Nucleus, a single nucleus found instead of two through failure of the first or second division of meiosis. $v$. Ameiosis. Rosenberg I927.

RESTRAINT, a linitation of the random arrangement of combinations in an experimental design so that error variation, while still capable of being estimated without bias, is reduced or potentially reduced.

Reversion, Atavism.

Rings. I. At mitosis, chromosomes with no ends.

2. At meiosis, chromosomes associated in rings, usually by terminal chiasmata, and in twos, fours, sixes, etc.

Salivary Chromosomes, the chromosomes in the nuclei of the salivary glands of Diptera. $v$. Polytene.

SALTANT, mutant of a fungus or bacterium especially in culture. 
SAMPLE (RANDOM), a finite series of observations or individuals taken at random from the hypothetical infinitely large population of potential observations or individuals.

Satellite, a short segment of a chromosome, separated from the rest by one long constriction if terminal or two if intercalary.

SECOND Division, the second of two divisions of Meiosis, formerly known as the homotypic or equation division.

Sectorial Chimaera, v. Chimaera.

SEgment, a portion of a chromosome considered as a unit for a given purpose, $v$. Differential Segment, Pairing Segment, Interchange. Belling I927.

Segregation, separation at meiosis of the chromosomes, or parts of chromosomes such as genes, of paternal and maternal origin. Bateson 1902.

Effective Segregation, that which gives viable gametic or zygotic combinations. Darlington I93 I.

Preferential Segregation, the non-random assortment of a particular chromosome or segment with respect to the four cells produced at meiosis, or to a non-homologous chromosome, or in Polysomics (or Allopolyploids) to a particular homologue.

Secondary Segregation, the segregation in an allopolyploid of differences between its ultimate diploid parents. Darlington 1928.

Somatic Segregation, the formation by mitosis of cells differing from one another, either through Mutation or Somatic Crossing-over in the nucleus, or through an unequal assortment of cytoplasmic determinants such as is not required by normal differentiation. $v$. Sport.

Selection. I. (statistical) discrimination in sampling or in arrangement. Opposed to randomness.

2. (biological) any non-random process which will lead to individuals of different genotypes being represented unequally by their progeny in later generations of a population of self-propagating units. Can be Natural or Artificial. Darwin i 858. Natural Selection is responsible for the Survival of the Fittest (Herbert Spencer I86I).

Mass Selection (in plant and animal breeding), where a group of selected individuals are pooled for breeding purposes. As distinguished from individual selection. Cf. Le Couteur i 836.

Cell Selection, where genetically distinct cells are selected within an individual. Darlington 1937.

Selection Pressure, the measure of the action of selection in tending to alter the frequency of a gene in a given population. Cf. Mutation Pressure. Wright $\mathrm{I} 92 \mathrm{I}$. 
Selective Advantage, that genotypic condition of a cell or individual or genctic class of individuals which increases its chances, relative to others, of representation in later generations of cells or individuals. Self-Fertilization (Selfing), $v$. Fertilization.

Self-Propagation, Reproduction.

Self-Sterility, self-incompatibility. $v$. Incompatibility. Sometimes incorrectly deduced from mere sterility and vice versa.

Semi-Heterotypic Division, a first division of meiosis which gives rise to a Restitution Nucleus following defective pairing. Rosenberg 1927. Semi-Lethal Gene, $v$. Lethal Gene.

Semi-Sterile, of gene heterozygotes or structural hybrids in which approximately half the male and female gametes are inviable. Belling I9I4.

Sensitive Period, the period of development during which the action of a gene is sensitive to the influence of external conditions.

Sensitive Volume, that hypothetical volume in which the occurrence of an ionization must produce a given mutation. Timoféeff-Ressovsky et al. 1939

Separation, $v$. Reductional Separation.

Set of Chromosomes, $v$. Chromosome Set.

Sex (Sexual Differentiation), the production by an individual or group of individuals, of gametes of two types, differing in size and mobility (Eggs and Sperm) such that the one can fuse only with the other. The individual or organ producing eggs is FEMALE, and that producing sperm is MALE. $v$. also Dioecy, Incompatibility, Relative Sexuality.

Sex Chromosome, one whose distribution to one and not to another of the products of meiosis determines the difference in sex of the offspring. Wilson 1906.

Sex Determination, the process by which a spore (haploid) or egg (haploid or diploid) comes to develop the properties of one or other sex.

Environmental Sex Determination, where the determining conditions are external to the spore or egg, e.g. Bonellia.

Genotypic Sex Determination, where the determination is by the genotype of the spore or egg.

Haplo-diploid Sex Determination, where the male arises from a haploid egg and the female from a diploid, e.g. in Hymenoptera.

Maternal Sex Determination, where the determination is by the genotype of the mother, e.g. Sciara.

Progamic Sex Determination, where the determination is by a size difference of the eggs, e.g. Dinophilus. 
SEX-Limited, of the Inheritance of differences which are expressed in one sex only or at least in the two sexes differently. Morgan igr4.

SeX-Linkage, $v$. Linkagc.

SEX Ratio, the ratio of numbers of the two sexes at conception, at birth, at a given age or in the total population, usually expressed in terms of the females as 100 .

SeX-Reversal, the change of a mature or nearly mature individual from having the appearance or function of one sex to having the appearance or function of the other. $v$. Intersex.

Sexual Isolation, genetic isolation by the sexual mechanism. $v$. Isolation. Dobzhansky 1937.

Sexual Reproduction, $v$. Reproduction.

Sib(s) or Sibling(s), progeny of the same parents derived from different eggs.

SiB MATing, the mating of sibs.

SiGNIFCANCE, the measure of reliability of a difference between observation and expectation. A difference is said to be Significant if, on the hypothesis being tested, the probability of obtaining one as large or larger is lower than some chosen level, termed the LEVEL OF SignifiCANCE.

TEST OF Significance, a test designed to assess significance, and so to distinguish differences due to sampling error from differences indicating discrepancies between obscrvation and hypothesis.

Simplex, v. Nulliplex.

SKEwNESS, asymmetry in a Frequency Distribution.

Somatic, of all parts of an individual except, in plants, Germ Mother Cells or, in animals, Germ Track cells.

Somatic Crossing-Over, v. Crossing-over.

Somatic Mutation, $v$. Mutation.

Somatic Pairing, v. Pairing.

SPECIES, in Sexually Reproducing organisms, a maximum interbrceding or potentially interbrecding group, brceding true within its own limits in naturc. v. Apomixis, Brced, Caste, Clone, Race, Stock, Strain and Variety. Ray 1670.

The classification into the next three (Turesson 1922) is based on ecological adaptation of groups of individuals of decreasing importance.

COENO-SPECIES, incapable of genetic recombination with other similar units.

ECO-SPECIES, capable of genetic recombination with other similar units, but liable to reduced fitness or fertility from it. 
SPECIES (contimed)

ECOTYPE, capable of genetic recombination with other similar units within the Eco-species and not suffering from it.

Cryptic Species, such as are not distinguishable by external morphology. Darlington I940.

SUb-SPECIES, a sub-division of a species larger than a supposed Race.

SPERM, the Male gamete. $v$. Egg.

Spermatocyte, $v$. Mother Cell.

SPERMATOGENESIS, development of the sperm from its mother cell.

Spermatogonium, a cell giving rise to sperm mother cells either directly or by mitosis. La Valette St. George 1876.

SPINDLE, the axially differentiated crystalline (or "fibrous") part of the cytoplasm within which the centromeres of the chromosomes are orientated during metaphase and anaphase. Normally bipolar, exceptionally unipolar or multipolar.

Spindie Attachment, the position of the Centromere.

SPIRAL, a coil of the chromosome, chromatid or chromosome thread at mitosis or meiosis. Classified by Darlington 1935 as follows:-

External SPIral, coiling which is externally visible when the internal coiling is concealed. Includes Relic and Relational Spirals.

INTERNAL SPIRAL, a coil within a single chromatid between prophase and anaphase, or at meiosis jointly for two sister chromatids.

Major SPIRAL, the larger internal coil at meiosis.

Minor SPIRAL, the smaller internal coil at meiosis.

Molecular SPIral, the coiling within the chromosome thread which conditions internal and relational spirals.

Relational Spiral, coiling of two chromatids or chromosomes round one another.

ReIIC SPIRAL, the internal coiling which survives as external at telophase and prophase.

SPIRALization, the assumption of an internal (but not a relational) spiral within the chromatids in mitosis and jointly for two chromatids in meiosis. Darlington 1932.

SPORE, a single cell reproducing an organism but incapable of fertilization. In the higher plants it is a haploid cell produced by meiosis from a diploid and gives rise to a Gametophyte.

Sporocyte, Spore Mother Cell.

Sporophyte, an individual of the diploid generation in plants, normally producing the spores. 
SPORT, a dissinilar form of an individual, or part of an individual, arising directly or indirectly from gene, plastid, chromosome or nuclear (polyploid) Mutation. The indirect origins are due to Segregation of a recessive gene, cellular sorting-out of Plastids, and tissue sorting-out in Chimaeras. The name is usually confined to new and unforeseen forms.

SQUare, Latin SQuare, an experimental design involving two restraints. MEAN SQUARE, average of the squared deviation of observations from their mean. Found as the ratio of the Sum of Squares to the Number of Degrees of Freedom. Synonymous with Estimated Variance.

Method of LeAst Squares, a method of estimation depending on minimization of sums of squares, and widely used in Regression analyses.

Sum OF SQuares, the sum of squared deviations of observations from their mean.

Standard Deviation, $v$. Deviation.

Standard ERror, $v$. Deviation.

Statistic, the estimate of a Parameter arrived at from an observed sample. Bears the same relation to the sample as the parameter does to the population. $v$. Likelihood.

Consistent Statistic, one which tends to approach the parameter in value as the sample size increases.

Efficient Statistic, one which tends, as the sample size increases, to use all of the information available in the data.

InEFFicient Statistic, one which uses less of the information than an Efficient Statistic.

Sufficient Statistic, one which uses all the information available in the data, even as small samples. Not always available.

Stem Body (STEMm-KörPER), the part of a spindle between two groups of chromosomes separating at anaphase. Belar 1928.

Step Allelomorphism, the occurrence of a series of Multiple Allelomorphs with overlapping effects, which can supposedly be related to a linear order in the distribution of units of change within the gene. Deduced from the scute gene in Drosophila melanogaster. Serebrovsky 1930.

STERILITy, any failure (partial or complete) of an individual under given environmental conditions to produce effective gametic or viable zygotic progeny. May be due to environmental or genotypic defects or to segregation in a hybrid or polyploid. Genotypic defects in the gametes are due to segregation in a hybrid parent, and in zygotes to unbalance in the hybrid individual concerned. 


\section{STERILITy (continued)}

MALE or Female Sterility, failure of male and female organs, respectively, but not of the opposite sex.

Strictly, sterility should not include the failure of zygotes to mate effectively, merely owing to unlikeness (so-called CrossSTERIIITy) or failure of male gametes to achieve fertilization of an egg merely owing to likeness (Incompatibility).

STOck, an artificial Mating Group.

STRAIN, a natural or artificial Mating Group uniform in some particular.

Biological Strain, one detected by difference in parasitic adaptation. Sexual Strain (incorrect), in Heterothallic fungi, one differing from others in its incompatibility allelomorphs.

Structural Change, change in the genetic structure of one or more chromosomes. May be intra-radial or extra-radial with respect to arms; internal, fraternal or external with regard to chromosomes; eucentric or dyscentric with respect to the possession of a centromere or to the direction of a segment in relation to the centromere. Darlington I929, 1937.

Balanced (Reciprocal) Structural Change, Interchange and Inversion.

Unbalanced (or Non-Reciprocal) Structural Change, Deficiency, Deletion and Translocation. Reduplication is a condition arising by segregation from special types of interchange or by unequal crossingover, e.g. at the Bar locus in Drosophila melanogaster.

Secondary Structural Change, change in structure resulting from Illegitimate Crossing-over. Darlington 1932.

Structural Control, $v$. Genotypic Control.

Structural Hybrid, $v$. Hybrid.

STRUCTURE, the potentially permanent linear order of the particles, chromomeres, or genes in the chromosomes. $v$. Structural Change. Darlington 1929.

Sublethal Gene, $v$. Lethal Gene.

Subsexual Reproduction, $v$. Reproduction.

SuPER-Female, an individual with more $\mathrm{X}$ chromosomes than sets of autosomes in Drosoplita. Bridges I923.

Super-Gene, $v$. Gene.

SuPER-MALE, an individual with less $\mathrm{X}$ chromosomes than half the sets of autosomes in Drosoplita. Bridges I923.

SuPPRESSIVE, of a plasmagene which suppresses the cxpression of an alternative condition in a particular respect in a hybrid individual and its descendents. Darlington I944. 
Suppressor, a gene one of whose allclomorphs renders indetectable the difference determined by the allelomorphs of another gene. v. Epistasy.

SyNAPSIS (SyNDESIS), chromosome pairing at pachytene and zygotene. v. Asynapsis. Moore I 895 .

SyNDIPLOIDy, doubling, the fusion of sister nuclei to give a doubled chromosome number, particularly in the divisions immediately preceeding meiosis. Strasburger 1907.

SYNDROME, a group of symptoms characteristic of the same infection or abnormal genetic condition, but not necessarily all appearing together.

SYNGAMY, fusion of gametes.

SYNOECY, the condition in which male and female gametes are produced by the same haploid or diploid individual. Includes Monoecy and Hermaphroditism. Correns I928.

$t$, the ratio of an observed deviation to its estimated standard deviation. TELEGONY, an effect of a previous sire on later progeny of the same dam by another sire. Stockbreeder's myth.

Telocentric, of a chromosome or a chromatid having a terminal centromere. A condition arising by Misdivision or by X-Ray Breakage. Darlington 1939.

Telosynapsis, $\nu$. Parasynapsis.

Telophase, the stage of mitosis and meiosis during which the chromosomes re-form a nucleus.

Terminal Affinity, the property by which chromosomes are held together end to end from Diplotene till first Metaphase of Meiosis (by a Terminal Chiasma) or are brought together in this way at Metaphase. Darlington I932.

Terminal Chimasma, v. Chiasma.

Terminalization, expansion of the association of the two pairs of chromatids on one side of a chiasma at the expense of that on the other side. So called because the resulting "movement" of the chiasma is towards the ends of the chromosomes. Darlington I929.

Test Cross, a cross of a double or multiple heterozygote to the corresponding double or multiple recessive. Used to estimate linkage relationships or behaviour. Bridges 1934 .

Tetrad, quartet of cells formed by meiosis in a mother cell.

Tetraploid, $v$. Polyploid. Nemec igio.

Tetrasomic, $v$. Polysomic. 
TIME Limit, limitation of time available for zygotene pairing which causes Localization. Darlington I935.

Torsion Pairing, $v$. Pairing.

Trabant, Satellite.

Trait, Character.

Transgressive Segregation, the appearance in a segregating generation $\left(\mathrm{F}_{2}\right.$, backcross, etc.) of an individual or individuals falling outside the limits of variation set by the parents and $F_{1}$ of the cross in one or more characters. $C f$. Heterosis.

Translocation, change in position of a segment of a chromosome to another part of the same or of a different chromosome, especially non-reciprocally in which case the segment is always interstitial. v. Structural Change. Bridges I9I7.

Reciprocal (or Mutual) Translocation, Interchange.

Trigenic, v. Monogenic.

TrihyBrid, Trigenic Hybrid.

Triplex, $v$. Nulliplex.

Triploid, $v$. Diploid, Polyploid.

Triplo-Polyploid, a relatively triploid derivative of an allopolyploid. Janaki Ammal I94I.

Trisomic, $v$. Polysomic. Blakeslee ig2r.

SECONDARY TRISOMIC, a trisomic individual in which the extra chromosome has two identical ends (and is probably an Isochromosome). Belling 1927.

Tertiary TrIsomic, a trisomic individual in which the extra chromosome is the product of interchange between two standard chromosomes. Belling 1927.

Trivalent, $v$. Univalent.

TwINs, two embryos developed simultaneously within one uterus in mammals, within one vitelline membrane in other vertebrates or within one testa in phanerogams. Similarly Triplets, Quadruplets, etc., $v$. also Dizygotic, Monozygotic, Polyembryony.

UNFXABLE, Ever-sporting from seed.

UnILATERAL INHERITANCE, $v$. Inheritance.

UniseXuality, $v$. Dioecy.

UNIT Character, one whose difference from an alternative is transmitted as a unit in heredity. $C f$. Character, Factor, Gene. 
UNIVALENT, a body at the first meiotic division corresponding with a single chromosome in the mitotic complement, especially when umpaircd. Bivalent, Trivalent, Quadrivalent, Quinquevalent, Sexivalent, Septivalent, Octavalent, etc., are associations of chromosomes held together between diplotene and metaphase of the first division of meiosis usually by chiasmata. Trivalents and higher associations are collectively termed MuLtivaLents.

Unstable Gene, $v$. Gene.

VARIABILITY, the capacity of an individual or group of individuals to produce gametes having genotypic variation. Mather I94I; a priori the distinction can be made (Fisher 1930, Mather 1943) between:-

Free Variability, that which is expressed phenotypically, and

Potential Variability, that which is not expressed in the phenotypes, owing to dominance, etc.

FLOW OF VARIABILITY, the movement of variability in a population from Free to Potential and vice versa.

VARIANCE, Mean Square deviation of a variate from its mean. Estimated as the Mean Square. The square of the Standard Deviation. Though strictly a parameter the name is commonly applied to a statistic (i.e. as synonymous with mean square).

ANALYSIS OF VARIANCE, a technique for the isolation of particular components of variation for assessment by comparison with Error Variance. Especially applicable to the analysis of Factorial Experiments.

Variance Ratio (V.R.), the ratio of two estimated variances. Twice the natural antilog of $z$. Sometimes denoted by $F$.

VARIATE, a variable quantity whose mcasurements or frequencies form all or part of the data for analysis.

VARIATION, I. The occurrence of differences in the permanent (i.e. selfpropagating) structures of cells (genotypic and heritable). Darlington I929.

2. The occurrence of differences between individuals duc to differences between the permanent structures of their cells (phenotypic and heritable) or to differences in external conditions (phenotypic and non-heritable).

Discontinuous Variation, where gradations of difference are perceptible in the phenotype.

Continuous Variation, where gradations of difference are imperceptible in the phenotype. 


\section{VARIATION (continitied)}

Continuous variation is not to be taken to imply continuous differences in the genotype, since statistical analysis may reveal discontinuities in the determinants. $v$. Polygenes, Quantitative Inheritance.

VARIEGATION, the deprivation of a part of the normally green cells of a plant of their chlorophyll through the action of a marginal genotype, somatic mutation or infection. $v$. Plastogene.

VARIETY, a sub-division of a species owing its uniformity either to Genetic Isolation in nature, or to artificial propagation in cultivation (where a variety is often a Clone), $v$. Breed.

Vegetative Propagation (Reproduction), reproduction of the higher plants otherwise than by seed, either naturally (runners, bulbs, viviparous bulbils, etc.) or artificially (cuttings, grafts, etc.).

VIABILITY, Measure of, the number of individuals surviving in one class relative to another, standard, class.

VICTNISM, unexpected outcrossing. Balls I922.

VIRUs, an ultra-microscopic particle whose reproduction in the cell and transmission by natural infection gives characteristic reactions of cells and individuals. Cf. Plasmagene.

VIVIPARY, the release of offspring by the mother free from her membranes (as in egg or seed). In animals due to delayed egg-laying, in plants due to precocious germination and (usually) apomixis.

W Chromosome, sometimes used for the $\mathrm{X}$ chromosome, where the female is the Heterogametic Sex.

WEIGHT, a differential value assigned to an estimate of a quantity, relative to other estimates of the same quantity, for purposes of combining the estimates. Usually its Invariance or Amount of Information is taken as the weight for each estimate of a series.

WILD TYPE, of an organism or gene of the type predominating in the wild population.

$x$, Basic Number.

$\mathrm{X}$ Снromosome, with diploid sex differentiation, the sex chromosome in regard to which one sex is homozygous (the Homogametic Sex). With haploid sex differentiation (Bryophyta, etc.), the sex chromosome of the Female.

AtTACHed X Chromosomes, where the two X's of a female are fused at their terminal centromeres and so act as one mechanical unit in inheritance (in Drosophila melanogaster). L. V. Morgan 1922. 
XENI $\Lambda$, the effect of more distantly related, as contrasted with more closely rclated, pollen on the maternal tissue of a fruit. Originally applied (Focke I88I) to the effect on the endosperm, now known to be genetic and direct. Metaxenia has been used for true xenia in contrast to the xenia of Focke.

X-RAy Breakage, the following terms are used in describing the results of X-ray breakagc. They have also been applicd to cases of spontancous breakage:-

Chromosome Break, one which occurs in the chromosome before its division into the two chromatids observable at the following mitosis, when the break is recorded. As opposed to-

Chromatid Break, one which follows this division, and therefore breaks only one of the chromatids at any one point. Mather and Stone 1933 .

Isochromatid BrEAK, chromosome break in a cell otherwise apparently characterized by chromatid breaks. Lea and Catcheside 1942.

REUNION, the joining of broken ends of chromosomes or chromatids. Classified by Darlington and Upcott I94I into

- Restitution, where the pre-breakage structure is restored.

Chromosome Reunion, of the ends of chromosomes following chromosome breakage.

Chromatid Reunion, of the ends of chromatids following either chromosome or chromatid breakage.

Sister Reunion, union of the identical broken ends of sister chromatids following chromosome breakage.

Minute (Fragment), a fragment less than the diameter of the chromatid and hence spherical. May be double, or, following sister reunion, single. Muller I940.

Empirical Coefficient of Reunion (E.C.R.), the proportion of the recognizable breaks in the chromosomes or chromatids, of a sample of nuclei which have undergone recognizable reunion.

Y Cнromosome, with diploid sex differentiation, the sex chromosome that is present and pairs with the $\mathrm{X}$ chromosome in the sex heterozygote (Heterogametic Sex). With haploid sex differentiation (Bryophyta, etc.) the sex chromosome of the Male.

$z$, the natural logarithm of the ratio of the two estimated Standard Deviations. $v$. Variance Ratio.

$\mathrm{Z}$ Chromosome, sometimes used for the $\mathrm{Y}$ chromosome where the female is the Heterogametic Sex. 
ZOOSPORE, motile spore.

Zygote, cell formed by the fusion of Gametes, and the individual derived from it. v. Homozygote, Heterozygote.

ZygOTENE, the stage in which the chromosomes, as extended threads, pair in the prophase of meiosis. 


\section{SYMBOLS AND SYMBOLISM}

\section{Cytological}

\section{Genomes (Triticum, etc.)}

The chromosome sets of different diploid species are denoted by capital letters. An allotetraploid species can thus be tepresented as AA BB.

\section{INTERCHANGES}

Chromosome types are usually denoted by the similarities of their pairing segments. In Datura ( $v$. Prime Type) numbers are used, as I.2 or 2.3 (Blakeslee, 1932. Proc. 6th Int. Cong. Gentics I: I04-I20). In Oenothera numbers or capital letters are used, as $\mathrm{AB}-\mathrm{BC}-\mathrm{CD}-\mathrm{DA}$ for a ring of four. Interstitial segments may be shown by small letters representing complexes, as $\mathrm{AgB}-\mathrm{BvC}-\mathrm{CgD}-\mathrm{DvF}$, etc. (Darlington, I93 I. J. Gentet., 24: 405-74).

\section{CHromosomes}

In maize and Drosophila, chromosomes are known by Roman numerals. In purely cytological descriptions chromosomes are shown either alphabetically as capitals, e.g. A, B, C, D, in Crepis with index letters for the species, or by initials for types as L, M and S, for long, medium, and short types in Hyacinthus.

\section{Gentical}

The convention of denoting the dominant allelomorph by a capital letter and the recessive allelomorph by the corresponding small letter was introduced by Mendel; but he did not attach specific letter pairs to each of his unit differences, or factors, so that his symbolism was of a general nature. This refinement, of reserving each letter pair for the representation of a given gene, or factor, was, however, brought into use soon after the rediscovery of Mendel's paper, and the system of symbolism thereby developed has been very widely used. It is the form adopted by Bateson in all his works, and is well illustrated in his Mendel's Principles of Heredity.

The letter used to denote a given gene was generally chosen for its relation to the effect of the dominant allelomorph. Thus $C$ was used for the dominant allelomorph giving colour as opposed to albinism $(c)$ in mice, and $B$ for the dominant producing bluer colour, as opposed to red $(b)$ in the flowers of Primula sinensis. Where a number of genes were described, if all the more appropriate letters had already been claimed, letters which bore no relation to the character expression of the gene were used, rather than a two-letter symbol, e.g. $Z-z$ for the normal-claw leaf gene in Primula sinensis.

In Drosophila genetics a somewhat different system has been developed. The wild-type appearance of the fly is taken as the standard, and all genes are described and symbolized by references to the departures which they cause from this 
standard type. Since most of these variants are due to recessive genes, the emphasis is thus generally on the effect of the recessive allelomorph. For example, a gene which causes a yellowing of the body colour is called yellow and designated by $\gamma$. Where, however, the effect of the mutant gene is detectable in the heterozygote,

STRUCTURAL CHANGES ARISING FROM IRRADIATION, CHEMICAL TREATMENT OR SPONTANEOUSLY (FROM DARLINGTON AND LA COUR, 1945. J. Genet., 46, 180-267, AND DARLINGTON AND KOLLER, 1947. Heredity, 1, 187-222)

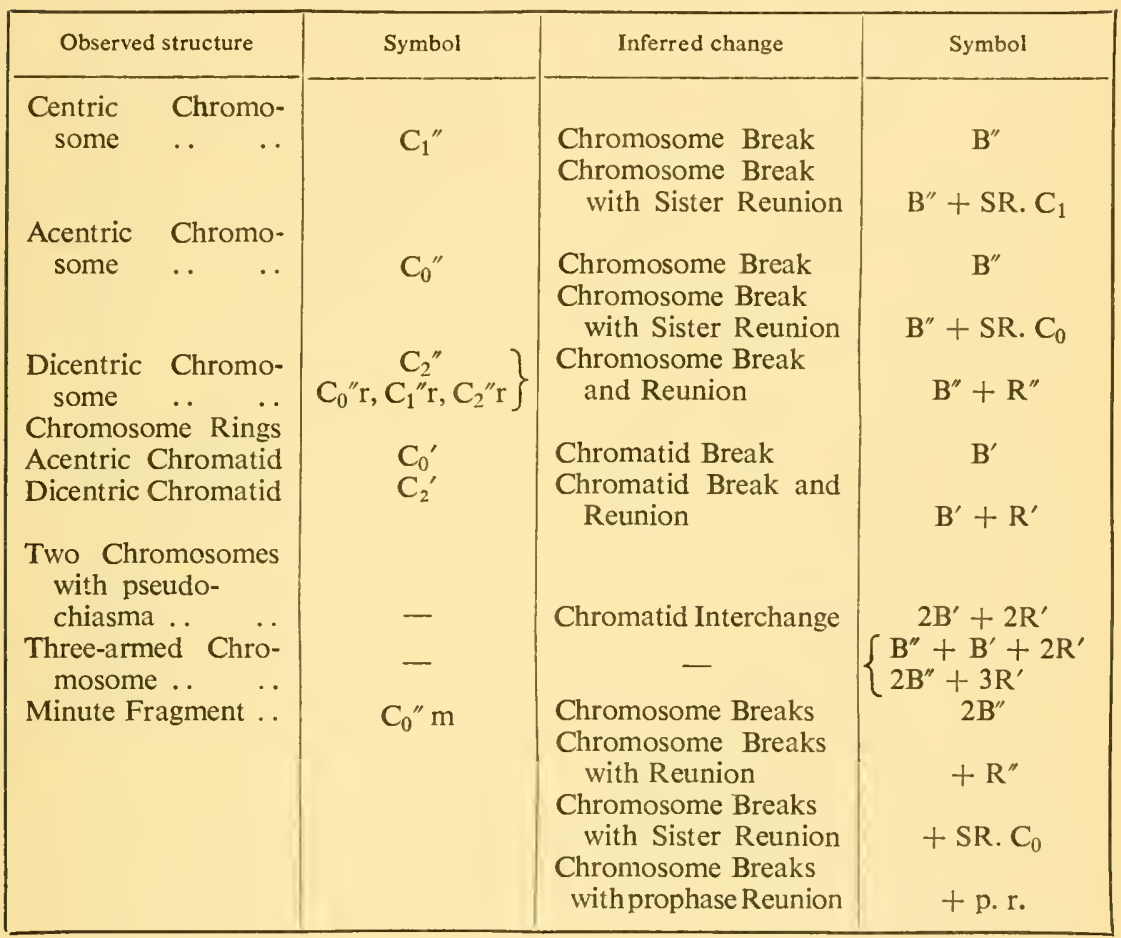

the gene is described as a dominant (though it may not be dominant in the mendelian sense of giving a heterozygote indistinguishable from the mutant homozygote). It is named from the appearance of the heterozygote and is given a capital letter suggesting its name. Thus $H$ indicates the gene Hairless which in the heterozygous condition removes certain hairs from the fly, though when homozygous it has a lethal effect. Two and even three letters are of necessity sometimes used as symbols. Thus $c=$ curved (wings), $c a=$ claret (eye colour) and $c a r=$ carnation (eye colour).

In contradistinction to the earlier system the wild-type allelomorph of a mutant gene is not denoted by the same letter, but in different case, as that for the mutant. 
Instead + is used as a gencral symbol for the wild type. Thus $y c v a t /+$ implies a fly heterozygous for all three genes, yellow (body colour), cross-veinless (wings) and cut (wings), the single stroke indicating linkage of the genes. Where it is necessary to specify the wild-type allelomorph of a particular gene the symbol for that gene is combined with + , as $+^{\nu}$ or $v^{+}$in the case of the wild-type allelomorph of vermilion (cye colour). This enables two different genes to be designated by one letter in different cases. For example $b=$ black (body coloura recessive variant), while $B=\mathrm{Bar}$ (eye shape-a dominant variant). The Drosophila system is also used for maize and some other species. Its elastic and comprehensive nature is well illustrated in the introduction to Drosophila Information Service, 9.

In both systems multiple allclomorphs are indicated by superscripts to the basic symbol. Some members of the set may have becn named before their multiple allelomorphism was discovered. This superscript then often reflects the expression of the allelomorph involved, e.g. in Drosophila $w$ is white (eye colour), $w^{e}$ is eosin (eye colour), $w^{a}$ is apricot (eye colour), $w^{c o}$ is coral (cye colour) and so on. In other examples a mere stroke is used as in $A^{\prime}$ for Alexandra-eye in Primula sinensis, or a symbol indicating the date of discovery or the name of the discoverer as $y^{31 d}$ (the allelomorph of yellow discovered in April 193I) or $B^{R}$ (the Bar allelomorph discovered by Rapoport) in Drosophila melanogaster.

With the Drosophila system the key symbol retains the case appropriate to the first allclomorph for which is was used, but the superscript can be used to indicate whether the allelomorph in question is dominant or recessive to wild type. In the other system the use of the letter pairs to distinguish the dominant and recessive allelomorphs breaks down with a multiple allelomorph scries, especially where a number of the allelomorphs are incompletely dominant in various combinations. It is then customary to denote those allelomorphs which are dominant, or partially dominant, to the dominant member of the pair first described, by the capital letter with a suitable superscript, the rest taking the small letter with appropriate superscripts.

One special case of multiple allelomorph symbolism which departs from the above practice is that of the series controlling incompatibility in various plants. $S-s$ is generally used to denote the incompatibility gene where it is associated with distyly (when only two allelomorphs are involved); but with homomorphic incompatibility as encountered in Nicotiana, Veronica, etc., the symbols $S_{1}, S_{2}$, $S_{3}$. . ., etc (with subscripts) are generally used. A compatibility allelomorph is then $S_{f}$.

Non-allelomorphic genes of similar effect are generally given names indicating their similarity and symbolized accordingly. A particular example of this is Lawrence's use of $L$ and $E$ for two complementary dominant lethal genes. Such genes may alternatively be given the same name with a distinguishing number which is also used in the symbol, either as an equal part or as a subscript. This is especially the case for lethals and Minutes in Drosophila.

Genes or chromosomes of related species, known or suspected of being homologous, are generally given the same symbol with, where necessary, a distinguishing 
subscript or superscript. The same practice may be adopted for homologous chromosomes from different strains of a species.

In Drosophila genetics the special symbols $I n$ and $\mathrm{Tr}$ arc used to indicate inversions and interchanges. The chromosome or chromosomes involved and the special name of the structural change in question are appended, e.g. $\operatorname{In}(1) d l-49$, is the delta-49 inversion in the X (or Ist) chromosome. Special conposite chromosomes may also receive special names and symbols as in the case of the $\theta$ compound X chromosome.

The German investigators of Antirrhinum and other organisms have adopted a system whereby each gene is given a three-letter symbol. In the case of a multiple allelomorph the superscript also has three letters. Thus the peloric gene is pel and the tincta allelomorph in the pallida series is pal tin. While achieving uniformity of symbolism this system is apt to defeat its own ends since it is easier to write, for example, tincta than palin.

For Lathyrus, Punnett proposed a symbolism based on linkage relations. Each chromosome was given a letter, each gene taking the letter of its chromosome and a subscript number indicating its place in the order of genes known to be borne on that chromosome. The difficulties arising from insufficient knowledge of linkage relations and the placing of new genes inside an established linkage group require no stressing. The system has achieved little popularity.

In human genetics the symbolism is so varied as to verge on the chaotic. Many character differences are known to be hereditary without, however, the exact mode of inheritance being clear. No symbolism is then possible. Even where the mode of inheritance is clear it is often portrayed in the form of pedigree diagrams with empty, hatched or filled circles or squares to denote the phenotypes and assumed genotypes. Sometimes symbols according with one or other of the regular systems are allotted; but often, even where symbols are used, the usage bears no relation to any wider scheme. Thus with the blood groups the three main allelomorphs are $\mathrm{A}, \mathrm{B}$ and $\mathrm{O}$, and when two $\mathrm{A}$ type allelomorphs are distinguished it is by subscripts as $A_{1}$ and $A_{2}$, not by superscripts. With the Rhesus blood group allelomorphs the haphazard symbolisms which had grown up independently in Great Britain and the United States are now being superseded by a more rational system due to Fisher, which takes into account the antigens and antisera concerned. Increasing genetic research in man must in time demand a similar rationalization of symbolism for other genes whose differences are commonly observed.

Various moves have been made towards a codification of genetical symbolism, the question having been discussed especially by de Haan (1932, Genetica, I5: I-2I, 2I9-223). No universal system has, however, been developed and adopted as such; though, as will have been seen, the two most widely used systems have very much in common.

\section{Statistical}

The various letters, Greek and Latin, are frequently used to suit the convenience of the moment in statistical accounts. Certain conventions are nevertheless widely 
recognized in addition to those which are standard throughout mathematics, such as the use of $\pi$ for the ratio of the circumference of circle to its diameter, and $e$ for the natural base of logarithms. A most useful general rulc has been proposed by Fisher, viz. that Greek letters should be used to denote parameters and the corresponding Latin letters to denote the corresponding statistics.

In addition the following special usages are widely, though by no means universally, adopted:-

$\Sigma$ or S to indicate summation, with superscripts and subscripts to show the range of summation where necessary.

$\mathrm{n}$ for the number of individuals or observations.

$p$ for a probability or proportion, with $q=I-p$.

$x$ and $y$ for variates, with $x$ as the independent and $y$ as the dependent variate in regression analysis.

$\overline{\mathrm{x}}$ for the mean of $\mathrm{x}$.

$\sigma$ or $\mathrm{s}$ for the standard deviation or standard error. N.B. $\mathrm{s}$ is preferable for estimated and $\sigma$ for theoretical standard deviations on Fisher's scheme. A subscript is used to denote the nature of the standard deviation, thus $s_{\mathrm{x}}$ is the standard deviation of $\mathrm{x}, \mathrm{s}_{\overline{\mathrm{x}}}$ that of $\overline{\mathrm{x}}$, etc.

$\mathrm{V}$ for the variance, with the appropriate subscript, as with $\mathrm{s}$ and $\sigma$.

$I=\left(\frac{I}{V}\right)$ for the amount of information yielded by a body of data.

$\mathrm{i}\left(=\frac{\mathrm{I}}{\mathrm{n}}\right)$ for the average information yielded by one observation or individual.

$\rho$ for the theoretical correlation coefficient.

$r$ for the estimate of $\rho$.

b for a regression coefficient.

t for the ratio of an observed deviation to the corresponding estimated standard deviation.

$\chi^{2}$ for the ratio of an observed sum of squares to the corresponding variance fixed by hypothesis.

$z$ for half the natural logarithm of the ratio of two estimated variances. V.R. or $F$ for the ratio of two estimated variances.

Variations of these conventions are used in agronomical and other statistical work as well as, or sometimes more especially than, in genetico-statistical analyses. 


\section{GENETICAL BOOKS PUBLISHED IN THE ENGLISH LANGUAGE}

What wants there to such a towardly and pregnant soil but wise and faithful labourers to make a knowing people:

Milton: Areopagitica, 1644.

AGAR, W. E. 1920. Cytology with Special Reference to the Metazoan Nucleus. London. ALtenburg, E. 1946. Gentetics. New York and London.

вавсOCK, E. B., and CLAUSEN, R. E. 1918, 1927. Genetics in Relation to Agriculture. New York.

BABCOCK, E. B., and Collins, J. L. I9I8. Genetics Laboratory Manual. New York. BaILeY, L. H., and Gilbert, A. W. I917. Plant Breeding. New York. BATESON, W. I 894. Materials for the Study of Variation. London. Bateson, w. 1908. The Methods and Scope of Genetics. Cambridge. Bateson, w. 1909, 1930. Mendel's Principles of Heredity. Cambridge. Bateson, w. 1913. Problems of Genetics. London. BAUR, E., FISCHER, F., and LENZ, F. I93 I. Human Heredity. London. BOWER, F. O., KERR, J. G., and AGAR, W. E. 19I9. Sex and Heredity. London. BurLingame, L. L. I940. Heredity and Social Problems. New York. CARPENTER, G. D. H., and FORD, E. B. 1933. Mimicry. London. CASTLE, W. E. I9I. Heredity in Relation to Evolution and Animal Breeding. New York and London.

Castle, W. E. 1916, 1930. Genetics and Eugenics. Cambridge, Mass. CASTLE, W. E. I930. Genetics of Domestic Rabbits. Cambridge, Mass. CAStle, W. E. I94C. Mammalian Genetics. Cambridge, Mass. CASTLE, W. E. et alii. 1912. Heredity and Genetics. Chicago. Chandrasekharan, s. N., and parthasarathy, s. v. I948. Cytogenetics and Plant Breeding. Madras. COCKAyne, E. A. 1933. Inherited Abnormalities of the Skin and its Appendages. Oxford. Colin, E. C. I94I. Elements of Genetics. Philadelphia. CONGER, G. P. 1929. New Views of Evolution. New York. COUlTer, J. M. I9I4. Evolution of Sex in Plants. Chicago. COULTER, J. M. I9I4. Fundamentals of Plant Breeding. New York and Chicago. COUlter, M. C. I923. Outline of Genetics. Chicago. COWDRY, E. v. (Editor). 1924. General Cytology. Chicago. CRANE, M. B., and LAWrence, w. J. C. I934, I938, 1947. The Genetics of Garden Plants. London. CREW, F. A. E. 1925. Animal Gentetics. Edinburgh and London. CREW, F. A. E. 1927. The Genetics of Sexuality in Animals. Cambridge. CREW, F. A. E. 1927. Organic Inheritance in Man. London. 
CREW, F. A. E. 1928. Heredity. London.

CREW, F. A. E. 1933. Sex Deternination. London.

CREW, F. A. E. I947. Genetics in Relation to Clinical Medicine. Edinburgh.

CREW, F. A. E., and LAMY, R. 1935. Genetics of the Budgerigar. London,

CUTLER, D. W. 1925. Evolution, Heredity and Variation. London.

DAHLBERG, G. I942. Race, Reason and Rubbish. London.

DARbishire, A. D. I9I I. Breeding and the Mendelian Discovery. London.

Darlington, C. D. 1932. Chromosomes and Plant Breeding. London.

Darlington, C. D. 1932, 1937. Recent Advances in Cytology. London.

Darlington, C. D. 1939, 1947. The Evolution of Genetic Systems. Cambridge.

DARLington, C. D., and LA COUR, I. F. 1942, 1947. The Handling of Chromosomes.

London.

Darlington, C. D., and JANAKi Ammal, E. K. I945. Chromosome Atlas of Cultivated Plants. London.

DARWIN, C. I859. The Origin of Species. London.

DARWIn, C. I 868. Antimals and Plants under Domestication. London.

DARWIN, C. I871. Descent of Man and Selection in Relation to Sex. London.

DARWIN, C. I876. The Effects of Cross- and Self-Fertilization. London.

DARWIN, C. I877. The Different Forms of Flowers. London.

DAVENPORT, C. B. 1910. Eugenics. New York.

DAVenport, C. B. I9II. Heredity in Relation to Eugenics. New York.

DAVenport, E. 1907. Principles of Breeding. New York.

DEMEREC, M. et alii. I941. Cytology, Gentetics and Evolution. Philadelphia.

DOBZHANSKY, TH. 1938, I94I. Genetics and the Origin of Species. New York. DonCaster, L. I9IO. Heredity in the Light of Recent Research. Cambridge.

DONCASTER, L. I914. The Determination of Sex. Cambridge.

DONCASter, I. 1920. An Introduction to the Study of Cytology. Cambridge.

DONkin, H. B. I9I I. The Inheritance of Mental Characters. London.

DOWNING, E. R. I928. Elementary Eugenics. Chicago.

DUNN, L. C., and DoBzhansKy, TH. 1946. Heredity, Race and Society. Pelican Books.

New York.

EAST, E. M., and JONES, D. F. I919. Inbreeding and Outbreeding. Philadelphia.

FASTEN, N. 1935. Principles of Genetics and Eugenics. New York.

FISIIER, R. A. I925-1947. Statistical Methods for Research Workers. Edinburgh.

FISHer, R. A. 1930. The Genetical Theory of Natural Selection. Oxford.

FISHER, R. A. I935-I947. The Design of Experiments. Edinburgh.

FISHER, R. A., and YATES, F. 1938, I943, 1946. Statistical Tables. Edinburgh. FORD, E. B. 1934, I940, 1945. Mendelism and Evolution. London.

FORD, E. B. I938. The Study of Heredity. London.

FORD, E. B. I942. Gentics for Medical Students. London.

FRASER-ROBERTS, J. A. 1940. An Introduction to Medical Gentics. Oxford.

Gager, C. S. 1920. Heredity and Evolution in Plants. Philadelphia.

GALTON, F. I 889. Natural Inheritance. London.

Galton, F. I 892. Hereditary Genius. London.

GALTON, F. 1909. Essays in Eugenics. London. 
GARROD, A. E. I909, I923. Inborn Errors of Metabolism. London. GATES, R. R. I9IS. The Mutation Factor in Evolution. London.

GATES, R. R. I923. Heredity and Eugenics. London.

GATES, R. R. I930. Heredity in Man. London.

GATES, R. R. I946. Human Genetics. New York.

GLASS, B. I943. Genes and the Man. New York.

Goldschmidt, R. 1938. Physiological Gentetics. New York.

goldschmidt, R. I940. The Material Basis of Evolution. New York and London. goldsmith, w. M. 1922. Laws of Life, Principles of Evolution, Heredity and Genetics.

Boston.

Gríneberg, H. 1943. The Gentics of the Mouse. Cambridge.

Grüneberg, H. 1947. Animal Gentics and Medicine. London.

GUN, W. T. J. 1928. Studies in Hereditary Ability. London.

GUYER, M. F. I927. Being Well-born, an Introduction to Heredity and Eugenics. London. Hagedoorn, A. L. I944. Animal Breeding. London.

HAgedoorn, A. L., and HAgedoorn, A. C. I921. The Relative Value of the Processes

Causing Evolution. Hague.

HALDANE, J. B. S. 1932. The Causes of Evolution. London.

HALDANE, J. B. S. I938. Heredity and Politics. London.

haldane, J. B. S. I94I. New Paths in Genetics. London.

HARLAND, s. C. 1939. The Genetics of Cotton. London.

HART, D. B. I9IO. Phases of Evolution and Heredity. London.

haYeS, H. K., and GARBer, R. J. I92I, I927. Breeding Crop Plants. New York.

HAYES, H. K., and IMmer, F. R. I942. Methods of Plant Breeding. New York.

HERBERT, S. I9I0, I919. The First Principles of Heredity. London.

herbert, S. I9I3, I920. The First Principles of Evolution. London.

Hogben, L. 1931. Genetic Principles in Medicine and Social Science. London.

HogBeN, L. I933. Nature and Nurture. London.

Hogben, L. 1946. An Introdiction to Mathematical Genetics. New York.

Holmes, s. J. I92I. Trend of the Race. New York and London.

holmes, s. J. I923. Studies in Evolution and Genetics. New York and London.

Holmes, s. J. 1926, I931. Life and Evolution. New York and London.

holmes, s. J. I936. Human Genetics and its Social Import. New York.

hunter, H., and LeAke, H. M. I933. Recent Advances in Agricultural Plant Breeding.

London.

HURST, C. C. 1925. Experiments in Genetics. Cambridge.

HURST, C. C. 1932. The Mechanism of Creative Evolution. Cambridge.

HURST, C. C. I935. Heredity and the Ascent of Man. Cambridge.

hutchinson, J. B., silow, R. A., and stephens, s. G. I947. The Evolution of

Gossypium. Oxford.

HuXley, J. S. (Editor). I940. The New Systematics. Oxford. HUXLEY, J. S. I942. Evolution, the Modern Synthesis. London.

ILTIS, H. I932. Life of Mendel. London.

JEnnings, H. S. I920. Life and Death, Heredity and Evolution in Unicellular Organismis.

London. 
Jennings, H. S. 1935. Genetic Variations in Relation to Evolution. Princeton.

JENnings, II. S. I935. Genetics. London.

JONes, D. F. 1925. Genetics in Plant and Animal Improvement. New York.

JONES, D. F. 1928. Selective Fertilization. Chicago.

Julı, M. A. 1932, 1940. Poultry Breeding. New York.

kalmus, H. 1948. Genetics. Pelican Books.

KELlOGg, v. I924. Evolution. New York and London.

KERR, J. G. I926. Evolution. London.

KNight, M. M., Peters, I. L., and BLANChard, P. I921. Taboo and Genetics. New

York.

LAWRenCE, W. J. C. I937, I945. Practical Plant Breeding. London.

LEA, D. E. I946. Actions of Radiations on Living Cells. Cambridge.

Lidbetter, E. J. 1933. Heredity and the Social Problem Group. London.

Lindsey, A. W. I931. The Problems of Evolution. New York.

LINDSEY, A. W. 1932. A Textbook of Gentics. New York.

LOCK, R. E. I906, I9II. Recent Progress in the Study of Variation, Heredity and Evolution. London.

LULL, R. S. 1929. Organic Evolution. New York.

LuSh, J. L. 1938, 1943. Animal Breeding Plans. Ames, Iona.

macfarlane, J. M. I9I8. Causes and Course of Organic Evolution.

Malthus, T. R. I 803. An Essay on the Principles of Population. London.

manson, J. S. 1928. Observations on Human Heredity. London.

MATHER, K. 1938. The Measurement of Linkage in Heredity. London.

MATHER, K. 1943, 1946. Statistical Analysis in Biology. London and New York.

MATHER, K. I949. Biometrical Genetics. London.

matsuURA, H. 1929, 1933. A Bibliographical Monograph on Plant Genetics. Tokyo.

MAYR, E. I942. Systematics and the Origin of Species. New York.

MOHR, O. L. 1943. Heredity and Disease. New York.

MORGAN, T. H. I903. Evolution and Adaptation. New York.

MORgan, T. H. I9I4. Heredity and Sex. New York.

morgan, T. H. I9I6. A Critique of the Theory of Evolution. Princeton.

MORGan, T. H. I919. The Physical Basis of Heredity. Philadelphia and London.

Morgan, T. H. I925. Evolution and Genetics. Princeton.

Morgan, T. H. 1926, I928. The Theory of the Gene. New Haven.

Morgan, T. H. 1932. The Scientific Basis of Evolution. New York.

Morgan, T. H. I934. Embryology and Genetics. New York.

MORGAN, T. H., STURTEVANT, A. H., MULLER, H. J., and BRIDGES, C. B. I9I5, 1923.

The Mechanism of Mendelian Heredity. New York.

MUller, H. J., Little, C. C., and SNyDer, L. H. I947. Genetics, Medicine and Man. New York.

needham, J. E., and Green, D. E. 1937. Perspectives in Biochemistry. Cambridge. NEwman, H. H. 1917. The Biology of Twins. Chicago.

NEWMAN, H. H. I92 I, 1932. Readings in Evolution, Genetics and Eugenics. Chicago. NEWMan, H. H. 1923. The Physiology of Twinning. Chicago.

NEWMan, H. H. 1926. Gist of Evolution. New York. 
NEWMAN, H. H. I932. Evolution Yesterday and To-day. Baltimore.

Newman, H. H. I940. Multiple Human Births. New York. Republished in 1942 as

Twins and Super-Twins. London.

Newman, H. H., FREeman, F. N., and Holzinger, K. J. I937. Twins: A Study of

Heredity and Environment. Chicago.

NICHOLS, J. E. I944. Livestock Improvement. Edinburgh.

ORMEROD, J. A. I908. On Heredity in Relation to Disease. London.

osborn, F. I940. Preface to Eugenics. New York and London.

PEARL, R. I9I5. Modes of Research in Genetics. New York.

PEARL, R. I922. The Biology of Death. Philadelphia.

PEARL, R. I923. Introduction to Medical Biometry and Statistics. Philadelphia.

PEARL, R. I925. Biology of Population Growth. New York.

PEARL, R. 1939. Natural History of Population. London.

PINCHER, C. 1946. The Breeding of Farm Animals. Harmondsworth. Penguin.

POPENOE, P. I929. Child's Heredity. Baltimore.

pOPEnOE, P. 1930. Practical Applications of Heredity. Baltimore.

popenoe, P., and Johnson, R. H. 1933. Applied Eugenics. New York.

PUNNETT, R. C. I905, I927. Mendelism. London.

PUNNETT, R. C. I9I5. Mimicry in Butterflies. Cambridge.

PUNNETT, R. C. 1923. Heredity in Poultry. London.

REID, G. A. I90I. Alcoholism, a Study in Heredity. London.

REID, G. A. I905. The Principles of Heredity. London.

ReID, G. A. I9IO, I9II. The Laws of Heredity. London.

RIDE, L. 1938. Genetics and the Clinician. Bristol.

ROBerts, H. F. 1929. Plant Hybridization Before Mendel. Princeton.

ROBSON, G. C. I928. The Species Problem. Edinburgh and London.

RUSSELL, E. S. I930. The Interpretation of Development and Heredity. Oxford.

SANSOME, F. W., and PHILP, J. 1932, I939. Recent Advances in Plant Genetics. London. SCHEINFELD, A. I939. You and Heredity. London.

SCHRADER, F. I944. Mitosis. New York.

SCHRÖDINGER, E. I944. What is Life? Cambridge.

scotT, w. B. I9I7. Theory of Evolution. New York.

SHARP, L. W. I92I, I926, I934. An Introduction to Cytology. New York.

SHULl, A. F. 1936. Evolution. New York.

SHULl, A. F. I938. Heredity. New York and London.

Simpson, G. G. I944. Tempo and Mode in Evolution. New York.

SINNOTT, E. W., and DunN, L. C. I925, I932, I939. Principles of Genetics. New York. SNYDER, L. H. I929. Blood Grouping in Relation to Clinical and Legal Medicine.

Baltimore.

SNYDER, L. H. I935. Principles of Heredity. London.

SPENCER, H. I 887. Factors of Organic Evolution.

sturtevant, A. H., and Beadle, G. W. I939. An Introduction to Genetics. Philadelphia.

THOMSON, J. A. 1908, 1926. Heredity. London.

DE VRIES, H. I905. Species and Varieties, their Origin and Mutation. Chicago.

DE VRIES, H. I907. Plant Breeding. Chicago. 


\section{APPENDIX 3}

Waddington, C. 11. 1939. An Introduction to Modern Genetics. London. Waddington, C. H. I940. Organizers and Genes. Cambridge.

WALKER, C. E. I9IO. Hereditary Characters and their Mode of Transmission. London. WALKER, C. E. I936. Evolution and Heredity. London.

Wallace, A. R. I889. Darwinism. London.

WALTER, H. E. I9I3-I938. Genetics. New York.

WATKINS, A. E. I935. Heredity and Evolution. London.

Weismann, A. I882. Studies in the Theory of Descent. London.

weismann, A. I891. Essays upon Heredity. Oxford.

weismann, A. I 893. The Germplasm: a Theory of Heredity. London.

WEISMann, A. 1904. The Evolntion Theory. London.

WHEELER, W. F. 1936. Inheritance and Evolution. London.

wheldale, M. I916. The Anthocyanin Pigments in Plants. Cambridge.

whetham, w. C. D., and whetham, C. D. I912. Heredity and Society. London.

WHITE, E. G. I940. Principles of Genetics. London.

white, M. J. D. I937, 1942. The Chromosomes. London.

white, M. J. D. I945. Animal Cytology and Evolution. Cambridge.

willis, J. C. 1922. Age and Area. Cambridge.

WILson, E. B. I 898, 1906, 1925. The Cell in Development and Heredity. New York. Winters, L. M. I925. Animal Breeding. New York.

WRIEDT, C. I930. Heredity in Livestock. London.

zIRKLe, C. 1935. The Beginnings of Plant Hybridization. Philadelphia. 


\section{INDEX}

(excluding the Appendices: pp. 375 sqq. and all References at ends of Chapters)

Abutilon, 208, $2 \mathrm{II}, 2 \mathrm{I} 7$

acentrics, IOO, I 3 I

Acetabularia, I7, I68, I90, 228

Acridium, 335

Adalia, 295

adaption, 273, 322, 327

fermentative, I 8 I

additive effects, 79,80, I 58

adjustment, levels of, 227

Aesculus, 138

agouti, I I 8, 160

Àkerman, 19

albinism, I 57, I69

alcaptonuria, I 62

algae, I 7, 29, 44

allelomorph, 40, 48, I06, I52

multiple, II 4 sqq.

allergy, 349

Allium, 30, I 52

Allonyces, 240

allopolyploid(y), I22, I 34, 3 I0, 3 I 8

ambilinearity, I 85

amorph, I 52

anaemia, I94, 352

anaphase, 25, I3 I

Andersson-Kottö, I86, I90, 327

anthocyanin, I63

anthropology, 354 sqq.

antibody, I 54, 208

antigen, I54, 165, 21 3, 227

antimorph, I 52

Antirrhinum, 84 sqq., I 10, I58, 259, 308, 327

aphides, $263,264,310$

apomixis, $263 s q q ., 270,309,318,344$

apospory, $20 \mathrm{I}$

Apotettix, 335

apple, 96, 126, 218

armadillo, I6

Artemia, 100, 265

aryan language, 360

Ascaris, 97, I97

Aspergillus, 343

Astbury, 146

asynapsis, I Io, 288, 289

attraction, 30, 33

autopolyploidy, 3 II

autosexing, so autosome, 48,347

autotetraploid, I 35

back-cross, 40

bacteria, I 46, 275

bacteriophage, 208 sqq., 275

balance, 96, I04, I23 sqq., I 28, 275

change of, I4I

in hybrids, $229 \mathrm{sqq}$.

internal, 305

loss of, 293,305

of polygenic system, 291, 292

relational, 293, 306

Barber, 198

Barigozzi, 265

barley, 240, 24I

bars to crossing, 307,308

basic number, 126

Bateson, 38, 4I, I09, III, I55, I 82

Baur, I I I, 2 I I

Bawden, 209

Beadle, I 62, 289

Belar, 197

Belling, I 34

beetles, 344

Billingham, 216

biparental progenies, 89

birds, 52, 227, 253

bivalent, 30

Blair, 2 I 7

blastula, 197

blending inheritance, 273

blood cells, I 94

blood group, I 53, 349, 362

Bombyx, II 3

Bonnier, 365

Bounure, 197

Boveri, I 8, 26, 35, 197

Boyd, 349

Brachet, I47

breakage of chromosomes, 100, 33I

breeder, 94

breeding systems, 237, 239 sqq., 269, 354

Bridges, 43

Bumpus, 282

Burgeff, 327, 328

Campanula, 104, 126, 134, 262, 316 sqq. 
cancer (tumour), 147, 210 sqq., 350

Capsella, 252

carcinogen, 213

Caspersson, 147, 329

caste, 355 sqq.

cat, 49,346

cell, I6 sqq.

competition, 198

lineage, 2 I2 sqq.

see also gradient and pollen

centromere, 25 sqq., 44 sqq., I00 sqq., 330

characters, 36

capital and subordinate, 300

chemical mutation, $v$. mutation

chemical tests, I45, I 59

cherry, $v$. Prunus

chiasma, 30 sqq., 44 sqq., I2 I

frequency, $137,234,288$

chimaera, I I I

Chlamydomonas, 44

chloroplast, 44, I69 sqq.

chromatid, $25,30,45$

chromomere, 27, 33, II9, I47, 329

chromosome

breakage, 100

chemistry, I45

coiling, 30

homology, 33, 102, I35

mitosis, 23

numbers, 324

orientation, 123

pairing, $27,30,110,135$

supernumerary, I47, I 50, 329 theory, 45

Chuksanova, I23, I24

Cimex, 150

Claude, 207

cleavage, 197

cleistogamy, 24I

cline, $35 \mathrm{I}, 364$

clone, 16

Cockayne, 347

colchicine, 95

colonization, 140

colour-blindness, 49

competition

cell, 198

in sexual reproduction, 268

plasmagene, I79

pollen-tube, 253

precursor, I6s

co-operation

of genes, 158

of nuclei, 199

of viruses, 209
Coprinus, 248

corolla length, 70, 7 I

correlation

coefficient, 61,62

fraternal, 90

parent-offspring, 90

correlated response, 298 sqq.

covariance, 60, 78, 82 sqq.

Crane, III

Crataegomespilus, 228

Crepis, IO5, 123 sqq., 324

Crocus, 26, 324

cross-breeding, $v$. outbreeding

crossing-over, 30 sqq., 45 sqq., I00 sqq., $107 \mathrm{sqq}$., $12 \mathrm{I}$

reciprocal, 52,132

suppression, 132

Culex, 346

Cytisus, 228

cytoplasm, I7, I49, I68, I89, 2 I6

Dahlberg, 347

Dahlia, 164

Darwin, 242, 248, 273, 300, 305, 328, 360,370

Datura, 96, 104, I26 sqq., 3 15, 316

dauermodification, 183

deficiency, I03, I 52

Delbrück, 274

delinquency, 349

development, I6, 37, I12, 127, I 57 sqq., I68 sqq., I89 sqq.

De Vries, 62, 263

diakinesis, 30

dicentric, 132

Diehl, 349

differential segment, 52, 133,346

differentiation, I6, I50, I89

diminution, 197

dioecy, 240, 243, 252, 258

diploid, 24, 29

diplotene, 30

Diptera, 290

disease resistance, $210,349,353,358$

Dobzhansky, 292, 312, 31 3, 337

dominance, 37, 80 sqq., I09, I I 4 sqq., I 56

Drosophila

abdominal hairs (chaetae), 73 sqq., 298

Bar gene, 107 sq9., I 52

chromosome assays, 76

chromosomes, 26

crossing over, 46,290

cubitus interruptus gene, I60

cytoplasmic heredity, 2 I 5

development, 190, 202 
Drosophila-continued

discriminative mating, 253

eycless gene, I 59, 275

fertility, I05, 126

genes, I06, II9

gynandromorph, I I 2

intersexes, 258

linkage, 42, 47

meiosis, 46

Minute genes, 107

mutation, I52, I55, 327

Notch gene, 107

polytene, 27, I06, II9

scute gene, I06, I 52, 33 I

scutellar bristles, 285, 297

sex chromosomes, 49, I 5 I, 330

sex determination, 23 I, 34I

sperm, ros

triploid, 96, I2 I

wild-type, 43, I 52, I 59, I6 I

Drosophila simulans, I3 I, 253, 323

azteca, 337, 338

miranda, 3 I 3 sqq. 320

persimilis, 232, 3 I 3 sqq., 320, 338

pseudoobscura, 230, 292, 312 sqq., 320, 335 sqq.

subobscura, I93, 272

duplication, IO3

East, 65, 70, 7x, 245

echinoderm, I8, I68, I9I

Echinus, 18

egg, 24, 28, 96 sqq., I I 3

embryology

animal, I I3, I I6, 197

plant, I95, 200 sqq.

environment, I 5, 64, 359

and selection, 294

enzyme, 179

Ephestia, 193

Ephrussi, I66, 3 I I

Epilachna, 69

Epilobium, 175, 228

epistasy, I 57, I63

equational separation, 31,52

euchromatin, 32, I46, 329

evolution, 273, 328, 344

expressivity, I 59

factor, 40

effective, 92

Fankhauser, 99

fatuoid, $v$. oats

fern, I 86, I 89 fertility, 95 sqq., I2 I, I34, I93, 283

allelomorph, 259

fcrtilization, 24, 28, 97 sqq.

Festuca, 234

Filzer, 245

finches, 305

fish, 50, 253, 297

Fisher, I I 8, I6I, 274, 333, 336

fitness, 276, 284 sqq.

in man, 353 sqq.

Flemming, 25

flexibility, 284 sqq., 318

fowl, 49, I57, 2 I 3

Fragaria, 168

fragment chromosomes, I00, 197

fragmentation, $32 \mathrm{I}, 323$

frequency distribution, 56

Fritillaria, 30, 104, 200, 289

Funaria, I73

fungi, 29, 162

fusion, 322

Galeopsis, 293, 294, 297

Galton, 60, 61, 93, 282

gamete, 28, I25, I29

gametic differentiation, 239

gametophyte, 29

Gammarus, 166

Garrod, 347

gene, 42,327 sqq. action, time of, I9I balance, Ios

complementary, 156 dosage, I05, I 52

duplicate, I 56 expression, I 59

inert, $15 \mathrm{I}$ interaction, I 54 sqq.

lethal, I06, I I6, I 57, 202

major, I 52

reproduction, 146

switch, 258, 259, 34I

generations alternation of, 189

genetic system, 237

change of, 296

genotype, I5

geographical variation, 364

germ cell, $v$. gamete, pollen, egg

germ line, I97

Goldschmidt, II 3, I66, I84

Gordon, I60

gradient

cell, I94 sqq.

population, $v$. cline 
graft, 2 I 3, 21 7,227

graft-liybrid, III, 227, 228

grasshoppers, 322

Grüncberg, II 6, 202

Gudjonsson, 266, 267

guinea pig, 216

gymnosperm, $20 \mathrm{I}$

gynandromorph, I I 2

\section{Habrobracon, II 3}

Hadorn, 202

haemophilia, 49

Haldane, 19, 229

Hämmerling, I7, 190

haploid, I8, 24, 98

haploid generation, Io5

Harrison, 73

Hartung, 269

Hemiptera, 52

heredity, I5, I 49

ambilinear, 183

matrilincar, I 70

herpes, 217

heterocaryon, $163,342,343$

heterochromatin, $32,47,146,161$, I95 sqq., 329

heterosis, 237

heterostyly, 248 sqq.

heterothally, 240, 247, 248

heterozygote, $38,102,110,276$

hexaploid, 140

Hieracium, 201

Hildebrand, 248, 255

hinduism, 356

Hodson, 365

Hoffmann, I 84

Hogben, 347

Holmes, 2 IO

homology, $v$. chromosome

homostyle, 259

homozygote, $38,102,276$

Hordeum, I7 I

host-parasite relation, 274

Huskins, 339

Hyacinthus, 123, 127

hybrid, I 8

incapacity, 307,308

interchange, 133

mendelian, 36

numerical, 127

specics, I68, I9I

structural, 128

truc-breeding, 260

twin, 26I hybridity optimum, 237, 259, 269

equilibrium, 24 I

hypergamy, 357

Hypericum, I30, 317

hypomorph, I 52

\section{Imai, I7I}

inbreeding, $275,282,283 \mathrm{sqq}, 297,318$ and isolation, 309

depression, 235, 292

in man, $353 \mathrm{sqq}$.

mechanisms, 24I

incest, $252,253,355$

incompatibility allclomorphs, 332, 334

sclf, I $64,174,243$ sqq., 255 sqq, .357

individuality, 16,34

inertia, 297, 300

inertness, $\nu$. gene

infection, $207 \mathrm{sqq}$.

inheritance, $v$. heredity

insects, 227,308

pollination, 242

interchange, 102, 128 sqq., 3 II, 3 I 5, 3 I 8 , 323,335

interference, 45

intergenic change, 106

intersexes, 258

inversion, 102, 131,312 sqq., 318, 323, 331,335

isochromosome, I03, 330

isolating mechanisms, 307

isolation, $305 \mathrm{sqq}$., 356

Janssens, 34

Johannsen, I 5, 42, 62 sqq., I71, 274, 373

Johansson, 284

Johnson, 2 I 8

Jollos, 183

Jones, D. F., 339, 340

Jones, Sir William, 360

Jorgensen, I I I

Kallmann, 350

Karpechenko, 135

Koller, IOI, 215,348

Kühn, I93

La Cour, I95, I96, 32 I

Lamarckian

effects, I 82, 2 I I

inheritance, 274

language, 360 
Lathyrus, 158,218

Lavatera, 2 I I

Lawrence, I64, 254

Lebiste's, 50, 336, 340, 34I, 346

Lens, I68

Lepidoptera, 52

lethal, $\nu$. gene

Levit, 366

Lewis, 248, 332

L'Héritier, 2 Is

life cycle, 24, 29, I89 squ.

Lilium, I39, 288

Limnea, I92

Lindegren, I 80

linear order, 43

linkage, 4I, 68, 85 sqq., I 30, 299

and variability, 287 sqq.

linkage map, I Io, I I4, I 34

Linum, I 74

localization, 30, 289, 335

Lolium, 234

Lotis, 3 I I

Luria, 210, 275

Lycopersicum, 95, I 35, 242, 255, 293

Lymantria, 166, 258

Lythrum, 2 so sqq.

McClintock, 330

Macklin, 350

McLennan, 354

Magnoliales, 324

Maheshwari, 200

maize $v$. Zea Mays

male-sterility, $v$. sterility

mammals, 49, 227, 253

man, 49, sI sqq., 9I, I 53, 253, 282, 346 blood, 194

map, linkage, 45, 47

Marchantia, 327

marriage, 347 sqq.

mating

continuum, 302 sqq.

discrimination, 252 sqq., 298

legitimate and illegitimate, 249

premature, 260

random, 277, 282

mean, $58,78,8 \mathrm{I}$

Mecostethus, 289

Medawar, 216

medical genetics, 352

meiosis, 24, 28, 53, 97, I2 I, I48, 290 cause of, 196

in polyploids, 122,140

Melandrium, 52, 204, 256

Mendel, 36, 55, 93, 262, 274, 369 mendelian experiment, $36,55,132,150$, 346

inheritance, $55,66,68$

method, 36 sqq., 66, 68

Menzies-Kitchen, 284

merogon, I8, I68

metaphase, 23, 3I

metastasis, $2 \mathrm{I} 2$

microsome, 207

mid-parent, $8 \mathrm{I}$

millet, $v$. Sorghum

misdivision, I03, 104

mitochondria, 207

mitosis, 23 sqq., I 45, 194

Moewus, 44

monoecy, 242

monosomic, IOS

Morgan, 42, I I2, I 97

Morris, 219

mosquitoes, 339

mosses, 189

See also Funaria

mother cell, 29, 45

mouse, II 6, II $8,160,2 I_{3}$

Mucor, 240

Muller, 45, II9, I52, 2 I0, 33 I

mustard gas, 100

mutafacience, I 73, 2 I I

mutant, 95, 327 in Oenothera, 263

mutation, I06, I I 4, I 52, 272

chemical, I 84,2 I 2

lethal, 272

plastid, I 7 I sqq.

rate, ISS

somatic, IIO, 214,227

theory of evolution, 263

virus, 2 IO, 219

Nabours, 335

Narcissus, 251

natural selection, $273,275,282,305,306$ in $\operatorname{man}, 359$

neomorph, I52

Neurospora, I62, 342

Nicandra, 104

Nicotiana, 70, 71, 168, 174, 245, 256

Nilsson-Ehle, 62, 64, 65

Nishiyama, 338

non-disjunction, 128

normal curve, 57,58

nuclear membrane, 203, 22I

nucleic acid, I45, I55, 194, 207, 268

nucleoli, 23 sqq., 32, I 46, 2 I I, 330

nucleotide, 148 
nuclcus, I7, 28, I 45 sqq., I 82

nullisomic, IO5

nutrition, I60 sqq.

oats, I6, 64, I 58

fatuoid, 338, 339

Oenothera, 30, 106, 130, 170, 246, 290,

- 309, 316, 332, 34I complexes, 261 sqq.

embryo sac, 201

orientation, 3I, I23

Orthoptera, 52, 290

osmosis (social), 358

outbreeding, 282 sqq., 297

bias, 247

devices, 242

restriction of, $305 \mathrm{sqq}$. species, 320

Oxalis, $25 \mathrm{I}$

pachytene, 29, I22, I2 8

Paconia, 32 I, 322

Painter, 27, 35

pairing segment, 52, I 33, 346

pangenesis, 274

Paramecium, I75 sqq., 19I, 203

parameters, 56

Paratettix, 335, 336

Paris, 290

parthenogensis, 98

cyclical, 264

Pasteur, 2 IO

Pätau, I 3 I

paternity, $35 \mathrm{I}$

pattern, I 57

pea, v. Lathyrus, Pisum

Pearson, 6I, 62

Pediculopsis, 24I, 260, 297

Pelargonium, I I I

penetrance, I60

Penicillium, 343

pepsin, I 45

Peto, 234

Petunia, I74, 253, 255, 305, 332

Pharbitis, I6 4

Phaseolus, 62, 72, 184, 218,239

phenocopy, I 84

phenotype, I5, 156, 204

optimum, 283

philology, 360

phonetics, 360

Pickford, 49

pigmentation, I 55 sq9., 163, I93, 2 I6

pigs, 237,283

pin and thrum, 249 piracy, 222

Pisum, 36, 55, I 82, 216, 240

plasmagenc, I73 sqq., 203, 216, 344

plastid, 149, I69

plastogene, I69

plciotropy, i 5

Pnentrococcus, 2 Io

Poa, 268, 269

point mutation, I Io

polar body, 97

pollen, 96, I05, I 23 sqq., I 39

differentiation, i9s sqq.

gene action in, I9I mechanism, 24 I

polygamy, 358

polygenes, 66,78 , I 50, 329, 330

polygenic system, 66 sqq., 279

polymeric genes, 68

polymerization, I 45

polymitosis, 198, 2 I 2

polymorphism, 335 sqq.

polypeptide, I 45

polyploidy, 95, I 2 I, I 34, 32 I

in blood, 194

secondary, 98,324

polysomy, 95

polytene, 26,47, I06, I 3 I

chemistry, I 45

Pomoideae, 324

Pontecorvo, 343

populations, I 53, 35I, 364

randomly breeding, 89,90

position effect, IO6, I I 8 , I 52, I 55, 330

potato, III, 2 I 8

poultry, $v$. fowl

precursor, I62 sqq., I 77 cells, 194

Preer, I77, 203

Prell, 245

presence and absence, I06

primrose, $\nu$. Primula

Primula, I 4 4, I35, 169, 231, 249 sq9., 3 IO

prophase, 23

protandry, 242, 258

protein, I45 sqq., 207 sqq., 219, 268

protogyny, 242

protozoa, 29

provirus, 2 I 4

Prunus, 243, 247

pure line, 62

Pyrethrum, 200

Pyrus v. apple

quadrivalent, I 26, I 37

quantitative effects, I 5 I 


\section{INDEX}

rabics, 210

Race, R. R., 333

race theory, 353

Raphano-brassica, I35, 23 I, 232

rat, 202

ratio, mendelian, 37 , I 56

recessive characters, 347

reciprocal crosses, $48,125,126$

recombination, 33, 4I sqq., II 7, I32, 237, 304 sqq.

and correlated response, 297

and natural selection, 290

reduction, 33

reductional separation, $3 \mathrm{I}, 52$

regeneration, $\mathrm{I} 73$, I 89

regression coefficient, $6 \mathrm{I}$

Rendel, 272

Renner, I70, 20I, 260

effect, 201, 268, 272, 3 II, 342

reproduction, $v$. sexual

Rhesus blood-groups, 333

$$
\text { v. self-propagation }
$$

Rhoeo, 130, 3 I 7

Rick, 99, 242

ring-formation, I 29

breakdown of, 263

Rischkov, 208

Risley, 354

rodents, I 57

rogue, I 82

See also mutant

root-cutting, I I I

Rosanoff, 349

Rous, 2 I3

Rudbeckia, I 59

rye, $237,260,297$

\section{Saccliaromyces, I79}

Salaman, 2 I 8

salivary gland, $v$. polytene

Sax, 72, 206

scales, 58,78 sqq.

tests of, 79

Schnarf, $20 \mathrm{I}$

Schrödinger, $37 \mathrm{I}$

Sciara, 193, 252

Scilla, I97

Scolopendrium, I86, I90, 327

sea urchin, $v$. echinoderm

Secale, $v$. rye

segregation, $38,45,68, I 27,237$

somatic, 187

Seiler, 264

selection, 272 sqq., 302

correlated response to, 298 selection-continued

discriminative action of, 294

self-fertilization, 36

diploid, 240

haploid, 240

self-propagation, 28, I49, 203

serology, $v$. blood group

sex-chromosomes (X and $\mathrm{Y}$ ), 46 sqq.

sex determination, 46 sqq., I66, I93, 204, 256, 339 sqq., 346

sexes, homogametic and heterogametic, 49,229

sex-linkage, 48 sqq.

partial, so, 346

sex-ratio, 275,337

super-gene, 336,337

sexual differentiation, $v$. dioecy

sexual reproduction, I6, 24, 29, 99

breakdown of, 268

shibboleth, 360

Sismanidis, 285, 297

Smith, K. M., 209

snail, I9I

Solanum, II I, 228

Sonneborn, I75

Sorghum, I04, I5I, I95

sparrows, 282

species crosses, I 35, I9I

speech, 360

speltoid, $v$. wheat

sperm, 24, 28, 5I, 105, 216

spermathecae, 298

spider, 52

Spiegelman, I79

spindle, 23

spiralization, I 46

spore, 29, 32, 44

sporophyte, 29

sport, $v$. mutation

standard deviation, 59

sterility, $v$. fertility

genotypic, $23 \mathrm{I}$

hybrid, 235

male, I74, I90

segregational, 232

Stern, C., I6I

Stern, F. C., 322

Stizolobium, I34

strawberry, 2 I 8

Streptocarpus, 228, 253

structural change, Ioo sqq.

structural hybrid, I22

Sturtevant, I32, 3 I 3

subsexual reproduction, 266

sugar beet, 237 


\section{INDEX}

super-gene, 46, II 8, I33, 312, 335 sqq. supernumerary, $v$. chromosome suppressiveness, I79

suppressor, 158

syndrome, 348

syphilis, 358

systematist, 323

tabu, 253

Talaeporia, 264

Taraxacum (dandelion), 265 sqq., 344

telocentric, I03, 104

telophase, 25,146

temperature, effect of, 95, 100, I6I, I78, 195, 198, 216

template theory, I46, 2 I I

tetrad, 32

tetraploid, 95, I22 sqq., I 34 sqq.

Thomas, 2 I2

Thyanta, 323

Timoféeff-Ressovsky, 159, 295

tissue specificity, 202

tomato, $v$. Lycopersicum

Torula, 2 I2

Tradescantia, 289

transformations, 80

translocation, I02, I06, I 37

transplantation, I66, I9I, 202, 2 I 2

See also graft

Trichoniscus, 265

Trifolium, 246

triploid, 96, 265

trisomic, 96 in Oenothera, 263

Triticum, $v$. wheat

Triton, 30, 99, I68, 290

Triturus, 99

trivalent, I2 I

tuberculosis, 349

Tulipa, I00, I25 sqq., I 39, 208, 3 I I

tumour, $v$. cancer

twins, 269, 270, 349

unisexual brood, 252

unity of heredity, I 30 sqq.

See also gene

univalent, I2 I, 137

unreduced germ cell, 34

Upcott, I25, I 37 sqq.

Uvularia, I98

vaccinia, 207

variability, 276

and mutation, 295, 296

fixed, 278

flow of, 277, 285 variability-continued

free, 276

frozen, 284

potential 276, 279, 280, 287, 296

rescrvoir of, 296

states of, 28 I

variance, $59,78,82$ sqq.

variation, $34,272,276$

continuous, 56, 6I, 349

cryptic, 323

discontinuous, 55, 6I

in $\operatorname{man}, 364$

non-heritable, 65

spectrum of, 65,66

variegation, I 70, I 86,2 I I

vector, $\nu$. virus

vegetative propagation, I6

Verbascum, 328

Veronica, 245

versatile reproduction, 269

Verschuer, 349

Vicia, 168, 288

Vinca, 209

Viola, 24 I

virus, I46, I49, 207 vector, 208 sqq.

vitamin, I60 sqq.

Wallace, 273

wart, 2 I 4

Weismann, 29, 35

Wettstein, I 73

wheat, 64, I $58,259,338$ speltoid, 338

Whiting, II 3

wild-type, $v$. Drosophila

Wilson, I97

Winge, 205, 256, 340

Winkler, I I I

woman, 347 sqq.

Wright, 203

$\mathrm{X}$ chromosome, $46,258,330,34 \mathrm{I}, 346$

Xiphophorus, 51

$X$-rays, I00, I46, I 52, 2I3, 327, 33I, 332

Y chromosome, 46, I 47, I5 I, 329, 330, $340,34 \mathrm{I}, 346$

yeast, 179,212

Zea Mays, 30, 45, I26, I47, 235, 242, 329, $330,339,340$

asynaptic, $2 \$ 8,289$

embryo sac, 200

mutation, 155

pollen, I9I, I98

zygote, 28 , I 45 




